GÖTTINGER SCHRIFTEN ZUR INTERNETFORSCHUNG

Hg.: S. Hagenhoff, D. Hogrefe, E. Mittler, M. Schumann, G. Spindler, V. Wittke

\title{
Internetökonomie der Medienbranche
}

Svenja Hagenhoff Herausgeber 

This work is licensed under the Creative Commons License 2.0 "by-nd", allowing you to download, distribute and print the document in a few copies for private or educational use, given that the document stays unchanged and the creator is mentioned. You are not allowed to sell copies of the free version. 
erschienen als Band 1 in der Reihe „Göttinger Schriften zur Internetforschung“ im Universitätsverlag Göttingen 2006 


\section{Svenja Hagenhoff (Hg.) \\ Internetökonomie der \\ Medienbranche}

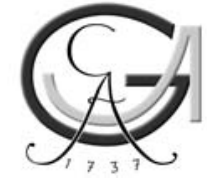

Universitätsverlag Göttingen 2006 


\section{Bibliographische Information der Deutschen Nationalbibliothek}

Die Deutsche Nationalbibliothek verzeichnet diese Publikation in der Deutschen Nationalbibliographie; detaillierte bibliographische Daten sind im Internet über $<$ http://dnb.ddb.de $>$ abrufbar.

\section{Herausgeber des Bandes}

Svenja Hagenhoff, Institut für Wirtschaftsinformatik, Abteilung Wirtschaftsinformatik 2, Georg-August-Universität Göttingen, Platz der Göttinger Sieben 5, 37073 Göttingen, shagenh@uni-goettingen.de.

\section{Reihe}

„Internetökonomie der Medienbranche“ ist der erste Band der Reihe „Göttinger Schriften zur Internetforschung“" in der qualitätsgeprüften Sparte des

Universitätsverlags Göttingen.

Herausgeber der Reihe: Svenja Hagenhoff, Dieter Hogrefe, Elmar Mittler, Matthias Schumann, Gerald Spindler und Volker Wittke.

\section{Mitwirkung}

Entstanden im Rahmen des Forschungsprojekts „Mediaconomy“ der Georg-AugustUniversität Göttingen. Mediaconomy wird im Rahmen des Schwerpunktprogramms „Internetökonomie“ durch das BMBF gefördert.

Sprecher : Prof. Dr. Matthias Schumann.

\section{Beteiligte}

Siehe Autorenliste

GEFÖRDERT VOM

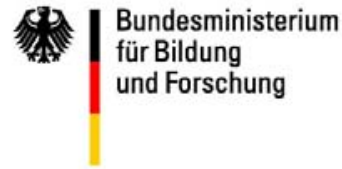

(C) 2006 Universitätsverlag Göttingen

Umschlaggestaltung: Martin Kaspar und Margo Bargheer

http:/ / univerlag.uni-goettingen.de

ISSN 1863-0944

ISBN-10 3-938616-29-6

ISBN-13 978-3-938616-29-1 


\section{Vorwort}

Produkte bzw. Leistungen der Medienbranche lassen sich vollständig digitalisieren und in digitalisierter Form distribuieren. Hierdurch ist die Medienbranche wie kaum eine andere von Innovationen der Informations- und Kommunikationstechnik tangiert. Folgende exemplarische Punkte verdeutlichen das Veränderungspotenzial holzschnittartig:

Mit neuen Distributionswegen über z. B. Peer-to-Peer-Netzwerke werden ganze Wertschöpfungsstufen (teilweise illegal) umgangen. Dies ist nicht nur im Bereich der Musikindustrie (Napster) zu beobachten, sondern auch im Falle wissenschaftlicher Publikationen (z. B. die Ginsparg-Plattform als Drehscheibe für Physik-Aufsätze). Mit einer weltweiten dezentralen virtuellen Bibliothek für alle wissenschaftlichen Veröffentlichungen versucht die Open Archive Initiative die Quasi-Monopolstellung der wissenschaftlichen Verlage aufzubrechen.

Unternehmen aus der Telekommunikations- und Informationstechnologie streben mit neuen Angeboten in die Kernmärkte der Medienindustrie. Es ist dabei das erklärte strategische Ziel großer Anbieter, möglichst alle Stufen der medialen Wertschöpfungskette mit eigenen Ressourcen oder mindestens über stabile Kooperationen (sog. Business Webs) abzudecken.

Die Anbieter und Nutzer von Medienprodukten sehen sich einer wachsenden Anzahl von Endgeräten und Trägermedien (z. B. E-Book, Digitales Fernsehen) konfrontiert, die spezifische Übertragungs- und Speichermedien erfordern. Für Me- 
dienunternehmen gilt es, relevante Endgeräte bzw. Medien und Vertriebsformen für entsprechende Themenschwerpunkte und Zielgruppen zu identifizieren und die Frage zu beantworten, welche Optionen die neuen Technologien im Hinblick auf eine Veränderung der bestehenden Produktpalette bieten. Die potenziellen Nutzer dieser Optionen müssen vom Nutzen und der Zweckmäßigkeit der neuen Angebote überzeugt werden.

Neue Technologien verändern Prozesse der Leistungserstellung, die bisher nur mit großem Aufwand durchführbar gewesen sind. Beispielhaft zu nennen ist das Printing on Demand, wodurch der originäre Druckvorgang digital unterstützt ablaufen kann. Ziel ist es, von einem traditionellen Auflagendruck weg hin zu einer bedarfsgesteuerten Produktion zu kommen und darüber hinaus mit individualisierten Produkten (z. B. individuell zusammengestellter Reiseführer) Kundenbedürfnisse besser zu befriedigen. Durch besondere Formen der Datenhaltung wird es möglich, Inhalte auf jedem vom Kunden gewünschten Trägermedium zu distribuieren.

Diese kurzen Ausführungen zeigen, dass die Medienbranche als Erklärungsgegenstand für die Phänomene der Internetökonomie hervorragend geeignet ist. Das vom BMBF im Rahmen des Schwerpunktes Internetökonomie geförderte Göttinger Forschungsvorhaben Mediaconomy (www.mediaconomy.de) widmet sich der Themenstellung Internetökonomie der Medienbranche aus der Sicht der fünf verschiedenen Disziplinen Bibliothekswissenschaft, Informatik, Jura, Soziologie und Wirtschaftsinformatik. In insgesamt neun Teilprojekten in den Analyseschwerpunkten Mobiles Internet einerseits und Wissenschaftskommunikation andererseits gilt es, Erklärungsmodelle für die Phänomene der Internetökonomie zu erarbeiten sowie Gestaltungsempfehlungen aussprechen zu können.

Das vorliegende Buch ist der erste Band der Reihe Göttinger Schriften zur Internetforschung, welche vom Mediaconomy-Team in der qualitätsgeprüften Sparte des Universitätsverlages der Niedersächsischen Staats- und Universitätsbibliothek herausgegeben wird. Der vorliegende Band präsentiert erste Ergebnisse der interdisziplinären Forschung. Zusätzlich zu den Autoren des Mediaconomy-Teams konnten mit Thomas Hess und Barbara Rauscher zwei Kollegen des intermediaProjektes der Ludwig-Maximilians-Universität München gewonnen werden. Ein weiterer externer Autor ist Hans Roosendaal von der University of Twente in the Netherlands.

Der erste Teil des Bandes präsentiert Grundlagen. Thomas Hess und Barbara Rauscher fokussieren in ihrem Überblicksartikel die Themenfelder Mobile Dienste 
und Wissenschaftskommunikation und zeigen Auswirkungen von Internettechnologien auf die Medienbranche auf.

Lutz Seidenfaden ergänzt die Grundlagen mit einem Artikel über die Distribution von digitalen Gütern. Er zeigt die Charakteristika dieser Güter auf und diskutiert verschiedene Distributionsmodelle.

Der zweite Teil des Buches ist dem Themenfeld Mobiles Internet gewidmet. Christian Kaspar zeigt grundsätzliche Möglichkeiten der Individualisierung von Medienprodukten auf mit dem Ziel, dem Nachfrager die bedürfnisexakte individuelle Befriedigung der Kundenwünsche zu jeder Zeit an jedem Ort zu ermöglichen.

Dieter Hogrefe, Andre Riedel und Marco Zibull beschäftigen sich mit der Ökonomie des drahtlosen, breitbandigen Internetzugangs zu digitalen Medienprodukten. Sie stellen in ihrem Beitrag den State of the Art der Technologie dar.

Heidemarie Hanekop und Volker Wittke ergänzen das Kapitel um eine soziologische Perspektive. Sie zeigen die Entwicklung neuer Formen der Kommunikation und Mediennutzung auf.

Matthias Mönch liefert abschließend Überlegungen aus juristischer Sicht. Er diskutiert datenschutzrechtliche Aspekte personalisierter mobiler Dienste.

Der dritte Teil des Buches führt in das Themenfeld der Wissenschaftskommunikation ein.

Hans Roosendaal gibt mit seinem Beitrag Scientific Communication: The Split between Availability and Selection einen Überblick über dieses noch relativ junge Forschungsgebiet und den Stand der Diskussion.

Margo Bargheer diskutiert anschließend, ob Open Acess-Modelle und Universitätsverlage Wege aus der so genannten Publication Crisis weisen können.

Heidemarie Hanekop und Volker Wittke übernehmen wieder den soziologischen Part und zeigen auf, welche Veränderungen das Internet in der Wissenschaftskommunikation hervorrufen kann.

Joachim Dorschel trägt eine juristische Perspektive bei, indem er Open AcessModelle mit dem bekannten Phänomen Open Source vergleicht und Fragen zum Urheberrecht aufwirft.

Der vierte Teil des Bandes widmet sich abschließend empirischen Befunden. Carmen Lanfer und Kai Marquardsen untersuchen das Thema Internet und Be- 
schäftigung und geben eine erste Analyse quantitativer Effekte in der Medienbranche.

Björn Ortelbach hat sich das Thema Controlling der Internetökonomie zur Aufgabe gemacht und gibt in einem ersten Schritt einen Überblick zu Studien, die einen empirisch fundierten Beitrag zum Thema Controlling von Start-up-Unternehmen liefern.

Göttingen, im April 2006 


\section{Inhaltsverzeichnis}

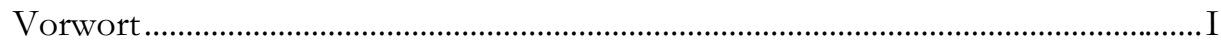

Inhaltsverzeichnis …………………………………………………………. V

Abkürzungsverzeichnis.................................................................................... VII

\section{Erster Teil: Grundlagen}

Internettechnologien in der Medienbranche: Mobile Dienste und

Wissenschaftskommunikation im Fokus .................................................................. 1

Thomas Hess / Barbara Rauscher

Absatz digitaler Produkte und Digital Rights Management: Ein Überblick 19 Lutz Seidenfaden

\section{Zweiter Teil: Mobiles Internet}

Individualisierung von Medienprodukten 51

Christian Kaspar 
Ökonomie des drahtlosen, breitbandigen Internetzugangs zu digitalen

Medienprodukten

Dieter Hogrefe / André Riedel / Marco Zibull

Die Entwicklung neuer Formen mobiler Kommunikation und

Mediennutzung

Heidemarie Hanekop / Volker Wittke

Datenschutzrechtliche Aspekte personalisierter mobiler Dienste

Matthias Mönch

\section{Dritter Teil: Wissenschaftskommunikation}

Scientific Information: The Split between Availability and Selection 161 Hans E. Roosendaal

Open Access und Universitätsverlage: Auswege aus der Publication Crisis? ... 173 Margo Bargheer

Das wissenschaftliche Journal und seine möglichen Alternativen:

Veränderungen der Wissenschaftskommunikation durch das Internet

Heidemarie Hanekop / Volker Wittke

Open Access und Urheberrecht: Open Source in neuem Gewand? Joachim Dorschel

\section{Vierter Teil: Empirische Befunde}

Internet und Beschäftigung: Quantitative Effekte in der Medienbranche 267

Carmen Lanfer / Kai Marquardsen

Controlling in Start-Up-Unternehmen: Empirische Untersuchungen zum

Verhältnis von Planung und Unternehmenserfolg

Björn Ortelbach

Autoren 345

Buch- und Reihenherausgeber 349 


\section{Abkürzungsverzeichnis}

a.a.O. am angegebenen Ort

AAA Authentication, Authorizing and Accounting

AAC Advanced Audio Coding

ABS Australian Bureau of Statistics

AES Advanced Encryption Standard

AGB Allgmeine Geschäftsbedingungen

AöR Archiv für öffentliches Recht (Zeitschrift)

AP Access Point

AVI Audio Video Interface

B2B Business-to-Business

BAG Bundesarbeitsgericht

BDSG Bundesdatenschutzgesetz

BER Bit Error Rate

$\mathrm{BGH} \quad$ Bundesgerichtshof

BGHZ Bundesgerichtshof / Zivilsachen 
BOAI Budapest Open Access Initiative

BS Basisstation

BSS Basic Service Set

BT-Drucks. Bundestags-Drucksache

BTX Bildschirmtext

BVerfGE Bundesverfassungsgericht - Entscheidungen

C2C CC Car-to-Car Communication Consortium

CA Collision Avoidance

CD Compact Disk

CERN Conseil Européen pour la Recherche Nucléaire

COS Content Owner Sponsoring

CPE Costumer Premise Equipment

CSMA Carrier Sense Multiple Access

CSS Cascading Stylesheet

CWLAN Consumer/SOHO Wireless Local Area Network

DCF Distributed Coordination Function

DINI Deutsche Initiative für Netzwerkinformation e. V.

DRM Digital Rights Management

DRMS Digital Rights Management System

DS Distribution System

DSL Digital Subscriber Line

DSSS Direct Sequence Spread Spectrum

DTAG Deutsche Telekom AG

DTP Desktop-Publishing

DVB Digital Video Broadcasting

DVB-C Digital Video Broadcasting Cable

DVB-H Digital Video Broadcasting for Handhelds

DVB-S Digital Video Broadcasting Satellite

DVB-T Digital Video Broadcasting Terrestrial 
DVD Digital Versatile Disk

EAP Extensible Authentication Protocol

E-Commerce Electronic-Commerce

EDCF Enhanced Distributed Coordination Function

EDGE Enhanced Data Rates for GSM Evolution

EGU European Geosciences Union

Einl. Einleitung

EIRP Effectiv / Equivalent Isotropic Radiated Power

EIRP Effective Isotropic Radiated Power

EMBO European Molecular Biology Organization

EMMS Electronic Media Management System

ESA Entomological Society of America

ESS Extended Service Set

EWLAN Enterprise Wireless Local Area Network

FDD Frequency Division Duplexing

FHSS Frequency Hopping Spread Spectrum

FTP File Transfer Protocol

GAP German Academic Publishers

GG Grundgesetz

$\mathrm{GHz} \quad$ Gigahertz

GPL General Public License

GPRS General Packet Radio Service

GPS Global Positioning System

GRUR Gewerblicher Rechtsschutz und Urheberrecht (Fachzeitschrift)

GSM Global System for Mobile Communication

HPI Highspeed Portable Internet

HRG Hochschulrahmengesetz

HSCSD High Speed Circuit Switched Data 
HTML Hypertext Markup Language

HTTP Hypertext Transfer Protocol

IBSS Independent Basic Service Set

ICT Information and Communication Technology

IDEA International Data Encryption Algorithm

iDTV interaktives digitales Fernsehen

IEEE Institute of Electrical and Electronics Engineers

IETF Internet Engineering Task Force

IETF Internet Engineering Task Force

IFWA Immediate Free Web Access

IP Internet Work Protocol

ISBN Internationale Standard-Buchnummer

ISI „Institute for Scientific Information“ von Thomson ISI, Dienstleister für Wissenschaftsinformation

ISM Industrial-Scientific-Medical

ISP Internet Service Provider

ITU International Telecommunication Union

IuK Informations- und Kommunikationstechnik

JDF Job Definition Format

KSK Künstlersozialkasse

KSVG Künstlersozialversicherungsgesetz

LAN Local Area Network

LBS Location Based Services

LG Landgericht

LLC Logical Link Control

MAC Media Access Control

Mbps Megabit per Second

MBWA Mobile Broadband Wireless Access

M-Commerce Mobile Commerce 
MDStV Medienstaatsvertrag

$\mathrm{MHz} \quad$ Megahertz

MMR Multimedia und Recht (Fachzeitschrift)

MMS Multimedia Message Service

MOV Apple Quicktime Video Format

MP3 Moving Picture Expert Group 1.0 Layer 3

MPEG Moving Picture Expert Group

MPG Max-Planck-Gesellschaft

MS Mobile Station

MT Mobile Terminal

NAR Nucleic Acids Research

NJW Neue Juristische Wochenschrift (Fachzeitschrift)

NJW-RR Neue Juristische Wochenschrift - Rechtsprechungsreport

NVwZ Neue Verwaltungsrecht-Zeitung (Fachzeitschrift)

NZA Neue Zeitschrift für Arbeitsrecht (Fachzeitschrift)

OA Open Access

OAI Open Archive Initiative

ODC Online Delivered Content

ODRL Open Digital Rights Language

OFDM Orthogonal Frequency Division Multiplex Modulation

OFDMA Orthogonal Frequency Division Multiple Access

OLG Oberlandesgericht

OPAC Online Public Access Catalogue

P2P Peer-to-Peer

P3P Platform for Privacy Preferences Project

PC Personal Computer

PDA Personal Digital Assistent

PDF Portable Document Format

PDU Protocol Data Unit 
PHY Physical Layer

PLoS Public Library of Science

PNAS Proceedings of the National Academy of Sciences in the USA

PWLAN Public Wireless Local Area Network

QoS Quality of Service

RADIUS Remote Authentication Dial-In User Service

RBÜ

Revidierte Berner Übereinkunft

RegTP Regulierungsbehörde für Telekommunikation und Post

REL Rights Expression Language

RFC Request for Comments

RG Reichsgericht

RGZ Reichsgericht / Zivilsachen

RSVP Resource Reservation Protocol

RTCP Real Time Control Protocol

RTP Real Time Transport Protocol

RTSP Real Time Streaming Protocol

Rz. Randziffer

S/W Schwarz/Weiß

SDU Service Data Uni

SIM Subscriber Identity Module

SMS Short Message Service

SOHO Small Office/Home Office

SPARC The Scholarly Publishing and Academic Resources Coalition

SS Subscriber Station

STM Science, Technology, Medicine

TC Traffic Category

TCP Transmission Control Protocol

TDD Time Division Duplexing

TDDSG Teledienstedatenschutzgesetz 
TDG

TDM

TIME

TK

TKG

TKIP

TOBIAS

TRIPS

TU

TV

UDP

UMTS

UrhG

URL

USASBE

VLAN

VLB

VoIP

VoWLAN

WCT

WECA

WEP

WHO

WiFi

WiMAX

WIPO

WISP
Teledienstegesetz

Time Division Multiplexing

Telekommunikation, Informationstechnologie, Medien und Entertainment

Tele-Kommunikation

Telekommunikationsgesetz

Temporal Key Integrity Protocol

Tübinger Online Bibliotheksinformations- und Ausleihsystem

Trade Related Aspects of Intellectual Property Rights

Technische Universität

Television

User Datagram Protocol

Universal Mobile Telecommunications System

Urheberrechtsgesetz

Uniform Resource Locator

United States Association for Small Business and

Entrepreneurship

Virtuelles Local Area Network

Verzeichnis lieferbarer Bücher

Voice over Internet Protocol

Voice over Wireless Local Area Network

WIPO Copyright Treaty

Wireless Ethernet Compatibility Alliance

Wired Equivalent Privacy

World Health Organisation

Wireless Fidelity

Worldwide Interoperability for Microwave Access

World Intellectual Property Organisation

Wireless Internet Service Provider 
WISU Das Wirtschaftsstudium (Fachzeitschrift)

WLAN Wireless Local Area Network

WMAN Wireless Metropolitan Area Network

WME Wireless Media Extensions

WML Wireless Markup Language

WPA Wireless Protected Access

WPPT WIPO Performances and Phonograms Treaty

WSP Web Standards Project

WWW World Wide Web

XML Extensible Markup Language

XSL Extensible Stylesheet Language

XSLT Extensible Stylesheet Language Transformation

ZR Zivilrecht 


\section{Erster Teil: \\ Grundlagen}





\section{Internettechnologien in der Medienbranche: Mobile Dienste und Wissenschaftskommunikation im Fokus}

Thomas Hess / Barbara Rauscher

\section{Auswirkungen von Internettechnologien auf die Medienbranche: ein Einstieg}

Die Medienbranche ist noch immer von technologischen Entwicklungen und daraus resultierenden Veränderungen des ökonomischen Umfelds geprägt. Die derzeit relevanteste technologische Entwicklung ist die Verbesserung von Internettechnologien hin zu zunehmender Mobilität, Ubiquität und Bandbreite von Datennetzen. Eine zunehmende Rückkanalfähigkeit von Datennetzen und Endgeräten ermöglicht eine für die Medienbranche - mit ihren überwiegend auf Broadcasting basierenden Geschäftsmodellen - ungewohnte Interaktivität und Integration der Rezipienten. Auch auf Seiten des ökonomischen Umfelds ist eine Entwicklung weg vom reinen Broadcast-Modell hin zur Anpassung an kleinere, individualisierte Zielgruppen zu verzeichnen. Hinsichtlich dieser Zielgruppen wird zudem auch deutlich, dass die Nutzerakzeptanz auch immer mehr zum kritischen 
Internettechnologien in der Medienbranche

Erfolgsfaktor für neuartige Angebote von Medienunternehmen wird. Daneben stellen erfolgreiche branchenfremde Neueintritte in klassische Medienmärkte die bisherigen Geschäftsmodelle von Medienunternehmen zunehmend in Frage.

Unter dem Einfluss dieser technologischen und ökonomischen Treiber stehen viele Medienunternehmen der Herausforderung gegenüber, sowohl ihre Geschäftsmodelle als auch ihr Selbstverständnis als „Medienunternehmen“ anpassen und neu definieren zu müssen.

Innerhalb der Medienbranche jedoch sind sowohl die technologischen Treiber als auch die branchenspezifischen Geschäftsmodelle sehr heterogen. Eine Betrachtung der Auswirkungen von Internettechnologien auf Medienunternehmen muss daher fokussiert auf relevante technologische Treiber oder einzelne betroffene Teilbranchen erfolgen. In diesem Beitrag erfolgt daher - angelehnt an die Themenschwerpunkte des Workshops „Internettechnologien in der Medienbranche ${ }^{\text {"1 } 1}$ - eine fokussierte Betrachtung der Technologie „mobile Dienste“ einerseits und der Teilbranche „Wissenschaftskommunikation“ andererseits. Mobile Dienste bieten Potentiale für neue Vertriebswege und Geschäftsmodelle für Medienunternehmen, da sowohl Übertragungswege als auch notwendige Endgeräte bereits weit verbreitet sind und weitere technische Entwicklungen eine zunehmende Multimedialität ermöglichen. Der Fokus auf die Teilbranche der Wissenschaftskommunikation ist derzeit besonders relevant, da die Geschäftsmodelle von Wissenschaftsverlagen durch Internettechnologien, die eine freie Verfügbarkeit von wissenschaftlichen Artikeln begünstigen, bedroht sind. Für Verlage stellt sich daher die Frage, ob sie sich eher defensiv verhalten sollten und wie die Musikindustrie vor allem auf rechtliche Schutzmaßnahmen setzen, oder ob sie offensiv neue Geschäftsmodelle entwickeln sollten.

Zunächst wird auf die mögliche Verwendung von mobilen Technologien für Medien-Unternehmen eingegangen. Dabei werden die wesentlichen technologischen Treiber, die aktuelle Situation mobiler Datendienste und medienrelevante Entwicklungstrends betrachtet. Anschließend wird die Medienteilbranche der Wissenschaftskommunikation mit den dort vorherrschenden technologischen Treibern, der aktuellen Branchensituation und aktuellen Entwicklungstrends genauer beleuchtet. Im Fazit werden schließlich Fragestellungen, die sich für Medienunternehmen aus den identifizierten technologischen Treibern und daraus resultierenden Entwicklungstrends ableiten lassen, zusammenfassend dargestellt.

\footnotetext{
1 Der Workshop wurde durchgeführt durch das vom Bundesministerium für Bildung und For-
} schung (BMBF) geförderte Forschungsprojekt mediaconomy (www.mediaconomy.de). 


\section{Auswirkungen mobiler Dienste auf die Medienbranche}

Das stationäre Internet wurde von Medienunternehmen erst spät als zusätzlicher Vertriebskanal erkannt und gerade in der Musikbranche wurde in den letzten Jahren deutlich, dass eine frühzeitigere Anpassung der Geschäftsmodelle sinnvoll gewesen wäre. Um derartige Fehler zukünftig zu vermeiden, sollten die Potentiale von Technologien des zunehmend breitbandigen und mobilen Internets auf ihren Nutzen für Medienunternehmen hin untersucht werden.

Die Potentiale mobiler Dienste sind konzeptionell vergleichbar mit denen des stationären Internets - digitale Inhalte können mehrfach genutzt werden und durch cross-mediale Angebote können direkte und indirekte Nutzeneffekte wie z. B. Verkaufserlöse oder eine höhere Kundenbindung entstehen. Diesen Potentialen stehen allerdings auch Risiken gegenüber. Im stationären Internet gab es bisher wenige Geschäftsmodelle, die die beschriebenen Effekte tatsächlich erreichen konnten. Zudem wird der Zugang zu den Kunden, gerade auch für das Angebot mobiler Datendienste, derzeit von Netzbetreibern dominiert. Aus diesen Gründen stellt sich für Medienunternehmen die Frage, ob Investitionen in zusätzliche mobile Vertriebswege auch tatsächlich ökonomisch sinnvoll sind.

Daher werden im Folgenden die relevanten technologischen Treiber in diesem Bereich vorgestellt, die aktuelle Situation mobiler Datendienste ausführlicher dargestellt und schließlich vertieft mit kontextsensitiven Diensten und DVB-H (Digital Video Broadcast - Handheld) zwei für die Medienbranche möglicherweise relevante Anwendungsfelder aufgegriffen.

\subsection{Technologische Treiber bei mobilen Diensten}

Technologische Treiber für mobile Dienste sind mobile Übertragungstechnologien einerseits und mobile Endgeräte andererseits (vgl. zu diesem Abschnitt Turowski / Pousttchi 2004).

Hinsichtlich der mobilen Übertragungstechnologien ist bisher der Mobilfunk dominierend. ${ }^{2}$ Der wichtigste Mobilfunkstandard ist derzeit noch GSM (Global System for Mobile Communications), der auch eine konzeptionelle und technische Datenbasis für weitere Standards wie GPRS (General Packet Radio Service) und UMTS (Universal Mobile Telecommunications System) bildet. Dieser Standard verwendet digitale Übertragungstechnologien und ist neben der Sprachübertragung prinzipiell auch für die Übertragung von Daten geeignet, aufgrund der niedrigen Bandbreite allerdings nur bedingt tatsächlich dafür einsetzbar. Verbessert wird die Fähigkeit zur Datenübertragung durch die Paketvermittlung von Daten in den

2 Zunehmend wichtiger werden aber auch drahtlose Technologien wie bspw. WLAN und/oder Bluetooth. 
weiterentwickelten Standards GPRS und EDGE (Enhanced Data Rates for Global Evolution) bzw. durch Kanalbündelung in HSCSD (High Speed Circuit Switched Data), der Softwareerweiterung von GSM. Neuere Netze unterstützen u. a. höhere Übertragungsraten und Multimedia-Anwendungen. In Deutschland kommt dabei der Standard UMTS zum Einsatz, in Japan und USA werden ähnliche technologische Standards verwendet. Die für Deutschland relevanten Übertragungstechnologien mit ihren zentralen Charakteristika sind in Abbildung $1 \mathrm{im}$ Überblick dargestellt.

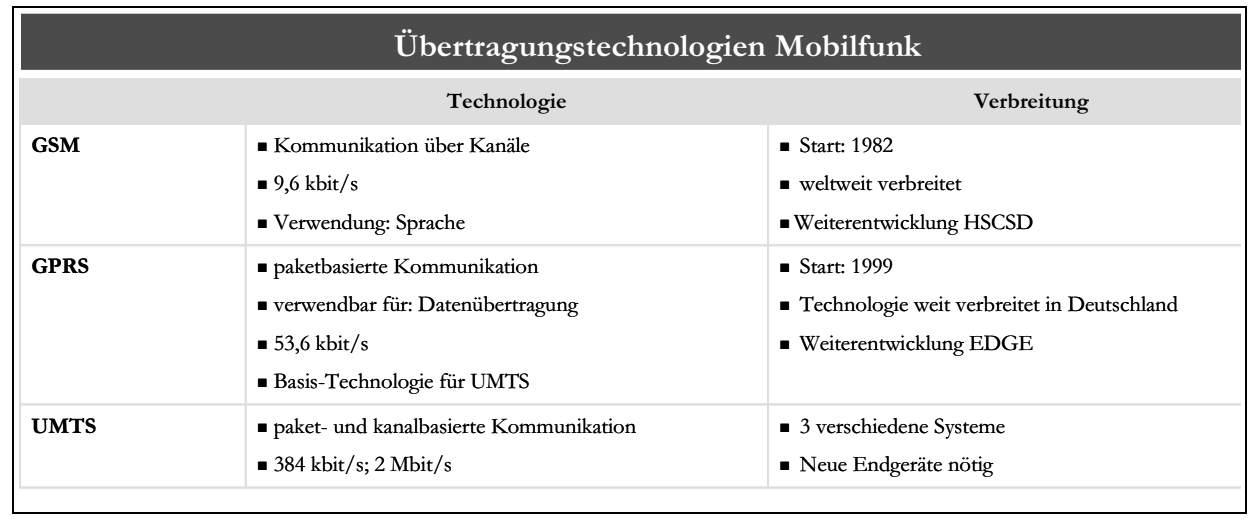

Abbildung 1: Übertragungstechnologien im Mobilfunk

Neben den Übertragungstechnologien sind aber auch Endgeräte für mobile Dienste von Bedeutung. Die Endgeräte mit der höchsten Verbreitung sind derzeit Mobiltelefone, die ursprünglich nur auf Sprachdienste spezialisiert waren, allerdings zunehmend auch über weitere Funktionalitäten wie z. B. den Versand von MMS oder Java-Anwendungen verfügen.

Da im Bereich der Endgeräte zunehmend Organizer-Funktionen von PDAs (Personal Digital Assistant) mit den klassischen Mobiltelefonen verschmelzen, wird eine zunehmende Konvergenz der Endgeräte erwartet. Wie Abbildung 2 zeigt, integrieren so genannte Smartphones die bisherigen Funktionen von Mobiltelefonen und PDAs und beinhalten zusätzliche Multimedia-Funktionen wie bspw. digitale Fotografie oder das Abspielen von Musik. 


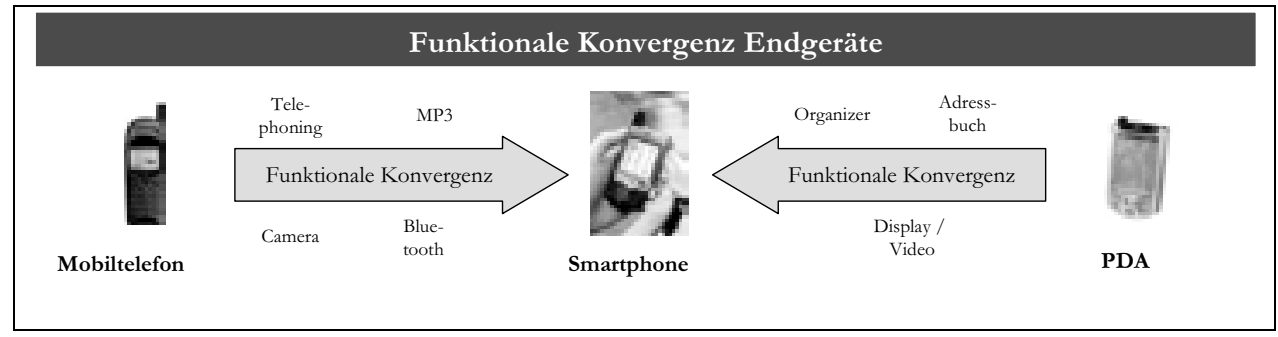

Abbildung 2: Funktionale Konvergenz bei Endgeräten

\subsection{Aktuelle Situation mobiler Dienste}

Auch wenn die technologischen Voraussetzungen für das erfolgreiche Angebot von mobilen Datendiensten vorhanden sind, so konnten sich bisher multimediale Datendienste, die für Medienunternehmen in ihrer Funktion als Content-Provider interessant wären, noch nicht durchsetzen. Dies zeigt sich einerseits an Nutzungsbzw. Umsatzzahlen der Mobilfunkbetreiber als auch am relativ geringen Angebot mobiler Datendienste bei Medienunternehmen.

Während im Jahr 2003 in Deutschland insgesamt ein Mobilfunk-Umsatz von 21,3 Mrd. € erreicht wurde, betrug der Anteil der Datenumsätze am Gesamtumsatz lediglich ca. 18\%. Derzeit werden 90\% dieser Datenumsätze noch durch SMS (Short Message Service) erzielt (vgl. Büllingen / Stamm 2004). Neben der SMS sind wirtschaftlich erfolgreiche und damit für Medienunternehmen interessante mobile Datendienste derzeit fast ausschließlich die Download-Angebote von Klingeltönen und Logos. Abb. 3 zeigt diese Verteilung der Anteile am Gesamtumsatz der Mobilfunkbranche in Deutschland im Jahr 2003 mit den absoluten Umsatzzahlen.

Ähnlich ernüchternde Ergebnisse zeigten sich in einer quantitativen Inhaltsanalyse der Online-Angebote ${ }^{3}$ von deutschen und US-amerikanischen Tageszeitungen, in der elektronische Zusatzangebote zu klassischen Print-Produkten untersucht wurden (vgl. Thallmayer 2004). Ein wichtiges Ergebnis war dabei einerseits, dass alle untersuchten Zeitungen über einen Online-Auftritt verfügten. Im Gegensatz dazu konnte das Angebot von Inhalten über mobile Vertriebswege (,,mobile Content") nur bei einem sehr geringen Anteil der Zeitungen festgestellt werden, der in Deutschland bei lediglich 7\% und in den USA bei 16\% lag.

3 Bei der Untersuchung wurde davon ausgegangen, dass ein Hinweis auf mobile Zusatz-Angebote auf den Websites der Tageszeitungen zu finden ist. 


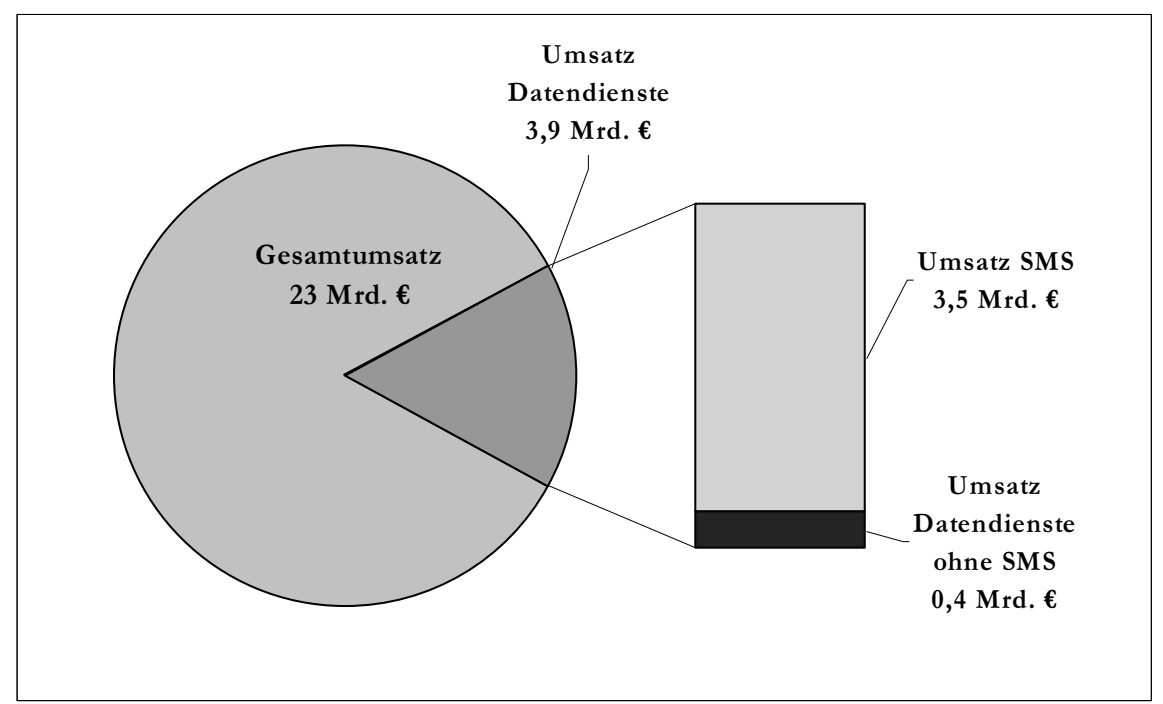

Abbildung 3: Umsatzanteile der Datendienste mit und ohne SMS am deutschen Mobilfunkmarkt (vgl. Büllingen / Stamm 2004)

Insgesamt deuten diese Zahlen darauf hin, dass nur ein begrenztes Potential für das Angebot mobiler Datendienste durch Medienunternehmen vorhanden ist und dieses bisher auch nur in geringem Umfang genutzt wird.

\subsection{Trends bei mobilen Diensten}

Wie in Kap. 2.2 dargelegt, ist die bisherige Nutzung von Datendiensten sowohl allgemein als auch in der Medienbranche noch nicht sehr weit verbreitet. Daher werden im Folgenden zwei neue Anwendungsfelder dargestellt, mit denen sich diese Situation möglicherweise verändern könnte.

\section{Anwendungsfall 1: Kontextsensitive Dienste}

Im Hinblick auf mobile Dienste wird Kontext verstanden als „,...) any information that can be used to characterize the situation of an entity. An entity is a person, place, or object that is considered relevant to the interaction between a user and an application, including the user and applications themselves" (Dey 2001, S. 3). Demnach ist jede Information, durch die eine Situation eines Users im Rahmen eines Kommunikationsvorgangs charakterisiert werden kann, Kontext.

Als kontextsensitive Dienste werden also Anwendungen bezeichnet, die Veränderungen in der Umwelt eines Users entdecken und entsprechend reagieren 
können. Informationen über diese Veränderungen werden als Kontextinformationen bezeichnet und sind in Ort, Identitäten von Objekten und Personen in der Umgebung und Veränderungen dieser aufgeteilt (vgl. Schilit / Theimer 1994).

Abb. 4 zeigt das grundlegende Funktionsprinzip kontextsensitiver Dienste sowie deren aktuelle Anwendungsgebiete. Bisher wurden vor allem Dienste, die die Kontextinformation „Ort“ nutzen, umgesetzt. Dabei beinhalten die Anwendungsmöglichkeiten meist ortsabhängige Verwaltungs- und Suchdienste oder Informationsdienste mit Ortsbezug.

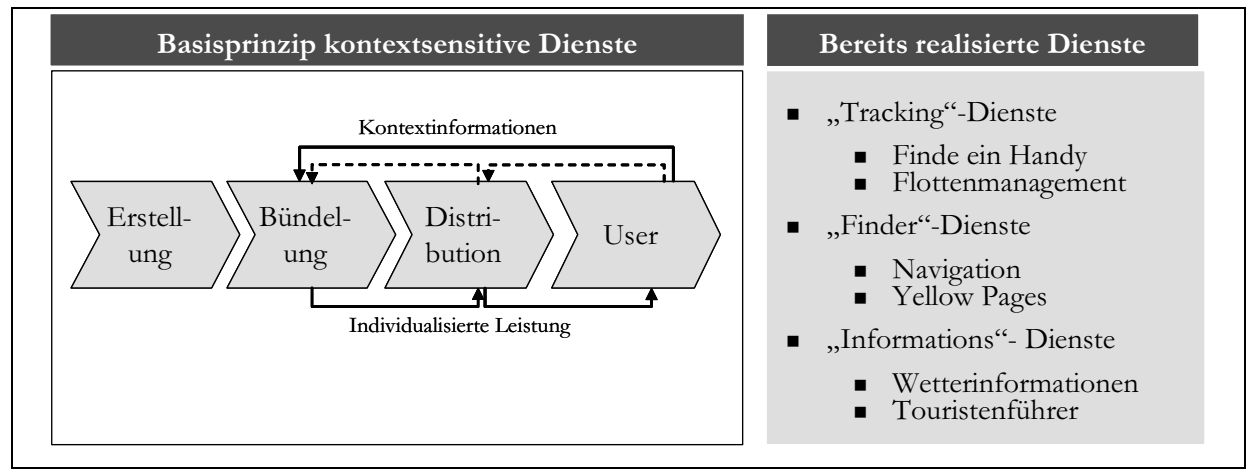

Abbildung 4: Überblick Funktionsprinzip und bisherige Anwendungsbereiche kontextsensitiver Dienste

Die Bereitstellung eines kontextsensitiven, also auf die Situation eines Konsumenten angepassten Dienstes, ist von wettbewerbsstrategischer Sichtweise aus als Individualisierung der Leistungserbringung zu verstehen. Das Konzept der kontextsensitiven Leistungen kann interessant sein auf weitgehend gesättigten Märkten, in denen auf individuelle Präferenzen und Bedürfnisse zugeschnittene Leistungen den größten Kundennutzen generieren können. Dieser Kundennutzen wiederum sorgt über direkte und indirekte Einflussgrößen, wie z. B. gestiegenen Umsatz durch höhere Zahlungsbereitschaft und höheren Absatz oder gestiegene Kundenbindung und -loyalität, für den Erfolg eines Unternehmens, und kann daher auch für Medienunternehmen einen Anreiz darstellen, in kontextsensitive Technologien zu investieren. Für die Nutzung kontextsensitiver Technologien sind allerdings auch Anpassungen bei technologischer Infrastruktur und Geschäftsmodellen verbunden, so dass eine Investitionsentscheidung auch unter Berücksichtung möglicher negativer Effekte getroffen werden muss.

\section{Anwendungsfall 2: Digitales mobiles Fernsehen über DVB-H}

Mit Digital Video Broadcasting (DVB) werden standardisierte Verfahren für die digitale Übertragung von Inhalten bezeichnet, die eine Alternative für die bisheri- 
Internettechnologien in der Medienbranche

ge analoge Rundfunkübertragung darstellen. Es existieren vier Ausprägungen von DVB, die anhand ihrer Übertragungswege unterschieden werden in Digital Video Broadcasting- Satellite (DVB-S), Digital Video Broadcasting-Cable (DVB-C), Digital Video Broadcasting-Terrestrial (DVB-T) ${ }^{4}$ und Digital Video BroadcastingHandheld (DVB-H).

Für den Mobilfunk ist der wesentliche Standard DVB-H, der wie DVB-T über terrestrische Senderketten bzw. Antennen ausgestrahlt wird, aber für mobile Endgeräte optimiert ist und eine asynchrone Übertragung von Inhalten ermöglicht. Mit diesem Standard sind hohe Video-Auflösungen bei gleichzeitig hohen Übertragungsraten möglich. Für den Empfang von DVB-H sind neue Endgeräte notwendig, die voraussichtlich in Form von funktionsintegrierten Mobiltelefonen oder von Steckkarten für PCs bzw. Notebooks vorliegen werden.

Im Raum Berlin wurde 2004 in einem gemeinsamen Pilotprojekt von Nokia, Philips, Universal Studios Networks Germany und der vodafone Pilotentwicklung ein Feldtest zur Nutzung von DVB-H- und DVB-H- inklusive DVB-T-Diensten für Mobiltelefone bzw. tragbare Fernsehgeräte durchgeführt, wobei auch interaktive Zusatzleistungen wie z. B. die Abstimmung über Musik-Clips oder die Bestellung von Kino-Tickets möglich waren. Nach Angaben der Projektleitung war die Akzeptanz dieser Dienste insgesamt sehr hoch, wobei Nachrichten, Sport und Musik zu den am meisten nachgefragten Inhalte-Kategorien zählten, aber auch interaktive Dienste häufig genutzt wurden (vgl. Bmco 2005).

Für Medienunternehmen würde ein Sendebetrieb mit DVB neben Investitionskosten für den Aufbau dieser neuen Übertragungswege auch eine Steigerung der laufenden Kosten für den Sendebetrieb bedeuten. ${ }^{5}$ Für eine Refinanzierung dieser Kosten stehen mit der neuen Technologie aber auch Möglichkeiten für neue Geschäftsmodelle zur Verfügung. Diese beinhalten veränderte Leistungsmodelle wie z. B. interaktive Angebote sowie neue Erlösmodelle wie z. B. pay-per-viewVerfahren (vgl. Bisenius / Siegert 2002, S. 89).

\section{Auswirkungen von Internettechnologien auf die Wissenschaftskommunikation}

Der Markt für Wissenschaftskommunikation ist, wie bereits eingangs dargestellt, ein Teilbereich der Print-Branche und wird derzeit größtenteils noch von Fachverlagen dominiert. Trotz spezifischer Eigenheiten des Marktes steht aber auch dieser

4 Bisher ist in Deutschland nur in Ballungsräumen der Empfang von DVB-T möglich, soll aber bis 2010 den analogen Fernsehempfang über Antenne vollständig ersetzt haben, vgl. DVB (2005).

5 Nach einer groben Schätzung würden die Kosten für den Sendebetrieb für DVB um 30\% (öffentlich-rechtliche Sendeanstalten) bzw. 100\% (private Sendeanstalten) über den jetzigen Sendekosten liegen, vgl. Bisenius / Siegert (2002), S. 87-88. 
Teilbereich der Medien- und Printindustrie ebenfalls unter dem Einfluss neuer und sich stetig weiterentwickelnder technologischer Treiber. Diese werden im Folgenden vorgestellt. Im Anschluss wird die aktuelle Situation des Marktes für Wissenschaftskommunikation beschrieben sowie zwei sich derzeit andeutende Entwicklungs-Szenarios aufgezeigt.

\subsection{Technologische Treiber auf dem Markt der Wissen- schaftskommunikation}

Als wesentliche technologische Treiber für Veränderungen im Bereich der Wissenschaftskommunikation gelten die Digitalisierung von Produktion bzw. Produkt, Internet-Technologien und Digital-Rights-Management-Systeme (vgl. Abb. 5), die in ihrem Zusammenwirken den Markt der Wissenschaftskommunikation beeinflussen.

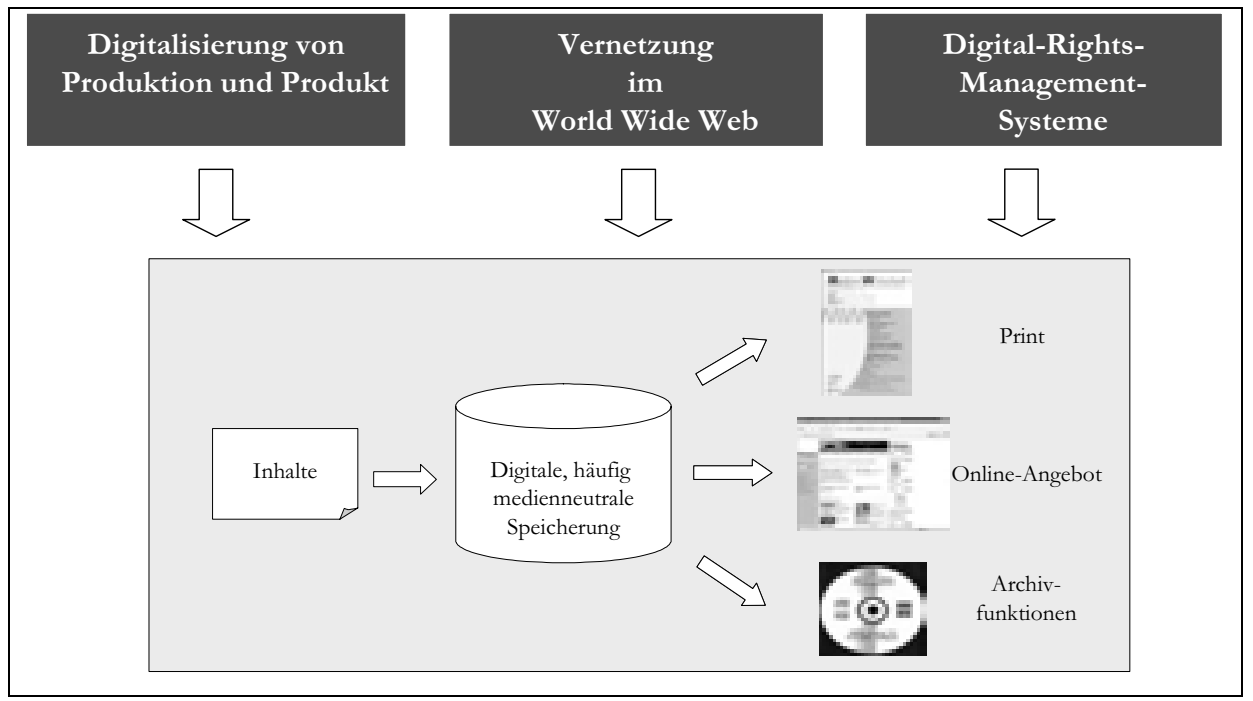

Abbildung 5: Technologische Treiber auf dem Markt der Wissenschaftskommunikation

Die Produktion von Inhalten kann heute weitestgehend digital erfolgen. Sowohl Texte als auch Bilder werden digital erstellt; auch die Bündelung und LayoutGestaltung erfolgt mit technischer Unterstützung. Durch die Digitalisierung von Produkten können Inhalte - unabhängig vom Print-Medium Zeitschrift - online zur Verfügung gestellt werden. Damit besteht technisch die Möglichkeit, den gesamten Prozess der Wissenschaftskommunikation ausschließlich digital zu gestalten. 
Von zentraler Bedeutung für die technische Unterstützung eines digitalen Prozesses der Wissenschaftskommunikation sind die Technologien des WWW. Die elektronische Speicherung und Archivierung von Dokumenten vereinfacht den $\mathrm{Zu}$ gang zu wissenschaftlichen Publikationen. Häufig bieten Verlage ihre Publikationen zusätzlich zu den Print-Ausgaben auch in digitaler Form an, zu denen der Leser entweder über Publikations-Portale des Verlags selber oder über elektronische Datenbanken von Bibliotheken Zugang erhält. Zusätzlich erweitern auch digitale Such- und Verlinkungsfunktionen über Online-Portale oder -Plattformen die Möglichkeiten der wissenschaftlichen Recherche.

Mit der Nutzung des WWW für die Wissenschaftskommunikation wird aber auch ein zusätzlicher technischer Schutz der Eigentumsrechte an den digitalen Inhalten zunehmend wichtiger. Mit Digital Rights Management Systemen (DRMS) soll die Weitergabe von Inhalten kontrollierbar gestaltet werden, wodurch sowohl Verlage als auch Wissenschaftler bei der Sicherung ihrer jeweiligen Copyrights unterstützt werden können. Wenn - wie bisher - Verlage die Rechte an publizierten Artikeln erhalten, können diese mit Hilfe von DRMS vor allem auch online geschützt werden. Zudem können aber auch alternative Konzepte wie beispielsweise neue Abrechnungsfunktionen oder die Differenzierung von Preisen und Zugriffsrechten umgesetzt werden (vgl. Hess / Ünlü 2004, S. 274).

\section{Aktuelle Situation der Wissenschaftskommunikation}

Obwohl die vorgestellten Technologien auch auf andere Sektoren der Medienbranche ähnliche Einflüsse ausüben, so ist der Markt für Wissenschaftskommunikation dennoch von drei wesentlichen Besonderheiten geprägt. Im Fokus der folgenden Betrachtungen liegt daher die qualitätsgesicherte Wissenschaftskommunikation, insbesondere das Segment der Inhalteanbieter; der bilaterale Austausch von Forschungsergebnissen $z$. B. wird dabei vernachlässigt.

Zunächst sind Wissenschaftler sowohl Produzenten als auch Konsumenten der Wissenschaftskommunikation und stellen somit - zumindest innerhalb von Forschungs-Communities - ihre eigene Zielgruppe dar. Dies spiegelt sich auch in dem Prozess der Auswahl von Beiträgen und der Qualitätssicherung wider, der durch so genannte Peer-Review-Verfahren organisiert wird. In diesem Prozess werden eingereichte Beiträge von fachlich versierten Wissenschaftlern „blind“, also ohne Kenntnis der Autoren, beurteilt und gemäß den qualitativen Anforderungen der Forschungs-Community zur Veröffentlichung angenommen oder abgelehnt. Die Publikation in diesen qualitätsgesicherten Zeitschriften bildet eines der wesentlichen Ziele von Wissenschaftlern, deren Arbeit und Image zu einem großen Teil anhand der Anzahl ihrer qualitativ hochwertigen Veröffentlichungen bewertet wird. 
Zudem unterscheidet sich aber auch das Produkt, das in Form von wissenschaftlichen Ergebnissen und Berichten vorliegt, teilweise von anderen Medienprodukten wie z. B. Musikstücken oder Fernseh-Shows. Während letztere häufig nur zu Unterhaltungszwecken genutzt werden, dienen wissenschaftliche Ergebnisse der Vergrößerung des allgemeinen Wissensstandes und haben somit höhere gesellschaftliche Bedeutung. ${ }^{6}$

Als dritte wesentliche Besonderheit ist die Markt-Organisation der Wissenschaftskommunikation zu bezeichnen, die von spezifischen Akteuren und Prozessen geprägt ist (vgl. Abb. 6).

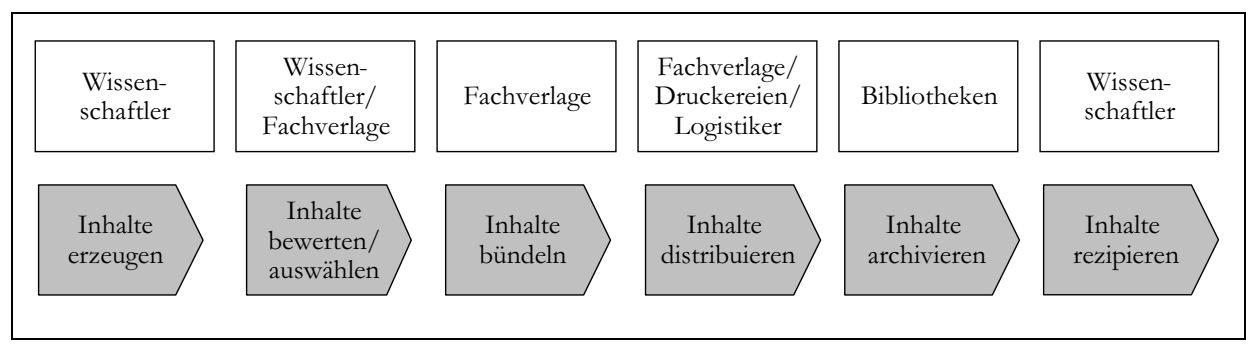

Abbildung 6: Wertschöpfungskette und Akteure der Wissenschaftskommunikation

Wissenschaftler erzeugen hierbei zunächst Inhalte in Form von wissenschaftlichen Beiträgen. Für deren Auswahl, Organisation der Bewertung, Aufbereitung, Verwertung und Verbreitung sind Fachverlage zuständig, wobei die Auswahl und Bewertung an Herausgeber und Gutachter abgegeben und in eingangs beschriebenen „Double Blind Review“-Verfahren durchgeführt werden. Auch der eigentliche Herstellungsprozess und die Distribution der Fachpublikationen werden meist an Druckereien bzw. externe Logistikunternehmen ausgelagert. Die Nutzung der Inhalte erfolgt wiederum durch die Wissenschaftler selbst oder über die Bibliotheken, die dieses Wissen sammeln, ordnen und für eine möglichst breite Verfügbarkeit sorgen.

Aus diesen drei wesentlichen Besonderheiten des Marktes für Wissenschaftskommunikation werden die beiden folgenden Problemfelder hinsichtlich seiner zukünftigen Entwicklung ersichtlich.

Zunächst wird die „freie“ Verfügbarkeit von wissenschaftlichen Ergebnissen derzeit vorrangig durch staatlich finanzierte Bibliotheken gewährleistet. Durch steigende Preise für Fachpublikationen sowie gleichzeitig sinkende öffentliche Etats für Bildungseinrichtungen ist die Fortführung dieses Systems auch schon ohne den Einfluss neuer technologischer Treiber gefährdet.

6 Dies wird mit dem Begriff „Wissensallemende“ bezeichnet (vgl. Müller-Litzkow 2004, S. 193). 
Durch diese technologischen Treiber aber können, organisiert durch die Forschungs-Community selber statt durch den Fachverlag, die durchschnittlichen Kosten für einen publizierten Artikel signifikant gesenkt werden. ${ }^{7}$ Daher stellt sich sowohl aus Sicht eines individuellen Nutzenkalküls von Wissenschaftlern, Verlagen und weiteren Einrichtungen wie Bibliotheken als auch aus Sicht gesamtwirtschaftlicher Wohlfahrtsüberlegungen die Frage, ob der Markt der Wissenschaftskommunikation nicht effizienter organisiert werden könnte.

\subsection{Trends in der Wissenschaftskommunikation}

Derzeit zeichnen sich zwei Szenarios hinsichtlich einer möglichen Restrukturierung des Marktes für Wissenschaftskommunikation ab. Das erste Szenario „Open Access“ steht für den freien Zugang zu wissenschaftlichen Publikationen, der durch wissenschaftliche Institutionen selbst organisiert wird. Das zweite vorgestellte Szenario „Open Choice“ stellt die bisher wesentlichste Reaktion der Verlage auf die „Open Access“ Entwicklungen dar.

\section{Szenario I: „Open Access“}

Auf Basis der in Kap. 3.1 vorgestellten technologischen Treiber entstanden in den vergangenen Jahren vermehrt Initiativen für eine freie digitale Verfügbarkeit von wissenschaftlichen Publikationen und gegen die bisherigen Marktstrukturen, die von wirtschaftlichen Zielen der Fachverlage geprägt sind. Eines der aktuellsten Beispiele aus Deutschland ist die Göttinger Erklärung von 2004, in der Gesetze für ein wissenschaftsfreundlicheres Urheberrecht gefordert werden (vgl. Aktionsbündnis „Urheberrecht für Bildung und Wissenschaft“ 2004).

Diese und ähnliche Initiativen werden unter dem Begriff „Open Access“, also öffentlicher Zugang zu wissenschaftlichen Erkenntnissen, zusammengefasst. „Open Access“-Initiativen verfügen meist über die folgenden drei Eigenschaften:

Die Eigentumsrechte an den Artikeln, die bisher für die Publikation in wissenschaftlichen Journals an die jeweiligen Fachverlage abgetreten wurden, sollen nun bei den Autoren der Artikel selbst verbleiben. Das bedeutet, ein Autor kann seinen Artikel online frei zur Verfügung stellen, behält aber das Recht an seinen „Erkenntnissen“. Dennoch können andere Wissenschaftler kostenfrei auf die vorliegenden Ergebnisse zugreifen.

Hinsichtlich technischer Anforderungen bedeutet „Open Access“, dass von wissenschaftlichen Institutionen selber oder von zentralen Stellen, wie z. B. Bibliotheken, Publikationsserver und entsprechende Websites eingerichtet werden müs-

7 Hilf (vgl. Hilf 2004) identifiziert sogar ein Kostensenkungspotential von 90\%. 
sen, die als Plattform für die Speicherung, Verwaltung und Suche von digitalen Publikationen dienen. Daneben muss die Sicherung des dauerhaften Zugriffs auf die Publikationen technologisch gewährleistet werden.

Auch die Sicherung der wissenschaftlichen Qualität von Beiträgen wird im „Open Access“-Szenario neu organisiert. Die Organisation und Unterstützung des Review-Prozesses unterliegt dabei nicht mehr wie bisher dem Verlag bzw. Herausgebern, sondern wird von der wissenschaftlichen Community selbst übernommen.

Zusammengefasst lassen sich diese Ansätze in der in Abb. 7 dargestellten Veränderung der Wertschöpfungskette vor allem hinsichtlich der beteiligten Akteure der Wissenschaftskommunikation abbilden.

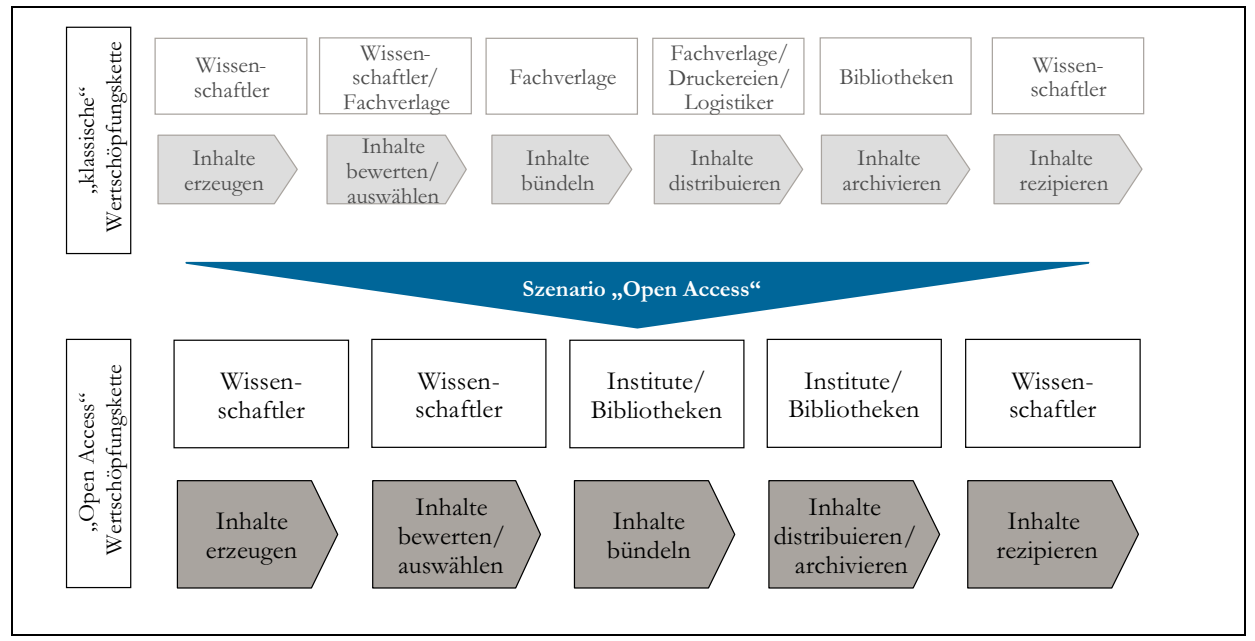

Abbildung 7: Veränderungen der Wertschöpfungskette und Akteure im Szenario „Open Access"

Im Hinblick auf die Effizienz der Prozesse der Wissenschaftskommunikation bieten „Open Access“ Modelle wesentliche Verbesserungspotentiale. Durch Nutzung von Technologien des WWW kann sowohl die Effizienz von ReviewProzessen gesteigert als auch der gesamte Publikationsprozess vom Erstellen bis zum Lesen eines Artikels wesentlich verkürzt werden. In einem rein digitalen Prozess müssen zudem weder Zeit noch Kosten für Druck und Auslieferung der Hard Copy aufgebracht werden. Auch können wissenschaftliche Artikel durch eine Vernetzung von Zusatz- oder Zitationsinformationen oder durch zusätzliche Bewertungs- oder Kommentarfunktionen verbessert werden.

Jedoch entstehen in diesem Szenario auch negative Auswirkungen für die beteiligten Akteure. Wissenschaftler bzw. wissenschaftliche Institutionen müssen mit höherem Ressourcenbedarf für die Verwaltung der notwendigen Technologien 
und des Publikationsprozesses rechnen. Wesentlich größere Herausforderungen ergeben sich jedoch in diesem Szenario für die Fachverlage: da ihre bisherigen Aufgaben von anderen Akteuren übernommen werden, ist ihr gesamtes bisheriges Geschäftsmodell gefährdet.

\section{Szenario II: „Open Choice“}

Die Reaktion der Fachverlage auf die veränderten technologischen Gegebenheiten beschränkte sich bisher größtenteils auf den zusätzlichen Verkauf digitalisierter Versionen der gedruckten Publikationen über Publikationsverzeichnisse bzw. über eigene elektronische Publikations-Plattformen.

Zumindest aus Sicht der Fachverlage ist dies eine verständliche Vorgehensweise, denn bislang können sie aufgrund hoher Abonnementpreise für Journals sehr attraktive Margen erzielen. Zudem verfügen sie über etablierte Infrastrukturen für Review-Prozesse, Druck- und Vertriebsorganisation sowie eine hohe Markenbekanntheit.

Um diese Vorteile weiterhin nutzen zu können, sich aber dennoch den Herausforderungen der „Open Access“-Initiativen stellen zu können, kann man anhand von „Open Choice“-Modellen nun doch erste Reaktionen der Fachverlage erkennen. In diesen Modellen werden die Kosten für den Publikationsprozess von den Autoren getragen, die aber dafür die Verwertungsrechte an ihren Artikeln behalten und diese dann u.a. online frei zur Verfügung stellen können.

Dieses Modell wird im deutschsprachigen Raum derzeit vor allem von Springer präferiert (vgl. Haank 2004), auch wenn der Umfang der realen Nutzung noch unklar ist. In diesem Ansatz kann der Autor nach einem „klassisch“ verlaufenden Einreichungs- und Begutachtungsprozess neben dem traditionellen Modell auch das „Open Choice“-Modell wählen. Dies bedeutet, dass er für einen - angenommenen - Artikel z. B. $3.000 €$ an den Verlag bezahlt, der dann den Artikel neben der normalen Veröffentlichung in der Print-Ausgabe auch auf dem Online-Portal von Springer kostenfrei zur Verfügung stellt.

Während in diesem Szenario im Gegensatz zu dem zuerst vorgestellten Szenario „Open Access“ die Wertschöpfungsstruktur der Wissenschaftskommunikation unverändert bleibt, ändert sich hier das Geschäftsmodell der Wissenschaftsverlage (vgl. Abb. 8). Von dieser Änderung sind vorrangig Wissenschaftler in ihrer Rolle als Autoren und Rezipienten betroffen. 


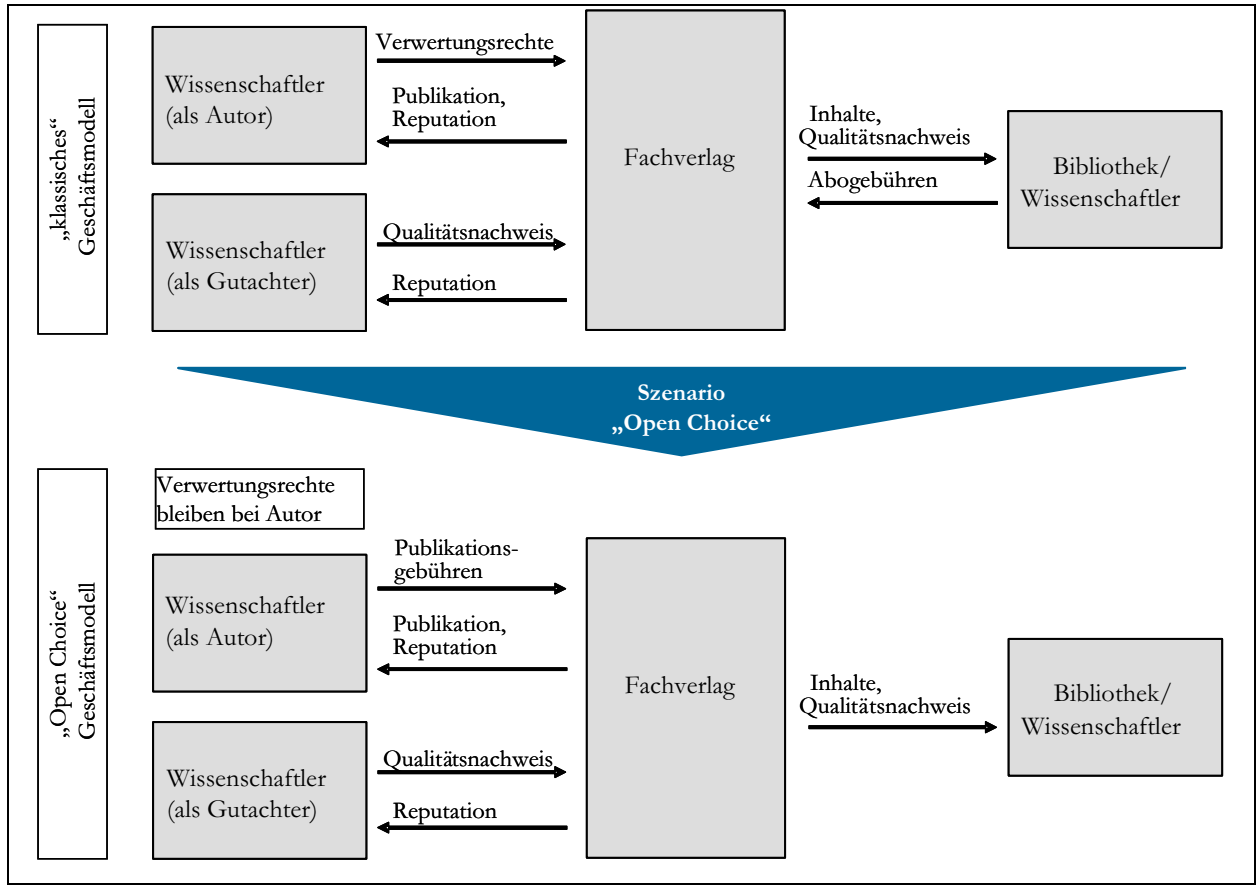

Abbildung 8: Veränderungen des Geschäftsmodells der Fachverlage im Szenario „Open Choice“ (in Anlehnung an Meier 2003, S. 36)

Ob viele Wissenschaftler sich von den Vorteilen dieses Ansatzes überzeugen lassen, und vor allem, ob sie das dafür notwendige Budget aufbringen können und möchten, ist fraglich. Das Szenario scheint vielmehr einen Versuch der Fachverlage darzustellen, den alternativen Modellen des „Open Access“ entgegenzutreten.

\section{Internettechnologien in der Medienbranche: Zukünftige Fragestellungen}

Sowohl hinsichtlich des übergreifenden technologischen Trends der mobilen Dienste als auch in der Teilbranche der Wissenschaftskommunikation wird deutlich, dass die skizzierten Entwicklungen einerseits für Medienunternehmen nützlich sein können, andererseits aber auch deren ökonomische Grundlagen gefährden können. Zudem ist in beiden Bereichen noch nicht abzusehen, welche der Entwicklungslinien sich durchsetzen wird bzw. welche Trends sich doch als irrelevant für die Medienbranche herausstellen werden. 
Hinsichtlich dieser Unsicherheiten werden in Abb. 9 die in nächster Zukunft relevanten Fragestellungen für Medienunternehmen bezüglich mobiler Dienste dargestellt.

\section{Ökonomisch}

- Wertschöpfungsstrukturen: Werden MNOs zukünftig „Gate-Keeper“ sein oder reiner Datenkanal?

- Geschäftsmodelle: Eignen sich mobile Dienste als Zusatzsangebotefür Medienunternehmen im Rahmen von MultiChannel-Strategien?

\section{Technologisch}

- Welche Übertragungsstandards werden sich durchsetzen?

- Wie werden sich Endgeräte entwickeln hinsichtlich Display, Batterieleistung und Ausmessungen?

\section{Nutzerseitig}

- Werden Konsumenten im Gegensatz zum statiorären Internet höhere Zahlungsbereitschaft aufweisen?

- Wovon hängt die Akzeptanz neuer mobiler Angebote $a b$ ?

\section{Abbildung 9: Zukünftige Fragestellungen hinsichtlich mobiler Dienste}

Die Fragestellungen ergeben sich dabei sowohl in den eher als exogen zu betrachtenden Bereichen der Technologieentwicklung und Nutzeraspekte als auch hinsichtlich der ökonomischen Ausgestaltungsoptionen für Medienunternehmen in den Bereichen ihrer Geschäftsmodelle und den daraus resultierenden Wertschöpfungsstrukturen.

In denselben Bereichen können auch für die Wissenschaftskommunikation relevante Fragestellungen abgeleitet werden (s. Abb. 10).

\section{Ökonomisch}

- Wertschöpfungsstrukturen: Werden Funktionen der Verlage zukünftig durch andere Akteure ausgeführt? Entfällt die Stufe ,Druck“ und physische Distribution?

- Geschäftsmodelle: Sind Erlöse durch Autoren generierbar oder weiterhin durch Leser/Bibliotheken?

\section{Technologisch}

- Wie kann eine dauerhafte und sichere elektronische Archivierung gewährleistet werden

- Wie kann ein effizienter Zugriff auf Dokumente realisiert werden?

- Werden innovative Verfahren wie Print-onDemand zukünftig eine Rolle spielen?

\section{Nutzerseitig}

- Müssen Wissenschaftler für ihre eigenen Publikationen oder für Publikationen anderer bezahlen?

- Wer übernimmt die Selektion von Publikationen und sichert so die wissenschaftliche Qualität? 
Es bleibt abzuwarten, wie sich einerseits die beeinflussenden Größen entwickeln werden und wie sich andererseits Medienunternehmen verhalten werden, um sich diesen anzupassen oder auch gestaltend auf sie einzuwirken.

\section{Literatur}

Aktionsbündnis „Urheberrecht für Bildung und Wissenschaft“ (2004): Göttinger Erklärung zum Urheberrecht für Bildung und Wissenschaft, 05.07.2004, http://www.urheberrechtsbuendnis.de/GE-Urheberrecht-BuW-Mitgl.pdf, Abruf am 14.02.2005.

Bisenius / Siegert (2002): Bisenius, J.-C., Siegert, W.: Multi Media Mobil: Mobile Dienste in digitalen Rundfunk- und Telekommunikationsnetzen - Analysen und Perspektiven, Berlin.

bmco (2005): Website des DVB-Projekts, www.bmco-berlin.com, Abruf am 13.02.2005.

Büllingen / Stamm (2004): Büllingen, F., Stamm, P. (2004): Mobile MultimediaDienste: Deutschlands Chance im globalen Wettbewerb, http://www.bmwi.de/Redaktion/Inhalte/Pdf/M-O/mobil-multimediamanagement-summary,property $=$ pdf,bereich $=$ bmwi, sprache $=$ de, $r w b=$ true.pdf, Abruf am 07.05.2006.

Dey (2001): Dey, A. K.: Understanding and Using Context. In: Journal of Personal and Ubiquitous Computing, 5. Jg., Nr. 1, S. 4-7.

DVB (2005): Website des DVB-Projekts, www.dvb.org, Abruf am 13.02.2005.

Haank (2004): Haank, D.: Evolution statt Revolution: Springer Open Choice. In: MedienWirtschaft, 1. Jg, Nr. 3, 2004, S. 143-144.

Hess / Ünlü (2004): Hess, T., Ünlü, V.: Systeme für das Management digitaler Rechte, in: Wirtschaftsinformatik, 46. Jg., Nr. 4, S. 273-280.

Hilf (2004): Hilf, E. R.: Zehn Jahre Open Access - und nun die wirtschaftliche Nutzung? In: MedienWirtschaft, 1. Jg., Nr. 3, 2004, S. 146-148.

Meier (2002): Meier, M.: Returning science to the scientists: der Umbruch im STMZeitschriftenmarkt unter Einfluss des Electronic Publishing, München.

Müller-Lietzkow (2004): Müller-Lietzkow, J.: „Open Science“-Paradigmawechsel in der Wissenschaftskommunikation. In: MedienWirtschaft, 1. Jg., Nr. 4, 2004, S. 193-203.

Schilit / Theimer (1994): Schilit, B., Theimer, M.: Disseminating Active Map Information to Mobile Hosts. In: IEEE Network, 8. Jg., Nr. 5, S. 22-32. 
Thallmayer (2004): Thallmayer, T.: Personalisierungsstrategien im Zeitungssektor: Entwicklungen und Perspektiven der Nutzung von Kontextinformationen. Diplomarbeit am Institut für Wirtschaftsinformatik und Neue Medien, LMU München.

Turowski / Pousttchi (2004): Turowski, K., Pousttchi, K.: Mobile Commerce: Grundlagen und Techniken, Berlin u.a. 


\section{Absatz digitaler Produkte und Digital Rights Management: Ein Überblick}

\section{Lutz. Seidenfaden}

\section{Einleitung}

Mit der Zunahme von Internetanschlüssen weltweit hat die Distribution digitaler Produkte an Bedeutung gewonnen. Hervorzuheben sind vor allem die Möglichkeiten der Disintermediation, also der Ausschaltung von Handelsstufen bzw. Intermediären im Distributionsprozess und den damit verbundenen Veränderungen in der Wertschöpfungskette.

Populäre Peer-to-Peer-Applikationen wie WinMx (http://www.winmx.com), Gnutella (http://www.gnutella.com) oder KaZaa (http://www.kazaa.com), mittels derer Inhalte beinahe jeglicher Art bezogen werden können, haben zudem den Ruf nach Mechanismen zur Wahrung des Urheberrechts laut werden lassen. Die wirtschaftlichen Auswirkungen der nahezu kostenlosen Distribution digitaler Güter sind anhand sinkender Abverkaufszahlen und damit einhergehenden Umsatzeinbrüchen in der Entertainmentbranche eindrucksvoll dargelegt (vgl. Ifpi 2004, S. 1). Es gibt jedoch kritische Stimmen, die behaupten, dass die Umsatzeinbußen nicht nur durch Raubkopieren (,piracy“) sondern ebenfalls durch Innovationsde- 
Absatz digitaler Produkte und Digital Rights Management

fizite im Hinblick auf Organisations- und Vertriebsformen seitens der Unterhaltungsindustrie verursacht werden (vgl. dazu Kuhlen 2003, S. 118ff).

Nicht nur die Entertainmentbranche ist von den neuen Vertriebsmöglichkeiten betroffen. So stellt sich z. B. im Bereich der Wissenschaftskommunikation die Frage, welche Rolle Bibliotheken und Verlage zukünftig spielen werden, wenn die Inhalte auch schnell und günstig vom Endnutzer in elektronischer Form über Netzwerke zu beziehen sind.

Ebenso wie bei Musikstücken oder Filmen stellt sich im Hinblick auf wissenschaftliche Beiträge die Frage nach dem Schutz der Autorenrechte. Unter dem Schlagwort Digital Rights Management (DRM) sind zahlreiche Initiativen gegründet worden, die den Schutz des Urheber- oder Verwertungsrechts von digitalen Produkten zum Ziel haben ${ }^{8}$. Da eine zu starke Einschränkung der Nutzung zugunsten der Rechteinhaber innovations- und wachstumshemmende Folgen haben kann, die derzeit noch nicht absehbar sind, empfiehlt das $\mathrm{CED}^{9}$ ein behutsames Vorgehen der Rechteinhaber. Hier gilt es, eine rechtliche Regelung zu finden, die sowohl Autoren- als auch Nutzerinteressen ausbalanciert.

Der vorliegende Beitrag klärt in Kap. 2 zunächst die grundlegenden Begriffe, Eigenschaften und Technologien im Zusammenhang mit digitalen Gütern, um dem Leser eine Orientierung zu bieten. Nachfolgend werden Erlösmodelle der Distribution digitaler Güter dargestellt (vgl. Kap. 3). Das Themengebiet Digital Rights Management wird in Kap. 4 behandelt. Dazu werden eine Begriffsdefinition gegeben, grundlegende Konzepte vorgestellt und aktuelle Technologien erörtert. Auf eine Darstellung juristischer Aspekte des Themenfeldes wird verzichtet. Stattdessen werden aktuelle DRM-Systeme im Überblick dargestellt. Der Beitrag schließt mit einer Zusammenfassung (vgl. Kap. 5).

\section{Grundlagen: Digitale Produkte}

Die Verwendung des Begriffs ,digitale Produkte“ ist in der wissenschaftlichen Literatur uneinheitlich, da sich dieser auf digitale Güter, digitale Dienstleistungen und Informationsgüter beziehen kann. Um ein allgemeines Verständnis des Untersuchungsgegenstandes zu ermöglichen, wird in 2.1 eine (mögliche) Definition (vgl. 2.1.1) vorgenommen und grundlegende Eigenschaften digitaler Produkte erläutert (vgl. 2.1.2).

8 z. B. Content Reference Group (http://www.crforum.org/), Trusted Computing Platform Association (http://www.trustedcomputing.org/home), Trusted Computing Group (http://www.trustedcomputing group.org/).

9 "The [...] CED is an independent, nonpartisan organization of business and education leaders dedicated to policy research on the major economic and social issues of our time and the implementation of its recommendations by the public and private sectors." (http://www.ced.org/about/mission.shtml). 


\subsection{Definition und Begriffsabgrenzung}

Digitale Produkte sind allgemein recht eindeutig gegenüber physischen Produkten abgrenzbar. Das grundlegendste Unterscheidungskriterium zwischen physischen und digitalen Produkten ist die Immaterialität (vgl. Löbbecke 1999, S. 1).

Physische Produkte werden hier nicht betrachtet, da sie keinen digitalen Anteil haben, d.h. nicht durch Datenströme repräsentiert werden können. Sie sind folglich materiell. Vielmehr sollen an dieser Stelle die digitalen Güter betrachtet werden, die aufgrund ihrer Immaterialität vollständig elektronisch handelbar sind.

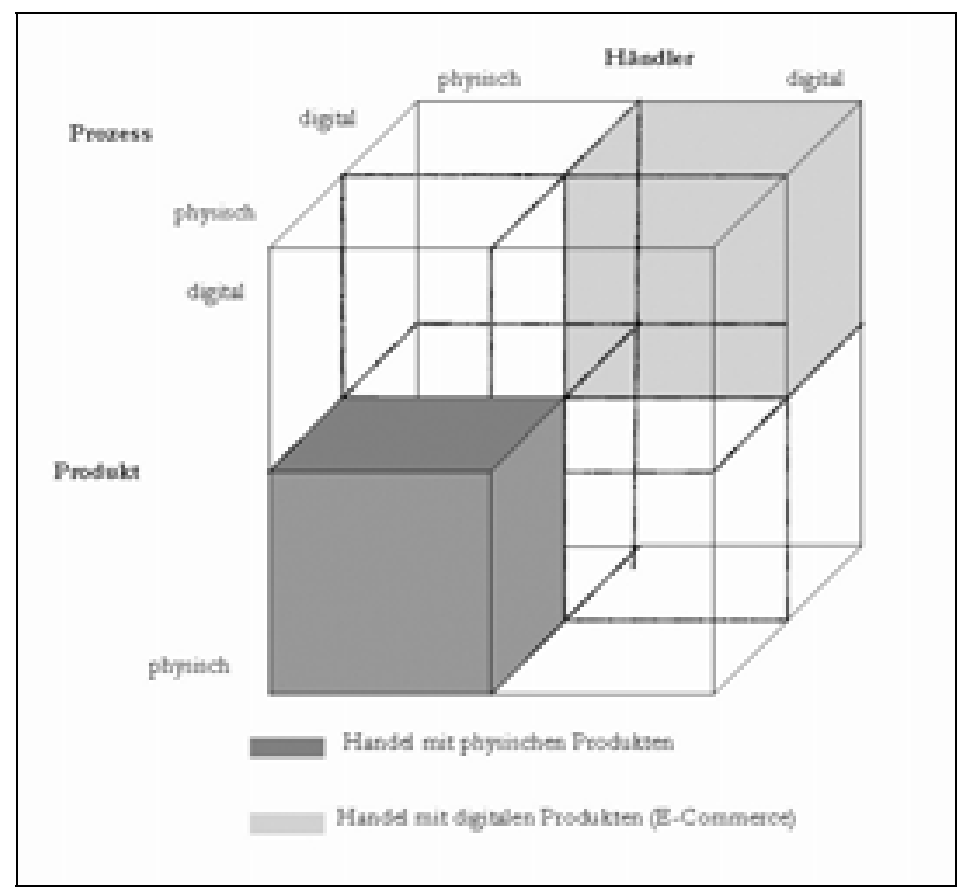

Abbildung 11: Einordnung von vollständig digitalem und traditionellem Handel (vgl. Löbbecke 1999, S. 2)

Dies bedeutet, dass neben dem gehandelten Gut (z. B. ein Musiktitel), auch der Handelsprozess (z. B. Suche, Kaufabwicklung und Distribution über das Internet) und der Makler (z. B. ein elektronisches Wertpapierhandelssystem) elektronisch sind (vgl. Abbildung 11).

Dieses Verständnis deckt sich weitgehend mit dem Begriff des Online Delivered Content (ODC), der als „data, information, and knowledge traded on the internet or other online means" (Löbbecke 1999, S. 1) verstanden wird und sich auf Online-Magazine, -Datenbanken und (Online-)Beratung, etc. bezieht. In beiden Definiti- 
onen ist die, durch die Digitalisierbarkeit bedingte, Möglichkeit der Distribution über ein Netzwerk das entscheidende Kriterium (ähnlich Brandtweiner 2000, S. 33). Dies impliziert eine Loslösung des Inhalts von einem physischen Trägermedium.

Die Definition des ODC fasst die Produktgruppen digitale Informationsgüter, digitale Güter und digitale Dienstleistungen zusammen, deren Unterschiede erklärungsbedürftig erscheinen:

Digitale Informationsgüter werden ausschließlich ihres Inhalts wegen gekauft (vgl. Brandtweiner 2000, S. 37). Das Trägermedium ist dabei unerheblich. Beispiele sind wissenschaftliche Publikationen, eBooks, Tageszeitungen und Musik in elek. tronischer Form. Digitale Güter im engeren Sinn sind immaterielle Güter, die ihrer Funktion wegen gekauft werden, der Inhalt jedoch keine oder nur eine untergeordnete Rolle spielt. Als Beispiel kann Software angeführt werden, die dem Nutzer eine gewisse Funktionalität bietet, dieser jedoch nicht an der der Funktionalität zugrunde liegenden Programmierung interessiert ist. Digitale Dienstleistungen benötigen, wie traditionelle Dienstleistungen auch, die Mitwirkung des Kunden („externer Faktor"). Darin unterscheiden sich die digitalen Dienstleistungen von den digitalen Produkten und digitalen Informationsgütern. Beispiele hierfür sind Beratungsdienste z. B. von Versicherungen und Banken, die online geführt werden. Ohne die Angabe der Kundenwünsche dürfte eine sinnvolle Beratung nicht möglich sein.

Im Folgenden wird der Fokus der Untersuchung auf die digitalen Informationsgüter und die digitalen Güter gelegt, die unter dem Term „digitale Produkte“ subsumiert werden. Sie weisen einige charakteristische Eigenschaften auf (vgl. Luxem 2001, S. 24; Brandtweiner 2000, S. 34):

Erstens sei die Nicht-Abnutz̧barkeit genannt. Dies bedeutet, dass digitale Güter keinerlei Abnutzung unterliegen, wie es üblicherweise bei physischen Produkten zu beobachten ist. Die Unterscheidung zwischen neuem und altem (z. B. Second Hand) Produkt entfällt, da die Produktqualität mit der Zeit nicht abnimmt.

Zweitens ist die vergleichsweise einfache Veränderbarkeit von digitalen Produkten hervorzuheben. Diese bezieht sich jedoch nicht auf Abnutzung durch Benutzung, sondern auf willentliche Veränderungen wie z. B. die gezielte Veränderung von Programmcode. Dadurch wird es möglich, mit geringem Aufwand Produktvarianten zu erstellen, die neue Nachfrage auf dem Absatzmarkt nach sich ziehen.

Drittens ist die einfache Reproduzierbarkeit von digitalen Produkten zu nennen. In der Literatur wird in diesem Zusammenhang von „first copy costs“ gesprochen, also jenen Kosten, die bei der Erstellung des ersten Exemplars des digitalen Produktes entstehen (vgl. Varian 1998, S.5; Brandtweiner 2000, S.146; Grimm 2003, S. 94). Die Grenzkosten für die Erstellung von weiteren Kopien des digitalen Produktes sind, im Gegensatz zu physischen Produkten, vernachlässigbar gering 
(bzw. tendieren gegen Null) (vgl. Grimm 2003, S. 96). Ökonomisch bedeutet dies, dass sich eine Fixkostendegression einstellt, da sich alle hergestellten Stücke nur noch die Produktionskosten teilen und daher die Kosten pro Stück drastisch abnehmen („economies of scale“). Dieser Effekt ist bei digitalen Gütern umso gröBer, da die Investitionen in Produktionsanlagen für digitale Produkte im Gegensatz zu Anlagen für die Herstellung physischer Produkte relativ gering sind. Da (fast) keine Kapazitätsgrenze existiert, entfallen zudem noch weitere Investitionen, die sonst bei einer Ausweitung der Produktion bei physischen Produkten notwendig wären.

Durch diese Eigenschaften ergibt sich eine Reihe von ökonomischen Fragestellungen. Zunächst muss geklärt werden, ob digitale Produkte als knappe, private und damit marktfähige, oder als öffentliche bzw. freie Güter einzuordnen sind. Für eine Einordnung als freie Güter spricht die einfache Reproduzierbarkeit, die es ermöglichen sollte, die Nachfrage nach einem Gut zu einem gegebenen Zeitpunkt zu befriedigen. Es entsteht also kein Knappheitseffekt, wie dies bei den meisten physischen Gütern der Fall ist. Für eine Einordnung als öffentliches Gut spricht, dass die Nutzung des Gutes unabhängig von der Anzahl der Nutzer ist, bzw. die Nutzung des Gutes durch eine Person nicht die Nutzung des Gutes durch eine andere Person einschränkt („Nichttrivalität beim Konsum“). Damit digitale Produkte marktfähig werden, d.h. den Charakter von knappen und privaten Gütern annehmen, erscheint eine unterstützende Gesetzgebung (z. B. Urheberrechte) und die Durchsetzung dieser Rechte notwendig.

Ein anderer Aspekt betrifft die Bestimmung der Angebotsmenge für digitale Produkte. Die mikroökonomische Theorie unterstellt bei einem degressiven Kostenverlauf, dass das Gewinnmaximum einer Unternehmung in der Kapazitätsgrenze liegt. Da diese durch die Eigenschaft der einfachen Reproduzierbarkeit digitaler Güter jedoch wegfällt, ist die Theorie auf digitale Güter nicht anwendbar.

Der Markt für digitale Produkte muss folglich als unvollkommen angesehen werden (vgl. dazu ausführlich Varian 1998), da der Preis für digitale Produkte über deren Grenzkosten liegt, staatliche Eingriffe notwendig und die digitalen Produkte nicht homogen sind (für Merkmale eines unvollkommenen Marktes vgl. Kortmann 2002).

\section{2 Überblick über Distributionstechnologien}

Distributionsarchitekturen für digitale Inhalte lassen sich allgemein in ,infrastucture-based“ und „Peer-to-Peer" Content Distribution unterteilen (vgl. Padmanabhan et al. 2002, S. 1). Erstere fügt sich in das traditionelle Client/Server-System ein, indem sie die zu verteilenden Inhalte auf einem oder mehreren zentralen Server(n) vorhält, von dem die Inhalte vom Benutzer bei Bedarf abgerufen werden. 
Absatz digitaler Produkte und Digital Rights Management

Letztere funktionieren in der Regel ohne Zentralinstanz, da die Inhalte auf den teilnehmenden Peers verteilt vorgehalten werden („Peer-provided Content") (vgl. Miller 2001, S. 20). Die Verschiedenartigkeit der Konzepte lässt vermuten, dass die Modelle sich in ihren Anwendungsgebieten und den eingesetzten Technologien unterscheiden.

\section{Distributionstechnologien in der zentralen Architektur}

Ein Beispiel für eine Technologie im Rahmen der zentralen Architektur stellt „Streaming Media“ dar, welche sich wie folgt beschreiben lässt: „Der Begriff Streaming Media bezeichnet eine Internet-Technologie, bei der Filme oder Sprache bzw. Musik in Echtzeit direkt aus dem Inter- oder Intranet abgespielt werden können" (Wegner/Bachmeier (2000), S. 13). Hierbei werden die Multimediadaten (z. B. Audio- und Videodaten) als stetige Datenströme (Streams) erzeugt und verarbeitet. Die Daten liegen hierbei auf den zentralen Servern, die sie auf Anfrage von Nutzern bereitstellen. Streaming stellt hohe Anforderungen an die Hardware (insbesondere Bandbreite) und kann selbst die Kapazität großer Server schnell erschöpfen (vgl. Gehrke et al. 2003, S. 2). Dies resultiert vor allem daraus, dass Streaming Media an besondere Anforderungen hinsichtlich der Servicequalität (Quality of Service, QoS) gebunden ist (vgl. dazu Coulouris et al. 2002, S. 714):

Bandbreite: Unter Bandbreite versteht man die Geschwindigkeit mit der ein Multimedia-Stream transportiert wird. Die Bandbreite sollte bei der Distribution ausreichend und kontinuierlich hoch sein, um eine flüssige Übertragung zu gewährleisten.

Laten₹: Hierunter wird die Transportlaufzeit verstanden, also die Zeit, die ein Stream zwischen Sender und Empfänger unterwegs ist. Die Latenz von Multimedia-Streams sollte möglichst klein sein und nicht zu stark schwanken.

Verlustrate: Die Verlustrate gibt an, wie viel Datenelemente verworfen werden, da sie nicht in der geplanten Auslieferungszeit den Empfänger erreicht haben und somit wertlos sind. Um Verlustraten möglichst gering zu halten, sind ausreichend große Zwischenspeicher (Buffer) vorzuhalten, welches häufig eine nicht akzeptable Inanspruchnahme von Ressourcen auf Clientseite darstellt.

Insbesondere um die Beanspruchung der Bandbreite (ca. 120 Mbps für einen TV-Video-Stream) während der Übertragung zu reduzieren, ist es üblich, Streams vor dem Versand zu komprimieren. Dies geschieht mit Hilfe so genannter Codecs (kurz für compression/decompression), die Informationen über die Verarbeitung und Ausgabe der Streamdaten beinhalten und die Komprimierung auf Senderund Empfängerseite vornehmen (vgl. Abb. 12). 


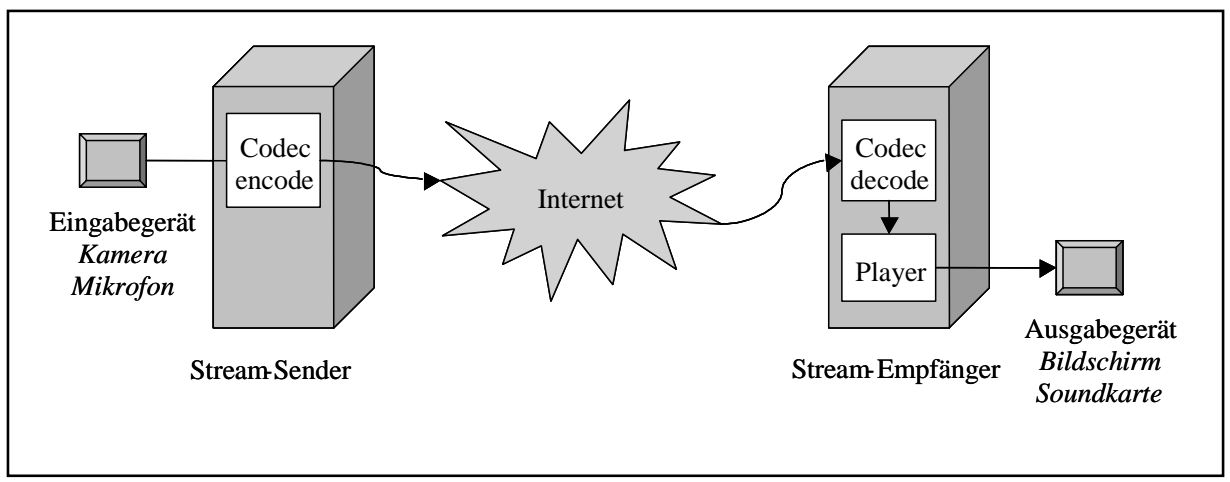

Abbildung 12: Versand eines Multimedia-Streams (vgl. Gehrke et al. 2003, S. 4)

Gängige Medienformate wie MPEG-1,2,4 (Motion Picture Expert Group), AVI (Audio Video Interface), MOV (Apple Quicktime), DivX im Videobereich sowie MPEG-3 und AAC (Advanced Audio Coding) im Audiobereich, können so die Bandbreitenanforderungen um den Faktor 10 bis Faktor 100 reduzieren. Dies wird allerdings mit erhöhtem Rechenbedarf für die Kodier- bzw. Dekodiervorgänge bei Versand und Wiedergabe erkauft. Dies geschieht i.d.R mit Hilfe eines meist properitären Audio-Video-Players (Quicktime Player, Realplayer, DivX Player oder Windows Media Player), der das Abspielen von Audio- und Videodateien ermöglicht.

Für die Übertragung selbst werden spezielle, meist auf TCP/IP oder UDP/IP aufsetzende Protokolle benutzt. Diese Notwendigkeit ergibt sich, da TCP/IP in Ermangelung geeigneter Mechanismen zur Bandbreitenreservierung die nötige Servicequalität nicht bereitstellen kann (ähnlich Coulouris et al. 2002, S. 99). Folgende Protokolle sind im Zusammenhang mit Streaming zu nennen:

- RTP (vgl. RTP 2003 sowie Schulzrinne et al. 1996, S. 1-13): Das paket. orientierte Real Time Transport Protocol überträgt Informationen zum Typ des Streams (Audio/Video) zur Abspielzeit und weiteren Timing Anforderungen, d.h. dass es kein reines Transportprotokoll ist. Es kann jedoch die zeitgerechte Auslieferung der einzelnen Pakete eines Streams nicht garantieren, so dass die Servicequalität nicht garantiert werden kann.

- RTCP (vgl. Schulzrinne et al. 1996, S. 15 -17): Das Real Time Control Protocol kontrolliert die Übertragung von RTCP-Paketen in einer RTPSession und ermöglicht Feedback vom Empfänger an den Sender. Dies bedeutet, dass mit Hilfe von RTCP Informationen über den Datenfluss ausgetauscht werden.

- RTSP (vgl. RTSP 2004): Das Real Time Streaming Protocol ist ein IETF Proposed Standard. RTSP ist auf der Applikationsebene angesiedelt, auf 
der es Multimedia-Streams eröffnet und kontrolliert. RTSP bietet Funktionen, die im Zusammenhang mit Streaming-Systemen von Bedeutung sind. Dazu zählen das Zwischenspeichern (Caching) von Daten (ähnlich wie bei HTTP), das Wechseln des Servers innerhalb eines Streams (load balancing) und Synchronisation verschiedener Streams von mehreren Servern.

- RSVP (vgl. RSVP 2004): Das Real Time Reservation Protocol ist für die Reservierung von Bandbreiten zuständig. Dafür wird auf den Routern, die zwischen Sender und Empfänger liegen, die benötigte Bandbreite reserviert, so dass eine bestimmte Servicequalität garantiert werden kann. Einschränkend ist zu bemerken, dass die bisher im Internet eingesetzten Router die Ressourcenreservierung nicht unterstützen (vgl. Gehrke et al. 2003, S. 5; Ripeanu et al. 2003, S. 6).

Die vorgestellten Protokolle arbeiten dergestalt zusammen, dass per RTSP die Verbindung geöffnet wird, dann mittels RTP oder RTCP die Übertragung vorgenommen wird. RSVP würde, wenn es zum Einsatz kommt, die Servicequalität durch die Reservierung benötigter Bandbreite gewährleisten. Der Durchbruch der Streaming Technologie wird in diesem Jahr erwartet, da dann die Anzahl der Breitbandanschlüsse genügt, um Skaleneffekte auf Seiten der Streaminganbieter realisieren zu können (vgl. Lainee et al. (2002), S. 3). Dementsprechend erwartet man einen Anstieg des Traffic, der durch „Rich Media“-Anwendungen (zu denen Streaming zählt) hervorgerufenen wird (vgl. Abb. 14).

Neben Streaming zählt natürlich auch das Beziehen von Inhalten jeglicher Art (z. B. per Download oder Email) von einem zentralen Anbieterserver zu den zentralen Distributionstechnologien. Die Anforderungen an Hardware und Bandbreite sind jedoch bei weitem nicht so hoch wie bei Streaming Media. Beispielsweise spielt beim Download einer Softwaredatei die Reihenfolge des Eintreffens der einzelnen Datenpakete keine Rolle, da die Software vor dem vollständigen Herunterladen ohnehin nicht verwendungsfähig ist. Daher werden in diesem Zusammenhang meist keine speziellen Protokolle (sondern TCP/IP oder FTP) verwendet, weshalb an dieser Stelle nicht darauf eingegangen werden soll.

\section{Technologien in der dezentralen Architektur}

Als dezentrale Distributionstechnologie ist z. B. das Peer-to-Peer-Filesharing zu nennen. Die Popularität von Filesharing-Applikationen war mit Beginn ihres Aufkommens sehr hoch, daher wird der Traffic nach Schätzungen in naher Zukunft nur marginal ansteigen. Beim Filesharing liegen die Inhalte auf den teilnehmenden Peers verteilt. Jedes Peer stellt Funktionen zum Dateiversand, Empfang und zur Dateisuche bereit, ist also Client und Server zugleich. In dieser Architektur erhöht sich, durch den Wegfall einer zentralen Koordinationsinstanz hervorgerufen, der 
Koordinationsaufwand zwischen Peers und damit die Belastung des Netzes (vgl. dazu Messungen im Gnutella Netz von Ripeanu et al. 2003, S. 6-7, Barkai (2002), S. 278). Das Problem im Gnutella-Netz lag in dessen Kommunikationsprotokoll begründet, welches durch ineffiziente Suchalgorithmen die Bandbreite überbeanspruchte.

Neuere Protokolle, wie z. B. Chord, implementieren allerdings Algorithmen, die Suchanfragen sehr effizient in logarithmischer Laufzeit durchführen und so die Belastung des Netzes minimieren (vgl. Stoica et al. 2002). Allgemein lassen sich die aktuellen Peer-to-Peer-Kommunikationsprotokolle in properitäre und offene Protokolle unterteilen (vgl. Tabelle 1).

Tabelle 1: Übersicht gängiger Peer-to-Peer-Protokolle

\begin{tabular}{|l|l|l|l||}
\hline Protokoll & properitär & offen & Client-Anwendung \\
\hline Napster & $\mathrm{X}$ & & Napster \\
Fasttrack & $\mathrm{X}$ & $\mathrm{X}$ & KaZaa \\
JXTA & & JXTA \\
Gnutella & & $\mathrm{X}$ & Gnutella \\
OpenFT & & $\mathrm{X}$ & beliebiger Client \\
Jabber & & $\mathrm{X}$ & beliebiger Client \\
eDonkey & $\mathrm{X}$ & & eDonkey \\
WinMX & $\mathrm{X}$ & & WinMX \\
\hline \hline
\end{tabular}

Während der Quellcode der properitären Protokolle nicht öffentlich und an bestimmte Client-Applikationen gebunden ist, sind die Protokollspezifikationen der offenen Protokolle frei zugänglich.
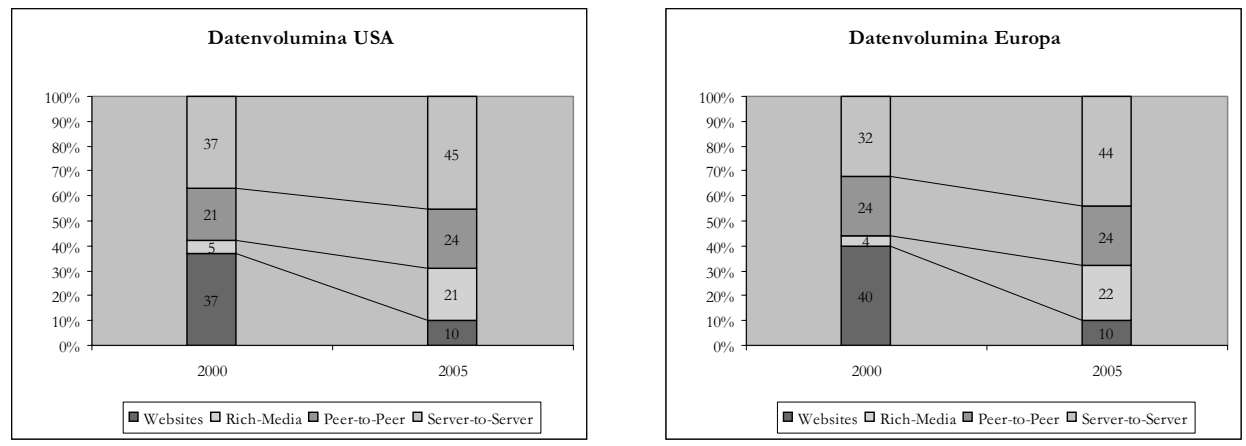

Abbildung 13: Übersicht über Traffic-Aufkommen in Europa und USA entnommen aus Lainee et al. (2002), S. 2 
Absatz digitaler Produkte und Digital Rights Management

Neben der zusätzlichen Bandbreite, die durch den erhöhten Koordinationsaufwand hervorgerufen wird, sind auch die Bereiche Sicherheit, Vertrauen und Urheberrechtsverletzungen (vgl. Kap. 3) Problembereiche, da sich die dezentralen Strukturen schlechter kontrollieren lassen als ein zentralisiertes System. Einige Autoren sehen in der schweren Kontrollierbarkeit von P2P-Strukturen durch Autoritäten aber auch gerade den Vorteil von P2P-Distribution (vgl. Miller 2001, S. 4-14; 25).

Vor dem Hintergrund der Entwicklung von P2P-Anwendungen zu einem kommerziell anerkannten, d.h. legalen Distributionskanal für digitale Inhalte ist fraglich, ob die mangelnde Kontrollierbarkeit dieser Entwicklung förderlich ist. Dass die beiden genannten Distributionsarchitekturen und die eingesetzten Technologien keine Antagonisten sind, zeigen Bestrebungen, Peer-to-Peer Umgebungen zum Verteilen von Streaming Media Datenströmen zu nutzen (vgl. z. B. Gehrke et al. 2003 sowie Padmanabhan et al. 2002).

\section{Grundlegende Erlösmodelle der Distribution digitaler Produkte}

Ein Erlösmodell ist der Teil einer modellhaften Beschreibung eines Geschäfts (Geschäftsmodell), in dem dargelegt wird, aus welchen Quellen die Einnahmen des Unternehmens generiert werden (vgl. Stähler 2001, S. 41). Die Arbeit konzentriert sich auf Erlösmodelle, da im Rahmen dieses Beitrags die Distribution erörtert wird und somit z. B. das die vorgelagerte Wertschöpfungsstufe beschreibende Produktionsmodell außer Betracht gelassen werden kann.

Aufgrund der etablierten Marktstrukturen besteht hinsichtlich der branchenspezifischen Geschäftsmodelle oft nur wenig Spielraum (vgl. Luxem 2001, S. 77). Die oben erläuterten technischen Möglichkeiten der Distribution digitaler Produkte (vgl. 2.2) eröffnen den Produzenten eine Chance, aus diesen etablierten Marktstrukturen auszubrechen und neue Erlösformen zu entwickeln, die erst durch digitale Produkte ermöglicht werden (z. B. Micropayment für Anzeigen und Inhalte im Internet, Pay-per-View-Modelle im digitalen Fernsehen). Allgemein können direkte und indirekte Erlösformen unterschieden werden, wie nachfolgend dargestellt wird. Aufgrund der Eigenschaften digitaler Produkte, insbesondere der einfachen Reproduzierbarkeit (vgl. 2.1.2) und der daraus resultierenden Möglichkeit zur kostengünstigen Distribution, wird angenommen, dass ein großer Teil der digitalen Produkte kostenfrei erhältlich sein wird (vgl. Dyson 1995, S.1-2, Grimm 2003, S. 96). Dies bringt die Anbieter dazu, einen Großteil des Erlöses über nichtdigitale Güter und Dienstleistungen, wie Beratung, Softwaretraining (sofern dieses nicht über Internet erfolgt) und kostenpflichtige Suchmaschinen zu erbringen, da diese nicht den Kostenstrukturen digitaler Produkte unterliegen (vgl. 2.1.1) und man sich dadurch eine Generierung von Erlösen erhofft. 


\subsection{Direkte Erlösmodelle}

Im Rahmen von direkten Erlösmodellen werden die Erlöse aus dem Verkauf der digitalen Produkte generiert. Als Beispiele hierfür sind der Vertrieb von Lizenzen, die volumenabhängige Abrechnung und Abonnements zu nennen. Beim Vertrieb von Lizenzen werden Nutzungsrechte an verschiedenen Varianten des digitalen Produkts an die Nutzer verkauft. Hierbei sind (teilweise aufwendige) Mechanismen zu installieren, die den Missbrauch der Lizenzen durch Unautorisierte verhindern sollen. In dem Modell der Superdistribution (vgl. zur Superdistribution im Kontext von Peer-to-Peer Rosenblatt et al. 2002, S. 29) werden die digitalen Produkte zwar über eine unsichere öffentliche Infrastruktur schnell und einfach verteilt, idealerweise kann die Nutzung aber erst erfolgen, wenn man einen zum Produkt passenden Schlüssel (Lizenz) vom Hersteller oder einem Clearing Center gegen Entgelt bezogen hat.

Somit wird eine schnelle Verbreitung der Produkte gewährleistet, ohne die Kontrolle der Nutzung aufzugeben (vgl. o.V. 1998). Die Komplexität der Lizenzmodelle reicht von den einfachen Pay-per-view bzw. Pay-per-use-Modellen (z. B. für Videofilme), die nur variable Kosten beinhalten, bis hin zu Modellen, die die Nutzung von Produkten mehrfach und zeitabhängig erlauben.

Die volumenabhängige Abrechnung stellt dem Nutzer den Umfang des von ihm genutzten Produkts in Rechnung. Der Umfang lässt sich z. B. anhand des Datenvolumens, der Anzahl unterschiedlicher Produkte oder der Anzahl von Abfragen quantifizieren. Im Gegensatz zum Lizenzmodell erfolgt in diesem Fall die Abrechnung nicht für ein einzelnes Produkt, sondern über ein, oft zeitraumbezogenes, Produkt- bzw. Leistungsbündel. Dies setzt die detaillierte Protokollierung der Inanspruchnahme sowie die Verwaltung von Kundenkonten voraus (vgl. Luxem 2001, S. 81).

Als ein von traditionellen Medien (insbesondere Printmedien) bekanntes Erlösmodell wurde das Abonnentenmodell in die Distribution digitaler Produkte integriert. Als einzigem der hier vorgestellten Erlösmodelle liegt dem Abonnentenmodell eine dauerhafte Geschäftsbeziehung zwischen Anbieter und Nutzer des digitalen Produktes zugrunde. Der Abnehmer (Abonnent) zahlt für die Nutzung zuvor determinierter Leistungen des Anbieters eine feste Gebühr pro Abrechnungszeitraum. Das Maß der Inanspruchnahme der Leistungen durch den Abonnenten ist dabei für die Abrechung unerheblich. Der Vorteil dieses Modells liegt in der Einfachheit der Abrechnung sowie der Regelmäßigkeit der Zahlungseingänge (vgl. Luxem 2001, S. 82). Weiterhin ist aufgrund der dauerhaften Beziehung zu identifizierbaren Abnehmergruppen eine gute Möglichkeit zur Personalisierung des Angebots gegeben, sofern vom Anbieter neben den Stammdaten auch Profile der Abnehmer generiert und verwaltet werden. In der Personalisierung (z. B. Begrenzung des Abonnements auf bestimmte Themengebiete) besteht die eigentliche 
Neuerung der Übertragung des Abonnentenmodells auf digitale Produkte, da eine Personalisierung von z. B. physischen Printmedien nicht wirtschaftlich zu realisieren war.

\subsection{Indirekte Erlösmodelle}

In den Bereich der indirekten Erlösmodelle, bei denen die Erlöse nicht direkt aus dem Verkauf des produzierten digitalen Produkts generiert werden, fällt die Angebotsfinanzierung durch Werbung. Bei diesem Modell versucht der Anbieter seine Kosten durch Werbeeinnahmen zu decken. Hierbei steht sein Angebot auf dem Werbe- und Anzeigenmarkt in Konkurrenz zu anderen Anbietern, die ebenfalls im Wettbewerb um Werbekunden stehen.

Um die Attraktivität eines digitalen Angebots zu messen, werden neben Website-Besuchen (Hits) auch die Zahl der Kunden sowie ggf. auch deren soziodemographische Merkmale herangezogen. Am attraktivsten für einen Werbekunden ist folglich das Angebot, das ihm eine garantierte Zahl von Nutzern mit den von ihm gewünschten Merkmalen bereitstellt. Die Erlöse können auf unterschiedliche Weise generiert werden. Zum einen kann mit den Werbekunden ein Festpreis für die Einblendung im Angebot des Anbieters ausgehandelt werden. Der Festpreis hängt, wie oben erwähnt, von der Besucherzahl und der Attraktivität der Nutzer als Zielgruppe für den Werbekunden ab. Zum anderen kann eine variable Bezahlung vereinbart werden, die z. B. sich nach der Anzahl der Besuche des Angebots des Werbekunden bemisst, die über die geschalteten Werbebanner im Angebot des Anbieters vermittelt wurden. Nachteil dieses Modells ist die Tatsache, dass man mit seinem Angebot nicht nur auf einem Rezipientenmarkt, sondern zusätzlich noch auf einem Werbe- und Anzeigenmarkt im Wettbewerb steht („doppelter Markt $^{\circ}$ ) (vgl. Schumann / Hess 2000, S. 20). Hierzu ist zu bemerken, dass der Erfolg auf dem Rezipientenmarkt oft auch eine hohe Attraktivität auf dem Werbeund Anzeigenmarkt nach sich zieht, da eine entsprechend große Nutzerschaft in der Regel auch eine attraktive Zielgruppe für Werbemaßnahmen des Massenmarketing darstellt.

Durch eine entsprechend große Nutzerschaft wird ein weiteres indirektes Geschäftsmodell ermöglicht, das als „Erlöse aus dem Verkauf von demographischen Daten“ beschrieben werden kann. Die Erlöse werden hierbei durch den Verkauf von anbieterseitig gesammelten demographischen Daten der Nutzer an z. B. Marktforschungsinstitute generiert. Der Anbieter tritt hier als so genannter Infomediär auf (vgl. z. B. Rose 1999, S. 164ff.; 173). Bei dem Verkauf sind nationale juristische Beschränkungen zu beachten, so dass nicht jede Kombination von Daten rechtmäßig verkauft werden kann. 
Das Content Owner Sponsoring (COS) stellt ein neueres Geschäftsmodell dar (vgl. Clarke 1999). Der Besitzer bzw. Hersteller des digitalen Produkts bezahlt für die Distribution seines Produkts, um einen möglichst hohen Verbreitungsgrad zu erreichen. Dies ist immer dann sinnvoll, wenn man erwartet, dass das digitale Produkt erhebliche positive Netzeffekte auslösen kann. Beispielsweise kann ein Hersteller sein Softwareprodukt bei upload.com (vgl. http://wwww.upload.com) gegen eine Gebühr registrieren lassen.

Tabelle 2: direkte und indirekte Erlösmodelle

\begin{tabular}{||l|l|l|l||}
\hline \hline \multicolumn{2}{||l||}{ Direkt } & \multirow{2}{*}{ Indirekt } \\
\hline $\begin{array}{l}\text { nutzungäng- } \\
\text { hängig }\end{array}$ & nutzungsunabhängig & \\
\cline { 2 - 4 } & einmalig & wiederholend & \\
\hline $\begin{array}{l}\text { Pay-per-View } \\
\text { Pay-per-Use }\end{array}$ & $\begin{array}{l}\text { einmalig zu } \\
\text { erwerbende } \\
\text { Lizenzen }\end{array}$ & $\begin{array}{l}\text { Abonnement } \\
\text { bzw. zeitraum- } \\
\text { bezogene Li- } \\
\text { zenzen }\end{array}$ & $\begin{array}{l}\text { Content Owner Sponsoring } \\
\text { Verkauf von Nutzerdaten } \\
\text { Werbe-finanzierte Angebote }\end{array}$ \\
\hline \hline
\end{tabular}

Die Software steht dann auf beliebten Websites (z. B. CNET Networks' download library mit ca. 2,5 Mio. Zugriffen täglich) zum Herunterladen bereit. Diesen Weg beschritt Adobe (http://www.adobe.com) mit dem Produkt „Adobe Acrobat Reader", der kostenlos erhältlich ist und das Portable Document Format (PDF) zum De-facto-Standard für den Dokumentenaustausch etabliert hat. Im Zuge dessen wurde die Software „Adobe Acrobat“ zum Erstellen von PDF-Dokumenten ein kommerzieller Erfolg. Tabelle 2 fasst die vorgestellten Geschäftsmodelle überblicksartig zusammen.

\section{Digital Rights Management}

Im Rahmen der Distribution digitaler Produkte hat die Frage des Urheberechtschutzes an Bedeutung gewonnen. Insbesondere das P2P-Filesharing unterläuft diesen systematisch und hat bereits zu Einbußen in der Musikindustrie geführt. Aus diesem Grund ist diese sehr stark an funktionsfähigen DRM-Systemen (DRMS) interessiert, die es ihr erlaubt, ihre bisherigen Geschäftsmodelle auch in einer digitalen Umwelt durchzusetzen. Aber auch in Business-to-Business (B2B)Bereichen dürften DRMS künftig stärker zum Einsatz kommen.

In diesem Abschnitt wird zunächst der Begriff „Digital Rights Management“ (kurz: DRM) erläutert. Anschließend werden Anforderungen an DRM und die aktuell eingesetzten Technologien vorgestellt. 


\subsection{Begriffsklärung}

Die Definitionen von DRM in der Literatur stellen sich recht heterogen dar. Während ein Autor DRM als ,type of server software developed to enable secure dis. tribution - and perhaps more importantly to disable illegal distribution - of paid content over the Web [...]“ (O.V. (2002), S. 1) definiert, fasst Grimm den Begriff weiter und stellt fest:

„unter DRM versteht man Verfahren, die helfen Rechte an digitalen Waren so zu schützen, wie wir das von den an physische Medien gebundenen intellektuellen Erzengnissen her gewöhnt sind. Kopie und Weitergabe sollen an die Regeln des Rechteinhabers, also des W arenanbieters (Content Provider) gebunden sein" (Grimm (2003), S. 97).

Diese Definition präzisierend führt Ianella an: „DRM covers the description, identification, trading, protecting, monitoring and tracking of all forms of usages over both tangible and intangible assets [...]” (Ianella 2001, S. 1) oder „Rights Management that uses digital technology and applies to intellectual property in digital form“. In diesem Zusammenhang ist Rights Management zu verstehen als: „,business processes that for legal and commercial purposes track rights, rights-holders, licenses, sales, agents, royalties and associated terms and conditions" (Rosenblatt et al. 2002, S. 4).

Der ersten Definition ist allerdings zu bescheinigen, dass sie zu kurz greift, da sie im Gegensatz zu der von Ianella das Umfeld, in welchem DRM eingesetzt wird, außer Acht lässt. DRM kann bzw. muss (in verschiedenen Varianten) auf allen Stufen der Wertschöpfungskette, von der Produktion des digitalen Gutes bis zu dessen Bezahlung und während der anschließenden Benutzung eingesetzt werden, wie dies in der Definition von Grimm anklingt. In anderen Worten: DRM umfasst alle Handlungen die jemand mit dem jeweiligen Inhalt im Rahmen von Distribution und Nutzung vornimmt (vgl. Rump 2003, S. 4, Rosenblatt et al. 2002, S. IX). $\mathrm{Zu}$ der Definition von Ianella ist ergänzend anzumerken, dass der Rechteinhaber weitere Distributoren zwischenschalten kann, die im Rahmen der ihnen verliehenen Rechte weitere verschiedene Rechte für den Inhalt definieren können. Es ist denkbar, dass der Rechteinhaber einem Distributor die Kopie und Weitergabe für einen Inhalt überträgt, dieser jedoch in Abhängigkeit von der Kundengruppe (Geschäfts- oder Privatkunden) die ihm übertragenen Rechte weiter einschränkt. Der Warenanbieter determiniert also nicht in jedem Fall die Regeln allein.

Aus den obigen Feststellungen lassen sich generische Aufgabenbereiche für DRM ableiten. Zum einen das Verwalten von digitalen Rechten, zum anderen das Durchsetzen von Urheberrechten mit digitalen Mitteln. In den erstgenannten Aufgabenbereich fallen die Identifizierung des Inhalts (zur eindeutigen Zuordnung zu Rechteinhabern), das Sammeln von Metadaten (zum Auffinden des ge- 
wünschten Inhalts durch den Benutzer), sowie das Entwickeln von Geschäftsmodellen für den Vertrieb der Inhalte.

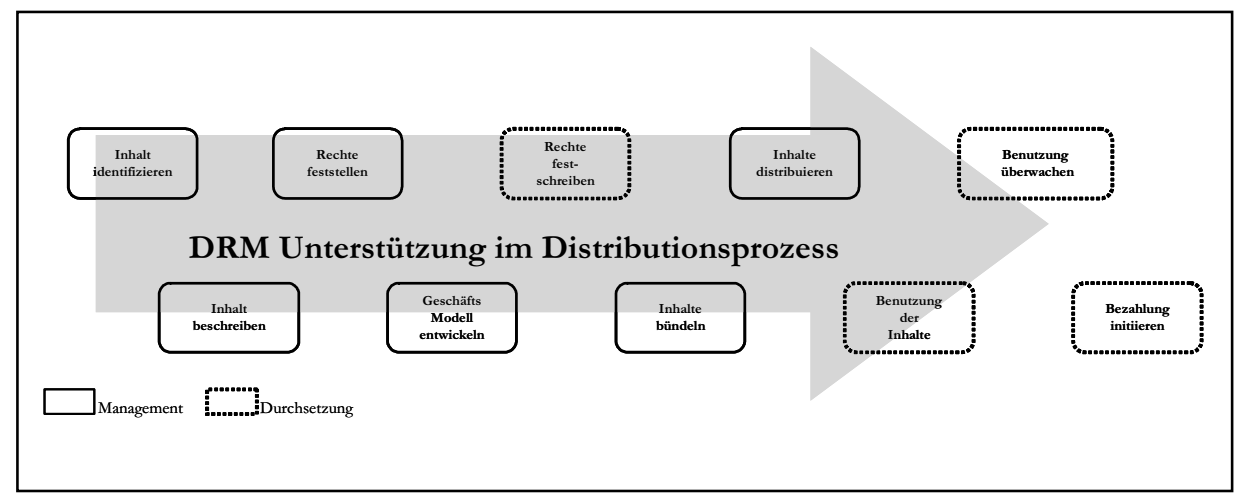

Abbildung 14: Schritte in der Distribution von Inhalten und DRM-Unterstützung (in Anlehnung an Rump 2003, S. 4)

Abbildung 14 zeigt die Schritte in der Distribution von digitalen Produkten, die von DRM Systemen unterstützt werden müssen und ordnet sie dem entsprechenden Aufgabenbereich zu.

In den Bereich der digitalen Durchsetzung von Urheberrechten fällt das Kontrollieren der Benutzung und Weitergabe des Inhalts durch Nutzer und Händler. Das heißt, dass ein DRM die Verwendung gemäß den Vorgaben des Rechteinhabers ermöglichen muss, andere Verwendungen und Manipulationen jedoch strikt zu verhindern hat.

\subsection{Anforderungen an DRM-Systeme (DRMS)}

Aus den oben identifizierten Aufgabenbereichen lassen sich zunächst einige grundlegende (vgl. zu grundlegenden Anforderungen Gooch 2003, S. 5 ff.) Anforderungen an DRMS ableiten (für einen umfangreichen Anforderungskatalog für Forschung und Bildung vgl. Martin et al. 2002).

Aus der Notwendigkeit zur begleitenden Unterstützung der gesamten Distributionskette resultiert die Forderung nach Interoperabilität von DRMS. Dies bedeutet, dass bestimmte Funktionen des DRMS auf den verschiedensten Plattformen, vom Multimediaserver beim Content Provider bis zum Handheld beim Endnutzer, zur Verfügung stehen müssen, um eine kontrollierte Distribution zu garantieren. Weiterhin muss es für Nutzer möglich sein, den Inhalt auf verschiedenen Endgeräten zu verwenden (z. B. im Netzwerk zu Hause), ohne dass die Inhalte sofort der unkontrollierten Distribution per Internet zur Verfügung stehen. Da 
bisherige Geräte nur Software- und kaum bzw. keine Hardware-Unterstützung für DRM bieten, ist DRM zunächst auf den PC-Bereich beschränkt. Die Interoperabilität kann daher als derzeit nicht befriedigend gelöstes Problem angesehen werden, der Einführungsprozess der Hardware-Unterstützung steht erst am Anfang.

Weiterhin ist anzunehmen, dass DRMS - wie heutiger Kopierschutz auch Ziel von Attacken sein wird. Da die Attackierungsmöglichkeiten sich ebenfalls über die gesamte Distributionskette erstrecken, müssen DRM besonders hohe Standards in punkto Sicherheit erfüllen.

Ziel dieser Attacken ist meist der unbefugte Zugriff (,injection“) (vgl. dazu Biddle et al. 2003, S. 352) auf den Inhalt unter Umgehung des Kopierschutzes bzw. der Zugriffsrechte. Dies ist zu verhindern, da bereits eine unerlaubte Kopie des Inhalts in einem Netzwerk ausreicht, um eine große Anzahl von Raubkopien zu ermöglichen. Dies ist unter anderem auf die einfache Reproduzierbarkeit und nahezu kostenfreie Distribution digitaler Güter zurückzuführen (vgl. 2.1.2). Sicherheitsexperten haben daher folgende Kriterien identifiziert (vgl. Gooch 2003, S. 21), die jedes DRMS erfüllen sollte:

1. es darf nicht auf einem globalen Geheimnis (z. B. einem allgemeingültigen Schlüssel) beruhen,

2. ein Versagen einzelner Systemteile darf nicht zur Unsicherheit des Gesamtsystems führen (d.h. kein „Single Point of Failure") sowie

3. es besitzt die Fähigkeit, nach einem erfolgreichen Angriff die Sicherheitsmechanismen zu erneuern.

Eine weitere Anforderung an ein DRMS ist Flexibilität. Sie resultiert aus den verschiedensten Laufzeitumgebungen auf denen DRMS eingesetzt werden sollen. Dies wird u.a. durch immer kleiner werdende mobile Endgeräte forciert (vgl. Sander 2002, S. 85). Die (im Vergleich zum PC) beschränkten Ressourcen derartiger Endgeräte sind in diesem Kontext zu berücksichtigen. Die Austauschbarkeit der Schutzmechanismen (z. B. Verschlüsselungsverfahren) ist neben dem Sicherheitsaspekt ebenfalls unter dem Gedanken der Flexibilität zu sehen. Während ältere DRMS die Lizenzen mit dem Inhalt bündelten und somit inflexibel waren, setzen neuere DRMS auf die Trennung von Lizenzen und Inhalt (vgl. zu Nachteilen der Bündelung von Inhalten und Rechten Rosenblatt et al. 2002, S. 81 ff.).

Bei allen Sicherheitsanforderungen ist zu bedenken, dass ein DRM nur die Akzeptanz der Nutzer findet, wenn die Sicherheitsmechanismen nicht einschränkend wirken. Das DRMS muss dem Nutzer den Umgang mit geschütztem (,managed“) Inhalt ebenso einfach ermöglichen, wie dies z. B. in einem ungeschützten P2PNetzwerk der Fall ist. Diese Anforderung kann unter dem Stichwort Nutzerfreundlichkeit angesiedelt werden, wobei ein Zielkonflikt zwischen Sicherheit bzw. Zugriffsschutz und der Nutzerfreundlichkeit zu unterstellen ist. 


\subsection{Technische Grundlagen}

Nachdem die Anforderungen an DRM-Systeme (DRMS) definiert wurden (vgl. 3.2) sollen nun Technologien vorgestellt werden, die die Grundlage für die noch vorzustellenden DRMS bilden. Streng genommen reichen die derzeitigen Anwendungsgebiete von DRMS von einfachen registrierungspflichtigen Angeboten (z. B. bei http://www.handelsblatt.com) über zahlungspflichtige Downloadangebote (z. B. http://www.proquest.co.uk) bis hin zu eher komplexen Print-on-Demand Angeboten. Diese Angebote beschreiben Rechte, implementieren jedoch keine Mechanismen zu ihrer Durchsetzung, sondern überlassen dies traditionellen Lizenzvereinbarungen. In diesem Abschnitt soll eine generische Architektur erörtert werden, die eine kontrollierte Distribution, d.h. die Durchsetzung von Rechten mittels Technologie ermöglicht. Die dabei eingesetzten technologischen Verfahren sind Verschlüsselungsmechanismen, digitale Wasserzeichen und Sprachen zur Modellierung von Rechten.

\section{DRM-Referenz-Architektur}

Zunächst soll eine technische DRM-Referenz-Architektur (vgl. Abb. 15) in ihre einzelnen Komponenten zerlegt und erörtert werden. Die identifizierbaren Komponenten sind (entnommen aus Rosenblatt et al. 2002, S. 80-83):

\section{Inhalteserver}

Der Inhalteserver umfasst folgende drei Komponenten:

- Inhalte Repository: Hier werden die eigentlichen Inhalte und die sie beschreibenden Metadaten (z. B. interne Revisionsnummern o.ä.) vorgehalten. Die Inhalte können entweder schon in einem DRM-Format zur Distribution bereitstehen oder können auf Abruf in ein solches übertragen werden. Es ist hierbei denkbar, dass DRMS die simultane Unterstützung mehrerer Distributionskanäle vorsehen (z. B. Print und Internet).

- Produktinformationen: Daten zu den einzelnen Produkten des Anbieters wie z. B. Preise, Marketingdaten, Formate, etc. sind hier abgelegt. Es bietet sich eine Integration von DRMS mit Shopsystemen an, so dass Eingaben in das Shopsystem direkt auch im DRMS verfügbar sind.

- DRM Packer: Die Vorbereitung des Inhalts für die Distribution findet hier statt, d.h. er wird in ein DRMS-konformes Format übersetzt. Dies kann entweder vor Einlagerung des Inhalts in das Inhalte Repository stattfinden oder bei Abruf des Inhalts („on the fly“). Zusätzlich zum eigentlichen Inhalt enthält das fertige Paket noch DRMS bezogene Metadaten. Dazu gehören z. B. eine eindeutige Paketnummer zur Identifikation, Informati- 
onen zum Auffinden des Inhalts sowie die Spezifikation zum Umgang mit dem Inhalt. Eine zweite wichtige Aufgabe des DRM Packers ist also die Definition von Rechten, die der Anbieter dem Nutzer einräumen will.

\section{Lizenzserver}

Die Informationen über den Nutzer oder das Endgerät, welches den Inhalt benutzen möchte, die Identifikationsdaten des Inhalts sowie die dazugehörigen Rechte und deren Spezifikationen werden in Lizenzen festgehalten. Die Lizenzen werden vom DRM Packer erstellt und an den Lizenzserver weitergegeben. Gleichzeitig wird ein Schlüsselpaar generiert, das der späteren Identifikation von Nutzern sowie der Entschlüsselung von Inhalten dient. Beides wird separat gespeichert und ist über die oben genannten Identifikationsdaten eindeutig Inhalten zugeordnet. Weiterhin werden sog. Identitäten vorgehalten. Dies sind Daten über Nutzer, die gerade Rechte auf bestimmten Inhalten ausüben (z. B. ein Musikstück anhören oder einen Film anschauen). Aus diesen Daten werden dann die einzelnen Lizenzen generiert und an die jeweiligen Nutzer versandt.

\section{DRM Client}

Die Komponenten des DRM auf Nutzerseite sind der Controller, die Applikation zur Nutzung (Wiedergabe) des Inhalts und der Identifikationsmechanismus.

Die Controller-Komponente kann als eigenständige Software, innerhalb einer Applikation oder als Hardware realisiert werden. Ihre Aufgaben umfassen:

- Empfangen und Weiterleiten von Benutzeranforderungen

- Identitätsinformationen über den Nutzer sammeln und eine entsprechende Lizenz vom Lizenzserver holen

- Authentifikation der Wiedergabeapplikation auf Nutzerseite

- Management der Verschlüsselungsmechanismen, insbesondere Entschlüsselung des Inhalts

Die Wiedergabeapplikationen können grob in zwei Klassen unterteilt werden: die speziell für DRM geschriebenen Applikationen und die Applikationen, die das DRM zunächst modifizieren muss, um die Durchsetzung der Rechte zu garantieren. Erstgenannte haben den Vorteil dass sie mittels spezifischer Befehle relativ leicht „sicher“ zu gestalten sind. Dem stehen jedoch zwei gewichtige Nachteile gegenüber. Erstens muss die Applikation an die Nutzer distribuiert werden, zweitens ist eine Lernphase nötig, bevor die Nutzer die Applikation bedienen können. $\mathrm{Zu}$ letzteren zählen vor allem Plug-Ins, die für eine Reihe von Standardsoftware erhältlich sind. Mit ihrer Hilfe können aus DRM-Sicht unerwünschte Funktionen zur Modifikation bzw. Weiterverwendung des Inhalts (z. B. Drucken) unterdrückt werden. Der Vorteil dieses Ansatzes liegt vor allem darin, dass der Nutzer die ge- 
wohnte Applikation weiterbenutzen kann und damit sowohl die Distribution der kompletten Applikation, da nur das vergleichsweise kleine Plug-in distribuiert werden muss, als auch die Lernphase entfallen. Nachteilig ist zu bemerken, dass ein Plug-in nicht so sicher gestaltet werden kann wie eine speziell für DRM programmierte Applikation (vgl. Rosenblatt et al. 2002, S. 85).

Die Identifikationskomponente identifiziert Geräte z. B. anhand einer vom DRM auslesbaren eindeutigen Seriennummer, die Hardwarehersteller in ihre Geräte einstellen (vgl. exemplarisch Fischer et al. 1999). Ein anderer Weg ist das generieren einer eindeutigen GeräteID aus den einzelnen Hardwarekomponenten des Endgeräts (vgl. dazu das Konzept der "Next Generation Secure Computing Base" (NGSCB) von Microsoft (www.microsoft.com/resources/ngscb/default.mspx)). Eine Identifikation von Endgeräten anhand ihrer IP-Adresse kann in Zeiten dynamischer Adresszuweisungen durch die Internet Service Provider (ISP) vernachlässigt werden. Ein idealtypischer Ablauf zum Abspielen einer Audio-/Videodatei kann wie folgt beschrieben werden:

- Der Nutzer erhält (z. B. per Download) den von ihm gewünschten Inhalt in einem verschlüsselten Container.

- Um den Inhalt benutzen zu können, wird eine Anfrage an den Lizenz Server gestellt, mit der Bitte bestimmte Rechte auf dem Inhalt ausüben zu dürfen. Diese Anfrage wird vom DRM Controller, unter Verwendung der Identitätsdaten sowie des Inhalts, generiert.

- Der DRM Controller kontaktiert den Lizenzserver und sendet die Anfrage.

- Der Lizenz Server überprüft den Nutzer anhand der gespeicherten Daten. Weiterhin werden die Rechte, die der Nutzer ausüben darf, anhand der Identifikationsdaten des Inhalts überprüft.

- Wenn nötig, wird eine finanzielle Transaktion veranlasst.

- Der Lizenzgenerator benutzt die gesammelten Identitätsdaten, Rechteinformationen und die Verschlüsselungsmechanismen, um eine Lizenz für den Nutzer zu generieren. Diese Lizenz ist verschlüsselt, um Missbrauch auszuschließen.

- Die Lizenz wird an den Client gesendet, auf dem weitere Authentifizierungsschritte ausgeführt werden (z. B. eine Überprüfung der Wiedergabeapplikation).

- Wenn die Authentifizierung abgeschlossen ist, kann der DRM Controller die Lizenz entschlüsseln. Mit den in der Lizenz enthaltenen Schlüsseln wird der Container mit dem Inhalt geöffnet. 
- Der Inhalt wird von dem DRM Controller an die Wiedergabeapplikation übergeben und dort abgespielt.

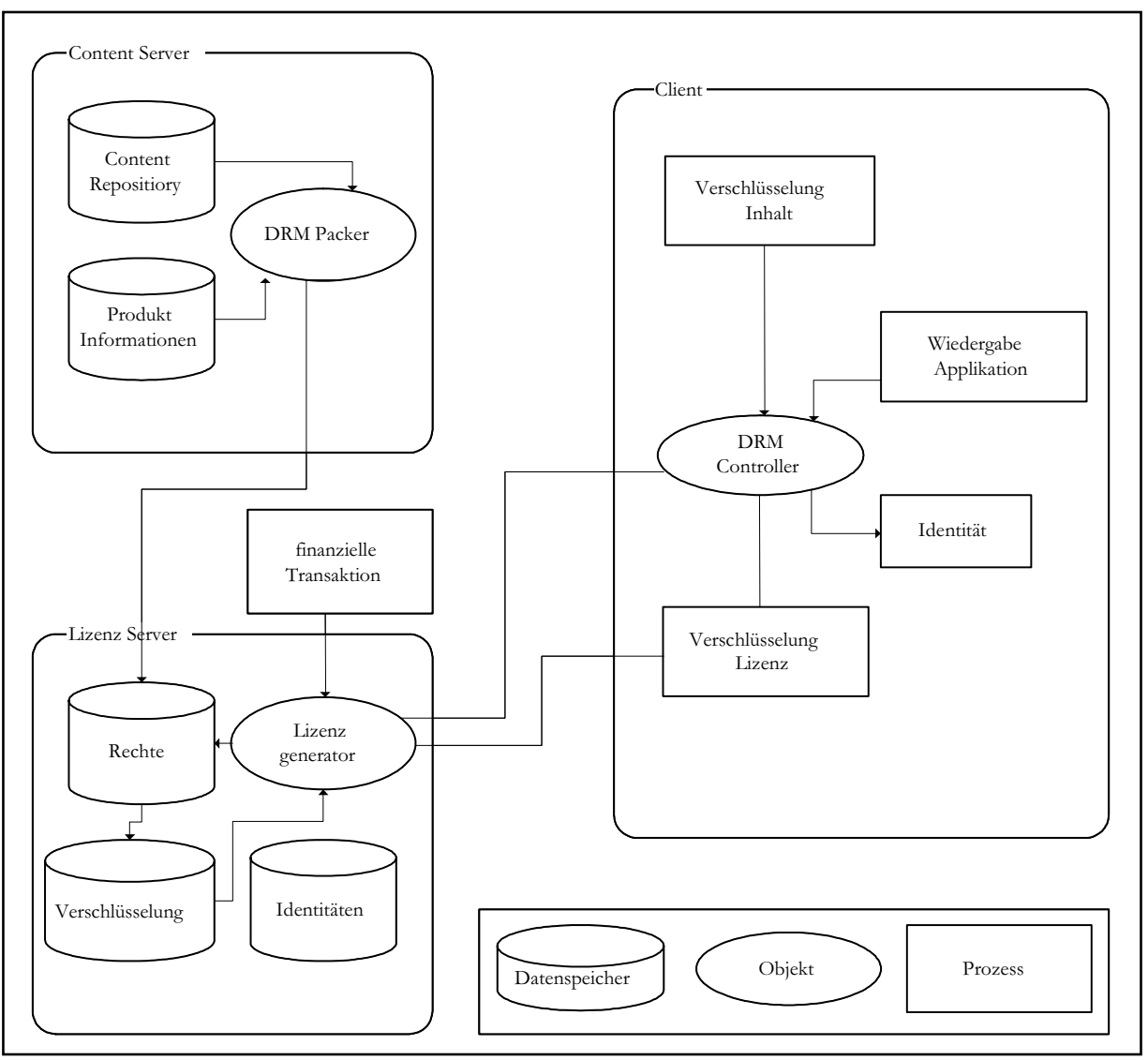

Abbildung 15: DRM Referenzarchitektur

\section{Sprachen zur Modellierung von Rechten}

Um eine kontrollierte Distribution digitaler Inhalte zu gewährleisten, ist es notwendig die Nutzungsrechte an digitalen Gütern informationstechnisch zu erfassen. Dies erfolgt mit Hilfe einer Rights Expression Language (REL), die vom DRM interpretierbar ist. Die z. Zt. bedeutsamsten REL sind die MPEG-21 REL sowie die Open Digital Rights Language (ODRL), die beide auf der Extensible Markup Language (XML) basieren. 


\section{MPEG-21 REL}

Die MPEG-21 REL besteht aus den Modulen REL Core, REL Standard Extension und REL Content Extension. Das REL Core Modul stellt die grundlegenden Elemente für die Funktionalität der Sprache bereit, während die Standard Extension nützliche Erweiterungen bietet. Die REL Content Extension stellt die Multimediafähigkeit sicher.

Rechte werden mit Hilfe des Lizenzkonzepts abgebildet. Eine Lizenz kann als Container verstanden werden, der Berechtigungen, die ein Lizenznehmer von einem Lizenzgeber erhält, bündelt. Diese ermächtigen den Lizenznehmer bestimmte Rechte auf einem Produkt auszuüben, wenn die vorher definierten Bedingungen erfüllt sind. Einfache Bedingungen können Zeiträume oder Häufigkeit der Benutzung sein. MPEG-21 stellt ebenfalls Mechanismen zur eindeutigen Identifizierung von Berechtigten, d.h. Endnutzer oder Endgeräte bereit. Des Weiteren werden Zahlungsabwicklung, Verschlüsselung von Inhalten und digitale Signaturen unterstützt.

\section{Open Digital Rights Language}

ODRL modelliert Rechte mit Hilfe der Elemente Werk (Asset), Recht (Right) und Beteiligter (Party). Die Werke umfassen physische und digitale Inhalte. Rechte setzen sich aus Nutzungsrechten, Beschränkungen, Anforderungen sowie Bedingungen zusammen. Der Rechtebegriff bezieht sich im Gegensatz zur MPEG-21 REL nicht nur auf Nutzungsrechte, die für einen bestimmten Inhalt freigegeben wurden, sondern ist weiter gefasst. Unter Beteiligten versteht man Rechteinhaber und Konsumenten. Durch diese Elemente können Angebote, Übereinkünfte und Widerrufe abgebildet werden. Angebote werden den Konsumenten von Rechteinhabern unterbreitet und sind an bestimmte Werke gebunden. Sind die Beteiligten sich handelseinig, entsteht eine Übereinkunft, die der Lizenz in MPEG-21 ähnelt. Widerrufe können sich sowohl auf Angebote als auch auf Übereinkünfte beziehen. Da die Reihenfolge der Interaktionen beliebig ist, können mit ihrer Hilfe viele Geschäftsmodelle abgebildet werden. ODRL unterstützt einen einfachen Verschlüsselungsmechanismus zum Schutz von Werken und Rechtedefinitionen.

\section{Mechanismen zum Schutz von digitalen Produkten}

Um die modellierten Rechte wirksam durchsetzen zu können, bedarf es Schutzmechanismen. Nachfolgend werden allgemein die Verschlüsselung sowie digitale Wasserzeichen vorgestellt.

\section{Verschlüsselung}

Wie bereits erwähnt, treffen die digitale Musik und die Lizenz in verschlüsselter Form beim Nutzer ein. Eine Verschlüsselung der digitalen Musik ist eine Voraus- 
setzung für den Einsatz von DRMS, da ansonsten ungehindert eine direkte Wiedergabe mittels eines Hard- oder Softwaregerätes unter Umgehung des DRMS stattfinden kann.

Die Verschlüsselung kapselt die digitale Musik in ein Behältnis, sog. „Secure Container", zu dem nur ein geheimer Schlüssel Zugang gewährt (vgl. Günnewig / Hauser 2002, S. 182, Spenger 2003, S. 79). Dieser Schlüssel ist als Inhaltsschlüssel zu bezeichnen und üblicherweise selbst in verschlüsselter Form in der Lizenz enthalten. Aufgrund der Laufzeiteigenschaften ist der Inhaltsschlüssel symmetrisch. Gebräuchlich sind Blockverschlüsselungsverfahren wie Triple-DES. Somit ergibt sich vorliegend ein hybrides System. Es sind auch rein symmetrische Kryptosysteme für DRMS denkbar, besonders in individualisierten Geschäftsmodellen bieten hybride Systeme aber Vorteile (vgl. Spenger 2003, S. 78). Der Inhaltsschlüssel in der Lizenz muss zwingend selbst verschlüsselt sein. Für die Rechte in der Lizenz ist dies jedoch nicht erforderlich, hier genügt eine digitale Signatur, um Manipulationen auszuschließen. Manipulationen an der verschlüsselten Musikdatei sind durch Einweg-Hashfunktionen aufzudecken. ODRL benutzt einen durch den SHA-1-Algorithmus ermittelten „Digest Value“ (vgl. Ianella 2002, S. 24).

Die vorliegend eingesetzten Algorithmen sind bewährt und stellen in Kombination mit einer auf den Verwendungszweck abgestimmten Schlüssellänge hinreichend sichere Verfahren dar (vgl. Schneier 1996, S. 507). Werden andere bekannte und öffentlich verifizierte Algorithmen, wie z. B. AES oder IDEA, für die aktuell keine Kryptoanalyseverfahren bekannt sind, korrekt implementiert, so bieten diese ebenfalls ein akzeptables Sicherheitsniveau. Wird das gesamte Verschlüsselungsverfahren vom Hersteller geheim gehalten und nicht nur der jeweilige Schlüssel, sog. Blackbox-Verfahren, kann dessen Qualität ohnehin nicht überprüft werden. In diesem Zusammenhang ergibt sich eine besondere Problematik, da die Wirksamkeit einer technischen Schutzmaßnahme, zu denen die Verschlüsselung zu zählen ist, nach geltendem Recht ( $\$ \int 95 a, 108 b$ Abs.1 UrhG) objektiv zu bewerten ist. Der Rückgriff auf eine unabhängige Public-Key-Infrastruktur (Guth 2003, S. 153) erscheint aus denselben Gründen notwendig. Die Anwendung von Verschlüsselung in DRMS verfolgt konkurrierende Ziele. Dem Nutzer soll der Zugriff auf Daten erlaubt und verboten werden. In der Theorie lässt sich die Zielkonkurrenz durch die jeweils nur zeitweilige Erteilung von Zugriffserlaubnissen bzw. -verboten auflösen, in der Praxis gibt es konkrete Umsetzungsprobleme, die technisch nur schwer zu lösen sind. Das Hauptproblem liegt in der Schlüsselverwaltung und nicht in den verwendeten Algorithmen. Im konkreten Beispiel des von ODRL verwendeten „Encrypted Key“ bedeutet dies, dass „Encrypted Key“ rekursiv auf einen weiteren „Encrypted Key“ verweisen kann. Die Rekursion ist jedoch endlich. Also muss ein Schlüssel existieren, mit dem „Encrypted Key“ entschlüsselt wird, ein sog. „key-encryption-Schlüssel“ (vgl. Schneier 1996, S. 208). Dieser Schlüssel kann grundsätzlich beim Musikanbieter oder in der Nutzersphäre 
aufbewahrt werden. Eine Aufbewahrung beim Anbieter ist auszuschließen, da eine sichere, abgeschirmte Online-Verbindung zu diesem aufgebaut werden müsste, was z. B. bei tragbaren MP3-Playern nicht möglich ist.

Verbleibt die Möglichkeit der Verwahrung in der Nutzersphäre, d.h. in seinem Wiedergabegerät, im DRMS Controller oder in anderer Soft- oder Hardware. Die Wahl des Verwahrungsortes muss verschiedene Aspekte berücksichtigen. Aus Sicherheitsgründen sollten Schlüssel häufiger ausgetauscht werden können, um das Risiko der Kompromittierung zu minimieren (vgl. Schneier 1996, S. 216f.). Eine Unterbringung in Software verursacht geringe Kosten, in Hardware entstehen hingegen hohe Kosten (vgl. Feigenbaum 2003, S. 16). Es ist möglich, Aufspaltungsverfahren für Schlüssel zu verwenden, bei denen Schlüssel in verschiedene Teile zerlegt werden um sie dann auf Hard- und Software aufzuteilen. Damit lassen sich sowohl die Kosten für den Austausch senken als auch die Sicherheit reiner Hard- oder Softwarelösungen erhöhen (vgl. Schneier 1996, S. 213).

Ein weiteres Problem besteht in der Anwendung der Verschlüsselung auf langfristig gespeicherte Daten. Wird ein „key-encryption-Schlüssel“ kompromittiert, muss er ausgetauscht werden. Alle betroffenen Lizenzen müssen erneut ausgestellt, alle Audiodaten müssen vernichtet, erneut übertragen und gespeichert werden. Deshalb muss die Schlüsselverwahrung auch über lange Zeit sehr sicher sein, ein vollständiger Schlüsselaustausch muss aus praktischen Gründen ausgeschlossen werden. Waren die Daten einmal, wenn auch nur kurzzeitig, unverschlüsselt verfügbar, ergeben sich für die Zukunft Möglichkeiten für „known-plaintextAngriffe" (Schneier 1996, S. 260). Bei einem hohen Sicherheitsniveau muss die Entschlüsselung der digitalen Musik den Laufzeitanforderungen der konkreten Anwendung genügen, besonders darf sie eine qualitativ hochwertige Wiedergabe nicht beeinträchtigen.

\section{Digitale Wasserzeichen}

Digitale Wasserzeichen stellen einen speziellen Schutz in DRMS dar. Sie wirken unabhängig von den vorgenannten allgemeinen Schutzmechanismen (vgl. Fraunhofer 2002, S. 1) und haben ihren Ursprung in der Steganographie (vgl. Dittmann 2000, S. 16). Ihr Einsatz ist deshalb notwendig, weil jedes Audiosignal letztlich die geschützte Sphäre des DRMS verlassen muss, damit der Nutzer es hört. „Ein nicht wahrnehmbares Wasserzeichen stellt ein transparentes, nichtwahrnehmbares Muster dar, welches in das Datenmaterial eingebracht wird. Dieses Muster wird dazu benutzt, entweder das Vorhandensein einer Kennzeichnung anzuzeigen oder Informationen zu codieren. Das Wasserzeichenverfahren nutzt geheime Informationen, wie zum Beispiel Schlüssel, und besteht aus einem Einbettungsprozess (Einbettungs- oder auch Markierungsalgorithmus) und einem Abfrageprozess (Abfragealgorithmus, Auslesen der Markierung). Das Wasserzeichenmuster ist meist ein Pseudorauschmuster und codiert die Wasserzeicheninformation" (vgl. 
Fränkl / Karpf 2004, S. 20). Während digitale Wasserzeichen im visuellen Bereich schon seit Beginn der neunziger Jahre Gegenstand zahlreicher Publikationen sind, wurden bisher in Bezug auf Audiodaten vergleichsweise wenige Verfahren veröffentlicht. Für digitale Wasserzeichen existieren zahlreiche Anwendungsmöglichkeiten. Im Kontext von Audiodaten können folgende Einsatzmöglichkeiten identifiziert werden:

1. Die Überwachung von Radiostationen, bei der dem Abstrahlsignal Informationen hinzugefügt werden.

2. Die Gewährleistung von Authentizität und Integrität der Audiodaten, wobei sog. fragile Wasserzeichen zum Einsatz kommen (vgl. Bechtold (2002), S. 79). Etwaige Veränderungen am Wasserzeichen deuten auf Manipulationsversuche an den Daten hin.

3. Die Zugriffskontrolle auf Audiodaten, die den Abspiel- bzw. Kopiervorgang in einem Hard- oder Softwaregerät nur dann zulässt, wenn ein authentisches Wasserzeichen existiert und der Nutzer dazu berechtigt ist.

4. Die Überwachung und Rückverfolgung von Urheberrechtsverletzungen, wobei Audiodaten, die außerhalb der gültigen Distributionspfade entdeckt werden, in Wasserzeichen Informationen über den Urheber enthalten. Im Falle von Transaktionswasserzeichen ist hierin auch die Quelle der illegalen Kopien codiert.

Die erste Anwendung ist in der Praxis verbreitet, aber hier nicht relevant. Für DRMS kommen die Anwendungen (2) bis (4) infrage. Informationen über die Lizenz sind durch Wasserzeichen in die Audiodaten eingebettet und somit fest mit den Audiodaten verbunden. Das DRMS erlaubt eine Nutzung nur bei unversehrtem Wasserzeichen unter den in der Lizenz festgelegten Bedingungen. Gelangen Audiodaten aus der Sphäre des DRMS, können der Urheber und letzte Lizenzinhaber dennoch eindeutig identifiziert werden. Auf digitale Fingerabdrücke wird hier nicht eingegangen.

Grundlegende Anforderungen an Audio-Wasserzeichen sind (vgl. Dittmann 2000, S. 25ff.): die Robustheit, die Unhörbarkeit, die Komplexität und die Kapazität. Demnach bedeutet Robustheit, dass die eingebrachte Information ,zuverlässig aus dem Datenmaterial ausgelesen werden kann, auch wenn das Datenmaterial modifiziert (aber nicht vollständig zerstört) wurde" (Dittmann 2000, S. 25). Beispiele für konkrete Modifikationen sind: Kompression und Quantisierung, Einbringen von Rauschen, Filtern, Samplepermutationen, Time-Stretching, Skalierung, nichtlineare Transformationen, Entfernen oder Einfügen einzelner Samples, Umwandlung in ein analoges Signal und Rückwandlung in ein digitales Signal, Verzerrung, Einbringen von Echoeffekten oder eine Veränderung der Frequenzanteile (vgl. Dittmann 2000, S. 89). Die Unhörbarkeit ist gegeben, „wenn ein 
durchschnittliches [...] Hörvermögen nicht zwischen markiertem Datenmaterial und Original unterscheiden kann“. „Komplexität: Beschreibt den Aufwand, der erbracht werden muss, die Wasserzeicheninformationen einzubringen und wieder auszulesen“ (Dittmann 2000, S. 26). Die Kapazität gibt an, „wie viel Informationen in das Original eingebracht werden können und wie viele Wasserzeichen parallel im Datenmaterial zugelassen bzw. möglich sind“" (Dittmann 2000, S. 27). Die Anforderungen stehen teilweise in einem konkurrierenden Verhältnis zueinander. Die Kapazität hängt von der Audiocharakteristik ab und geht zulasten der Unhörbarkeit und der Robustheit. Weiterhin kann die Komplexität in Konkurrenz zu den Laufzeitanforderungen der konkreten Einbettungsanwendung oder zum Abfrageprozess stehen (vgl. Petitcolas 2003, S. 7). Für die Verwendung in DRMS kann zusätzlich gefordert werden, dass eine Erkennung, Entfernung oder Fälschung von Wasserzeichen durch etwaige Angreifer nicht möglich ist und dass eine unparteiische Überwachung stattfindet (vgl. Petitcolas 2003, S. 7, Dittmann 2000, S. 26ff. Cox et al. 2002, S. $31 \mathrm{ff}$.$) .$

\section{4 Überblick über aktuelle DRM Systeme}

Die Software iTunes 4 dient seit April 2003 für Apple- und seit Oktober für Windows-Nutzer als Client für den Apple Music Store und ermöglicht Musik auf das mobile Endgerät iPod zu übertragen. Geschützte Dateien vom Apple Music Store gelangen in MPEG-4-Containern zum Konsumenten. Das Format MPEG-4 ermöglicht über Erweiterungen ein DRM. Die Lizenzvergabe erfolgt über den Music Store. Zwar ist ein Abspielen geschützter Dateien nur durch iTunes bzw. QuickTime möglich, doch es können bis zu drei verschiedene Computer gleichzeitig dazu autorisiert werden, erworbene Dateien zu spielen. Brennvorgänge sind mit unverändertem Inhalt nur je zehnmal gestattet. Der Schutz der digitalen Musikdateien geht dabei aber verloren, so dass eine anschließende Umwandlung in ein ungeschütztes Format möglich ist. Eine direkte Umwandlung geschützter Dateien in MP3 innerhalb von iTunes ist dagegen nicht möglich.

Ein bereits in der Musikindustrie eingesetztes System stellt IBM's Electronic Media Management System (EMMS) dar, welches weitgehend auf offenen Standards (z. B. Java und XML) aufbaut.

Helix DRM von Real Networks soll so ausgelegt sein, dass es jedes andere Medien-Format unterstützen kann. Dadurch ist es nicht mehr notwendig, für jedes Format einen Player mit properitärem DRM auf den Endgeräten zu installieren. Helix DRM bietet dazu zwei Varianten an, eine native Unterstützung als auch die Möglichkeit, Daten in einen sicheren Speicher zu transferieren. Geräte mit direkter Unterstützung können dann Inhalte direkt von Helix-DRM-Lizenz-Servern 
Absatz digitaler Produkte und Digital Rights Management

beziehen, der beispielsweise von Internet Service Providern (ISP) oder Musikanbietern betrieben wird.

Ein ähnliches Konzept verfolgen Philips und Sony, die von Intertrust ein DRMS entwickeln lassen, das ebenfalls endgeräteunabhängig arbeitet.

\section{Zusammenfassung}

Die Distribution digitaler Produkte nimmt stetig zu. Die technischen Möglichkeiten hierzu können grundsätzlich in zentralisierte und dezentralisierte Technologien unterteilt werden. Im Zusammenhang mit zentralisierten Technologien ist Streaming Media zu nennen, welches hauptsächlich zum Vertrieb von „Rich Media" Anwendungen (z. B. Videodaten) eingesetzt wird. Es ist anzunehmen, dass diese Technologie im kommerziellen Bereich weiterhin (z. B. im Marketingbereich durch Streaming-Werbung) an Bedeutung zunehmen wird. Im Gegensatz dazu sind dezentralisierte Peer-to-Peer-Systeme derzeit hauptsächlich in illegalen Tauschbörsen im Einsatz. Beide Distributionstechnologien können kombiniert werden, wobei derartige Systeme sich zumeist noch im Prototypenstadium befinden.

Traditionelle Erlösmodelle des Handels mit digitalen Produkten wie Abonnements, Lizenzmodelle und Erlöse durch Werbung scheinen bedroht bzw. können in den derzeitigen Absatzkanälen wegen mangelnder Kontrolle nicht durchgesetzt werden. Zu diesem Zweck werden Digital Rights Management Systeme (DRMS) entwickelt, die digitale Inhalte vor unerlaubter Nutzung, Manipulation und Weiterverbreitung schützen sollen. Deren Ziel ist die Wahrung des Urheberrechts durch kontrollierbare Absatzkanäle.

Die technologischen Grundlagen dieser Systeme sind Verschlüsselungsmechanismen und digitale Wasserzeichen, mit deren Hilfe der Inhalt sowie die vom Rechteinhaber mittels REL definierten Rechte, geschützt werden können. Das größte Problem von DRMS ist in erster Linie die Einschränkung der Verwendungsmöglichkeiten des Inhalts und damit eine geringere Nutzerfreundlichkeit.

Weitere Forschungsvorhaben sollten das Ziel haben, neue Geschäftsmodelle zu entwickeln, die eine kommerzielle Distribution über zentrale, im Kontext illegaler P2P Tauschbörsen, vor allem aber über dezentrale Technologien ermöglichen. Diese Geschäftsmodelle können DRM integrieren, jedoch scheint dies nicht in allen Bereichen zwingend notwendig, da mit DRM immer auch eine Nutzungseinschränkung einhergeht. Bei zu starker Einschränkung können anreizkompatible Erlösmodelle einen höheren Erfolg versprechen. 


\section{Literatur}

Barkai (2002): Barkai, D.: Peer-to-peer computing: technologies for sharing and collaborating on the net, Hilsboro, Or., 2002.

Bechtold (2002): Bechtold, S.: Vom Urheber- zum Informationsrecht: Implikationen des Digital Rights Management, München, 2002.

Biddle et al. (2003): Biddle, P., England, P., Peinado, M., Willman, B.: Darknet and the Future of Content Protection, in: E. Becker (Hrsg.): Digital Rights Management Technological, Economic, Legal and Political Aspects, Berlin [u.a.], 2003, S. $344-365$.

Brandtweiner (2000): Brandtweiner, R.: Differenzierung und elektronischer Vertrieb digitaler Informationsgüter, Düsseldorf, 2000.

Clarke (1999): Clarke, R.: Electronic Services Delivery: From Broschure-Ware to Entry Points, http://www.anu.edu.au/people/Roger.Clarke/EC/ESD.html, 1999, Abruf am 2004-03-16.

Coulouris (2002): Coulouris, G., Dollimore, J., Kindberg, T.: Verteilte Systeme: Konzepte und Design, München, 2002.

Cox et. al. (2002): Cox, I.J., Miller, M.L., Bloom, J.A.: Digital watermarking, San Francisco, Calif. [u.a.], 2002.

Dittmann (2000): Dittmann, J.: Digitale Wasserzeichen: Grundlagen, Verfahren, Anwendungsgebiete, Berlin [u.a.], 2000.

Dyson (1995): Dyson, E.: Intellectual Value, http://www.wired.com/wired/archive/ 3.07/dyson.html, 1995.

Feigenbaum (2003): Feigenbaum, J.: Digital Rights Management: ACM CCS-9 workshop, DRM 2002, Washington, DC, USA, November 18, 2002, Berlin [u.a.], 2003.

Fischer et. al. (1999) Fischer, S., Mi, J., Teng, A.: Pentium ${ }^{\circledR}$ III Processor Serial Number Feature Applications, http://www.intel.com/technology/itj/q21999/ pdf/serial_number.pdf, 1999, Abruf am 16.03.2004.

Fränkl / Karpf (2004): Fränkl, G., Karpf, P.: Digital Rights Management Systeme Einführung, Technologien, Recht, Ökonomie und Marktanalyse, München, 2004.

Fraunhofer (2002): Fraunhofer Institut Integrierte Schaltungen: Audio Watermarking, http://www.iis.fraunhofer.de/amm/download/watermark.pdf, 2002, Abruf am 16.03.2004.

Gehrke et. al. (2003): Gehrke, N., Burghardt, M., Schumann, M.: Divide et impera ein Peer-to-Peer basiertes Geschäftsmodell für Streaming Media, in: Wirtschaftsinformatik 2003, (2003), S.467-490.

Gooch (2003): Gooch, R.: Requirements for DRM Systems, http://www.springerlink.com/media/m37uj6789cnqxha124e7/Contributions/ A/9/3/7/A937V9P96C9FRM7R.pdf, 2003, Abruf am 16.03.2004. 
Grimm (2003): Grimm, R.: Digital Rights Management: Technisch-organisatorische Lösungsansätze, in: A. Picot (Hrsg.): Digital Rights Management, Berlin [u.a.], 2003, S. 93-107.

Günnewig / Hauser (2002): Günnewig, D., Hauser, T.: Musik im Hochsicherheitstrakt - Digital Rights Management - Stand der Dinge, in: c't, (2002), S.182-185.

Guth (2003): Guth, S.: A Sample DRM System, in: Becker, E., Buhse, W., Günnewig, D., Rump, N. (Hrsg.): Digital Rights Management - Technological, Economic, Legal and Political Aspects, Berlin [u.a.], 2003, S. 150-160.

Ianella (2001): Ianella, R.: Digital Rights Management (DRM) Architectures, http://www.dlib.org/dlib/june01/iannella/06iannella.html, 2001, Abruf am 16.03.2004.

Ianella (2002): Ianella, R.: Open Digital Rights Language (ORDL) Version 1.1 - Specification, http://ordl.net/1.1./ORDL-11.pdf, 2002, Abruf am 2004-03-16.

Ifpi (2004): ifpi: Bundesverband der phonographischen Wirtschaft e.V., http://www.ifpi.de/, 2004.

Kortmann (2002): Kortmann, W.: Mikroökonomik: anwendungsbezogene Grundlagen, Heidelberg, 2002, Abruf am 16.03.2004.

Kuhlen (2003): Kuhlen, R.: Medienprodukte im Netz - Zwischen Kommerzialisierung und freiem Zugang, in: Picot, A. (Hrsg.): Digital Rights Management, Berlin [u.a.], 2003, S. $107-132$.

Lainee et. al. (2002): Lainee, F., de Boeck, P., Wilshire, M.: Internet Protocol Services: Vom Backbone zur Peripherie, http://www.digitaltransformation.de/pdf/ 2838988_digital_transformation_mdul5_isp.pdf, 2002, Abruf am 16.03.2004.

Löbbecke (1999): Löbbecke, C.: Electronic Trading in On-line Delivered Content, http://www.mm.uni-koeln.de/loebbecke_pdf/Conf-036-1999-

Electronic\%20trading\%20in\%20On-Line.pdf, 1999, Abruf am 16.03.2004.

Luxem (2001) Luxem, R.: Digital Commerce: Electronic Commerce mit digitalen Produkten, Lohmar [u.a.], 2001.

Martin et. al. (2002): Martin, M., Agnew, G., Boyle, J., McNair, J., Page, M., Rhodes, W.: DRM Requirements for Research and Education Discussion Paper, 2002.

Miller (2001): Miller, M.: Discovering P2P, San Francisco, 2001.

o.V. (1998): o.V.: A Piece of the Tick - Supporting the Commercial Redistribution of Electronic Information through Value Chains, http://www.intertrust.com/ media/pdf/tick.pdf, 1998, Abruf am 16.03.2004.

o.V. (2002): o.V.: Digital Rights Management, http://searchcio.techtarget.com/ sDefinition/0,sid19_ gci493373,00.html, 2002, Abruf am 16.03.2004.

Padmanabhan (2002): Padmanabhan, P., Venkata, N., Wang, H.J., Chou, P.A.: Distributing Streaming Media Content Using Cooperative Networking, http://www.research.microsoft.com/ padmanab/papers/msr-tr-2002-37.pdf, 2002, Abruf am 16.03.2004. 
Petitcolas (2002): Petitcolas, F.A.P.: Digital watermarking: first international workshop, Seoul, Korea, November 21 - 22, 2002, Berlin [u.a.], 2003.

Ripeanu (2003): Ripeanu, A., Foster, I., Iamnitichi, A.: Mapping the Gnutella Network: Properties of Large-Scale Peer-to-Peer Systems and Implications for System Design, http://people.cs.uchicago.edu/ matei/PAPERS/ic.pdf, 2003, Abruf am 16.03.2004.

Rose (1999): Rose, F.: The economics, concept, and design of information intermediaries: a theoretic approach, Heidelberg [u.a.], 1999.

Rosenblatt et al. (2002): Rosenblatt, W., Trippe, W., Mooney, S.: Digital rights management: business and technology, New York, NA [u.a.], 2002.

RSVP (2004): RSVP Protocol Overview, http://www.isi.edu/div7/rsvp/ overview.html, 2004, Abruf am 16.03.2004.

RTP (2003): About RTP and the Audio-Video Transport Working Group, http://www.cs.columbia.edu/ hgs/rtp/, 2003, Abruf am 2004-03-16.

RTSP (2004): Real Time Streaming Protocol (RTSP) Information and Updates, http://www.rtsp.org/, 2004, Abruf am 16.03.2004.

Rump (2003): Rump, N.: Digital Rights Management Technological Aspects, in: Becker, E., Buhse, W., Günnewig, D., Rump, N. (Hrsg.): Digital Rights Management Technological Economical Legal and Political Aspects, Berlin [u.a.], 2003, S. $3-16$.

Sander (2002): Sander, T.: Security and privacy in digital rights management: revised papers, Berlin [u.a.], 2002.

Schneier (1996): Schneier, B.: Angewandte Kryptographie: Protokolle, Algorithmen und Sourcecode in, Bonn [u.a.], 1996.

Schulzrinne et al. (1996): Schulzrinne, H., Casner, Frederick, Jacobson: RTP: A Transport Protocol for Real-Time Applications, http://www.ietf.org/rfc/ rfc1889.txt?number=1889, 1996, Abruf am 16.03.2004.

Schumann / Hess (2000): Schumann, M., Hess, T.: Grundfragen der Medienwirtschaft, Berlin [u.a.], 2000.

Spenger (2003): Spenger, G.: Authentication, Identification Techniques, and Secure Containers - Baseline Technologies, in: Becker, E., Buhse, W., Günnewig, D., Rump, N. (Hrsg.): Digital Rights Management: Technological, Economic, Legal and Political Aspects, Berlin [u.a.] 2003, S. 62-80.

Stähler (2001): Stähler, P.: Geschäftsmodelle in der digitalen Ökonomie: Merkmale, Strategien und Auswirkungen, Lohmar [u.a.], 2001.

Stoica et al. (2002): Stoica, I., Morris, R., Liben-Novell, D., Karger, D., Kaashoek, F., Dabek, F., Balakrishnan, H.: Chord: A scalable Peer-to-Peer Lookup Protocol for Internet Applications, http://www.pdos.lcs.mit.edu/papers/chord: sigcomm01/chord_sigcomm.pdf, 2002. 
Varian (1998): Varian, H.R.: Markets for Information Goods, http://www.sims.berkeley.edu/ hal/Papers/japan/index.html, Abruf am 07.05.2006.

Wegner et. al. (2000): Wegner, R., Bachmeier, C.: Streaming Media im BusinessBereich: Echtzeitverfahren für das WWW, Boston. 
Zweiter Teil:

Mobiles Internet 



\section{Individualisierung von Medienprodukten}

\section{Christian Kaspar}

\section{Einleitung}

Die Möglichkeiten der digitalen Erzeugung und Distribution von Inhalten haben für Medienunternehmen sowohl wettbewerbstrategische als auch ressourcenbezogene Implikationen: Zum einen können digitalisierte Inhalte im Vergleich zu den Vertriebsformen traditionell erzeugter Inhalteformate (bspw. Printprodukte) kostengünstig distribuiert werden. Dies mindert aus wettbewerbstrategischer Sicht bestehende Zutrittsbarrieren von Medienmärkten und eröffnet neuen Konkurrenten Marktzutrittschancen. Die Folge ist, dass das vor allem in Onlinemedien resultierende inhaltliche Überangebot derzeit, wenn überhaupt, nur noch geringe direkte Erlöse im Inhaltevertrieb erlaubt (vgl. Mings/White 2000, S. 64 f.). Zum anderen schafft die Digitalisierbarkeit von Inhalten aus Ressourcenperspektive die Voraussetzung für einen effizienteren Umgang mit Inhalten im Rahmen der Erzeugung von Medienprodukten. Einmalig erzeugte digitale Inhalte können grundsätzlich mehrfach in unterschiedlichen Bündelungs- und Distributionsformaten genutzt und angeboten werden. Dadurch kann eine höhere Variantenvielfalt des Inhalteangebots bei nahezu gleich bleibendem Kostenniveau angeboten werden. Die Interaktivität von Onlinemedien erlaubt zudem eine nahtlose und effiziente Integration von Interaktions- und Feedbackdaten aus dem Rezipientendialog in die 
Inhaltebündelung und Distribution. Dadurch können Inhalte potenziell auf individueller Ebene an wahrgenommene Nutzerbedürfnisse und das wahrgenommene Rezeptionsverhalten angepasst werden.

Aktuelle Untersuchungen zeigen, dass Rezipienten zwar auch in Onlinemedien grundsätzlich bereit sind, für das Inhalteangebot zu bezahlen, jedoch nur dann, wenn damit für sie hinsichtlich ihres Informationsstands bzw. der Bequemlichkeit der Informationsselektion und -rezeption ein Zusatznutzen verbunden ist (vgl. u.a. Hass 2002, S. 137; ECIN 2002; ECIN 2003). Im Folgenden wird untersucht, inwiefern Medienkonsumenten durch die Individualisierung von Medienprodukten ein solcher Kaufanreiz geboten werden kann. In Kapitel 2 werden Möglichkeiten der Mehrfachnutzung von Inhalten als Grundlage einer Individualisierung von Medienprodukten vorgestellt. In Kapitel 3 werden technische Aspekte einer Bereitstellung individueller Medienprodukte erläutert. In Kapitel 4 wird schließlich der Mehrwert denkbarer Individualisierungsformen in der Medienbranche aus Rezipientensicht analysiert. Kapitel 5 fasst die Ergebnisse des Beitrags zusammen.

\section{Mehrfachnutzung von Inhalten}

Das Prinzip der mehrfachen Nutzung von Inhalten besitzt in der Medienbranche eine lange Tradition. Beispiele für eine herkömmliche Mehrfachnutzung bilden im Verlagswesen die mehrfache Verwertung von Buchtiteln als gebundene und als Taschenbuchausgabe oder in der Filmindustrie die mehrfache Vermarktung von Filmen in Kino, Video, Bezahlfernsehen und Rundfunk. Diese Spielart der Mehrfachverwertung wird auch als „Windowing“ bezeichnet (vgl. u. a. Schumann / Hess 2002, S. 74; Vizjak / Ringlstetter 2001; Schumann 2003). Durch Digitalisierung von Inhalten entstehen jedoch zusätzliche Möglichkeiten einer mehrfachen Nutzung von Inhalten, die weitgehend ohne zusätzliche Erzeugungskosten realisiert werden können. Dabei ist zu unterscheiden zwischen Möglichkeiten einer mehrfachen Vermarktung von Inhalten, der sog. Mehrfachverwertung (vgl. Abschnitt 2.1), und den Möglichkeiten einer mehrfachen Verwendung von Inhalten in unterschiedlichen Produktvarianten (vgl. Abschnitt 2.2).

\subsection{Mehrfachverwertung digitaler Inhalte}

Trotz Computer- und Systemunterstützung fallen bspw. im Bereich der Printmedien etwa $50 \%$ der Herstellkosten eines Medienprodukts im Bereich der Inhalteerzeugung und Bündelung an (vgl. Hess 2004, S. 60). Digital erzeugte Inhalte sind jedoch anders als traditionell erzeugte Inhalte nicht an ein spezifisches Trägermedium gebunden, sondern können auf jedem digitalen Träger vertrieben werden. Dadurch können mit digitalisierten Inhalten zusätzliche Skaleneffekte der Distri- 
bution durch eine mehrfache Verwertung des digitalen Inhalts in verschiedenen Trägermedien, bspw. als digitale Druckvorlage einer Zeitschrift oder als Onlineausgabe der gedruckten Zeitschrift, realisiert werden (vgl. nachstehende Abbildung).

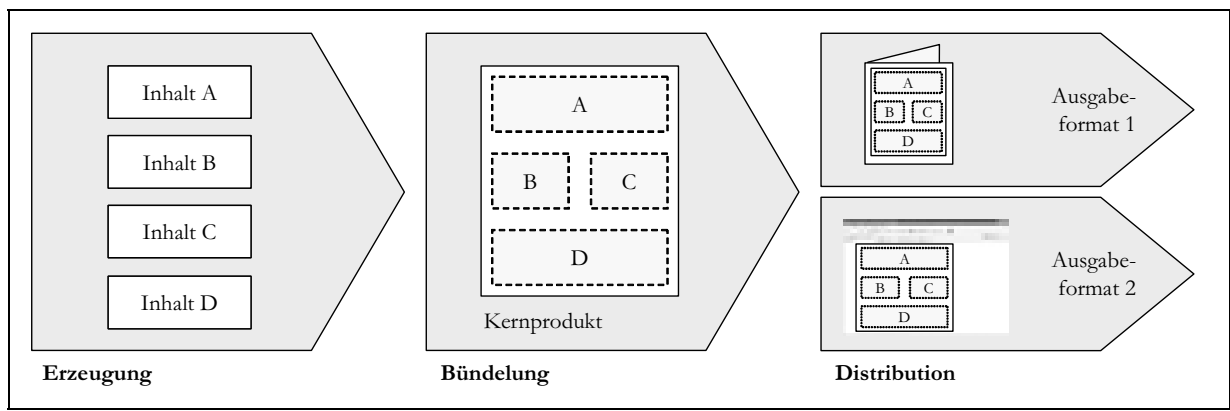

\section{Abbildung 16: Das Prinzip der Mehrfachverwertung}

In der Regel erzeugen und bündeln Medienunternehmen einzelne Inhalteelemente speziell für einen bestimmten Produkttyp, bspw. eine Zeitung, eine Zeitschrift oder eine Rundfunksendung. Dieses Vorgehen wird auch als „kernproduktorientiert" bezeichnet (vgl. Rawolle 2002, S. 149 f.). Um ein solches Kernprodukt in verschiedenen Distributionskanälen verwerten zu können, müssen die gebündelten Inhalte in einem medienneutralen Datenformat gespeichert werden. Nicht jedes digitale Datenformat eignet sich gleichermaßen für den Einsatz in verschiedenen Zielmedien. Bspw. werden im Printbereich in der Regel Formate wie Quark Express, Adobe Framemaker oder Microsoft Word verwendet, während im netzbasierten Vertrieb Auszeichnungssprachen wie HTML oder WML zum Einsatz kommen (vgl. Schumann 2003, S. 119). Als viel versprechend für eine verwendungsneutrale Datenformatierung gelten die im Umfeld der Auszeichnungssprache XML angesiedelten Spezifikationen (vgl. W3C 2004). XML ist ebenso wie HTML eine strukturierte Auszeichnungssprache (vgl. Weitzel / Harder / Buxmann 2001, S. 18). Während jedoch die HTML-Syntax vordefinierte Datentypen zur Inhaltebeschreibung besitzt, können die Datentypen bei XML durch sog. Formattyp-Definitionen (wie bspw. auf Grundlage der Document Type Definition, DTD) flexibel sowohl hinsichtlich Struktur als auch hinsichtlich ihres Wertebereichs definiert werden (vgl. Phillips 2000, S. 13; Rawolle 2002, S. 52; Hess 2004, S. 63 ff.). Dadurch kann die Verwertung eines Medienprodukts sowie sein Darstellungsformat unabhängig vom Inhalt durch typgebundene Verwendungsanweisungen festgelegt werden. 


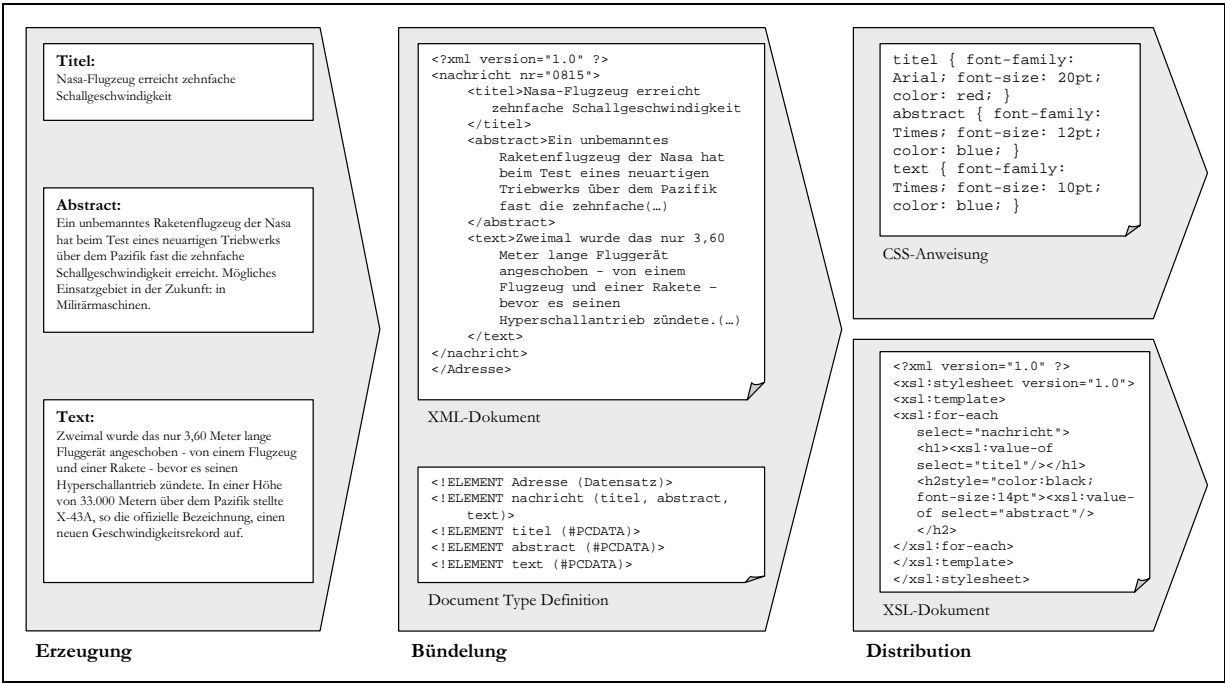

Abbildung 17: Mehrfachverwertung auf der Grundlage von XML

Die vorstehende Abbildung zeigt schematisch die Möglichkeiten einer Mehrfachverwertung auf der Grundlage von XML am Beispiel einer textbasierten Nachricht. Zunächst werden die einzelnen Inhalte zu einer Nachricht gebündelt. Diese Nachrichtenbündelung wird im XML Format gespeichert und die Formattypdefinitionen festgelegt. Das Ausgabeformat kann bspw. durch Darstellungsanweisungen in einem Cascading Stylesheet $\left(\mathrm{CSS}^{10}\right)$ oder durch Anweisungen in der Extensible Stylesheet Language (XSL ${ }^{11}$ ) festgelegt werden. Inhalte, die auf einem gültigen XML-Format beruhen, können automatisch in unterschiedliche Ausgabeformate transformiert werden. Dieser Vorgang wird auch als „Transcoding“ bezeichnet (vgl. Oesterdiekhoff 2004). Eine solche Transformation erfolgt in der Regel im Zuge der Definition des Ausgabeformats und kann bspw. durch die zur XSL-Spezifikation gehörenden Programmiersprache Extensible Stylesheet Language Transformation (XSLT) umgesetzt werden.

10 Cascading Style Sheet ist ein einfacher Mechanismus, um Layoutangaben und Inhalte einer Webseite zu trennen. Die Spezifikation der CSS Syntax wurde 1996 vom W3C verabschiedet (vgl. W3C 1999).

11 Die Extensible Stylesheet Language ist eine Sprachfamilie zur Definition von Layoutangaben auf der Grundlage von XML. XSL besteht aus drei Sprachbestandteilen: Die hier im Beispiel verwendete Objektformatierungssprache XSL Formatting Objects (XSL-FO), die Verweissprache XML Path Language (XPath) und die Transformationssprache XSL Transformations (XSLT). Die Spezifikation der XSL-FO Syntax wurde 2001 vom W3C verabschiedet (vgl. W3C 2001). 


\subsection{Mehrfachverwendung digitaler Inhalte}

Eine kernproduktorientierte Erzeugung von Medienprodukten stößt in der Regel auch bei mehrfacher Verwertung auf der Wertschöpfungsstufe der Distribution an kostenbedingte Varietätsgrenzen. Insofern muss sich ein Medienunternehmen üblicherweise entscheiden, ob entweder eine hohe Zahl von Produktvarianten und dadurch ein hoher Differenzierungsgrad des Angebots, oder eine Kostenführerschaft im Marktsegment durch eine Standardisierung des Angebots angestrebt werden soll. Einem Unternehmen, dem es nicht gelingt, seine Strategie in eine dieser Richtungen zu entwickeln, sitzt sinnbildlich zwischen zwei Stühlen, da für Kostenführerschaft Skaleneffekte einer Massenproduktion ausgenutzt werden müssen, deren Ausbringungsvolumina bei einer hohen Leistungsvarietät jedoch nicht erreicht werden können (vgl. Porter 1980, S. 79). Das traditionelle Prinzip der Massenkommunikation von Inhalten in der Medienbranche mit einem geringen Varietätsgrad trägt diesem Strategieverständnis Rechnung.

Der vor allem im industriebetrieblichen Bereich diskutierte Ausweg aus dem Varietätsdilemma bildet die Reduktion der internen Produktionskomplexität einer hohen Angebotsvarietät durch Modularisierung der Fertigung. ${ }^{12}$ In der Medienbranche kann eine solche Modularisierung in begrenzter Form durch verwertungsneutrale Auszeichnung von Inhalteformaten ermöglicht werden, indem bspw. bei unterschiedlichen Verwertungsformaten nur bestimmte Formatbereiche des Inhalts verwertet werden. Für eine über diese begrenzte Modularisierung auf der Distributionsebene hinausgehende Modularisierung bieten sich in der Medienbranche datenbankgestützte Lösungen für eine mehrfache Verwendung von Inhalten auf der Wertschöpfungsstufe der Erzeugung und Bündelung von Inhalten. Solche, auch als „Content Management Systeme“ bezeichneten Lösungen trennen die Erzeugung und Speicherung von Inhalten einerseits strikt von ihrer Verwendung im Rahmen der Produktbündelung und andererseits von ihrer Verwertung im Rahmen der Distribution. Dazu werden Inhalte zunächst in Datenbanken gespeichert und dynamisch in bestimmten Bündelungsformaten, die als Formatvorlagen, sog. „Templates“, vorproduziert werden können, referenziert (vgl. nachstehende Abbildung). Damit ist es möglich, denselben Inhalt mehrfach in verschiedenen Bündelungsformaten zu verwenden. Durch die Verwendung von Formatvorlagen auf der Grundlage verwertungsneutraler Formattypdefinitionen, können diese Templates darüber hinaus in verschiedenen Distributionskanälen verwertet werden.

12 Beispiele für Unternehmen, die eine große Reihe von Produktvarianten traditionell auf der Grundlage von vorgefertigten Modulen anbieten, bilden im Fotokopierbereich Canon und Xerox, im Werkzeugbereich Black\&Decker oder Sony im Bereich der Unterhaltungselektronik (vgl. Piller 2001, S. 228 f.). 


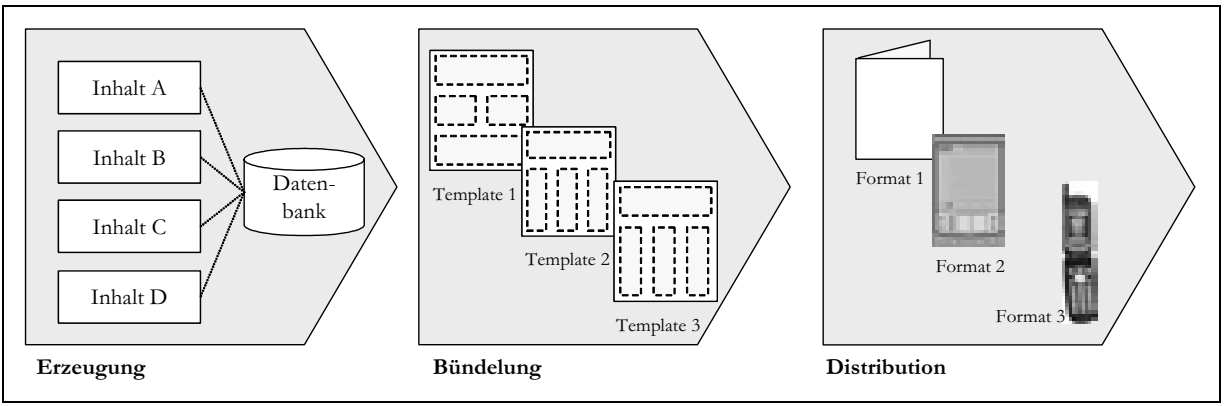

Abbildung 18: Mehrfachverwendung von Inhalten

Die Vorteilhaftigkeit einer Mehrfachverwendung beruht darauf, dass Medienprodukte üblicherweise als Produktbündel gekoppelter Informationsgüter angeboten werden, um im Vertrieb Größen- und Verbundeffekte ausnutzen zu können. Eine Zeitung repräsentiert bspw. ein Bündel aus redaktionellen Artikeln, Werbe- und Kleinanzeigen. Während digitale Bündelungsformate auf der Grundlage von XML eine mehrfache Verwertung von Inhalten im Rahmen der Distribution ermöglichen, erlauben es datenbankgestützte Erzeugungsformen von Inhalten, Medienprodukte dynamisch aus unterschiedlichen Inhaltebestandteilen zu kombinieren. Sind Medienunternehmen in der Lage, inhaltliche Nachfragebedürfnisse zu identifizieren, kann im Rahmen einer solchen Mehrfachverwendung von Inhalten ein Produkt selbst auf individueller Ebene entsprechend dieser Bedürfnisse konfiguriert und angeboten werden - und zwar zu einem Kostenniveau, das näherungsweise dem der Massenfertigung entspricht.

\section{Bereitstellung individueller Medienprodukte}

Die traditionelle Marktforschung zielt darauf ab, über Umfragen und Beobachtungen aus der breiten Schicht der potenziellen Käuferschaft statistische Aussagen über die Struktur relevanter Kundenpräferenzen zu ermitteln. Digitale Onlinemedien eröffnen darüber hinaus die Möglichkeit, Kunden und ihr Verhalten mit einer Präzision zu identifizieren, die über traditionelle statistische Methoden hinausgeht. In Abschnitt 3.1 werden die Möglichkeiten einer individuellen Identifikation der Rezipienten von Onlineinhalten erläutert. Die individuelle Identifikation eröffnet aus Sicht der Medienbranche ein Potenzial, Medienprodukte im Rahmen einer Mehrfachverwendung von Inhalten Medienprodukte dynamisch entsprechend individueller Merkmale für den jeweiligen Nutzer anzupassen. Diese Anpassung wird auch als „Individualisierung“ bezeichnet. In Abschnitt 3.2 wird das technische Prinzip von Anwendungssystemen erläutert, die eine solche Individualisierung automatisch vornehmen können. 


\subsection{Nutzeridentifikation in Onlinemedien}

Die im Internet mittlerweile geläufigste Form der Erhebung von individuellen Kundenprofilen bildet die explizite Befragung von Nutzern in Verbindung mit einer zwar kostenfreien, jedoch häufig obligatorischen Registrierung eines Benutzerkontos. Mangels Kontrollierbarkeit durch den Anbieter ist jedoch die Qualität freiwilliger Angaben von Nutzern in der Regel sehr schlecht (vgl. Shapiro/Varian 1999, S. 35). Zudem sind die per einmaliger Befragung und Registrierung ermittelten und gespeicherten Profile im Zeitablauf statisch. Neben freiwilligen Authentifizierungsangaben kann ein Nutzer jedoch auch anhand seines Verhaltens auf einer Website identifiziert werden (vgl. Mobasher 2002; Mobasher/ Cooley/Srivastava 1999). Jeder Webserver verfügt über eine Protokollkomponente, die sämtliche Serveraktivitäten registriert und chronologisch in meist unterschiedlichen anwendungsorientierten Protokolldateien, den so genannten „Server Logfiles", abspeichert. Abbildung 19 zeigt einen typischen Eintrag einer Protokolldatei.

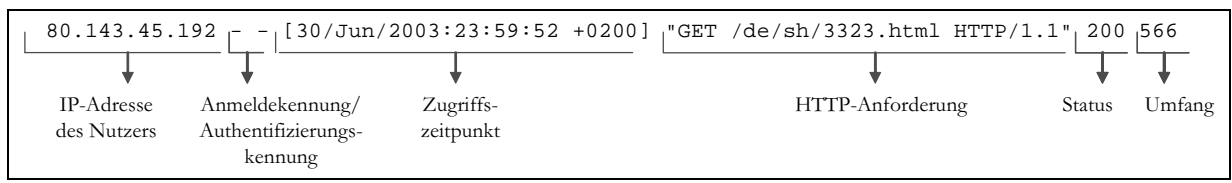

Abbildung 19: Eintrag einer Webserver-Protokolldatei

Anhand des Serverprotokolls kann bestimmt werden, welche manipulativen Handlungen in Bezug auf welche Ressourcen eines Webauftritts vollzogen wurden. Um diese Handlungen einem individuellen Nutzer zuordnen zu können, muss dieser eindeutig identifiziert werden. Die Identifizierung zwischen zwei Kommunikationspartnern im Internet erfolgt grundsätzlich über den 32-Bit Zahlencode der IP-Adresse. Während eines Kommunikationsvorgangs muss jedem Sender und Empfänger eine eindeutige Adresse zugeordnet sein. Speziell privaten Anwendern, die über einen Dienstanbieter auf das Internet zugreifen, wird allerdings in der Regel für jede Zugangssitzung eine dynamische IP-Adresse aus einem Adresspool des Zugangsdienstleisters vergeben, wodurch sie sitzungsübergreifend nicht mehr eindeutig allein anhand der IP-Adresse identifiziert werden können. Technisch bieten sich derzeit zwei alternative Verfahrensweisen für eine automatische Nutzeridentifizierung an: die Verwendung clientseitiger Cookies oder die Wiedererkennung des anbieterseitig gespeicherten Kundenprofils durch eine clientseitige Erweiterungssoftware des Browsers, bspw. auf Grundlage der P3P-Protokolle.

Sind einem individuellen Nutzer eindeutig bestimmte Handlungen im Serverprotokoll zugeordnet, können diese Handlungen als Gegenstand systematischer Untersuchungen genutzt werden, um Rückschlüsse auf die Bedürfnisse und Präferenzen des Nutzers zu ziehen. Dieser Vorgang der Datenanalyse im Serverprotokoll wird auch als „Web Usage Mining“ bezeichnet (vgl. Spiliopoulou 2001, 
S. 491). Das Web Usage Mining repräsentiert eine Sonderform des Data Mining und beruht im Wesentlichen auf statistischen Verfahren zur Erkennung von Datenmustern. Zum Instrumentarium gehören Klassifikationen bspw. auf der Grundlage von Diskriminanzanalysen oder induktiven Lernverfahren, lineare und nichtlineare Regressionen, Segmentierungen durch Gruppenbildung auf der Grundlage von statistischen Verfahren oder maschinellem Lernen sowie Abhängigkeits- und Abweichungsanalysen (vgl. Bensberg 2001, S. 96.). Ziel der Mustererkennung durch Web Usage Mining ist es, im Serverprotokoll auffällige Verhaltensmuster, bspw. häufige Abrufe von Inhalten eines bestimmten Typs, zu bestimmen, die dann als Maße für das Interesse des Nutzers herangezogen werden können.

\subsection{Individualisierungssysteme für Medienprodukte}

Um digitale Inhalte automatisch entsprechend wahrgenommener Handlungen oder Profilmerkmale eines Nutzers anpassen zu können, werden so genannte „Individualisierungssysteme“ eingesetzt. Den Kern eines Individualisierungssystems bildet eine regelbasierte Software, die die Art und Weise festlegt, wie Informationen und Angebote dem Kunden präsentiert werden (Grimm/Jüstel/Klotz 2002, S. 181). Derartige anpassungsfähige Softwaresysteme stützen sich auf ein breites Feld von Forschungsaktivitäten und werden in der Informatik im Zusammenhang mit der Entwicklung „künstlicher Intelligenz“ bereits seit Ende der 1950er Jahre untersucht (vgl. Russel/Norvig 2003). Ein Individualisierungssystem, das digitale Inhalte individuell für einen Rezipienten entsprechend seiner Bedürfnisse bündelt, muss über drei Komponenten verfügen (vgl. Abbildung 20):

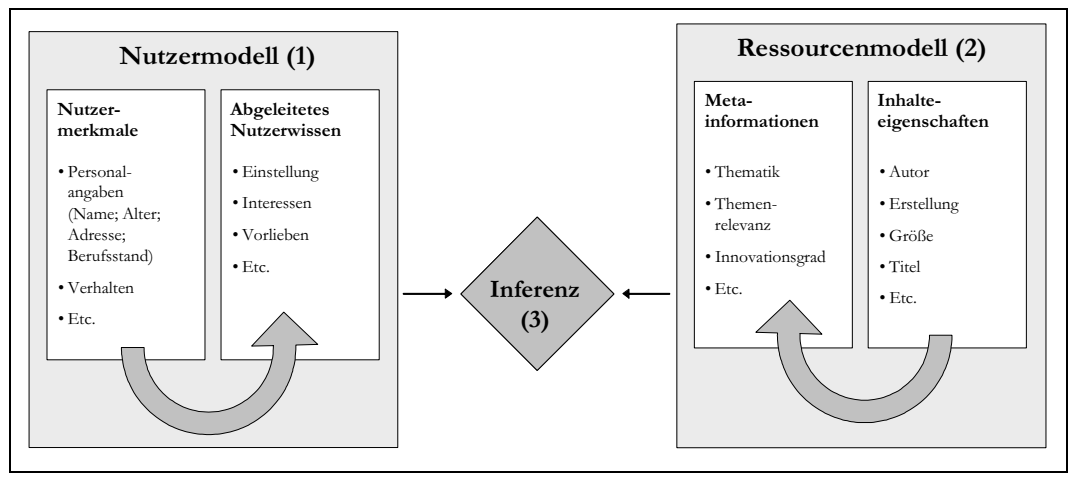

Abbildung 20: Aufbau eines Individualisierungssystems (angelehnt an Schackmann / Link 2001)

ein Nutzermodell, in dem die Bedürfnisse und Präferenzen des Rezipienten gespeichert sind (1); ein Ressourcenmodell, das verfügbare Inhalte auf der Grundlage von 
Inhalteeigenschaften kategorisiert (2); ein automatisches Verfahren, einen sog. „Inferenzmechanismus", der die individuelle Zuordnung zwischen Ressourcen und Kunden vornimmt (3).

(1) In der Regel kann ein einzelnes identifiziertes Nutzermerkmal, wie bspw. eine dem Nutzer zugeordnete Handlung in der Protokolldatei eines Webservers, nicht automatisch in ein maschinenverständliches Ereignis umgewandelt werden, anhand dessen die individuelle Anpassung eines Medienprodukts vorgenommen werden kann. Das zentrale Problem von Individualisierungssystemen im Rahmen der Nutzermodellierung besteht vielmehr darin, die maschinell erfassbaren nutzerbezogenen Rohdaten zu Bedürfnissen und Präferenzen in Bezug auf die verfügbaren Inhalte zu verdichten. Ein Individualisierungssystem erzeugt daher auf der Grundlage maschinell erfassbarer Nutzermerkmale Annahmen über den Nutzer, die in einem Nutzermodell gespeichert werden. Auf Basis der Annahmen des Nutzermodells wird die weitere Interaktion des Nutzers an dessen individuelle Bedürfnisse und Präferenzen angepasst. Das Nutzermodell enthält das gesammelte spezifische Wissen über einen individuellen Nutzer für das jeweilige Medienprodukt (vgl. Fink/Kobsa 2000). Die Rohdaten der Nutzermodellierung können, wie auch schon in Abschnitt 3.1 erläutert, aus Beobachtungen der unmittelbaren manipulativen Interaktionen eines Nutzers, aus expliziten Nutzerbefragungen oder aus Analysen der durch den Nutzer nachgefragten Informationen gewonnen werden. Eine Möglichkeit der Kombination von Verhaltensbeobachtung und Informationsanalyse bildet die Speicherung gewichteter Schlagwortlisten, die auf einer statistischen Auswertung der Seiteninhalte besuchter Webseiten beruhen (vgl. Kaspar/Hagenhoff 2004). Dazu werden zunächst Verhaltensprofile in Form häufig besuchter URL-Kombinationen bspw. anhand von Cluster- oder Assoziationsanalysen der Protokolldateien ermittelt. Diesen Verhaltensprofilen können dann auffällige Textmerkmale oder inhaltsbezogene Metadaten der besuchten Adressen zugeordnet werden.

(2) Damit ein Individualisierungssystem eine individuelle Auswahl an Inhalten für einen Rezipienten vornehmen kann, müssen zu den in der Datenbank verfügbaren Inhalten Metainformationen auf Grundlage vordefinierter Kategorien als Auswahlkriterien gespeichert werden (vgl. Hess 2004). Eine solche Inhaltekategorisierung kann entweder durch den Autor, bspw. im Zuge der Inhalteerstellung, oder durch automatische Textanalyse und -klassifikationsinstrumente erfolgen. Der Vorteil, aber auch das Risiko, einer automatischen Textkategorisierung besteht in einer Unabhängigkeit der Einordnung eines Inhalts von dem vom Autor als plausibel erachteten Themenfeld. Beispiel für Textanalyseverfahren bilden Techniken zur Erkennung von Textstrukturen, die Erstellung von Stopplisten sowie Wortanalysen - beispielsweise durch Stammformbildung, durch Analyse des Bedeutungskontextes bei Mehrdeutigkeiten oder durch Ermittlung von Mehrworttermen. Auf der Grundlage solcher Textanalysen können Inhalte in logisch zu- 
sammengehörige Partitionen gruppiert werden. Die hier verfügbaren Techniken können anhand von zwei Dimensionen unterschieden werden (vgl. Frants/Shapiro/Voiskunskii 1997): Zum einen nach dem gegenseitigen Ausschlussgrad der zulässigen Kategorien in nicht-exklusive bzw. überlappende Gruppierung und exklusive Gruppierung. Zum anderen nach dem Überwachungsgrad der Kategorien durch den Autor, in extrinsische (fremdbestimmte) und intrinsische (automatische) Gruppierung.

(3) Als Inferenzmechanismen zum Abgleich zwischen Nutzerprofil und den zu individualisierenden Inhalten können zwei verschiedene Verfahren eingesetzt werden: Eigenschaftsbasierte Filterverfahren ziehen einen direkten Vergleich zwischen den Profilmerkmalen eines Nutzers und den Eigenschaften verfügbarer Inhalte und treffen eine Auswahl der Inhalte, die mit den Nutzerbedürfnissen am besten übereinstimmen. Eigenschaftsbasierte Filterverfahren verwenden in der Regel statistische Klassifikationsalgorithmen, um Inhalte entsprechend den jeweiligen Nutzermodellen zu gruppieren (van Meteren/van Someren 2001). Im Rahmen solcher Klassifikationsalgorithmen können verschiedene Verfahren zum Einsatz kommen wie bspw. Vektorraummodelle (vgl. Salton/Wong/Yang 1975), Verfahren auf Grundlage kombinierter Wahrscheinlichkeiten (vgl. Pazzani/Billsus 1997), Entscheidungsbäume oder neuronale Netze (vgl. Yang/Liu 1999). Collaborative Filtering Verfahren leiten dagegen individualisierte Angebote indirekt, anhand struktureller Gemeinsamkeiten in den Präferenzen verschiedener Nutzern ab (vgl. Herlocker/Konstan/Borchers/Riedl 1999; Melville/Mooney/ Nagarajan 2001; Schafer/Konstan/Riedl 2002). Anstelle einer direkten Ähnlichkeitsüberprüfung zwischen Inhalteeigenschaften und Nutzerpräferenzen werden zunächst signifikante Präferenzähnlichkeiten zwischen verschiedenen Nutzern bestimmt. Auf der Grundlage solcher Präferenzähnlichkeiten treffen Collaborative Filtering Verfahren für einen Nutzer eine Auswahl an Inhalten, die ein anderer Nutzer mit ähnlichen Präferenzen nachgefragt hat.

Individualisierungssysteme können im Zusammenhang mit Medienprodukten in zweierlei Form eingesetzt werden: Zum einen kann ein Individualisierungssystem, insbesondere bei einem großen und unübersichtlichen Angebot an unterschiedlichen für den Massenmarkt gefertigten Medienprodukten, Kaufempfehlungen entsprechend der jew. Nutzerpräferenzen aussprechen. Das bekannteste Beispiel eines solchen Empfehlungssystems für Medienprodukte bildet die persönliche Kaufempfehlung auf der Webseite des Onlinebuchhandels Amazon. Zum anderen kann ein Individualisierungssystem sowohl die Tiefe, als auch die Breite einzelner inhaltlicher Bestandteile eines Medienprodukts gezielt auf Kundeninteressen abstimmen. Derzeit bereits in der Praxis verfügbare Beispiele solcher individuellen Produktformen bilden individuell konfigurierbare Onlinezeitungen wie das Onlineangebot des amerikanischen Wall Street Journal oder der deutsche Nachrichtendienst TOPIX der Wirtschaftszeitung Handelsblatt. 


\section{Nutzen individueller Medienprodukte}

Einleitend wurde festgestellt, dass das Überangebot von Medienprodukten in den Medienmärkten zu einer sinkenden Zahlungsbereitschaft der Rezipienten geführt hat. Im Folgenden wird aus Sicht eines Medienunternehmens erläutert, in wiefern Konsumenten durch Berücksichtigung individueller Konsumpräferenzen dennoch ein Kaufanreiz für ein Medienprodukt geboten werden kann. In Abschnitt 4.1 werden Bedingungen der Entscheidung eines Rezipienten für den Konsum von Inhalten erläutert und ein ökonomisches Nutzenkalkül formuliert. Auf dessen Grundlage wird in Abschnitt 4.2 untersucht, welche Auswirkungen eine Individualisierung der Bestandteile eines Medienprodukts auf die Zahlungsbereitschaft des Rezipienten besitzt.

\subsection{Nutzenkalkül eines Rezipienten}

Ein Medienprodukt wird in erster Linie wegen seines Inhalts konsumiert (vgl. Brandtweiner 2000, S. 41; Sjurts 2002, S. 8; Schumann/Hess 2002, S. 39). Ein Rezipient wählt im Rahmen seiner Konsumentscheidung immer dasjenige Medienprodukt aus, von dessen Inhalten er sich den höchsten Nutzen verspricht. Dabei ist ein Rezipient bereit, für ein Medienprodukt nur gerade den Differenzwert aus dem für ihn erwarteten Nutzwert der Inhalte dieses Medienprodukts und den für ihn durch Auswahl und Rezeption entstehenden Kosten zu bezahlen (vgl. nachstehende Abbildung).

\begin{tabular}{|c|c|c|c|}
\hline Vorleistungen & \multirow{4}{*}{ 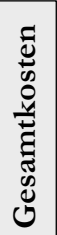 } & \multirow{4}{*}{$\leq$} & \multirow{4}{*}{$\begin{array}{l}\text { Nutzwert des } \\
\text { Medienprodukts }\end{array}$} \\
\hline Auswahlkosten & & & \\
\hline Nutzungskosten & & & \\
\hline Kaufpreis & & & \\
\hline
\end{tabular}

Abbildung 21: Notwendige Bedingung des Medienkonsums

Die Digitalisierbarkeit von Inhalten schafft - wie in Kapitel 2 erläutert wurde zwar neue Möglichkeiten im Rahmen der Inhaltebündelung und -distribution, der kreative Prozess im Rahmen der Inhalteerzeugung und dadurch der objektive Nutzwert der resultierenden Inhalte bleibt jedoch weitgehend unverändert. Sollen digitalisierte Inhalte einen Kaufanreiz für Rezipienten bieten, muss daher ihr Bündelungs- oder Distributionsformat gegenüber bestehenden Formaten einen geringeren Rezeptionsaufwand besitzen. Dabei lassen sich im Rahmen der Rezeption von Medienprodukten vier Aufwandsarten unterscheiden (vgl. Fehr 2003, S. 29 f.): 
der nominelle Kaufpreis des Medienprodukts sowie Aufwendungen für Vorleistungen, Auswahl und Nutzung.

Der Kaufpreis eines Medienprodukts kann einmaliger oder wiederkehrender Natur sein. Wiederkehrende Kaufpreise hängen von der Nutzungsintensität nachgefragter Inhalte ab. Neben nutzungsabhängigen nominellen Kaufpreisen, wie sie bspw. bei Tageszeitungen im Straßenverkauf anfallen, können beim Erwerb von Onlineinhalten weitere wiederkehrende Kosten bspw. durch den Aufwand der Zahlungsabwicklung anfallen.

Ein einmaliger Kaufpreis, wie er bspw. bei einem Zeitungsabonnement erhoben wird, stellt dagegen eine Vorleistung für den Konsum von Inhalten in Form eines Erwerbs von ggf. längerfristigen Inhaltebezugsrechten dar. Neben einmaligen Aufwendungen für den Erwerb solcher Bezugsrechte können im Falle von Onlineinhalten für die Inhalterezeption weitere Vorleistungen in Form von Sachund Lerninvestitionen zur Gewährleistung der Nutzung des Mediums anfallen. Derartige Sachinvestitionen umfassen bspw. die Anschaffung eines PCs sowie der Unterhalt eines Internetanschlusses. Lerninvestitionen bestehen demgegenüber bspw. in der Aneignung von Kenntnissen, die zum Umgang mit PC und Internet erforderlich sind.

Der Aufwand im Zuge der Auswahl eines Medienprodukts ist zweifacher Natur: Die Auswahl bedürfnisgerechter Inhalte erzeugt durch die Dauer der Suche erstens einen zeitlichen Aufwand. Bereits 1961 stellte der später u. a. für diese Untersuchung mit dem Nobelpreis geehrte amerikanische Ökonom George Stigler fest, dass die Marktpreise selbst bei homogenen Gütern wie Steinkohle zum Teil erheblich streuen können (vgl. Stigler 1961). Ursache dieser Preisstreuung bilden Informationsasymmetrien zwischen den Marktnachfragern, die entstehen, weil der Vergleich von konkurrierenden Preisen und Eigenschaften unterschiedlicher Angebote einen zeit- und damit kostenintensiven Vorgang darstellt. Dieses allgemeine Auswahlproblem wird im Falle von Medienprodukten zweitens dadurch verstärkt, dass ein Rezipient den genauen Produktnutzen angebotener Inhalte vor dem Kauf bestenfalls unvollständig kennt (vgl. Heinrich 2001, S. 99). Um das resultierende Rezeptionsrisiko zu minimieren, entsteht für den Rezipienten ein zusätzlicher Suchaufwand im Rahmen der Nutzung von Bewertungssubstituten für ein Medienprodukt, bspw. in Form von Kaufempfehlungen oder redaktionellen Kritiken.

Die Inhalterezeption selbst kann durch ihren Zeitverzehr einen Aufwand für den Rezipienten darstellen. Der Nutzungsaufwand wird zum einen beeinflusst durch performative und nutzungsökonomische Merkmale des jeweiligen Distributionsformats. Üblicherweise erfolgt die Vermittlung kurzer, stark aggregierter Inhalte tendenziell in elektronischen, bspw. audio-visuellen Formaten, die Vermittlung von detaillierten Hintergrundinformationen in textbasierten Formaten. Digi- 
tale Distributionsformate wie Webseiten oder DVDs erlauben potenziell eine Senkung des durchschnittlichen Nutzungsaufwands durch die Verknüpfung audiovisueller und textbasierter Vermittlung. Ob tatsächlich der Nutzungsaufwand für ein Medienprodukt reduziert werden kann, hängt dabei insbesondere von der Gestaltung der Inhaltepräsentation, bspw. in Form einer intuitiven Bedienbarkeit, sowie insbesondere bei Onlineinhalten von einer akzeptablen Bereitstellungsdauer und Darstellungsqualität ab. Zum anderen können speziell bei werbefinanzierten Inhalten Werbeelemente den Nutzungsaufwand erhöhen, besonders dann, wenn Werbung parallel mit den rezipierten Inhalten wahrgenommen wird. Je mehr Werbebanner bspw. auf einer Seite platziert sind, desto zeitaufwändiger ist die Rezeption des gewünschten Inhalts, da die Nutzeraufmerksamkeit abgelenkt wird.

Zusammenfassend kann das Entscheidungskalkül eines Rezipienten (j) als Budgetrestriktion seines Einkommens (E) in Abhängigkeit vom nutzungsunabhängigen Aufwand für Kaufpreis (pNOM), Auswahl ( $\mathrm{p}_{\text {INF }}$ ) und Vorleistungen (pADP) sowie vom nutzungsabhängigen Aufwand für Einzelpreise von Inhalten (qNOM) und dem inhaltebezogenen Nutzungsaufwand (qusE) in Abhängigkeit von der Nutzungsintensität (v) eines Inhalts (i), sowie seinen Ausgaben für alle anderen Konsumgüter (H) formuliert werden als (vgl. Fink 2002, S. 71 f.):

$$
E=H+(\underbrace{p_{N O M}+p_{I N F}+p_{A D P}}_{p})+\underbrace{\left(q_{N O M}+q_{U S E}\right)}_{q} \cdot \sum_{i=1}^{n} v_{i}
$$

Im Modellfall einer Cobb-Douglas-Nutzenfunktion lässt sich der von einem Rezipienten aus Inhalte- und sonstigem Güterkonsum erzielbare Nutzen (U) des Einkommens in Abhängigkeit von der relativen Präferenz des Medienkonsums $(\alpha)$ im Vergleich zu sonstigen Konsummöglichkeiten $(\mathrm{H})$ formulieren als:

$U=H^{\left(1-\sum_{i=1}^{n} \alpha_{i}\right)} \cdot \prod_{i=1}^{n} v_{i}^{\alpha_{i}}$

bzw. als deren monotone Transformation:

$U=\left(1-\sum_{i=1}^{n} \alpha_{i}\right) \ln (H) \cdot \sum_{i=1}^{n} \alpha_{i} \ln \left(v_{i}\right)$

\subsection{Konsumentscheidung eines Rezipienten}

Die Konsumentscheidung eines Inhalterezipienten repräsentiert ein zweistufiges Optimierungskalkül in Bezug auf die Nutzenfunktion in Ausdruck 4.1-2b unter 
der Bedingung der Budgetrestriktion in Ausdruck 4.1-1 (vgl. Fink 2002, S. 74 ff.). Zunächst muss ein Konsumplan für die optimale Aufteilung des verfügbaren Einkommens zwischen der Nachfrage (v) nach Inhalten (i) und allen übrigen Konsummöglichkeiten aufgezeigt werden. Die zugehörige Lagrangefunktion für das Optimierungsproblem der Budgetaufteilung lautet:

$$
L=\underbrace{\left(1-\sum_{i=1}^{n} \alpha_{i}\right) \ln (H) \cdot \sum_{i=1}^{n} \alpha_{i} \ln \left(v_{i}\right)}_{\text {Nutzenfunktion }}+\underbrace{\lambda\left(E-H-p-q \sum_{i=1}^{n} v_{i}\right)}_{\text {Budgetrestriktion }} \rightarrow \max !
$$

Die Lösung der Lagrangefunktion liefert die bedingte optimale Nachfragefunktion für die Menge (v) eines Inhalts (i) in Abhängigkeit der Konsumpräferenzen ( $\alpha$ ) des Nutzers für den jeweiligen Inhalt, seinem Einkommen sowie dem Rezeptionsaufwand (p bzw. q):

$$
v_{i}=\frac{\alpha_{i}(E-p)}{q}
$$

Im zweiten Schritt muss überprüft werden, ob der optimale Konsumplan in Ausdruck 4.2-2 die in Abbildung 21 dargestellte Bedingung eines positiven Nutzwerts erfüllt. Diese Bedingung ist dann erfüllt, wenn der Nutzen aus der Aufteilung des Budgets in Inhaltekonsum und dem Konsum aller anderen Güter gemäß Ausdruck 4.2-2 einen höheren Nutzwert erzeugt, als die Verwendung des gesamten Budgets ausschließlich für andere Güter. Diese Bedingung eines positiven Nutzwerts spiegelt sich formal in der Ungleichung in Ausdruck 4.2-3 wieder:

$$
\underbrace{\left(1-\sum_{i=1}^{n} \alpha_{i}\right) \ln (H)}_{\substack{\text { Nutzen des reduzierten } \\
\text { Konsums anderer Güter }}}+\underbrace{\sum_{i=1}^{n}\left(\alpha_{i} \cdot \ln \left(v_{i}\right)\right)}_{\begin{array}{c}
\text { Nutzen des } \\
\text { Inhaltekonsums }
\end{array}}-\underbrace{\left(1-\sum_{i=1}^{n} \alpha_{i}\right) \cdot \ln (E)}_{\begin{array}{c}
\text { Nutzen des ausschl. } \\
\text { Konsums anderer Güter }
\end{array}} \geq 0
$$

Durch einsetzen des optimalen Konsumplans in Ausdruck 4.2-2 in die Konsumbedingung in Ausdruck 4.2-3 kann ein positiver Nutzwert (S) als Bedingung aus der nutzungsabhängigen Zahlungsbereitschaft und dem nutzungsunabhängigen Aufwand der Inhalterezeption formuliert werden wie dargestellt in Ausdruck 4.2-4. 


$$
\left.S_{j}=\sum_{i=1}^{n}\{\underbrace{\alpha_{i}\left[\ln \left(\frac{\alpha_{i}}{q}\right)-1\right]}_{\substack{\text { Bruttonutzen eines } \\ \text { Inhalts } i}}\}-p \stackrel{!}{\geq 0}\right\}
$$

Aus der Konsumbedingung in Ausdruck 4.2-4 können zwei Schlussfolgerungen gezogen werden: Zum einen kann ein positiver Nutzwert nur dann entstehen, wenn der nutzungsabhängige Aufwand (q) der Inhalterezeption möglichst klein ist. Zum anderen steigt der kumulierte Nutzwert eines Nutzers (j) in Abhängigkeit von seinen inhaltebezogenen Präferenzen $(\alpha)$ mit der Anzahl (n) der rezipierten Inhalten (i).

\subsection{Einfluss von Individualisierung auf die Konsumentscheidung eines Rezipienten}

Wie bereits in Abschnitt 2.2 angesprochen wurde, werden Medienprodukte üblicherweise aus anbieterseitigen Kostengründen als Bündel verschiedener Inhalte zu einem nominellen Einheitspreis ( $\mathrm{p}_{\mathrm{NOM}}$ ) ohne zusätzliche Gebühren für die Einzelinhalte $\left(\mathrm{q}_{\mathrm{NOM}}=0\right)$ angeboten. Vor dem Hintergrund der Schlussfolgerungen aus Ausdruck 4.2-4 erzeugt eine Bündelung von Inhalten jedoch nicht nur anbieterseitige Skaleneffekte des Vertriebs, sondern kann zudem zu einer nachfrageseitigen Senkung des Rezeptionsaufwands führen und damit durch den Anbieter als Preisgestaltungsinstrument eingesetzt werden. Die Bündelung von Gütern als Instrument einer Preisdiskriminierung wurde erstmalig ausführlich untersucht von Adams und Yellen (1976), erweitert von Schmalensee (1984) sowie von Olderog und Skiera (1998). Unter der Annahme normalverteilter Reservationspreise verschiedener Rezipienten (ausgedrückt durch den Präferenzparameter $\alpha$ ) nähert sich im Falle einer standardisierten Bündelung von Inhalten für den Massenmarkt die durchschnittliche Zahlungsbereitschaft für das Inhaltebündel an den Erwartungswert der gemeinsamen Reservationspreisverteilung aller Rezipienten an (vgl. Adams / Yellen 1976; Schmalensee 1984; Olderog / Skiera 1998).

Wie in Kapitel 3 erläutert wurde, fungiert ein Individualisierungssystem als Filtermechanismus, der von einem Medienunternehmen dafür eingesetzt werden kann, für einen Rezipienten aus verfügbaren Inhalten diejenigen auszuwählen, die am besten zu seinen Interessen passen. Indem nur solche Inhalte angeboten werden, die den Interessen des Rezipienten entsprechen, kann ein Individualisierungssystem, das eingesetzt wird, um bspw. im Rahmen einer Onlinezeitung ein individuelles Inhaltebündel zu konfigurieren, dazu beitragen, den Nutzungsaufwand im Rahmen der Rezeption im Vergleich zu einem unkonfigurierten Bündel zu senken (quse $\downarrow$ ). Darüber hinaus kann ein individuelles Empfehlungssystem den nutzungs- 
unabhängigen Suchaufwand nach wunschgemäßen Medienprodukten senken und gleichzeitig dazu beitragen, das angesprochene Auswahlrisiko in Bezug auf die Inhaltequalität zu senken ( $\left.\mathrm{p}_{\mathrm{INF}} \downarrow\right)$ ). Allerdings setzt eine effektive Senkung des Auswahlaufwands voraus, dass der Rezipient mit der Funktionsweise des, der Empfehlung zugrunde liegenden Filtermechanismus vertraut ist und seiner Empfehlung Vertrauen schenkt. Dies erfordert einen gewissen Lernaufwand, sowohl in Bezug auf die Erweiterung der Fähigkeiten des Rezipienten, als auch ggf. in Bezug auf eine gewisse Trainingsdauer des verwendeten Filteralgorithmus (pADP $\uparrow$ ).

Vor dem Hintergrund der einleitenden Fragestellung, in wiefern Individualisierungssysteme einen Beitrag leisten, um die Zahlungsbereitschaft von Rezipienten zu steigern, kann der Einfluss einer Individualisierung auf die Konsumentscheidung des Rezipienten gemäß der Ungleichung in Ausdruck 4.3-1 zusammenfasst werden:

$p_{N O M} \leq \sum_{i=1}^{n}\left\{\alpha_{i}\left[\ln \left(\frac{\alpha_{i}}{q_{U S E} \downarrow}\right)-1\right]\right\}-\left(p_{I N F} \downarrow+p_{A D P} \uparrow\right)$

Ein Rezipient wird nur dann bereit sein, für individuelle Inhalteangebote zu bezahlen (ausgedrückt durch $\mathrm{p}_{\mathrm{NOM}}>0$ ), wenn die Summe des Nutzens aus den nachgefragten Inhalten seinen Such- und Lernaufwand übersteigt. Die Bedingung der relativen Vorteilhaftigkeit individualisierter Angebote gegenüber nichtindividualisierten Inhaltebündeln kann dementsprechend gemäß der Ungleichung in Ausdruck (4.3-2) formuliert werden.

$$
\sum_{i=1}^{n}\left\{\alpha_{i}\left[\ln \left(\frac{\alpha_{i}}{q_{U S E_{B}}}\right)-1\right]\right\}-\left(p_{I N F_{B}}+p_{A D P_{B}}\right) \leq \sum_{i=1}^{m}\left\{\alpha_{i}\left[\ln \left(\frac{\alpha_{i}}{q_{U S E_{I}}}\right)-1\right]\right\}-\left(p_{I N F_{I}}+p_{A D P_{I}}\right)
$$

Individualisierung ist im Vergleich zu einer standardisierten Bündelung immer dann von Vorteil, (1) wenn entweder die nutzungsunabhängigen Kosten eines individuell angebotenen Bündels ( $\mathrm{p}_{\mathrm{I}}$ ) kleiner sind als im Falle des Massenmarktprodukts $\left(\mathrm{p}_{\mathrm{B}}\right)$, oder (2), wenn ein individuelles Bündel beim Nutzer einen höheren Bruttonutzen $\left(\mathrm{S}_{\mathrm{I}, \mathrm{br}}\right)$ als ein nichtindividualisiertes Bündel $\left(\mathrm{S}_{\mathrm{B}, \mathrm{br}}\right)$ erzeugt.

(1) In Ausdruck 4.3-1 wurde zusammengefasst, dass ein individuelles Empfehlungssystem zwar die nachfrageseitigen Suchkosten ( $\mathrm{P}_{\mathrm{INF}, \mathrm{I}}$ ) reduzieren kann, jedoch zu einem höheren Lernaufwand ( $\mathrm{p}_{\mathrm{ADP}, \mathrm{I}}$ ) führt. Die Vorteilhaftigkeitsbedingung in Un- 
gleichung 4.3-2 ist ceteris paribus immer dann erfüllt, wenn der nutzungsunabhängigen Kosten eines individuellen Bündels ( $\mathrm{p}_{\mathrm{I}}$ ) geringer ausfällt als der des Massenmarktprodukts $\left(\mathrm{p}_{\mathrm{B}}\right)$. Dies ist immer dann der Fall, wenn die Differenz der Suchkosten von individuellem und Massenmarktbündel höher ist als die Differenz der Lernkosten (vgl. Ausdruck 4.3-3).

$$
\begin{aligned}
& p_{I N F B}+p_{A D P B}>p_{I N F_{I}}+p_{A D P I} \Longleftrightarrow p_{I N F_{B}}-p_{I N F_{I}}>p_{A D P_{I}}-p_{A D P B} \\
& \text { Differenz der Differenz des } \\
& \text { Suchkosten Lernaufwands }
\end{aligned}
$$

(2) Der nutzungsbezogene Bruttowert eines Inhaltebündels ist abhängig vom Präferenzparameter $(\alpha)$ eines Nutzers und den Opportunitätskosten (qusE) des Konsums der im Bündel enthaltenen Inhalte. Vor dem Hintergrund der vereinfachenden Annahme normalverteilter Konsumpräferenzen kann der Präferenzparameter als nutzerinvariant und dadurch sowohl im Falle individualisierter als auch nichtindividualisierter Bündelung vereinfachend als gleichförmig verteilt angenommen werden. Insofern Konsumenten in der Regel nur einen kleinen Teil ihres Budgets in den Medienkonsum investieren, kann der Erwartungswert für das Präferenz$\mathrm{maß}$ als klein angenommen werden. Ebenso bilden die Opportunitätskosten der Inhalterezeption eines Inhalteaggregats unabhängig von dessen Individualisierungsgrad üblicherweise lediglich einen kleinen Aufwand im Cent-Bereich. Somit ergibt die in Ausdruck 4.3-1 enthaltene Schlussfolgerung einer Reduzierung der Opportunitätskosten der Inhalterezeption durch Individualisierung der Aggregation einzelner Elemente eines Inhaltebündels, vor dem Hintergrund der getroffenen Annahmen, ein zwingendes Vorteilskriterium. Abbildung 22 veranschaulicht den Verlauf der Bruttonutzenfunktion für ein individualisiertes Inhaltebündel mit $n=8$ Elementen bzw. ein nichtindividualisiertes Inhaltebündel mit $\mathrm{n}=10$ Elementen. ${ }^{13}$

Beide abgebildeten Nutzenfunktionen besitzen unter den in Ausdruck 4.2-4 zugrunde gelegten Annahmen eine streng monoton steigende Form. Die Vorteilhaftigkeit individueller Inhaltebündel wird gemeinhin jedoch insbesondere dann unterstellt, wenn das Überangebot an Inhalten ein Auswahlproblem für den Nutzer erzeugt. Vor diesem Hintergrund ist davon auszugehen, dass bei einem individuellen Inhaltebündel ab einer gewissen Bündelgröße (n) der durch das Vorselektieren relevanter Inhalte geschaffene Mehrwert konterkariert wird. Es ist demnach ab einer gewissen Bündelgröße von einem sinkenden Grenznutzen zusätzlicher Elemente eines individuellen Inhaltebündels auszugehen.

13 Als Zahlenbeispiel wurden angenommen: $\mathrm{E}(\alpha)=0,1 ; \mathrm{quSE}, \mathrm{B}=0,3 ; \mathrm{quSE}, \mathrm{I}=0,2$. 


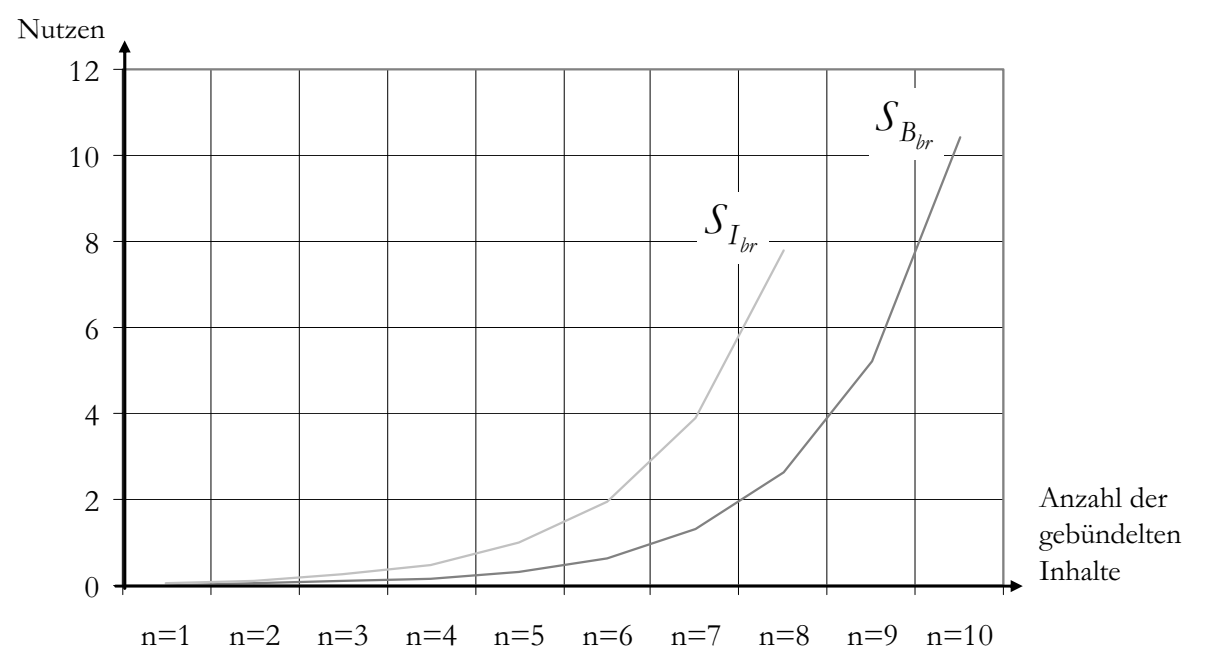

Abbildung 22: Bruttonutzenfunktionen für ein individualisiertes und ein nichtindividualisiertes Bündel

\section{$5 \quad$ Fazit}

Im zweiten Kapitel wurde dargelegt, dass die traditionelle Alternativhypothese in Bezug auf Kostenoptimierung und Varietätsgrad, durch die Standardisierbarkeit digital und medienneutral erzeugter Inhalten, in der Medienbranche nur noch bedingt Gültigkeit besitzt. Insbesondere gilt der durch hohe First-Copy-Costs begründete Zwang zu Massenkommunikation für Medienunternehmen nicht mehr uneingeschränkt. Wenn Medienunternehmen die Wünsche ihrer Kunden individuell bestimmen können, dann kann eine individuelle Produktvariante auf der Grundlage standardisierter Module zu einem Kostenniveau erzeugt werden, das dem der Massenfertigung entspricht. Eine solche Individualisierung von Medienprodukten erfordert den Einsatz von Individualisierungssystemen, die überblicksartig in Kapitel 3 vorgestellt wurden. Die Individualisierung des Angebots von Medienprodukten besitzt immer dann gegenüber alternativen Inhalteangeboten einen relativen Mehrwert, wenn dadurch der für ihn mit der Rezeption verbundene Aufwand gesenkt werden kann. Wie in Kapitel 4 gezeigt wurde, ist die Vorteilhaftigkeit von Individualisierungsformen immer dann gegeben, wenn zum einen im Rahmen der Auswahl der betreffenden Inhalte ein hoher Suchaufwand besteht und zum anderen der Aufwand der Rezeption einzelner Inhalte gesenkt wird.

Das Ergebnis der Vorteilhaftigkeit von Individualisierungsformen in der Medienbranche in Kapitel 4 wurde unter stark vereinfachenden Annahmen ermittelt. Aus Gründen der Vereinfachung wurden in der Praxis sicher existierende Effekte 
wie ein sinkender Grenznutzen der Rezeption zusätzlicher Inhalte bei steigender Zahl gemeinsam angebotener Inhalte oder nachfrageseitige Budgetrivalitäten zwischen Medienprodukten unterschiedlicher Distributionsformate vernachlässigt. Dennoch konnte gezeigt werden, dass eine Individualisierung des Angebots von Inhalten unter gewissen Bedingungen, wie hohen Such- und Selektionskosten, für Rezipienten einen Mehrwert bieten kann. Somit kann der Möglichkeit zur Individualisierung von Medienprodukten, vor dem Hintergrund der einschränkenden Vorteilhaftigkeitsbedingungen, das Potenzial für einen radikalen Paradigmenwechsel in der Medienbranche beschieden werden: War bislang die absolut generierbare Reichweite das dominante ökonomische Zielkriterium des Medienmanagements, kann nun der individuelle Kundenwertbeitrag als strategisches Ziel verfolgt werden, wobei der Endkunde individuell den Wert der wirtschaftlichen Aktivitäten bestimmt. Denkbare Hebel für eine solche strategische Neuausrichtung bilden eine Verlängerung der Kundenbeziehungsdauer durch Steigerung der Kundenloyalität sowie eine cross-mediale Erfassung und Berücksichtigung der Kundenbedürfnisse.

\section{Literatur}

Adams/Yellen (1976): Adams, W., Yellen, J.: Commodity Bundling and the burden of monopoly. The Quaterly Journal of Economics; Vol. 90, Issue 3 (Aug. 1976), S. 476-498.

Bensberg (2001): Bensberg, F: Web Log Mining als Instrument der Marketingforschung. Deutscher Universitätsverlag, Wiesbaden 2001.

Brandtweiner (2000): Brandtweiner, R.: Differenzierung und elektronischer Vertrieb digitaler Informationsgüter. Symposion Publishing, Düsseldorf 2000.

Ecin (2002): Ecin: Gute Perspektive für Paid Content. http://www.ecin.de/news/ 2002/12/16/05190/, Abruf am 01.12.2004.

Ecin (2003): Ecin: Steigende Akzeptanz für kostenpflichtige Inhalte; http://www.ecin.de/news/2003/02/04/05336/, Abruf am 01.12.2004.

Fehr (2003): Fehr, H.: Paid Content erfolgreich verkaufen. Ein Leitfaden zur Erstellung eines individuellen Konzepts zum Verkauf von digitalen Inhalten in Onlineportalen. Hansebuch Verlag, Hamburg 2003.

Fink/Kobsa (2000): Fink, J., Kobsa, A.: A Review and Analysis of Commercial User Modeling Servers for Personalization on the World Wide Web. In: User Modeling and User-Adapted Interaction; Nr. 10 2000; S. 209-249.

Fink (2002): Fink, L.-R.: Economies of Aggregation - Internetportale als Informationsgüterbündel und die Zahlungsbereitschaft der Nachfrager, Dissertation, Universität Köln, Köln 2002.

Frants/Shapiro/Voiskunskii (1997): Frants, V., Shapiro, J., Voiskunskii, V.: Automated Information Retrieval. Academic Press; San Diego 1997. 
Grimm/Jüstel/Klotz (2002): Grimm, R., Jüstel, M., Klotz, M.: Methoden zur Personalisierung im M-Commerce. In: Gora, W. / Röttger-Gerigk, S. (Hrsg.): Handbuch Mobile-Commerce. Springer Verlag, Berlin 2002; S. 177-190.

Hass (2002): Hass, B.: Geschäftsmodelle von Medienunternehmen - Ökonomische Grundlagen und Veränderungen durch neue Informations- und Kommunikationstechnik. Gabler, Wiesbaden 2002.

Heinrich (2001): Heinrich, J.: Medienökonomie. Bd.1: Mediensystem, Zeitung, Zeitschrift, Anzeigenblatt. Westdeutscher Verlag, Wiesbaden 2001.

Herlocker/Konstan / Borchers / Riedl (1999): Herlocker, J., Konstan, J., Borchers, A., Riedl, J.: An Algorithmic Framework for Performing Collaborative Filtering. Proceedings of the 22nd annual international ACM SIGIR conference on research and development in information retrieval 1999; S. 230-237.

Hess (2004): Hess, T.: Medienunternehmen im Spannungsfeld von Mehrfachverwertung und Individualisierung - eine Analyse für statische Inhalte. In: Zerdick, A., Picot, A., Schrape, K., Burgelman, J.-C., Silverstone, R., Feldmann, V., Heger, D., Wolff, C. (Hrsg.): E-Merging Media - Kommunikation und Medienwirtschaft der Zukunft. European Communication Council Report; Springer Verlag, Berlin 2004.

Kaspar/Hagenhoff (2004): Kaspar, C., Hagenhoff, S.: Individualization of a mobile news service - a simple approach. In: Proceedings of the VIIth SAM/IFSAM World Congress, Göteborg, 2004.

Melville/Mooney/Nagarajan (2001): Melville, P., Mooney, R., Nagarajan, R.: Content-Boosted Collaborative Filtering. Proceedings of 18th national conference on artificial intelligence 2001; S. 187-192.

Mings/White (2000): Mings, S., White, P.: Profiting from Online News: The Search for Viable Business Models. In: Kahin, B. / Varian, H. (Hrsg.): Internet Publishing and Beyond - The Economics of Digital Information and Intellectual Property. The MIT Press, Cambridge 2000; S. 62-96.

Mobasher (2002): Mobasher, B.: WebPersonalizer: A Server-Side Recommender System Based on Web Usage Mining. http://facweb.cs.depaul.edu/ research/TechReports/ TR01-004.pdf, Abruf am 01.12.2004.

Mobasher/Cooley/Srivastava (1999): Mobasher, B., Cooley, R., Srivastava, J.: Automatic Personalization Through Web Usage Mining. Technical Report No. TR99010; Department of Computer Science, DePaul University. http://maya.cs.depaul.edu/ classes/ect584/papers/mobasher.pdf, Abruf am 01.12.2004.

Oesterdiekhoff (2004): Oesterdiekhoff, B.: Transcoding von Webinhalten. In: Informatik Spektrum, Band 27, Nr. 5, Oktober 2004, S. 448-452.

Olderog/Skiera (1998): Olderog, T., Skiera, B.: Vorteilhaftigkeit von Preisbündelungsstrategien. http://www.wiwi.tu-freiberg.de/wi/courses/im/bundelung.pdf, Abruf am 01.12.2004. 
Pazzani/Billsus (1997): Pazzani, M., Billsus, D.: Learning and Revising User Profiles: The Identification of Interesting Web Sites. Machine Learning, Nr. 27; Kluwer Academic Publishers, 1997; S. 313-331.

Piller (2001): Piller, F.: Mass Customization. Deutscher Universitätsverlag, Wiesbaden 2001.

Phillips (2000): Phillips, L.: Using XML. Macmillan, London 2000.

Porter (1998): Porter, M.: Wettbewerbsstrategie. Campus Verlag, Frankfurt 1998.

Rawolle (2002): Rawolle, J.: Content Management integrierter Medienprodukte - Ein XML-basierter Ansatz. Gabler, Wiesbaden 2002.

Russel/Norvig (2003): Russel, S., Norvig, P.: Artificial Intelligence, a modern approach. Pearson Education, New Jersey 2003.

Salton/Wong/Yang (1975): Salton, G., Wong, A., Yang, C.: A vector space model for automatic indexing. Communications of the ACM; Vol. 18, Issue 11, 1975; S. $613-620$.

Schackmann/Link (2001): Schackmann, J., Link, H.: Mass Customization of Digital Products in Electronic Commerce. Diskussionspapier WI-97; Lehrstuhl für Betriebswirtschaftslehre, Wirtschaftsinformatik \& Financial Engineering, Universität Augsburg 2001.

Schafer/Konstan/Riedl (2002): Schafer, B., Konstan, J., Riedl, J.: E-Commerce Recommendation Applications, Data Mining and Knowledge Discovery. Kluwer Academic Publishers, Vol. 5 2002, Issue 1-2; S. 115-153.

Schmalensee (1984): Schmalensee, R.: Pricing of Product Bundles. http://www.jstor.org/view/00219398/di993838/99p0133h/0, Abruf am 01.12.2004.

Schumann (2003): Schumann, M.: Möglichkeiten der Mehrfachverwertung von Inhalten bei Medienunternehmen - technische, organisatorische und ökonomische Betrachtung. In: Ehrenberg, D. / Kaftan, H.-J. (Hrsg.): Herausforderungen der Wirtschaftsinformatik in der Informationsgesellschaft, 1. Auflage, Leipzig, 2003.

Schumann/Hess (2002): Schumann, M., Hess, T.: Grundfragen der Medienwirtschaft. Springer Verlag, Berlin 2002.

Shapiro/Varian (1999): Shapiro, C., Varian, H.: Information Rules - A strategic guide to the network economy. Harvard Business School Press, Boston 1999.

Sjurts (2002): Sjurts, I.: Strategien in der Medienbranche - Grundlagen und Fallbeispiele; Gabler, Wiesbaden 2002.

Spiliopoulou (2001): Spiliopoulou, M.: Web Usage Mining: Data Mining über die Nutzung des Web. In: Hippner, H., Küsters, U., Meyer, M., Wilde, K. (Hrsg.): Handbuch Data Mining im Marketing, Wiesbaden 2001, S. 489-510.

Stigler (1961): Stigler, G.: The Economics of Information. In: The Journal of Political Economy, Vol. 69, Nr. 3; S. 213-225. http://www.jstor.org/view/00223808/ di950876/95p0002d/0, Abruf am 01.12.2004. 
van Meteren/van Someren (2001): van Meteren, R., van Someren, M.: Using Content-Based Filtering for Recommendation. www.ics.forth.gr/ potamias/ mlnia/paper_6.pdf, Abruf am 01.12.2004.

Vizjak/Ringlstetter (2001): Vizjak, A., Ringlstetter, M.: Medienmanagement: Content gewinnbringend nutzen. Gabler, Wiesbaden 2001.

W3C (1999): Cascading Style Sheets. W3C Recommendation 17 Dec 1996, revised 11 Jan 1999; elektronisch veröffentlicht: http://www.w3.org/TR/REC-CSS1; Abruf am 01.12.2004.

W3C (2001): Extensible Stylesheet Language (XSL), Version 1.0. W3C Recommendation 15 October 2001; http:/ / www.w3.org/TR/xsl/; Abruf am 01.12.2004.

W3C (2004): Extensible Markup Language (XML) 1.0. http://www.w3.org/ TR/REC-xml; Abruf am 01.12.2004.

Weitzel/Harder/Buxmann (2001): Weitzel, T., Harder, T., Buxmann, P.: Electronic Business und EDI mit XML. Dpunkt Verlag, Heidelberg 2001.

Yang/Liu (1999): Yang, Y., Liu, X.: A Re-Examination of Text Categorization Methods. Proceedings of the 22nd Annual International ACM SIGIR Conference on Research and Development in Information Retrieval, August 1999; S. 42-49. 


\section{Ökonomie des drahtlosen, breitbandigen Internetzugangs zu digitalen Medienprodukten}

Dieter Hogrefe / André Riedel / Marco Zibull

\section{Einleitung}

Der Gesamtmarkt für Telekommunikationsdienste in Deutschland wächst in diesem Jahr voraussichtlich um 3,3 Prozent auf insgesamt 62,9 Milliarden Euro. Sowohl im Festnetz als auch im Mobilfunk werde das Marktwachstum wesentlich von den neuen Wettbewerbern getrieben, die ihren Anteil am Gesamtumsatz bis Ende 2005 um voraussichtlich 2,5 auf 28,7 Milliarden Euro steigern können - so eine Studie des Verbandes der Anbieter von Telekommunikations- und Mehrwertdiensten (VATM e.V.) (Dialog Consult 2004).

Mit einem Umsatzvolumen von 18,9 Milliarden Euro machen dabei die Mobilfunkdienste fast zwei Drittel des Umsatzvolumens der Wettbewerber im Telekommunikationsmarkt aus. Auch der DSL-Wettbewerb erhalte durch die hohe Nachfrage nach breitbandigen Internetdiensten neue Impulse. Trotz des seit Juli 2004 möglichen Vertriebs von DSL-Anschlüssen auf Resale-Basis gewinne dieser aber nur langsam an Fahrt. Aufgrund des bestehenden Anschlussmonopols im Ortsnetz sei die Vormachtstellung der Deutschen Telekom dort allerdings weiterhin ungebrochen (Bundesamt 2003). 
Dass der TK-Markt durch Wettbewerb wächst, ist kein Geheimnis - im Zuge sich entwickelnder drahtloser Zugangstechniken, die es dem Kunden immer mehr ermöglichen werden, ortsungebunden und mit hohen Geschwindigkeiten Zugang zum Internet zu bekommen, wird dieser Wettbewerb weiter entscheidend angefacht (Galambos / Abrahamson 2002). Die monopolistische Stellung der Deutschen Telekom AG (DTAG) kann, sofern die technischen und rechtlichen Rahmenbedingungen geklärt sind, fallen oder zumindest stark geschwächt werden. Die sehr dynamischen Entwicklungen im Rahmen der Schwächung der Monopolstellung der DTAG und im Zuge der stets verbesserten Technologien ergeben sich interessante Fragestellungen insbesondere, ob die Entwicklungen neue Nutzungsformen des Internets bzw. der Medien zur Folge haben werden. Darüber hinaus ist zu untersuchen, inwiefern sich neue Nutzer als Individuen in die moderne IT-Gesellschaft integrieren werden - insbesondere unter dem Aspekt der Mobilität und dessen Subformen und Ausprägungen (Reichwald et al. 2002). Weiter ist zu betrachten und zu analysieren, wie sich die Rahmenbedingungen im Geschäftsfeld der TK-Anbieter verändern und ob sich Synergieeffekte mit heutigen Drahtlos-Technologien ergeben oder ob diese bei der Realisierung entsprechender Lösungen bzw. Szenarien derzeit noch an ihre Grenzen stoßen und ferner, wie künftige sich noch in der Entwicklung bzw. Planung befindliche Technologien vorhandene Defizite mindern werden können.

Dazu ist es zunächst notwendig - im Sinne einer state-of-the-art-Analyse - die aktuellen und kommenden Technologien, die mit hoher Wahrscheinlichkeit in zukünftigen Entwicklungen beteiligt sein werden, darzulegen, um die oben gestellten Fragen zumindest im Ansatz beantworten zu können. Welche Technologien daran maßgeblich beteiligt sein werden, welche möglichen Szenarien daraus entstehen können sowie eine aktuelle Marktübersicht der entsprechenden Technologie- und Dienstanbieter, wird im Folgenden gegeben.

\section{Wireless-LAN nach 802.11}

Funk-LANs bzw. Wireless-LANs (WLANs) basierend auf dem 1997 vom Institute of Electrical and Electronics Engineers (IEEE) definierten Standard IEEE 802.11 bieten die Möglichkeit, mit geringem Aufwand drahtlose lokale Netzwerke aufzubauen oder bestehende drahtgebundene Netzwerke zu erweitern.

802.11 ist eine Normen-Familie für WLANs. Am 25. Juni 2004 waren vier Normen verabschiedet: 802.11, 802.11a, 802.11b und 802.11g. Zur Verabschiedung vorgesehen noch für 2004 waren 802.11e und 802.11i. Für Details der einzelnen Substandards siehe die folgenden Abschnitte.

Aufgrund der einfachen Installation werden Funk-LANs auch für temporär zu installierende Netze (z. B. auf Messen) verwendet. Darüber hinaus besteht die 
Möglichkeit, an öffentlichen Plätzen wie Flughäfen oder Bahnhöfen Netzwerkzugänge, so genannte Hotspots, anzubieten, um den mobilen Nutzern Verbindungen in das Internet oder in ihr Home-Office zu ermöglichen. Die Mehrzahl der derzeit am Markt verfügbaren Funk-LAN Systeme basieren auf der 1999 vom IEEE verabschiedeten Erweiterung 802.11b des Standards 802.11. Die HerstellerVereinigung WiFi-Alliance (vormals WECA (Wireless Ethernet Compatibility Alliance)) dokumentiert die Kompatibilität zum Standard 802.11b durch die Vergabe des WiFi-Zertifikats. Seit November 2002 sind in Deutschland auch Frequenzen im 5-GHz-Bereich freigegeben, sodass auch Systeme der Standards 802.11a bzw. 802.11g zum Einsatz kommen.

\subsection{Architekturen}

Funk-LANs können in zwei verschiedenen Modi betrieben werden. Im Ad-hocModus (siehe Abb. 23) kommunizieren zwei oder mehr mobile Endgeräte, die mit einer Funk-LAN-Karte ausgestattet sind (Clients), direkt miteinander.

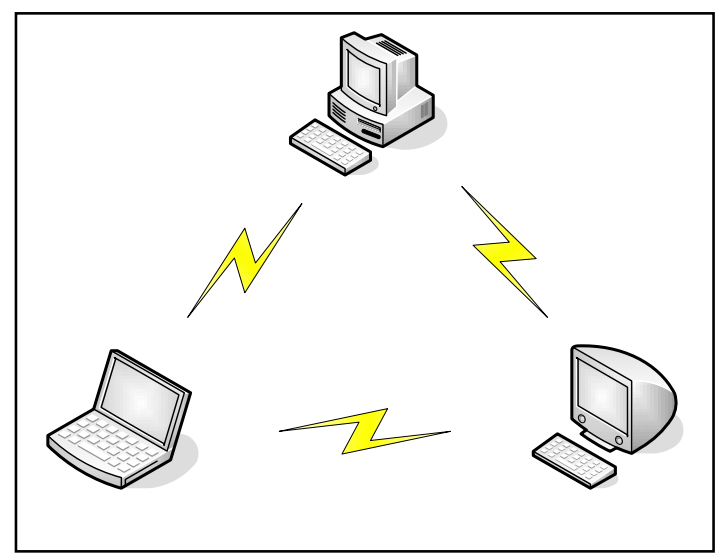

Abbildung 23: Ad-hoc-Modus (Peer-to-Peer Kommunikation)

In den meisten Fällen wird ein Funk-LAN im Infrastruktur-Modus betrieben, d.h., die Kommunikation der Clients erfolgt über eine zentrale Funkbrücke, den so genannten Access-Point (AP) (siehe Abb. 24). Über diesen erfolgt auch die Verbindung in kabelgebundene LAN-Segmente (siehe Abb. 25). 


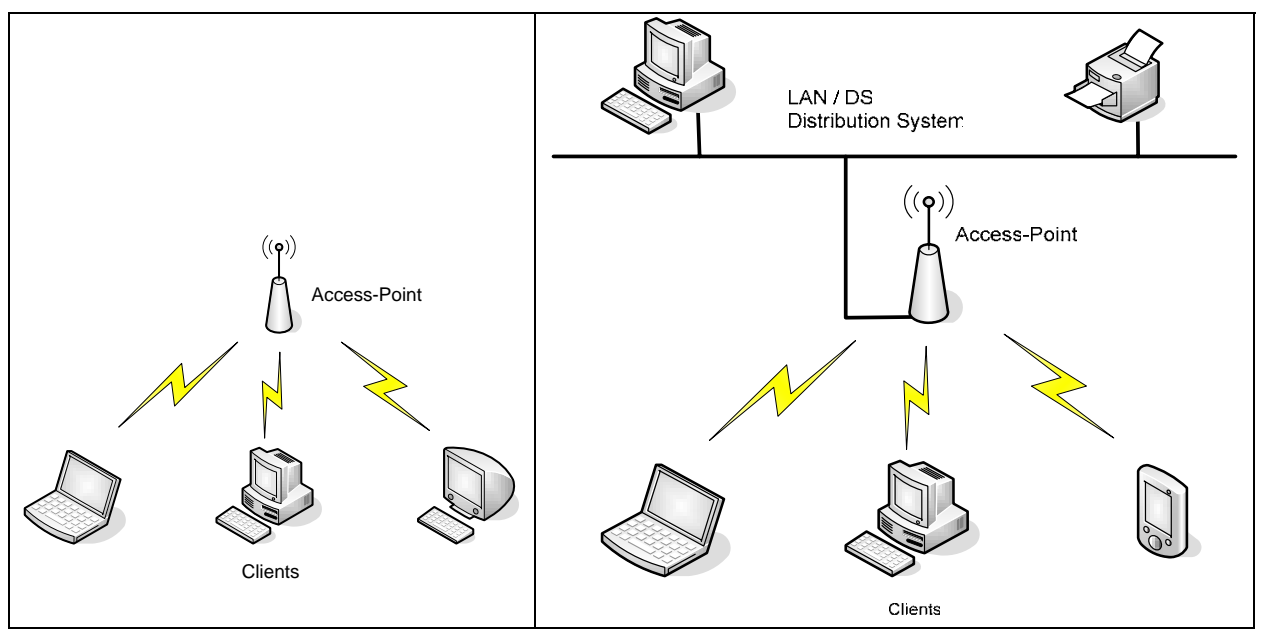

Abbildung 24: Infrastruktur-Modus (Clients u. Access-Point)

Abbildung 25: Infrastruktur-Modus (Anbindung an kabelgebundenes LAN)

Der Infrastruktur-Modus lässt mehrere Einsatzvarianten zu:

- Mittels mehrerer Access-Points können überlappende Funkzellen installiert werden, sodass beim Übergang eines Clients in die nächste Funkzelle die Funkverbindung aufrechterhalten werden kann (Roaming). Auf diese Weise können große Bereiche flächendeckend versorgt werden. Die Reichweite einer Funkzelle ist in erhöhtem Maße abhängig von den Umgebungsbedingungen und liegt im Bereich zwischen ca. 10 - 150 Meter.

- Zwei Access-Points können auch als Brücke (Bridge) zwischen zwei leitungsgebundenen LANs eingesetzt werden. Ebenso ist der Einsatz eines Access-Points als Relaisstation (Repeater) zur Erhöhung der Reichweite möglich.

- Bei der Verwendung entsprechender Komponenten (Richtantennen) an den Access-Points kann ein Funk-LAN auch zur Vernetzung von Liegenschaften eingesetzt werden. Hier können laut Herstellerangaben Reichweiten im Kilometerbereich erreicht werden. Die Access-Points können dabei als Relaisstation oder Brücke betrieben werden.

Innerhalb des Standards werden auch die Bezeichnungen Independent Basic Service Set (IBSS) für Funk-Netzwerke im Ad-hoc-Modus und Basic Service Set (BSS) für Konstellationen im Infrastruktur-Modus mit einem Access-Point verwendet. Mehrere gekoppelte BSS werden als Extended Service Set (ESS) bezeichnet, das koppelnde Netzwerk wird Distribution System (DS) genannt (Kopp 2004). 


\section{$2.2802 .11 b$}

Diese 1999 verabschiedete Erweiterung zum ursprünglichen Standard IEEE 802.11 verwendet im Gegensatz zu diesem ein verändertes Verfahren zur physikalischen Übertragung und erreicht damit eine Bruttodatenrate von 11 Mbps. Dies erlaubte es erstmals, multimediale Daten in Echtzeit ${ }^{14} \mathrm{zu}$ übertragen. Zusätzlich zu einer erhöhten Bandbreite bietet das System erweiterte Verfahren zur Netzwerksicherheit, die im Abschnitt über die Sicherheit drahtloser Netzwerke genauer erläutert werden. Mit zunehmender Entfernung zum Sender wird die Bandbreite adaptiv angepasst und beträgt dann 5,5 Mbps bzw. 2 Mbps.

Bei der Planung eines 802.11b-Netzes ist darauf zu achten, dass die Anzahl verfügbarer Kanäle, die insgesamt 14 beträgt, auf der Welt unterschiedlich reglementiert wurde. So sind in Deutschland die Kanäle 1-13 nutzbar, während in den USA nur die Kanäle 1-11 zur Verfügung stehen. Dies ist insbesondere von Bedeutung, da einige Firmen damit begonnen haben, ihr WLAN-Equipment auf Grund der Einheitlichkeit nur mit den Kanälen 1-11 auszustatten, was zu Problemen führen kann, sollte das weitere Equipment dieser Einschränkung nicht unterliegen (Friedrich 2002).

Im 2,4-GHz-Frequenzbereich stehen in Deutschland 13 Frequenzkanäle mit einem Frequenzabstand von $5 \mathrm{MHz}$ für die Funkübertragung nach 802.11b zur Verfügung. Bei einer Kanalbandbreite von ca. $22 \mathrm{MHz}$ können jedoch nur maximal 3 Kanäle gleichzeitig überlappungsfrei genutzt werden, beispielsweise die Kanäle 1, 7 und 13 (siehe Abb. 26).

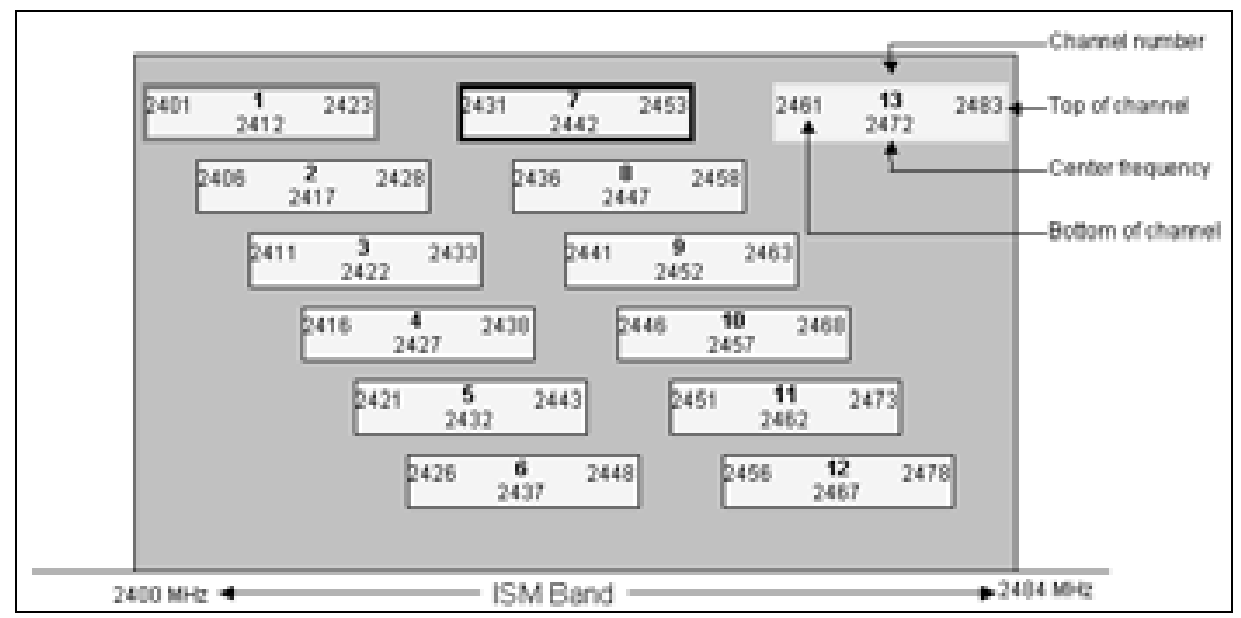

Abbildung 26: 802.11b Kanalraster, überlappungsfreie Kanäle

${ }_{14}$ Datenverarbeitung, bei der die Ergebnisse so schnell produziert werden, dass keine nennenswerte Zeitverzögerung gegenüber der absoluten Zeit auftritt. 
Dies wirkt sich unmittelbar auf die maximal zur Verfügung stehende Bandbreite an einem Zugangspunkt für die Endkunden aus und stellt somit eine Einschränkung auf theoretisch $33 \mathrm{Mbps}$ pro AP dar.

Neben der maximal verfügbaren Bandbreite spielt gerade für den Einsatz in Unternehmen die Reichweite des verfügbaren Netzes eine Rolle. Dazu stellt Abbildung 27 (Ohrtmann 2004) in einem Diagramm dar, welche Reichweiten im Durchschnitt für WLAN (802.11a/802.11b) möglich sind. Dies ist jedoch nur ein grober Wert, da bedingt durch physikalische Gesetze, lokale Gegebenheiten wie die Anzahl und die Beschaffenheit der Wände in einem Gebäude entscheidend auf die Reichweite Einfluss haben.

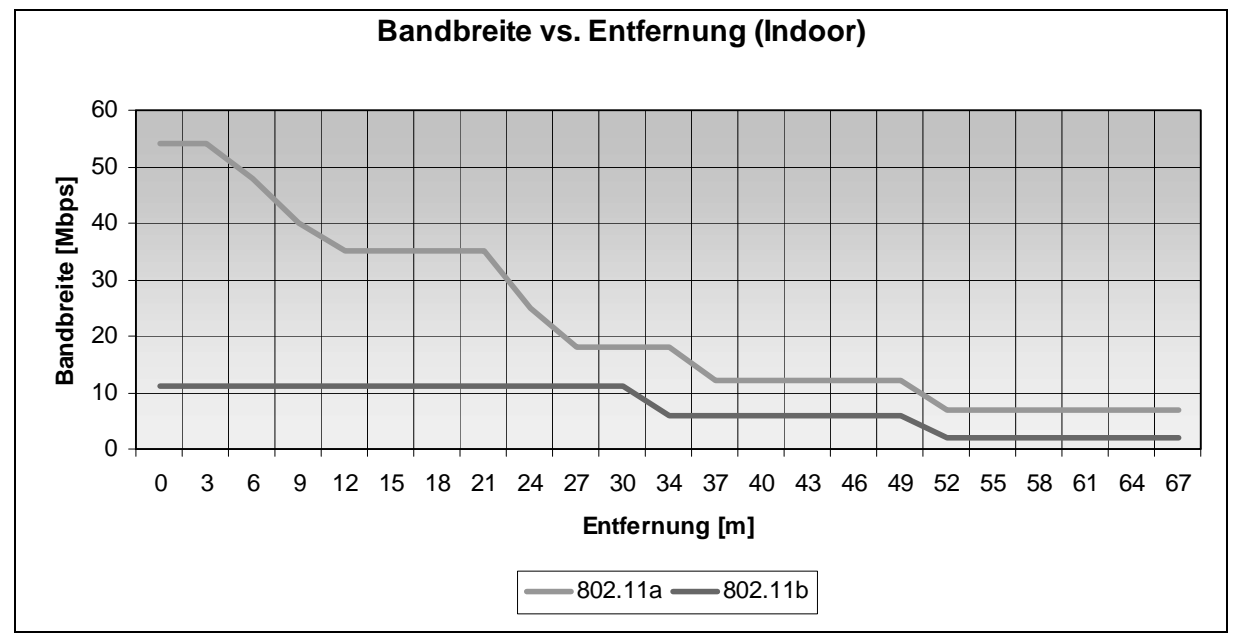

Abbildung 27: Bandbreite bei bestimmter Entfernung zum AP

Die in Deutschland und in fast allen Staaten Europas zugelassenen Funk-LANSysteme nach 802.11 und $802.11 \mathrm{~b}$ nutzen das ISM-Frequenzband (IndustrialScientific-Medical) zwischen 2,4 und 2,48 GHz, das gebührenfrei und ohne zusätzliche Genehmigung verwendet werden kann. Die Sendeleistung ist laut RegTP auf maximal 100 mW EIRP (Effective Isotropic Radiated Power) begrenzt. Systeme des Standards 802.11 übertragen die Daten mit einer Rate von 1 bzw. 2 Mbps mittels Bandspreizverfahren - entweder mittels Frequenzsprung- (FHSS) oder Direct-Sequence- (DSSS) Verfahren. Der Vollständigkeit halber sei erwähnt, dass 802.11 auch eine Infrarot-Übertragung definiert, die bisher aber in der Praxis bedeutungslos geblieben ist.

Die aktuellen Systeme nach Standard $802.11 \mathrm{~b}$ verwenden nur das DSSSVerfahren. Die zu übertragenden Daten werden mit einem festen Code gespreizt, um die Übertragung unempfindlicher gegen Störungen zu machen. Der Zugriff auf den Funkkanal erfolgt, wie bei allen Systemen der 802.11 Standards, nach ei- 
nem zufallsgesteuerten Verfahren - genannt Carrier Sense Multiple Access with Collision Avoidance (CSMA/CA). Die Brutto-Datenübertragungsrate beträgt bei 802.11b maximal 11 Mbps. Die Übertragungsraten können, wie bei allen Systemen der 802.11 Standards, nicht garantiert werden - sie hängen ab von der Anzahl der Clients und der Qualität der Funkübertragungsstrecke.

\section{$2.3 \quad 802.11 \mathrm{a}$}

Im Jahre 1999 wurde neben dem IEEE 802.11b-Standard die Erweiterung IEEE 802.11a vorgestellt. Diese arbeitet im Gegensatz zum originalen 802.11-Standard sowie der Erweiterung 802.11b im 5-GHz-Band. Trotz der weitaus höheren Bandbreite von bis zu 54 Mbps sowie weiteren Funktionen (z. B. Qualitätssicherung der Verbindung), konnte sich dieser Standard in Deutschland nicht verbreiten, da das entsprechende Frequenzband nicht frei verfügbar war. Dies wurde erst im Jahre 2003 geändert, sodass heute bereits Hersteller Equipment für diesen Standard anbieten.

Aufgrund des gänzlich anderen Frequenzbandes besteht zwischen dem IEEE 802.11a- und dem IEEE 802.11b-Standard keinerlei Kompatibilität. Die höheren Datenraten werden durch Anwendung eines gänzlich anderen Frequenzmodulationsverfahrens erreicht, das neben einer verbesserten Ausnutzung der Kanäle zusätzlich qualitätssichernde Eigenschaften bietet. So ermöglicht 802.11a unterschiedliche Datenraten zwischen 6 und 54 Mbps je nach Entfernung zwischen Sender und Empfänger.

\section{$2.4 \quad 802.11 \mathrm{~g}$}

In den letzten Jahren ist das aufkommende Datenvolumen auf Grund einer immer besseren Anbindung von Firmen und Unternehmen an das Internet stetig gewachsen. Da sich eine Entspannung bei der Freigabe der IEEE 802.11aErweiterung nicht abzeichnete, wurde im April 2003 eine weitere Erweiterung der IEEE 802.11g-Standard verabschiedet. Vorteil dieser Erweiterung ist die generelle Abwärtskompatibilität zum IEEE 802.11b-Standard auf Grund des gleichen Frequenzbandes. Daher kann auch diese Erweiterung lizenzfrei verwendet werden, bietet aber mit $54 \mathrm{Mbps}$ die gleiche Brutto-Bandbreite wie der IEEE 802.11aStandard. Als nachteilig erweist sich jedoch, dass keine zusätzlichen Eigenschaften (wie die bereits angedeutete Qualitätssicherung im 802.11a-Standard) existieren. Zusätzlich verwenden sowohl der 802.11g- als auch der 802.11b-Standard das 2,4-GHz-Band, sodass eine noch stärkere Auslastung der vorhandenen Frequenzen und somit eine geringe Netto-Datenrate zu erwarten ist, als dies mit dem 802.11a-Standard möglich wäre. Die Bandbreite wird jedoch trotzdem auf höherem Niveau liegen, als sie der 802.11b-Standard anbieten kann, sodass die Erwei- 
terung 802.11g eine ernstzunehmende Alternative ist und Netzwerke mit maximal elf Mbps in Zukunft nach und nach ersetzen wird. Dieser Trend zeichnet sich bereits heute ab (Kopp 2004).

\begin{tabular}{|c|c|c|}
\hline & $2,4 \mathrm{GHz}$ & $5 \mathrm{GHz}$ \\
\hline Vorteile & $\begin{array}{l}\text { - kostenfreies Frequenzband, } \\
\text { dadurch höhere Verbreitung } \\
\text { - etwas höhere Reichweite, da } \\
\text { diese Frequenzen weniger } \\
\text { stark gedämpft werden }\end{array}$ & $\begin{array}{l}\text { - bessere Verbindungsqualität } \\
\text { (subjektiv); weniger Verbin- } \\
\text { dungsabbrüche } \\
\text { - in Deutschland } 19 \text { (bei } \\
\text { RegTP-Zulassung) sich nicht } \\
\text { überlappende Kanäle }\end{array}$ \\
\hline Nachteile & $\begin{array}{l}\text { - Frequenz muss mit anderen } \\
\text { Geräten geteilt werden (Mik- } \\
\text { rowellenherde, Babyphones, } \\
\text { etc.), dadurch Störungen } \\
\text { - effektiv nur } 3 \text { brauchbare (sich } \\
\text { nicht überlappende) Kanäle }\end{array}$ & $\begin{array}{l}\text { - höhere Kosten, dadurch ge- } \\
\text { ringere Verbreitung } \\
\text { - etwas weniger Reichweite, da } \\
\text { diese Frequenzen stärker ge- } \\
\text { dämpft werden }\end{array}$ \\
\hline
\end{tabular}

Abbildung 28: Vor- und Nachteile bei der Verwendung der typischen Frequenzbereiche

\subsection{Reichweite und Antennen}

Die Antennen handelsüblicher 802.11 Endgeräte lassen 30 bis 100 Meter Reichweite zu. Mit externen Rundstrahlantennen lassen sich bei Sichtkontakt 100 bis 300 Meter überbrücken. Leichtbauwände mindern die Reichweite, sind aber einzeln kein Hindernis; dagegen werden Stahl und Beton nicht durchdrungen. Bäume, insbesondere dicht belaubte, sind ebenfalls Hindernisse für WLANVerbindungen. WLAN nach 802.11b oder 802.11g funkt im 2,4-GHz-Band mit einer Wellenlänge von ca. $13 \mathrm{~cm}$. Damit werden alle Gegenstände ab einer Dicke von $13 \mathrm{~cm}$ zu starken Hindernissen und es gilt zusätzlich die Gesetzmäßigkeit: je stärker die elektrische Leitfähigkeit des Materials, desto stärker der Effekt. Außerdem können leitende Gegenstände in der Nähe von Antennen deren Richtcharakteristik stark beeinflussen.

WLAN nach 802.11a funkt im 5-GHz-Band, in dem ein größerer Frequenzbereich (455 MHz) zur Verfügung steht. Auch dieser Frequenzbereich ist in Deutschland lizenzfrei nutzbar, jedoch sind strenge Auflagen notwendig, um die in diesem Bereich arbeitenden Satelliten und Radargeräte nicht zu stören. Dies und die höheren Kosten der Hardware auf Grund der höheren Frequenz bewirken, dass sich 802.11 a noch nicht gegen 802.11 b oder $g$ durchgesetzt hat. Es ist zu 
erwarten, dass dies erst geschieht, wenn eine Frequenzknappheit im 2,4-GHzBand aufkommt (Sinn 2002).

Mit speziellen Richtfunkantennen lassen sich bei Sichtkontakt Strecken von mehreren km überbrücken. Antennen bringen einen Sende- wie Empfangsgewinn (Antennengewinn, in $\mathrm{dBi}$ ), indem sie elektromagnetische Wellen bündeln. Rechtlich darf die Sendeleistung in Deutschland 100mW (EIRP) nicht übersteigen. WLAN verwendet eine größere Bandbreite als der Amateurfunk, daher dürfen selbst gebaute Geräte nur mit Sondergenehmigung betrieben werden. Generell dürfen nur Geräte verwendet werden, die von RegTP abgenommen und unverändert sind. Für diese Anlagen besteht keine Meldepflicht. (Mangold 2001)

\subsection{QoS (Quality of Service) und Sicherheit in 802.11}

Erweiterungen und Verbesserungen bezüglich der Qualitätssicherung werden von den Ergebnissen der Arbeitsgruppe 802.11e erwartet, die sich der Quality of ServiceProblematik (QoS) gewidmet hat, um Voice- und Multimedia-Anwendungen besser unterstützen zu können. Der neue Wireless-Standard soll unter dem Namen Wireless Media Extensions (WME) vermarktet werden.

Im 802.11 wird unter anderem festgelegt, wie der Medienzugriff von Funkstationen für verschiedene Übertragungsfunktionen und Frequenzbänder durchgeführt werden muss. Die so genannte Distributed Coordination Function (DCF) als Basis der Protokolle von 802.11b arbeitet mit einem dezentralen Carrier Sense Multiple Access/Collision Avoidance (CSMA/CA) Verfahren, welches nicht in der Lage ist, Dienstgüte bei den Anwendungen, z. B. garantierte Übertragungsraten, kontrollierte Laufzeiten, minimale Laufzeitschwankungen oder minimale Verluste von Daten, zu unterstützen. Die in der Arbeitsgruppe 802.11e entwickelten Verfahren, die zentral koordinierende Hybrid Coordination Function (HCF) und die darin enthaltene Enhanced DCF (EDCF) sollen Lösungen darstellen (Dufft 2004). Eine dedizierte Vergabe von Kapazitäten an die jeweiligen Funkstationen wird mithilfe der HCF und damit die Belegung eines Funkkanals zu beliebigen Zeiten möglich. Die EDCF leistet eine schwache Unterstützung von Dienstgüte durch die Einführung von Prioritäten mittels unterschiedlicher Fenstergrößen im CSMA. Die Prioritäten werden durch bis zu acht Traffic Categories (TCs) bestimmt, deren Parameter dynamisch verändert werden können.

In den heutigen WLAN-Systemen kommt überwiegend das WEP-Verschlüsselungsverfahren (Wired Equivalent Privacy) zum Einsatz. Die Schwachstellen dieses Verfahrens sind allerdings bereits seit langem bekannt (Fluhrer et al. 2001), sodass im Herbst 2003 zur besseren Absicherung der Funknetze der Verschlüsselungsstandard Wireless Protected Access (WPA) spezifiziert wurde und im Vergleich zu WEP als wesentlich sicherer gilt. Der im Juni 2004 vom IEEE verab- 
schiedete 802.11i-Standard beinhaltet die erwartete Sicherheitsspezifikation WPA2, die eine Weiterentwicklung des WPA-Standards darstellt und jetzt ein Bestandteil des 802.11i-Standards ist. Während WPA das Temporal Key Integrity Protocol (TKIP) und den Verschlüsselungsalgorithmus RC4 benutzt, setzt WPA2 auf AES (Advanced Encryption Standard) und bietet zusätzlichen Schutz durch dynamische Schlüssel, die auf dem TKIP basieren, und erfordert die Anmeldung von Nutzern über das Extensible Authentication Protocol (EAP) (Schneier 1996). Bei WPA2 wird zwischen einer Personal- und einer Enterprise-Variante unterschieden. Bei einer WLAN-Verbindung im WPA2-Personal-Modus nutzen beide Endstellen ein zuvor vereinbartes Passwort. Im Enterprise-Modus erfolgt die Authentifizierung in der Regel über einen RADIUS-Server (RFC 2865) mittels IEEE802.1X-Verfahren und EAP. Erste WPA2-kompatible und zertifizierte WLANProdukte werden von Atheros, Broadcom, Cisco, Intel und Realtek angeboten.

\subsection{11 - Märkte}

Die beschriebene Technologie zur Bereitstellung drahtloser Internetverbindungen ist für verschiedene Kundengruppen interessant. Man unterscheidet nach Nutzerklassen drei Märkte:

- Public WLAN / Hotspot:

PWLAN

- Consumer/SOHO (small office/home office) WLAN:

CWLAN

- Enterprise WLAN:

EWLAN

Die Nutzung von PWLAN lässt sich im Wesentlichen in folgende Szenarios unterteilen:

- Die Nutzung von Reise- und Wartezeiten: Die Nutzung von Reise- und Wartezeiten bei etwas längeren Aufenthalten, um komfortabel auf einem eigenen Endgerät zu arbeiten. Handelt es sich um sehr kurze Wartezeiten, dürften jedoch Mobilfunklösungen der Nutzung eines PWLAN vorgezogen werden.

- Die Arbeit an bestimmten Orten: Die Arbeit an bestimmten Orten, wie z. B. Messen oder Pressecentern, für die ein schneller Zugang zum Internet erforderlich ist. Die häufig an diesen Orten vorhandenen LAN-Anschlüsse sind jedoch eine ernst zu nehmende Alternative.

- Die flexible Arbeit an jedem Ort. Die flexible Arbeit an jedem Ort, wenn zum Beispiel Freiberufler oder Studenten ihren Arbeitsplatz flexibel an verschiedenen Orten wie Bibliotheken oder Cafés einrichten möchten, an denen kein LAN-Zugang existiert. Flexible und angemessene Preismodelle sind aufgrund der begrenzten Zahlungswilligkeit dieser Nutzergruppen allerdings besonders wichtig. 
- Datentankstelle: Die Nutzung eines PWLANs als Datentankstelle, wenn Mitarbeiter unterwegs Daten mit dem Unternehmen abgleichen müssen. PWLANs werden sich hier allerdings nur schwer gegenüber Mobilfunklösungen durchsetzen können.

- Digital Lifestyle: Die Nutzung durch Personen, für die das Internet integraler Bestandteil des täglichen Lebens ist und unabhängig vom Internetanschluss zu Hause genutzt werden soll, zum Beispiel im Urlaub oder in der Freizeit. Auch hier werden die Preisgestaltung des Netzzugangs und die ausreichende Ausstattung der Endkunden mit Notebooks eine entscheidende Rolle für den Erfolg von PWLANs spielen.

Folgende Akteure des PWLAN-Marktes lassen sich identifizieren:

- Endkunden

- Provider

- Betreiber

- Location-Besitzer und

- Technologie-Anbieter.

Die folgende Grafik (Berlecon 2003) zeigt die Beziehungen der Akteure des PWLAN-Marktes.

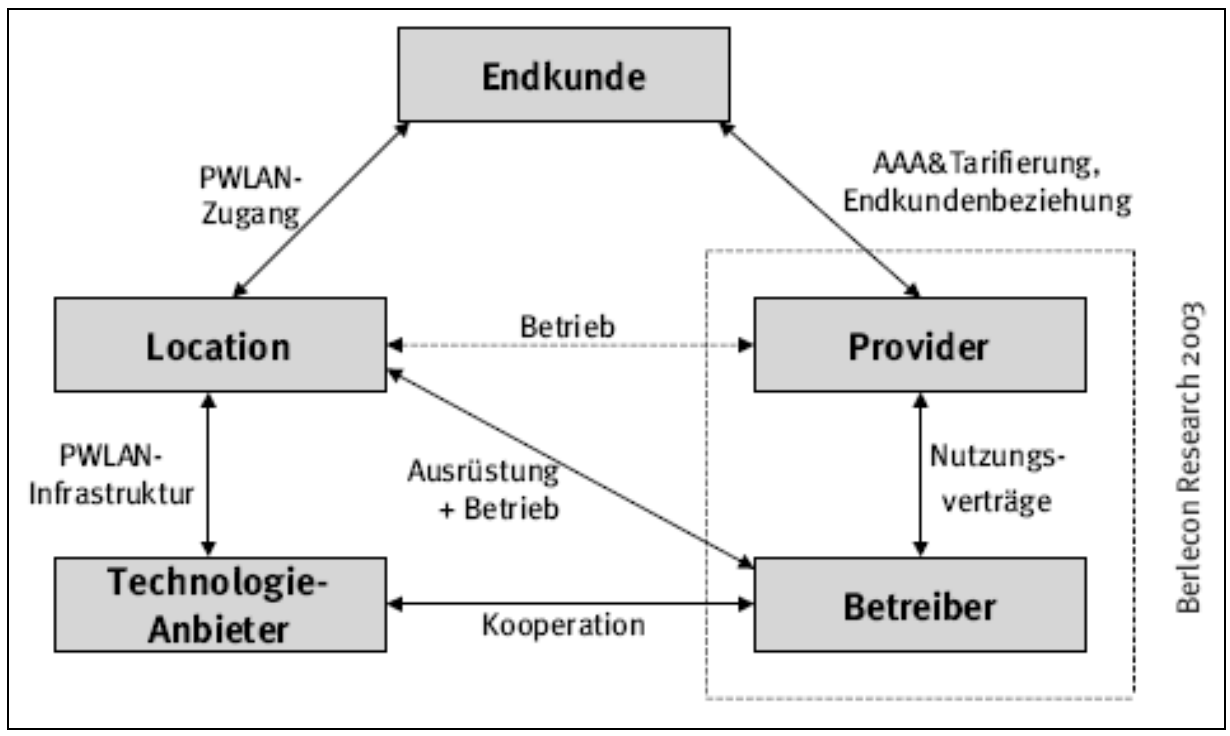

Abbildung 29: Beziehungen der Akteure des PWLAN-Marktes

Am Beispiel der Location-Besitzer und Technologie-Anbieter lassen sich bezüglich möglicher Strategien die Erfolgsfaktoren der PWLAN-Akteure abschätzen. 
Location-Besitzer: Bisher sind viele interessante Hotspot-Locations noch nicht ausreichend über die realistischen Potenziale des WLAN-Marktes informiert, was eine realistische Einschätzung des Kosten-Nutzen-Verhältnisses von Hotspots erschwert. Deswegen verfolgen die Location-Besitzer noch keine klare PWLANStrategie. In kleinen Locations sind zwar die Kosten für die Einrichtung eines Hotspots niedrig, dafür dürften diese Hotspots aber auch nur von vergleichsweise wenigen Kunden mit begrenzter Zahlungsbereitschaft genutzt werden. In den meisten Top-Locations mit vielen zahlungskräftigen Hotspot-Kunden ist dagegen der Aufwand für die Einrichtung eines PWLANs mit hohen Investitionen verbunden und erfordert eine komplexe technische Infrastruktur. Hotspots werden nur für wenige Locations ein wirklich lukratives Zusatzgeschäft sein. Trotzdem können PWLANs einen kleinen, aber kontinuierlichen Strom an Zusatzeinnahmen erzeugen und für einen wirtschaftlichen Betrieb eine ausreichende Anzahl an Kunden anlocken.

Ausrïster und Technologieanbieter: Technologieanbieter, die sich als Ausrüster am PWLAN-Markt etablieren, haben den Vorteil, dass ihr WLAN-Geschäftsmodell sich nicht auf den Public-WLAN-Markt beschränkt. Sie richten in der Regel sowohl Corporate- als auch Public-WLANs ein und sind damit weniger stark von der Entwicklung dieses einzelnen Marktsegments abhängig als beispielsweise WISPs. Da die Anzahl lukrativer Locations am Public-Hotspot-Markt jedoch relativ klein ist, reicht eine Fokussierung auf Orte mit überzeugendem Business Case nicht aus, um bei einer großen Anzahl an Technikanbietern für Umsatz zu sorgen. Dagegen ist die Anzahl weniger lukrativer Hotspot Locations relativ hoch. Aufgrund des begrenzten Erlöspotenzials aus dem PWLAN-Zugang muss hier die Ausrüstung jedoch zu relativ geringen Kosten erfolgen. Dazu muss auch die große Anzahl kleinerer Locations mit weniger klarem Business Case davon überzeugt werden, Hotspots einzurichten. Dies erfordert jedoch das Angebot günstiger Lösungen, die es den Locations erlauben, über niedrige Tarife möglichst viele private Nutzer anzulocken. Niedrigere Kosten können vornehmlich über Größenvorteile erzielt werden. Somit dürfte die Ausrüstung von Hotspots mittelfristig für den größten Teil der Technikanbieter zum Massengeschäft mit niedrigen Margen werden (Mahr 2004).

\subsection{Aktuelle Preismodelle}

Werden Hotspots direkt von einem Wireless Network Operator betrieben, steht der Zugang in der Regel nur den jeweiligen Kunden zur Verfügung, beziehungsweise denen von Roaming-Partnern. Die Verrechnung erfolgt dabei in der Regel minutenweise über die Mobilfunkrechnung des Kunden. Alternativ gibt es aber auch Preismodelle, bei denen nach dem übermittelten Datenvolumen abgerechnet wird. So wird oftmals in Verbindung mit der kombinierten GPRS/UMTS/ 
WLAN-Karte einen volumenbasierter Tarif angeboten, das heißt nur die übertragene Datenmenge wird in Rechnung gestellt - nicht aber die Verbindungszeit. Größere Auswahl erlauben in der Regel die Hotspots, die von national oder international operierenden Betreibern angeboten werden. Hier ist der Zugang den Kunden mehrerer Mobilfunkbetreiber und/oder weiterer Internetprovider möglich. Außerdem stehen diese Hotspots den Roaming-Partnern des Betreibers und so genannten Aggregatoren zur Verfügung.

Es treten auch Anbieter auf, die speziell für regelmäßige Hotspot-Benutzer Zugangsabonnements anbieten, die in allen angeschlossenen Lokalitäten gelten und bis zum Ablauf der Vertragsdauer gelten. Darüber hinaus können zum Beispiel Hotels für ihre Kunden spezielle Angebote gestalten, wie z. B. Login-Zeit für eine bestimmte Zeitperiode (Pauschalpreis während des Aufenthalts, für 24h, etc.) oder die Benutzung des Anschlusses in den Zimmerpreis integrieren. Es kann also festgehalten werden, dass in diesem offenen, jungen Markt mehrere Preismodelle konkurrieren, wobei hauptsächlich zwischen zeit- und volumenbasierenden Schemata unterschieden werden muss. Es existieren aber auch Pauschalangebote (Flatrate) und Mischformen (Schulte 2004a).

Die weiter unten aufgeführten neuen Szenarien werden nur realisierbar sein, wenn breitbandiger, drahtloser Internetzugang über größere Entfernungen bzw. Abdeckungsflächen möglich wird. Die maßgebliche Technologie verbirgt sich hinter dem Kürzel 802.16 und wird im Folgenden beschrieben.

\section{$3 \quad 802.16$}

IEEE 802.16 ist eine 2001 vom IEEE standardisierte Netzwerkwerktechnologie für breitbandige Punkt-zu-Mehrpunkt-Netze mit lokaler und regionaler Reichweite (Wireless Metropolitan Area Network, WMAN). Bekannt ist 802.16 auch unter dem Namen des ihn unterstützenden Industrie-Forums Worldwide Interoperability for Microwave Access (WiMAX). WiMAX bringt Festnetzzugang via Funk zu Gebäuden, die mit zentralen Basisstationen (BS) kommunizieren.

Der Standard IEEE 802.16 nutzt die Frequenzbänder bei 10-66 GHz und 2-11 $\mathrm{GHz}$ in lizenzierten und unlizenzierten Bereichen mit Kanalbreiten von $25 \mathrm{MHz}$ in den USA und $28 \mathrm{MHz}$ in Europa. Die Übertragungsgeschwindigkeit ist mit bis zu 70 Mbps 23-mal höher, als bei den schnellsten DSL-Anschlüssen (3072 Mbps im Downstream) beziehungsweise mehr als 1.300-mal so schnell wie GPRS.

In einer Punkt-zu-Mehrpunkt-Konfiguration kommuniziert die WiMAXBasisstation (BS) einerseits mit den öffentlichen Festnetzen andererseits mit den angeschlossenen Teilnehmern auch als Subscriber Station (SS) bzw. Mobile Station (MS) oder Mobile Terminal (MT) bezeichnet. Die Basisstation ordnet, je nach Bedarf, den einzelnen Stationen entsprechend priorisierte Bandbreite zu. Die Luft- 
schnittstelle ermöglicht die Echtzeit-Übertragung von Daten oder MultimediaInformationen aller Art und mit voller QoS-Unterstützung (Quality of Service).

Derzeit sind drei Frequenzbereiche für WiMAX vorgesehen: 3400 bis $3600 \mathrm{MHz}, 5725$ bis $5850 \mathrm{MHz}$ und 2500 bis $2690 \mathrm{MHz}$. Der erste Frequenzbereich wird in über 100 Ländern genutzt werden können, unterliegt dort aber jeweils einer Regulierung bzw. Frequenzzuteilung. Der zweite Bereich ist in den USA lizenzfrei, in vielen anderen lizenziert. Der dritte Bereich ist zumindest in Europa auch für andere Technologien vorgesehen (Klinkenberg 2004).

Für die lizenzierten Frequenzbänder sind drei Schnittstellen vorgesehen: Eine Single-Carrier-Modulation, die Nutzung der Orthogonal-Frequency-DivisionMultiplex-Modulation (OFDM) und der Einsatz von OFDMA (Orthogonal Frequency Division Multiple Access). Diese Variationen geben dem Netzbetreiber die Möglichkeit, auf die Einsatzbedingungen und Kundenwünsche entsprechend zu reagieren. In den lizenzfreien Bändern (5,725-5,850 U-NII und 2,4 GHz ISM) besteht die Möglichkeit der Interferenz mit anderen Wireless MANs oder WLANs sowie mit anderen Nutzern desselben Frequenzbandes. Als Lösung für dieses Problem wird die dynamische Frequenzauswahl benutzt.

Die geforderten Dienste der Teilnehmer umfassen beim 802.16-Standard Time Division Multiplexing (TDM) für Voice und Daten, IP Connectivity und Packetized Voice (VoIP). Zu diesem Zweck müssen sowohl bündelartiger Datenverkehr als auch kontinuierliches Datenaufkommen unterstützt werden. Zwischen den Basisstationen ist mit diesem Standard kein Handover oder Roaming möglich, d.h. keine Mobilität der Endgeräte. Zwar benötigt die Technologie größere Sender als bei WLAN-Hotspots üblich. Aber diese Basisstationen sind deutlich kleiner als jene herkömmlicher Handy-Netze und vor allem viel günstiger: etwa 10.000 USDollar, schätzen Experten (WiMAX 2004), werden WiMAX-Basisstationen kosten. Über einen Sender ließen sich nach Aussage von Intel (WiMAX 2004) hunderte Privathaushalte mit schnellen Internetanschlüssen versorgen. Da die Endgeräte anfangs noch vergleichsweise groß sein werden, dürfte WiMAX zuerst das Festnetz-Geschäft revolutionieren: die de facto Monopole auf Ortsnetze von Europas einstigen staatlichen Telefongesellschaften könnten so bald mit günstiger Technik umgangen werden (Telephony 2004).

Geplante Anwendungen sind die PC-Anschlüsse im Heimbereich oder im Bereich von Small Office/Home Office (SOHO). WiMAX stellt damit eine Art drahtloses DSL als Ersatz für Breitbandkabel auf der letzen Meile in Gegenden mit unzureichender Verkabelung dar. Die Netzbetreiber können über WiMAX schnell und im Vergleich zum Kabel relativ preiswert breitbandige Zugänge für viele Kunden schaffen. Der WiMAX-Funkstandard 802.16a dürfte schon bald neuen Anbietern von Festnetz- und Mobilfunk-Leistungen den Weg ebnen und als eine Alternative zur verkabelten letzten Meile sein (WiMAX 2004). 
Zur Unterstützung der Entwicklung des Standards IEEE 802.16 und zur weltweiten Vermarktung der entsprechenden Breitband-Wireless-Produkte und Dienste schlossen sich im Jahr 2003 mehr als einhundert Firmen und Organisationen zu einer gemeinnützigen Industrieorganisation, dem WiMAX-Forum, zusammen. Zusätzlich werden hier Tests zur Zertifizierung der IEEE-802.16-Produkte bereitgestellt, um eine problemlose Kompatibilität und Interoperabilität sicherzustellen. WiMAX soll die Implementierung breitbandiger und drahtloser Zugangsnetze vorantreiben, dies durch die Nutzung eines globalen Standards und der Zertifizierung der Interoperabilität der entsprechenden Produkte.

Die zu den WiMAX-Mitgliedern zählenden führenden Firmen der IT- und Telekommunikationsindustrie wie Alcatel, AT\&T, Intel, Motorola und Siemens beteiligen sich ebenso wie die Netzbetreiber British Telecom und France Telecom. Bei WiMAX laufen die Bemühungen zur Schaffung eines Standards auf Hochtouren, sodass WiMAX schneller zur Marktreife kommen könnte (Friedewald / Zoche 2004, Gerbert / Witzki 2004, BWCS 2004).

Die folgende Grafik (Alvarion 2003) zeigt die erwarteten Umsätze aus Dienstleistungen, die durch breitbandigen drahtlosen Internetzugang geliefert werden.

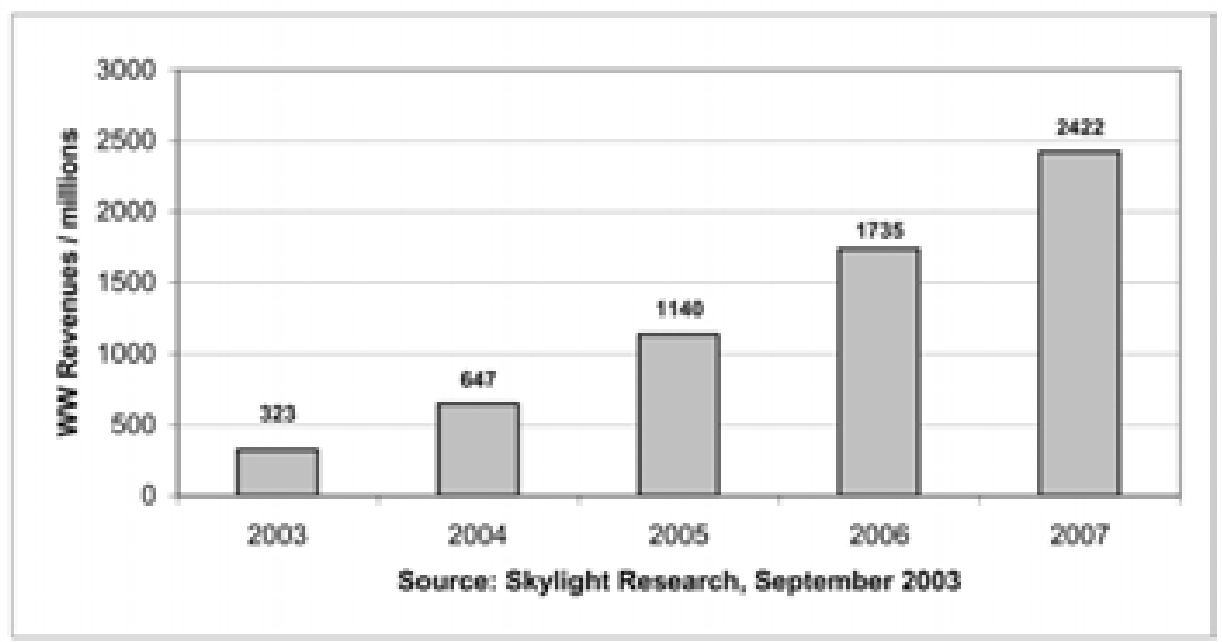

Abbildung 30: Umsatzerwartungen für Dienstleistungen auf Basis breitbandiger Internetzugänge

\subsection{Geschäftsmodelle und Nutzungsszenarios}

Einer Studie der Telekom- Beratungsunternehmen BWCS London and Senza-Fili Consulting Washington zufolge soll der IEEE-Standard 802.16 allein in den USA 
bis 2009 ein jährliches Marktvolumen von 3,7 Milliarden Dollar erreichen (ITReseller 2004).

Für den neuen Wireless-Standard WiMAX sind folgende drei Geschäftsmodelle vorstellbar:

- FWiMAX (Fixed WiMAX): fester Zugang, wobei WiMAX die Funktion übernimmt, die derzeit Kabel oder DSL haben

- NWiMAX (Nomadic WiMAX): mobiler Zugang in bestimmten Zonen, ähnlich einem sehr großen Hotspot

- MWiMAX (Mobile WiMAX): vollkommen mobiles System, für den Einsatz im Mobilfunkbereich

FWiMAX stellt eine Alternative zu DSL dar. In Regionen, wo Glasfasernetze vorhanden sind, die für die derzeitige DSL-Technologie nicht eingesetzt werden können, und solche, wo es kein DSL gibt, könnte die Funktechnologie WiMAX sinnvoll eingesetzt werden, meinen die Experten (WiMAX 2004); WiMAX könnte eingesetzt werden, um das DSL-Monopol der DTAG zu brechen (Marktanteil 86\%). Im westfälischen Selm ${ }^{15}$ entsteht momentan das erste kommerzielle WiMAX-Netz in Deutschland. Für FWiMAX sind folgende Nutzungsszenarios vorstellbar:

- High Speed Internet Zugang für Haushalte und SOHO: Dieses Marktsegment hängt heutzutage vor allem von der Verfügbarkeit von DSL oder Kabel ab. In manchen Regionen dürfte das verfügbare Serviceangebot den Kundenansprüchen in puncto Leistungsfähigkeit, Verlässlichkeit und Kosten nicht genügen. In den meisten ländlichen Gegenden sind die Haushalte auf low speed dial-up Services angewiesen. In Entwicklungsländern besteht in vielen Regionen überhaupt kein Internetzugang.

- Kleine und mittlere Unternehmen: Dieses Marktsegment ist mit Ausnahme der hoch kompetitiven städtischen Gebiete oft unterversorgt. WiMAX stellt nicht nur in schwach besiedelten Gebieten eine kostengünstige Lösung dar, sondern bietet auch in den städtischen Regionen eine Alternative zu DSL oder Kabel.

- WLAN Hotspot Backhaul: Hotspots verbreiten sich weltweit mit hoher Geschwindigkeit. Eine Hürde für das anhaltende Hotspot-Wachstum stellt die Verfügbarkeit kostengünstiger backhaul-Lösungen mit großer Kapazität dar. Neben dieser Aufgabe kann WiMAX auch helfen, Abdeckungslücken zwischen Hotspots zu schließen.

- Zelluläres Backhaul: In den USA wird der Großteil des Backhaul durch das Leasing von T1-Leitungen von den etablierten Netzwerkbetreibern bewäl-

\footnotetext{
15 www.wimax-selm.de.
} 
tigt. Mit Hilfe von WiMAX könnten Mobilfunkbetreiber ihre Abhängigkeit von Wettbewerbern reduzieren. Außerhalb der USA wird vorwiegend point-topoint microwave als mobile backhaul genutzt. WiMAX stellt in diesem Fall zumindest eine günstige Möglichkeit der Kapazitätserweiterung dar.

- Öffentliche Sicherheit und private Netzwerke: WiMAX kann dazu dienen in Krisensituationen kritische Kommunikationskanäle aufrechtzuerhalten. Private Netzwerke von Gewerbezentren, Universitäten etc. stellen ebenfalls potenzielle Einsatzbereiche dieser Technologie dar.

Der Einsatz von WiMAX-Netzen scheint in erster Linie in solchen Ländern interessant, deren großflächige Städte wegen einer geringen Dichte in der Telefonversorgung noch nicht so engmaschig verkabelt sind wie in Europa - also Länder wie China, Russland, Indien oder die Türkei. In hoch industrialisierten Ländern wird diese Technik auch noch Hemmnisse zu überwinden haben, die mit dem Erwerb passender Funklizenzen und dem Erhalt von Installationsgenehmigungen für die Antennenstationen mit hoher Sendeleistung an angestrebten Standorten verbunden sind.

Bereits 2005 sollen laut Intel Broadband Wireless Division die Preise für Basisstation zwischen 10000 und 20000 Dollar und die Sende-/Empfangsstation den Enduser (Customer Premise Equiment, CPE) 150 Dollar kosten. Ab 2006 sollen sich die Preise den heute erhältlichen WLAN-Ausrüstungen angenähert haben (Proxim 2004).

Industrieexperten gehen davon aus, dass WiMAX nicht vor 2007 marktfähig sein wird, laut Swisscom Mobil wird es ab 2005 Anbieter geben, die WiMAXfähige Endgeräte anbieten werden. Es wird erwartet, dass WiMAX in fünf Jahren auf weltweit sieben Mio. Kunden kommen wird. Die Technologie soll dabei, so die Vorhersage, zuerst in Europa und Asien Fuß fassen, bevor sie auch die USA erreicht. Analysten gehen davon aus, dass bis 2009 3,6\% aller Breitbandanbindungen an das Internet ohne Kabel überbrückt werden.

Zur Frage des möglichen Erfolgs entsprechender WiMAX-Datendienste stellen sich unter Kenntnisnahme der Existenz tausender WLAN-Hotspots, deren Nutzung deutlich unter den Erwartungen liegen, viele kritische Fragen. Von der Seite der WLAN-Nutzer werden beispielsweise oft Probleme artikuliert, die mit der Nutzung in diversen WLAN eingesetzten Vouchern verbunden sind. Die Voucher sind an den Standorten, wo sie nicht erworben wurden nicht verwendbar. Zusätzliche WiMAX-Netze könnten diese Situation für den Verbraucher noch verschlimmern. Intel geht dennoch davon aus, dass WiMAX ein Erfolg wird. Es ist vorgesehen, in zwei bis drei Jahren neben WLAN auch WiMAX in den Nachfolger der derzeitigen Centrino-Technologie zu integrieren. Das bedeutet aber nicht automatisch, dass WiMAX auch genutzt wird. Somit ist der wirtschaftliche Erfolg für Ausrüster und TK-Unternehmen noch nicht sicher (Schulte 2004). 


\section{$3.2 \quad 802.20$}

Zum Jahresende 2002 etablierte das Institute of Electrical and Electronics Engineers (IEEE) eine Arbeitsgruppe IEEE 802.20 - Mobile Broadband Wireless Access (MBWA) mit dem Ziel, einen neuen WMAN-Standard für eine effiziente, paketbasierte Luftschnittstelle zu entwickeln. Der Standard soll den Transport von IP-Daten und entsprechende Dienste unterstützen und ergänzt den Standard IEEE 802.16. Der neue WMAN-Standard 802.20 soll im lizenzierten 3,5-GHz-Band mit Kanalbreiten von 25 bis $30 \mathrm{MHz}$ arbeiten und eine Datenübertragungsrate von größer als $1 \mathrm{Mbps}$ bereitstellen. Die Mobilität soll entsprechend ITU-R M.1034-2 für Fahrzeuge bis $250 \mathrm{~km} / \mathrm{h}$ gegeben sein (Schulte 2004b).

Die Luftschnittstelle soll eine Inter-Zell bzw. eine Inter-Sektor-Handoff-Prozedur für die angegebene Fahrzeuggeschwindigkeit unterstützen. Hiermit sollen der Paketverlust und die Latenzzeit bei einer robusten und nahtlosen IP-Paketübertragung minimiert werden. Sektor bedeutet hier ein Paar des Sende- bzw. Empfangskanals von einer Basisstation an einen Empfänger. Die Datenübertragungsrate für die Teilnehmer soll für den Downlink, das heißt BS zur MT, größer als 1 Mbps und für den Uplink, also MT zu BS, größer als 300 Kbps betragen. Die Unterstützung der Luftschnittstelle für Voice over IP (VoIP) soll durch entsprechende QoS-Parameter sichergestellt werden. Im 802.20-Standard wird auch die Bitübertragungs- und die Sicherungsschicht für eine neue Luftschnittstelle spezifiziert. Sie wird auch als Highspeed Portable Internet (HPI) bezeichnet. Die Übertragungsraten bei HPI sind deutlich höher als z. B. bei dem mehr sprachorientierten UMTS.

In der Arbeitsgruppe 802.20 wurden fünf Kriterien definiert und als Leitfaden für die weitere Entwicklung des geplanten Standards beschlossen. Dies sind:

- Die Anwendungen, auf IPv4 bzw. IPv6 basierend, nutzen ein breites Spektrum von Terminals und Diensten, die international standardisiert sind.

- Die Verträglichkeit und die Koexistenz mit vorhandenen Terminals, Protokollen und Diensten, wie z. B. IEEE 802.1D MAC-Bridges oder IEEE 802.1Q VLAN (Virtuelles LAN), müssen gewährleistet sein.

- Ein dedizierter Standard mit genau definierten Funktionen für die Bitübertragungsschicht (PHY) und die MAC-Teilschicht ist hier gefordert. Für die Bitübertragungsschicht sind als Betriebsweise sowohl das Time Division Duplexing (TDD) - die Up- und Downlink-Verbindung teilen sich hierbei einen Kanal - als auch das Frequency Division Duplexing (FDD) - Upund Downlink arbeiten auf getrennten Kanälen - vorgesehen.

- Die Demonstration der technischen Machbarkeit soll durch proprietäre Systeme, die zurzeit im Versuch bzw. in der Entwicklung sind, unterstützt werden. Ein ausreichendes Testen soll vor Beginn des öffentlichen Dienstes sicher- 
gestellt werden. Die Zuverlässigkeit des Betriebs soll durch die Netzbetreiber demonstriert werden.

- Der Nachweis der Wirtschaftlichkeit soll sowohl für den Dienst als auch für die Komponenten der Terminals bzw. der Basisstationen erbracht werden. Die Kosten sollen sich an der Leistung der Dienste für die Endteilnehmer orientieren, das heißt, mehr Leistung (Bandbreite) bedeutet höhere Entgelte.

Das Referenzmodell für Wireless MAN umfasst die Data- und die Control Plane zum Transfer der Steuer- und Nutzerdaten. Die Sicherungsschicht bei IEEE 802.20 in der Data/Control Plane umfasst zurzeit zwei Teilschichten:

- Die Teilschicht Logical Link Control (LLC), spezifiziert in IEEE 802.2, ist nicht Teil der WMAN-Standards. Auf LLC setzt die Vermittlungsschicht mit dem Internet-Protokoll in der Version 4 oder 6 auf.

- Die MAC-Teilschicht empfängt die Service Data Unit (SDU) der LLCTeilschicht und erstellt die Protocol Data Units (PDU) für die MACTeilschicht des Empfängers. Der Standard spezifiziert hier die MAC-Dienste wie Connect- und Terminate-Request für den Verbindungsauf- und -abbau. Die MAC-Teilschicht in der Data/Control Plane ist weiterhin u.a. zuständig für die Zuordnung der benötigten Bandbreite, die Verschlüsselung der Nutzlast nach dem MAC-Header (MAC PDU) und das Berechnen der Prüfsumme (CRC) zur Sicherstellung einer korrekten Übertragung. Die MACTeilschicht sollte mehr als 100 aktive Sitzungen pro Sektor unterstützen.

Der Projektplan für die Entwicklung von IEEE 802.20 zeigt die geplante Verfügbarkeit des neuen Standards im vierten Quartal 2006.

Es existieren bereits Aktivitäten zur Realisierung des Highspeed Portable Internet (HPI), die auf dem neuen IEEE 802.20-Standard basieren. HPI ist eine drahtlose Technologie mit deutlich höheren Übertragungsraten als UMTS, die aber dennoch eine gewisse Mobilität ermöglicht. HPI wird derzeit von Unternehmen wie Navi$\mathrm{ni}^{16}$, Flarion ${ }^{17}$, Samsung oder Motorola vorangetrieben. Der HPI-Dienst ist komplett IP-basiert und bietet Übertragungsraten von bis zu $10 \mathrm{Mbps}$ an. Die HPIVerbindung erfolgt drahtlos im 2,3-GHz-Band, wobei eine Weiterleitung zwischen den Basisstationen ohne Unterbrechung der Session analog zum heutigen Mobilfunk gewährleistet wird. HPI befindet sich derzeit bei den koreanischen Festnetzanbietern Korea Telecom ${ }^{18}$ und Hanaro ${ }^{19}$ in der Erprobungsphase.

\footnotetext{
16 Navini Networks: www.navini.com.

17 Flarion: www.flarion.com.

18 Korea Telecom: www.kt.co.kr.

19 Hanaro Telecom Inc.: www.hanaro.com.
} 
In Australien bietet der alternative Netzbetreiber Unwired Australia Pty Ltd bereits das erste drahtlose Breitbandangebot namens „Unwired Broadband in Wide Area Wireless Network“ an, das die gesamte Region Sydney umspannt. Es basiert auf der „Ripwave“-Ausrüstung von Navini (angelehnt an IEEE 802.20), ist technologisch aber deutlich weniger leistungsfähig als HPI. In den USA startet Nextel, der kleinste der fünf nationalen US-Mobilfunker, einen Feldversuch mit den neuen Technologien, in diesem Fall mit Flarion (Gerbert/Witzki 2004).

\section{UMTS}

UMTS (Universal Mobile Telecommunication System) wurde als Weiterentwicklung von GSM bzw. GPRS eingeführt, um den zukünftigen Erwartungen an Kommunikationsnetze den Weg zu ebnen, die unter anderem mehr Datenverkehr umfassen sollen. Die Standardisierung von UMTS wurde von der ITU (International Telecommunication Union) durchgeführt und neben den Hardwareherstellern Ericsson und Nokia auch von dem weltweit größten Mobilfunk-Diensteanbieter NTT DoCoMo vorangetrieben.

Es gibt für UMTS verschiedene Übertragungsverfahren, wobei momentan das Wichtigste wohl das so genannte WCDMA-Verfahren (Wideband Code Division Multiple Access) ist, das im Gegensatz zu GSM, welches sich auf ein Zeitmultiplex-Verfahren stützt, die Daten innerhalb einer Funkzelle, die auf derselben Frequenz gesendet werden, zum gleichen Zeitpunkt überträgt. Die Trennung der einzelnen Datenströme erfolgt über unterschiedliche Codewörter, mit denen das Signal codiert und als Summensignal übertragen wird. Die Codes werden vor der Übertragung zwischen Sender und Empfänger ausgehandelt, damit beide den gleichen verwenden. Die verschiedenen Frequenzbänder, die für die Übertragung verwendet werden können, liegen für den Uplink - das heißt vom mobilen Endgerät zur Basisstation - zwischen $1920 \mathrm{MHz}$ und $1980 \mathrm{MHz}$, sowie für den Downlink, also die Gegenrichtung, zwischen $2110 \mathrm{MHz}$ und $2170 \mathrm{MHz}$. Ähnlich wie bei GPRS, das als Weiterentwicklung von GSM den paketorientierten Datenaustausch ermöglicht, teilen sich so die Teilnehmer dynamisch, je nach Auslastung der Funkzelle und Entfernung zwischen Sender und Mobilterminal, die insgesamt zur Verfügung stehende Bandbreite.

Der entscheidende Unterschied zwischen der GSM- und der UMTSTechnologie liegt in der Bandbreite der genutzten Frequenzen. In den D- und ENetzen betragen diese etwa $200 \mathrm{KHz}$, bei UMTS hingegen sind es $5 \mathrm{MHz}$. Nur diese großen Frequenzspektren ermöglichen die schnellen Datenübertragungsraten. UMTS kann theoretisch Übertragungsraten bis zu 2 Mbps anbieten. In der Praxis planen die Netzbetreiber derzeit Bandbreiten von bis zu 384 Kbps. T-Mobile und O2 haben ihre Netze derzeit sogar auf 128 Kbps begrenzt, was der Performance von zwei ISDN-B-Kanälen entspricht. Für ländliche Regionen ist auch eine 
Basisversorgung mit 64 Kbps angedacht. Diese bringt nur kleine Geschwindigkeitsvorteile gegenüber High Speed Circuit Switched Data (HSCSD) und General Packet Radio Service (GPRS), vor allem sorgt die Ausweichmöglichkeit auf UMTS in diesem Fall aber für eine Entlastung der bisherigen Netzinfrastruktur.

UMTS-Netze zeichnen sich durch eine neuartige Zellenstruktur aus. Die kleinste Zelle ist die Pikozelle mit einem Durchmesser von unter hundert Metern. Mit Pikozellen werden Hotspots, Bürogebäude, Hotels, Flughäfen, Messen usw. abgedeckt. Die Mikrozelle mit einer Ausdehnung von bis zu mehreren Kilometern versorgt ganze Stadtbereiche. Für Vororte gibt es die Makrozelle mit einer Reichweite von über 20 Kilometern. Hyper- und Umbrella-Zellen, die im globalen Konzept von UMTS auch als Weltzellen bezeichnet werden, haben eine Ausdehnung von bis zu mehreren hundert Kilometern.

Diese Zellen bestimmen unter anderem die mögliche Übertragungsrate und die erlaubte Fortbewegungsgeschwindigkeit. Die Charakteristika der Zellen sind auf die jeweiligen Anwendungsbereiche zugeschnitten. So sind in den Pikozellen, die eine ungefähre Reichweite von $15 \mathrm{~m}$ haben werden, nur Geschwindigkeiten bis zu $10 \mathrm{~km} / \mathrm{h}$ erlaubt, wobei aber Datenübertragungsraten von bis zu $2 \mathrm{Mbps}$ erreicht werden können. Die in nächster Zeit wohl wichtigste Zelle, die Mikrozelle, hat eine Reichweite von durchschnittlich $300 \mathrm{~m}$, wobei hier dann auch schon höhere Fortbewegungsgeschwindigkeiten bis $\mathrm{zu} 120 \mathrm{~km} / \mathrm{h}$ erlaubt sind, was die Übertragungsrate auf $384 \mathrm{Kbps}$ sinken lässt. Die Makrozellen, die vor allem für Vororte und ländliche Gebiete vorgesehen sind, lassen in ihrem Funkbereich von bis zu $20 \mathrm{~km}$ eine Datenrate von bis zu $144 \mathrm{Kbps}$ zu. Die erlaubte Fortbewegungsgeschwindigkeit des Endgerätes liegt hier dafür schon bei $500 \mathrm{~km} / \mathrm{h}$, was nur von den Globalzellen mit bis zu $1000 \mathrm{~km} / \mathrm{h}$ übertroffen wird, die bei gleicher maximaler Übertragungsrate den Rest der Welt über Satelliten versorgen sollen. Aktuell werden lediglich Mikro- und Makrozellen aufgebaut (Erdle/Schneider 2004).

Wie gut eine Verbindung im UMTS-Netz ist, hängt auch von der Quality-ofService-Klasse ab, die beim Anfordern zwischen Endgerät und UMTS-Netz ausgehandelt wird. Es sind vier verschiedene QoS-Klassen implementiert, die je nach erlaubter Reaktionszeit und erforderlicher Datenintegrität gewählt werden. Für Sprache und Videotelefonie zum Beispiel sind eine isochrone Datenübertragung sowie eine sehr kleine End-to-End Zeitverzögerung wichtig. Bei E-Mail oder SMS ist die Gewährleistung der Datenintegrität beziehungsweise eine geringe Bitfehlerrate als wichtiger einzustufen. Diese Güteklassen gelten hierbei sowohl für Sprach- als auch für Datenübertragungen.

Der Mobilfunkstandard UMTS hat seine größten Vorteile in der mobilen, genauer gesagt, bewegten Kommunikation. Durch die sehr guten HandoverVerfahren im Netzbereich von UMTS sollte es möglich werden, eine Verbindung über die verschiedensten Netze und Zellen aufrecht zu erhalten, was viel Komfort 
mit sich bringt und auch die Möglichkeit bietet, zum Beispiel auf einer Zugfahrt ein Gespräch zu führen, bei dem man in einer UMTS-Mikrozelle startet, über eine Makrozelle in einen GSM-Bereich wechselt, um dann eventuell über eine globale Zelle wieder in eine UMTS-Makrozelle einzusteigen.

Für die meisten Mobilfunkunternehmen bietet UMTS einen großen neuen Markt, dem jedoch bisher noch eine Killer-Applikation fehlt, die den Umstiegshype auslösen könnte. Als einfache Ablösung für GSM/GPRS würde UMTS auf keinen Fall genug Gewinn einbringen, um die horrenden Kosten, die für die Lizenzen und den Netzaufbau angefallen sind, in akzeptabler Zeit wieder einzubringen. Wie aus der geschichtlichen Entwicklung des Mobilfunks hervorgeht, ist UMTS aus Vorgängernetzen entstanden, die nur für Sprachdienste gedacht waren, sodass leicht einsichtig wird, dass UMTS vorrangig auch dafür vorgesehen ist (Turowski/Pousttchi 2004). Um Mobile Computing weiter verbreiten zu können, muss eine Möglichkeit gefunden werden, einen einfachen Übergang von UMTSin WLAN-Netze und umgekehrt zu ermöglichen. Falls es möglich werden sollte, Endgeräte zu verwenden, die UMTS und WLAN vereinen und Sprache sowie Daten sinnvoll einsetzen, wird daraus mit hoher Wahrscheinlichkeit der Bedarf am Mobile Office erwachsen.

Da alle Dienste, die von UMTS angeboten werden, auch von bestehenden GSM/GPRS und WLAN mit niedrigeren Kosten und ohne Neues zu lernen, geliefert werden können, lässt den Schluss zu, dass ohne eine wirkliche KillerApplikation kein massenhafter Umstieg auf UMTS in naher Zukunft erfolgen wird (Erdle/Schneider 2004).

\section{Synergien zwischen UMTS-Netzen und WLAN- Infrastrukturen}

Obwohl es keine grundsätzliche Konkurrenz zwischen der UMTS- und der WLAN-Technologie gibt, existieren sehr wohl Synergieeffekte an bestimmten Standorten. Ein Konkurrenzverhältnis zwischen UMTS und WLAN ergibt sich vor allem im Zugangsgeschäft zum Internet, wobei UMTS einen deutlichen Vorteil in Bezug auf die Flächenabdeckung aufweist, während WLAN-Zugänge schneller und kostengünstiger sind (Friedewald/Zoche 2004). Aus technischer Sicht gibt es unterschiedliche Möglichkeiten zur Integration beider Technologien, die jeweils in Abhängigkeit vom Geschäftsmodell des Betreibers ihre Relevanz haben.

Anbieter, die sowohl im UMTS- als auch im WLAN-Geschäft tätig sind, können in naher Zukunft eine enge Kopplung beider Netze realisieren und so ihren Kunden die Vorzüge beider Technologien anbieten. Bei diesem Szenario könnte die eingeschränkte Mobilität bei der WLAN-Nutzung aus Benutzersicht durch das Mobilfunk-Kernnetz realisiert werden. Dienste können aus einer Hand angeboten 
werden. Voraussetzung dafür wäre allerdings ein für den Nutzer transparenter Wechsel zwischen den Zugangsnetzen, was z. B. auch die Verwendung einer gemeinsamen AAA-Infrastruktur (Authentication, Authorization and Accounting) bedeutet. Eine lose Kopplung der Netze ermöglicht unabhängigen Anbietern den Zugang zum Mobilfunknetz, Hotspots können schnell und einfach integriert werden.

Inwieweit das UMTS-Diensteangebot durch andere Funkzugangsnetztechnologien substituiert oder ergänzt werden kann, richtet sich technisch betrachtet hauptsächlich nach folgenden Kriterien: Mobilitätsgrad, Frequenzverfügbarkeit, Kompatibilität der Geräte, Verträglichkeit mit anderen Funkanwendungen, Verfügbarkeit über Landesgrenzen hinaus, Übertragungsrate einschließlich Fehlerschutz und Datensicherheit (Kurth 2002).

UMTS wurde in der Vergangenheit in der Regel mit höheren Bandbreiten gleichgesetzt. Doch die dritte Mobilfunk-Generation bringt einen weiteren und mindestens ebenso wichtigen Vorteil mit sich: die Einführung einer IP-basierten Übertragungsplattform, welche die Bereitstellung interaktiver, multimedialer Dienstleistungen erlaubt. Dies ist auch für das Vermittlungssystem eine Herausforderung. Zwar wird der gegenwärtige, auf leitungsvermittelter Übertragung beruhende GSM-Standard noch lange Zeit in Koexistenz mit dem UMTS-Mobilfunk-Netz bestehen, doch schon heute arbeiten die wichtigen Akteure in Forschung und Industrie an IP-basierten Mobilfunknetzen (Küfner/Hofmann/Vogt 2004).

Die Entwicklung zum konvergenten Netz, das alle verfügbaren TeilnehmerZugangsnetze in eine alles übergreifende Netzarchitektur mit einem einheitlichen IP-basierten Kernnetz integriert, soll sich als schrittweise Evolution vollziehen. Im Zusammenspiel all dieser Komponenten können ganz neue, einfach zu nutzende und über IP nahtlos integrierbare Dienste für den professionellen Nutzer und den privaten Endkunden entstehen. Selbst wenn bestimmte Dienste sich keine inhaltliche Konkurrenz machen, besteht ein grundsätzlicher Wettbewerb zwischen den unterschiedlichen Diensten um die limitierten Zeit- und Geldressourcen der Nutzer. Auch wenn grundsätzlich gilt, dass der Wettbewerb zwischen den Anbietern entscheiden soll, welches Angebot sich letztlich beim Nutzer durchsetzt, kommt es dennoch darauf an, für alle Anbieter vergleichbarer Dienste auch gleiche Marktchancen zu schaffen.

Neben der punktuellen Konkurrenz ergeben sich aber auch Möglichkeiten zur Schaffung neuer komplementärer Dienstangebote, die auf der Kombination zweier und mehrerer Technologien erwächst. So erlaubt die Kombination von UMTS und WLAN die Realisierung von Synergieeffekten. Dies betrifft insbesondere die Möglichkeit, für Anwendungen mit unterschiedlichen Anforderungen (insbesondere Bandbreite) die optimale Übertragungstechnologie in Bezug auf Ressourcennutzung oder Kosten nutzen zu können. (Intel 2005, Kaufmann 2004) 
Nachdem die wesentlichen technischen Aspekte der beteiligten Technologien an der Ausgestaltung neuer und aus ökonomischer Sicht interessanter Szenarios dargestellt wurden, werden diese nun näher beschrieben und einer kritischen Betrachtung unterzogen.

\subsection{Szenario: $802.11 / 802.16$}

Der Wireless-Markt etabliert sich in zunehmendem Maße und gewinnt immer mehr an Dynamik. Davon betroffen sind alle Hierarchieebenen von Netzwerken, die die Vorteile der drahtlosen Systeme nutzen wollen. Für die lokale bzw. metropolische Ebene zeichnen sich insbesondere die IEEE-Standards 802.11 respektive 802.16 verantwortlich.

Mit WiMAX - unter dem Kürzel 802.16 bekannt, tritt ein FunktechnikStandard an, der über Reichweiten von bis zu 50 Kilometern bei Datenübertragungsraten von ca. $70 \mathrm{Mbps}$ pro Sektor einer Basisstation verfügt, die üblicherweise 6 Sektoren versorgt $(\mathrm{Sw03})$. Mit der großen Abdeckung durch die WiMAXBasisstationen, hohen Bandbreiten und den vergleichsweise niedrigen Investitionskosten in die notwendigen Infrastrukturen - derzeit wird mit Preisen von rund 10.000 Dollar für eine Basisstation gerechnet - bietet sich WiMAX für 4GMobilfunknetze und auch für stationäre Breitbandzugänge an (WiMAX 2005).

In Deutschland werden derzeit über 4000 Hotspots betrieben. Die HotspotBetreiber sind bei der Bereitstellung des Internetzugangs für ihre Kunden ihrerseits auf die High-Speed-Internet-Zugänge angewiesen und müssen sich mit dem Problem der Standortanbindung auseinandersetzen.

Auch nach der Öffnung des Telefonmarktes für private Anbieter in Deutschland, die bis heute viele positive Auswirkungen für die Geschäfts- und Privatkunden gezeigt hat, besitzt die Deutsche Telekom AG (DTAG) bei der Verfügung über die Leitung in das Haus des Kunden - die Strecke zwischen Telefonzentrale und Hausanschluss - die so genannte letzte Meile über eine nahezu ungebrochene Monopolstellung (Schatz 2000). Die Mitbewerber der Telekom, die Fernmeldedienste anbieten wollen, müssen ihr eigenes Netz mit jenem der DTAG verbinden oder Leitungen des ehemaligen Monopolisten mieten. Die DTAG verlangt für die Anmietung von den privaten Anbietern hohe Entgelte, obwohl die DTAG verpflichtet ist, ihren Konkurrenten den Teilnehmeranschluss und Mietleitungen zu kostenorientierten Preisen anzubieten. Die Preisgestaltung bzw. entsprechende Rahmenbedingungen werden von der RegTP überwacht und vorgegeben. Die hohen Interkonnektionspreise der DTAG stellen für das Wachstum des 802.11WSP-Marktes ein schwerwiegendes Hemmnis dar und setzen dem Wettbewerb auf diesem Markt Grenzen, da sie viele potenzielle Betreiber und Investoren vom 
Einstieg in diese Sparte des TK-Marktes abschreckt. Aus diesem Grund wird fortlaufend nach Lösungen zur Überbrückung der letzten Meile gesucht.

Eine mögliche Alternative neben existierenden technischen Verfahren, wie Satellitenanbindung, Nutzung der Kabel-TV-Infrastruktur und des Stromnetzes, stellt der Ansatz, per drahtlosem Anschluss (Wireless Local Loop) den kabelbasierten Breitbandzugang per Funk nutzbar zu machen. Die Familie der IEEE 802.16 Standards ist die zurzeit wohl vielversprechendste neue DrahtlosTechnologie am Markt, die Hotspots, die einen Internetzugang auf Basis des IEEE 802.11-Standards bieten, mit den Internet-Backbones verbindet und einen kostengünstigen, effektiven, drahtlosen Breitband-Zugang als Alternative zu bestehenden DSL- bzw. Kabelverbindungen für die letzte Meile erlaubt.

Die vorangegangene Betrachtung hat gezeigt, dass durch den Einsatz der WiMAX-Technologie das Quasi-Monopol der DTAG bezüglich der so genannten letzten Meile erhebliche Konkurrenz erhalten kann. Das angedachte Szenario ist in Abbildung 31 dargestellt. Offensichtlich bietet der kombinierte Ansatz aus der 802.11und der 802.16-Technologie neue ökonomische Spielräume, die insbesondere in dem kurzfristigen Handel mit Bandbreite Ausdruck finden können. Unter der Annahme, dass sich die Hotspot-Landschaft insofern entwickelt und es zu Bandbreitenüberschüssen aber auch zu Bandbreitenengpässen kommen kann, bietet sich durch die großflächige Abdeckung der WiMAX-Basisstationen die Möglichkeit, ähnlich wie auf Spotmärkten (vgl. Rohölmarkt - (Kasten 2001, Hensing 1994)) mit Bandbreitenkapazitäten Handel zu betreiben, sodass sich sowohl für den 802.11-WSP als auch für den Endkunden attraktive Preise bzw. -modelle realisieren lassen.

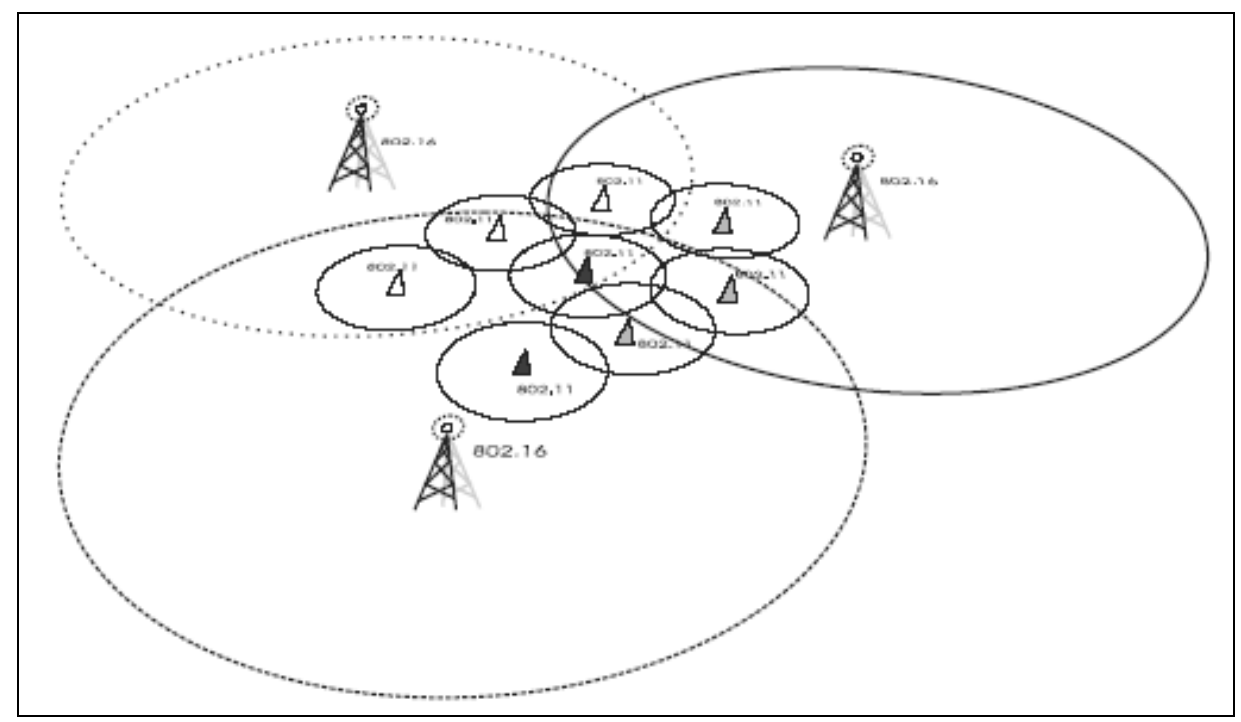

Abbildung 31: Wi-Fi/WiMAX Use-Case 
Neben den zahlreichen Vorteilen, die das oben beschriebene Szenario mit sich bringt, treten auch Probleme auf, die einer Darstellung bedürfen.

Drahtlose Kommunikation unterliegt der Problematik der Fehleranfälligkeit, die sich beispielsweise durch eine hohe BER (Bit Error Rate) auszeichnet. Werden aktuelle drahtgebundene Hotspot-Anschlussmöglichkeiten mit der drahtlosen 802.16-Technik verglichen, ergibt sich offensichtlich das zusätzliche Fehlerpotenzial durch die zweite drahtlose Schnittstelle zwischen dem 802.11-Hotspot und dem drahtgebundenen Internet, das direkt die 802.16-Basisstationen über ein entsprechendes Backbone-Netzwerk versorgt.

Insbesondere neue multimediale mobile Dienste mit Echtzeitanforderungen verlangen geradezu garantierte und zugesicherte Qualitäten. Dabei ist es nicht einmal wesentlich, dass hohe Bandbreiten zur Verfügung stehen, sondern möglichst kurze Paketlaufzeiten und geringe Paketlaufzeitschwankungen das grundsätzliche Übertragungsverhalten charakterisieren - eine typische Anwendung ist z. B. Voice over Wireless LAN (VoWLAN).

Durch WiMAX könnten die Konkurrenten der Deutschen Telekom nicht nur die letzte Meile umgehen, sondern auch die Datenübertragungsraten der heutigen DSL-Technik um ein Vielfaches übertreffen. Die WiMAX-Anbieter können die Technologie auch einsetzen, um ihre Flächenabdeckung zu vervollständigen, da WiMAX-Breitbandanbindungen auch dort möglich sind, wo DSL aus technischen oder ökonomischen Gründen nicht angeboten werden kann. Ein weiterer Vorteil von WiMAX sind die geringen Kosten für die Einrichtung einer entsprechenden Infrastruktur. Der besondere Vorteil für WiFi-WSPs besteht in der Möglichkeit, flexibel und dynamisch Bandbreite abhängig vom Auslastungsgrad des Hotspots zusätzlich zur bestehenden Anbindung von einem oder mehreren 802.16-WSPs zu ordern.

In den vergangenen Jahren wurden in Erwartung hoher Traffic-Zuwachsraten starke Investitionen in den Ausbau der Glasfaser-Infrastruktur in Deutschland getätigt. Mehr als ein Dutzend Unternehmen haben dabei etwa 286.000 km hochleistungsfähige Glasfaserkabel verlegt, wobei die wichtigsten Strecken in und zwischen Ballungsgebieten versorgt wurden, wo Leitungen mit hohen Übertragungsraten bis $\mathrm{zu} 2,5 \mathrm{Gbps}$ angeboten werden. Infolgedessen besteht ein enormes Überangebot an Bandbreite auf den Hauptstrecken, wobei mehr als 90 Prozent, der verlegten Leitungen ungenutzt sind. Die strukturschwachen Regionen sind in dieser Hinsicht allerdings stark unterentwickelt. Das Wachstum des 802.16Marktes würde den Glasfaser-Carriern zu zusätzlichem Umsatz verhelfen.

Die Weitergabe günstiger Preise für die Bandbreitenanbindung mit 802.16 durch 802.11-WSPs an die Endkunden ist förderlich für die Entwicklung des Marktes mobiler Sprach- und Datendienste, wovon natürlich auch die Contentund Service-Provider profitieren können. 
Abbildung 32 zeigt ein mögliches Szenario bezogen auf Unternehmensnetzwerke, das den Mitarbeitern mobilen Internetzugang ermöglichen kann. Maßgeblich beteiligte Technologien sind auch hier wieder die IEEE Standards 802.11 und 802.16. Verschiedene Einsatzmöglichkeiten werden aktuell diskutiert. Diese reichen von logistischen Lösungen bis hin zum allgegenwärtigen Internetzugang mit hoher zur Verfügung stehender Bandbreite - beispielsweise um den Mitarbeitern größere und schnelle Datentransfers zu ermöglichen.

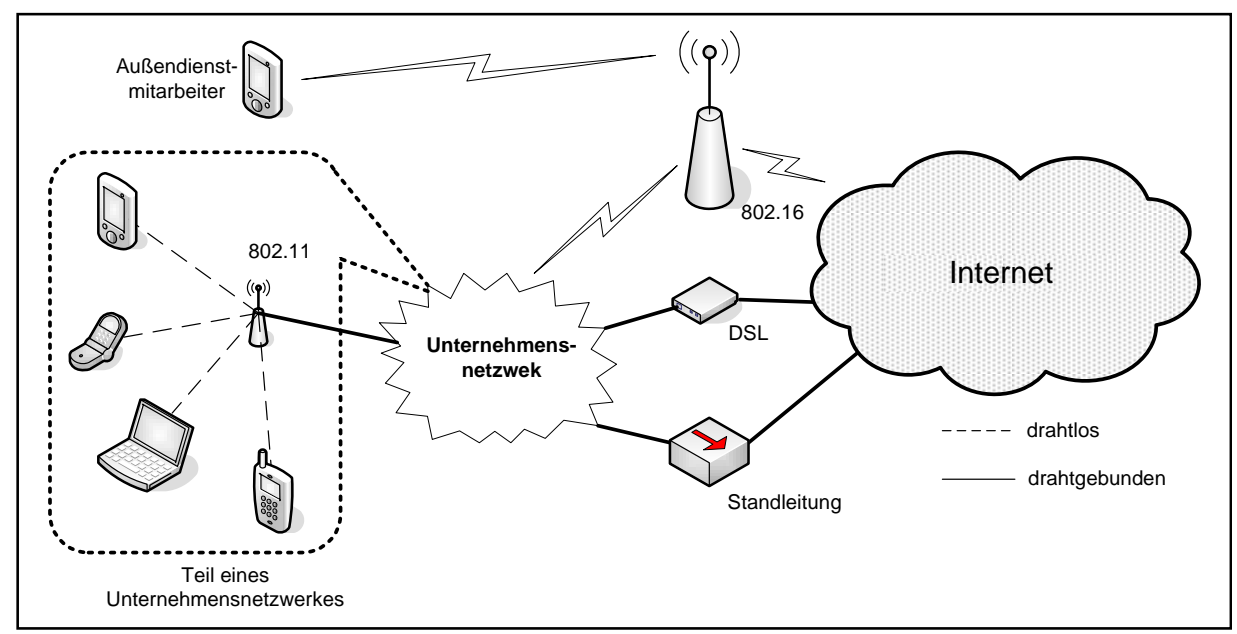

Abbildung 32: Szenario 802.11 / 802.16

Der Erfolg des vorgestellten Szenarios wird entscheidend davon abhängen, wann die Netzausrüstung für die Betreiber verfügbar, wie schnell und wie engmaschig das Netz der 802.16-Basisstationen sein wird und wie hoch die tatsächlichen Übertragungsgeschwindigkeiten der WiMAX-Netze in der Praxis sein werden. Ebenso wichtig sind aber auch die dabei tatsächlich erzielbaren „Zellgrößen“ mit der WiMAX-Technologie.

\subsection{Szenario: $802.16 / 802.20$}

Eine weitere interessante Anwendungsmöglichkeit der 802.16-Technologie liegt in der Backboneanbindung der 802.20-Basisstationen durch die auf 802.16-Standard basierende Infrastruktur. Dies kann durch folgendes Szenario verdeutlicht werden.

Mobilität ist ein entscheidender Faktor unserer modernen Wirtschaft. Wenn man die gegenwärtige Entwicklungsdynamik mobiler Gesellschaften und des Automobils als zentralen Bestandteil des Mobilitätssystems betrachtet, entdeckt man zahlreiche Entwicklungslinien, die interessante Innovationsfantasien und Zukunftsperspektiven eröffnen und neue Herausforderungen stellen (Klein 1998, Seiffert 2000). 
Mobilität ist unmittelbar mit der Notwendigkeit eines häufigen Ortswechsels verbunden, den man heutzutage mit Hilfe von Kraftfahrzeugen realisiert. Während der Fahrt werden kontinuierlich Informationen aus der unmittelbaren Fahrzeugumgebung aufgenommen und verarbeitet. Neben diesem Informationsbedarf, welcher das Lenken eines Fahrzeuges und somit insbesondere den Fahrer betrifft, besteht bei den Passagieren häufig der Wunsch nach Informationen über den Ort, an dem man sich vorbei bewegt. Zur Beschaffung solcher Informationen und Realisierung einer Palette neuer Dienstleistungen, wie zum Beispiel Flottenmanagement, dynamische Navigationshilfe, Verkehrsinformationen, ortsspezifische Dienstleistungen, M-Commerce ist neben der Kombination von schnurloser Kommunikation und Ortungstechnologie in Nutzfahrzeugen und Pkws ein brauchbarer mobiler terrestrischer Internetzugang vom Fahrzeug aus notwendig (Ehmer 2002).

Durch den zukünftigen Einsatz des WMAN-Standards 802.20 wird ein mobiler terrestrischer Internetzugang für Fahrzeuge bis $250 \mathrm{~km} / \mathrm{h}$ möglich sein. Zwar ist WiMAX auch für mobile Anwendungen konzipiert, der mobile Zugriff bei höheren Geschwindigkeiten ist aber im 802.20-Standard festgelegt. 802.20 erlaubt durch die Unterstützung der Luftschnittstelle einer Inter-Cell bzw. einer Inter-Sektor-HandoffProzedur für die angegebene Fahrzeuggeschwindigkeit die Minimierung des Paketverlustes und der Latenzzeit bei einer robusten und nahtlosen IP-Paketübertragung. Dank dieser Möglichkeit wird die Verbindung von Internet, Multimedia und VideoAnwendungen begünstigt und ein enormes Marktpotenzial erschließbar.

Zur Anbindung von Fahrzeugen an das Internet unter Nutzung stationärer 802.20 Basisstationen (BS), die entlang der stark befahrenen Trassen, wie z. B. Autobahnen, sind entsprechende Mobile Stationen (MS) für Fahrzeuge erforderlich. Die Reichweite der 802.20 Basisstationen zur Durchführung eines bidirektionalen Datenaustausches mit der MS in einem beweglichen Fahrzeug soll bis zu $20 \mathrm{~km}$ betragen, sodass eine BS etwa für den gleichen Straßenabschnitt theoretisch ausreichen würde. Da aber die Datenübertragungsrate für die Teilnehmer in der „Zelle" für den Downlink, d. h. von BS zur MTs, insg. bis $16 \mathrm{Mbps}$ und für den Uplink, also MSs zu BS, etwa 3,2 Mbps betragen soll (pro Teilnehmer jeweils $1 \mathrm{Mbps}$ und 0,3 Mbps im 1,25-MHz-Band; im 5-MHz-Band: entsprechend 4 Mbps und 1,2 Mbps), ist es allerdings zu erwarten, dass die Dichte der BS pro Straßenabschnitt höher sein wird, um einer größeren Anzahl Teilnehmern gleichzeitig einen breitbandigen Internetzugang zu ermöglichen.

Als wichtige Argumente für den Einsatz dieser Technologie im Bereich der Fahrzeugtelematik kann man die Kosten einer BS, die laut Expertenmeinungen deutlich unter denen für eine UMTS-BS liegen werden, und die Möglichkeit der Bereitstellung der Bandbreite von bis zu 1 Mbps pro Teilnehmer nennen. 
Für die Bereitstellung des mobilen Internetzugangs auf Basis von 802.20 ist eine nahtlose Abdeckung der Verkehrswege durch Wirkungsbereiche der BS erforderlich. Dem Ausbau dieser Infrastruktur würden allerdings oftmals die mangelnden Anbindungsmöglichkeiten der 802.20-BS an die Backbone-Netze im Wege stehen. Eine konventionelle Netzanbindung durch Kabel wird oft - nicht nur aus Kostengründen - nicht durchführbar sein. Die Notwendigkeit der Installation der 802.20-BS mit möglichst geringer Entfernung von den Verkehrsadern erfordert insbesondere in ländlichen Gegenden die Suche nach einer Alternative. Diese Alternative ist durch die Anwendung des bereits vorhandenen 802.16-Standards für drahtlosen, stationären Breitbandzugang zum Internet in sehr naher Zukunft möglich. So würde die Verwendung einer 802.16-BS mit maximaler Reichweite von $50 \mathrm{~km}$ eine Anbindung von mehreren 802.20-BS eines oder mehrerer WISPs mit bis zu 70 Mbps problemlos ermöglichen. Die Position der 802.16-BS kann somit unter Aspekten der Standortkonfliktminimierung anhand zahlreicher Faktoren, wie z. B. Einhaltung von Emissionsschutzverordnungen oder Berücksichtigung naturgeschützter Flächen, flexibel optimiert werden. Auf diese Weise ist es möglich, relativ kostengünstig eine Infrastruktur, die einen mobilen terrestrischen Internetzugang vom Fahrzeug aus ermöglicht, basierend auf der Kombination der beiden Standards aufzubauen.

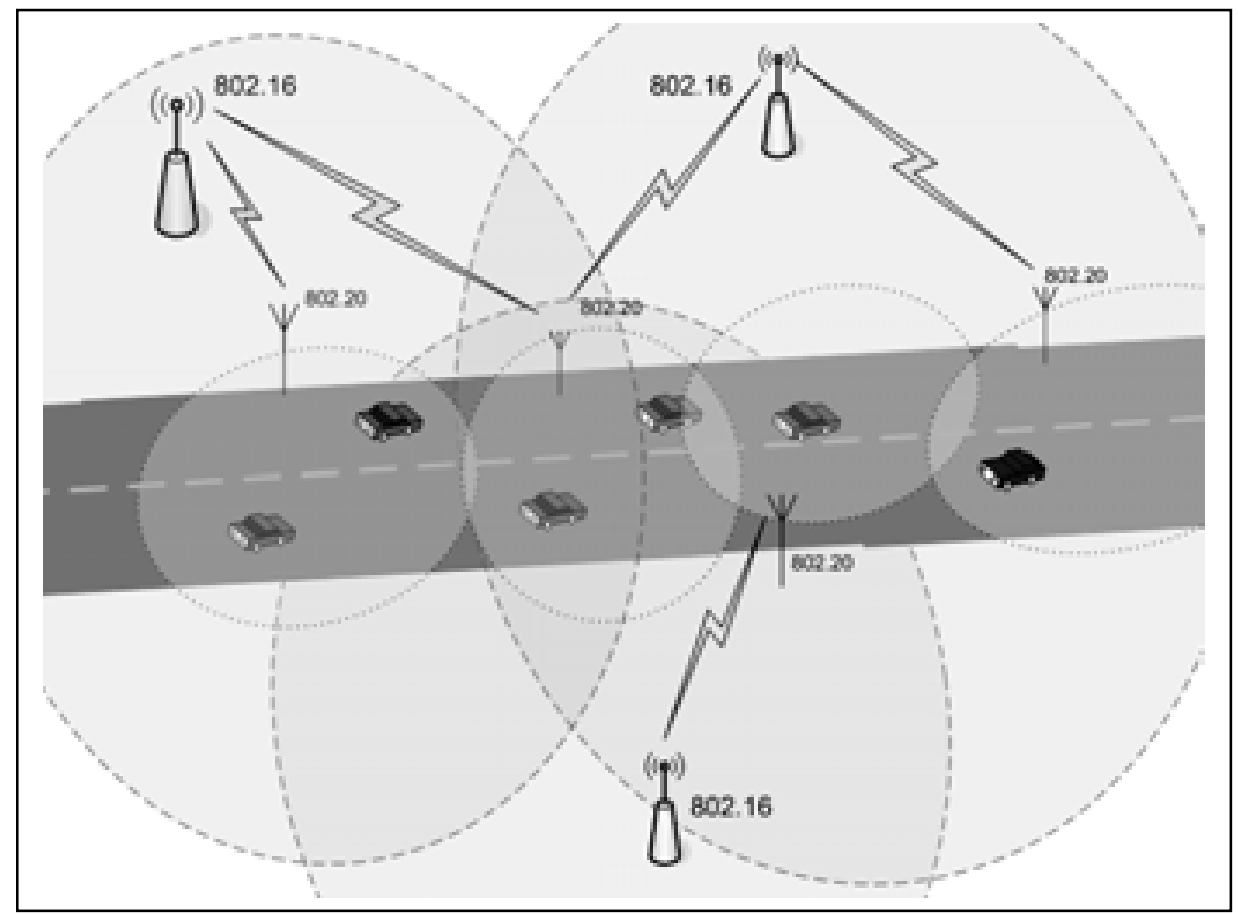

Abbildung 33: 802.16-802.20 Szenario 
Während der Fahrt wird im Nutzungsfall eine Verbindung von der MS im Fahrzeug zur nächsten 802.20-BS aufgebaut, in deren Abdeckungs-/Wirkungsbereich sich das Fahrzeug gerade befindet. Die Netzanbindung der 802.20-BS ist durch die drahtlose Verbindung mit der 802.16-BS, die eine drahtgebunde BackboneAnbindung mit erforderlicher Anbindungsbandbreite, realisiert.

Bei dem Aufbau der Verbindung ist eine kundenfreundliche und sichere Authentifizierung des Nutzers gegenüber dem Anbieter zum Zwecke der Abrechnung der bereitgestellten Dienstleistung sowie ein optimales Abrechnungsverfahren erforderlich (Reichwald et al. 2002). Die Spezifik der Nutzungsbedingungen, die durch eine hohe Geschwindigkeit der Fahrzeuge und der erwarteten Dienstanspruchsnahme von unterschiedlichen WISPs gegeben ist, erfordern neue Modelle und Konzepte für die Realisierung der Authentifizierungs- und Abrechnungsmechanismen. Die heute beim Betrieb der WLAN-Hotspots eingesetzten Mechanismen zeigen deutliche Schwächen bezüglich des Nutzungskomforts, Nutzerakzeptanz, Flexibilität und bei den Möglichkeiten der Ertragsteigerung.

$\mathrm{Zu}$ den bekannten und vor allem für WLAN-Roaming relevanten Authentifikationstechnologien gehören heute SIM (Subscriber Identity Module), RADIUS (Remote Authentication Dial-In User Service) und die von der GSMA empfohlene EAP SIM Technologie. EAP SIM steht derzeit bei der IETF (Internet Engineering Task Force) vor der Verabschiedung als Standard. Beim Authentifizierungsverfahren EAP SIM kommt, wie der Name schon andeutet, eine SIM-Karte wie sie beim Mobilfunk eingesetzt wird, zum Einsatz, über die sich der Nutzer dem Netzbetreiber ausweist. EAP SIM stellt ein sicheres und zweckmäßiges Verfahren zur Authentifizierung dar. Für die EAP SIM-Technologie muss der Markt erst einmal vorbereitet werden, man rechnet damit, dass die kunden- und betreiberfreundliche SIM-Lösung auch mittelfristig dank den Standardisierungsanstrengungen zum Erfolg führen wird und Nachfrage erfährt.

Europa gehört im Bereich der Mobilkommunikation zur Weltspitze. Der Markt für mobile Anwendungen bleibt jedoch bisher hinter den Erwartungen zurück. Bei den potenziellen Anwendern gibt es noch zu viel Skepsis im Blick auf Investitionsrentabilität und Betriebskosten. Die Betreiber sind besorgt um das Auslastungsniveau und die Umsätze, die sich mit mobilen Anwendungen erwirtschaften lassen. Laut einer Studie der Unternehmensberatung Frost \& Sullivan (Frost/Sullivan 2004) wird der Datenverkehr im Geschäftskundensegment kontinuierlich zunehmen, was entscheidende Möglichkeiten bietet, Umsatz mit mobilen Anwendungen zu erwirtschaften. Das Volumen des Datenverkehrs ist im Vergleich zur Sprach- und zur privaten Datenkommunikation noch begrenzt, dieser beansprucht dennoch schon heute nennenswerte Netzkapazitäten.

Die bisherigen Tarifstrukturen in diesem Sektor tragen allerdings noch nicht direkt zur Steigerung des durchschnittlichen Umsatzes pro Anwender bei. Der 
verstärkte mobile Datenverkehr - (zukünftig auch von Fahrzeugen aus) in Verbindung mit der Anpassung der heute üblichen Flatrate-Preissysteme, die es dem Betreiber erlauben werden, den Wert des verstärkten Geschäftsdatenverkehrs für sich besser zu realisieren - kann zu einer stärkeren Nutzung mobiler Dienste durch Geschäftskunden führen.

\subsection{20 und mobile Ad-hoc-Netze für die Fahrzeug- Fahrzeug-Kommunikation}

Das oben skizzierte Szenario erlaubt auch eine interessante Kombination mit den Lösungen zum Einsatz von mobilen Ad-hoc-Netzen für die Fahrzeug-zu-Fahrzeug-Kommunikation. In diesem Forschungsbereich sind aktuell starke Aktivitäten zu verzeichnen (Bsp.: Forschungsprojekte Network On Wheels ${ }^{20}$, Connected Drive $\left.^{21}\right)$. Ziel der Aktivitäten ist es, Lösungen zu entwickeln, wie Autos künftig direkt miteinander kommunizieren und drahtlos Informationen zur aktuellen Verkehrslage sowie Gefahren durch Glatteis, Nebel oder Unfälle austauschen können. Auch eine Reihe von Automobilherstellern, wie Audi, BMW Group, Daimler Chrysler, Fiat, Renault und Volkswagen gründeten ein Car-2-Car Communication Consortium (C2C CC). Ziel dieses Gremiums ist es, auf europäischer Ebene einen offenen Industriestandard für die Kommunikation zwischen Fahrzeugen und Infrastruktureinrichtungen zu erarbeiten.

Innerhalb von Ad-hoc-Netzwerken übernehmen die Fahrzeuge dabei die Rolle als Sender, Empfänger oder Vermittler. Kommt ein Auto beispielsweise in einen Stau, erkennt das Navigationssystem die Situation und der Bordcomputer gibt eine aktuelle lokale Verkehrsmeldung an andere Fahrzeuge in der Umgebung weiter. Die Hauptschwierigkeit bei den mobilen Ad-hoc-Netzen besteht in der dynamischen Veränderung der Netztopologie, was vom Routingansatz einen hohen Grad an Adaptivität im Hinblick auf die Dynamik des Netzes sowie gute Skalierbarkeit fordert. Der Bandbreitenbedarf vieler Anwendungen aus dem Bereich der Kommunikations- und Internetdienste nimmt ständig zu, die Kommunikationsreichweite hängt entscheidend von der Position des Kommunikationspartners ab. Dieses Kommunikationsmodell ist allerdings auf eine robuste und breitbandige Internetanbindung angewiesen, sodass die Realisierung dieses Konzepts wirkungsvoll durch die Ansätze aus dem beschriebenen Szenario unterstützt werden kann (Enkelmann/Thienhaus 2005).

\footnotetext{
20 Network-on-Wheels: www.bmbf.de/press/1338.php.

21 BMW Connected Drive: www.bmw.com/generic/com/de/fascination/technology/connecteddrive.
} 


\subsection{Anwendungen}

Die intelligente Verbindung von Fahrzeug-Informationssystemen - mitsamt geografischer Positionierung - mit Informationsnetzwerken mittels drahtloser Kommunikationssysteme ermöglicht eine Vielfalt neuer ortsspezifischer Dienstleistungen, wie z. B. Straßennotfalldienste, dynamische Navigation, Kommunikationsund Internetdienste und kundenspezifisches M-Commerce.

Das im Szenario beschriebene Konzept der stationären Basisstationen am Straßenrand erlaubt beispielsweise die gezielte Übertragung von Marketinginformationen zu den Fahrzeugen und somit an potenzielle Kunden, die durch den Ortsbezug für den Nutzer einen hohen Mehrwert bieten. Einkaufszentren oder Schnellrestaurants können so ihre Kunden über aktuelle Angebote informieren. Auf diese Weise werden auch neue Angebotsformen für mobile Dienstleistungen mit Sponsoring-Aspekten denkbar. Durch die Anbindung der Fahrzeuge durch die 802.20-Technologie werden auch Anwendungen, die höhere Anforderungen an das Kommunikationssystem im Hinblick auf Bandbreite und Verzögerung stellen, wie Videoübertragung oder Live-Streaming aus dem bewegten Fahrzeug möglich. Weitere Anwendungen umfassen die Übertragung von geschäftlichen Daten aus Nutz- oder gewerblich genutzten Fahrzeugen. Im Gegensatz zur statischen Darstellung von Firmeninformationen können in Zukunft dynamische Informationen an interessierte Insassen in Fahrzeugen verschickt werden (Zobel 2001).

Das skizzierte Modell stellt aus heutiger Sicht ein Trend-Szenario dar, das mit hoher Wahrscheinlichkeit eintreten wird, wenn die bisherigen technologischen Entwicklungen sich fortsetzen. Die Vorhersehbarkeit der Zukunft ist allerdings unmöglich, man kann sich aber auf eine ungewisse Zukunft vorbereiten, indem verschiedene mögliche Entwicklungen in Szenarien durchdacht und dahinterstehende Abläufe verstanden werden.

\section{Zusammenfassung und Ausblick}

Die dargestellten möglichen Szenarien des Einsatzes bekannter und neuer WLAN-Technologien - 802.11 bzw. 802.16 und 802.20 - sowie mögliche Synergieeffekte in den Bereichen der klassischen Mobilkommunikation - UMTS/ GPRS/GSM - sowie Ad-hoc-Netzwerktopologien deuten auf ein vorhandenes, hohes Potenzial für neue, interessante Möglichkeiten im Bereich der TK-Branche hin. Zur Realisierung dieser Szenarien ist es nicht nur erforderlich, noch einige technische Problemstellungen zu lösen bzw. alltagstauglich auszugestalten, sondern es muss auch die Bereitschaft der TK-Branche vorhanden sein, in die neuen Technologien zu investieren. 
In diesem Zusammenhang ist es für die Betreiber solcher Infrastrukturen von hoher Bedeutung, Geschäftsstrategien auszuarbeiten und Mechanismen zur Umsetzung dieser zu besitzen, um einen möglichst wirtschaftlichen Betrieb gewährleisten zu können. Bei solchen Mechanismen handelt es sich um Lösungen zur zeitlich optimalen, bedarfsgerechten Ausnutzung der Zugangspunkte in Verbindung mit optimalen Abrechnungs- und Preisbestimmungsmodellen. Mit der 802.16Technologie wird eine Möglichkeit geschaffen, am jeweiligen Zugangspunkt angepasst an ein entsprechendes Managementmodell die laufenden Kosten zu minimieren, indem Mechanismen zur Bandbreitenallokation eingesetzt werden, um Bandbreitendefiziten und -überschüssen entgegenzuwirken bzw. diese positiv ausnutzen zu können.

Die IP-basierte Telefonie wird möglicherweise eine der ersten Anwendungen sein, die in künftigen heterogenen Netzen großes Kundenpotenzial ansprechen wird. Dabei entsteht die Problematik der schwankenden zur Verfügung stehenden Bandbreite, so dass hier eine praktikable Lösung des Problems erarbeitet werden muss. Die erfolgreiche Entwicklung eines adaptiven, skalierbaren Sprachcodecs stellt in diesem Zusammenhang eine Möglichkeit dar, um diesen Bandbreitenschwankungen entgegenzuwirken, sodass eine netzunabhängige, hochwertige Sprachqualität erzielt werden kann.

Schon heute bieten VoIP-Anbieter sehr häufig preislich attraktivere Angebote als die klassischen Telefonieanbieter an. Dieses lässt die Vermutung zu, dass sich ähnliche Preisstrukturen auch unter mobilen Bedingungen realisieren lassen. VoWLAN - also die drahtlose IP-basierte Telefonie wird mit den klassischen Mobilkommunikationstechnologien verschmelzen. Dieses wirft einerseits technische Frage- und Problemstellungen auf - andererseits ergeben sich aber auch insbesondere ökonomisch interessante Fragestellungen, die es zu untersuchen gilt. Dabei gilt es - wie bei allen mobilen Dienstleistungen, also auch unabhängig vom speziellen Anwendungsfall VoWLAN - den Bandbreitenhandel, benutzerspezifische Anpassungen aber auch die Gestaltung optimaler Preisbildungs- und entsprechende Distributionsmechanismen zu entwerfen.

Der Einsatz der WiMAX- und MBWA-Technologie kann die Entscheidung neuer Betreiber/Provider in den TK-Markt einzutreten begünstigen. Genauso kann mit hoher Wahrscheinlichkeit prognostiziert werden, dass die Aufnahme ins Leistungsportfolio bestehender Bereiter insbesondere zur Ergänzung bereits vorhandener Netzzugangstechnologien, sich als eine Entscheidung von großer Bedeutung erweisen kann. Eine signifikante Anzahl von Antworten zu den identifizierten Fragestellungen zu finden, sowie Handlungsalternativen bei der Realisierung aufzuzeigen und gegeneinander abzuwägen wird weiterhin verfolgt werden. Insbesondere die Abbildung auf reale Bedingungen und gegebene Marktkonstellationen wird dabei im Vordergrund stehen. 


\section{Literatur}

Alvarion (2003): Alvarion: Introducing WiMAX - The next wave on the wireless agenda, Alvarion.

Berlecon Research (2003): Marktanalyse Public Wireless LAN, Berlecon Research $\mathrm{GmbH}$.

Bundesamt (2003): Bundesamt für Sicherheit in der Informationstechnik: Sicherheit im Funk-LAN (WLAN, IEEE 802.11), Bonn.

BWCS Report (2004): Wi-Fi, WiMAX and 802.20 - The Disruptive Potential of Wireless Broadband, BWCS.

Dialog Consult, VATM (2004): Sechste gemeinsame Marktanalyse zur Telekommunikation - Ergebnisse einer Befragung der Mitgliedsunternehmen im „Verband der Anbieter von Telekommunikations- und Mehrwertdiensten e.V.“ im Juli/August 2004, Bonn.

Dufft (2004): Dufft, N.: E-Interview: Erfolgsstory Wireless LAN!, Berlecon Research $\mathrm{GmbH}$, NetSkill AG.

Ehmer (2002): Ehmer, M.: Mobile Dienste im Auto - Die Perspektive für Automobilhersteller, In: Mobile Kommunikation, Ralf Reichwald (Hrsg.), Gabler Verlag.

Enkelmann / Thienhaus (2005): Enkelmann, W., Thienhaus, W.: Nutzen dezentraler Ad-hoc-Kommunikation für vernetzte Fahrzeuge, Uni Dortmund.

Erdle / Schneider (2004): Erdle, C., Schneider, T.: WLAN Hotspots Bedrohungspotential für UMTS oder Ergänzung?, TU München.

Fluhrer / Mantin / Shamir (2001): Fluhrer, S., Mantin, I., Shamir, A.: Weaknesses in the Key Scheduling Algorithm of RC4, Selected Areas in Cryptography SAC2001, Lecture Notes in Computer Science 2259. Springer Verlag.

Friedewald / Zoche (2004): Friedewald, M., Zoche, P.: Wechselseitiges Verhältnis hochbitratiger Funknetze in künftigen Telekommunikationsmärkten, Endbericht an das Bundesministerium für Wirtschaft und Arbeit (BMWA), Karlsruhe und Berlin.

Friedrich (2002): Friedrich, J.: Sprachübertragung in IEEE 802.11 Wireless Local Area Networks, RWTH-Aachen.

Frost \& Sullivan 2004: Analysis Of Opportunities In The Emerging Mobile Applications Market (Report B437), Frost \& Sullivan.

Galambos / Abrahamson (2002): Galambos, L.., Abrahamson, E.: Anytime, Anywhere - Enterpreneurship and the Creation of Wireless World, Cambridge University Press.

Gerbert / Witzki (2004): Gerbert, P., Witzki, A.: Breitband der Zukunft, In: Funkschau Ausgabe 14. 
Hensing (1994): Hensing, I.: Terminmärkte als Form internationalen Rohstoffhandels - dargestellt am Beispiel von Mineralöl und Erdgas, In: Umwelt- und Ressourcenökonomik, Bd. 3, Hrsg. Wolfgang Pfaffenberger/Wolfgang Strobele, Munster/Hamburg.

Intel (2005): Ethernet - Drahtlose Netzwerke, In: intel.com.

IT-Reseller (2004): WiMax auf dem Weg zum Massenmarkt, IT-Reseller Nr. 9/10.

Kasten/Klepper (2001): Kasten, C., Klepper, G.: Verschwörung oder Marktwirtschaft? Was bestimmt die Benzinpreise? Kieler Arbeitspapier Nr. 1048, Institut für Weltwirtschaft Kiel.

Kaufmann (2004): Kaufmann, J.: WiMAX verändert den TK-Markt, ZDNet Deutschland News.

Klein (1998): Klein, H.: Wie mobil wollen Menschen sein?, In: Mobilität und Kommunikation, J. Speidel (Hrsg.), Heidelberg.

Klinkenberg (2004): Klinkenberg, F.: Wimax-Funknetz: Das Aus für DSL und UMTS? - Alternative zur "Letzten Meile", In: macwelt.de.

Kopp (2004): Kopp, H.: Leitfaden Einsatz von WLAN in Unternehmen, Electronic Commerce Center Mecklenburg-Vorpommern (Hrsg.), Rostock.

Küfner / Hofmann / Vogt (2004): Küfner, T., Hofmann, D., Vogt, C.: Mobility Management Approaches for 4G Networks, Conference of Computer Science 2004, Sofia.

Kurth (2002): Kurth, M.: UMTS und/oder WLAN, Pressekonferenz am 9. Juli 2002, RegTP.

Mahr (2004): Mahr, W.: PWLAN - hot or not?, In: Intercai AG Bulletin.

Mangold: Mangold, S.: IEEE 802.11e Dienstgüte in Wireless LANs, RWTH-Aachen.

Ohrtmann (2004): Ohrtmann, F.: Voice Over 802.11, Artech House Publishers.

Proxim: White Paper - Evolution of the WiMAX Standard, Proxim Wireless Networks.

Reichwald et al. (2002): Reichwald, R. et al.: Die mobile Ökonomie - Definition und Spezifika, In: Mobile Kommunikation, Ralf Reichwald (Hrsg.), Gabler Verlag.

Schatz (2000): Schatz, K.-W.: Telekommunikation - Liberalisierung trägt Früchte, Institut der deutschen Wirtschaft, Köln.

Schneier (1996): Schneier, B.: Applied Cryptography 2nd Edt., John Wiley \& Sons.

Schulte (2004): Schulte, S.: Schnell, kabellos - und auch mobil, die IEEE-Standards 802.16 und 802.20 für Funk-Metronetze; In: NET Zeitschrift für Kommunikationsmanagement, Ausgabe 12/04, NET Verlagsservice GmbH.

Schulte (2004): Schulte, W.: Schnell, kabellos - und auch mobil - Die IEEEStandards 802.16 und 802.20 für Funk-Metronetze, NET, 12.2004.

Schulte (2004): Schulte, W.: Schnell, kabellos - und auch mobil - Die IEEEStandards 802.16 und 802.20 für Funk-Metronetze, In: net-im-web.de. 
Seiffert (2000): Seiffert, U.: Mobilität: Begriff, Leitbilder und Geschichte, In: Mobilität 21, M. Bohnert/K. Lompe (Hrsg.), Braunschweig.

Sinn (2002): Sinn, W.: Drahtlose Übertragungssysteme, In: Mobile Kommunikation, Ralf Reichwald (Hrsg.), Gabler Verlag.

Sweeney (2003): Sweeney, D.: WiMax Operator's Manual: Building 802.16 Wireless Networks. Apress.

Telephony (2004): TELEPHONY's Complete Guide To WiMAX - The Business Case For Service Provider Deployment, TELEPHONY.

Turowski / Pousttchi (2004): Turowski, K., Pousttchi, K.: Mobile Commerce Grundlagen und Techniken, Springer.

WiMAX (2005): Broadband Wireless Access Technology, In: intel.com.

WiMAX Forum (2004): Business Case Models for Fixed Broadband Wireless Access based on WiMAX Technology and the 802.16 Standard, WiMAX Forum.

Zobel (2001): Zobel, J.: Mobile Busness und M-Commerce: Die Märkte der Zukunft erobern, München. 


\section{Die Entwicklung neuer Formen mobiler Kommunikation und Mediennutzung}

Heidemarie Hanekop / Volker Wittke

\section{Einführung}

Das Internet als „Netzwerk der Netzwerke“ hat es für Millionen von Nutzern möglich gemacht, von ihrem Schreibtisch oder ihrem Wohnzimmer aus weltweit mit anderen Nutzern zu kommunizieren oder auf Inhalte zuzugreifen. Der ortsunabhängige Zugriff auf Content und Kommunikationspartner macht geradezu den Kern der durch das Internet ausgelösten Revolution des Informations- und Kommunikationsverhaltens aus. Dieser galt allerdings zunächst unter einer Bedingung: Der Nutzer selbst unterlag den räumlichen - und damit zugleich auch zeitlichen - Restriktionen, die mit den Konditionen seines Zugangs zum Internet verbunden sind. Die Allermeisten nutzten für den Internetzugang bis vor kurzem entweder das Equipment an ihrem Arbeitsplatz oder den häuslichen PC. Hielt man sich an anderen Orten auf oder war gar mit Auto, Flugzeug oder Bahn unterwegs, war man von den Zugangsvoraussetzungen abgeschnitten und damit notgedrungen „offline“. 
Seit rund fünf Jahren befindet sich nun weltweit eine Infrastruktur für drahtlose Zugänge im Aufbau, die eine Emanzipation der Internetnutzung von diesen räumlichen und zeitlichen Beschränkungen ermöglicht. Drahtlose Übertragungstechnologien (GPRS, UMTS und WLAN) sind nicht nur technisch entwickelt, sondern mittlerweile auch in einer Reihe von Ländern - wie in Deutschland - für den ortsunabhängigen Internetzugang verfügbar. Mehr und mehr werden jetzt auch Endgeräte angeboten, mit denen die Nutzer ständig und überall E-Mails empfangen und schreiben, im WWW surfen oder Musik downloaden können. Kurz gesagt: Die technischen und infrastrukturellen Voraussetzungen sind mittlerweile gegeben, um das Internet ,anywhere“ und „,anytime“ zu nutzen.

Weit weniger eindeutig lässt sich hingegen die Frage beantworten, was mobiles Internet hinsichtlich der damit verbundenen - neuen - Nutzungsformen und Anwendungsszenarien heißt. Verbreitet existiert der Eindruck fehlender Nachfrage auf Seiten der Anwender nach den neuen mobilen Internettechnologien. Jedenfalls schien sich keine mit der breitflächigen Diffusion des stationären Internet vergleichbare Massennachfrage zu entwickeln, welche die Grundlage für die Refinanzierung der Investitionen in die mobile Infrastruktur hätte bieten können. Zumindest in Deutschland hat diese Einschätzung offenbar zum zögerlichen Ausbau und zur zurückhaltenden Vermarktung der UMTS-Netze seitens der Netzbetreiber beigetragen. Zu konstatieren wäre demnach ein „time lag“ von Nutzungsformen und Anwenderinteressen im Vergleich zur Entwicklung der technischen Möglichkeiten. Und ganz auf dieser Linie bewegt sich die vereinte Suche von Netzbetreibern, Geräteherstellern und Content-Anbietern nach attraktiven Applikationen, mit denen sich dieser ,time lag“ auflösen ließe.

Diese Einschätzung der Lage mag zutreffend sein, soweit man neue mobile Nutzungsformen daran festmacht und zugleich darauf beschränkt, dass die genannten Übertragungstechnologien und Netzwerke sowie die für diese Technologien entwickelten Endgeräte zum Einsatz kommen. Allerdings, so unsere Ausgangsthese, würde eine solche Verengung der Betrachtungsperspektive auf die Anwendung der neuen Netzwerks- und Übertragungstechnologien wichtige Entwicklungen im Bereich der Nutzungsformen und Anwendungsszenarien außer Acht lassen. Erweitert man den analytischen Fokus um die mobile Nutzung von IuK-Technologien unabhängig von der Art und Weise, wie die verwendeten Endgeräte mit dem Internet vernetzt sind, lässt sich der Eindruck dilatorischer Konsumenten nicht aufrecht erhalten. Vielmehr haben sich, so unser Argument, bereits zeitlich parallel zum Aufbau der Infrastruktur für den drahtlosen Internetzugang neue Formen der mobilen Nutzung vernetzter IuK-Technologien herausgebildet. Dies gilt in besonderer Weise für die Mobilkommunikation, aber auch für mobile Mediennutzung (beispielsweise mobilen Musikkonsum) lassen sich diese Nutzungsformen bereits nachzeichnen. 
Charakteristisch für die Genese dieser neuen Kommunikations- und Medien. nutzungsformen ist es dabei, dass die Nutzer unter Adaption der neuen Technologien ihre bisherigen Gewohnheiten und Gebrauchsweisen, ihre Art zu kommunizieren und Musik zu hören, verändert haben. Dieser Veränderungsprozess ist dabei nur als Prozess der sozialen Aneignung der neuen Technologien adäquat analysier- und beschreibbar.

Für unseren Kontext, der Frage nach Nutzungsformen des mobilen Internet, sind diese Veränderungsprozesse aus zwei Gründen von Belang: Zum einen treffen die neuen drahtlosen Übertragungstechnologien und Endgeräte nicht auf ein unstrukturiertes Terrain, sondern auf bereits neu entstandene mobile Nutzungsformen. Teilweise könnten sich diese Nutzungsformen als empfänglich für die Adaption der neuen Übertragungstechnologien und Endgeräte erweisen; der kritische Punkt ist hier, dass sich ein spezifischer „Mehrwert“ der neuen Technologien ausmachen lässt, der zur Entwicklungsrichtung mobiler Kommunikationsformen passt. Teilweise ist die Anschlussfähigkeit aus heutiger Sicht allerdings zumindest offen, gerade weil ein vergleichbarer „Mehrwert“ der neuen Übertragungstechnologien und Endgeräte nicht ohne weiteres evident ist (wie wir am Beispiel des mobilen Musikkonsums argumentieren werden).

Zum andern wollen wir mit den Beispielen zeigen, dass mobile Nutzung ganz unterschiedliche Implikationen für die technische Vernetzung der Nutzer und ihrer Endgeräte haben kann. Soweit es um Mobilkommunikation geht, ist beispielsweise die ständige Sende- und Empfangsbereitschaft der Kommunikationspartner (,,always on“) eine zentrale Voraussetzung für die neuen Nutzungsformen. Dies galt schon für Mobilkommunikation auf Grundlage der GSM-Technologie und wird auch für mögliche weitere Veränderungen des Kommunikationsverhaltens etwa durch die Adaption mobiler E-Mail-Kommunikation - gelten. Wenn es hingegen um mobile Mediennutzung geht, ist „always on“ als Feature weit weniger zwingend. So können beispielsweise Content-Download und Content-Konsum durchaus räumlich und zeitlich auseinander fallen. Dies gilt insbesondere in Fällen wie dem mobilen Musik-Konsum, in denen der zeitlichen Aktualität des Contents keine primäre Bedeutung zukommt.

Zusammenfassend: Wenn beim mobilen Internet gegenwärtig ein „mismatch“ zwischen Technologieentwicklung und Entwicklung der Nutzungsformen zu konstatieren ist, dann liegt dieser - so unsere These - nicht in einem ,time lag“ der Nutzungsformen gegenüber der Technologieentwicklung. Eher ist es so, dass der Pfad, entlang dessen sich neue Formen mobiler Kommunikation und Mediennutzung entwickeln, nicht in ähnlicher Weise mit dem bisherigen Technologieentwicklungspfad des Internets kompatibel ist, wie dies in der Phase rascher Diffusion des stationären Internets der Fall war. Oder anders formuliert: Der Adaptionspfad mobiler Nutzungsformen hat eine eigene Logik. Solange man die 
Suche nach neuen mobilen Nutzungsformen auf die mobile Nutzung etablierter Internetdienste beschränkt, kommt man leicht zu dem Schluss, dass die Dynamik enttäuschend ist und sich wenig Neues abzeichnet.

Wir wollen den Blick für mobile Nutzungsformen öffnen, die sich parallel dazu entwickeln, Charakteristika und Triebkräfte der Entfaltung unterschiedlicher mobiler Nutzungsformen beschreiben und Anknüpfungsmöglichkeiten für die Adaption der neuen mobilen Technologien diskutieren. Wir sehen dabei gegenwärtig Anknüpfungspunkte vor allem bei der Mobilkommunikation und bei mobiler Mediennutzung. Wir wollen untersuchen, welche neuen Nutzungsformen erkennbar bzw. welche Keimformen im Entstehen begriffen sind, um damit zur Überwindung des oben skizzierten „mismatch“ zwischen Technologieentwicklung und der Entfaltung mobiler Nutzungsformen beizutragen.

Der Beitrag gliedert sich in zwei Teile. Zunächst werden die Entwicklung interpersonaler mobiler Kommunikationsformen dargestellt und ihre Entwicklungstrends skizziert. Dabei können wir uns auf internationale Forschungsergebnisse stützen. Im zweiten Teil wird die Entwicklung der mobilen Mediennutzung untersucht, um daraus Anknüpfungspunkte für die Adaption der neuen Technologien und Endgeräte zu gewinnen. Zusammenfassend diskutieren wir die Entwicklungstrends und die sich darin abzeichnenden Pfade ${ }^{22}$ der Adaption mobiler Nutzungsformen.

\section{Mobile interpersonale Kommunikation}

Neben dem Internet ist der Mobilfunk die zweite große Innovation der 1990er Jahre. Dabei wurde die Reichweite der damit verbundenen sozialen Innovation zunächst unterschätzt. Denn im Vergleich zum Internet, das mehrere neue Dienste hervorgebracht hat (vor allem E-Mail und WWW), schien der Mobilfunk eher eine inkrementelle denn eine radikale Innovation zu sein: Neu ist zwar die mobile Übertragungstechnologie, doch der Dienst selbst - das Telefonieren - schien nichts gänzlich anderes als bisher. Mobiles Telefonieren sei - so die ursprüngliche Erwartung - das Gleiche wie Festnetztelefonieren nur unter erschwerten räumlichen Bedingungen, zu höheren Preisen und mit schlechterem Empfang. Mobiltelefonie, so die Erwartung, werde besonderen Anlässen (vor allem beruflicher Nutzung), wichtigen Mitteilungen und speziellen Nutzergruppen (wie Geschäftskunden) vorbehalten sein. Die Möglichkeit einer breiten alltäglichen Nutzung wurde kaum ernsthaft in Betracht gezogen. Aus dieser technikbezogenen Perspektive

22 In Anlehnung an Theorien zur Pfadabhängigkeit technologischer und gesellschaftlicher Entwicklungen (David 1985, North 1990) gehen wir davon aus, dass die Entwicklungsoptionen für die Adaption von neuen Technologien und Diensten durch existierende Praxen, Routinen und Erwartungen geprägt werden. 
überraschte dann das rasante Wachstum des Mobilfunks in der zweiten Hälfte der 1990 er Jahre.

Die überraschende Wachstumsdynamik des Mobilfunks inspirierte in der Folge die Vision des mobilen Internets, welche die vom Mobilfunk ausgelösten Innovationen erneut unterschätzte. Denn einen erneuten Innovationsschub erwartete man sich davon, dass das Internet nunmehr mobil nutzbar sein würde. Die Potentiale des Mobilfunk-Entwicklungspfades wurden hingegen vernachlässigt. Tatsächlich wurde die Entwicklung der Datenkommunikation jedoch technisch betrachtet von vergleichsweise primitiven Diensten (wie der SMS, den Downloads von Klingeltönen etc.) beherrscht. WAP entpuppte sich demgegenüber als Flop. Wir wollen eine positive Entwicklung mobiler Internetdienste nicht grundsätzlich in Frage stellen. Im Gegenteil, der Erfolg des Blackberry und vergleichbarer mobiler E-Mail-Dienste spricht dafür, dass sich mobile Internetdienste unter bestimmten Bedingungen dynamisch entwickeln. Unser Argument weist in eine andere Richtung: Die spezifische Eigendynamik der durch den Mobilfunk ausgelösten Innovation wird tendenziell unterschätzt, insbesondere dann, wenn man sie vornehmlich aus der technischen Perspektive beurteilt. Denn sie lässt sich nur durch die tief greifende soziale Innovation erklären, die dadurch ausgelöst wurde und die - so unsere These - auch die weitere Entwicklung entscheidend prägen wird.

In der sozialwissenschaftlichen Debatte hat sich ein Forschungsansatz etabliert, der die überraschende Entwicklungsdynamik des Mobilfunks durch die spezifische Aneignungsform mobiler Kommunikationstechnologien erklärt. Mobilkommunikation ermöglicht es den Menschen, soziale Bindungen und emotionale Nähe trotz wachsender Mobilität und räumlicher Distanz aufrecht zu erhalten, die Kommunikation in primären Alltagskontexten, d. h. in der Familie, unter Freunden und im engen Kollegenkreis zu verdichten und trotz räumlicher Mobilität und zeitlicher Flexibilität den gemeinsamen Alltag zu organisieren. Insofern ist die Herausbildung dieser neuen Nutzungsform eng mit Veränderungen der Lebensweise und des Alltags verknüpft. Unsere These ist, dass sich dieser Aneignungspfad auch bei den neuen mobilen Technologien fortsetzen und dabei zur Herausbildung neuer Dienste, Anwendungen und Nutzungsformen führen wird.

\subsection{Die soziale Innovation der Mobilkommunikation: „Absent Presence“}

Mobile Kommunikationstechnologie ermöglicht prinzipiell die jederzeitige Erreichbarkeit von Personen gleichgültig an welchem Ort sie sich gerade aufhalten (anytime and anywhere). Entscheidend für das Verständnis des sozialen Innovationsprozesses ist die Bedeutung, die die jederzeitige Erreichbarkeit für die Nutzer im Aneignungsprozess erhält. Von Giddens (Giddens 1995, S. 119f) stammt der 
Hinweis, dass in Fällen physischer Ko-Präsenz von Personen ein Gefühl der Nähe nicht nur durch die Kommunikation selbst, sondern bereits durch die jederzeitige Möglichkeit hierzu vermittelt wird. Die von Giddens für diese Möglichkeit eigentlich unterstellte Bedingung - physische Ko-Präsenz - gilt bei Mobilkommunikation nun gerade nicht, und dennoch eröffnet diese Technologie die jederzeitige Möglichkeit zur Kommunikation. Häufige und (fast) jederzeit mögliche Kontakte per Handy bilden die Grundlage einer Kommunikationsform, die eine neue - medial vermittelte - Form von Anwesenheit konstituiert. Sie vermittelt ein Gefühl von Nähe trotz räumlicher Distanz, denn die betreffende Person ist per Handy zumindest akustisch in Reichweite oder mit anderen Worten: Sie ist vermittelt über das Handy anwesend.

In der sozialwissenschaftlichen Forschungsliteratur wird diese mobile Kommunikationsform durch die Metapher „Absent Presence“ (Gergen 2002) bezeichnet. Katz und Aakhus sprechen vom „Perpetual Contact“ (Katz 2002) und Burkart (2000) beschreibt die soziale Konstruktion eines neuen Kommunikationsraums, der vom physischen Raum abgekoppelt ist und dennoch oder gerade deswegen die Poren des Alltags durchdringen kann. Denn mobile Kommunikation verlängert - so die These - die Anwesenheit in primären sozialen Kontexten (Familie, Freunde, enge Kollegen) in solche Phasen des Alltags hinein, in denen man sich zwar an getrennten Orten aufhält, aber dennoch miteinander in Kontakt bleibt. Mobil telefonieren erhält hierdurch etwas von dem Charakter alltäglicher Face-to-face-Kommunikation. Insofern kompensiert sie bis zu einem gewissen Grad die physische Abwesenheit, allerdings ohne sie gänzlich unnötig zu machen bzw. zu verdrängen.

Die Herausbildung mobiler Kommunikationsformen ist eingebettet in Veränderungen von Lebensformen und Alltagswelten, durch die die Zeiten physischer (face-to-face) Ko-Präsenz in den primären Alltagskontexten immer kürzer und flexibler werden. Die für moderne Gesellschaften typische Mobilität und Flexibilität der Lebensweise begrenzt die Zeit der Anwesenheit in alltäglichen sozialen Beziehungen - immer länger ist man hingegen in Situationen, in denen man üblicherweise gerade nicht kommuniziert, weil man die dort Anwesenden nicht kennt und oft genug auch nicht näher kennen lernen will: auf dem Weg zur Arbeit, zu einem Termin oder in einer fremden Stadt. Man kann die Art und Weise, wie mobile Kommunikationstechnologien im privaten Alltag angeeignet werden daher auch als Reaktion auf die Mobilitäts- und Flexibilitätsanforderungen moderner Gesellschaften interpretieren. Gergen beschreibt diesen Zusammenhang an folgendem Beispiel:

"Because the automobile invites a high degree of mobility, and because dual-income families are becoming the norm, few denizens of the face-to-face community are present on a roundthe-clock basis. Most suburban communities stand relatively empty during the day. In effect, 
there are only remnants of the face-to-face community remaining for which the telephone may serve as a prosthesis" (Gergen 2002, S. 237).

Ein weiteres Merkmal mobiler Kommunikationsformen ist, dass sich diese intensive, jederzeit mögliche Kommunikation auf Personen konzentriert, die im realen Alltag eine wichtige Rolle spielen. In der Regel besteht eine mehr oder weniger enge Face-to-face Beziehung zu dem mobilen Gesprächspartner; Mobilkommunikation ist für enge soziale Beziehungen „reserviert“. Gergen spricht von einer Erweiterung und Revitalisierung der Face-to-face-Beziehungen (Gergen 2002, S. 237).

"The realities and moralities of the face-to-face relationship are revitalized. This is not only because of the perpetual connection that a mobile phone allows. But the very fact that the $u$ ser is rendered vulnerable to calls at any time of the day or night invites carefull selection of those who will be granted access to one's number. Such access is typically limited to those who are otherwise 'close' in the traditional sense: family, intimate friends, close colleagues and the like" (Gergen 2002, S. 237).

Das dritte Merkmal mobiler Kommunikation betrifft ihren Inhalt: Es handelt sich vornehmlich um Alltägliches bis hin zu scheinbar Belanglosem. Zum einen werden Absprachen oder Verabredungen in Bezug auf den gemeinsamen Alltag getroffen. Erlebtes wird spontan mitgeteilt, und es geht um die Vermittlung emotionaler Nähe. Höflich (Höflich 2003: 39ff) bezeichnet diese Form des Austauschs von eigentlich inhaltsleeren Mitteilungen als „Rückversicherung“, man möchte wissen, dass der Andere noch „,da“ ist oder zeigen, dass man an die betreffende Person denkt, ihr ,nah“ ist.

Ling / Ytrri (Ling / Ytrri, 2002) kommen in ihren Studien über norwegische Jugendliche zu ähnlichen Befunden. Sie unterscheiden zwischen Microcoordination des Alltags und Hypercoordination, womit sie eine ähnliche Dimension wie die Höflichsche Rückversicherung meinen. Als Hypercoordination bezeichnen sie die emotionale, expressive Dimension der jederzeitigen Erreichbarkeit. Hierbei geht es weniger um den eigentlichen Inhalt der Kommunikation, sondern vielmehr darum, dass Kommunikation stattfindet. Microcoordination hingegen dient der Feinabstimmung von Alltagsabläufen durch Just-in-time-Absprachen, z. B. zur Organisation des modernen Familielebens bei berufstätigen Eltern. In Studien über die Nutzung im Familienkontext wird der Begriff „Remote Mothering“ benutzt, um die neuen Möglichkeiten der Koordination zu beschreiben, die erweiterte Spielräume und mehr Flexibilität sowohl für die (vielfach berufstätigen) Mütter als auch für die Kinder bringen können. Sie werden daher auch als Befreiung von der Notwendigkeit zur ständigen Anwesenheit erlebt, wie Feldhaus et al. ebenfalls an Beispielen aus dem Familienalltag belegen (Feldhaus et al. 2004). Ein weiterer, häufig beschriebener Fall von Microcoordination ist die Art und Weise, wie Verabredungen in jugendlichen Peer Groups getroffen werden (Spiegel 10/2005, S. 96). Im Folgenden untersuchen wir, wie sich diese spezifische Form mobiler Kommunikation in der konkreten Aneignung des Endgerätes widerspiegelt. 


\section{Das Handy als persönliches Gerät oder „extension of the body“}

Die jederzeitige Erreichbarkeit über das Handy setzt nicht nur technische Features voraus, sondern auch die entsprechenden Nutzungspraktiken: Um jederzeit persönlich erreichbar zu sein, muss man über ein eigenes Handy verfügen, es immer bei sich tragen und zudem muss es angeschaltet sein. Diese Nutzungspraktiken scheinen heute selbstverständlich, weil sie den meisten Nutzern zur Routine geworden sind. In der Anfangsphase waren sie dies jedoch keineswegs. Zunächst nahmen viele das Handy nur gezielt mit, wenn ein bestimmter Grund zum Telefonieren absehbar war. Unser Argument ist, dass das Handy erst durch die neue Kommunikationsform der „Absent Presence“ zum persönlichen „Immer-dabei“und „Always on"-Gerät geworden ist. Diese spezifische Form der Aneignung des mobilen Telefons lässt sich am Vergleich mit dem Gebrauch des Festnetztelefons veranschaulichen:

Im Unterschied zum Festnetzanschluss ist das mobile Telefon ein persönliches Gerät, über das sein Besitzer unmittelbar persönlich erreichbar ist. Dem gegenüber wird der Festnetzanschluss üblicherweise gemeinsam genutzt, entweder in der Familie oder am Arbeitsplatz. Wenn ein anderes Familienmitglied oder ein Kollege am Apparat ist, muss der Anrufer sich erst mit der gewünschten Person verbinden lassen. Das jeweilige soziale Umfeld nimmt von dem Gespräch Kenntnis oder kann es sogar mitverfolgen - es ist nicht immer wirklich vertraulich. Die Fesselung des Telefons und damit des Telefonierenden an den Raum beinhaltet daher auch ein Element der sozialen Kontrolle. ${ }^{23}$ Mobiles Telefonieren eröffnet neue Möglichkeiten, sich sozialer Kontrolle zu entziehen. Dies spielt insbesondere bei Jugendlichen in der Phase der Ablösung vom Elternhaus und des Aufbaus eigener Beziehungen eine wichtige Rolle. Die Möglichkeit sich sozialer Kontrolle zu entziehen ist ein Grund für die stürmische Aneignung mobiler Kommunikationstechnologie durch Jugendliche (vgl. Feldhaus 2004, Ling / Yttri 2002).

Die Adaption des Mobiltelefons als persönliches Gerät ist eine entscheidende Voraussetzung dafür gewesen, dass sich Dienste und Funktionen entwickeln können, durch die sich auf dem Handy sehr persönliche Daten ansammeln, die man in einem auch für andere zugänglichen Medium vermutlich nicht speichern würde. Ein Beispiel hierfür ist das persönliche Telefonbuch. Wie bereits erwähnt, hat die verbreitete Nutzung des individuellen Telefonbuchs mit der Konzentration der mobilen Kommunikationsform auf eng vertraute Personen zu tun. Aus diesem Grund sind Mobiltelefonnummern üblicherweise nicht öffentlich. Um Zugriff auf die entsprechenden Telefonnummern zu haben, führt fast jeder Anwender ein eigenes, privates Telefonbuch im Handy, in das er die persönlichen Gesprächspartner einträgt. Das Telefonbuch bildet auf diese Weise die sozialen Beziehungen ab.

23 Insofern war die Einführung schnurloser Telefone bereits ein Fortschritt, weil der Angerufene sich nun zum Telefonieren begrenzt zurückziehen konnte. 
Die Weitergabe der Mobiltelefonnummer gilt als Erlaubnis, die jeweilige Person mobil anrufen zu dürfen. Sie signalisiert gleichsam die Aufnahme in den engeren Kreis. Insbesondere unter Jugendlichen hat der Eintrag ins Telefonbuch daher einen hohen Symbolcharakter. Mit solchen Features ${ }^{24}$ entsteht im Handy ein dokumentiertes Abbild der sozialen Beziehungen seines Besitzers. Mit der Speicherung von SMS auf dem Handy werden darüber hinaus auch Kommunikationsinhalte dokumentiert. Diese Funktionen tragen entscheidend zur Attraktivität des persönlichen Gerätes bei, gleichzeitig enthalten sie sehr sensible Daten über seinen Besitzer. Die Intimität des Handys ist vielleicht am besten mit der einer Brieftasche vergleichbar. Dementsprechend unüblich ist es, das eigene Telefon zu verleihen oder ein „fremdes“ Telefon zu benutzen.

Ein weiteres Merkmal mobiler Nutzungspraxen ist, dass man das Mobiltelefon (fast) immer bei sich trägt, denn sonst wäre man ja nicht jederzeit erreichbar. Zunächst war auch dies keineswegs selbstverständlich; in der Anfangsphase haben viele das Gerät nur bei einem zu erwartenden Bedarf mitgenommen. Erst mit der Zeit wurde es zum unverzichtbaren Begleiter im Alltag, dessen Fehlen Störungen alltäglicher Abläufe und Konflikte in den alltäglichen Beziehungen fast unvermeidlich erscheinen lässt. Gergen charakterisiert das Handy daher auch als eine Art von Prothese (s.o.). In einer finnischen Untersuchung wird von Jugendlichen berichtet, die das Handy als Teil des eigenen Körpers thematisieren:

"The unreserved, everyday attitude of Finnish teenagers is visible in the fact that children do not refer to their phones as 'mobile phones', but use the word 'känny', which can be interpreted as meaning an extension of the hand"(Oksman / Rautiaineen 2003, S. 294).

"The common view of the teens taking the ubiquitous presence of their mobiles for granted was well illustrated in a statement where the mobile phone is compared to personal commodities that are carried everywhere without a second thought: 'Your mobile is like your shoes" " (dies., S. 298).

Unsere These ist, dass dieser Aneignungspfad des mobilen Endgerätes als sehr persönliches Gerät und gleichzeitig als Gerät, das man immer dabei hat, weit reichende Implikationen für die Entwicklung neuer Features und Dienste hat. Die Erweiterung dieses „Immer dabei“-Geräts ist bisher eine starke Triebkraft gewesen und wird dies nach unserer Einschätzung auch zukünftig sein. Anknüpfungspunkte sehen wir einerseits in Bezug auf die individuelle Gestaltung, wie z. B. durch Klingeltöne, persönliche Bilder etc. Zukünftig können dies vielleicht ganze

24 Die Funktion des Telefonbuchs wurde durch eine Reihe Features erweitert, z. B. die Möglichkeit den Anrufer zu identifizieren, um ihn in einer Anruferliste zu dokumentieren oder um Anrufe nach ihrer individuellen „Wertigkeit“ entweder anzunehmen oder auf den Anrufbeantworter umzuleiten. Die Anruferliste bietet die Möglichkeit, den Anrufer zurückzurufen, sofern man ihn verpasst hat. Voraussetzung für diese Funktionalität ist die Rufnummernweitergabe als weitere technische Neuerung, die eigentlich nichts mit mobiler Technologie zu tun hat (sondern vielmehr mit der digitalen Vermittlungstechnologie), die aber erst im Zusammenhang mit mobiler Nutzung als nützliches Feature entdeckt wurde. 
Bilderalben sein oder auch persönliche Videos. Hinzu kommen solche Inhalte, die emotional hoch aufgeladen sind, die man gerade besonderes mag oder mit denen man sich identifiziert. Auch hierbei ist wichtig, dass man sie nach außen demonstrieren und den Freunden zeigen kann, weil man sie ja im Handy immer dabei hat. Dies können Prominentenbilder und Clips sein, Cartoons und Clips aus TVSerien usw. Downloads mit unterschiedlichen Inhalten sind bereits heute die wichtigsten Inhalte, die mobil gekauft werden.

Ein weiterer Aspekt des Adaptionspfades als persönliches „Immer dabei“Gerät ist die ständige Erweiterung der Leistungsfähigkeit und der Funktionen des mobilen Endgerätes durch die Integration von zusätzlichen Hardwarekomponenten (z. B. immer leistungsfähigere Kameras, bessere Lautsprecher für das Musikhören, Speichermedien) sowie weiterer Softwaremodule (z. B. Büroanwendungen, MP3-Player, Videoplayer usw.). Das Mobiltelefon ist auf dem Weg, zu einem mobilen Universalgerät zu werden. Hieraus könnte sich eine Entwicklungsdynamik ähnlich der des PC ergeben. Fraglich scheint uns allerdings, ob die bisherige Entwicklungsstrategie von Herstellen und insbesondere von den großen Providern, die nach unserem Eindruck weitgehend auf geschlossene und proprietäre Systeme setzen, geeignet ist, eine dem PC vergleichbare Entwicklung des mobilen Universalgerätes auszulösen - insbesondere deshalb, weil diese gerade nicht modulare und geschlossene Architektur und Systemsoftware dem Anwender wenig Möglichkeiten zur modularen Gestaltung und Konfiguration lässt.

Bisher haben wir unsere Argumentation auf den Aspekt der mobilen Kommunikationsformen konzentriert, der mit der Möglichkeit zur Fern-Anwesenheit zusammenhängt. Die jederzeitige Erreichbarkeit hat jedoch auch eine Kehrseite: Mobiles Telefonieren fordert die Aufmerksamkeit des Telefonierenden, er entzieht sich dabei der realen Situation vor Ort bzw. stört diese durch das Klingeln des Telefons und das anschließende Gespräch. Mit den Konsequenzen der medial vermittelten Abwesenheit beschäftigen wir uns im folgenden Abschnitt.

\subsection{Die Kehrseite jederzeitiger Erreichbarkeit: mentale Abwesenheit trotz physischer Anwesenheit}

Technisch vermittelte Fern-Anwesenheit hebt die reale Anwesenheit „vor Ort“ nicht auf, sondern tritt neben sie - häufig genug plötzlich und unerwartet. Der mobile Anruf kann den Angerufenen in (fast) jeder Situation „,treffen“: während der Zugfahrt, beim Einkaufen, in der Schule, mitten in einer wichtigen Arbeit oder beim Essen mit Freunden. Häufig genug reißt das Klingeln des mobilen Telefons den Angerufenen aus einer anderen sozialen Situation heraus. Zweifellos treten solche Situationen auch beim Festnetztelefonieren auf, aber sie sind räumlich und zeitlich begrenzt. Zudem weiß der Anrufer beim Festnetz, an welchem Ort sich 
der Gesprächspartner befindet, z. B. zu Hause, im Büro oder an einem anderen bestimmten Ort. Mit dem Ort verbindet er zumindest rudimentäre Vorstellungen über die Situation, in die er mit seinem Anruf eindringt und kann sich dementsprechend verhalten. Diese Möglichkeit der präventiven Konfliktvermeidung entfällt beim mobilen Telefonieren, der Anrufer erfährt bestenfalls im Gespräch, wo der Gesprächspartner gerade ist und was er tut. ${ }^{25}$

In der sozialwissenschaftlichen Literatur wird dieser potentielle Konflikt als „Verdopplung“ diskutiert. Meyrowitz argumentiert, dass Fern-Anwesenheit zur Verdopplung des Ortes führt, an dem Personen gleichzeitig anwesend sind (Meyrowitz 2003). Bei Gergen hat die Metapher „Absent Presence“ eine doppelte Bedeutung: Einerseits meint sie medial vermittelte Anwesenheit trotz physischer Abwesenheit, andererseits mentale/emotionale Abwesenheit trotz physischer Anwesenheit.

"We are present but simultaneously rendered absent; we have been erased by an absent presence" (Gergen 2002, S. 227).

Der Telefonierende ist zwar physisch anwesend, aber für Kommunikation „vor Ort" nicht erreichbar, solange er in das Telefongespräch vertieft ist. Höflich analysiert diesen Konflikt unter Bezug auf Goffman als Verdopplung des Rahmens der Kommunikation (Höflich 2005). Jede soziale Kommunikation benötigt einen Rahmen, auf den sich die Personen mit ihren Erwartungen beziehen können. Aus diesem Rahmen ergeben sich soziale Regeln, Normen sowie Handlungs- und Rollenmuster für die jeweilige Situation. Kommunikation funktioniert, wenn die beteiligten Personen in ihrer Definition des Rahmens der Kommunikationssituation übereinstimmen und sich dementsprechend verhalten, d. h. insbesondere die aus dieser Rahmendefinition ableitbaren Erwartungen bedienen. Dies kann in mobilen Kommunikationsformen zum Konflikt führen, da zwei unterschiedliche Rahmen (und die daraus abgeleiteten Erwartungen) gleichzeitig berücksichtigt werden müssen. Die Kommunikationssituation des mobil Telefonierenden wird einerseits durch den realen Ort der Anwesenheit und die dort geltenden sozialen Regeln und Rollenerwartungen bestimmt, sowie andererseits durch die begründeten Erwartungen nahe stehender Personen, die ihm über das Handy vermittelt werden.

'Using a mobile phone means to be part of two frames simultaneously, confronted with the interference of different rule systems. This requires a new arrangement of situational communicative behaviour"'(Höflich 2003, S. 39).

Die Verdopplung des Rahmens führt potentiell zu einer Situation, in der die an die Person gerichteten Erwartungen miteinander konkurrieren. Dieser Konflikt wird dadurch verschärft, dass am mobilen Telefon vornehmlich nahe stehende Perso-

25 Eine der häufigsten Fragen zu Beginn eines Gesprächs am Handy ist daher: „Wo bist du gerade?“. Damit versucht sich der Anrufer auf die „Vor Ort“"- Situation des Angerufenen einzustellen. 
nen anrufen, wodurch der mobile Anruf eine gewisse Priorität bekommt und den Angerufenen möglicherweise in ein intensives Gespräch verwickelt.

"The efficacy of the cell phone in extending the power of endogenous realities is partially reflected in the resentment many feel toward those using them in their presence. It is not simply that one's reverie may be interrupted by a nearby conversation; it is the fact that such conversation actively excludes one from participation. Cell phone conversation typically establishes an 'inside space' ('we who are conversing') vs. an 'outside space' constituted by those within earshot but prevented form participation. The fact that 'it doesn't matter whether you listen or not' underscores the impotent insignificance of the outsider" (Gergen 2002, S. 238).

Was tut z. B. eine Mutter in einer angespannten Arbeitssituation, wenn gleichzeitig der Anruf des Kindes auf dem Handy eine möglicherweise dringende Anforderung signalisiert oder der Freund im überfüllten Zug beim Anruf seiner Freundin? Wie reagieren die Kollegen beim gemeinsamen Essen in der Mittagspause auf ein längeres Telefongespräch mit dem Lebenspartner? Was tut der Schüler, dessen Freund während der Schulstunde per SMS eine Verabredung für die nächste Pause treffen will?

Die Beispiele verdeutlichen, dass die Verdopplung der Anwesenheit soziale Konflikte provoziert. Im Folgenden beschäftigen wir uns damit, wie der Umgang mit diesen Konflikten die Adaption der mobilen Kommunikationstechnologien prägt.

\section{Veränderung von Normen und Nutzungspraktiken zur Konfliktver- meidung}

Telefonieren in der Öffentlichkeit - also auf der Straße, im Zug, im Restaurant, im Kino - hat zunächst heftige Kontroversen über angemessenes Verhalten in der Öffentlichkeit ausgelöst. Burkart (2000) beschreibt mobiles Telefonieren in öffentlichen Räumen als Verletzung von Höflichkeitsregeln.

„Die bisherigen Regeln der Kommunikation im öffentlichen Raum werden, so die Grundannabme, durch das Mobiltelefon in verschiedener Hinsicht verletzt oder in Frage gestellt. [...] Telefonieren galt bisher als intime Praxis. Man telefonierte bevorzugt ungestört, allein, zu Hause. Wer es in der Öffentlichkeit tun musste, konnte sich in die 'Zelle' begeben. Mit dem Handy verliert das Telefonieren seine Intimität, das Private dringt in die Öffentlichkeit. [...] Mobiles Telefonieren im öffentlichen Raum ist deshalb häufig eine Verletzung von Intimitätsregeln. [...] Wer bei Anwesenheit anderer mobil telefoniert, verletət Höflichkeitsregeln, insbesondere die Regel Aufmerksamkeit und Priorität für Anwesende" (Burkart 2000, S. 218).

Höflich hat die Praxis beim Telefonieren in der Öffentlichkeit etwa drei Jahre später untersucht und kommt zu dem Ergebnis, dass sich diese Normen verändert und neue Konventionen herausgebildet haben, um solche Konflikte zu vermeiden: 
"Some kind of new calibration of the private in the public is going on, especially when looking at the ongoing discussions of emerging rules. Solutions can be more powerful (as the explicit probibition of using mobile phones at certain places) or technical. [...] The appropriation of the mobile phone is a reciprocal one. Not only does the user have to make arrangements, but the public does as well" (Höflich 2003a, S. 44).

"Using the mobile phone means a further step towards bringing the private sphere into the public. This may lead to the blurring of boundaries between public and private" (ders., S. 42). "The mobile phone is embedded in public communication and it is changing public communication" (ders., S. 50).

Solche Konventionen zur Konfliktvermeidung sind bspw. zur Seite zu gehen, um zu telefonieren, leise zu reden, den Vibrationsalarm zu nutzen, das Klingeln leise zu stellen usw. Zur Vermeidung von nicht akzeptierten Störungen an öffentlichen Orten haben sich Normen für den Umgang mit dem Mobiltelefon herausgebildet, z. B. dass man das Klingeln des Handys im Kino, Theater oder auf Veranstaltungen unterbindet. Die Etablierung solcher Normen und Konventionen ist aus soziologischer Perspektive ein wichtiges Merkmal der Entwicklung mobiler Kommunikationsformen, denn sie haben es erleichtert, dass das Telefonieren in der Öffentlichkeit im Prinzip akzeptiert wird. Dies war wichtig, denn gerade Situationen, in denen man sich in der Öffentlichkeit aufhält, bieten sich besonders zum Telefonieren an, z. B. beim Weg zur Arbeit, auf der Strasse, in der Bahn oder in Wartesituationen. Solche ungenutzten Zeiten, in denen man sich unter Fremden aufhält, werden nun für private Kontakte zu vertrauten Personen genutzt.

Zusammengefasst ist das Argument hier, dass der potentiellen Ausdehnung der Erreichbarkeit (anytime und anywhere) Grenzen gesetzt werden müssen. Diese Grenzen werden normativ eingefordert. Insofern sind die neuen Normen und Konventionen ein wichtiges Element der mobilen Kommunikationsformen. Die daraus erwachsenden Anforderungen an die Nutzer spiegeln sich in der Nutzungspraxis wider und formen die Entwicklung der technischen Features. Diese Features können von Anwendern genutzt werden um die jederzeitige Erreichbarkeit zu steuern.

\section{Die Adaption von technischen Features zur Steuerung der Erreich- barkeit}

Die Steuerung der Erreichbarkeit ist ein zentrales Merkmal des technischen Entwicklungspfades mobiler Kommunikationsdienste. Anders als beim Festnetztelefon kann man Handys schon immer an- und ausstellen. Diese primitive Steuerungsmöglichkeit wurde in vielfacher Hinsicht verfeinert. Um die Belästigung anderer Personen durch das Klingeln zu beschränken, kann man die Lautstärke des Klingeltons reduzieren, Vibrationsalarm benutzen oder Musiksequenzen als Klingelton verwenden. Um den Zeitpunkt des Telefonats auf später zu verschieben, 
kann man den automatischen Anrufbeantworter verwenden oder die automatisch generierte Liste der eingegangen Anrufe für den Rückruf nutzen. Der Angerufene kann dann den Zeitpunkt des Telefonats unter Berücksichtigung der Vor-OrtSituation selbst bestimmen. Der Anrufer erwartet allerdings eine zeitnahe Reaktion. Die Konvention, sobald wie möglich zurückzurufen, erleichtern beiden - dem Anrufer und dem Angerufenem - den Umgang mit dieser Form von Asynchronität. Für die Steuerung der mobilen Erreichbarkeit ist charakteristisch, dass die Kommunikation so zeitnah und direkt wie möglich bleibt, aber gleichzeitig den Kommunikationspartnern Entscheidungsspielräume eröffnet.

Die Möglichkeiten zur Steuerung der Erreichbarkeit betreffen nicht nur den Zeitpunkt des Telefonats, sondern auch die Person des Anrufers. Der Angerufene kann mittels automatischer Telefonnummernübermittlung sehen, wer anruft und kann so die Anrufe in Abhängigkeit von der Person des Anrufers selektieren. Anruferbezogene Selektionsmechanismen werden von einigen Handys technisch über Prioritätenlisten unterstützt, die vom Anwender konfiguriert werden können. Sie bilden die ganz persönliche Hierarchie von mehr oder weniger wichtigen Personen im Leben der betreffenden Person ab.

Die Adaption und Nutzung solcher Features wie Rufnummernanzeige und Anruferliste ist charakteristisch für die spezifische Formung des Entwicklungspfades mobiler Kommunikationstechnologien. Sie werden genutzt, um die Erreichbarkeit in Bezug auf den Zeitpunkt und in Abhängigkeit von der Person des Anrufers zu kontrollieren. Das eigentlich synchrone Telefonieren wird hierdurch asynchron, wobei der Anwender selbst die Asynchronität kontrolliert. Er kann bei Bedarf asynchron reagieren. Genauso gut kann er auch sofort antworten. Daneben hat sich auf Basis der SMS eine neue asynchrone Kommunikationsform entwickelt.

\section{Die Entdeckung einer neuen Kommunikationsform: „Absent Pre- sence" per SMS}

SMS ist eine schriftliche und asynchrone Kommunikationsform, die in besonderer Weise dem oben beschriebenen Spannungsverhältnis der "Absent Presence“ Rechnung trägt. Denn per SMS kann man eine andere Person jederzeit erreichen, jedoch ohne die Situation „,vor Ort" unmittelbar zu stören oder die Aufmerksamkeit des Adressaten unmittelbar zu erzwingen. Das Lesen einer SMS geschieht lautlos, es geht schnell (da die Zahl der Zeichen technisch begrenzt ist) und kann asynchron erfolgen, d. h. dann, wenn der Adressat es will. Gleichzeitig hat es sich zumindest in der Gruppe intensiver SMS-Nutzer als Norm herausgebildet, dass man zeitnah, d. h. so bald wie möglich antworten sollte. Insbesondere in engen Beziehungen wird eine schnelle Reaktion erwartet, Verzögerungen verursachen tendenziell emotionale Verstimmungen bzw. sind erklärungsbedürftig. Diese Nutzungspraxis der SMS ermöglicht einerseits die jederzeitige Erreichbarkeit, andererseits 
zwingt sie den Empfänger nicht zur sofortigen Unterbrechung seiner Aktivitäten „,vor Ort“. Zudem ist die SMS weniger störend für andere vor Ort anwesende Personen als die mündliche Kommunikation, so dass das Konfliktpotential der SMS-Kommunikation als vergleichsweise gering betrachtet werden kann.

Provider und Inhaltsanbieter schätzen die SMS mittlerweile als „,cash cow“, doch entdeckt und adaptiert wurde sie durch meist jugendliche Anwender, die bis heute die Hauptnutzergruppe bilden. ${ }^{26}$ Die Besonderheiten der Adoleszenzphase erklären die besondere Attraktivität der SMS für Jugendliche. Denn die SMS ist ein ideales Medium für intensive, aber verdeckte Kommunikation. Sie ist so kurz, dass man sie nebenbei lesen oder schreiben kann (sofern man über das notwendige Training verfügt). Durch die Schriftform ist sie zudem vertraulicher als das (öffentlich) gesprochene Wort, mitzuhören ist unmöglich. SMS werden genutzt, z. B. um die Kommunikation in der Peer Group auszudehnen und zu intensivieren. Per SMS nehmen Jugendliche am Geschehen in der Gruppe teil, auch wenn sie in der Familie oder in der Schule sind. Verabredungen werden per SMS getroffen; sogar für das Anbahnen von Freundschaften oder Liebesbeziehungen gibt es SMSbasierte Konventionen. Gleichzeitig kann man die SMS-Kommunikation leicht vor anderen verbergen und damit sozialer Kontrolle ausweichen. Besonders wichtig ist dieser Aspekt in Bezug auf die soziale Kontrolle durch Erwachsene, z. B. in der Familie. Selbst in der Peer Group spielen soziale Kontrolle und die Möglichkeiten, ihr auszuweichen eine wichtige Rolle. Die SMS passt daher besonders gut zur Lebensphase der Jugendlichen, was erklärt, dass sich die intensive Nutzung von SMS im Wesentlichen auch auf Jugendliche und junge Erwachsene beschränkt.

Im nächsten Abschnitt beschäftigen wir uns mit zwei aktuellen Entwicklungstrends und diskutieren, ob sich hier Anknüpfungspunkte an den Entwicklungspfad der „Absent Presence“ wiederfinden. Auch in der weiteren Entwicklung spielt - so unsere These - das Spannungsverhältnis zwischen dem Wunsch nach jederzeitiger Erreichbarkeit oder Fern-Anwesenheit einerseits und dem Bedürfnis dieser Erreichbarkeit andererseits auch Schranken zu setzen eine wichtige Rolle.

\subsection{Entwicklungstrends bei mobilen Kommunikations formen}

Durch die neuen Übertragungstechnologien und Endgeräte eröffnen sich Möglichkeiten zur Erweitung mobiler Kommunikationsformen. Bisher ist allerdings noch nicht klar, welche neuen Dienste und Anwendungen sich letztendlich etab-

26 Die Entdeckung bestand darin, dass man einen ursprünglich für Störungsmeldungen bei militärischer Nutzung vorgesehenen Kanal für wechselseitige zugesandte Kurznachrichten nutzbar machte. 
lieren werden. Wir werden uns im Folgenden auf zwei Entwicklungstrends konzentrieren - mobile E-Mail-Dienste sowie visuelle Kommunikationsmöglichkeiten (Bilder, Videos und Videotelefonie). Beide Trends schließen - so unsere These in unterschiedlicher Weise an den bisherigen Adaptionspfad an. Mobile E-Mail ermöglichen die jederzeitige Erreichbarkeit des Adressanten, auch wenn dieser die Mail möglicherweise nicht sofort lesen und beantworten kann. Visuelle Kommunikationsformen erweitern die interpersonale Kommunikation um eine Wahrnehmungsdimension, die möglicherweise in engen sozialen Beziehungen von besonderer Bedeutung ist.

\section{„Absent Presence“ per E-Mail?}

Die SMS haben wir als Kommunikationsform beschrieben, die jederzeitige und dabei doch asynchrone Erreichbarkeit ermöglicht. Die klassische E-Mail landet zwar ebenfalls fast sofort nach dem Verschicken im elektronischen Briefkasten des Absenders. Doch der Zugang zum elektronischen Briefkasten ist an die Verfügbarkeit eines stationären, ortsgebundenen Internetzugangs geknüpft. Insofern führt der stationäre E-Mail-Dienst nicht zur Erreichbarkeit der Person ,anytime“ und ,anywhere“. Die Vorteile der ortsunabhängigen und damit jederzeitigen Erreichbarkeit per E-Mail werden seit Jahren propagiert, doch erst mit dem modifizierten E-Mail-Dienst des Blackberry beginnt sich E-Mail auch als mobile Kommunikationsform zu etablieren. Der Blackberry E-Mail-Dienst unterscheidet sich in einigen, für die mobile Nutzung entscheidenden Features von der traditionellen Form der E-Mail. Dies betrifft nicht nur die mobile Übertragungstechnologie, sondern auch den Dienst selbst. Die E-Mail als Push-Dienst wird unmittelbar an das „Always on“-Endgerät des Empfängers ausgeliefert. Der Absender kann davon ausgehen, dass der Empfänger die Mail erhalten hat und erwartet, dass er sie sobald wie möglich zur Kenntnis nimmt. Dienst und Endgerät sind speziell für die mobile Nutzung konzipiert. Der bisherige Erfolg des Blackberry scheint auf dieser speziellen Kombination von Dienst, Endgerät und der daraus resultierenden Bedienungsfreundlichkeit in mobilen Nutzungssituationen zu beruhen. Oder anders formuliert: Der mobile E-Maildienst entfaltet sich bisher als Spezialanwendung auf einem Spezialgerät (statt als klassischer E-Maildienst auf einem UniversalPDA oder Smartphone). Ein wesentlicher Grund für diesen Trend scheint der höhere Bedienungs- und Nutzungskomfort zu sein. Hierzu passt, dass sich die Nutzung dieses mobilen E-Mail-Dienstes bisher vor allem auf berufliche Anwender konzentriert hat. Deren Anforderungen an die technischen Features sind höher als die von Jugendlichen und sie sind bereit und in der Lage, die Kosten hierfür zu tragen. Auch wenn der Blackberry als Spezialgerät möglicherweise auf diese Nutzergruppe begrenzt bleibt, würden wir vermuten, dass sich diese Form der mobilen Push-E-Mail etablieren kann - möglicherweise jedoch auf einem (kostengünstigeren) Universalgerät. Wir vermuten, dass sich - ähnlich wie beim Telefo- 
nieren - auch beim E-Mailen durch die mobile Nutzung neue Nutzungspraxen entstehen. Hier ergeben sich aus unserer Sicht interessante Forschungsfragen.

Die Alternative zur E-Mailnutzung mit Hilfe von PDA, Smartphone oder Handy ist der „normale“ E-Maildienst auf dem Laptop mit UMTS oder WLAN. Auch wenn es bisher kaum Nutzungsdaten in Bezug auf die einzelnen Dienste gibt, kann man davon ausgehen, dass diejenigen, die bereits UMTS oder WLAN nutzen dies auch für E-Mails benutzen. Stetig steigende Verkaufszahlen von UMTS-Karten sprechen dafür, dass sich der drahtlose Internetzugang über den Laptop etabliert. Unsere These ist allerdings, dass sich die Nutzung mittels Laptop gravierend von der mobilen Nutzung unterscheidet. Um die Differenz deutlich zu machen, bezeichnen wir sie als portable Nutzungsform. Denn im Unterschied zum E-Mailen auf einem kleinen Endgerät nutzt man den Laptop nicht „anytime and anywhere", sondern nur an Orten, die für diese Tätigkeit geeignet erscheinen, an denen man sich z. B. hinsetzen kann, akzeptable Lichtverhältnisse hat und an denen man sich zumindest für eine bestimmte Zeit aufhalten wird. Dies hat u. E. Implikationen für die weitere Entwicklung: Erstens vermuten wir, dass die portable Internetnutzung eine gelegentliche Nutzung bleibt. Wer den Laptop häufig mit sich trägt, wird ihn auch für die klassischen Internetanwendungen einsetzten. Dabei ist es nicht zuletzt eine Frage es Preises, wie sich die Nutzung auf mobile vs. stationäre Zugänge verteilen wird. Dennoch erwarten wir, dass die portable Nutzung auf bestimmte Situationen begrenzt bleibt. Zweitens gehen wir davon aus, dass aus der portablen Nutzung im Unterschied zur mobilen Nutzung keine wesentlich neuen Nutzungsformen entstehen. Diese Vermutung wird durch Ergebnisse einer Untersuchung der WLAN-Nutzung durch Studierende der GeorgAugust-Universität Göttingen gestützt, die große Ähnlichkeiten zwischen portabler und stationärer Nutzung des Internets zeigt. Der wesentliche Unterschied besteht darin, dass der Internetzugang nunmehr an unterschiedlichen Orten möglich wird, was die Studierenden dazu veranlasst, den Laptop häufiger mitzunehmen, wenn sie wissen, dass ihnen drahtlose Internetzugänge zur Verfügung stehen werden (Hanekop et al. 2004).

\section{Erweiterte „Absent Presence“ durch visuelle Kommunikation?}

Medial vermittelte mündliche und schriftliche Kommunikationsformen können in sozialen Beziehungen emotionale Nähe vermitteln, allerdings fehlen ihnen die visuellen Ausdrucksmöglichkeiten der Face-to-face Kommunikation. Die neuen Kommunikationstechnologien und Endgeräte können auch Bilder und Videos übertragen. Damit wird eine mobile Kommunikationsform möglich, die visuelle Fern-Anwesenheit erlaubt. Man könnte vermuten, dass dies der face-to-faceKommunikation näher kommt als die reine Sprach- oder gar Schriftkommunikati- 
on. ${ }^{27}$ Insbesondere in engen sozialen Beziehungen könnten Emotionen und Intimität intensiver erfahrbar werden. Auch die Koordination des Alltags könnte von der Visualisierung profitieren, wie die bekannten Werbespots über Fern-Beratung beim Einkaufen veranschaulichen. Das Kaufobjekt wird visuell präsentiert ohne das der Gesprächspartner körperlich präsent sein muss.

Die einfachste Form der Visualisierung ist das Bild. Üblicherweise benutzt man Bilder, um etwas für später festzuhalten und um es Nicht-Anwesenden zu zeigen. Beides ist heute mit den neuen Endgeräten möglich. Bisher wenig verbreitet ist hingegen das Versenden dieser Bilder, z. B. per MMS. Allerdings ist die technische Entwicklung und das Dienstangebot in Europa noch nicht sehr attraktiv (vergleichsweise teuer). In Japan ist das Versenden von Bildern ein populärer Dienst (Büllingen/Stamm 2004).

Die Entwicklung konkreter Anwendungen, Dienste und Nutzungsformen ist aus unserer Sicht noch offen. Allerdings könnten visuelle Kommunikationsformen gut an den Adaptionspfad der „Absent Presence“ in alltagsrelevanten, nahen Lebenskontexten anschließen. Diese Vermutung lässt sich noch nicht durch reale Entwicklungen erhärten. Wir vermuten, dass auch mobilen visuellen Kommunikationsformen geeignete technische Features für die Bewältigung der aus der jederzeitigen Fern-Anwesenheit potentiell resultierenden Spannungen (als Kehrseite noch intensiverer Fern-Anwesenheit) gefunden werden müssen. Dies wäre ein wichtiger Gesichtspunkt, der bei der Gestaltung von technischen Features, Diensten und Anwendungen zu berücksichtigen wäre.

Für mobile Kommunikationsformen ist die jederzeitige Erreichbarkeit der Personen entscheidend. Drahtlose Übertragungstechnologien und der „Always-on“Zugang sind daher unabdingbar. Dies gilt nicht in gleicher Weise für mobile Mediennutzung. Denn die jederzeitige Verfügbarkeit von Inhalten kann auf sehr unterschiedliche Weise gewährleistet werden. Nicht zwangsläufig benötigt man dazu jederzeit verfügbare Übertragungstechnologien. Denn im Unterschied zu Menschen kann man Inhalte vervielfältigen, verkleinern und einfach mitnehmen.

\section{Mobile Mediennutzung}

Eingangs haben wir die These formuliert, dass eine Entwicklung mobiler Formen der Mediennutzung zu beobachten ist, diese jedoch nicht dem vorherrschenden Technologieentwicklungspfad (GPRS, UMTS, WLAN) folgt. Im Unterschied zur mobilen Kommunikation hat die mobile Mediennutzung konventionelle Vorläufer, die seit langem etabliert sind. Eine Reihe von Medien lassen sich auch ohne mobile Übertragungstechnologien unterwegs nutzen. So kann man in der Straßenbahn die

27 Diese Idee ist keineswegs neu - bereits die ISDN-Entwicklung war mit hohen Erwartungen an die Bildtelefonie verknüpft. 
Zeitung oder im Zug ein spannendes Buch Zug lesen bzw. während des Joggens Musik hören. Diese traditionelle mobile Mediennutzung funktioniert offline. Die Inhalte sind dadurch unterwegs verfügbar, dass der Nutzer diese in materieller Form mit sich trägt - ob als Printprodukt oder auf einem elektronischen Speichermedium.

Diese traditionelle Art der Verfügbarkeit von Medien hat wichtige Konsequenzen für die mobile Nutzung: „Mitnehmen“ setzt voraus, dass man die Inhalte bereits hat. Damit fallen Beschaffung und Nutzung medialer Inhalte notwendigerweise zeitlich wie räumlich auseinander. Um beim Beispiel zu bleiben: Zeitung oder Buch müssen vor Fahrtantritt erworben sein. Das „Mitnehmen“ setzt der mobilen Verfügbarkeit von Medien damit zugleich deutliche Grenzen. Da man nicht alles mitnehmen kann, muss vorab entschieden werden, welche Inhalte man später nutzen will. Der Zwang zur Vorauswahl schränkt den mobilen Konsum konventioneller Medien ein und birgt zudem das Risiko, dass man im Zweifel das Falsche mitgenommen hat. Auf der anderen Seite reduziert diese Einschränkung aufwändige Such- und Auswahlprozesse in mobilen Situationen, wodurch unterwegs auch kurze Zeitfenster sinnvoll für den konventionellen Medienkonsum genutzt werden können.

Durch Online-Medien werden diese Grenzen der Verfügbarkeit im Prinzip aufgehoben. „Mitnehmen“ muss der Nutzer nicht mehr an materielle Träger gebundene Inhalte, sondern für die mobile Mediennutzung geeignete Endgeräte. Entsprechend der Vision des mobilen Internet wäre damit ein jederzeitiger Zugang zu medialen Inhalten möglich. Die Einschränkung mobiler Mediennutzung durch den Zwang zur Selektion und Vorauswahl entfiele. Die Nutzer müssten sich nicht mehr vorher festlegen, Beschaffung und Nutzung medialer Inhalte könnten eine räumliche und zeitliche Einheit bilden. Dadurch würde nicht nur die Realisierung spontaner, vorab nicht antizipierter Nutzungswünsche möglich; online wären auch der Text, das Spiel oder der Film verfügbar, den man nicht „,mitgenommen“ hat. Darüber hinaus ermöglicht der mobile Internetzugang prinzipiell den Zugriff auf Informationen, nach denen der Bedarf typischerweise erst unterwegs entsteht: Also etwa Informationen über die Straßenverkehrslage und alternative Routen (Ausweichstrecken, nächstgelegene Bahnhöfe, Haltestellen und Fahrpläne), für den Ortsunkundigen Informationen zur Orientierung am Aufenthaltsort (Geldautomaten, Tankstellen, Apotheken, Supermärkte oder Schwimmbäder, Cafes, Restaurants oder Hotels) oder komplexere Informationen über den Aufenthaltsort (Reiseführer, Stadtführer). Schließlich könnte der mobile Onlinezugang den vom stationären Internet gewohnten Zugriff auf aktuelle Informationen (von Nachrichten bis hin zur Entwicklung laufender Auktionen bei Ebay) nunmehr ortsund zeitunabhängig - jederzeit und überall - ermöglichen.

In der Vision vom mobilen Internet werden diese Potentiale durch die neuen drahtlosen Übertragungstechnologien in Verbindung mit neuen Endgeräten reali- 
siert. Dabei wird in Analogie zur Mobilkommunikation üblicherweise unterstellt, dass der orts- und zeitunabhängige Internetzugang erweiterte der Nutzungsmöglichkeiten bietet, denen keine ernsthaften Einschränkungen gegenüber stehen. Wir behaupten demgegenüber, dass diese Annahme trügerisch ist und den Blick auf Schwierigkeiten und mögliche Lösungsperspektiven bei der Entwicklung neuer Formen mobiler Mediennutzung verstellt. Im Folgenden wollen wir zeigen, dass auch ohne mobile Übertragungstechnologien und ohne „always on“ neue Formen mobiler Mediennutzung möglich sind, deren Potential in der Debatte häufig unterschätzt wird. Wir werden dieses Argument am Beispiel des iPod deutlich machen. Und wir wollen belegen, dass „always on“ im Fall mobiler Mediennutzung für die Nutzer sehr viel ambivalenter ist als im Fall der Mobilkommunikation, weil die Schwierigkeiten und Probleme unterschätzt werden, die mit der mobilen Nutzung der klassischen, stationären Internetdienste verbunden sind. Wir werden dies am Beispiel der mobilen WWW-Nutzung deutlich machen. Abschließend diskutieren wir auf Entwicklungsperspektiven mobiler Mediennutzung ein.

\subsection{Weiterentwicklung des traditionellen Adaptionspfades mobiler Mediennutzung: Das Beispiel des iPod}

Die neue Qualität der Verfügbarkeit liegt beim iPod darin, dass man seine Musiksammlung ,immer dabei“ hat. Man kann den iPod als einen neuen Typ eines mobilen IuK-Endgeräts betrachten, der das Mitnehmen einer nicht mehr wesentlich eingeschränkten Menge digitaler, persönlicher Inhalte ermöglicht, z. B. kann die eigene Musiksammlung problemlos im Gerät mitgeführt werden.

Bei dieser Nutzungsform präferieren Musikkonsumenten „ihre“ Musik. Sie wollen unterwegs die Musik genießen, die sie auch Zuhause gern hören. Dass dies häufig dieselben Stücke sind, spricht in diesem Fall für und nicht gegen die Sammlung. Da diese Präferenzen gerade durch die besondere Auswahl und Zusammenstellung der Titel nach dem persönlichen Geschmack des Nutzers befriedigt werden, wenn also der eigene Musikgeschmack im Vordergrund steht, ist das „Mitnehmen“ die angemessene Form der mobilen Verfügbarkeit. Die Beschaffung der Musik kann von der eigentlichen Nutzung - also dem Hören der Musikstücke abgekoppelt werden, ohne dass dies vom Nutzer als Nachteil empfunden wird. Der mit der Zusammenstellung und der Beschaffung der Musikstücke verbundene Selektionsprozess ${ }^{28}$ kostet den Nutzer Anstrengung und Zeit, insofern entlastet das Mitnehmen der vorausgewählten Musikstücke die mobile Nutzungssituation.

28 Beim Musikhören am Radio findet hingegen keine Selektion durch den Nutzer statt, denn er hört die gleiche Musik wie die anderen Zuhörer dieses Senders; die Auswahl ist Sache des Redakteurs. Musikhören am Radio ist insofern eine andere Nutzungsform, bei der gerade nicht der eigene Geschmack im Vordergrund steht, sondern man einfach nebenbei (im Hintergrund) Musik hören möchte. Diese sollte dem eigenen Geschmack nicht zuwiderlaufen. Aber welche Stücke dies im 
Die aktiv gehörte Sammlung wird zwar regelmäßig, aber typischerweise ausgesprochen inkrementell und langsam um neue Stücke ergänzt. Diese Gewohnheit ist offen dafür, die Pflege der Sammlung nicht unterwegs, sondern zuhause durchzuführen. Zwar beziehen die meisten Nutzer einen Großteil ihrer neuen Musikstücke aus dem Internet, aber das spricht noch nicht unbedingt dafür, die Downloads unterwegs auszuführen. Der jederzeitige Online-Zugriff ist als ,add on“ zwar möglicherweise interessant, aber nicht als Ersatz für die Verfügbarkeit der gesamten Musiksammlung im Gerät. Im Zweifel ist der mobile Onlinezugang verzichtbar. Umso wichtiger ist die Vernetzung des iPod mit einem PC oder Laptop mit Internetzugang.

Die jederzeitige, spontane und unbegrenzte Verfügbarkeit der persönlich präferierten Musik wird mit dem iPod ohne mobilen Online-Zugang realisiert. Insofern handelt es sich um mobile Offline-Nutzung. Die Beschaffung der Musikstücke hingegen erfolgt über den Internet-Musikshop i-tunes ${ }^{29}$, - zumindest ist dies der vom Anbieter vorgesehene Weg der Beschaffung. Der iPod ist zwar nicht über das Internet online vernetzt, dafür aber mit einem PC oder Laptop.

Vernetzung ist ein weiteres konstitutives Element der iPod-Nutzung wie auch generell der neuen mobilen Nutzungstrends. Die direkte Vernetzungsmöglichkeit von Gerät zu Gerät scheint ganz allgemein ein wichtiges Feature für neue mobile Nutzungsformen zu werden ${ }^{30}$. Der vernetzte PC oder Laptop ist beim iPod universeller Speicher und Administrationstool für die Beschaffung, Vervielfältigung und Zusammenstellung der Musikstücke; das Internet wird oder ist bereits der wichtigste Bezugskanal für Musik. Die Organisation der eigenen Musiksammlung wird hierdurch wesentlich vereinfacht.

Im Fall des iPod ist das Gerät auf Musik spezialisiert. Demnächst soll ein iPod auf den Markt kommen, mit dem man auch Telefonieren kann. Andere Hersteller statten Smartphones und PDAs mit iPod-Funktionalität aus. Das interessante an diesem Trend ist aus unserer Sicht, dass sich ein Einwicklungspfad herauskristallisiert, bei dem das mobile Endgerät selbst den jederzeitigen Zugriff auf digitalisierte, persönliche Inhalte garantiert - da es letztlich zum mobilen Datenträger wird. Diesen Trend hatten wir auch bereits beim Handy konstatiert - das immer mehr zur digitalen Brieftasche avanciert, in die nur sehr viel mehr persönliche Dinge hineinpassen. Letztlich geht es auch dabei um die Möglichkeit, mehr persönliche Dinge mitnehmen zu können. Ob es zur Integration in einem mobilen Universalgerät kommt oder doch mobile Spezialgeräte für das Musikhören, Telefonieren

Einzelnen sind, ist nicht so wichtig. Radiohören ist das wichtigste Begleitmedium für unterwegs (Klingler/Müller 2004).

29 http://www.apple.com/de/itunes

30 Wichtig ist der direkte Datenaustausch von Gerät zu Gerät z. B. auch bereits beim Austausch von Fotos, die mit der Handykamera gemacht wurden. 
und Fernsehen entstehen, ist aus unserer Sicht noch nicht entschieden. Vermutlich wird es beides geben (siehe dazu auch unten zum Universalgerät).

\subsection{Mobile Online-Mediennutzung}

Mobile Mediennutzung lässt sich freilich nicht durchgängig nach dem Muster des iPod vorstellen. Trotz weit reichender Digitalisierung der Inhalte und zunehmend leistungsfähiger Datenspeicher decken selbst technisch avancierte Formen des „Mitnehmens“ von Inhalten in vielen Fällen die Interessen an mobiler Mediennutzung nicht ab. Wenn es um die mobile Nutzung von Texten statt von Musik geht (seien es Bücher, Zeitschriften oder andere Schriften), ist die für die Nutzer relevante Datenbasis sehr viel größer und die Wahrscheinlichkeit wiederholter Nutzung deutlich geringer (die meisten Texte werden nur einmal gelesen). Anders als im Fall der Musik gibt es hier keine sukzessive aufgebaute und in einem leistungsfähigen Datenspeicher abgelegte persönliche „Sammlung“, aus der dann „Lesestücke“ ausgewählt werden. ${ }^{31}$ Von daher ist das Interesse am zeit- und ortsunabhängigen Zugriff auf Texte größer als bei Musik. Für den Zugriff auf aktuelle Informationen ist der Online-Zugang ,hier und jetzt“ sogar unabdingbar, ebenso für Informationen, deren Bedarf erst spontan - z. B. aus einer Situation unterwegs entsteht. Beschaffung und Konsum lassen sich in diesen Fällen räumlich und zeitlich nicht gut entkoppeln. Demnach wäre zu erwarten, dass sich neben dem traditionellen Pfad mobiler Mediennutzung ein Entwicklungspfad herausbildet, der diese Wünsche nach spontan jederzeit Online zugänglichen Inhalten erfüllt. Die verbreite Annahme war, dass das WWW bzw. WAP als mobile Variante der dafür prädestinierte Dienst sein würde.

\section{Mobile WWW-Nutzung}

Charakteristisch für die Nutzung des WWW ist gerade die Suche nach solchem Content, den der Nutzer noch nicht hat. In der Interaktivität des WWW ist Suche, Beschaffung, Auswahl und Rezeption von Content eng ineinander verwoben, sie lassen sich kaum voneinander trennen. Die Navigation im WWW kann in diesem Zusammenhang als ein ständiger Wechsel von Selektion und Rezeption betrachtet werden. Allerdings ist die mobile WWW-Nutzung bislang kaum empirisch vorfindbare Realität, die verfügbaren Technologien und Dienste werden erst in Ansätzen mobil genutzt (Eimeren et al. 2004). WAP, der seit Jahren verfügbare Vorläufer des mobilen WWW, hat sich trotz erheblicher Anstrengungen von Inhalts-

31 Die private Bibliothek, die der Musiksammlung noch sehr nahe kommt, stellt für die Mehrzahl der Nutzer nur einen Bruchteil des Fundus der von ihnen gelesenen Texte dar. Zudem gilt auch hier, dass die meisten Bücher nur einmal gelesen werden. 
anbietern und Providern als Flop erwiesen. Diesen Misserfolg könnte man mit den unzulänglichen technischen Features erklären. Die neuen mobilen Übertragungstechnologien und Endgeräte bieten verbesserte Möglichkeiten zur mobilen Nutzung des WWW. Die Übertragungsgeschwindigkeit wird vervielfacht, man kann mit UMTS oder WLAN ebenso schnell im WWW surfen wie zu Hause. Auch die Endgeräte werden immer leistungsfähiger, und die Displays sind häufig zwar immer noch klein, aber auch sie werden immer leistungsfähiger. Zudem bietet eine wachsende Zahl von Anbietern speziell angepasste Webseiten für mobile Endgeräte an.

Doch allein aus den technischen Möglichkeiten folgt nicht zwangsläufig, dass sich die mobile WWW-Nutzung nun stürmisch ausbreiten wird. Wir vermuten, dass gerade der skizzierte Vorteil mobiler WWW-Nutzung (die tendenziell unbegrenzte Möglichkeit, Inhalte zu suchen und zu beschaffen) in vielen Kontexten mobiler Nutzung zugleich ein Nachteil ist. Bei der Nutzung des WWW sind die Suche, Beschaffung, Auswahl und Nutzung von Inhalten in einem komplexen Navigations- und Rezeptionsprozess vereinigt. Man muss die gewünschten Inhalte in der mobilen Nutzungssituation suchen, bevor man sie rezipieren kann. Anders gesagt: Die Nutzung des WWW erfordert komplexe Such- und Selektionsprozesse, die vom Nutzer Aufmerksamkeit, Konzentration und häufig auch einen beachtlichen Zeitaufwand fordern. Diese hohe Eigenaktivität der Nutzer in der mobilen Situation impliziert weit reichende Anforderungen an den Nutzer (Hanekop / Wittke 2005). Komplexe Such- und Selektionsprozesse stehen allerdings häufig in Widerspruch zu den Eigenheiten mobiler Mediennutzung. Zusätzlich ist die Navigation im WWW durch die kleineren mobilen Endgeräte erschwert. ${ }^{32}$ Die äußeren Rahmenbedingungen für komplexe Navigationsprozesse sind in mobilen Situationen vielfach unzureichend und auch die Zeitstruktur ist stärker restringiert als im Büro oder Zuhause. Auch wenn die Nutzer in mobilen Situationen am raschen und unkomplizierten Zugriff auf fehlende Informationen oder Inhalte interessiert sind erschweren die Rahmenbedingungen die Suche anstatt ihn zu erleichtern. Der Vorteil mobiler WWW-Nutzung, die Möglichkeit auf einen breiten ContentFundus ohne einschränkende Vorauswahl zugreifen zu können, geht im Kontext mobiler Nutzungsbedingungen mit erheblichen Nachteile einher, die immer wieder unterschätzt wurden. Dies muss nicht prinzipiell gegen die mobile WWWNutzung sprechen. Doch für die weitere Entwicklung wird es wichtig sein, ob und wie das Such- und Selektionsproblem in mobilen Nutzungssituationen gelöst werden kann.

32 Zwar ließe sich argumentieren, dass diese Probleme durch die Nutzung eines Laptops mit mobilem Internetzugang vermieden werden. Das ist zwar grundsätzlich richtig und tatsächlich hat sich die drahtlose Internetnutzung über GPRS/UMTS-Karten seit ihrer Einführung positiv entwickelt. Laptops mit mobilem Internetzugang ermöglichen zwar die Mediennutzung an einer Reihe zusätzlicher Orte, allerdings nur mit gravierenden Einschränkungen (Hanekop / Lanfer / Wittke 2004). 


\subsection{Entwicklungstrends mobiler Online-Mediennutzung}

Ausgangspunkt vieler mobiler Online-Anwendungen oder Anwendungsideen ist die Überlegung, dass der mobile Anwender spezifische Informationsbedarfe haben könnte, die aus der räumlichen Mobilität entstehen, z. B. Informationen über den ihm fremden Ort. Der Umfang der angebotenen Informationen könnte hierauf begrenzt, anstatt den Anwender mit der Suche im Universum der weltweit im Web verfügbaren Inhalte zu belasten. Mobile Anwendungen sollten dem Kunden die Informationen liefern, die er gerade benötigt: aktuelle, individuelle und situationsbezogene Informationen. Der Vorteil individueller Auswahlmöglichkeiten als eine der wichtigsten Stärken des WWW würde genutzt ohne sich gleichzeitig den oben beschriebenen Nachteil komplexer Such- und Navigationsprozesse einzuhandeln. Das Grundprinzip solcher Anwendungen ist auch wieder die VorSelektion. Hierzu existieren unterschiedliche Modelle, die wir an den folgenden Beispielen diskutieren wollen.

Viele dieser Anwendungen beziehen sich auf Orientierungsbedürfnisse im weitesten Sinne, von Informationen über die nächste Apotheke, Hinweise auf interessante Kneipen, das Kinoprogramm bis hin zu kompletten Verkehrsinformationssystemen. Die sind Anwendungen darauf ausgelegt dem Kunden komplexe Suchund Navigationsvorgänge während der mobilen Nutzung abzunehmen. Eine Lösungsvariante mit automatischer Vor-Selektion sind Location Based Services (LBS). Sie nutzen die Lokalisierungsfunktion von UMTS oder GPS um den genauen Standort des Anwenders automatisch zu bestimmen. Mit dieser Information kann der Anbieter eine Vorauswahl treffen, z. B. die nächstgelegene Apotheke vorschlagen oder einen Stau auf der geplanten Reiseroute ankündigen und eine Ausweichroute vorschlagen. Der Nutzer bekommt diese Informationen ohne sie selbst suchen zu müssen. Das Selektionsproblem ist für den Kunden durch den Einsatz der neuen die Technologie entschärft, er erhält individuell und situativ angepasste Vorschläge zur Lösung seines Orientierungsproblems. Das Problem solcher Anwendungen ist die realistische Antizipation der Informationsbedürfnisse von potentiellen Anwendern. Zudem ist ihre Reichweite dadurch begrenzt, dass es sich i. d. R. um spezielle Situationen handelt.

Reine Navigationsanwendungen (GPS) realisieren eine andere Lösungsvariante. Hier ist der Grunddatenbestand, auf den die Nutzer zurückgreifen, auf dem Endgerät gespeichert (offline). Das Gerät holt die darüber hinaus benötigten Information selbsttätig (über GPS oder/und GPRS/UMTS) ein. Der Kunde muss eigentlich unterwegs nicht viel tun - er teilt seinem Navigationsgerät lediglich sein gewünschtes Ziel mit. Zudem ist der Aktualisierungsbedarf relativ begrenzt. Navigationsanwendungen sind so gesehen ein Beispiel, dass ähnlich wie der iPod nur begrenzt in den Online-Adaptionspfad passt. 
Zeitkritische Anwendungen, wie z. B. eine laufende Ebay-Auktion, erzeugen Informationen, die der Nutzer ortsunabhängig erhalten möchte, um auch unterwegs weiter mitbieten zu können. Das Suchproblem tritt hier nicht auf, denn der Anwender interessiert sich nur für die bestimmte WWW-Seite, die er leicht nebenbei verfolgen kann. ebay ist bezeichnenderweise ein Anbieter, der mobile WWWSeiten betreibt, die auch genutzt werden. Ein anderer Fall für zeitkritische Informationen sind Sportevents oder andere Großereignisse. Auch hier ist das Interesse des Nutzers von vorn herein begrenzt. In beiden Beispielen wird die Auswahl einmal getroffen, die Aktualisierung erfordert i. d. R. keine weiteren Selektionsaktivitäten des Nutzers.

In anderen Fällen wird die Selektionsentscheidung des Anwenders in der mobilen Nutzungssituation durch das Anlegen eines individuellen Nutzerprofils vorbereitet (siehe hierzu den Aufsatz von Kaspar in diesem Band). In einem solchen Nutzerprofil werden individuelle Präferenzen einmal festgelegt, die dann später zur automatischen Vorauswahl herangezogen werden können. Diese Nutzerprofile werden in der Regel am PC vorab konfiguriert. In der mobilen Nutzungssituation wird von der Anwendung darauf zurückgegriffen um eine automatische Vorauswahl zu treffen und dem Nutzer gezielte Vorschläge unterbreiten zu können (Hanekop/Wittke 2005).

Die Vorgehensweise dieser mobilen WWW-Anwendungen beruht auf der Aufteilung der Selektionsentscheidung in eine Vorbereitungsphase, die vorab am PC durchgeführt wird, sowie der Entscheidung über eine begrenzte Anzahl konkreter Vorschläge in der mobilen Nutzungssituation. Interessanterweise zeigen sich hier Parallelen zur traditionellen Mediennutzung und zum iPod-Beispiel. Fraglich ist allerdings, ob durch die Vorentscheidungen die spätere Auswahl soweit eingeschränkt und determiniert wird, dass der Vorteil des jederzeitigen Onlinezugriffs und der spontanen Auswahlmöglichkeiten zu stark eingeengt wird. Ob also in dem Spannungsfeld zwischen situativem Bedarf nach Informationen einerseits und den hohen Anforderungen an die Suche und Navigation erstere nicht zu stark eingeengt werden. Die Reduktion der Navigationserfordernisse durch Vorabauswahl birgt möglicherweise die Gefahr, dass der Vorteil spontaner, selbst gesteuerter Auswahl aus einem breiten Informationsangebot nicht mehr gewährleistet wird. Alternative Lösungen könnten darin bestehen für das mobile WWW einfachere Navigationsmöglichkeiten zu entwickeln und den Aufbau mobil genutzter Seiten zu vereinfachen.

\section{Mobiles TV}

Ein anderer Entwicklungspfad mobiler Medienangebote zielt auf Unterhaltungsinhalte. Anbieter setzen gegenwärtig hohe Erwartungen in Online-Games und in mobiles TV. TV als klassisches, massenmediales Unterhaltungsmedium stellt - 
anders als das WWW - keine hohen Anforderungen an die Such- und Selektionsleistung des mobilen Nutzers. Technisch bedingte Einschränkungen der Nutzungsqualität werden durch hochauflösende Farbdisplays und breitbandige Übertragungstechnologien reduziert, so dass der Vorteil orts- und zeitunabhängiger Verfügbarkeit für Anwender erfahrbar und eine Triebfeder mobiler Nutzung werden könnte. Unsere Vermutung ist, dass TV als mobiles Medienangebot zwar die oben geschilderten Probleme mobiler WWW-Nutzung vermeidet, allerdings damit auch wesentliche Vorzüge vermissen lässt. Denn TV ist zwar ein elektronisches Medium, für dessen mobile Nutzung neue Übertragungstechnologien entwickelt werden $^{33}$, aber TV als Broadcast-Medium kennt nur eine Richtung: Vom Anbieter zum Rezipienten. In dem Spannungsverhältnis zwischen individueller Selektion und spontaner (anytime, anyplace) Verfügbarkeit von bestimmten Inhalten einerseits und Aufwand bzw. Anstrengung in der mobilen Nutzungssituation andererseits ist TV eindeutig zuzuordnen. Die Programmgestaltung des Anbieters definiert, was der Rezipient wann sehen kann. Die Auswahl reduziert sich auf die begrenzte Zahl an Kanälen. Das übliche Zappen durch die Programme ist dafür nebenbei ohne großen Aufwand zu bewältigen und daher vermutlich mit mobilen Situationen kompatibel. Defizite erwachsen in diesem Fall vielmehr aus den beschränkten Steuerungs- und Selektionsmöglichkeiten des Nutzers. Der feste Ablauf von TV-Programmen und klassischen TV-Formaten fordert vom Rezipienten, dass er seine sonstigen Aktivitäten an die vorgegebene Zeitstruktur anpasst. In vielen mobilen Situationen ist dies jedoch nicht oder nur sehr begrenzt möglich. Die Sendezeit der morgendlichen Nachrichten stimmt z. B. nicht mit dem Fahrplan der S-Bahn überein; die Sendezeit der Lieblingssoap liegt möglicherweise mitten in einem wichtigen Termin. Somit scheint fraglich, inwieweit die traditionelle Form des Fernsehens auf mobile Nutzungsformen übertragbar ist, ob mobiles Fernsehen aus der Nutzerperspektive möglicherweise etwas grundlegend anderes ist als Fernsehen zu Hause. Für diese Annahme spricht auch, dass bereits Ansätze diskutiert werden, die geeignet sein könnten, diese Probleme zu verkleinern, z. B. durch Zwischenspeichern der gesendeten Inhalte auf dem Endgerät oder durch neue Selektions- bzw. Filtermöglichkeiten. Welche Nutzungsformen sich herausbilden werden wird sich letztlich erst im Verlauf des Adaptionsprozesses, also nach der Markteinführung zeigen. ${ }^{34}$ Gegenwärtig sind Akteure aus dem Feld der TV-Medien (Contentanbieter und Medientechnik) sowie TK-Provider dabei, größere Pilotvorhaben vorzubereiten, deren Start für 2006 erwartet wird.

33 Mobile TV-Übertragungstechnologien setzen auf den Technologien des digitalen Rundfunks und Fernsehens auf, bedürfen aber zusätzlicher Anpassungen, die die Nutzung auf mobilen Endgeräten (Handy, Smartphone oder PDA) ermöglichen. Es werden zwei Technologien mit unterschiedlichen technischen Merkmalen eingeführt: DVB-H setzt auf dem europäischen TVStandard DVB-T auf, während DMB eine Weiterentwicklung des digitalen Rundfunkstandards $\mathrm{DAB}$ ist, die bereits in Korea eingesetzt wird, aber auch als europäischer Standard anerkannt ist.

34 Wir planen hierzu mehrere Nutzungsanalysen, um die Entwicklung mobiler TV-Nutzungsformen prozessbegleitend zu untersuchen. 
Die zweite Art des mobilen TV vermeidet die geschilderten Einschränkungen von Broadcast-TV. Sie basieren auf UMTS-Technologien und individuellem Streaming. D. h. jeder Nutzer/Kunde erhält den Inhalt individuell zugeschickt. Somit kann der Kunde sowohl den Inhalt als auch die Zeit auswählen. UMTS-TV ist daher nicht nur eine neue Übertragungstechnologie, sondern vermutlich auch eine neue Form der TV-Nutzung, bei der der Rezipient selbst den Inhalt auswählt und den Zeitpunkt des TV-Konsums bestimmt. Allerdings dürfte sich auch die Kostenstruktur wesentlich von der des klassischen Broadcast-TV unterscheiden. Dies wird in dem preissensitiven Massenmarkt nicht ohne Wirkung auf Art und Weise der Nutzung bleiben. Darüber hinaus ist zu fragen wie die Auswahloptionen gestaltet sind und welche Anforderungen sie an den Nutzer stellen. Hiervon könnte es abhängen ob und in welchen Situationen mobiles TV aus Anwendersicht attraktiv ist. UMTS-TV weist daher Analogien zum interaktiven Fernsehen der 90er Jahre auf. Interaktives TV hat sich nicht wirklich etablieren können, obwohl in den USA viele Kabelnetze rückkanalfähig ausgebaut wurden. Zwar stellt sich bei mobiler Nutzung nicht die Frage, ob der „Couch-Potato“ zu der beim interaktiven TV erforderlichen Eigenaktivität bereit ist, dennoch ist die Art der Selektion auch bei mobilem UMTS-TV vermutlich eine wichtige Frage.

\section{Der Trend zum mobilen Universalgerät}

Mit der Herausbildung der neuen mobilen Kommunikationsform der „Absent Presence“ ist das Handy zum „immer dabei“ Gerät geworden. Es liegt daher nahe, das Handy so aufzurüsten, dass man es gleichzeitig auch für die mobile Mediennutzung verwenden kann. Die Schwelle für mobile Mediennutzung würde reduziert werden, wenn man das dafür notwendige Endgerät bereits aus anderen Gründen dabei hat. Die Leitidee eines multifunktionalen mobilen Endgerätes wird insbesondere von den Geräteherstellern mit großem Ideenreichtum vorangetrieben. Die Vorstellung, dass auf dem Handy alle anderen Medien mobil werden, ist aus Anwenderperspektive weniger überzeugend, wenn dieses deswegen größer, schwerer und unübersichtlicher in der Bedienung wird. Dennoch ergeben sich durch die Verkopplung mobiler Mediennutzung mit dem Handy, Smartphone oder PDA möglicherweise Anschlussmöglichkeiten. Z. B. könnte die gelegentliche, spontane Mediennutzung in mobilen Situationen erleichtert werden. Dies wäre sowohl in Bezug auf Informationsinhalte im WWW interessant, wie auch für Unterhaltungsinhalte, z. B. um eine unerwartete Wartezeit zu überbrücken. Aus einer solchen gelegentlichen Nutzung könnten sich neue Formen der mobilen Mediennutzung entwickeln. Ein weiterer Anknüpfungspunkt ergibt sich möglicherweise daraus, dass das mobile Universalgerät als Datenträger für persönliche Inhalte eingesetzt werden kann. Das mobile Endgerät nimmt nicht nur selbst gemachte Bilder oder die eigene Musiksammlung auf, sondern zukünftig möglicherweise auch die letzte Version der Fernsehserie, die im Fernsehen gesendet wurde. 


\subsection{Mobile Mediennutzung - ein vorläufiges Fazit}

Neue Formen mobiler Mediennutzung haben es nicht leicht, sich zu etablieren. Dies betrifft bisher vor allem das WWW und WAP. Anders als erwartet, lassen sich die Erfahrungen und Lehren der Mobilkommunikation nicht bruchlos auf mobile Mediennutzung übertragen, denn für die Erreichbarkeit von Personen ist der mobile „Always-on“-Zugang unerlässlich. Nicht so für die mobile Verfügbarkeit von Inhalten, deren Verbesserung auch ohne mobilen Online-Zugang möglich ist. Dies zeigt das Beispiel des iPod. Die überraschend dynamische Weiterentwicklung etablierter Formen (offline) der mobilen Mediennutzung könnte eine ernsthafte Konkurrenz für mobile Online-Medien sein.

Es liegt auf der Hand, dass die beiden Adaptionspfade mobiler Mediennutzung unterschiedliche Anforderungen an Technik, Endgerät und Dienst stellen - zum Mitnehmen benötigt man den mobilen „Always-on“-Zugang nicht, dafür aber ein Endgerät mit Speicher- und Verarbeitungskapazität. Beim WWW ist ein leistungsfähiger mobiler „Always-on“-Zugang eine Grundvoraussetzung für mobile Nutzung. Die Frage, ob und in welchen Fällen mobiler Mediennutzung der OnlineZugang zusätzliche nützliche Optionen ins Spiel bringt, ist aus Anwendersicht noch nicht beantwortet.

\section{Literatur}

Burkart (2000): Burkart, G.: Mobile Kommunikation: Zur Kulturbedeutung des „Handy“. In: Soziale Welt: Zeitschrift für sozialwissenschaftliche Forschung und Praxis, 2/2000, S. 209-231.

Büllingen / Stamm (2004): Büllingen, F., Stamm, P.: Mobile Multimedia-Dienste. Bad Honnef; www.mobilmedia.de.

David (1985): David, P.A.: Clio and the economics of QWERTY. In: American Economic Review 75 (2), S. 332-337.

van Eimeren / Gerhard / Frees (2004): van Eimeren, B., Gerhard, H., Frees, B.: ARD/ZDF-Online-Studie 2004. Internetverbreitung in Deutschland: Potential vorerst ausgeschöpft? In: Media Perspektiven 8, 2004, S. 350-370.Feldhaus / Nave-Herz (2004): Feldhaus, M., Nave-Herz, R.: Mobile Kommunikation im Familiensystem. Ergon, Würzburg.

Gergen (2002): Gergen, K. J.: The challenge of absent presence. In: James E. Katz, Mark Aakhus: Perpetual Contact. Cambridge University Press. 2002, S. 227-241.

Giddens (1995): Giddens, A.: Die Konstitution der Gesellschaft. Campus, Frankfurt a.M.. 
Hanekop / Lanfer / Wittke (2004): Hanekop, H., Lanfer, C., Wittke, V.: Auf dem Weg zur „Notebook University“? Zur Veränderung von Lehre und Lernen durch den Einsatz neuer Informations- und Kommunikationstechnologien an Präsenzhochschulen. In: SOFI-Mitteilungen Nr. 32, Dezember.

Hanekop / Wittke (2005): Hanekop, H., Wittke, V.: Der Kunde im Internet. In: Heike Jacobsen, Stephan Voswinkel, Stephan (Hrsg.): Der Kunde in der Dienstleistungsbeziehung; VS Verlag, Wiesbaden.

Hess / Figge / Hanekop / Hochstaller / Hogrefe / Kaspar / Rauscher / Richter / Riedel (2005): Hess, T., Figge; S., Hanekop, H., Hochstaller, I., Hogrefe, D., Kaspar, C., Rauscher, B., Richter, M.., Riedel, A.: Technische Möglichkeiten und Akzeptanz mobiler Anwendungen - eine interdisziplinäre Betrachtung; In: Wirtschaftsinformatik Heft 1/05, S. Heft 1/05, S. 6-16.

Höflich (2005): Höflich, J. R.: An mehreren Orten zugleich: Mobile Kommunikation und soziale Arrangements. In: Joachim R. Höflich (Hrsg): Mobile Kommunikation. Lang, Ffm.

Höflich (2003): Höflich, J. R. (Hrsg): Vermittlungskulturen im Wandel. Brief, E-Mail, SMS. Lang, Ffm.

Höflich (2003a): Höflich, J. R.: Part of Two Frames: Mobile Communication and the Situational Arrangement of Communicative Behaviour. In: Nyiri, Kristof (Hrsg.): Mobile Democracy: Essays On Society, Self And Politics. Passagen Verlag, Vienna. 2003, S. 31-51.

Katz / Aakhus (2002): Katz, J. E., Aakhus, M.: Introduction: framing the issues. In: James E. Katz, Mark Aakhus: Perpetual Contact. Cambridge University Press. 2002, S. 1-14.

Klingler / Müller (2004): Klingler, W., Müller, D.: Aktuelle Daten zur Hörfunknutzung in Deutschland. ma 2004 Radio II: Hörfunk behauptet Stärke. In: Media Perspektiven 9, 2004, S. 410-420.

Ling / Yttri (2002): Ling, R., Yttri, B.: Hyper-Coordination via Mobile Phones in Norway. In: James E. Katz, Mark Aakhus: Perpetual Contact. Cambridge University Press. 2002, S. 139-169.

Meyrowitz (2003): Meyrowitz, J.: Global Nomads in the Digital Veldt. In: Nyiri, Kristof (Hrsg.): Mobile Democracy: Essays On Society, Self And Politics. Passagen Verlag, Vienna. 2003, S. 91-102.

North (1990): North, D.C.: Institutions, institutional change and economic performance. Cambridge.

Oksman / Rautiaineen (2003): Oksman, V., Rautiaineen, P.: „Perhaps it is a Body Part": How the Mobile Phone Became a Organic Part of the Everyday Lives of Finnish Children an Teenagers. In: James E. Katz (Hrsg.): Machines That Become Us. The Social Context of Personal Communication Technology. Transaction Publishers, New Brunswick. 2003, S. 293-310. 



\section{Datenschutzrechtliche Aspekte personalisierter mobiler Dienste}

\section{Matthias Mönch}

\section{Einführung}

Die ortsbezogenen Dienstleistungen, die sog. „Location Based Services“ werden als eine der Hauptattraktionen des M-Commerce angeführt. ${ }^{35}$ Hier ist es im Gegensatz zum E-Commerce theoretisch möglich, für etwaige an den Nutzer gerichtete Angebote nicht nur das bisherige Kaufverhalten des Kunden, sondern darüber hinaus noch seine derzeitige geografische Position fruchtbar zu machen. So verlockend diese zusätzliche Möglichkeit für die Unternehmer sein mag, so ergeben sich hier doch erhebliche datenschutzrechtliche Schwierigkeiten.

$\mathrm{Ob}$ und in welchem Umfang diese Informationen überhaupt ausgewertet und im Wege personalisierter mobiler Dienste verwertet werden dürfen, soll im Folgenden unter datenschutzrechtlichen Gesichtspunkten beleuchtet werden.

35 Grapentin, in Bräutigam/Leupold, 2003, Online-Handel, B X., Rz. 88. 


\section{Datenschutz im Allgemeinen}

Eine einheitliche Regelung des Datenschutzes ist dem deutschen Recht fremd, auch wenn ursprünglich die Absicht bestand, alle datenschutzrechtlichen Belange in einem einzigen Gesetz zu regeln. ${ }^{36}$ Da jedoch schnell erkannt wurde, dass auf diese Weise der Schutz des Bürgers vor Missbrauch seiner Daten nicht umfassend gewährleistet werden konnte, entstanden neben dem Bundesdatenschutzgesetz (BDSG) weitere datenschutzrechtliche Regelungen, ${ }^{37}$ die je nach dem einschlägigen Sachverhalt zur Anwendung kommen. ${ }^{38}$ Dabei gehen diese bereichsspezifischen Regelungen dem BDSG vor. Dies ergibt sich bereits aus $\int 1$ Abs. 3 BDSG, wonach andere Rechtsvorschriften des Bundes, soweit sie auf personenbezogene Daten Anwendung finden, gegenüber dem BDSG leges speciales sind. Da das Bundesdatenschutzgesetz demzufolge lediglich die grundsätzlichen und allgemeinen Reglungen zum Datenschutz enthält, ${ }^{39}$ bleibt es weiterhin subsidiär anwendbar, soweit die spezielleren Gesetze für bestimmte Probleme keine Regelung enthalten. ${ }^{40}$

Aufgrund dieser Nachrangigkeit im Hinblick auf bereichsspezifische Datenschutzregelungen kommt das BDSG in vielen Datenschutzfragen nicht mehr unmittelbar zur Anwendung, sondern prägt diese nur noch durch seine grundlegende Regelungsstruktur und die von ihm verwendeten Begriffe. ${ }^{41}$

Für den Bereich des Internet und dem folgend auch für den Bereich des M-Commerce sind die spezifischen datenschutzrechtlichen Regelungen im Telekommunikationsgesetz (TKG), dem Teledienstedatenschutzgesetz (TDDSG) und dem Mediendienstestaatsvertrag (MDStV) enthalten.

\section{Abgrenzungen der Anwendungsbereiche}

Je nachdem, in welcher Funktion - telefonieren, Lokalisierung des Endgeräts oder einkaufen - ein Mobiltelefon genutzt wird, finden unterschiedliche Gesetze mit ihren unterschiedlichen datenschutzrechtlichen Regelungen Anwendung. Im Folgenden soll kurz skizziert werden, wann welches Gesetz einschlägig ist:

\footnotetext{
36 Niedermeier, in Gounalakis, Rechtshandbuch Electronic Business, 2003, § 19 Rz. 8.

37 Niedermeier a.a.O. \19 Rz. 8; Roßnagel, in Roßnagel/Banzhaf/Grimm, Datenschutz im Electronic Commerce, 2003, S. 123.

38 Gola/Schomerus, BDSG, 8. Aufl. 2005, 』 1 Rz. 24.

39 Niedermeier a.a.O. \19 Rz. 11; Roßnagel, Handbuch Datenschutzrecht, 2003, 1. Rz. 67.

40 Köhler/Arndt, Recht des Internet, 4. Aufl. 2003, S. 281; Niedermeier a.a.O. \19 Rz. 5, 26; Gola/Schomerus, BDSG, 8. Aufl. 2005, \1 Rz. 24; Roßnagel a.a.O. S. 136.

41 Niedermeier a.a.O. \19 Rz. 11; Roßnagel, Handbuch Datenschutzrecht, 2003,1. Rz. 67.
} 


\subsection{Telekommunikationsgesetz}

Das TKG findet im Bereich der Telekommunikation Anwendung. Dabei ist der Begriff der „Telekommunikation“ in \ 3 Nr. 22 TKG als der „technische Vorgang des Aussendens, Übermittelns und Empfangens von Signalen mittels Telekommunikationsanlagen“ legaldefiniert. Unter „Telekommunikationsanlagen“ nach $₫ 3$ Nr. 23 TKG versteht man hierbei „technische Einrichtungen oder Systeme, die als Nachrichten identifizierbare elektromagnetische oder optische Signale senden, übertragen, vermitteln, empfangen, steuern oder kontrollieren können“".

\subsection{Teledienstegesetz}

Demgegenüber ist gemäß $\int 2$ Abs. 1 TDG das Teledienstegesetz einschlägig, wenn es sich um einen elektronischen Informations- und Kommunikationsdienst handelt, der für eine individuelle Nutzung kombinierbarer Daten wie Zeichen, Bilder oder Töne bestimmt ist und dem eine Übermittlung mittels Telekommunikation zugrunde liegt.

\subsection{Mediendienste}

Durch $₫ 2$ Abs. $1 \mathrm{MDStV}$ schließlich werden Mediendienste als an die Allgemeinheit gerichtete Informations- und Kommunikationsdienste in Text, Ton oder Bild, die unter Benutzung elektromagnetischer Schwingungen ohne Verbindungsleitungen oder längs oder mittels eines Leiters verbreitet werden, definiert.

\subsection{Abgrenzung}

Aufgrund dieser nicht ganz einfachen und, im Fall der Tele- und Mediendiensten auch noch ähnlichen, Definitionen, kommt es immer wieder zu Abgrenzungsschwierigkeiten. Daraus resultiert die Frage, in welchem gesetzlichen Bereich man sich im konkreten Einzelfall gerade bewegt. Um die Abgrenzung handhabbar zu machen, sollen im Folgenden einige Faustregeln dargestellt werden.

\section{Telekommunikation}

Telekommunikation unterscheidet sich von den Tele- und Mediendiensten dadurch, dass dem Empfänger keinerlei Inhalte präsentiert werden, sondern allein der Transport der Information vom Sender zum Empfänger im Vordergrund 
steht; ${ }^{42}$ für den Vorgang der Datenübertragung ist es demnach charakteristisch, dass er von Inhalt und physischen Details der zu übertragenden Daten abstrahiert zu betrachten ist, so dass nicht zwischen der Übermittlung bestimmter Daten aus verschiedenen Anwendungen differenziert wird. ${ }^{43}$

\section{Tele- und Mediendienste}

Dies ist bei den Tele- und Mediendiensten anders: Hier kommt es nicht auf den Transport der Information als solche, sondern auf deren Inhalt an, ${ }^{44}$ der mit Hilfe der Telekommunikation übertragen wird. Es werden somit alle anwendungsspezifischen Vorgänge, die auf der Grundlage der Telekommunikation erfolgen, erfasst. ${ }^{45}$

Ein für die Anwendung des TDG wesentliches Merkmal ist gemäß $\ 2$ Abs. 1 TDG die individuelle Nutzung der Daten, wohingegen sich die Mediendienste nach $\int 2$ Abs. 1 MDStV an die Allgemeinheit richten. ${ }^{46}$

Aufgrund der zunehmenden Konvergenz der Medien lässt sich mit Hilfe dieser Faustformel jedoch keine trennscharfe Abgrenzung erreichen. Für eine genauere Differenzierung wird somit auf die redaktionelle Gestaltung zur Meinungsbildung abzustellen sein, wie sie in $\int 2$ Abs. 4 Nr. 3 TDG zur negativen Abgrenzung zwischen Tele- und Mediendiensten angeführt ist. ${ }^{47}$ Dabei ist diese in Anlehnung an die im Presserecht entwickelten Maßstäbe als das Sammeln und Aufbereiten von verschiedenen Informationen oder Meinungen mit Blick auf den potentiellen Empfänger zu sehen. ${ }^{48}$ Ist eine solche redaktionelle Gestaltung nicht gegeben, wie dies zum Beispiel bei einer reinen Zusammenstellung von Informationen der Fall ist, so ist das TDG einschlägig. ${ }^{49}$

Darüber hinaus muss die redaktionelle Gestaltung im Sinne der Mediendienste „zur Meinungsbildung für die Allgemeinheit dienen“. Dies ist üblicherweise dann der Fall, wenn eine große Anzahl von Mitteilungen an beliebig viele, anonyme

42 Schuster in Geppert, Beck'scher TKG-Kommentar, 3. Aufl. 2005, \ 3 Rz. 21a, \4 Rz. 4; Moritz/Scheffelt, in: Hoeren/Sieber, Handbuch Multimedia Recht, 2004, Kap. 4 Rz. 8; Roßnagel a.a.O. S. 132.

43 Roßnagel a.a.O. S. 132.

44 Schuster a.a.O. \ 3 Rz. 21a, \ 4 Rz. 4; Roßnagel a.a.O. S. 132; Spindler in Spindler/Schmitz/Geis, TDG, 2004, \ 2 TDG Rz. 22.

45 Roßnagel a.a.O. S. 132.

46 Schuster a.a.O. \4 Rz. 4; Moritz/Scheffelt a.a.O. Kap. 4 Rz. 12; Spindler a.a.O. \ 2 TDG Rz. 5.

47 Beucher/Leyendecker/v. Rosenberg, Mediengesetze, 1999, \ 2 MDStV Rz. 1; Spindler a.a.O. \ 2 TDG Rz. 11.

48 Gounalakis/Rhode, CR 1998, 487 (490); Spindler, in Roßnagel, Recht der Multimedia-Dienste, 2004, Teil 2, § 2 TDG Rz. 31; Spindler a.a.O. \ 2 TDG Rz. 11.

49 Spindler a.a.O. $₫ 2$ TDG Rz. 11. 
Empfänger verbreitet werden ${ }^{50}$ und sonach also eine Ausrichtung an der Öffentlichkeit erfolgt. ${ }^{51}$

Schwierigkeiten mit dieser Abgrenzung ergeben sich jedoch insofern, als dass $\int 2$ MDStV nicht wie $\int 2$ Abs. 4 Nr. 3 TDG auf eine redaktionelle Gestaltung zur Meinungsbildung sondern allein darauf abstellt, dass sich die Informations- und Kommunikationsdienste an die Allgemeinheit richten. ${ }^{52}$

Um zu einer klaren Abgrenzung bezüglich der Anwendungsbereiche des TDG und des MDStV zu gelangen, ist zunächst zu prüfen, ob ein Teledienst im Sinne des $\int 2$ Abs. 1, 2 TDG vorliegt. Anschließend ist zu kontrollieren, ob die negative Generalklausel des $\int 2$ Abs. 4 Nr. 3 TDG eingreift und das vorliegende Angebot nicht doch dem Mediendienstestaatsvertrag unterwirft. ${ }^{53}$ Nur in diesem Fall findet der MDStV Anwendung, auch wenn nach \ 2 Abs. 1, 2 MDStV ein Mediendienst gegeben wäre. ${ }^{54}$

Leider ist auch diese Abgrenzung nicht immer eindeutig, was sich an dem Beispiel eines Chats oder einer Telefonkonferenz zeigt. Hier wird man auf eine wertende Gesamtbetrachtung abstellen müssen. ${ }^{55}$

\section{Vorliegen mehrerer Anwendungsbereiche}

Ein weiteres Problem ergibt sich in den Fällen, in denen mindestens zwei der genannten Bereiche parallel vorliegen. Im Bereich des Internet ist dies zum Beispiel der Fall, wenn ein Anbieter den Zugang zum Internet vermittelt und gleichzeitig auf der Startseite auch noch redaktionell bearbeitete Inhalte anbietet. In diesen Konstellationen ist umstritten, auf welche Art und Weise bestimmt wird, welche der einschlägigen gesetzlichen Regelungen zur Anwendung kommt.

Nach einer Ansicht ist auf den Schwerpunkt der erbrachten Leistung abzustellen. ${ }^{56}$ Die jeweilige Leistung wäre somit, je nach ihrem Schwerpunkt, einheitlich als Telekommunikationsdienstleistung, als Tele- oder Mediendienst einzuordnen. ${ }^{57}$ Diese Art der Abgrenzung führt jedoch insofern zu unschönen Ergebnissen, als

\footnotetext{
50 BVerfG, Beschl. v. 3.10.1969 - 1 BvR 46/65, BVerfGE 27, 71 (83); BVerfG, Urt. v. 24.1.2001 1 BvR 2623/95, 1 BvR 622/99, BVerfGE 103, 44 (60); Löffler/Ricker, Handbuch des Presserechts, 5. Aufl. 2005, \1 Rz. 19; Bullinger in Löffler/Wenzel/Sedelmaier, Presserecht, 4. Aufl. 1997, Einl. Rz. 9, 11.

51 Beucher/Leyendecker/v. Rosenberg, Mediengesetze, 1999, \2 MDStV Rz. 1; Gounalakis, NJW 1997, 2993 (2994).

52 Spindler a.a.O.Teil 2, \ 2 TDG Rz. 26; Spindler a.a.O. \ 2 TDG Rz. 18.

53 Spindler a.a.O.Teil 2, $\int 2$ TDG Rz. 29.

54 Spindler a.a.O.Teil 2, \ 2 TDG Rz. 29.

55 Schuster a.a.O. \3 Rz. 21a, \4 Rz. 4; Spindler a.a.O.Teil 2, \2 TDG Rz. 19.

56 Moritz/Scheffelt a.a.O. Kap. 4 Rz. 10.

57 Waldenberg, MMR 1998, 124 (125).
} 
dass ein Anbieter, der sowohl Telekommunikation als auch Teledienste in einem Angebot darbietet, nur unter eine Regelung fällt, obwohl er sich im Falle des getrennten Angebotes im Anwendungsbereich beider Regelungen bewegen würde. ${ }^{58}$ Ferner dürfte zum Teil auch die Ermittlung des jeweiligen Schwerpunkts Schwierigkeiten bereiten. Das größte Problem an diesem Ansatz ist jedoch, dass auf diese Art und Weise durch eine entsprechende Gestaltung des Angebots die Einschlägigkeit von einzelnen Normen umgangen werden kann, ${ }^{59}$ was diese ins Leere laufen lassen und aushebeln würde.

Richtig ist vielmehr der Ansatz, nach dem zur Abgrenzung zwischen den einzelnen Diensten auf eine funktionale Betrachtung abzustellen ist. ${ }^{60}$ Folglich ist zwischen dem technischen Vorgang des Aussendens, Übermittelns und Empfangens auf der einen und dem durch diesen technischen Vorgang transportierten Inhalten auf der anderen Seite zu unterscheiden. ${ }^{61}$ Dies hat zur Konsequenz, dass für einen Dienst, der beide Komponenten enthält, dieser in seine Bestandteile aufgeteilt und das für den jeweiligen Teil einschlägige Gesetz angewendet werden muss. ${ }^{62}$ Die oben angeführte Diskrepanz entsteht somit erst gar nicht.

\section{Anwendung auf personalisierte mobile Dienste}

Da auch der Bereich des M-Commerce den Regelungen des TKG, des TDG und dem folgend dem TDDSG und dem MDStV unterfällt, drängt sich im Zusammenhang mit personalisierten mobilen Diensten nunmehr die Frage auf, in welches konkrete Regelungswerk die einzelnen Aspekte der Location Based Services, sprich die Lokalisierung des mobilen Endgerätes und die Darbietung der Inhalte, fallen. Nach dem oben Gesagten ist für die Bestimmung der anwendbaren Regelung auf eine funktionale Betrachtung abzustellen. Folglich müssen für die personalisierten Dienste zwei Bereiche von einander getrennt untersucht werden. Zum einen handelt es sich hierbei um die Lokalisierung des mobilen Endgerätes zum anderen um das Darbieten der jeweiligen Inhalte.

\footnotetext{
58 Moritz/Scheffelt a.a.O. Kap. 4 Rz. 10.

59 OLG Hamburg CR 2000, 363 (365); Schuster a.a.O. \ 4 Rz. 4a; Moritz/Scheffelt a.a.O. Kap. 4 Rz. 10.

60 Schuster a.a.O. \4 Rz. 4; Moritz/Scheffelt a.a.O. Kap. 4 Rz. 10; Roßnagel a.a.O. S. 132.

61 OLG Hamburg, Urt. v.23.3.2000 - 3 U 80/99, OLG Hamburg CR 2000, 363 (364); Schuster a.a.O. \4 Rz. 4a; Engel-Flechsig/Maennel/Tettenborn, NJW 1997, 2981 (2983); Engel-Flechsig, RDV 1997, 59 (61); Roßnagel, NVwZ 2000, 622 (625); Schmitz, TDDSG und das Recht auf informationelle Selbstbestimmung, 2000, S. $70 \mathrm{ff}$.

62 Schuster a.a.O. \ 4 Rz. 4 a.
} 


\subsection{Lokalisierung}

Bei der Lokalisierung wird der geografische Standort des jeweiligen mobilen Endgerätes ermittelt. Im Falle von Mobiltelefonen kann sich hierfür netzinterner Verfahren bedient oder aber auf die Lokalisierung des Mobiltelefons durch sich selbst, zum Beispiel durch einen eingebauten GPS-Empfänger, zurückgegriffen werden. ${ }^{63}$

Bei der Lokalisierung eines Mobiltelefons mit Hilfe netzinterner Möglichkeiten werden dem Benutzer keinerlei Inhalte dargeboten. Auch, wenn in diesem Moment keine Nachrichten im klassischen Sinne übertragen werden, liegt doch ein „technischer Vorgang des Aussendens, Übermittelns und Empfangens von Signalen mittels Telekommunikationsanlagen", mithin Telekommunikation im Sinne des $\int 3$ Nr. 22 TKG vor.

Auch bei einer Lokalisierung des Mobiltelefons durch das Gerät selbst, also zum Beispiel im Falle der Positionsbestimmung via Global Positioning System (GPS), werden noch keine Inhalte an seinen Benutzer übermittelt. Auch hier erfolgt lediglich der ,technische Vorgang des Aussendens, Übermittelns und Empfangens von Signalen mittels Telekommunikationsanlagen“. Folglich bewegt man sich auch hier im Bereich der Telekommunikation.

Demnach sind bezüglich des Datenschutzes im Rahmen der Lokalisierung mittels netzinterner Möglichkeiten und mittels des Mobiltelefons selbst die datenschutzrechtlichen Regelungen des TKG anzuwenden.

\subsection{Inhalte}

Von der reinen Lokalisierung des Mobiltelefons, sei es nun durch netzinterne Möglichkeiten oder aufgrund des Mobiltelefons selbst, ist das Darbieten von Inhalten zu unterscheiden. Hier geht es nicht mehr um das Aussenden und Empfangen von Signalen, sondern vielmehr um elektronische Informations- und Kommunikationsdienste. ${ }^{64}$ Insofern bewegt man sich nach den oben angeführten Abgrenzungskriterien im Bereich der Tele- und Mediendienste. Eine genaue Zuordnung zu einem dieser beiden Bereiche ist damit allerdings noch nicht erreicht.

Bedient man sich zunächst der vorgehend erwähnten Faustformel, die aufgrund der Gesetzesformulierung der $\int 2$ Abs. 1 TDG und \ 2 Abs. 1 MDStV annimmt, dass bei einer individuellen Nutzung der Daten ein Teledienst, bei an die Allgemeinheit gerichteten Daten hingegen ein Mediendienst gegeben ist, so liegt schon aufgrund des Begriffs ,personalisierte mobile Dienste“ der Schluss nahe,

$63 \mathrm{Zu}$ den einzelnen Verfahren und ihren technischen Unterschieden siehe ausführlich Lehner, WISU 2/2004, 211 ff.; Rannenberg, DuD 2003, 546 ff.; Turowski/Pousttchi, Mobile Commerce, 2004, S. 76 ff.; Zogg/Zivadinocic, ct 2004, 222 ff.

64 S. BT-Drucks. 13/7358, S. 41 (43). 
dass die übermittelte Information auf den Empfänger persönlich zugeschnitten und nicht an die Allgemeinheit gerichtet ist. Hiernach läge demzufolge ein Teledienst vor.

Geht man über den Begriff „personalisierte mobile Dienste“ hinaus und betrachtet sich die entsprechenden Inhalte, wird schon fraglich, ob immer noch ein Teledienst vorliegt. Wie bereits dargelegt, ist für eine genauere Differenzierung auf das Vorliegen einer redaktionellen Gestaltung zur Meinungsbildung abzustellen, wobei eine redaktionelle Gestaltung beim Sammeln und Aufbereiten verschiedener Informationen oder Meinungen angenommen wird. Um festzustellen, ob es an einer solchen im Rahmen der personalisierten mobilen Dienste fehlt, ist nach der jeweiligen Art der zugesandten Information zu unterscheiden.

Allen personalisierten mobilen Diensten ist gemein, dass der Kunde aufgrund seiner geografischen Position Inhalte zugeschickt bekommt. Hierbei handelt es sich zumeist um Werbung oder mit der gegenwärtigen Position zusammenhängende Fakten, wie zum Beispiel die Temperatur an der entsprechenden geografischen Position.

Die bloße Zusammenstellung von Fakten reicht für eine redaktionelle Bearbeitung indessen nicht aus, ${ }^{65}$ so dass man sich hier im Bereich der Teledienste bewegt.

Anders kann es sich dagegen bei zugesandter Werbung verhalten. Regelmäßig wird es sich zwar bei der versandten Werbung um eine solche handeln, die für jeden als Werbung erkennbar ist. Hier hat keine redaktionelle Bearbeitung stattgefunden, so dass, ebenso wie bei der Zusammenstellung von Fakten, der Anwendungsbereich des MDStV versperrt ist. Darüber hinaus gibt es jedoch noch die redaktionell gestaltete Werbung. Hierunter sind Anzeigen zu verstehen, die wie ein redaktioneller Bericht aufgemacht oder gar wirklich redaktionelle Hinweise sind und folglich nicht ohne weiteres als Werbung erkannt werden können. ${ }^{66}$ Unter wettbewerbsrechtlichen Gesichtspunkten ist redaktionelle Werbung unzulässig, da hier die Gefahr einer Irreführung des Verbrauchers besteht. ${ }^{67}$ Diese den Schutz des Verbrauchers bezweckende wettbewerbsrechtliche Unzulässigkeit ändert jedoch nichts an der für die Abgrenzung von Tele- und Mediendiensten erforderlichen Einordnung in redaktionell und nicht redaktionell bearbeitete Beiträge. ${ }^{68}$ Ist somit eine Werbung redaktionell bearbeitet, so ist weiter zu untersuchen, ob sie auch zur Meinungsbildung für die Allgemeinheit geeignet ist. Spätestens hier fällt man auch bei der redaktionell bearbeiteten Werbung aus dem Anwendungsbereich des MDStV heraus, da sie dem potentiellen Kunden eben als Individuum aufgrund seiner derzeitigen Lokalisierung zugesandt wird und der potentielle Kunde

65 Vgl. Spindler a.a.O. \$2 TDG Rz. 14.

66 Löffler/Ricker, Handbuch des Presserechts, 5. Aufl. 2005, Kap. 75 Rz. 26.

67 Löffler/Ricker, Handbuch des Presserechts, 5. Aufl. 2005, Kap. 75 Rz. 25.

68 Spindler a.a.O.Teil 2, \2 TDG Rz. 32; Spindler a.a.O. \$2 TDG Rz. 16. 
eben kein Adressat einer Mitteilung eines individuell nicht abgrenzbaren Personenkreises ist. ${ }^{69}$

\section{Datenschutz im Besonderen}

Nachdem untersucht wurde, für welche Bereiche der personalisierten mobilen Dienste welcher datenschutzrechtliche Bereich einschlägig ist, ist es nunmehr erforderlich, sich im Folgenden mit den einzelnen in Betracht kommenden Regelungen auseinanderzusetzen.

\subsection{Telekommunikationsrecht}

Das TKG enthält in den $\iint 91 \mathrm{ff}$. einige datenschutzrechtliche Regelungen. Von besonderer Bedeutung ist im vorliegenden Zusammenhang die Vorschrift des $\int 98$ TKG, welche sich mit Standortdaten befasst. Wörtlich heißt es in $\int 98$ Abs. 1 S. 1 TKG:

„Standortdaten, die in Bequg auf die Nutzer von öffentlichen Telekommunikationsnetzen oder Telekommunikationsdiensten für die Öffentlichkeit verwendet werden, dürfen nur im zur Bereitstellung von Diensten mit Zusatznutzen erforderlichen Maß und innerhalb des dafür erforderlichen Zeitraums verarbeitet werden, wenn sie anonymisiert wurden oder wenn der Teilnehmer seine Einwilligung erteilt hat."

Dabei sind gemäß $\int 3$ Nr. 19 TKG Standortdaten solche Daten, die in einem Telekommunikationsnetz erhoben oder verwendet werden und die den Standort des Endgeräts eines Endnutzers eines Telekommunikationsdienstes für die Öffentlichkeit angeben. Damit fallen unter die Standortdaten des $\int 3$ Nr. 19 TKG auch die Daten des Aufenthalts eines Mobiltelefons zu einem bestimmten Zeitpunkt. Wie der Gesetzeswortlaut schon sagt, dürfen solche Daten nur anonymisiert oder mit der Einwilligung des Betroffenen erhoben werden.

Wie diese Einwilligung konkret ausgestaltet sein soll, lässt sich dem Gesetzestext nicht entnehmen. Lediglich aus dem Regierungsentwurf zu $\int 96$ TKG-E, dem jetzigen $\int 98$ TKG, geht hervor, dass die Art und Weise der Einwilligung sich im Einzelfall nach den Besonderheiten des jeweiligen Dienstes zu richten hat. Ferner weist die Begründung darauf hin, dass die Einwilligung nicht in jedem Fall ausdrücklich erfolgen muss, sondern auch in einem Rahmenvertrag erteilt werden kann. ${ }^{70}$ Dabei muss es nach \98 Abs. 2 TKG jedoch möglich sein, die einmal gegebene Einwilligung für jede Verbindung zum Netz oder jede Übertragung einer Nachricht auf einfache und unentgeltliche Weise und zeitweise zu untersagen. Schließlich ist dem Teilnehmer mitzuteilen, welche Arten von Standortdaten ver-

69 Vgl. Bullinger a.a.O. Einl. Rz. 9, 11.

70 BT-Drs. 15/2316, S. 89. 
arbeitet werden, ob die Standortdaten für Zwecke der Bereitstellung von Diensten mit Zusatznutzen an einen Dritten weitergegeben werden.

\section{Einwilligung bei Vertragsschluss}

In Anwendung des Gesetzeswortlauts und unter Berücksichtigung der Regierungsbegründung bedeutet dies für die Praxis, dass ein Mobilfunkanbieter sich bereits beim Vertragsschluss des Mobilfunkvertrages das Einverständnis des Kunden geben lassen kann, um die Standortdaten auslesen und verwenden zu können; da er zum Auslesen der Daten in jedem Fall der Einwilligung des Kunden bedarf, ist er andernfalls gezwungen, das Einverständnis zu einem späteren Zeitpunkt einzuholen. Darüber hinaus können diese Standortdaten dann für Zwecke von Diensten mit Zusatzleistungen, namentlich für eben jene personalisierten mobilen Dienste, auch an einen Dritten weitergegeben werden. Unerlässlich ist hierbei, dass gleichzeitig auch eine kostenlose Ruf- oder SMS-Nummer bereitgestellt wird und der Anbieter den Kunden darauf hinweist, dass er - sollte er die Lokalisierung einmal nicht wünschen - eben diese Nummer wählen kann, woraufhin die Lokalisierung dann für eine bestimmte Zeit zu unterbleiben hat. Dabei ist von Seiten des Mobilfunkbetreibers jedoch darauf zu achten, dass die Einwilligungserklärung dem Koppelungsverbot des $\int 95$ Abs. 5 TKG entspricht und alle geplanten Datenverwendungsarten ausdrücklich erwähnt werden.

Nach \95 Abs. 5 TKG, der die Regelung des \89 Abs. 10 S. 1 TKG und $\int 3$ Abs. 2 TDSV ablöst, und nun nach der Gesetzesbegründung der Regelung des $\int 3$ Abs. 4 TDDSG entspricht, ${ }^{71}$ darf die Erbringung von Telekommunikationsdiensten nicht von einer Einwilligung des Teilnehmers in eine Verwendung seiner Daten für andere Zwecke abhängig gemacht werden, wenn dem Teilnehmer ein anderer Zugang zu diesen Telekommunikationsdiensten nicht oder in nicht zumutbarer Weise möglich ist. Durch dieses Verbot soll die freie und eigenständige Willensbetätigung des Nutzers bei der Einwilligung geschützt werden. ${ }^{72}$ Das Verbot, die Nutzung von der Datenschutzerklärung abhängig zu machen, enthält somit zugleich das Gebot, dass der Anbieter den Zugang zu dem Telekommunikationsdienst auch dann anbieten muss, wenn der Nutzer nicht in die Datenerhebung einwilligt. ${ }^{73}$ Für den Bereich des Mobilfunks bedeutet dies konkret, dass der Kunde seinen Mobilfunkvertrag entweder mit der datenschutzrechtlichen Einwilligung

71 BT-Drs. 15/2316, S. 89.

72 Siehe zum alten TKG Löwnau-Iqbal, in Scheurle/Mayen, TKG, 2002, \ 89 Rz. 46; vgl. zu der entsprechenden Regelung im Bereich der Teledienste \ 3 Abs. 4 TDDSG BT-Drs. 13/7385, S. 22; Bizer, in Roßnagel, Recht der Multimedia-Dienste, 2004, Teil 3, \ 3 TDDSG Rz. 184; EngelFlechsig/Maennel/Tettenborn, NJW 1997, 2981 (2987); Schmitz, in Spindler/Schmitz/Geis, TDG, 2004, \ 3 TDDSG Rz. 34.

$73 \mathrm{Vgl}$. zu der entsprechenden Regelung im Bereich der Teledienste $\int 3$ Abs. 4 TDDSG EngelFlechsig/Maennel/Tettenborn, NJW 1997, 2981 (2987); Schmitz, a.a.O. \ 3 TDDSG Rz. 34. 
abgeben kann, so dass seine Standortdaten für personalisierte mobile Dienste ermittelt werden dürfen, oder er seine Datenschutzerklärung nicht abgeben möchte. Im letzteren Fall hat der Kunde nach dem Koppelungsverbot einen Vertrag zu erhalten, nach dem ihm zwar die ,gewöhnlichen“ Mobilfunkleistungen, wie telefonieren und Kurznachrichten verschicken und empfangen, zur Verfügung stehen, seine geografische Position aufgrund der fehlenden datenschutzrechtlichen Einwilligung jedoch nicht ermittelt werden darf. Die Nutzung bzw. die Bereitstellung von personalisierten mobilen Diensten ist in einem solchen Fall ausgeschlossen, da es an den notwendigen Positionsdaten fehlt.

Die genaue Reichweite des Koppelungsverbots war für \ 3 Abs. 4 TDDSG, dessen Wortlaut $\int 95$ Abs. 5 TKG entspricht, umstritten. In der Literatur herrschte Uneinigkeit darüber, ob sich die Beschränkung auf ein konkretes Angebot eines Diensteanbieters bezieht ${ }^{74}$ oder ob für das Eingreifen des Koppelungsverbots eine Monopolstellung des Anbieters für die entsprechende Leistung bestehen muss. ${ }^{75}$

Dieser Streit dürfte sich mit der Neuregelung des Koppelungsverbots in $\int 95$ Abs. 5 TKG erledigt haben, da die Gesetzesbegründung dieser Norm ausdrücklich darauf hinweist, dass durch das Koppelungsverbot die Ausnutzung einer eventuellen Monopolstellung eines Diensteanbieters verhindert werden soll. ${ }^{76}$ Dementsprechend greift das Koppelungsverbot des \95 Abs. 5 TKG in den Fällen nicht ein, in dem für den Kunden noch die Möglichkeit besteht, die erwünschte Leistung bei anderen Diensteanbietern in Anspruch zu nehmen. ${ }^{77}$ In Anbetracht dessen, dass es zumindest vier Mobilfunkanbieter in Deutschland gibt, wird das Koppelungsverbot in diesem Zusammenhang keine Rolle spielen. Folglich könnte die Datenschutzerklärung eines Mobilfunkanbieters mit dem restlichen Mobilfunkvertrag verbunden werden.

Dass alle geplanten Datenverwendungsarten in der Einwilligungserklärung angegeben werden müssen, resultiert aus dem das deutsche Datenschutzrecht prägenden und in $\int 4$ Abs. 1 BDSG festgeschriebenen Grundsatz des Verbots mit Erlaubnisvorbehalt. ${ }^{78}$ Demnach ist die Erhebung, Verarbeitung und Nutzung von Daten verboten, soweit nicht eine spezielle Erlaubnis durch eine Rechtsnorm oder den Betroffenen selbst gestattet ist. ${ }^{79}$ Legt man \ 98 TKG als die spezialgesetzliche datenschutzrechtliche Norm zugrunde, so ergibt sich, dass eine Datenerhebung gemäß \98 Abs. 1 TKG nur statthaft ist, wenn die Daten zuvor anonymisiert wurden oder der Betroffene eingewilligt hat. Eine Anonymisierung der Daten

\footnotetext{
74 So für die entsprechende Regelung im TDDSG Bizer a.a.O. Teil 3, \ 3 TDDSG Rz. 183 ff.; Dix, in Roßnagel, Recht der Multimedia-Dienste, 2004, Teil 3, \ 5 TDDSG Rz. 55.

75 Vgl. für die entsprechende Regelung im TDDSG Schmitz a.a.O. \ 4 TDDSG Rz. 35.

76 BT-Drs. 15/2316, S. 89.

77 Vgl. zum TDDSG Schmitz a.a.O. \ 3 TDDSG Rz. 35.

78 Gola/Schomerus, BDSG, 8. Aufl. 2005, § 4 Rz. 3.

79 Gola/Schomerus, BDSG, 8. Aufl. 2005, \ 4 Rz. 3.
} 
im Zusammenhang mit personalisierten mobilen Diensten ist allerdings wenig sinnvoll, da es hier gerade darum geht, den Verbraucher aufgrund seines Standortes und seiner Person anzusprechen. Da insoweit keine gesetzliche Erlaubnis zur Datenerhebung gegeben ist, kann es bei den personalisierten mobilen Diensten nur auf die konkrete Einwilligung in die geplanten Datenverwendungsarten in dem vom Kunden konkret abgesteckten Umfang ankommen.

\section{Elektronische Einwilligung; $\mathbb{9} 94$ TKG}

Darüber hinaus kann die Einwilligung gemäß \94 TKG auch elektronisch erteilt werden, soweit der Teilnehmer oder Nutzer seine Einwilligung bewusst und eindeutig erteilt hat, die Einwilligung protokolliert wird, der Teilnehmer den Inhalt der Einwilligung jederzeit abrufen kann und er die Einwilligung jederzeit mit Wirkung für die Zukunft widerrufen kann. Diese Norm entspricht, so die Gesetzesbegründung, weitgehend dem ehemaligen $\ 4$ TDSV und dem jetzigen $\ 4$ Abs. 2 , 3 TDDSG. ${ }^{80}$ Folglich können für die Frage nach den Anforderungen an eine wirksame elektronische Einwilligung die zu diesen Paragraphen entwickelten Grundsätze herangezogen werden.

\section{„Bewusste und eindeutige“ Einwilligung}

Da sich weder im Gesetz selbst, noch in seiner Begründung, Angaben darüber finden lassen, was mit einer „bewussten und eindeutigen“ Einwilligung gemeint ist, ist davon auszugehen, dass mit dieser Formulierung lediglich die Anforderungen wiederholt werden, die allgemein an rechtsgeschäftsähnliche Handlungen gestellt werden. ${ }^{81}$ Somit scheint es sachgerecht, auf die allgemeinen Grundsätze zur Abgabe elektronischer Willenserklärungen und der Einholung vorformulierter Erklärungen nach der Rechtsgeschäftslehre des $\mathrm{BGB}$ zurückzugreifen. ${ }^{82}$ Folglich kommen die Regeln über Willenserklärungen und Allgemeine Geschäftsbedingungen (AGB) zur Anwendung. ${ }^{83}$

Da der Diensteanbieter im Rahmen der elektronischen Einwilligung gemäß \94 Nr. 1 TKG sicherzustellen hat, dass diese bewusst und eindeutig erfolgt, muss er verhindern, dass sie zufällig und unbeabsichtigt abgegeben wird. Für eine bewusste Erklärung ist in Anwendung der allgemeinen Vorschriften über die Abgabe von Willenserklärungen erforderlich, dass der Erklärende ein subjektives Handlungs- und Erklärungsbewusstsein hat. ${ }^{84}$ Dieses kann nur vorliegen, wenn

\footnotetext{
80 BT-Drs. 15/2316, S. 88.

81 Vgl. so schon für das TDDSG Schmitz a.a.O. \4 TDDSG Rz. 17.

82 Schmitz a.a.O. \4 TDDSG Rz. 17.

83 Schmitz a.a.O. \4 TDDSG Rz. 17.

84 Königshofen, TDSV, 2002, §4 Rz. 4; Schmitz a.a.O. \4 TDDSG Rz. 15.
} 
der Benutzer weiß, welcher Art und welchen Umfangs seine Einwilligung ist. ${ }^{85}$ Darüber hinaus muss dieser Wille auch ausreichend deutlich als Einwilligung zu verstehen sein. ${ }^{86}$ Es muss also auch die objektive Komponente einer geschäftsähnlichen Handlung gegeben sein.

Besonders problematisch gestaltet sich in diesem Zusammenhang die zumutbare Kenntnisnahme der Einwilligungserklärung in entsprechender Anwendung des $\int 305$ Abs. 2 Nr. 2 BGB. Aus dieser Norm ergibt sich ein Mindeststandard für AGB. Demnach müssen die AGB in einer lesbaren Größe verfasst sein, ${ }^{87}$ es muss eine sinnvolle Gliederung gegeben und die Formulierungen müssen sprachlich und inhaltlich klar sein. ${ }^{88}$ Darüber hinaus ergeben sich jedoch durch die Verwendung von neuen Medien noch weitere Anforderungen an die Möglichkeit der zumutbaren Kenntnisnahme:

So wurde zunächst von der Rechtsprechung bei der Verwendung von AGB im Bereich des BTX angenommen, dass eine zumutbare Kenntnisnahme nur bei relativ kurzen Texten - einer Bildschirmseite - vorliegt. ${ }^{89}$ Begründet wurde dies damit, dass der Aufruf einer jeden BTX-Seite einige Zeit in Anspruch nahm und zudem auch noch mit Kosten verbunden war, die für jede neu aufgerufene Seite entrichtet werden mussten. Hält man sich dies vor Augen, so könnte man diese Rechtsprechung ohne weiteres auch auf die Verwendung von AGB im Internet anwenden, was zum Teil unter der zusätzlichen Begründung der Flüchtigkeit und schlechten Lesbarkeit am Bildschirm auch getan wird. ${ }^{90} \mathrm{Um}$ die Unzumutbarkeit der Kenntnisnahme zu begründen, wird es jedoch nicht ausreichen, lediglich auf die mit dem Aufrufen der AGB verbundenen Verbindungskosten im Internet abzustellen. Vielmehr ist dem Benutzer, der einen Vertragsschluss via Internet anstrebt, bewusst, dass er die Verbindungsentgelte für die Dauer des Vertragsschlusses zahlen muss. Wendet er jetzt ein, dass ihm die Kenntnisnahme der AGB aufgrund der Verbindungsentgelte unzumutbar sei, setzt er sich zu seinem bisherigen Verhalten in Widerspruch. ${ }^{91}$

Ob man im Bereich des M-Commerce im Allgemeinen bzw. der Einwilligung bezüglich der personalisierten mobilen Dienste im Hinblick auf die Kosten parallel zum E-Commerce argumentieren kann, erscheint zumindest fraglich. Sicherlich ist dem Kunden auch bei personalisierten mobilen Diensten bewusst, dass er die

85 Königshofen a.a.O. \4 Rz. 4.

86 Schmitz a.a.O. $₫ 4$ TDDSG Rz. 15.

87 BGH Urt. v. 3.2.1986 - II ZR 201/85, BGH NJW-RR 1986, 1311 m.w.N.

88 OLG Hamburg Urt. v. 26.3.1986-5 U 119/85, OLG Hamburg NJW-RR 1986, 1440; OLG Stuttgart Urt. v. 25.3.1988 - 2 U 155/87, OLG Stuttgart NJW-RR 1988, 786 (787).

89 OLG Köln Urt. v. 21.11.1997 - 19 U 128/97, OLG Köln NJW-RR 1998, 1277 (1278).

90 LG Aachen Urt. v. 24.1.1991 - 6 S 192/90, LG Aachen NJW 1991, 2159 (2160); Mehrings, BB 1998, 2373 (2378).

91 Köhler/Arndt, Recht des Internet, 4. Aufl. 2003,S. 86; Löhning, NJW 1997, 1688 (1689). 
für den Vertragsschluss anfallenden Verbindungsentgelte aufzuwenden hat. Insofern ergibt sich hier auf den ersten Blick kein Unterschied zum E-Commerce, so dass es vordergründig auch hier einen Widerspruch zum vorangegangen Tun darstellen könnte, würde der Kunde die Kosten für das Aufrufen der AGB als unzumutbar darstellen.

Diese Betrachtung greift jedoch zu kurz: Bei einem Mobiltelefon ist zu berücksichtigen, dass das Display eines solchen Gerätes um ein Vielfaches kleiner ist als der Bildschirm eines Computers. Die AGB, die bei einem Computer ohne Schwierigkeiten auf einer Bildschirmseite Platz finden, können bei einem Mobiltelefon leicht mehrere Bildschirmseiten füllen. Folglich vervielfacht sich proportional hierzu auch der vom Kunden zu betreibende Aufwand beim Lesen der gleichen AGB. Alleine schon unter dem Aspekt dieses Mehraufwandes scheint die Einbeziehung von AGB bei einem per Mobiltelefon geschlossen Vertrag fraglich. Hinzu kommt dann noch das Kostenargument, das im Bereich der Mobiltelefone im Gegensatz zum E-Commerce aufgrund der sehr viel höheren Verbindungsentgelte durchaus fruchtbar gemacht werden kann: Der Kunde muss für das Lesen der AGB auf seinem Mobiltelefon aufgrund des häufigeren Blätterns und des damit verbundenen Ladens der entsprechenden Seite wesentlich länger seine Verbindung aufrechterhalten, als dies an einem Computer der Fall wäre. Dies ist im Hinblick auf die Zumutbarkeit der Kenntnisnahme nicht mehr tragbar. Um demnach eine für den Kunden noch akzeptable Erklärung zu bekommen, muss bedingt durch das kleine Display und die hohen Kosten für den Abruf jeder weiteren WAP-Seite sicherlich auf lange Einwilligungstexte verzichtet werden. Einzig kurze Texte erscheinen in diesem Zusammenhang praktikabel und aus Sicht der Nutzer vor allen Dingen akzeptabel. Dementsprechend müsste die Einwilligungserklärung bezüglich der Ortung so kurz gefasst sein, dass sie lediglich ein bis drei Displayseiten füllt. Wobei sich dann natürlich wieder die Frage stellt, ob man eine Einwilligungserklärung in der Kürze abfassen kann.

Eine Möglichkeit, um die Einwilligung auch im Bereich von Mobiltelefonen umfangreicher zu gestalten, wäre, sich neuer Techniken zu bedienen, die eine schnellere und umfangreichere Datenübertragung gewährleisten. Ein weiterer Aspekt zumutbarer Kenntnisnahme ist allerdings, dass der Diensteanbieter den Kunden in die Lage zu versetzen hat, die erforderlichen Informationen unabhängig von der von ihm verwendeten Technik wahrzunehmen. ${ }^{92}$ Für den Bereich des E-Commerce bedeutet dies, dass der Kunde, egal welchen Browser er benutzt, die Informationen abrufen können muss. ${ }^{93}$ Übertragen auf den Bereich der Mobilfunkgeräte folgt daraus, dass die Informationen über Art und Umfang der Einwilligung mit jedem Mobiltelefon abrufbar sein muss. Demnach wird auf Seiten der Mobilfunkbetreiber auf die Verwendung der neuesten Techniken zu verzichten

92 Königshofen a.a.O. \4 Rz. 4.

93 Vgl. zum E-Commerce Königshofen a.a.O. \4 Rz. 4. 
sein, soweit sie noch nicht überall unter den Kunden Verbreitung gefunden haben. In concreto bedeutet dies, dass weiterhin nur eine Einwilligung per SMS oder durch das Aufrufen einer WAP-Seite möglich ist.

Will man auch im M-Commerce längere und ausführlichere Einwilligungen haben, ist schließlich über die Möglichkeit nachzudenken, die Einwilligung im Internet abgeben zu lassen. Allerdings erledigt die Eröffnung dieser Möglichkeit das Problem nur partiell, da nicht pauschal davon ausgegangen werden darf, dass jeder Mobilfunkkunde über einen Internetzugang verfügt. Ferner scheint die Bereitschaft der Abgabe einer solchen Erklärung im Internet mehr als fraglich, da sich hierzu noch eines anderen Mediums als dem des Mobiltelefons bedient werden muss. Zumal ist es unter den oben genannten Gesichtpunkten mehr als fraglich, ob dieser gedoppelte Weg der Kenntnisnahme noch zumutbar ist.

\section{Protokollierung und Abrufbarkeit der Einwilligung; \94 Nr. 2, 3 TKG}

Nach \ 94 Nr. 2 TKG hat eine Protokollierung der Einwilligung zu erfolgen. Diese erfolgt durch Speicherung der elektronischen Erklärung des Einwilligenden.9 ${ }^{94}$ Dadurch soll dem Kunden die Möglichkeit eröffnet werden, den Zeitpunkt und den Umfang der elektronischen Einwilligung zu erfahren. ${ }^{95}$ Dies korrespondiert mit dem Erfordernis des $\int 94$ Nr. 3 TKG, nach dem der Diensteanbieter sicherzustellen hat, dass der Kunde seine Einwilligung jederzeit wieder abrufen kann, um zu sehen, wann und in welchem Umfang er seine Einwilligung erteilt hat. Dabei muss die Erklärung aber nicht so gespeichert sein, dass der Kunde sie selbstständig abrufen kann. Es ist vielmehr ausreichend, dass dem Kunden die vollständige und ungekürzte Erklärung unverzüglich auf Anfrage mitgeteilt wird. ${ }^{96}$

\section{Jederzeitiger Widerruf der Einwilligung; $\$ 94$ Nr. 4 TKG}

Schließlich muss der Kunde nach \94 Nr. 4 TKG seine Einwilligung mit Wirkung für die Zukunft jederzeit widerrufen können. Ab diesem Zeitpunkt dürfen die Standortdaten des Verwenders nur noch anonymisiert bzw. im Rahmen der anderen gesetzlichen datenschutzrechtlichen Vorschriften des TKG verwendet werden.

\footnotetext{
94 Königshofen a.a.O. \4 Rz. 5.

95 Königshofen a.a.O. \4 Rz. 5.

96 Königshofen a.a.O. \4 Rz. 7.
} 


\subsection{Teledienstegesetz bzw. Teledienstedatenschutzver- ordnung}

Eine vergleichbare Regelung wie die des $\int 98$ TKG, der sich speziell mit Standortdaten auseinander setzt, existiert im Teledienstegesetz bzw. in der Teledienstedatenschutzverordnung nicht. Hier muss folglich auf Regelungen zurückgegriffen werden, die sich allgemein mit der Erhebung von Daten auseinandersetzen.

So bestimmt $\int 3$ Abs. 1 TDDSG, dass personenbezogene Daten, wie sie für personalisierte mobile Dienste benötigt werden, nur erhoben, verarbeitet und genutzt werden dürfen, soweit das TDDSG oder andere Rechtsvorschriften es erlauben oder der Nutzer eingewilligt hat. Aufgrund des strengen Zweckbindungsgebotes, das sich aus $\int 3$ Abs. 2 TDDSG ergibt und der immer im Zusammenhang mit $\int 3$ Abs. 1 TDDSG zu berücksichtigen ist, gilt eine gegebene Einwilligung immer nur für einen ganz bestimmten Zweck. ${ }^{97}$ Sollen Daten zu einem weiteren Zweck erhoben werden, muss eine neue Einwilligung eingeholt werden oder es bedarf einer anderen gesetzlichen Norm, die die Erhebung legalisiert. ${ }^{98}$ Gleiches gilt, wenn eine alte Einwilligung erweitert werden soll. Demnach bedarf es für die inhaltliche Verwendung der Daten des Kunden im Zusammenhang mit personalisierten mobilen Diensten entweder einer gesetzlichen Ermächtigung oder der Einwilligung durch den Benutzer. Darüber hinaus bedarf es gemäß \4 Abs. 1 TDDSG zu Beginn der Nutzung eines Teledienstes der Information des Nutzers seitens des Anbieters bezüglich Art, Umfang und Zweck der Erhebung, Verarbeitung und Nutzung seiner personenbezogenen Daten.

\section{Gesetzliche Erlaubnistatbestände}

Gesetzliche Erlaubnistatbestände im Sinne von \3 Abs. 1 TDDSG sind zunächst die besonderen Tatbestände des TDDSG, die sich mit der Verarbeitung von Bestands-, Verbindungs- und Abrechnungsdaten befassen. ${ }^{9}$ Soweit die Normen des TDDSG nicht einschlägig sind und keine abschließende Regelung darstellen, kommen als ,andere Rechtsvorschriften“, vorrangig solche des BDSG, in Betracht. ${ }^{100}$

Im Zusammenhang mit personalisierten mobilen Diensten ist der gesetzliche Erlaubnistatbestand des $\int 6$ Abs. 1 TDDSG von besonderer Bedeutung: Diese Norm gestattet es einem Diensteanbieter, die personenbezogenen Daten eines Nutzers auch ohne dessen Einwilligung zu erheben, zu verarbeiten oder zu nutzen, soweit dies erforderlich ist, um die Inanspruchnahme von Telediensten zu

\footnotetext{
97 Schmitz a.a.O. \ 3 TDDSG Rz. 2.

98 Schmitz a.a.O. $\int 3$ TDDSG Rz. 2.

99 Schmitz a.a.O. $\int 3$ TDDSG Rz. 3.

100 Schmitz a.a.O. $\int 3$ TDDSG Rz. 3.
} 
ermöglichen und abzurechnen. Die große Aussagekraft von Nutzungsdaten hinsichtlich der Interessen, Vorlieben und Verhaltensweisen des Nutzers führt hierbei dazu, dass der Begriff der „Erforderlichkeit“ grundsätzlich eng auszulegen ist.101 Ergibt sich jedoch aus dem konkreten Vertragsverhältnis zwischen Anbieter und Nutzer eine bestimmte Ausgestaltung des Teledienstes, so erfolgt die Erforderlichkeitsprüfung allein am Maßstab dieses Vertrages. ${ }^{102}$

„Nutzungsdaten“ sind dabei nach dem Gesetzeswortlaut insbesondere die Merkmale zur Identifikation des Nutzers (§ 6 Abs. 1 S. 2 Nr. 1 TDDSG), Angaben über Beginn und Ende sowie über den Umfang der jeweiligen Nutzung ( $\int 6$ Abs. 1 S. 2 Nr. 2 TDDSG) und Angaben über die vom Nutzer in Anspruch genommenen Teledienste ( $\int 6$ Abs. 1 S. 2 Nr. 3 TDDSG). Aus dem Wort ,insbesondere“ ergibt sich, dass diese Aufzählung nur beispielhaft sein soll. ${ }^{103}$ Nach der allgemeinen Definition sind Nutzungsdaten personenbezogene Daten, die dem Nutzer die Nachfrage nach Telediensten ermöglichen; es handelt sich dabei um Daten, die während der Nutzung eines Teledienstes, zum Beispiel der Interaktion des Nutzers mit dem Diensteanbieter, entstehen. ${ }^{104}$ Demnach unterfallen den „Nutzungsdaten" stets sowohl inhaltliche als auch identifizierende Komponenten. ${ }^{105}$

Somit gehören die im Rahmen der personalisierten mobilen Dienste anfallenden Daten zu den von $\int 6$ Abs. 1 TDDSG erfassten Nutzungsdaten und dürfen, soweit es für die Ermöglichung der Inanspruchnahme oder die Abrechnung von Telediensten erforderlich ist, erhoben werden. Haben die Parteien, also der Inhalteanbieter und der Nutzer, bereits einen Vertrag in Bezug auf die personalisierten mobilen Dienste geschlossen, so richtet sich die Erforderlichkeit der Datenerhebung nach diesem Vertrag. Liegt kein Vertrag vor, an dem sich die Erforderlichkeit orientieren kann, wird man \6 Abs. 1 TDDSG als Erlaubnisnorm ablehnen müssen und den Telediensteanbieter auf die Einwilligung des Benutzers verweisen müssen. Sinn und Zweck der personalisierten mobilen Dienste ist es, dem Kunden Produkte, die möglichste genau auf sein Benutzerprofil zugeschnitten sind, anbieten zu können. Zu diesem Zweck wäre eine Orientierung über das Kundenverhalten erforderlich, welche von $\int 6$ Abs. 1 TDDSG aber gerade nicht erfasst werden soll. ${ }^{106}$

\footnotetext{
${ }^{101}$ Dix/Schaar, in Roßnagel, Recht der Multimedia-Dienste, 2004, Teil 3, \ 3 TDDSG Rz. 97; Schmitz a.a.O. \$ 6 Rz. 10.

102 Schmitz a.a.O. $\int 6$ TDDSG Rz. 8, 12.

103 Dix/Schaar a.a.O. Teil 3, \6 TDDSG, Rz. 83; Schmitz a.a.O. \6 TDDSG Rz. 9.

104 BT-Drs. 13/7385, 24; Dix/Schaar a.a.O. Teil 3, \6 TDDSG Rz. 82.

105 Dix/Schaar a.a.O. Teil 3 \6 TDDSG Rz. 87.

106 Scholz, Datenschutzrechtliche Anforderungen, in Roßnagel, Datenschutz beim Online-Einkauf, 2002, S. 50.
} 


\section{Einwilligung}

Zur Einwilligung, die gemäß \ 3 Abs. 3 TDDSG unter den Voraussetzungen des $\int 4$ Abs. 2 TDDSG auch elektronisch erklärt werden kann, kann auf das oben im Rahmen des Telekommunikationsrechts Gesagte verwiesen werden. Die Anforderungen an eine elektronische Einwilligung sind im TDDSG in $\int 4$ Abs. 2, 3 TDDSG niederlegt.

\section{Literatur}

Beucher / Leyendecker / v. Rosenberg (1999): Beucher, Leyendecker, v. Rosenberg: Mediengesetze: Rundfunk, Mediendienste, Teledienste, Kommentar zum Rundfunkstaatsvertrag, Mediendienste-Staatsvertrag, Teledienstegesetz und Teledienstedatenschutzgesetz, Vahlen, München 1999.

Bräutigam / Leupold (2003): Bräutigam, Leupold (Hrsg.): Online-Handel: betriebswirtschaftliche und rechtliche Grundlagen, einzelne Erscheinungsformen des E-Commerce, Beck, München 2003.

Engel-Flechsig (1997): Engel-Flechsig: Die datenschutzrechtlichen Vorschriften im neuen Informations- und Kommunikationsdienste-Gesetz, Recht der Datenverarbeitung 1997, S. 59 ff.

Engel-Flechsig / Maennel / Tettenborn (1997): Engel-Flechsig, Maennel, Tettenborn: Das neue Informations- und Kommunikationsdienste-Gesetz, Neue Juristische Wochenschrift 1997, S. 2981 ff.

Geppert (2005): Geppert (Hrsg.): Beck'scher TKG-Kommentar, Beck, 3. Auflage München 2005.

Gola / Schomerus (2005): Gola, Schomerus: Bundesdatenschutzgesetz: BDSG, Kommentar, Beck, 8. Auflage München 2005.

Gounalakis (2003): Gounalakis (Hrsg.): Rechtshandbuch Electronic Business, Beck, München 2003.

Gounalakis (1997): Gounalakis: Der Mediendienste-Staatsvertrag der Länder, Neue Juristische Wochenschrift 1997, S. 2993 ff.

Gounalakis / Rhode (1998): Gounalakis, Rhode: Elektronische Kommunikationsangebote zwischen Telediensten, Mediendiensten und Rundfunk, Computer und Recht 1998, S. 487 ff.

Hoeren / Sieber (1999): Hoeren, Sieber (Hrsg.): Handbuch Multimedia-Recht: Rechtsfragen des elektronischen Geschäftsverkehrs, Losebl.Ausg., Beck, München 1999, Stand 2004. 
Köhler / Arndt (2003): Köhler, Arndt: Recht des Internet, Müller, 4. Auflage Heidelberg 2003.

Königshofen (2002): Königshofen: Telekommunikations-Datenschutzverordnung (TDSV): Kommen-tar, v. Decker, Heidelberg 2002.

Löffler, Ricker (2005): Löffler, Ricker: Handbuch des Presserechts, Beck, 5. Auflage München 2005.

Löffler / Wenzel / Sedelmaier (1997): Löffler, Wenzel, Sedelmaier (Hrsg.): Presserecht: Kommentar zu den Landespressegesetzen der Bundesrepublik Deutschland, mit einem besonderen Teil und einem Textanhang, Beck, 4. Auflage München 1997.

Löhning (1997): Löhning: Die Einbeziehung von AGB bei Internet-Geschäften, Neue Juristische Wochenschrift 1997, S. 1688 ff.

Mehrings (1998): Mehrings: Verbraucherschutz im Cyberlaw: Zur Einbeziehung von AGB im Internet Betriebs-Berater 1998, S. 2373 ff.

Rannenberg (2003): Rannenberg: Datenschutz und Datensicherheit 2003, S. 546 ff.

Roßnagel (2003): Roßnagel (Hrsg.): Handbuch Datenschutzrecht: die neuen Grundlagen für Wirtschaft und Verwaltung, Beck, München 2003.

Roßnagel (2003a): Roßnagel: Handbuch Datenschutzrecht, 2003.

Roßnagel (2003): Roßnagel: Recht der Multimediadienste 1998/1999, Neue Zeitschrift für Verwaltungsrecht 2000, S. $622 \mathrm{ff}$.

Roßnagel / Banzhaf / Grimm (2003): Roßnagel, Banzhaf, Grimm (Hrsg.): Datenschutz im electronic commerce: Technik, Recht, Praxis, Verl. Recht und Wirtschaft, Heidelberg 2003.

Scheurle / Mayen (2002): Scheurle, Mayen (Hrsg.): Telekommunikationsgesetz (TKG), Beck, München 2002.

Schmitz (2000): Schmitz: TDDSG und das Recht auf informationelle Selbstbestimmung, Beck, München 2000 (zugl. Jur. Diss. Univ. Mainz 1999).

Scholz (2003): Scholz, M. : Datenschutzrechtliche Anforderungen, in: Roßnagel (Hrsg.): Datenschutz beim Online-Einkauf: Herausforderungen, Konzepte, Lösungen, Viehweg Braunschweig 2003, S. 50 ff.

Spindler / Schmitz / Geis (2004): Spindler, Schmitz, Geis: Teledienstegesetz, Teledienstedatenschutzgesetz, Signaturgesetz: Kommentar, Beck, München 2004. 
Turowski / Pousttchi (2004): Turowski, Pousttchi: Mobile Economy Transaktionen, Prozesse, Anwendungen und Dienste, Ges. für Informatik, Bonn 2004.

Waldenberg (1998): Waldenberg: Teledienste, Mediendienste und die „Verantwortlichkeit" ihrer Anbieter, Multimedia und Recht - Zeitschrift für Informations-, Telekommunikations- und Medienrecht 1998, S. 124 ff. 


\section{Dritter Teil: Wissenschaftskommunikation}





\title{
Scientific Information: The Split between Availability and Selection
}

\author{
Hans E. Roosendaal
}

"The scientific information market will in future be based on a federated network of repositories of scientific information that conform to open standards, and an accommodating architecture that allows users the easiest and fastest possible access to information in all of these repositories.

The information available by such a network will not only comprise of scientific information material, but also of management information relating to this information.

The market is the scientific community. This federated network will be an open and global network" (Roosendaal / Geurts / Hilf 2004).

The vision states that users of scientific content will always strive to integrated access to publications in a particular domain and that these publications can be seamlessly searched irrespective of the mode of access. The vision is not new; it is just phrased in a language reflecting present-day technological opportunities. It could equally well apply to scientific information shared in ancient times by the Greek philosophers. An early expression of the vision with the aim to create a 
world wide communication vehicle for all science is the creation of the scientific journal in $1665^{107}$ making use of the new technologies of that time.

This universal vision has already for centuries driven the market for scientific information and, as a consequence, its value chain, and will continue to do so. Innovations allowing to better fulfilling this vision have lead and will always lead to concomitant changes in the value chain of the scientific information market.

These changes can result in new structures, new services and new service providers, private or public, non-commercial or commercial, if and when this will be appropriate, or even in new professions if needed. Such a development will have important consequences for the present stakeholders, be these universities, commercial or non-commercial publishers or other intermediaries in the scientific information system, in the existing value chain.

On a worldwide scale, scientific literature is growing at about $5 \%$ on an annual basis and has already been growing at roughly this rate for a period of several centuries. This means a doubling of the worldwide annual scientific output every 10 to15 years. This growth has primarily been the result of a growth in the number of scientists worldwide; on balance the productivity per scientist has not changed much (see De Solla Price 1968). In the western world growth in the number of scientists is now more or less exhausted and this growth nowadays comes mainly from Asia, from China and India. Growth in output in the western world can only result from an increase in efficacy and efficiency in the scientific process. This efficacy and efficiency in the process depends on the efficacy and efficiency of the information transfer, i.e. of the scientific information system. An effective and efficient scientific process in turn is indispensable for innovation and our modern society is nowadays particularly dependent on the success of innovation. This autonomous growth of scientific information has resulted in more and more journals to cover the expansion in research areas and existing journals have grown in volume. This makes them more expensive as part of the costs of journals is related to the volume of articles. The overall system will become more expensive if we want all this growing information to be widely available for science.

More and more expensive journals result in cancellations of these journals as libraries cannot afford to subscribe anymore due to necessary budget ceilings. This in turn results in loss of revenues for the publishers and in return these publishers raise prices additionally to compensate for this loss. This process has launched a pricing and cancellation spiral, and is known as the 'serial's crisis' (see Bargheer and Hanekop / Wittke in this book).

107 See the archives of the Royal Society, Bodleian Library, Oxford. 
This serials crisis endangered and still endangers the full availability of information and this availability is of utmost relevance, as

'authors want to publish more, readers want to read less' (Roosendaal / Geurts / van der Vet 2001),

stating that in scientific information the author, after claiming intellectual property for the work, wants most wide exposure for this work, (pre)selection is what the reader wants. Availability is evidently a prerequisite to selection. As expressed in the title of this preface and as will be expanded upon below, availability and selection will each lead to separate and sometimes even opposed requirements on the business model of scientific information. These demands for full availability of scientific information and concomitant power of selection drive change. Availability is crucial to empower the users of scientific information, and such empowerment is a necessary condition for integration into the everyday working processes of the scientists. Changes in technology will lead to major changes in the value chain, if and only if these changes will give the users, be this as author or reader, and as researcher, teacher or student, innovative, effective and efficient instruments for the sharing of scientific information. Sharing of information is needed for a healthy intellectual competitive research environment in which researchers want to work. For such an environment the general availability of and access to high-quality certified material currently provided by commercial and noncommercial publishers is of vital importance. ${ }^{108}$ However, seamless use of these information resources is impeded by the present and incompatible variety of access gateways and services.

The technological development of the past 10 years has already resulted in considerable changes in the scientific information world. Universities and publishers have both felt the need to invest in technological innovation, albeit that these investments were primarily focused on improving the power of selection of the reader. With respect to publishers these investments have next to other market considerations resulted in an ongoing clustering of publishers resulting in ever fewer publishers, but each of the remaining publishers controlling an everexpanding market share of published papers. No doubt that this development has strengthened the strategic position of the publishers and as a consequence their negotiation position vis a vis the scientific world, i.e. the universities.

What kind of strategies will universities develop and implement to their benefit? Only if science can build a strong position and will be a strong partner in the value chain, will it be possible to create a transparent market. Just complaining a-

108 In physics alone the meta-document service OAD Open Archive Distributed which collects information on all documents in distributed, central and commercial publishers archives, in as much as they are available, already shows 450.000 documents: http://www.dlib.vt.edu/OAD/ and http://www.isn-oldenburg.de/projects/OAD/. 
bout high prices is simply not sufficient. In many countries universities now create 'repositories of information' with the aim to integrate the information required for research, education and the management thereof in an in-house system to make the scientific process more effective and efficient. These repositories also give them opportunities for public relations activities as needed nowadays in an era of growing worldwide competition between universities. And not least, the development of repositories also provides universities with the chance to strengthen their joint position in the value chain versus the other stakeholders, in particular the publishers. The creation of these repositories reflects the opportunity to fulfil the vision in its aim for integration of the entire information provision of the university. Information provision has become, if it were not already, a core strategic activity of the university. It goes without saying that the library plays and will have to play a crucial role in this integration process. Scientific information provision is definitively on the move and this provides great opportunities for professional, commercial or non-commercial service providers. From such a perspective it becomes relevant to reanalyse the conditions for competition, and thus in terms of prospective value chains.

Next to the above described general strategic considerations a strategic approach will also have to meet the following specific conditions:

- in-depth understanding of the ICT-requirements of users of scholarly and educational information as a basis for further development and integration;

- development of network solutions, including middleware and specific applications, to provide the essential glue for components and functions of the integrated research and education information infrastructure;

- pro-active participation of stakeholders in global standardisation efforts in relevant areas including data formats, digital libraries, archival formats and digital preservation practices;

- promulgation and exchange of reference models and best practices for scientific information on topics including user ICT-requirements, middleware, data formats, and organisational frameworks;

- creation of legal arrangements, e.g. in areas such as copyright and licensing, between stakeholders in the research and educational information domain;

- collaboration of universities worldwide in creating a network of repositories.

These developments in the scientific information system may not only benefit universities and researchers and thus in an indirect way society, but may benefit society also in a direct way as the use of scientific information is both intensive and of a complex nature. As a corollary, the investments in scientific information may 
well prove to pay off through a wealth of spin-offs in other markets with a high demand of complex information.

What makes the above discussion on strategies related to the value chain of scientific information even more relevant? We are on the brink of creating an escience environment. E-science essentially means the sharing of scientific information in a federated network of research and higher education institutions. Escience means a further step towards full availability of scientific results, comprising also the primary results and other pertinent data, and thus a further step towards full integration of information into the scientific process. This development is not only relevant for the larger research establishments such as CERN in Geneva or the Max Planck Society in Germany, but is probably even more important for the smaller and medium sized institutions. It allows them to fully participate in research that is nowadays organised in a more and more programmatic approach in a national or international setting. Only when firmly embedded in e-science these institutions may be able to effectively and efficiently participate in projects of that scale. E-science means sharing of information at all levels in the scientific process, at the informal and at the formal level. Next to this demand for general availability, the demand for selection remains and this means a demand for services provided by other service providers as publishers and other intermediaries.

It may be worthwhile to have a brief look into some research carried out in the Netherlands in 2003/2004 with respect to digital services of publishers. The Dutch university libraries and the Royal Library, the publishers Elsevier and Kluwer Academic, and 'Surf ICT in research', jointly carried out this research as part of agreements that allowed the university libraries to offer their users access to complete journal collections in electronic form, e.g. from ScienceDirect and Kluwer Online. In this way full availability to the users for at least these journal collections was realised and this is a condition for comparative research. Overall, this research confirms the relevance of full availability and concomitant power of selection ${ }^{109}$.

Scientists appreciate full accessibility as a most important property of electronic journals: journals are nowadays available at any desired moment and can be consulted at the working place and from home, via the university network. Access to scientific information by the desktop has now become the rule.

The users of the Dutch university libraries have downloaded during the past years millions of articles from the platforms of the two publishers. In October 2001 the users downloaded on average 1 in 14 articles of these databases, in October 2003 this number had raised to an average of 1 in 10 . The number of down-

109 Report of the steering group, "Het gebruik van digitale diensten van uitgevers", December 2004, in Dutch. 
loads per journal varies substantially, from just a few downloads per month to more than a thousand downloads per month. From the Figure below we see that $20 \%$ of the journals do cover about $80 \%$ of the downloads, $40 \%$ of the journals is good for more than $90 \%$ and $60 \%$ of the journals even for more than $95 \%$ of the downloads. This download behaviour follows nicely the ' $80 / 20$ ' rule or the 'Pareto' distribution as was to be expected.

downloads/article

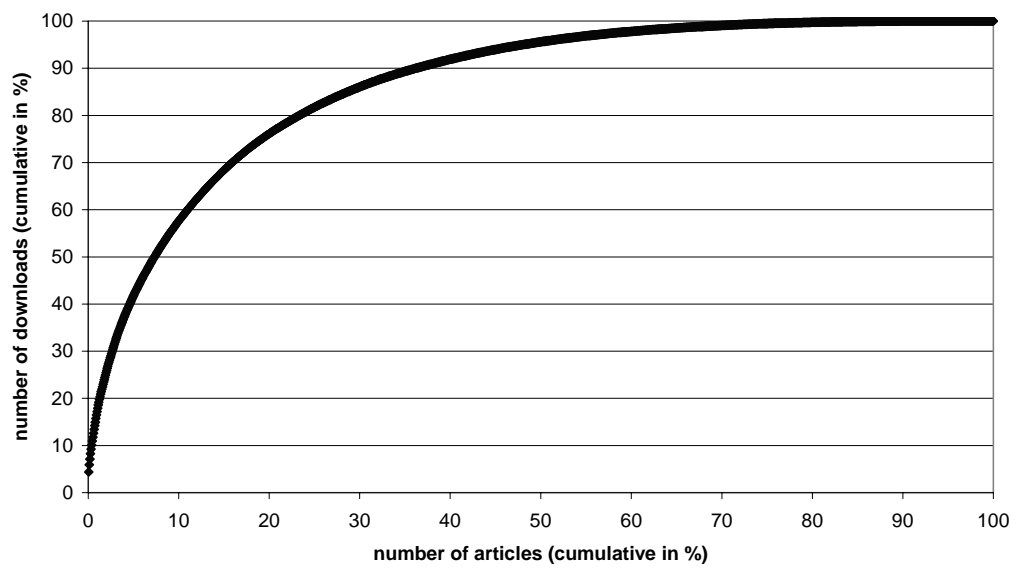

According to the scientists electronic journals do offer additional advantages for performing interdisciplinary research: the comfort at which one can inspect many different articles, e.g. by using specific search terms, allows scientists to find yet unknown sources and new angles that were not evident before. Research is also expected to speed up as the required information from disparate sources can be collected and integrated much faster. Again, full availability is required to be able to do this.

We have seen that the scientific process demands that scientific information must be widely available, and this in perpetuity. The latter is generally valid, but it is in particular valid for information that has been accepted for publication in scientific journals after the peer review process.

The above arguments lead to two basic requirements to any business model for scientific information:

- on the one hand side the model should account for wide availability anyhow, anywhere and anytime, and

- on the other side for the power of selection by the reader. This power of selection should ever continue to advance with technology. 
This leads to two basic models:

- The subscription model

- The 'open access' model.

In both models the author, as we have seen above, claims the intellectual property right to the work by publishing this work. Publishing in an acknowledged medium of publication, be this an institutional repository, a book, a journal or any other accessible medium is for the author the only way of acquiring this intellectual property right. Property rights are part of the copyright and the author will retain these property rights in perpetuity. The author can transfer the integral or partial exploitation rights of the copyright to a publisher, be this a university operating an institutional repository, a university publisher, a non-commercial or a commercial publisher. In return to transferring these commercial rights, the publisher is committed to protect next to its own commercial rights the intellectual property right of the author. Which commercial or exploitation rights are exactly transferred is just a matter of negotiation between the author and the publisher.

In the presently most used business model, the subscription model, it is the library that pays on behalf of the reader. The basic idea is that the overriding added value for which is paid is the power of selection by the reader. This was no doubt relevant with printed journals as here selection is mainly based on title choice. With electronic journals digital searching means searching over more than one title with the concomitant result that one can and will find new sources and new angles. This makes the journal title less important as a search tool, albeit that the journal and thus its title will remain important to the reader for the scientific and editorial policies it represents. As such, the journal title represents the flavour of the journal, the title is the brand. The subscription model puts the total financial responsibility and burden, and this includes the costs for availability, on the reader.

As we have seen, the costs for availability will continue to increase in the foreseeable future as there will be no end to the steady growth of worldwide scientific output. And indeed, such an end is not desirable from the point of view of the scientific process as it would signal that scientific progress has come to a stand-still. And scientific progress is badly needed in our society as an engine for innovation. This growth has given rise to a debate on the 'information overload', suggesting exorbitant growth of information. As we have seen this 'information overload' is just the result of the steady growth of scientific output as witnessed already for centuries and as is needed to sustain scientific progress. The solution to this 'information overload' is evidently not to bring scientific progress to a halt with the only objective to reduce the availability of information, this would be the world upside down, but to find ways to deal more effectively and efficiently with this ever growing pile of information. This calls for continuous innovation of the power of selection by the reader. Technology can and will help us here. Availability will and even must grow and as a consequence the costs for availability will continue to 
grow in a commensurate way. This means roughly a doubling in annual costs every 10 years or so for availability only, irrespective of other factors such as costs arising from additional added value, profits or inflation.

An alternative to the subscription model may be the 'open access' model. In this model availability is the overriding added value for which is paid. Basically, it means that the authors pay a one-time lump sum for the publication of their work, and the journals will be freely accessible for the readers. This model is not new, but recently reintroduced as a possible new solution. Not for long, most American journals used to have a somewhat similar system of 'page charges': authors paid to cover the fixed costs connected to a publication. The variable costs were covered by subscription costs paid by the reader. However, pressure of mainly nonAmerican authors forced many journals to abandon this system largely or partially in the seventies. In some research areas, such as economics, review charges are being levied as separate payment covering the costs of the reviewing process. In this way, the costs for the reviewing process were made independent from the revenues from subscriptions.

The open access model seems philosophically and ethically more acceptable for the scientific community than the subscription model as in this model the production of scientific output is appreciated as acquisition costs, i.e. as integral part, of the scientific process. This may well be a main reason for the revival of the model. Also present-day technology allows a much more sophisticated application of the model. It goes without saying that also this model has to account for the fact that the costs for availability will double every 10 years or so.

Technology also allows universities to make scientific information available via their repositories of information. This feels as scientific production coming back home to the alma mater. The university can also support or even act on behalf of the author in negotiating the exploitation rights for the work. Scientific quality control by peer review coupled to power of selection is then a clear separate added value for which independent segmentation or branding, e.g. by journal titles or other editorially controlled scientific information collections, is vital.

While it remains to be seen if authors will accept the model of 'open access' and if it will prove to reduce total costs, this model has one clear weakness: there is no real incentive to warrant an optimal power of selection by the reader. Optimal power of selection meaning that its continuous innovation using the latest technology will be guaranteed in future.

Combination of the two elements in one hand may well result in a painful split: either there will be problems with full availability or problems with less effective and efficient selection. 
Would it then not be desirable to separate the costs and pricing for availability from the costs and pricing for selection? Again this is not a new issue. A similar functional segregation is nowadays being introduced in the Netherlands for the energy market separating the responsibility for the infrastructure from the responsibility for the supply of electric power.

Following the above arguments on the different aspects of availability and selection as core added values of scientific information and the different roles they therefore play in the business model it follows to consider, at least from a theoretical point of view, two separate suppliers: one for availability and a second for the selection by the reader. These suppliers can be both universities and publishers. This may be an interesting development as we have already seen some evidence for a convergence of the roles of each stakeholder, the publisher, the library and the agent in their attempts to cater for the entire intermediary function in the scientific information system. Some pertinent examples to this convergence of roles are the creation of institutional repositories by universities, the integration of primary and secondary literature and the bundling of digital journal packages by agents, just to name a few.

A market segmentation with independent pricing for both forms of added value can increase the transparency of the market, and more transparency is badly needed in this presently rather imperfect market. Enhancing the transparency in the market requires strong partners and we have seen that universities, albeit somewhat hesitantly, are nowadays strengthening their strategic position by building repositories and by investing in e-science. Next to using these repositories for internal and other external purposes such as public relations in a more and more competitive world for universities, it also allows universities to assume a key role in providing the availability of scientific information.

A transparent market also requires transparency in cash flows, tariffs, revenues and all, also hidden, costs, and for all stakeholders. As a first step to support this development proactively and to bring the investments in repositories and escience to fruition the cash flows between research, education and information within the university will have to be transparent, and this still seems far away. More generally, further developing the scientific information market in a digital environment then requires developing and testing new value chains different from the present ones, and consequently new business and distribution models.

A prerequisite for the acceptance of new models is that they provide critical mass. Critical mass is also needed to be able to support a variety of value chains for the information market representing different organisational models, legal models and business models. The individual stakeholders will need this flexibility to effectively compete with their information products. 
Needless to state that these new business models should be developed between universities and the other partners in the value chain like publishers and other service providers. This process has begun with the experimentation of new license models, but these models can only be considered as a beginning of a long process still ahead of us.

Scientific information is one of the subjects dealt with in the Project Mediaconomy on which this book reports. The overall objective of Mediaconomy is "to investigate the consequences of altered market structures and modes of supply by internet-based and in particular mobile applications exemplified on the media market. The investigation uses different theoretical research perspectives and seeks to develop explanatory and conceptual models incorporating these different perspectives." Needless to state that within this objective the scientific information market is a particularly relevant object of study whereas at the same time this market may well benefit from such a novel approach.

This book marks perhaps the end of the beginning of this process of developing new value chains business models in its analyses of a number of issues relevant to the process from a novel perspective, such as:

- a knowledge-sociological approach that assumes scientific information as oscillating between a state of inalienable goods and commodities, and that analyses the effects of the information's shifting nature on the scientific information system at large, the relevant value chains and its stakeholders;

- 'open access' as seen from a media-scientific perspective and analysing in this way the above mentioned developments with respect to repositories and also university presses;

- open access as seen from a legal perspective looking for parallels to the open source phenomenon.

It is intended and much hoped that this book will spur this process of innovation of the scientific information market. 


\section{Literatur}

Roosendaal / Geurts / Hilf (2004): Roosendaal, H., Geurts, P., Hilf, E.: Pertinent Strategy Issues in Scientific Information and Communication in 2004 ,Invited review to be published in the book "Library Science - quo vadis?" edited by the Institute of Library Science at the Humboldt University Berlin, Berlin 2004.

De Solla Price (1968): De Solla Price, D.: "Little Science, big science... and beyond", Columbia University Press, New York, 1986.

Roosendaal / Geurts / van der Vet (2001): Roosendaal, H., Geurts, P., van der Vet, P.: Developments in scientific communication: Considerations on the value chain. Information Services and Use, 21 (2001) p. 13-32. 



\section{Open Access und Universitätsverlage: Auswege aus der Publication Crisis?}

\section{Margo Bargheer}

\section{Einführung}

In den letzten Jahren sind an deutschen Universitäten wieder Verlage gegründet worden, nachdem die Erstgründungen des 18. Jh. wie Herder oder Vandenhoek \& Ruprecht zusehends aus dem Einflussbereich der Universitäten gerückt waren und sich als privatwirtschaftliche Wissenschaftsverlage etabliert hatten (zur Geschichte der Universitätsverlage vgl. Jäger 2001, S. 406-421). Die rezenten Gründungen gehören zum Kontext der hochschuleigenen Veröffentlichungswege, die angesichts der Krise des wissenschaftlichen Publikationssystems aufgebaut werden. Warum hochschuleigene Verlage, tritt damit nicht die öffentliche Hand in Konkurrenz zu einem funktionierendem marktwirtschaftlichen System? Als sich in den 1990er Jahren das Internet durchsetzte, schien dies, zusammen mit den technischen Möglichkeiten der digitalen Publikation, einen Paradigmenwechsel für die wissenschaftliche Informationskultur zu bringen. Denn es stellt erstmals in der Wissensgeschichte ein dezentrales Kommunikationsmedium ohne Kontrollinstanz und inhaltliche Zweckbestimmung dar, das damit neue Publikationsmöglichkeiten bie- 
tet. Die traditionelle Trennung von Sender und Empfänger verschwindet, jeder kann die Medienproduzentenrolle einnehmen (siehe Klaus / Röttger 1998, S. 236) und unabhängig vom etablierten Markt Informationen verbreiten. Software wie DTP-Programme und das Aufsetzen auf bestehenden IT-Infrastrukturen machen die First-Copy-Publikationskosten erschwinglich, die Grenzkosten gehen im Internet nahezu gegen Null. Doch trotz der technischen Entfaltungsmöglichkeiten veränderte sich die grundlegende Strukturierung des Marktes nicht zugunsten des freieren Zugriffs auf wissenschaftliche Information. Vielmehr ist ein seit Jahren schwelender Strukturkonflikt verstärkt worden, der sich als die „Publication Crisis" manifestiert hat.

\section{Problematische Macht- und Kostenverhältnisse: die „Publication Crisis“}

In der traditionellen Wertschöpfungskette - Autoren produzieren Inhalte, Verlage bündeln thematisch, reichern die Inhalte (Qualitätsprüfung, technische Produktion) an und vertreiben sie, Bibliotheken erwerben Verlagserzeugnisse und stellen sie der wissenschaftlichen Gemeinschaft kostenfrei zur Verfügung (siehe Abb. 34) - waren bis zum Beginn der 1980er Jahre die Macht- und Kostenverhältnisse halbwegs ausbalanciert. Heute zeigen die weiter steigenden Bezugspreise für STMZeitschriften (Science, Technology, Medicine), dass weite ${ }^{110}$ Bereiche des Marktes dysfunktional sind. Der STM-Markt hatte 2000 ein Volumen von etwa 9,5 Milliarden US-Dollar (vgl. Meier 2001, S. 3) und wird von wenigen Verlagskonzernen wie Reed Elsevier (17\% Marktanteil), Blackwell, Whiley oder dem neugeordneten Springer Verlag dominiert. Nach der fortlaufenden Untersuchung „Brandon/Hill Selected List of Books and Journals for the Small Medical Library" liegt die Preissteigerungsrate deutlich über dem allgemeinen Niveau. Der sprunghafte Anstieg 1996-1999 von Ø 52\% zeigt sich mit den derzeitigen 32\% nur als abgeschwächt, wobei einzelne Titel seit 1994 über 200\% teurer wurden. Dies korrespondiert zwar mit Entwicklungskosten für elektronische Publikationen, die vehementen Preissteigerungen begannen aber schon vorher (vgl. Meier 2002, S. 31). Denn das wissenschaftliche Publikationswesen ist zum gewinnträchtigen Kapitalmarkt geworden. Dies hat sich in den Konzernbildungen und Umstrukturierungen gezeigt (vgl. hierzu McCabe 2002), etwa 2003 im kartellrechtlich bedenklichen Verkauf des Springer-Fachverlages an Cinven und Candover, eine Investorengemeinschaft, die zuvor schon Kluwer und den Wissenschaftsbereich von Bertelsmann erworben

110 In den Geisteswissenschaften ist die Situation weniger dramatisch, weil der deutsche Markt (noch) von mittelständischen Verlagen geprägt ist. Die Krise wirkt sich hier anders aus, indem die Absatzchancen geisteswissenschaftlicher Monographien seit 1990 um $\varnothing 60 \%$ gesunken und die Preise um $\varnothing 90 \%$ gestiegen sind. Bei Zeitschriften hat es einen $\varnothing$ Auflagenrückgang von 38\% gegeben (vgl. Füssel 2002). Dies liegt v.a. an Bibliotheksetatverlagerungen zum STM-Bereich. 
hatten. Preissprünge lassen sich zudem mit Verlagsaufkäufen parallelisieren, etwa beim Verkauf des Leipziger Verlagshauses Urban \& Fischer an den Reed Elsevier Konzern. Besonders der STM-Bereich ist weit entfernt von der Situation des vollkommenen Marktes, und typische Preisregulierungsmechanismen greifen nicht, weil wissenschaftliche Zeitschriften keine substituierbaren Güter sind. Der hohe Spezialisierungsgrad, begrenzte personelle Kapazitäten in einem Fachgebiet sowie der lange Weg bis zur Etablierung und Akzeptanz einer Zeitschrift lassen den Abnehmern von Wissenschaftsinformation kaum Wahlmöglichkeiten. Das Preisgefüge ist unelastisch, Preissteigerungen führen erst ab einer unerträglichen „Schmerzgrenze“ zum Erliegen der Nachfrage. Denn der Literaturversorgungsauftrag von Bibliotheken hindert diese, sich marktkonform zu verhalten, so dass die Abnehmerseite keine marktregulierende Wirkung zeigen kann. Paradoxerweise hat die Leistungsfähigkeit von Bibliotheken, trotz knapper Mittel die Wissenschaftsgemeinschaft weiterhin mit Literatur zu versorgen, als Faktor für die Dysfunktionalität des Marktes gewirkt. Preissteigerungsraten zwischen 1986 und 2001 von durchschnittlich 215\% haben nur zu etwa 5\% weniger Erwerbungen von Zeitschriften geführt (vgl. van Orsdel / Born 2003).

\section{Reziprozitätsmodell bei kostenpflichtigen Zeitschriften}
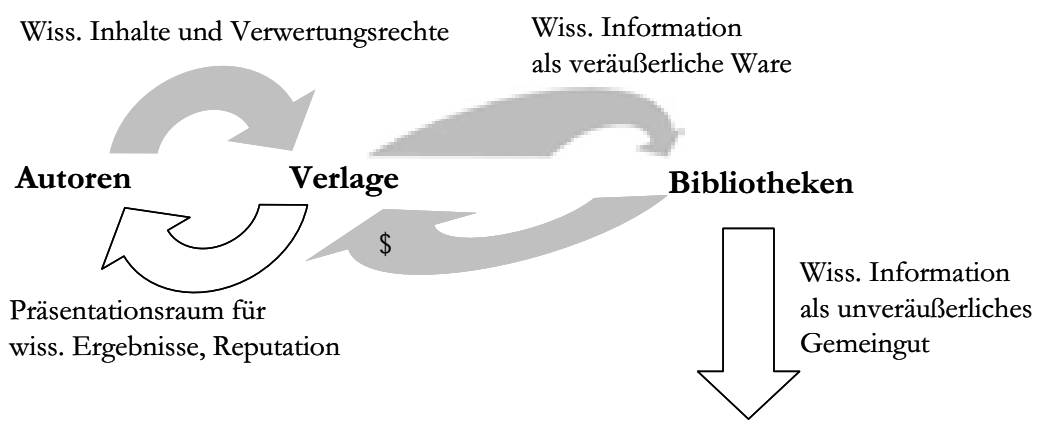

immaterielles Gut

Wissenschaftliche Gemeinschaft

materielles Gut

Abbildung 34: Herkömmliche Wertschöpfungskette und Reziprozitäten

Als beeinflussender Faktor für den Markt wirkt ebenso, dass Produzenten des Gutes „Wissenschaftsinformation“ keine interessensneutralen Akteure sind. Publizieren in etablierten Medien stellt eine Grundsäule von wissenschaftlicher Kommunikation und Fortschritt dar und ist damit unerlässlich für die berufliche Anerken- 
nung. Die immaterielle Anerkennung manifestiert sich als geldwerter Vorteil, wenn anhand wissenschaftlicher Reputation Mittel und Stellen verteilt werden (vgl. de Vries 2001, S. 238). Veröffentlichungen sind daher besonders lohnend in angesehenen Zeitschriften. Im wissenschaftlichen Incentive-Modell sind dies Medienprodukte mit einem hohen Impactfaktor, worunter die Zitierhäufigkeit der letzten zwei Jahre in anderen anerkannten Medien verstanden wird. Der Impactfaktor stellt jedoch keinen objektiven Parameter für die Qualität des einzelnen Artikels dar, sondern beruht auf der verbreiteten Gleichsetzung von Zeitschriftenrenommee mit wissenschaftlicher Qualität (vgl. Meier 2001, S. 48ff). So wird die Stellung von etablierten Fachtiteln gestärkt, deren Nachfrage nicht mehr nur von ökonomischen Faktoren abhängt und deren Preis sich nicht über Qualitätsmerkmale wie ein günstiges Preisleistungsverhältnis regulieren kann. Sozioökonomisch betrachtet, sind die monopolistischen Strukturverhältnisse durch das derzeitige Incentive-Modell noch perpetuiert worden, weil die geldgebenden Institutionen lange Zeit keinen Einfluss auf das System nahmen und Drittmittel anhand von Publikationsleistungen in etablierten Medien vergaben.

Medientechnologisch ist die Dysfunktionalität des wissenschaftlichen Publikationsmarktes im Aufkommen der elektronischen Zeitschriften und der gestiegenen Nachfrage nach der papierlosen Verbreitung begründet, entweder als Parallelausgabe zu bestehenden Printangeboten, als ablösendes Modell oder als E-Only Neugründung. Die erheblichen Investitionen in Hard- und Software sowie in die Entwicklung notwendiger Standards, die für leistungsfähige elektronische Zeitschriften notwendig sind, werden nur zum geringen Teil durch Einsparungen gegenüber der Verbreitung von Druckerzeugnissen (Porto, Herstellung etc.) gedeckt. Auf diesem Markt sind die finanzkräftigen global agierenden Verlagskonzerne gegenüber kleineren Verlagen erheblich im Vorteil, was deren Quasi-Monopolstellung weiter gestärkt hat.

Besonders die durch ,private equity“ gesteuerten Monopolisten unter den Wissenschaftsverlagen befinden sich in der wohl einmaligen Situation, dass sie trotz mehrfacher Finanzierung durch die öffentliche Hand (vgl. auch Bergstrom 2001, S. 20f) - Inhalte liefert die akademische Gemeinschaft kostenlos, durch Hochschulen finanzierte Herausgeber selektieren die Inhalte, öffentliche Bibliotheken sind die Hauptabnehmer der Medienprodukte - gegenüber der wissenschaftlichen Gemeinschaft eine unbalancierte Vormachtstellung besitzen. Die Folgen dieser Situation werden mit den Begriffen der „Serial Crisis“ und „Publication Crisis“ bezeichnet. In diesem Kräfteverhältnis ist die akademische „Scientific Community“ aus dem Zentrum der Wertschöpfungskette gerückt und trägt den Großteil der finanziellen Last, ohne dass sie damit korrespondierenden Nutzen oder Einflussmöglichkeiten hat. 
Die Vormachtstellung von Verlagen beruht medienökonomisch auf drei Säulen, zum einen dem Urheberrecht, wonach Verlage - geschützt durch das Copyright im angloamerikanische Rechtsraum und das Urheber- und Verwertungsrecht im deutschen Rechtsraum - weitgehende Ansprüche an dem veröffentlichten Werk erwerben. Die zweite Säule ist die redaktionelle Themenbündelung (bundling), die dritte die Qualitätssicherung durch Peer Review, worunter die Begutachtung durch Fachkollegen verstanden wird. Sie soll zum einen inhaltliche Mängel, redundante Forschung oder Plagiate aufdecken. Vor allem aber dient die Begutachtung der Selektion von Themen für spezifische Medienprodukte sowie dem Agenda-Setting für bestimmte fachliche Diskurse. Im Regelfall bleibt das Verfahren unveröffentlicht. Das System ist wegen seiner verzögernden Effekte auf den Publikationsprozess und unvermeidlichen menschlichen Schwächen in der Kritik (vgl. de Vries 2001, S. 231, 236ff.), genießt aber weiterhin hohe Anerkennung als Qualitätsprüfung. Durch den Erfolg des Internets jedoch sind alle drei Garanten für Wachstum und den Erhalt des Status Quo infragegestellt.

Welche wirtschaftliche Bedeutung etwa das Urheberrecht hat, verdeutlicht der Kampf um die Zulässigkeit der „Kopie für wissenschaftliche Zwecke“. Nach \$52a des neuen Urheberrechts ist die Kopie für wissenschaftliche Zwecke zulässig, und zwar auch in digitaler Form. Dieser Passus war bei Verlegern und dem Börsenverein auf heftigen Widerstand gestoßen, weshalb ein Wegfall der Klausel zum Jahr 2006 im Gesetz verankert wurde. Die Göttinger Erklärung zum Urheberrecht für Bildung und Wissenschaft vom 5. Juli 2004111 weist auf den potentiellen Schaden für die wissenschaftliche Gemeinschaft hin, der durch eine weitere Verschärfung des Urheberrechts entsteht.

Zur traditionellen Themenbündelung etablieren sich zusehends alternative Modelle. Die Themenselektion und -zusammenstellung ist bisher innerhalb von Medienprodukten, etwa einer Fachzeitschrift, vorgenommen worden. Der Fortschritt auf dem Gebiet von Metadaten, Harvesting- oder Semantic Web Technologien erlaubt verlässliche Selektion und Aggregierung von einzelnen Dokumenten zu bestimmten Themen, die keine kommerziellen Medienprodukte oder Verlagsstrukturen mehr benötigt. Die Qualitätssicherung über Peer Review impliziert bei den meisten Verlagen noch immer, dass nur unveröffentlichte Artikel angenommen werden (Ingelfinger-Regel, vgl. Harnad 2001) und das Verfahren geheim bleibt. Zur traditionellen Begutachtung vor der Veröffentlichung haben sich durch das Internet inzwischen Alternativen gebildet. Preprintserver wie die arXivServer ${ }^{112}$ etwa dienen als Plattform, auf dem Wissenschaftler ihre Ergebnisse offen mit ihrer „Scientific Community“ diskutieren können, bevor sie diese in endgültiger Form in Zeitschriften veröffentlichen. Die Herausgeber von „Electronic

\footnotetext{
$111 \mathrm{http}: / /$ www.urheberrechtsbuendnis.de/

112 http://arxiv.org/
} 
Transactions on Artificial Intelligence" ${ }^{\text {"113 }}$ betrachten den Begutachtungs- und Überarbeitungsprozess als Beitrag zur Forschung und veröffentlichen diesen zusammen mit einem akzeptierten Artikel.

\section{Open Access: Antwort auf die Krise?}

Eine Antwort auf die „Publication Crisis“ ist die „Open Access“ Bewegung (i. S. des freien Zugangs zu Wissenschaftsinformation). Sie beruht darauf, dass wissenschaftliche Autoren selten für finanzielle Entlohnung produzieren, das Internet neue Verbreitungswege bietet und gleichzeitig sich die bestehenden Strukturverhältnissen des Publikationsmarktes weiter zum Nachteil der wissenschaftlichen Gemeinschaft entwickeln. Bereits zu Beginn des neuen Jahrtausends empfahlen in Deutschland der Wissenschaftsrat ${ }^{114}$ und die Hochschulrektorenkonferenz (vgl. HRK 2002, S. 2), dass die wissenschaftliche Gemeinschaft wieder mehr ins Zentrum der Wertschöpfungskette gerückt werden müsse und Hochschulen eigene Publikationswege aufbauen. Hierbei sollen vor allem Open Access Publikationswege gefördert werden. Mit der im Februar 2002 ins Leben gerufenen Budapest Open Access Initiative (BOAI) trat die Bewegung verstärkt an die Öffentlichkeit. Verschiedene 2003 veröffentlichte Erklärungen von Wissenschaftsförderinstitutionen wie die „Berlin Declaration“115 zeigen, dass die Brisanz der „Publication Crisis“ erfasst und zum anderen erkannt wurde, dass eine grundlegende Veränderung des wissenschaftlichen Publikationsmarktes vonnöten ist. Der Begriff „Open Access" ist dabei im Fluss. Sind registrierungspflichtige Angebote „Open Access“? Müssen Autoren die Verwertungsrechte an die Öffentlichkeit abgeben, also Download, Spiegelung und Druck zulassen? Impliziert Open Access, dass die Verwertungsrechte beim Autor bleiben? Pragmatisch werden deshalb als Open Access Publikationen qualitätsgeprüfte wissenschaftliche Information verstanden,

- die im Internet kostenfrei ohne Zugangsbeschränkung zur Verfügung stehen

- die verlässlich und dauerhaft gespeichert werden

- bei denen die Verfasser (bzw. Rechteinhaber) dieser Art der Verbreitung zugestimmt haben.

Die Finanzierung der Medienprodukte und ihrer Verbreitung erfolgt dabei weniger durch Kostenflüsse von den Abnehmern zu den Verlagen, sondern vornehmlich durch die Seite der Informationsproduktion selbst, indem Wissenschaftler zu Medienproduzenten werden oder an Verlage für die Verbreitung ihrer Ergebnisse zahlen. Hier lassen sich zwei zentrale Veröffentlichungsstrategien ausmachen. Da

\footnotetext{
113 http://www.ida.liu.se/ext/etai/

114 http://www.wissenschaftsrat.de/texte/4935-01.pdf

115 http://www.zim.mpg.de/openaccess-berlin/berlindeclaration.html
} 
sind zum einen Open Access Medienprodukte, bei denen einzelne Beiträge auf ihre Qualität geprüft und von Verlagen (bzw. verlegerisch wirkenden Institutionen) thematisch gebündelt einer breiten Öffentlichkeit über verschiedene Nachweisinstrumente (Kataloge, OPACs, Datenbanken wie das Directory of Open Access Journals ${ }^{116}$ etc.) dauerhaft zugänglich gemacht werden. Sie werden von Fachgesellschaften, von Hochschulinstitutionen, von Not-For-Profit Verlagen, aber auch von kommerziellen Verlagen angeboten. Die zweite Säule, seit den Frühzeiten der Open Access Bewegung ,self archiving“ genannt, bilden frei zugängliche Veröffentlichungen auf persönlichen Homepages und Servern, wenn etwa Wissenschaftler ihre Verlagspublikationen vorab als Preprint oder parallel als Postprint veröffentlichen. Damit existieren solche Beiträge außerhalb eines Medienproduktes wie einer Zeitschrift. Die Praxis von Instituten, Hochschulen oder Fachgesellschaften, den elektronischen (qualitätsgeprüften) Output ihrer Mitarbeiter auf ihren Servern abzulegen, wird ebenfalls unter dem Terminus „self archiving“ verstanden. Es ist abzusehen, dass das individuelle und nichtstandardisierte ,self archiving“ zunehmend durch die Speicherung auf institutionellen Servern, den sog. „Institutional Repositories" ergänzt und abgelöst wird. Hierzu trägt die kürzlich geänderte Rechtehandhabung auch von Großverlagen wie Elsevier bei, wonach Autoren ihre eigene Version eines Artikels auf institutionellen Servern bereitstellen dürfen.

Was bis zur Jahrtausendwende vom etablierten Markt noch belächelt und mit dem Argument der mangelnden Qualität, Sichtbarkeit und Zitierfähigkeit abgetan werden konnte, hat sich dank der Unterstützung schlagkräftiger Initiativen wie der 1998 gegründeten SPARC (The Scholarly Publishing and Academic Resources Coalition ${ }^{117}$ ), der BOAI oder der Public Library of Science (PLoS) zu einem beeinflussenden Faktor für das gesamte System der wissenschaftlichen Veröffentlichung ausgewirkt. So ist die Diskussion inzwischen auf der politischen Ebene angekommen. Die Europäische Kommission hat kürzlich die Studie „An effective scientific publishing system for European research ${ }^{118 “}$ in Auftrag gegeben. Sie ist im Zusammenhang des EU-Ziels zu sehen, einen leistungsfähigen europäischen Forschungsraum zu etablieren. Das britische Unterhaus hatte 2003 eine Untersuchungskommission eingesetzt, deren Abschlussbericht „Scientific Publication: Free for All?" 119 auf das problematische Kräfteverhältnisse zwischen der wissenschaftlichen Gemeinschaft und dem etablierten Verlagswesen hinweist und die Stärkung von hochschul- und institutionseigenen Publikationswegen empfiehlt. Dies entfachte im August 2004 eine lebhafte Diskussion in britischen Tageszeitungen. Schlagzeilen wie „Reed Elsevier chief hits back in scientific publishing

\footnotetext{
116 http://www.doaj.org/

117 http://www.arl.org/sparc/

$118 \mathrm{http}: / /$ europa.eu.int/comm/research/press/2004/pr1506en.cfm

119 vgl. Poynder 2004 sowie http://www.publications.parliament.uk/pa/ cm200304/cmselect/cmsctech/ 399/399.pdf
} 
row“ oder „Reed boss denies 'profiteering"“ (vgl. Kaiser, Aldrick, Sabbagh, alle 2004) weisen darauf hin, dass die Open Access Bewegung den Markt deutlicher verändern kann, als es der bisherige Anteil von 1,5\% autorenfinanzierter wissenschaftlicher Open Access Veröffentlichung glauben macht. Der „House Ap-

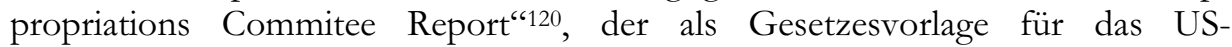
amerikanische Repräsentantenhaus dienen soll, sieht vor, dass Forschungsergebnisse, die durch das National Institute of Health gefördert wurden, der Öffentlichkeit frei und ohne Zugangsbeschränkung - spätestens sechs Monate nach Erscheinen in einem kommerziellen Medienprodukt - zur Verfügung stehen sollen. Die Vorlage zielt auf einen Ausgleich zwischen den berechtigten Verwertungsinteressen kommerzieller Verlage, dem Wunsch von Autoren, in verlagsgebundenen Medien zu veröffentlichen und der Nachfrage der (wissenschaftlichen) Öffentlichkeit nach freiem Zugang zu Forschungsergebnissen ${ }^{121}$. Eine Akzeptanz dieser Vorlage würde eine staatliche Regulierung des Marktes bedeuten, was aus Sicht der etablierten Verlage, besonders der Verlage von Fachgesellschaften ein ökonomisches Risiko darstellt (vgl. Kaiser 2004).

Die Open Access Bewegung wird bei weiteren Veränderungen dieser Tragweite die bisher übliche Rollen- und Kostenverteilung im wissenschaftlichen Publikationssystem in Frage stellen. Solche Veränderungen werden vielleicht die Gewinnmargen im kommerziellen STM-Verlagsmarkt mindern, aber keineswegs das Ende der privatwirtschaftlichen Verleger bedeuten, wie Diskussionsbeiträge um den Untersuchungsbericht „Scientific Publication: Free for All?" meinten. Dies zeigen nicht nur die Ökonomen McCabe und Snyder (2004), wonach für kommerzielle Verlage das Open Access Modell gegenüber dem klassischen Subskriptionsmodell unter bestimmten Bedingungen vorteilhafter sein kann, sondern vor allem die Verlage, die bereits erfolgreich Open Access veröffentlichen. Ergänzend sei jedoch die Kritik von Siebeck „Freibier für die Wissenschaft?“ im Börsenblatt Oktober 2004 genannt. Er sieht im Verlagsbereich der Rechtswissen-schaften den freien Wettbewerb durch die staatliche Förderung von Open Access gefährdet, weil damit die öffentliche Hand versuche, durch Regulierung die Fehlentwicklungen des STM-Bereichs aufzufangen.

\section{Geänderte Kosten- und Verwertungsmodelle}

Auch in der digitalen Welt verursacht Publizieren Kosten. Um den freien und kostenlosen Zugang zu gewähren, sind Modelle entstanden, bei denen wissenschaftliche Produzenten für die Präsentation ihrer Ergebnisse bezahlen. Ein grundsätzli-

\footnotetext{
120 http://www.aaas.org/spp/rd/nih05h1.pdf.

121 Vgl. hierzu die Diskussion im SPARC-Newsletter vom August 2004, http://www.earlham.edu/ $\sim$ peters/fos/newsletter/08-02-04.htm
} 
cher Unterschied des produzentenfinanzierten Open Access zum klassischen Subskriptionsmodell liegt in der Handhabung der Rechteübertragung. Aus Sicht der Open Access Bewegung muss für die „Scientific Community“ nicht nur der möglichst freie Zugang, sondern auch die Verfügungsgewalt über die eigenen Ergebnisse gewährleistet bleiben. Dementsprechend behalten bei fast allen Zeitschriften, die sich über die Produzentenseite finanzieren, Autoren die Verwertungsrechte an ihren Inhalten, welche sie unausgesprochen oder ausdrücklich ${ }^{122}$ der Rezipientenschaft einräumen. Verlage „erwerben“ unter diesem Modell nicht im Tausch für die Veröffentlichung eines Artikels das Recht, diesen kommerziell zu verwerten, sondern bieten gegen Bezahlung Dienstleistungen zur Anreicherung des Artikels (Qualitätsprüfung, Aufbereitung als hochwertiges Medienprodukt) und die qualifizierte Verbreitung desselben an. Im Bereich der Open Access Medienprodukte haben sich drei Ansätze entwickelt, die Finanzierung durch die Autoren, hybride Modelle mit einer Mischfinanzierung aus Gebühren für den sofortigen „Immediate Free Web Access“ (IFWA) und Subskriptionseinnahmen sowie die institutionelle Mitgliedschaft.

\subsection{Autorengebühren}

Beim Modell „Autor zahlt“ entrichten Autoren von angenommen Artikeln eine Gebühr, die für die PLoS-Produkte „PLoS biology“ und „PLoS medicine“ (als Alternative zu kostenpflichtigen Top-Titeln des STM-Bereichs platziert) bei 1.500 \$ liegt. Es gab bereits vor den Open Access Ansätzen Autorengebühren für Farbabbildungen oder Artikelüberlänge. Sie wurden jedoch für den zusätzlichen Produktionsaufwand erhoben, wobei die Sockelfinanzierung der Zeitschrift über die Subskriptionsgebühren gedeckt war. Open Access Zeitschriften wie PLoS biology bestreiten jedoch ihre laufenden Kosten über die Produzentenseite. Der innovative Charakter liegt in der umgekehrten Allokation für knappe Ressourcen. Für Verlage sind nun nicht mehr die Abnehmer (bzw. deren Finanzkraft) die knappe Ressource, um deren Erhalt sie kämpfen müssen, sondern die zahlungsfähigen Produzenten der Inhalte. Verlage können ihre zahlenden Produzenten nur dann binden, wenn sie die Qualität und entsprechende Verbreitungsraten ihrer Medienprodukte nachweisen können. Kommerzielle Wissenschaftsverlage waren bisher in der privilegierten Situation, dass als Gradmesser ihrer wissenschaftlichen Qualität weniger die tatsächliche Artikelnutzung durch die Leserschaft als die über den Impact-Faktor messbare Zitierungshäufigkeit der gesamten Zeitschrift galt.

122 „Open Access“ Bereitstellung hängt ohne eine Übertragung der Verwertungsrechte an die Abnehmerschaft vom Wohlwollen der Autoren ab. Im Bereich des Open Access beginnen sich deshalb Lizenzen zu etablieren, z. B. die Creative Commons Licence (http://creativecommons.org/) oder die Open Content Lizenzen der Centrums für eCompetence NRW (http://www.uvm.nrw.de/opencontent). 
Unter Open Access Modellen werden für Verlage strukturelle und technische Vorkehrungen zur Nutzerforschung deutlich an Bedeutung gewinnen.

\section{Reziprozitätsmodell bei autorenfinanzierter OA Zeitschrift}

Autorengebühr für IFWA

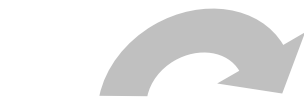

Autoren Verlage
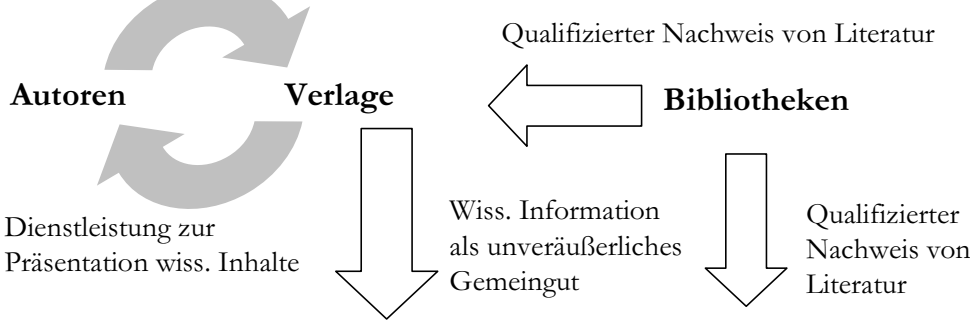
Qualifizierter Nachweis von Literatur

Dienstleistung zur

Präsentation wiss. Inhalte

Wissenschaftliche Gemeinschaft

immaterielles Gut

materielles Gut

\section{Abbildung 35: Geänderte Wertschöpfungskette und Reziprozitäten}

Weil Angebot und Nachfrage hier in einem direkten Verhältnis stehen (vgl. Abb. 35) und nicht durch den Intermediär „Bibliothek“ getrennt sind, - der, ähnlich wie eine Krankenkasse, durch einen gesellschaftlichen Auftrag gebunden ist können theoretisch marktwirtschaftliche Preisregulierungsmechanismen zum Zuge kommen. Ob dies in der Praxis geschieht, wird die Zukunft zeigen.

Die Veränderung des Finanzierungsmodells birgt die Gefahr, dass im Bereich von Open Access der wissenschaftliche Diskurs von den Finanzstarken bestimmt wird. Fast alle Anbieter auf diesem Feld bieten daher Gebührenerlass an, damit auch Autoren finanzschwacher Institutionen und aus Schwellen- und Entwicklungsländern publizieren können. Ausdrücklich ist in den Arbeitsabläufen der Anbieter verankert, dass der Erlass erst dann beantragt wird, wenn das Peer Review erfolgreich durchlaufen wurde. Die unproportionale, nationale Beteiligung am wissenschaftlichen Diskurs aber wird dadurch nicht aufgehoben. So wies Barbara Aronson $^{123}$ (WHO) darauf hin, dass ein Artikelgebührenerlass für Wissenschaftler in Schwellen- und Entwicklungsländern irrelevant ist, wenn dort schon die simple Teilnahme am Medium Internet eine enorme Hürde darstellt.

123 http://www.lub.lu.se/ncsc2004/. 


\subsection{Hybride Finanzierung}

Existierende Zeitschriften, die zum Open Access Geschäftsmodell wechseln wollen, gehen beträchtliches wirtschaftliches Risiko ein, wenn sie auf die kalkulierbaren Einnahmen der Subskription verzichten und sich nur auf die unsicheren Einnahmen von der Produzentenseite verlassen. Dies betrifft vor allem solche Verlage, die aufgrund ihrer engen Bindung an die akademische wissenschaftliche Gemeinschaft voranging kostendeckend arbeiten und sich als „not-for-profit“ verstehen. Im Editorial der Proceedings of the National Academy of Sciences in the United States of America (PNAS) ${ }^{124}$ verdeutlicht Cozzarelli (2004) dieses Risiko, in dem er den experimentellen Charakter des geplanten hybriden Finanzierungsmodells herausstreicht. So lässt sich für PNAS trotz einer Autorenumfrage nicht absehen, wie viele Autoren de facto bereit sind, die Autorengebühren von 1.000 \$ aufzubringen und ob weitere Förderinstitutionen solche Autorengebühren übernehmen werden. Ein hybrides Modell, bei dem Autoren auf Wunsch gegen Gebühr für ihre Artikel „Immediate Free Web Access“ (IFWA) erwerben und die Zeitschrift weiterhin gegen Gebühr vertrieben wird, kann dieses Risiko abfedern. Die Zeitschriften der Entomological Society of America (ESA) durchlaufen seit 1999125 einen Übergang von der Finanzierung über die Abnehmer hin zur Produzentenfinanzierung. Inzwischen werden ca. 60\% der Artikel per IFWA elektronisch bereitgestellt.

Die Preisspanne für den IFWA ist breit. Bei BioMed Central, einem Verlagshaus für medizinische Zeitschriften, beträgt die Autorengebühr 500 US\$, ESA berechnet für die Schnellveröffentlichung ca. 600 US\$ pro Artikel für Mitglieder. Der Springer Verlag bietet mit „Springer Open Choice“126 Autoren für 3.000 US\$ die Möglichkeit, ihre Artikel ohne Zugangsbeschränkung Open Access zu stellen. Als Gegenwert propagiert Springer die dauerhafte Bereitstellung über das leistungsfähige Nachweisinstrument Springer Link. Kritisiert wird neben dem hohen Preis, dass damit weiterhin die strikte Übertragung der Rechte an den Verlag verbunden ist. ${ }^{127}$ Bei hybriden Modellen bleibt die Frage, welche Verteilung bei der Onlinebereitstellung von frei verfügbaren und nur per Subskription angebotenen Artikeln markt- und sozialtauglich ist. Unklar ist, ab welcher Menge frei verfügbaren Artikeln innerhalb einer Zeitschrift Bibliotheken auf die kostenpflichtige Lizenz verzichten werden, womit die Sockelfinanzierung wegbräche. Wird der Erwerb von IFWA zu ,vanity publishing“ führen oder wird den stärker der Öffentlichkeit ausgesetzten Beiträgen automatisch eine besonders hohe Qualität und Bedeutung zugemessen? Fraglich ist ebenfalls, ob es zu weiteren ökonomisch beding-

\footnotetext{
124 http://www.pnas.org/cgi/content/full/101/23/8509.

125 http://csssrvr.entnem.ufl.edu/ walker/epub/esaepub.htm\#IFWAhistory.

$126 \mathrm{http}: / /$ www.springeronline.com/sgw/cda/frontpage/0,10735,5-40359-0-0-0,00.html.

127 http://www.earlham.edu/ peters/fos/2004_08_01_fosblogarchive.html.
} 
ten Gefällen im wissenschaftlichen Diskurs kommt - wenn die Finanzschwachen hinter der Wand der Kostenpflicht und die anderen frei im Internet publizieren.

\subsection{Institutionelle Mitgliedschaft}

Bei der Zeitschrift „Nucleic Acids Research“128 (NAR) stellt der Verlag „Oxford University Press“" nur für ein einzelnes Medienprodukt das Geschäftsmodell einer rein abnehmerfinanzierten Zeitschrift auf ein produzentenfinanziertes Modell um. Die NAR-Herausgeber haben nach einer Umfrage unter Autoren und Gutachtern beschlossen, die Inhalte ab dem 01. Januar 2005 frei ins Internet zu stellen und sich weitgehend über die Produzentenseite zu finanzieren. NAR bietet neben der Autorengebühr die institutionelle Mitgliedschaft an. Ihr Preis entspricht der vorherigen - institutionsgrößenabhängigen - Subskription, die zumeist die Bibliotheken aufgebracht haben.

\section{Reziprozitätsmodell bei OA Zeitschrift mit institutioneller Mitgliedschaft}

Autorengebühr für Immediate Free Web Access (IFWA)

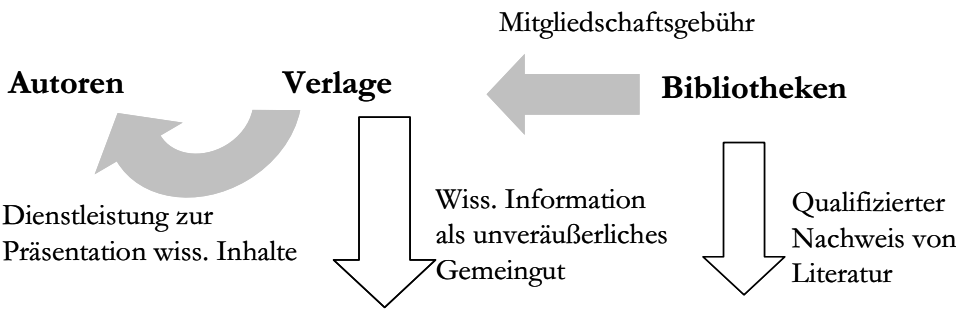

immaterielles Gut

Wissenschaftliche Gemeinschaft

materielles Gut

Abbildung 36: Wertschöpfungskette und Reziprozitäten bei OA Zeitschriften mit institutioneller Mitgliedschaft

Bibliotheken sichern in diesem Modell (vgl. Abb. 36) zu gleichen finanziellen Konditionen statt der Literaturversorgung die Publikationsmöglichkeit für ihre Nutzer. Ebenfalls ein vollständiges Finanzierungsmodell durch die Produzenten

${ }^{128}$ URL der Zeitschrift: http://nar.oupjournals.org/. Stellungnahme zum Open Access Übergang: http://www3.oup.co.uk/nar/special/14/default.html. 
verfolgt BioMed Central, die rund 150 qualitätsgeprüfte Open Access Zeitschriften herausbringen. Gegen eine größenabhängige Jahresgebühr können Institutionen ihren Mitarbeitern die kostenlose Publikation in allen BioMed Central Medienproduktenermöglichen. Das Angebot koppelt BioMed Central mit der Option, auch ohne institutionelle Mitgliedschaft pro Artikel zu zahlen.

\section{Marktveränderungen durch Open Access}

Was auf den ersten Blick so positiv aussieht - freier Zugang zu wissenschaftlicher Information - birgt neben zweifelsfreien Vorteilen auch Risiken für das strukturelle Gefüge des wissenschaftlichen Publikationsmarktes. Unklar bleibt zum Beispiel, wie beim Übergang auf produzentenfinanzierte Modelle die Lücke zu schließen ist, die durch den Wegfall der Subskriptionseinnahmen aus dem kommerziellen Wissenschaftssektor entsteht. Sie kann nicht durch vermehrte Autoreneinnahmen oder institutionelle Mitgliedschaften aufgefangen werden, da im Bereich der kommerziellen Wissenschaft die Wissensproduktion deutlich geringer als die -konsumption ist. Damit können finanzkräftige Sektoren wie die Pharmaindustrie zu öffentlich subventionierten „Trittbrettfahrern“" werden. Denkbar wäre ein Ansatz wie beim Download von Open Source Software, wonach die Inhalte zwar grundsätzlich frei zur Verfügung stehen, privilegierter Zugriff jedoch gegen Gebühr erworben werden kann. Übertragen auf wissenschaftliche Information stünden Inhalte grundsätzlich Open Access zur Verfügung, für die akademischwissenschaftliche Gemeinschaft jedoch IP-Adressen-gesteuert mit schnelleren Downloadraten und anderem Mehrwert (wie reichere Metadaten, Zusatzinformationen oder Crosslinking). Ein solcher Premium-Zugriff könnte von Nutzern auBerhalb der akademischen Gemeinschaft kostenpflichtig erworben werden.

Eine wissenschaftliche Zeitschrift sollte nach dem Ökonomen BERGSTROM weniger als Medienprodukt, sondern als die ,erfolgreiche Lösung eines Kommunikationsproblems“" verstanden werden, indem sich in ihr das Informationsbedürfnis der Rezipientenschaft und das Mitteilungsbedürfnis der Produzentenschaft langfristig treffen. Dies trifft insbesondere auf die Medienprodukte zu Spezialthemen zu, die idealiter im Zentrum ihres wissenschaftlichen Diskurses stehen - was sowohl die beteiligten Personen als auch die Theoriendiskussion und Themenbreite betrifft. So sind es häufig die wissenschaftlichen Fachgesellschaften, die (medial betrachtet) im Zentrum ihrer jeweiligen Disziplinen stehen und verlegerisch tätig werden. Die technologischen Möglichkeiten, die das elektronische Publizieren und das Internet als Verbreitungsmedium mit sich brachten, haben den grundsätzlichen Strukturkonflikt des wissenschaftlichen Publikationsmarktes zutage gefördert und in der Open Access Diskussion gemündet. 
Daraus resultiert ein gesellschaftlicher und ökonomischer Druck, der die am wissenschaftlichen Kommunikations- und Publikationsprozess Beteiligten zur Veränderung zwingt. Fachgesellschaften haben für die wissenschaftliche Gemeinschaft häufig eine ambivalente Funktion. Sie können für ihre Disziplinen die wichtigsten Verlage sein, die aufgrund ihrer prominenten Position zur wissenschaftlichen Gemeinschaft in ein gewinnorientiertes Verhältnis treten, wie die Diskussion 2004 um die angekündigten Preissteigerungen der EMBO-Zeitschriften zeigte ${ }^{129}$. Gleichzeitig aber finanzieren sie mit der verlegerischen Arbeit Aktivitäten wie Stipendien, Kongresse oder Forschungsschwerpunkte. Sie befinden sich damit im Spannungsfeld von Gewinnstreben und einem sozialen Optimum für die „Scientific Community". Die Forderungen nach Open Access haben daher zuerst die Verlage von Fachgesellschaften zu Änderungen in ihrem Geschäftsmodell bewogen. Dass dieses Umschwenken nicht nur Marktanpassung, sondern auch aktive Marktsteuerung darstellt, zeigt das Beispiel der Entomological Society ${ }^{130}$. Diese hatte bereits 1995 beschlossen, auf die Open Access Bereitstellung ihrer Verlagserzeugnisse hinzuarbeiten, als es noch keinen nennenswerten sozioökonomischen Druck in Richtung Open Access gab. Verlagskonzerne wie Reed Elsevier oder Springer hingegen haben erst nach den gewichtigen Erklärungen der „Scientific Community" der jüngsten Zeit Zugeständnisse gemacht.

Geschäftsmodelle, die auf dem Paradigma „Produzentenseite zahlt“ beruhen, sind davon abhängig, dass Autoren die finanzielle Verantwortung für die Veröffentlichung ihrer wissenschaftlichen Ergebnisse übernehmen, indem sie die Kosten selbst aufbringen oder einwerben können. Positiv ist daher das Votum der deutschen Fördergesellschaften zu werten, dass sie Veröffentlichungsgebühren gewähren werden. In gleichem Maße sind solche Zeitschriften auch davon abhängig, dass sie für Autoren den gleichen Stellenwert im wissenschaftlichen IncentiveModell erlangen wie die Medienprodukte des kommerziellen Verlagswesens. Eine Umfrage des Universitätsverlages Göttingen unter den Göttinger Wissenschaftlern zur Akzeptanz von OA-Zeitschriften zeigte, dass aus Unkenntnis (vor allem zu Rechteübertragung und Qualitätsprüfung) noch Vorbehalte gegenüber Open Access Veröffentlichungen bestehen.

Die Praxis der institutionellen Mitgliedschaft, in der Bibliotheken die Sockelfinanzierung von Zeitschriften übernehmen, ändert die finanzielle Lastenverteilung zunächst nicht. Langfristig kann sie jedoch - bei entsprechender Propagierung und Akzeptanz in der jeweiligen Hochschule - zu einer vermehrten Publikationstätigkeit in Open Access Zeitschriften führen, weil die finanzielle Verantwortung nicht beim einzelnen Autor sondern in einer Hand gebündelt liegt. Vermehrte Publikationstätigkeit ist dabei nicht immer im Sinne der Verlage. BioMed Central

\footnotetext{
129 http://www.library.yale.edu/ llicense/ListArchives/0401/msg00014.html.
}

130 http://csssrvr.entnem.ufl.edu/ walker/epub/esaepub.htm\#ESAHistory. 
hatte z. B. mit unvorhergesehen hohem Aufkommen von Artikeln zu kämpfen, für die nur die mit Bibliotheken pauschal vereinbarten Mitgliedsgebühren zur Verfügung stehen, und wird deshalb ab 2005 wieder vermehrt artikelabhängige Gebühren verlangen (vgl. Albanese 2004)

Die Etablierung von neuen Wissenschaftszeitschriften im Open Access Geschäftsmodell ist problematisch. Anders als in sich geschlossene Medienprodukte wie Monographien oder Sammelbände, die durch einen einmaligen Produktionskostenzuschuss vorfinanziert werden können, stellt eine Zeitschrift die „erfolgreiche Lösung eines Kommunikationsproblems“ dar, die bis zu ihrer Etablierung unter Umständen über mehrere Jahre bezuschusst werden muss. Dies gilt besonders für die Etablierung eines neuen Finanzierungsmodells wie die Autorengebühr. Der Erfolg der PLoS-Produkte hängt von der großzügigen Anschubfinanzierung der Moore-Foundation i.H.v. 9 Mio. \$ ab, die zur Propagierung der PLoS-Bewegung und der Markteinführung der Medienprodukte Marketing wie Broschüren und TV-Spots sowie erhebliche Präsentations- und Lobbyaktivitäten der Herausgeber ermöglicht hatte. Ebenso ist der Erfolg in der exzellenten technischen Qualität der Medienprodukte begründet, die einen beträchtlichen Initialaufwand erforderte. Das angesehene Editorial Board, etwa der Nobelpreisträger Harald Varmus, und die garantierte Qualität des Peer Review haben den Erfolg dieses Geschäftsmodells vorhersehbar gemacht. Die erstmalig erhobenen Impact-Faktoren für die PLoS Produkte, die im Sommer 2005 veröffentlicht wurden, bestätigten die Publikationsstrategie. Die hohe Akzeptanz der Medienprodukte in der wissenschaftlichen Gemeinschaft hängt zum einen vom Image der beteiligten Personen und zum anderen von fachspezifischen Besonderheiten ab und ist nur eingeschränkt übertragbar. Bei deutschen Universitätsverlagen liegt der verlegerische Schwerpunkt noch vorwiegend bei in sich geschlossenen Medienprodukten wie Büchern (vgl. Halle 2003). Die Verlegung von Periodika ist jedoch erklärtes Ziel, das auf Unterstützung hoffen darf, wenn das Projekt „German Academic Publishers“ (GAP) zum Ende 2005 praktische Ergebnisse vorlegt.

Open Access Zeitschriften haben aus Analysten-Sicht von PNB Paribas und Credit Suisse das Potential für nachhaltigen Erfolg - auch wenn solche Voraussagen nach dem Zusammenbruch des Neuen Marktes mit Vorsicht zu genießen sind. Open Access Modelle bringen sowohl für die einzelnen Medienprodukte und Verlage als auch für die Märkte der wissenschaftlichen Kommunikation und Publikation soziale und wirtschaftliche Unwägbarkeiten mit sich. Aus Sicht der akademischen wissenschaftlichen Gemeinschaft ist es daher ratsam, bei der Sicherung der wissenschaftlichen Kommunikation und Publikation nicht allein auf die Kräfte des Marktes zu vertrauen und zu hoffen, dass weitere Verleger auf Open Access Geschäftsmodelle wechseln. Das hieße, weiterhin auf die Aufgabenverteilung des traditionellen Publikationsmodells zu setzen, die Abb. 37 verdeutlicht. 


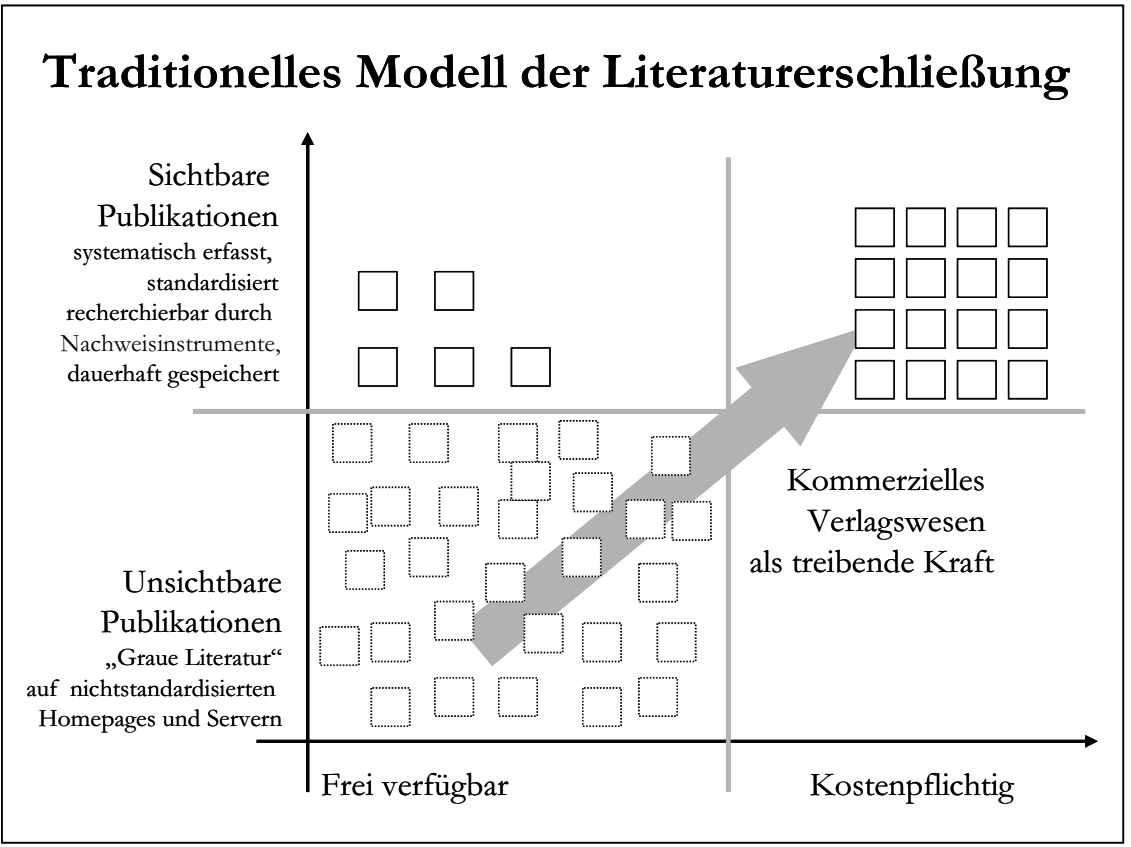

Abbildung 37: Erschließung als kostenpflichtige Verlagspublikationen

Besondere Chancen bietet der Bereich des systematischen „Self Archiving“ auf institutioneller Ebene durch den weiteren Aufbau von sog. „Institutional Repositories“" (vgl. ausführlich hierzu Crow 2002) und die stärkere Vernetzung dieser Infrastrukturen durch Kompatibilität, Standards wie Open Archive Initiative (OAI $\left.{ }^{131}\right)$, generische Metadaten, Harvesting- oder Semantic Web Technologien. Damit kann die Schwelle zur unsichtbaren Literatur und die Abhängigkeit vom kommerziellen Verlagswesen bei der Literaturversorgung gesenkt werden, wie Abb. 38 zeigt. Um dies zu erreichen, müssen digital vorliegende wissenschaftliche Beiträge systematisch erschlossen sowie versionsverlässlich und dauerhaft auf leistungsfähigen Servern zur Verfügung stehen, vor allem aber harvestfähig und in übergreifenden Nachweisinstrumenten wie der Dienst OAIster ${ }^{132}$ recherchierbar sein. Dokumente mit aussagekräftigen Metadaten (die Suchen nach Themen, Autoren, Referenzen oder Materialart erlauben) auf standardisierten Servern können publizistisches Potential auch ohne die Bündelung durch Verlage in bestimmten Medienprodukten erlangen und damit als eigenständige Entität bestehen, weil sie von Recherchierenden individuell zusammengestellt werden können. Für kommerzielle Verlage

\footnotetext{
$131 \mathrm{Zu}$ OAI gehören standardisierte Metadatenschemata (z. B. Titel, Autor, Datum, keywords etc.) für die einzelnen Dokumente sowie Schnittstellen, Übertragungsprotokolle und Serverstrukturen, die das automatische Metadatenharvesting zulassen.

132 http://oaister.umdl.umich.edu/o/oaister/.
} 
gibt es auch in diesem Modell weiterhin Betätigungsfelder, die zudem auf Kernkompetenzen wissenschaftlicher Verlage aufsetzen: liegt ein Großteil wissenschaftlicher Information frei im Internet vor, steigt der Bedarf an fachwissenschaftlicher Dienstleistung wie redaktionelle Aufbereitung, Themenbündelung, Review oder personalisierbarer Alertservice.

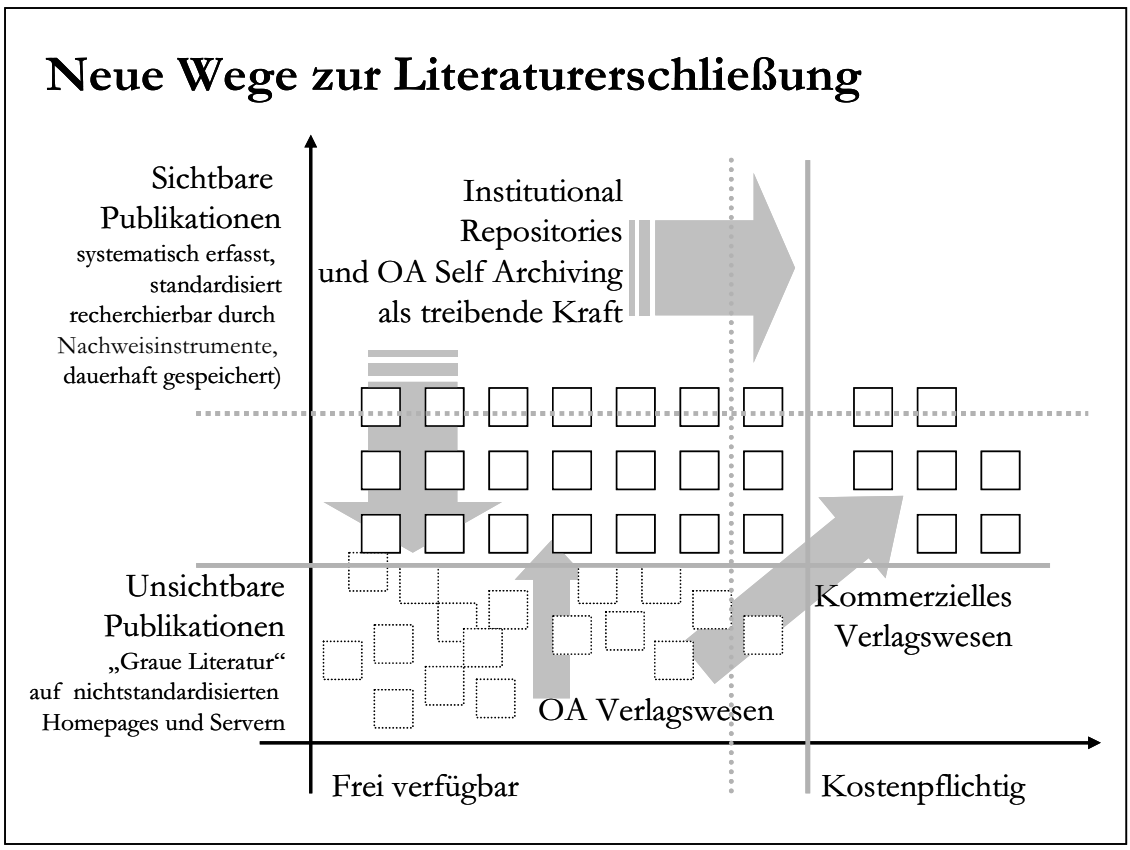

Abbildung 38: Verschiebung der Erschließungsgrenzen durch Open Access und institutionelle Publikationswege

\section{Hochschuleigene Publikationswege}

Schon mit dem Aufkommen des elektronischen Publizierens wurden an zahlreichen Universitäten in Deutschland eigene Publikationswege aufgebaut, wie etwa für die elektronische Veröffentlichung von Dissertationen und dauerhafte Sicherung der digitalen ,grauen Literatur“. Erste Ansätze für die systematische Erfassung und Bereitstellung elektronischer Dissertationen etwa stammen von 1995 und wurden schon 1997 in größerem Stile an deutschen Bibliotheken umgesetzt. (vgl. Degenhardt 1997, S. 4)

Diese Konzepte waren von Beginn an auf hochschul- und institutionsübergreifende Kompatibilität ausgerichtet und als Teil von integrativen Publikationssystemen konzipiert. An der Georg-August-Universität Göttingen wurde dies mit der 
Publikationspyramide umgesetzt (Abb. 39), der zentralen, vernetzten Vorhaltung von elektronischen Dokumenten, die über die Art der Ausgabe, des Zugriffs und der Qualitätsprüfung voneinander unterschieden werden. Wesentlicher Bestandteil des Publikationssystems ist der Universitätsverlag, den die Niedersächsische Staats- und Universitätsbibliothek im Auftrage des Präsidiums für die Hochschule betreibt. Die Art der Veröffentlichung erfolgt bedarfsorientiert - sei es gedruckt, auf Datenträgern oder „,born digital“ im Internet - im Sinne des medienneutralen Publizierens. Technisches Rückgrat des Publikationssystems ist ein Repository, das als System vernetzter Server arbeitet. Die zentral vorgehaltenen Inhalte sind über gemeinsame Funktionalitäten wie OPACs und servereigene Oberflächen recherchier- und erreichbar. Durch die OPAC-Integration in internationale Bibliotheksdatenbanken ist der Nachweis zudem nicht national beschränkt. Der Zugriff - etwa temporär im Intranet, passwortgeschützt oder IP-Adressen-gesteuert für begrenzte Nutzerkreise oder frei im Internet - erfolgt nach Maßgabe der Produzenten. Das Repository dient jedoch nicht ausschließlich der elektronischen Publikation, sondern ebenso als Archiv für medienneutrales Publizieren, etwa als hochwertiger Druck in kompletten Auflagen oder als Print on Demand in Menge 1.

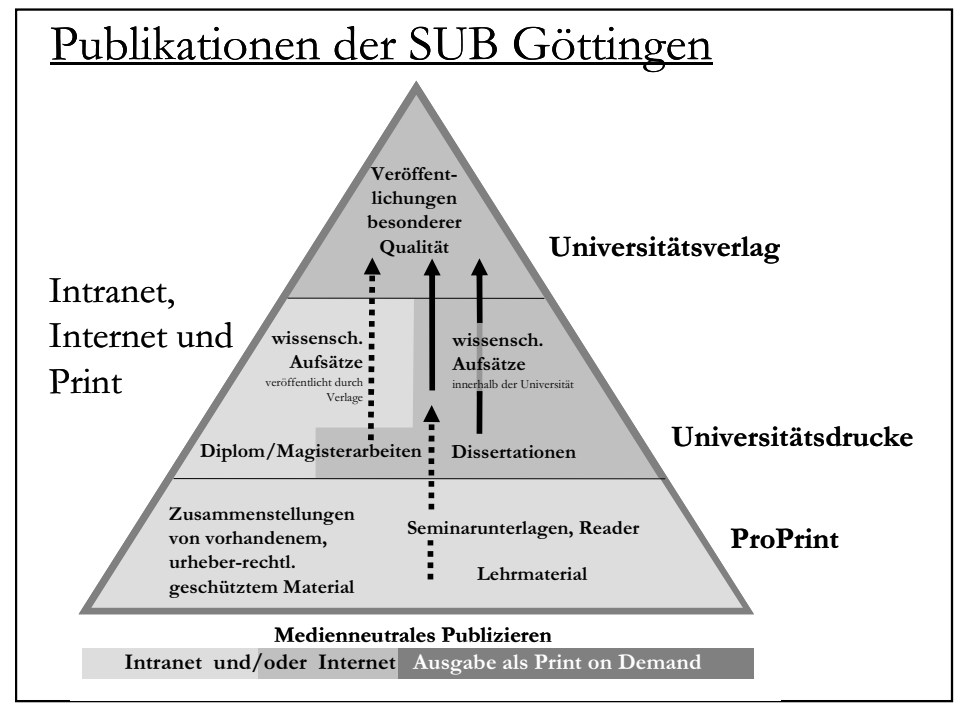

Abbildung 39: Publikationssystem für die Universität Göttingen mit dem Service ProPrint ${ }^{133}$

133 Der Service ProPrint wurde zusammen mit der HU Berlin als Print on Demand Dienst entwickelt. Frei zugängliche Dokumente oder Teile derselben auf Hochschul- und anderen Servern können damit zusammengestellt und bei lokalen Dienstleistern ausgedruckt werden. http://www.proprint-service.de. 
Die neuen Publikationssysteme gingen damit über die bloße Funktion als technische Plattform zur Speicherung von Dokumenten hinaus, sondern antizipierten die Potentiale des elektronischen Publizierens. Neben medienimmanenten Vorteilen wie Multimedialität, Crosslinking, Hypertext etc. bestehen diese in der zeitnahen Veröffentlichung, der kostengünstigen Verbreitung, der weltweiten und 24/365 Bereitstellung, der Vernetzungsmöglichkeit von verteilten Providern sowie der Interoperabilität der Dienste, die eine multidimensionale Recherche erlauben. Hierzu tragen Informationsintermediäre wie Google, MetaGer, RENARDUS, OAIster oder VASCODA sowie fachliche Nachweisinstrumente wie Clio online, der Anglistikguide oder die Virtuellen Fachbibliotheken ${ }^{134}$ bei.

Neben der klassischen Archiv- und der Publikationsfunktion werden Institutional Repositories als öffentlichkeitswirksames Medium der Selbstdarstellung eingesetzt. Das Jahrbuch der Max-Planck-Gesellschaft, traditionell veröffentlicht als kostenpflichtige Druckpublikation mit bibliographischer Datenbank auf CD, dient als Rechenschaftsbericht für die Leistungen der Gesellschaft. Die Publikationsleistungen der MPG-Mitarbeiter (bibliographische Angaben aller Veröffentlichungen sowie teilweise Volltexte) sind inzwischen durch den „eDocument Server“135 nachgewiesen. Mit diesem Projekt des setzt die MPG das Ziel um, ihren intellektuellen Output der breiten Öffentlichkeit zu präsentieren und für diese zu archivieren. An der Universität Tübingen ist mit „TOBIAS-lib“136 ein Publikationsserver entstanden, der Nutzern erlaubt, Dokumente nach Klassifikationen wie Autor, Titel, Thema, aber auch entlang der Organisationsstruktur der Universität zu suchen.

\subsection{Charakteristika von Hochschulverlagen}

Wenn hochschuleigene Publikationssysteme nicht nur „öffentliche Archive“ sondern leistungsfähige Komplementäre und Alternativen zum kommerziell dominierten Sektor darstellen sollen - die damit auch Wirkung auf den Markt ausüben -, müssen sie Funktionen des traditionellen Verlagswesens übernehmen. Das beinhaltet die Qualitätsprüfung, das Publizieren unter geklärten rechtlichen Rahmenbedingungen sowie die Erschließung der Inhalte in Nachweisinstrumenten und durch geeignetes Marketing. Der Bedarf für verlegerische Dienstleistung und gedruckte Werke ist mit dem Aufkommen des elektronischen Publizierens im Internet nicht verschwunden. Universitäten wie die TU Berlin, Kassel, Oldenburg, Siegen, Hildesheim, Göttingen, Karlsruhe und Potsdam haben daher ihre eigenen

\footnotetext{
134 http://www.clio-online.de, http://www.anglistikguide.de, http://www.virtuellefachbibliothek.de. $135 \mathrm{http}: / /$ edoc.mpg.de.

136 http://www.uni-tuebingen.de/ub/elib/tobias.htm. Einen Überblick über bereits bestehende Hochschulserver gibt die URL: http://www.e-teaching.org/didaktik/recherche/ quellen/dokserver/serverdirekt.
} 
Verlage gegründet, manche davon mit Buchumsätzen von über 10.000 Exemplaren pro Jahr. Sie sind angetreten, um kostengünstige Wege des Publizierens zu bieten, Hochschulen die Rechte an ihrem intellektuellen Output zu sichern und gleichzeitig einen möglichst freien Zugang zu wissenschaftlicher Information zu bieten. Trotz der Unterschiede im Betreibermodell, dem Verlagsprogramm oder der verlegerischen Philosophie lassen sich Gemeinsamkeiten dieser hochschuleigenen Verlage aufzeigen.

Sie stellen zumeist kleine Abteilungen dar, die vorwiegend mit externen Dienstleistern die Druckproduktion abwickeln, wohingegen die digitalen Angebote meist ,inhouse“ umgesetzt werden. Sie sind dort angesiedelt, wo an den Hochschulen die Kompetenz zum elektronischen Publizieren liegt - an Rechenzentren, in Abteilungen wie einem Computer- und Medienservice, oder wie häufig der Fall, an der Hochschulbibliothek. Die Anbindung an die Bibliothek gewährleistet beispielsweise die Integration der Verlagswerke in bibliothekarische Instrumente (Bibliographien, Verbundkataloge, Tauschnetzwerke) sowie die Unterstützung des laufenden Verlagsbetriebs durch bereits bestehende Geschäftsgänge (Finanzbuchhaltung, Vertrieb, Kontakte zu anderen Bibliotheken und Fachzeitschriften).

Als hochschuleigene Unternehmen arbeiten die Verlage nicht gewinnorientiert (vgl. Halle 2003, S. 244) und werden zumeist durch die Einbringung von universitären Eigenmitteln unterstützt. Sie arbeiten vornehmlich für die Angehörigen der Hochschule oder die Hochschule selbst. Der Universitätsverlag Göttingen ${ }^{137}$ bezieht besonderen Rückhalt an der Hochschule durch das Herausgebergremium der Universität, das aus Vertretern der Fakultäten zusammengesetzt ist. Das Gremium vertritt den Verlag in den Fakultäten, steuert seine programmatische Ausrichtung und gewährleistet für die oberste Sparte des Verlages die fachwissenschaftliche Qualität. Bei der Sparte Universitätsskripte wird nur Produktionsdienstleistung angeboten, bei den Universitätsdrucken wird die wissenschaftliche Qualität durch die Zugehörigkeit der Autoren zur Hochschule vorausgesetzt, bei der Sparte „Universitätsverlag“ wird jede Publikation durch die Fachvertretung im Herausgebergremium sowie zwei weitere Gutachter geprüft. Verlegt werden Einzelpublikationen, Dissertationen, Institutsreihen, Ringvorlesungen, Ausstellungskataloge oder Lehrmaterial. Aus bereits genannten Gründen stehen Zeitschriftenprojekte noch am Anfang der Umsetzung.

\subsection{Universitätsverlage am Markt}

Eine direkte Konkurrenzsituation zum privatwirtschaftlichen Sektor ist nach Halle (2003, S. 246) nicht gegeben. Dies liegt primär am diametral entgegengesetzten

137 http://univerlag.uni-goettingen.de. 
Unternehmensziel der Universitätsverlage, die zwar ökonomisch sinnvoll handeln müssen, deren vornehmliche Existenz aber nicht dem Markterfolg, sondern der Verbreitung von Wissenschaftsinformation dient. Daraus ergibt sich die besondere Art der Universitätsverlagsveröffentlichungen, etwa die Kopplung der Druckausgabe mit Open Access Bereitstellung auf einem zertifizierten Server, die für einen privatwirtschaftlichen Anbieter unrealisierbar oder kaum lohnend ist. Medienprodukte mit einem ausgeprägten Lokalbezug wie Vorlesungsbeilagen, Institutsschriften oder Tagungsbände werden von kommerziellen Verlagen häufig nur unter vollkommener Risikoübertragung an die Autoren angenommen. Die pessimistische Aussage von Halle (2003), „den Hochschulverlagen bl[iebe] gewissermaßen nur der Rest" von dem, was andere Verlage nicht wollen, darf - so die Erfahrung in Göttingen und Kassel - revidiert werden. An den Hochschulen wächst inzwischen das Verständnis, dass sich die Missverhältnisse des wissenschaftlichen Publikationssystems nur verändern, wenn Autoren den Mut haben, außerhalb der etablierten Strukturen zu veröffentlichen und dass die Zusammenarbeit mit einer hochschuleigenen Einrichtung (zeitnah, Service vor Ort) vorteilhaft sein kann.

Bei der Veröffentlichung sind Autoren von Universitätsverlagen - wie bei anderen Wissenschaftsverlagen auch - stark in den verlegerischen Prozess eingebunden. Von ihnen (oder durch ihre Vermittlung) stammen die unverzichtbaren Produktionskostenzuschüsse, die Vorschläge für Rezensenten, fachspezifische Kontakte für Marketingmaßnahmen, aber auch die publikationsreifen Vorlagen. Dies wird durch technische Entwicklungen erleichtert, Erstellungsprogramme wie LaTeX beinhalten Stylesheets für die Buchproduktion, die einfach an die Formatierungsvorgaben eines Verlages anzupassen sind und der gängigen Ästhetik im wissenschaftlichen Publikationsprozess entsprechen. Der Universitätsverlag Göttingen unterstützt seine Autoren mit „Do-it-yourself“ Hilfetexten und WordFormatierungsvorlagen. Im Gegenzug gelingt es den Hochschulverlagen, das PreisLeistungs-Verhältnis für die Autoren so günstig zu gestalten, dass die Entscheidung zur Stärkung des Verlages ihrer Alma Mater nicht mehr schwer fällt.

Denn die Philosophie des Open Access wird nicht nur durch den freien Zugriff im Internet umgesetzt, sondern schlägt sich in der Preisgestaltung der Druckwerke (sowohl beim Produktionskostenzuschuss als auch Verkaufspreis) nieder. Neue Arten der Preisgestaltung hat der digitale Druck ermöglicht, der die Auflagenhöhe durch Druck auf Bestellung ermöglicht. So wird im Universitätsverlag Göttingen bei Medienprodukten mit unklaren Absatzchancen die Auflagenhöhe anhand der Grundkosten für die Druckausgabe (ISBN, VLB, Datenpauschalen für dauerhafte Bereitstellung), der seitenpreisabhängigen Produktionskosten, der gewünschten Autorenexemplare und einer Startmenge an Verkaufsexemplaren kalkuliert. Die langfristige Lieferbarkeit gewährleistet die Zusammenarbeit mit einer zuverlässigen Druckerei, bei der die Druckvorlage für Nachbestellungen (auch 
in Menge 1) elektronisch gespeichert bleibt. War der digitale Druck zunächst nur für den einfachen S/W-Druck in „Copy-Shop Qualität“ einsetzbar, lassen sich inzwischen hochwertige Bücher im Print on Demand Verfahren herstellen. Die Druckmaschine Indigo UltraStream etwa, die beim Dienstleister des Universitätsverlages zum Einsatz kommt, erlaubt den Vierfarbdruck nahe an der Offsetqualität, sodass auch Farbseiten keine hohe Auflage erzwingen. Trotz des höheren Einzelpreises im digitalen Druck sinken die Kapitalbindung und damit das finanzielle Risiko für Verlag und Autoren.

\subsection{Universitätsverlage und Open Access}

Zum positiven Bild der Universitätsverlage trägt der Open Access Gedanke bei, der inzwischen in der wissenschaftlichen Gemeinschaft Fuß fasst. Dieser Gedanke ist für die Universitätsverlage programmatisch, indem er sich im direkten Angebot niederschlägt oder die Verlage eng mit den jeweiligen Publikationsservern verknüpft sind, so in Oldenburg, Potsdam, Weimar, Karlsruhe, Kassel oder Göttingen. Die „,kassel university press“ etwa bietet die verlegten Werke dreistufig an, als funktionsbeschränktes Open Access PDF (ohne Druck und Speicherung), kostenpflichtiges PDF (innerhalb der Universität Kassel Open Access) sowie als Buchausgabe. In Göttingen liegen viele gedruckt vorliegende Titel mit einer parallelen, kostenfreien elektronischen Vollversion auf, bei den restlichen ist mit den Autoren eine zeitversetzte Veröffentlichung der Open Access Ausgabe vertraglich vereinbart. Das Angebot von gedruckter Ausgabe und kostenfreier OnlineBereitstellung kann in Kannibalismus-Effekten münden, aber auch den Absatz des gedruckten Werkes fördern. Die „National Academic Press“ stellt ihre kostenpflichtigen Publikationen Open Access bereit. Der Absatz der gedruckten Werke, vor allem aus der Backlist, soll dadurch gestiegen sein.

Dieses Vorgehen entspricht den Ansätzen von Amazon ,search inside the book“ (siehe Seite 188) sowie dem neuen Service ,google print", wonach funktionsbeschränkter Open Access als Marketingmaßnahme für kostenpflichtige Vollversionen angeboten wird. Bei Dissertationen, für die es eine Publikations- und Verbreitungspflicht gibt und die aus Kostengründen zunehmend elektronisch auf Hochschulservern veröffentlicht werden, stellt sich die Frage, inwieweit der Publikationspflicht mit einer funktionsbeschränkten Onlineversion genügt wurde. Um den Absatz einer Druckausgabe zu gewährleisten, müssen daher andere Marketingmaßnahmen ergriffen werden, wenn die Prüfungsordnungen Vollversionen vorschreiben.

Die Alternative sind elektronische Dissertationsveröffentlichungen, bei denen Autoren nur die benötigten Pflichtexemplare für das Promotionsverfahren bezahlen und weitere Buchexemplare im Print on Demand Verfahren durch Verkaufs- 
bestellungen finanziert werden. An der Universität Göttingen wird dies durch Integration der elektronischen Ausgabe in den Print-on-Demand Service „ProPrint“ realisiert.

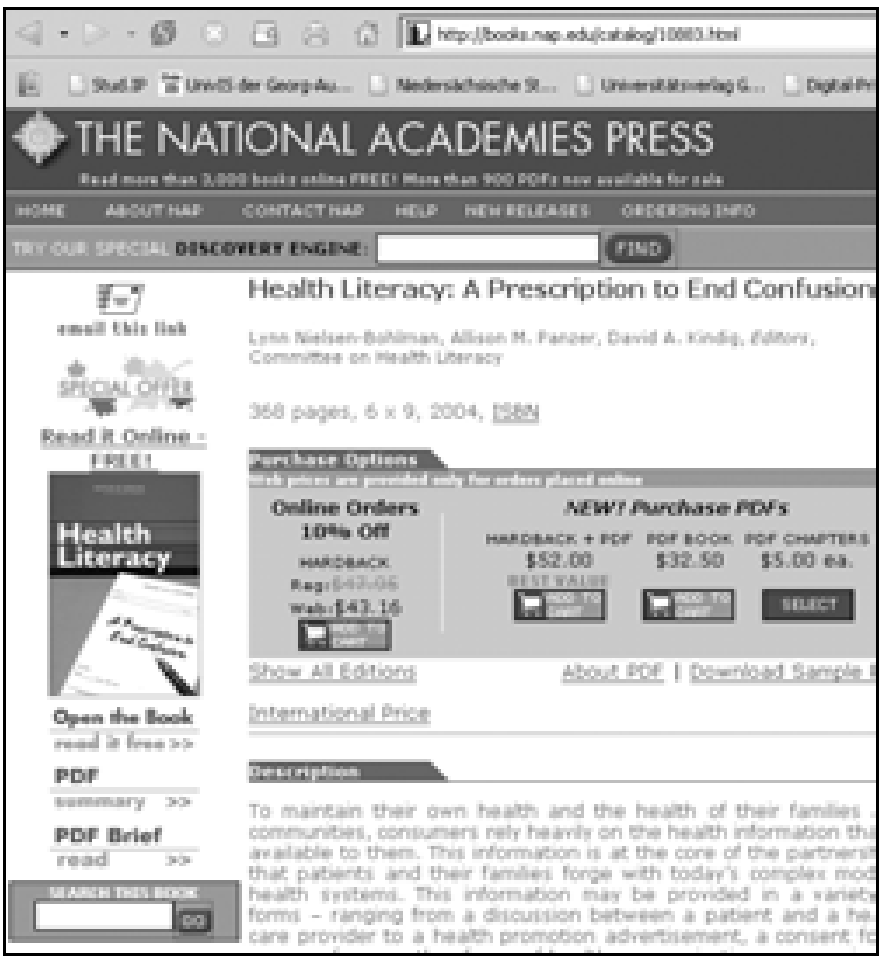

Abbildung 40: Katalogeintrag mit „Open the Book"138 und Bestelloption für das gebundene Buch sowie verschiedene PDF-Varianten

\subsection{Zukunftschancen von Universitätsverlagen}

Eine wirtschaftliche Bedeutung wie die Oxford University Press mit ihren weltweit 3.700 Mitarbeitern wird kein deutscher Universitätsverlag mehr erringen können, bietet doch der wissenschaftliche Publikationsmarkt kaum noch Entfaltungsmöglichkeiten und lässt sich die historische gewachsene Stellung des Oxforder Verlages nicht wiederholen. Dennoch kann die Zukunft der deutschen Uni-

${ }^{138}$ Die „Open the Book“ Option zeigt die einzelnen Buchseiten als Image. Dennoch ist eine Recherche im Volltext möglich, indem die Textebene des Original-PDFs - das allerdings nicht aufgerufen werden kann - durchsucht wird. Das Suchergebnis wird nur abschnittsweise in HTML dargestellt, so dass kein Kopieren größerer Textmengen möglich ist. Der Initialaufwand zum Aufsetzen des technischen Systems ist beträchtlich, die laufende Bereitstellung auf diesem Wege wäre jedoch auch für kleinere Verlage möglich. 
versitätsverlage positiv gesehen werden. Ihre Entfaltungsmöglichkeiten sind in ihrer Hochschulnähe und ihrer programmatischen Ausrichtung des Open Access begründet, indem sie in einem gewandelten wissenschaftlichen Publikationssystem eine Rolle besetzen, die nur sie einnehmen sollten. Das ist etwa die Sicherung der grundsätzlichen Verwertungsrechte an wissenschaftlichen Ergebnissen der Hochschule für die Hochschule, was die Vergabe von Lizenzen an kommerzielle Verlage nicht ausschließt. Auch die Bereitstellung von Infrastruktur für hochschuleigene Open Access Zeitschriften ist Aufgabe eines Hochschulverlags. Denkbar sind z. B. elektronische Zeitschriften, deren Druckausgabe im Print on Demand Verfahren zur gezielten Schonung des Literaturetats eingesetzt werden, indem damit Zeitschriften anderer Institutionen eingetauscht werden können.

Um die Medienproduktion nicht zur unrentablen Bindung von Eigenmitteln an Instituten oder Bibliotheken verkommen zu lassen, bedarf es der Standardisierung der Produktionsabläufe. Dies ermöglichen z. B. XML-Konverter, die medienneutrale Publikation aus gängigen Erstellungsformaten erlauben, Serverarchitekturen zum selbständigen Hochladen von Dokumenten durch Autoren oder elektronische Workflows zur Selbstorganisation von Herausgebern elektronischer Zeitschriften. Zurzeit herrschen die Dokumentformate PDF und Postscript vor, die von unterschiedlichen Erstellungsprogrammen gezogen werden. Hinzuarbeiten ist auf Formate wie XML, die Struktur, Layout und Inhalt voneinander differenzieren: so aufbereitete Dokumente können ohne Konvertierung für alle Arten des Publizierens vorgehalten werden und lassen sich langfristig ohne Informationsverlust auch für die Nachwelt archivieren.

Auf einer übergreifenden, technischen Ebene hängt der Erfolg der Hochschulverlage von ihrer weiteren Verknüpfung mit Institutional Repositories und Publikationsservern ab. Hierzu bedarf es der kooperativen Fortentwicklung von Standards (Persistent Identifier, kompatible Publikationssysteme, Metadatenschemata), Tools (XML-Konverter und Stylesheets für gängige Erstellungsprogramme) und nicht zuletzt der noch ungelösten Langzeitarchivierung.

Infrastrukturell gesehen, benachteiligt der derzeitige Umgang mit dem Urheberrecht und der Verteilungsmodus der Verwertungsgesellschaften (vgl. Mühlbauer 2004 und Ermert 2004) alternative Veröffentlichungsstrategien gegenüber den etablierten kommerziellen Verlagen. Für den Erfolg von hochschuleigenen Publikationswegen gilt es hierauf einzuwirken, wie es etwa das Aktionsbündnis „Urheberrecht für Bildung und Wissenschaft“" fordert ${ }^{139}$. Damit Open Access Verleger und hochschuleigene Publikationssysteme wirtschaftlich bestehen können, muss die generelle Zusage der Wissenschaftsförderinstitutionen, Publikationsbeihilfen zu gewähren und alternative Veröffentlichungen gleichberechtigt zu behandeln, weiter in die Tat umgesetzt werden. Auch neue Arten der Evaluierung von Publi-

139 http://www.urheberrechtsbuendnis.de/unterzeichnen.html. 
kationsleistung sind vonnöten wie die quantitative Nutzungserfassung sowie artikelbasierter „hit factor“ statt dem „Impact Factor“. Wo eine Publikationspflicht besteht, etwa bei Projektberichten oder Qualifizierungsarbeiten, sollten Persistent Identifier der elektronischen Version den gleichen Stellenwert haben wie bisher die ISBN als Garant für die Verbreitung als Buch.

Die Hochschulen haben einen besonderen Einfluss auf den Erfolg ihres Verlages. Dies beginnt bei der Vermittlung von Medienkompetenz für Lehrende und Studierende - nur wer publikationsgerecht produzieren kann, wird sich an alternativen Publikationswegen beteiligen, die eine höhere Eigenverantwortung für die Autoren mit sich bringen. Auch für das medienneutrale Publizieren per XML, das reibungslos laufend - den Publikationsprozess erheblich vereinfachen kann, ist die Autorenkompetenz ein kritischer Faktor. Wenn an Hochschulen der Open Access Gedanke verantwortungsbewusst aufgegriffen und propagiert wird, wächst die Akzeptanz für neue Veröffentlichungsstrategien und damit die Zukunftschancen für Hochschulverlage und ihre flankierenden digitalen Publikationsserver. Das privatwirtschaftliche Verlagswesen wird durch die Stärkung von hochschuleigenen Publikationswegen nicht vergehen, sondern erhält einen Komplementär, der für das gesellschaftliche System Wissenschaft dringend notwendig ist.

\section{Literatur}

Albanese (2004): Albanese, A.: BioMed Central Changes Tack - Flat fee becomes "per article published"; can institutions shift? In: Library Journal, 15. März 2004, http://www.libraryjournal.com/article/CA386750.

Aldrick (2004): Aldrick, P.: Reed boss denies 'profiteering'. Daily Telegraph, August 6, 2004, Onlineausgabe.

Bergstrom (2001): Bergstrom, T.: Free Labour for costly journals, 20. März 2001, URL: http://www.econ.ucsb.edu/ tedb/jep.pdf.

Cozzarelli (2004): Cozzarelli, N. R.: An open access option for PNAS. In: Proceedings of the National Academy of Sciences in the United States of America, June 8, 2004, vol. 101, no. 23, 8509 .

Crow (2002): Crow, R.: „The Case for Institutional Repositories“. SPARC-Papier, 2002, http://www.arl.org/sparc/IR/ir.html.

de Vries (2001): de Vries, J.: Peer Review: The Holy Cow of Science. In E.H. Fredriksson (ed.): A Century of Science Publishing. IOS Press, 2001, pp. 231-244.

Degenhardt (1997): Degenhardt, E.: "Elektronische Dissertationen" in Bibliotheken. Diplomarbeit an der FH Hannover 1997, veröffentlicht unter http://transfer.ik.fh-hannover.de/ik/person/bock/degenhardt/ediss.pdf.

Ermert (2004): Ermert, M.: Urheberrecht: Gesittet "Pfui" sagen. Heise online News 02.11.2004. http://www.heise.de/newsticker/meldung/52841. 
Füssel (2001): Füssel, S.: Geisteswissenschaften und digitale Medien. Von der Medienkonkurrenz zur Mediensymbiose. Aus: Börsenverein des Deutschen Buchhandels, der Bundesvereinigung Deutscher Bibliotheksverbände und der Deutschen Bibliothek (Hg.): Wissenschaftspublikation im digitalen Zeitalter. Wiesbaden 2001.

Grätzel von Grätz (2004): Grätzel von Grätz, P.: Britisches Unterhaus sagt "Ja, aber" $\mathrm{zu}$ Open Access in der Wissenschaft., URL: http://www.heise.de/tp/ r4/artikel/17/17927/1.html, Heise Online 23.07.2004.

Halle (2003): Halle, A.: Wissenschaftliche Publikationskultur und Hochschulverlage. In: Zeitschrift für Bibliothekswesen und Bibliographie, Jahrgang 50 (2003) 5, S. 243-250 (ZfBB).

Harnad (2000): Harnad, S.: Ingelfinger Over-Ruled: The Role of the Web in the Future of Refereed Medical Journal Publishing. In: The Lancet 256, S16ff. http://cogprints.ecs.soton.ac.uk/archive/00001703/00/harnad00.lancet.htm.

HRK (2002): HRK: Neuausrichtung des Informations- und Publikationssystems der Deutschen Hochschulen. Hochschulrektorenkonferenz, Bonn 2002.

Jäger (2001): Jäger, G.: Geschichte des deutschen Buchhandels im 19. und 20. Jahrhundert. Bd. 1. Frankfurt am Main 2001.

Kaiser (2004): Kaiser, J.: Seeking Advice on 'Open Access,' NIH Gets an Earful. In: Science, Vol 305, Issue 5685, 764 , 6 August 2004, [DOI: $10.1126 /$ science.305.5685.764b].

Klaus / Röttger (1998): Klaus, E., Röttger, U.: Medium, Organisation, Nutzung: Bedingungen erfolgreicher Öffentlichkeitsarbeit im Internet. In: Irene Neverla (Hg.): Das Netz-Medium. Kommunikative Potentiale eines Mediums in Entwicklung. Opladen 1998, S. 219-243.

McCabe / Snyder (2004): McCabe, M., Snyder, C.: The Economics of Open Access Journals. Preprint der eingereichten Veröffentlichung in "Journal of the European Economic Association“. Oktober 2004 http://www.prism.gatech.edu/ $\sim$ mm284/OA.pdf.

McCabe (2002): McCabe, M.: Journal Pricing and Mergers: A Portfolio Approach. In: American Economic Review, Vol. 92, No. 1, March, 2002. URL: http://www.prism.gatech.edu/ mm284/AER.pdf.

Meier (2002): Meier, M.: Returning Science to the Scientists. Der Umbruch im STMZeitschriftenmarkt unter Einfluss des Electronic Publishing. München 2002.

Mühlbauer (2004): Mühlbauer, P.: Zwischenbericht aus der Wurstfabrik (sic!) Heise online, 04.11.2004. http://www.heise.de/tp/deutsch/special/copy/18721/ 1.html.

Poynder (2004): Poynder, R.: British Politicians Call on U.K. Government to Support Open Access. Information Today, 11. August 2004, URL http://www.infotoday.com/newsbreaks/nb040726-1.shtml. 
Sabbagh (2004): Sabbagh, D.: Reed Elsevier chief hits back in scientific publishing row. In: Times Online, August 06, 2004, http://business.timesonline.co.uk/ article/0,9071-1204570,00.html.

Savenije (2001): Savenije, J. S. M.: The Roquade project: Towards new models in scientific communication. Paper presented at the IATUL Conference, Delft, 29 May 2001 http://www.library.uu.nl/staff/savenije/publicaties/IATUL2001.htm.

Siebeck (2004): Siebeck, G.: Freibier für die Wissenschaft? Open Access. Die öffentliche Hand verhindert einen fairen Wettbewerb. In: Börsenblatt 43-2004, S. 11.

Van Orsdel / Born (2003): Van Orsdel, L., Born, K.: Big Chill on the Big Deal? In: Online Ausgabe des Library Journal, 15.04.2003. http://www.libraryjournal.com. 



\section{Das wissenschaftliche Journal und seine möglichen Alternativen: Veränderungen der Wissenschafts- kommunikation durch das Internet}

Heidemarie Hanekop / Volker Wittke

\section{Einführung}

Das Internet hat die Art und Weise, wie Wissenschaftler miteinander kommunizieren und wie sie ihre Ergebnisse publizieren, weitreichend verändert. Emails, Mailinglisten und Homepages dienen der Beschleunigung und Intensivierung wissenschaftlicher Kommunikation. Neue Publikationstechnologien einerseits und die fortschreitende Globalisierung und weltweite Vernetzung des Wissensaustauschs andererseits bedingen und verstärken sich gegenseitig. Internetbasierte Publikationen führen zunehmend zu einem grundlegenden Formwandel wissenschaftlicher Publikationen. Insbesondere bei Periodika gewinnen derzeit digitale über das Internet zugängliche Publikationen rasch an Bedeutung. Auch etablierte Journale werden über das Internet angeboten, neue Journale werden zunehmend als E-Journale konzipiert. Allerdings ist der Wandel der formellen Wissenschaftskommunikation - der wissenschaftlichen Publikation - ein komplexer und langwieriger Prozess. Dies hängt damit zusammen, dass nicht nur wissenschaftsinterne 
Strukturen und Prozesse tangiert sind, sondern auch die Wertschöpfungsprozesse wissenschaftlicher Verlage. Mit dem Formwandel wissenschaftlicher Journale verändern sich auch die Geschäftsmodelle der Verlage und die Austauschbeziehungen und Interdependenzen zwischen Wissenschaft und Verlagen. In den letzten Jahren wachsen die Spannungen zwischen der Wissenschaft - repräsentiert durch die Bibliotheken als institutionelle Nachfrager stellvertretend für die Wissenschaftler als Leser - und den kommerziellen Verlagen. Sie entzünden sich an steigenden Preisen insbesondere für die wachsende Zahl an STM-Journalen, deren Finanzierung den Etat der Bibliotheken zu sprengen droht. Die Open Access Bewegung ist ein Ausdruck der wachsenden Kritik am bisherigen Publikationssystem. Kritisiert wird unter anderem, dass die Veröffentlichungstätigkeit der Verlage mit der beschleunigten Wissensproduktion nicht Schritt hält - festgemacht an der langen Dauer des Produktionsprozesses bis zur Veröffentlichung und an steigenden Ablehnungsquoten eingereichter Manuskripte (90\%ige Ablehnungsquoten bei STMJournalen). Auch das von den Verlagen organisierte Peer Review Verfahren wird zunehmend als dysfunktionales Nadelöhr thematisiert. Die schärfste Kritik an den großen Verlagen entzündet sich jedoch an den überproportionalen Preissteigerungsraten wissenschaftlicher Journale, die von Bibliotheken kleinerer oder finanzschwächerer Universitäten und Forschungseinrichtungen kaum mehr aufgebracht werden können. Der Zugang zu wissenschaftlichen Publikationen verschlechtert sich trotz oder gerade wegen der dynamisch wachsenden Wissensproduktion - so das Argument der Kritiker. Diese Entwicklung stehe in krassem Widerspruch zu den neuen technischen Möglichkeiten, die eine schnellere und kostengünstigere Distribution und verbesserte Zugangsmöglichkeiten erlauben. Der Zugang zu den neuesten wissenschaftlichen Ergebnissen wird somit ausgerechnet in einer Situation zum Problem, in der die wissenschaftliche Kommunikation durch weltweite Vernetzung, fortschreitende Spezialisierung und wachsende Anforderungen an den Wissenstransfers zwischen Wissenschaft, Wirtschaft und Gesellschaft (Gibbons 1994, Novotny 2004) immer wichtiger wird.

Die Forderung nach Open Access wird nicht zuletzt auch damit begründet, dass wissenschaftliche Ergebnisse, die im allgemeinen Interesse mit öffentlichen Mitteln finanziert werden, dem Leser aus Wissenschaft und Gesellschaft als öffentliches Gut frei zur Verfügung stehen sollten. Es sei nicht länger hinzunehmen, so die zugespitzte Argumentation der Open Access Protagonisten (z. B. Hanard 2001; Odlyzko 2000), dass die Wissenschaft das von ihr produzierte Wissen als immer teurere Ware von den Verlagen zurück kaufen müsse. Open Access impliziert daher sowohl den Einsatz avancierter internetbasierter Technologien für den uneingeschränkten weltweit und jederzeit verfügbaren Zugang, wie auch neue Publikationsmodelle. Damit stehen zentrale Elemente des bisherigen Publikationssystems zur Disposition. 
Unsere These ist, dass diese Veränderungen weder allein aus der Perspektive des Wissenschaftssystems noch allein aus der Logik der Wertschöpfung heraus erklärt und beurteilt werden können. In der Diskussion um zukünftige bzw. alternative Publikationsmodelle müssten daher die besonderen institutionellen Verschränkungen und gegenseitigen Abhängigkeiten zwischen Wissenschaft und Verlagen stärker berücksichtigt werden.

Wir wollen mit diesem Beitrag den theoretischen Bezugsrahmen aus wissenschaftssoziologischer Perspektive darlegen, um auf dieser Grundlage unterschiedliche Open Access Publikationsmodelle zu diskutieren. Im ersten Teil des Beitrags beschreiben wir den Produktionsprozess wissenschaftlicher Journale als Wertschöpfungsprozess einerseits und als Teil der Wissenskommunikation andererseits. Auf dieser Grundlage analysieren wir die bisherige institutionelle Verschränkung zwischen (kommerziellen) Verlagen und dem Wissenschaftssystem. Triebkräfte, die zum Aufbrechen dieser Verschränkung führen könnten, kommen im Konflikt um die so genannte Zeitschriftenkrise zum Ausdruck, aber auch im Wandel der Rezeptionsformen von Wissenschaftlern durch die Nutzung des Internets. Im letzten Abschnitt diskutieren wir alternative Open Access Publikationsformen.

\section{Die Besonderheiten des Wertschöpfungsprozesses wissenschaftlicher Journale}

Auf den ersten Blick wirkt der Wertschöpfungsprozess wissenschaftlicher Journale vergleichsweise unkompliziert: Hauptakteure sind Verlage, die wissenschaftliche Publikationen produzieren, vermarkten und vertreiben. Der Kern verlegerischer Tätigkeit konzentriert sich auf Contentverarbeitung, Auswahl und thematische Bündelung der von wissenschaftlichen Autoren gelieferten Aufsätze zu einem Journal mit inhaltlich und qualitativ definiertem Profil. Dies schließt die Akquise der Artikel ebenso ein wie die Organisation des Auswahlverfahrens durch wissenschaftliche Gutachter (peer review) und die Zusammenarbeit mit Herausgebern bei der Konzipierung des Journals und der Erstellung nicht artikelgebundener Bestandteile. Wissenschaftler sind als Contentlieferanten, Gutachter und Editoren beteiligt. Die Nachfrageseite konzentriert sich ebenfalls weitgehend auf Wissenschaftler als Leser wissenschaftlicher Publikationen. Bei genauerer Betrachtung ergeben sich allerdings einige Besonderheiten der Austauschbeziehungen zwischen den beteiligten Akteuren, die aus der Logik eines wirtschaftlichen Wertschöpfungsprozesses nicht zu verstehen sind.

Als erste Besonderheit fällt auf, dass Verlage nicht Contentproduzenten sind, sondern lediglich die Inhalte be- bzw. verarbeiten, die ihnen wissenschaftliche Autoren liefern. Das allein wäre nichts Besonderes, denn eine eigenständige Contentvorstufe ist bei der Medienproduktion nicht ungewöhnlich. Allerdings sind die 
Contentlieferanten üblicherweise an der Wertschöpfung beteiligt, ob als Arbeitnehmer, Auftragnehmer oder als Zulieferer. Wissenschaftliche Autoren hingegen produzieren weder im Auftrag der Verlage noch partizipieren sie an der ökonomischen Wertschöpfung der Verlage. Sie liefern einen maßgeblichen Beitrag zur Wertschöpfung obwohl sie nicht am Ertrag der Wertschöpfung durch die Verlage beteiligt werden. Auch die i. d. R. ebenfalls unentgeltliche Tätigkeit von Gutachtern, Editoren und Mitherausgebern leistet einen entscheidenden Beitrag zur Qualität und damit zum ökonomischen Erfolg der Publikation.

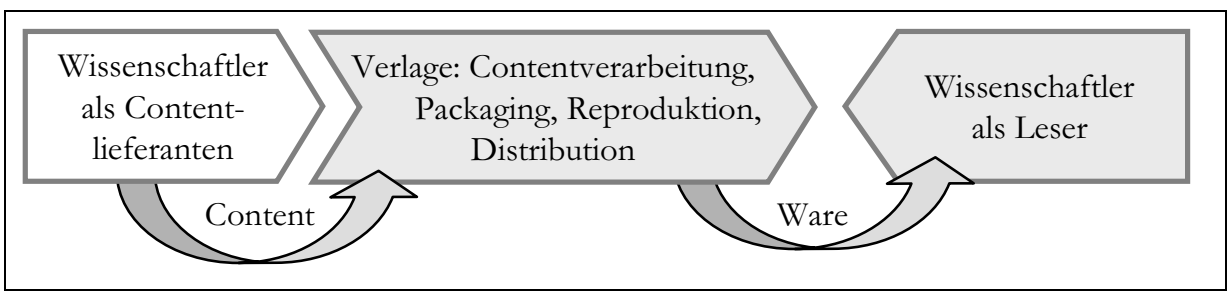

Abbildung 41: Wertschöpfungsprozess wissenschaftlicher Journale

Die Verlage sind darauf angewiesen, dass Wissenschaftler als Autoren ihnen das Rohmaterial in Form von wissenschaftlichen Aufsätzen auch ohne ökonomischen Anreiz liefern. Diese Art der Beziehung zwischen Verlagen und Wissenschaftlern ist wichtig, um zu verstehen, weshalb Verlage viel Energie auf die Pflege ihrer Beziehungen zu den Autoren verwenden. Denn für den ökonomischen Erfolg der Verlage ist es von entscheidender Bedeutung anerkannte Wissenschaftler als Autoren zu gewinnen und neue, wissenschaftlich relevante Aufsätze zu publizieren. Ebenso wichtig ist es renommierte Wissenschaftler als Editoren zu gewinnen und einen Kreis kompetenter Gutachter zu organisieren, die die Manuskripte der Autoren bewerten und auswählen, so dass sie dem wissenschaftlichen Stand entsprechen und die zentralen Themen der jeweiligen Community widerspiegeln.

Aus der Perspektive des Wertschöpfungsprozesses bleibt unverständlich, warum Autoren den Verlagen das Ergebnis ihrer wissenschaftlichen Arbeit unentgeltlich zur wirtschaftlichen Verwertung überlassen und weshalb Gutachter und Editoren ihre Arbeit ebenfalls unentgeltlich einbringen.

Eine zweite Besonderheit des Wertschöpfungsprozesses der Verlage resultiert aus der Doppelrolle der Wissenschaftler als Produzenten und Nachfrager. Als Produzenten arbeiten sie unentgeltlich, als Nachfrager jedoch sollen sie das von Kollegen produzierte Wissen als Ware kaufen. Diese Besonderheit spielt in der Diskussion um alternative Publikationsformen eine wichtige Rolle, denn analog zu anderen internetbasierten peer-to-peer Beziehungen (z. B. bei Musiktauschbösen) könnte man den Austausch wissenschaftlicher Publikationen zwischen Autoren und Lesern direkt, d. h. ohne Verlage organisieren. 
Betrachtet man die Erstellung wissenschaftlicher Publikationen von der Seite des Wertschöpfungsprozesses, so läst sich der Beitrag der Wissenschaftler nur schwer als rationales Handeln zu verstehen. Im nächsten Abschnitt werden wir zeigen, dass das Verhalten der Wissenschaftler nicht aus der Logik des Wertschöpfungsprozesses, wohl aber aus der Logik des Wissenschaftssystems erklärt werden kann. Im Folgenden werden wir daher untersuchen, welche Funktionen wissenschaftliche Publikationen aus der Perspektive des Wissenschaftssystems haben.

\section{Die Funktion der wissenschaftlichen Publikationen im Wissenschaftssystem}

Wissenschaftliche Publikationen erfüllen zentrale Funktionen bei der Produktion wissenschaftlichen Wissens. Wissenschaftliches Wissen zeichnet sich gegenüber anderen Wissensformen dadurch aus, dass es ausschließlich der wissenschaftlichen Wahrheit verpflichtet ist. Zudem soll es originell und relevant sein (Luhmann 1992, Merton 1985). Die Aufgabe des Wissenschaftssystems in modernen Gesellschaften besteht in der beschleunigten Produktion dieser Art von Wissen. Hierzu bedarf es einer besonderen Art der Steuerung, der die Produktion dieser Art von Wissen stimuliert ${ }^{140}$, sie privilegiert und seine private Aneignung verhindert. Dies rechtfertigt die relative Autonomie der Wissenschaft innerhalb moderner, kapitalistischer Gesellschaften.

Den besonderen Steuerungsmodus im Wissenschaftssystem hat Merton (Merton 1985, S. 86ff.) als normative Struktur der Wissenschaft beschrieben. Er formuliert vier Grundprinzipien (wissenschaftliche Normen): 1. die Universalität des Wissens, 2. die Kollektivität der Wissensproduktion, 3. die Uneigennützigkeit der Wissensproduzenten und 4. das Prinzip des organisierten Skeptizismus. Universalität meint, dass die Wissensproduktion ausschließlich wissenschaftlichen Kriterien (der Wahrheitsfindung) verpflichtet sein soll. Weder Religion, Rasse noch Geld sollen den wissenschaftlichen Erkenntnisprozess bestimmen. Die Norm der Kollektivität (oder des Kommunismus) definiert die Wissensproduktion als kollektiven Prozess, der darauf basiert, dass neue Erkenntnisse schnell verbreitet werden, damit sie von anderen Wissenschaftlern verwendet werden können. Konstitutiv ist daher gerade nicht private Aneignung und Verwertung, sondern uneigennütəige Weitergabe in der Scientific Community. Erst wenn der Wissenschaftler seine neuen Erkenntnisse durch Veröffentlichung aus der Hand gegeben hat, kann er dafür im Wissenschaftssystem eine Belohnung in Form von Anerkennung erwarten. Durch das auf Anerkennung beruhende Belohnungssystem wird die kollektive Wissens-

140 Kennzeichnend für die Wissensproduktion in modernen Gesellschaften ist ihr exponentielles Wachstum, das sich ebenso wie die Zahl der Wissenschaftler jeweils innerhalb von 15 Jahren verdoppelt (Solla Price 1974, S. 25). 
produktion privilegiert. Auch die spezifische Form von Intellectual Property Rights innerhalb des Wissenschaftssystems ist nicht auf ökonomische Verwertungsrechte ausgerichtet, sondern sie sichert durch die Publikation den Anspruch des Wissenschaftlers auf Priorität der Erkenntnis (den Anspruch, neues Wissen produziert zu haben). Organisierter Skeptizismus schließlich bildet die normative Grundlage für die dem Wissenschaftssystem eigene Form der Qualitätssicherung, die dafür sorgt, dass nur solche Ergebnisse (als wissenschaftliches Wissen) Geltung beanspruchen, die vor dem Urteil anderer Wissenschaftler Bestand haben (Beurteilung durch Peers). Dieses Prinzip hat zwei wichtige Implikationen:

Erstens: Steuerung und Qualitätskontrolle bei der Wissensproduktion sind ein Kommunikationsprozess. In der kritischen Auseinandersetzung unter den Wissenschaftlern der (jeweiligen) Scientific Communiy wird entschieden, was wahr und relevant ist. Oder anders formuliert: Wissenschaftskommunikation ist der entscheidende Steuerungsmechanismus der Wissensproduktion.

Zweitens: Die Autonomie der Wissenschaft manifestiert sich darin, dass kein der Wissenschaft externer Kontroll- und Steuerungsmechanismus anerkannt wird, sondern allein die kritische Bewertung der Wissenschaftler untereinander als Kontrollsystem fungiert.

Den Merton'schen Grundprinzipien des Wissenschaftssystems entspricht ein Anreizsystem zur Produktion neuen Wissens, dessen Währung nicht Geld ist, sondern Anerkennung. Reputation in der Scientific Community ist die Wäbrung mittels derer Status und Ressourcen verteilt werden. Die Währung Reputation hat einige Besonderheiten, die für das Verständnis des Steuerungsmodus im Wissenschaftssystem wichtig sind. Da nur solche wissenschaftlichen Erkenntnisse anerkannt werden können, die verbreitet (kommuniziert) wurden, steigen die Chancen auf wissenschaftliche Anerkennung mit verbesserten Möglichkeit ihrer Verbreitung. Die Entstehung von wissenschaftlichen Journalen fällt daher nicht zufällig mit der Entwicklung des Drucks zusammen. Gegenwärtig beobachten wir einen vergleichbaren Veränderungsprozess durch die neuen Verbreitungsmöglichkeiten des Internets.

Für wissenschaftliche Anerkennung reicht jedoch die Verbreitung neuer wissenschaftlicher Erkenntnisse allein reicht nicht aus, sie müssen auch gelesen werden. Reputation in der Scientific Community entsteht in einem durch selektive Aufmerksamkeit geprägten Kommunikationsprozess. Unter den vielen neuen Erkenntnissen werden immer nur wenige so herausragend wahrgenommen, dass sie bleibende Anerkennung finden. Die Dynamik der Wissensproduktion lebt davon, dass viele Wissenschaftler ihre Befunde vorstellen, von denen aber nur wenige die Anerkennung der Fachkollegen finden. Kennzeichnend für die Wissenschaftskommunikation ist daher die hohe Selektivität der Rezeption und Aufmerksamkeit. Das Zitat ist die formalisierte Form wissenschaftlicher Anerkennung. Die Bezugnahme auf vorgängiges Wissen durch das Zitat gehört zu den Grundregeln der 
Wissensproduktion. Das Zitat ist in gewisser Weise die normative Voraussetzung für die uneigennützige Weitergabe der neuesten Erkenntnisse durch den Autor, denn sie entschädigt ihn in Form von formalisierter Anerkennung und Reputation. Wissenschaftliche Anerkennung wird durch das Zitat messbar. Zitationsraten sind ein Messinstrument für Anerkennung, hohe Zitationsraten generieren Reputation im Wissenschaftssystem.

Eine weitere Besonderheit von Reputation resultiert aus dem Charakter des Kommunikationsprozesses: Aufmerksamkeit wird auch durch vorherige Kommunikation geweckt. Wenn über einen Kollegen und seine Arbeit bereits gesprochen wird, ist die Aufmerksamkeit und damit auch die Chance auf Anerkennung gröBer. Reputation wächst schneller, wenn sie an vorgängige Reputation anknüpfen kann. Merton beschreibt dieses Phänomen als Matthäus-Effekt. Wer bereits Reputation hat (z. B. ein berühmter Wissenschaftler), erhält bevorzugt weitere Reputation. Umgekehrt ist es für einen jungen unbekannten Wissenschafter schwieriger, Aufmerksamkeit auf sich zu ziehen und wissenschaftliche Anerkennung zu erlangen (Merton 1985, S. 147ff.). Wenn ein bekannter und ein junger Wissenschaftler gemeinsam einen interessanten Aufsatz veröffentlichen, wird dieser in der Wahrnehmung der Wissenschaftler üblicherweise dem bekannten Wissenschaftler zugeschrieben. Allerdings kann dessen Reputation auf den jungen Wissenschaftler abfärben, z. B. wenn dieser einen weiteren Aufsatz veröffentlicht, dem Aufmerksamkeit zukommt, weil er bereits einmal mit dem bekannten Wissenschaftler zusammen publiziert hat. Daraus folgt, dass Reputation akkumuliert und auch übertragen werden kann. Matthäus-Effekte lassen sich nicht nur für Wissenschaftler beschreiben, sondern auch für Forschergruppen und Forschungseinrichtungen. Wir werden unten zeigen, wie Verlage sich diesen Mechanismus bei der Etablierung einer Hierarchie von Journalen zunutze machen.

Für das Wissenschaftssystem nimmt das Journal eine zentrale Funktion bei der Verbreitung, Qualitätssicherung und Selektion ein. Von besonderer Bedeutung sind in unserem Zusammenhang jedoch die auf Journalen basierenden Mechanismen zur Generierung formalisierter Indikatoren für Anerkennung. Wissenschaftliche Anerkennung und Reputation wird in vielen Wissenschaftsfeldern ganz wesentlich durch Journale generiert (insbesondere in den so genannten STMWissenschaften). Diese Bedeutung von wissenschaftlichen Journalen erklärt - wie wir unten zeigen werden - warum sich Wissenschaftler am Wertschöpfungsprozess der Verlage beteiligen. Den Verlagen ist es gelungen eine Schlüsselstellung bei der formalisierten Messung von Anerkennung zu erringen, obgleich sie nicht zum Wissenschaftssystem gehören, sondern als Teil des Wirtschaftssystems nach ökonomischen Grundprinzipien agieren (müssen). 


\section{Die besondere institutionelle Interdependenz zwischen Wissenschaft und Verlagen}

Verlage haben wissenschaftliche Journale als eine zentrale Institution im Steuerungssystem der Wissenschaft etablieren können, indem sie die oben beschriebenen Grundprinzipien des Wissenschaftssystems für ihre Verwertungsinteressen instrumentalisieren. Das Verblüffende ist, dass die wissenschaftlichen Grundprinzipien und Normen eigentlich ökonomischen Verwertungsinteressen zu widersprechen scheinen. Wir wollen im Folgenden zeigen, wie dieser Widerspruch in einer besonderen Form der Interdependenz zwischen Wissenschaftssystem und Verlagen (bisher) aufgehoben wird.

Verlage nutzen das dynamische Wachstum der Wissensproduktion (Solla Price 1974) für ihre ureigenen Verwertungsinteressen. Es bietet ihnen die Möglichkeit mit der gleichen exponentiellen Geschwindigkeit immer neue Märkte für neue Journale zu erschließen. Das exponentiell wachsende Wissen wirkt wie ein beständiger Zufluss zum Wertschöpfungsprozess, der sich im exponentiellen Wachstum neuer Journale ausdrückt.

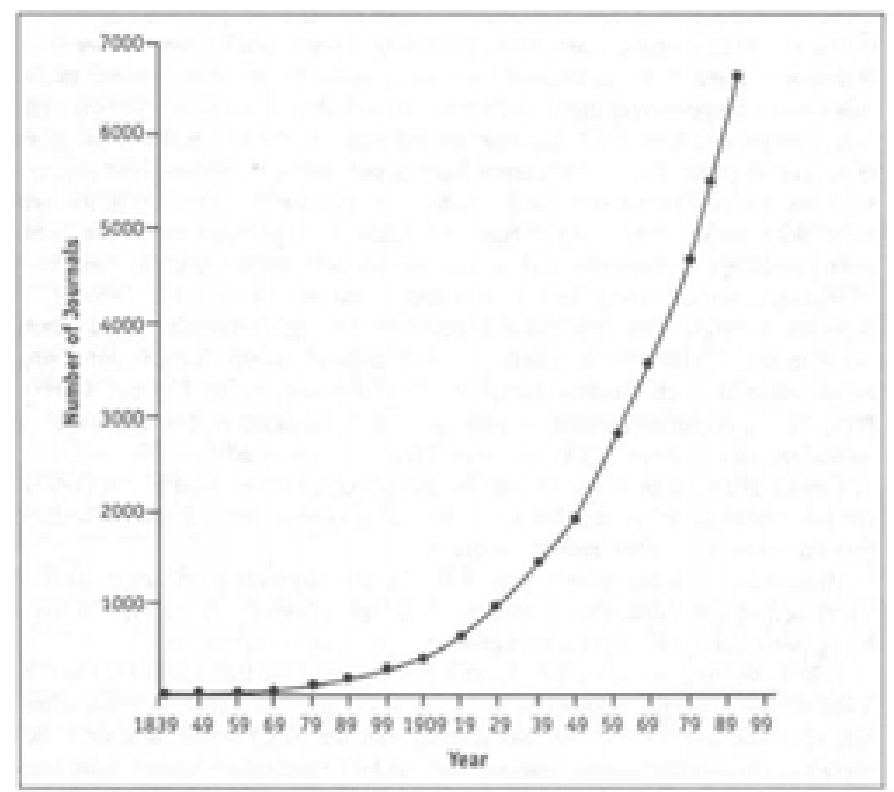

Abbildung 42: Exponentielles Wachstum wissenschaftlicher Journale 141

Die Verlage entwickeln aufgrund ihrer Verwertungsinteressen ein ureigenes Interesse an der Einführung neuer Journale und deren Verbreitung, weil sie sich da-

141 Quelle: King/Tenopir 2000; in Fortschreibung der Analyse von Solla Price 1974, S. 20. 
von zukünftige Erträge versprechen können. Diese Feststellung scheint überflüssig, weil sie so selbstverständlich klingt. Stellt man sich jedoch vor, dass die Verbreitung der Journale durch die Wissenschaft selbst hätte organisiert und finanziert werden müssen, erscheint die Leistung der Verlage zumindest in der (Print-)Vergangenheit in einem anderen Licht.

Die fortschreitende Ausdifferenzierung der Journale durch die Verlage war für die Entwicklung der Wissenschaft auch deswegen wichtig, weil sie die Ausdifferenzierung und Spezialisierung von Forschungsdisziplinen erleichtert hat. Oder anders formuliert: Jedes fachlich eigenständige Journal erleichtert den Konstituierungsprozesses einer neuen Fachdisziplin oder eines neuen thematischen Schwerpunkts. Das ökonomische Interesse der Verlage „passt“ daher zu dem Interesse von Wissenschaftlern, in neue Themenfelder vorzustoßen und neue Communities zu bilden ${ }^{142}$.

Verlage nutzen das wissenschaftliche Prinzip des organisierten Skeptizismus, also die Pflicht des Wissenschaftlers zur kritischen Kenntnisnahme der Ergebnisse von Fachkollegen, indem sie die Arbeit von wissenschaftlichen Gutachtern und Editoren beim Verfahren des Peer Review weitgehend unentgeltlich in Anspruch nehmen. Für Verlage ist die Begutachtung durch kompetente Wissenschaftler unter Verwertungsgesichtspunkten wichtig, weil das Peer Review die wissenschaftliche Qualität und damit die Nachfrage der Leser sichert. Durch die qualitätsgesteuerte Auswahl bündeln sie die Aufmerksamkeit von Wissenschaftlern. Gleichzeitig setzen sie Themen- und Schwerpunkte bei Journalen (Agenda Setting und Moderationsfunktion) und binden die Leser an ein Journal, indem sie in periodischer Folge relevante Beiträge zu bestimmten Themen herausbringen. Die Bündelung von Aufsätzen zu einem Themenspektrum lenkt die Aufmerksamkeit und erleichtert die Suche.

Verlage nutzen das Grundprinzip der uneigennützigen, kollektiven Wissensproduktion, um unentgeltlich an wissenschaftliche Aufsätze von den Autoren zu gelangen. Autoren bestehen nicht auf ökonomischen Verwertungsinteressen, weil dies den Grundprinzipien der Wissensproduktion widerspräche. Verlage nutzen dies, dennoch sind sie - damit der Austausch mit den Autoren zustande kommt zur Gegenleistung verpflichtet. Diese besteht nicht in Geld (der Währung des Wirtschaftssystems), sondern in der Chance auf Anerkennung und Reputation. Verlage fungieren als Vermittler von Reputationschancen ${ }^{143}$. Der scheinbar ungleiche Tausch zwischen Autoren und Verlagen funktioniert gerade, weil die Steue-

142 Mit der Ausdifferenzierung der Forschungsdisziplinen reagiert das Wissenschaftssystem nach Weingart (Weingart 2003, S. 37) auf sein eigenes exponentielles Wachstum. Denn ohne Ausdifferenzierung und Abspaltung neuer Disziplinen ist der auf Kommunikation beruhende Steuerungsprozess nicht zu bewältigen.

143 Prof. Spindler bezeichnet diese Funktion der Verlage treffend als „Repuationsagentur“ (Vortrag Spindler auf einem Mediaconomy-Workshop am 13.05.05). 
rungsmodi im Wissenschaftssystem andere sind als im Verwertungsprozess der Verlage. Autoren müssen nicht in der Währung Geld bezahlt werden, sondern in der Währung Reputation, die innerhalb des Wissenschaftssystems die Allokation von Status- und Ressourcen ermöglicht.

Die enge Verzahnung zwischen kommerziellen Verlagen und Wissenschaftssystem basiert darauf, dass die Verlage ein formalisiertes und damit bis zu einem gewissen Grad für die Autoren verlässliches System zur Verteilung von Reputationschancen aufgebaut haben. Relativ stabil und verlässlich ist das System der Verlage dadurch, dass es um wissenschaftliche Journale herum verschiedene Institutionen hervorgebracht hat, die im Wissenschaftssystem bei der Verteilung von Reputation, Status und Ressourcen eingesetzt werden (z. B. das ISI ${ }^{144}$ ).

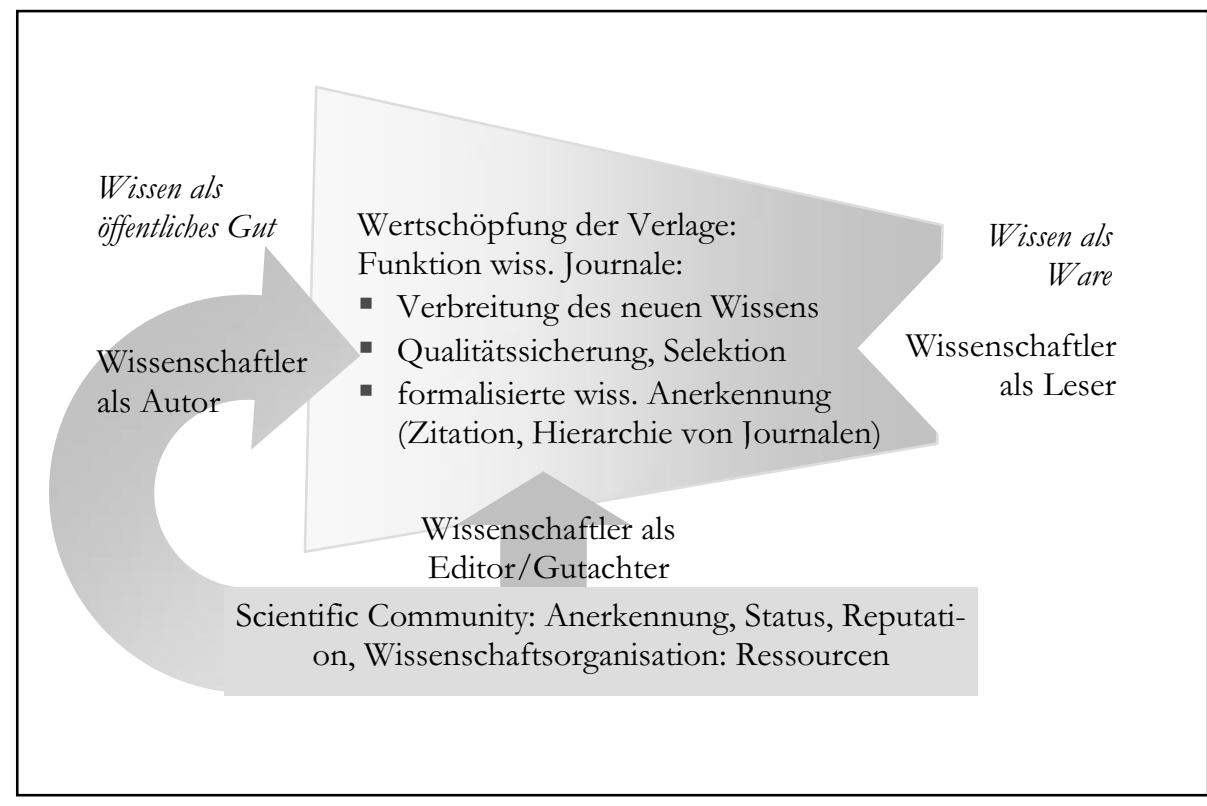

Abbildung 43: Die institutionelle Interdependenz zwischen Wissenschaft und Verlagen

Auf Basis einer internationalen Auswahl an Journalen wurde ein formalisierter Indikator zur Messung von Zitationen eingeführt. Das Instrument des Citation Index basiert darauf, dass Aufsätze generell ausgesprochen selektiv gelesen, rezipiert und zitiert werden. Rund 80\% aller Zitierungen entfallen auf 20\% aller Artikel (Weingart 2003: 37); weniger als 10\% der verfügbaren Periodika der zentralen Science Library in London entfielen 1956 auf 80\% der Anforderungen (Solla Price 1974, S. 86/87, siehe dort auch Verteilung der Benutzung von Zeitschriften nach

144 http://www.isinet.com/. 
Häufigkeit der Benutzung) ${ }^{145}$. Die Häufigkeit, mit der ein Aufsatz gelesenen und anerkennend zitiert wird, gilt als wichtiger Maßstab für Anerkennung durch die Fachkollegen und hat als Messverfahren zur Quantifizierung wissenschaftlicher Aufmerksamkeit wachsende (teilweise auch zweifelhafte) Bedeutung erlangt. Für die Verlage eröffnet die Berechnung von (durchschnittlichen) Zitierraten pro Journal - dem so genannten Impact Faktor - die Möglichkeit den Wert des Journals für Wissenschaftler zu steigern. Mit der Institutionalisierung von Regeln und Methoden zur Messung von Anerkennung, die am Journal „kleben“ ist es den Verlagen gelungen eine Schlüsselstellung bei der Verteilung von Reputation im Wissenschaftssystem zu erringen. Impact Faktoren sind gleichermaßen für Verlage wie für Autoren relevant, sie prägen die Veröffentlichungsentscheidungen der Autoren. Im Wissenschaftssystem werden sie bei der Evaluation eingesetzt und haben somit unmittelbare Effekte auf die Ressourcenverteilung. Diese Formalisierung und Institutionalisierung der Mechanismen zur Verteilung von Anerkennung durch die Verlage erleichtert einerseits die Steuerung innerhalb des Wissenschaftssystems, weil sie Anerkennung messbar und die Verteilungsregeln (zumindest scheinbar) transparent macht. Andererseits manifestiert sie Abhängigkeiten der Wissenschaft (genauer: ihrer Steuerungsmechanismen) von den Verlagen bzw. den von ihnen etablierten Journalen. Diese Abhängigkeit von Autoren und Wissenschaftsorganisationen von den etablierten Journalen der Verlage macht es - wie wir später zeigen werden - schwierig, alternative Publikationsformen zu implementieren. Denn ihre Akzeptanz bei den Autoren ist davon abhängig, ob sie diese oder ähnliche Institutionen zur Reputationsverteilung anbieten können. In diesem Sinne bedeutet die Institutionalisierung (wie immer bei Institutionen im soziologischen Sinne) nicht nur Stabilität im positiven Sinne, sondern sie erschwert Wandel, verzögert oder blockiert eigentlich gewollte Veränderungsprozesse.

Der von Merton (Merton 1985) in Bezug auf die Akkumulation und Übertragung von Reputation bei Wissenschaftlern beschriebene Matthäus-Effekt wurde von den Verlagen auf Journale übertragen: bekannte Autoren und Herausgeber bzw. Gutachter verschaffen dem Journal Reputation, die es wiederum auf Autoren übertragen kann, deren Aufsätze in diesem Journal publiziert werden. Wichtig ist dabei, dass Reputation (vermittelt über Impact Faktoren) durch die Journale quantifizierbar bzw. messbar wird: unterschiedliche Journale generieren unterschiedlich hohe Reputationschancen. Steigt die Attraktivität des Journals für Autoren, wirkt dies positiv auf die Menge und Qualität der eingesandten Artikel zurück. Denn Autoren streben danach, ihren Aufsatz in dem Journal zu veröffentlichen, dass ihnen die höchste wissenschaftliche Anerkennung verspricht.

145 Roosendaal (in diesem Band) zeigt, dass sich an der hohen Selektivität der Rezeption auch durch die neuen Technologien nichts ändert: $80 \%$ der Downloads bei den Dutch University Libraries im Oktober 2003 entfallen auf 20\% der Artikel. 
Einerseits erfüllen die Verlage - wie wir zeigen konnten - wichtige Funktionen für die Wissenschaft, andererseits verwandeln sie das im gesellschaftlichen Interesse und weitgehend mit öffentlichen Mitteln produzierte Wissen in eine Ware, die als Publikationen von den Wissenschaftlern (zurück) gekauft werden müssen. Damit treten die Verlage zu einer anderen wichtigen Funktion wissenschaftlicher Publikationen in Widerspruch: das neue Wissen soll der Gesellschaft, insbesondere aber den Wissenschaftlern als öffentliches Gut uneingeschränkt zugänglich sein.

\section{Die Achillesferse der institutionellen Interdependenz zwischen Wissenschaftssystem und Verlagen: die Rolle der Bibliotheken}

Unerlässliche Voraussetzung für das dynamische Wachstum der Wissensproduktion als kollektiver Prozess ist der freie Zugang der Wissenschaftler zu wissenschaftlichen Veröffentlichungen. Verlage verwandeln das neue Wissen in einen Ware, sodass der Zugang der Wissenschaftler von dessen finanziellen Ressourcen abhängig wäre. Um diese - die Funktionsfähigkeit des Wissenschaftssystems insgesamt gefährdende - Konsequenz der Kommodifizierung des neuen Wissens durch die Verlage zu verhindern, hat die Wissenschaft, bzw. haben Universitäten und Forschungseinrichtungen eine Institution geschaffen, die dieses Wissen wieder in ein öffentliches Gut zurück verwandelt (dekommodifiziert): die Bibliotheken. Die Funktion der Bibliotheken erschöpft sich längst nicht mehr in ihrer Archiv- und Katalogfunktion, bei der sie Publikationen sammeln und archivieren, um sie für zukünftige Generationen von Wissenschaftlern zu erhalten. Sondern sie gewähren auch den uneingeschränkten Zugang der Wissenschaftler zu neuen Publikationen, insbesondere auch zu wissenschaftlichen Journalen. Journale nehmen insofern eine Sonderstellung ein, als sie in den meisten Fachdisziplinen das Medium per se sind, in dem die neuesten wissenschaftlichen Ergebnisse zuerst veröffentlicht werden. Zudem zielt das Format von Zeitschriften darauf ab, Ergebnisse in kurzer, prägnanter Form zu veröffentlichen. Daher sind sie für Wissenschaftler von besonderer Bedeutung und bis in die zweite Hälfte des vergangenen Jahrhunderts (u. a. wegen der Kürze der Darstellung) auch erschwinglich gewesen. Die Bedeutung der Bibliotheken als Zugangsweg zu aktuellen Journalen ist jedoch in den vergangenen Jahrzehnten ständig gewachsen, weil Wissenschaftler die für sie wichtigen Journale nicht mehr selbst gekauft, sondern sich in der Bibliothek die für sie relevanten Aufsätze besorgt (und kopiert) haben. Immer mehr Wissenschaftler sind heute gerade bei Journalen auf den Zugang über die Bibliotheken angewiesen. Die Gründe hierfür sind vielfältig ${ }^{146}$ - wichtig ist in unserem Zusam-

146 Einerseits spielt hierbei die veränderte soziale Struktur der Wissenschaftler eine Rolle, insbesondere die wachsende Zahl von Angestellten in großen Forschungseinrichtungen. Gleichzeitig wächst die Zahl die Zahl der Journale, die der Wissenschaftler in seinem Fachgebiet zur Kenntnis 
menhang, dass Bibliotheken (auch) bei Journalen heute von entscheidender Bedeutung für die Versorgung der Wissenschaftler sind.

Die Besonderheit der intermediären Funktion von Bibliotheken besteht nun darin, dass sie tendenziell widersprüchliche Rollen miteinander vereinen sollen. Als Marktteilnehmer fungierten sie (formal) wie ein „,normaler“ Käufer auf einem „normalen“ Markt. Als institutionelle Nachfrager, die stellvertretend für die Wissenschaftler bzw. die Forschungseinrichtung agieren, sollen sie die Arbeitsfähigkeit der Wissenschaftler gewährleisten, d. h. sie sollen alle Publikationen verfügbar halten, die die Wissenschaftler für ihre Arbeit benötigen. Eigentlich darf der Preis der Publikation bei der Anschaffungsentscheidung keine entscheidende Rolle spielen, aus der Perspektive der Wissenschaft hat Bedarf der Wissenschaftler und die Qualität und Relevanz der Publikation im Vordergrund zu stehen.

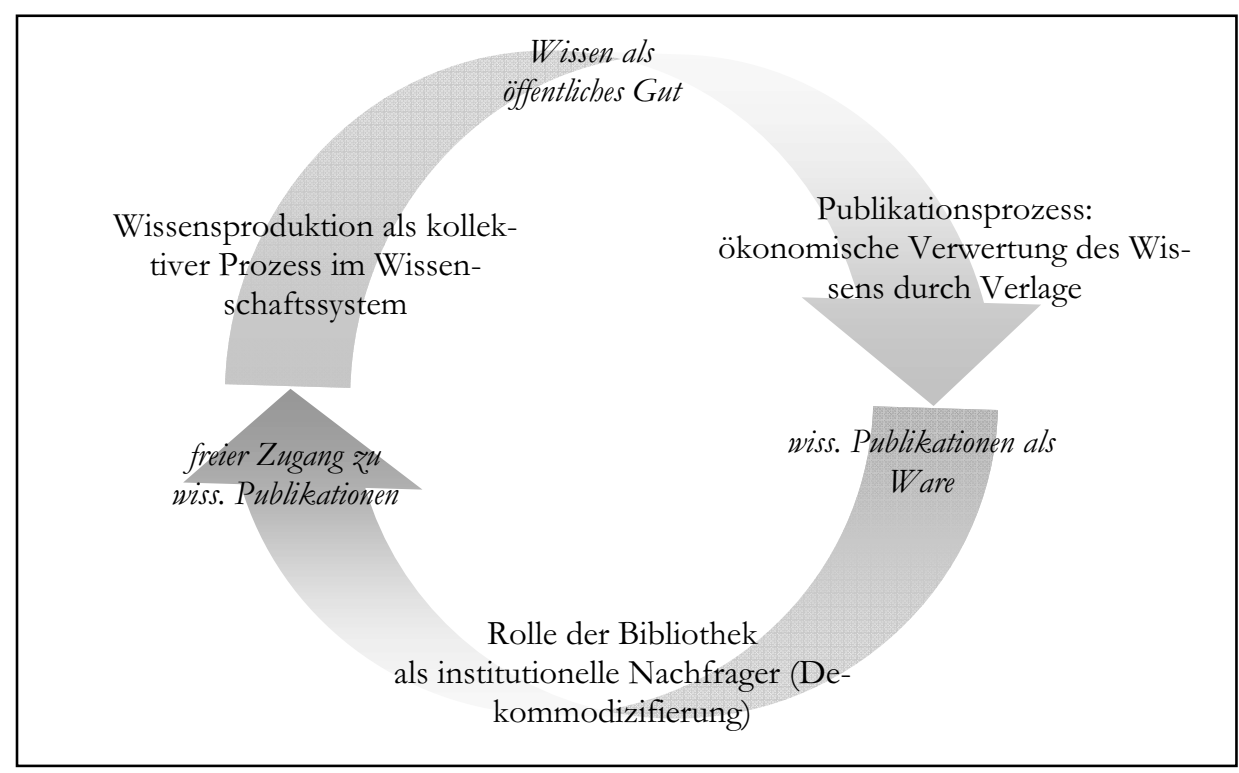

Abbildung 44: Die Rolle der Bibliotheken bei der Rückverwandlung des Wissens in ein öffentliches Gut (Dekommodifizierung)

In dieser Widersprüchlichkeit der Handlungsanforderungen an die Bibliotheken einerseits als institutioneller Nachfrager/Käufer der Publikation als Ware, andererseits als Institution im Wissenschaftssystem, die die Publikation als öffentliches Gut zugänglich machen soll - spiegelt sich der grundlegende Widerspruch des (bisherigen) Publikationssystems wider. Solange es den Bibliotheken gelingt die

nehmen muss. Vor allem aber steigen die Preise für Journale so stark an, dass sie für den einzelnen Wissenschaftler nicht mehr finanzierbar sind. 
beschriebene Rolle auszufüllen, also die widersprüchlichen Handlungsanforderungen auszutarieren, kommt der immanente Widerspruch zwischen Wissenschaft und Verlagen nicht zum Ausbruch. Wenn sie diese Funktion nicht mehr ausfüllen (können), ist das bisherige Publikationssystem aus der Perspektive der Wissenschaft nicht (mehr) akzeptabel. In der Vergangenheit sind Bibliotheken üblicherweise damit so umgegangen, dass Entscheidungen soweit wie möglich zugunsten der Anforderungen der Wissenschaftler fielen. Nicht ohne Grund, denn schließlich sind Bibliotheken primär ihren Kunden und Auftragebern verpflichtet. Doch in dem Maße, wie die intermediäre Funktion der Bibliotheken auch im Hinblick auf die wachsende Zahl der Journale immer wichtiger wurde, hat sich ihre Stellung gegenüber den Verlagen verändert. Einerseits werden sie bei vielen Verlagen zu den entscheidenden Marktteilnehmern (weil die individuelle Nachfrage drastisch zurück geht), andererseits können sie nur begrenzt wie ein ,normaler“ Marktteilnehmer - d. h. vor allem preissensitiv - reagieren. Sie werden immer mehr zum Stellvertreter der Wissenschaft auf dem Markt und gleichzeitig zum unverzichtbaren Zugangsweg der Wissenschaftler zu wissenschaftlichen Journalen.

Die Bibliotheken kommen dieser Rolle als institutionelle Nachfrage durch die Lizenzierung der von ,ihren“ Wissenschaftlern benötigten Journale nach. Sie können daher nur schwerlich den Kauf bestimmter Journale aufgrund von steigenden Preisen ablehnen, wenn dort wichtige Artikel veröffentlicht werden, die die für die Wissenschaftler ihrer Forschungseinrichtung unerlässlich sind. Daher können die Bibliotheken - im Unterschied zu individuellen Nachfragern - nur begrenzt preissensitiv reagieren. Bibliotheken haben in der Vergangenheit als Marktteilnehmer (fast) jeden Preis für ein wichtiges Journal gezahlt. Durch die Verlagerung der Nachfrage auf die Bibliotheken hat sich also die Marktkonstellation zu ungunsten der Wissenschaft verschoben. Verlage haben gegenüber den Bibliotheken Preise durchgesetzt, die bei individuellen Nachfragern nicht realisierbar gewesen wären. Nur weil Bibliotheken die sinkende individuelle Nachfrage durch höhere institutionelle Preise aufgefangen haben, konnten die kommerziellen Verlage für ihre Journale steigende Preise realisieren. Und dabei haben sie nach Ansicht von Kritikern Extra- bzw. Monopolprofite auf Kosten der Wissenschaft erzielt (Bergstrom 2004, McCabe 2004).

Auch gegenüber Wissenschaftlern hat sich die Rolle der Bibliotheken verändert. Wichtig ist in unserem Zusammenhang, dass sie nicht mehr nur den Zugang zu immer mehr Journalen gewähren, sondern in diesem Zusammenhang auch wesentliche Aufgaben bei der Verteilung und Vervielfältigung übernehmen. Zwar ist die marktbezogene Nachfrage nach Journalen gesunken, nicht jedoch die Rezeption der (relevanten) Journale durch die Wissenschaftler. Wissenschaftler benutzen die Bibliotheken zum Suchen und Recherchieren, die benötigten Aufsätze werden vervielfältigt (kopiert). Dies schlägt sich bei (Print-)Journalen darin nieder, dass die Verlage wenige Exemplare drucken, aber die Wissenschaftler viele Exemplare ko- 
pieren. Bibliotheken haben vielfältige Möglichkeiten ein einmal gekauftes Journal möglichst vielen Lesern zugänglich zu machen. Sie haben die Verfügbarkeit und den Umlauf erhöht, Kopiermöglichkeiten für die Leser und Kopierservices eingerichtet und vor allem haben sie den Austausch mit anderen Bibliotheken organisiert (Fernleihsysteme). D. h. Bibliotheken haben zunehmend Aufgaben der Distribution übernommen, die früher - als Wissenschaftler die von ihnen benötigten Journale noch weitgehend selbst gekauft haben - bei den Verlagen lagen. Die Distribution wissenschaftlicher Journale hat sich dabei grundlegend gewandelt. Dies bedeutet für den Wissenschaftler zunächst Mehrarbeit. Man muss in die Bibliothek gehen, suchen und kopieren, d. h. die Beschaffung kostet Zeit. Bibliotheken haben sich bemüht diese Arbeit zu erleichtern, Services anzubieten, vor allem aber haben sie IuK-Technologien eingeführt, um die Distributions-, Such- und Recherchetätigkeiten zu effektivieren. Damit haben sie die Herausbildung neuer Such- und Rezeptionsformen der Wissenschaftler erleichtert und beschleunigt (siehe unten).

Die neuen IuK-Technologien haben den Bibliotheken die alte Rolle des Archivs und die neue Rolle bei der Distribution erleichtert. Kataloge und Archivsysteme wurden digitalisiert und über das Internet (bzw. Campusnetze) zugänglich gemacht; sie wurden zu Datenbanken von Bibliotheksverbünden verschmolzen und für die überregionale Distribution genutzt. Dabei werden zunehmend zusätzliche bibliographische Informationen wie Inhalts- und Aufsatzverzeichnisse digital zur Verfügung gestellt bis hin zu Abstracts und Volltexten, die online abgerufen werden können.

Allerdings hatten alle diese Erfolge der Bibliotheken einen nicht intendierten Bumerangeffekt: Die Zahl der Lizenzen von wissenschaftlichen Journalen ist weiter gesunken (siehe unten) und die Preisspirale hat sich umso schneller gedreht. Der Vorteil von Einkaufsverbünden reduziert sich aus dieser Perspektive auf die Stärkung bzw. Bündelung der Verhandlungsposition der Wissenschaft als Nachfrager. Mittelfristig und in der Summe wird die Kostenexplosion beim Kauf der Journale nicht überwunden. Auch die verstärkte Zusammenarbeit in überregionalen Leihverbünden bringt i. d. R. zwar kurzfristige Entlastungen des eigenen Budgets, doch auch sie treibt letztendlich die Preisspirale weiter an. Die aus Sicht der einzelnen Bibliothek im Rahmen ihrer Handlungsmöglichkeiten sinnvollen Maßnahmen tragen im gegenwärtigen institutionellen Gefüge des Publikationssystems weiter zur Zuspitzung der Widersprüche bei. Daher suchen Bibliotheken und Forschungsorganisationen nach Alternativen, eine davon ist die aktive Beteiligung an Open Access Initiativen (z. B. im Rahmen von DINI - www.dini.de). 


\section{Zuspitzung der Widersprüche: die Zeitschriftenkrise}

Die so genannte Zeitschriftenkrise ist in unserem Verständnis nicht eigentlich eine Krise der Wertschöpfung der Verlage, sondern Ausdruck des Aufbrechens der immanenten Widersprüche der Logik des Wissenschaftssystems und ökonomischer Verwertungslogik. Die beschriebene Interdependenz zwischen Wissenschaft und Verlagen bei der Produktion droht hier an ihre Achillesverse - der intermediären Rolle der Bibliotheken - aufzubrechen. Kristallisationspunkt der Zeitschriftenkrise ist die scheinbar unaufhaltsame Spirale aus Preissteigerungen und sinkenden Auflagen, deren Folgen von den großen kommerziellen Verlagen auf die Nachfrager, stellvertretend für die Wissenschaft auf die Bibliotheken, abgewälzt werden.

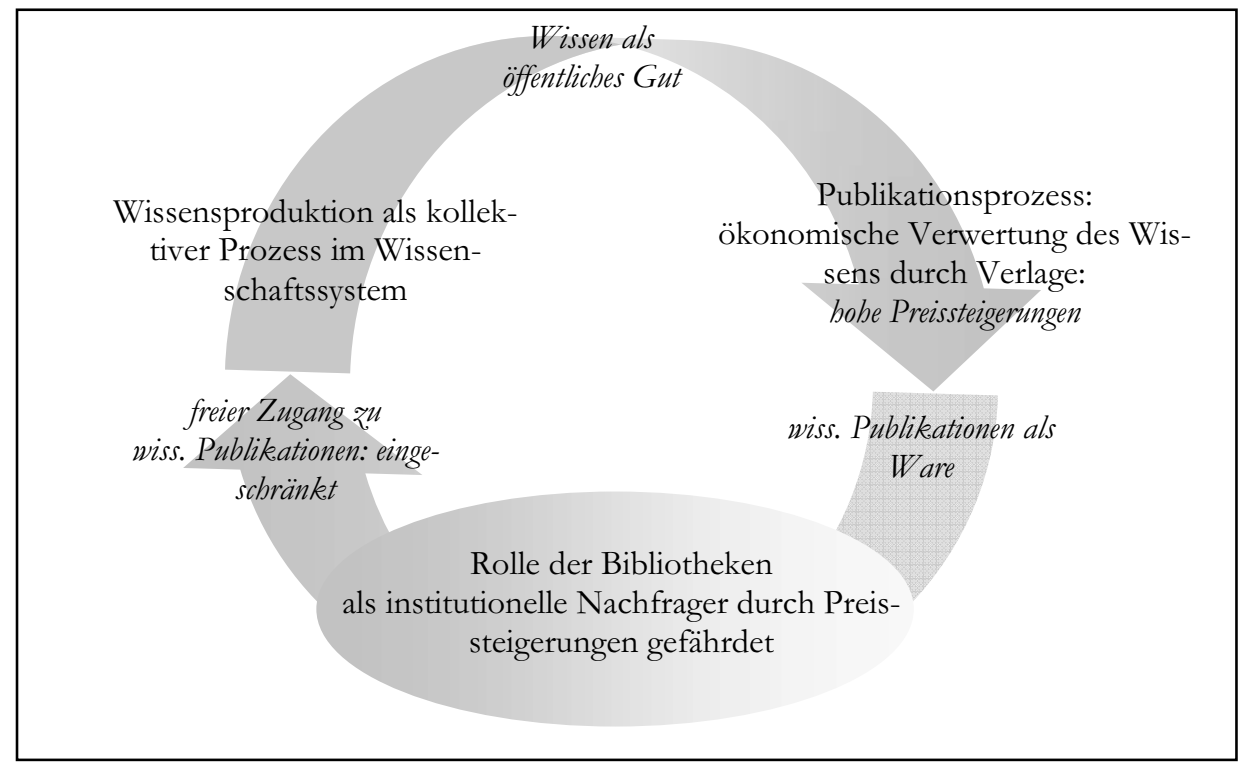

Abbildung 45: Die „Zeitschriftenkrise“ gefährdet die Rolle der Bibliotheken als institutionelle Nachfrager

Die Zeitschriftenkrise selbst und ihre Folgen sind von ökonomischer Seite vielfach analysiert worden ${ }^{147}$. Wir wollen uns daher auf einige zentrale Aussagen konzentrieren, die für unsere Argumentation wichtig sind. Ein Grund für die Preissteigerungen sind nach Tenopir / King die steigenden Fixkostenanteile der Verlage, die bei gleichzeitig sinkender Nachfrage zu exponentiell steigenden Kosten pro Einzellizenz führen. Sie beschreiben drei Trends, die für die Preisexplosion bei

\footnotetext{
147 Siehe hierzu die detaillierten Untersuchungen von King / Tenopir für US-Journale (King / Tenopir 2000); andere Untersuchungen aus den USA kommen zu ähnlichen Ergebnissen (Bergstrom 2004). Leider liegen vergleichbare Daten für deutsche bzw. europäische Journale nicht vor.
} 
wissenschaftlichen Journalen von 1975 bis 1995 verantwortlich waren: steigende Fixkostenanteile, sinkende individuelle Lizenzen von Wissenschaftlern und das damit verbundene Absinken der durchschnittlichen Auflagenhöhe.

Steigende Fixkostenanteile sind eine bekannte Folge des Einsatzes der neuen IuK-Technologien. Durch Digitalisierung und neue Distributionstechnologien sinken die variablen Kosten für Reproduktion und Distribution, somit steigt der relative Fixkostenanteil. Folgt man der Argumentation von Tenopir/King, so steigen die Fixkosten seit den 80er Jahren auch absolut, weil die verlegerischen Aufgaben bei der Auswahl, Qualitätssicherung und Bearbeitung der Artikel immer aufwendiger werden. Pro Journal werden immer mehr Aufsätze und Seiten publiziert. Die Zahl der eingereichten Manuskripte steigt, Ablehnungsquoten über 90\% sind bei STM-Journalen üblich; nicht zuletzt weil der Publikationsdruck auf die Wissenschaftler in den meisten Fachdisziplinen stetig zunimmt. Zudem investieren die Verlage in solche Tätigkeiten, die man im weiteren Sinne als community building bezeichnen kann, also Tätigkeiten die vornehmlich auf die Wissenschaftler als Produzenten - also auf Autoren und Peers - ausgerichtet sind und dazu dienen, die wissenschaftliche Qualität der Journale zu optimieren und die Aufmerksamkeit der scientific community für das Journal zu erhöhen. Ökonomisch gesehen sind steigende Fixkosten solange tragbar, wie sie sich auf viele Produkte verteilen - in unseren Fall also bei hohen Auflagen. Bei wissenschaftlichen Journalen sind jedoch in dem betrachteten Zeitraum die durchschnittlichen Auflagen gesunken ${ }^{148}$.

Gleichzeitig sind in diesem Zeitraum auch die (individuellen) Lizenzen von Wissenschaftlern drastisch gesunken. In den USA (vergleichbare Zahlen für Deutschland liegen uns nicht vor) ist die Zahl der Lizenzen, die ein Wissenschaftler durchschnittlich erwirbt, von 5,8 Lizenzen pro Wissenschaftler 1975 auf 2,7 pro Wissenschaftler im Jahr 1995 zurückgegangen (King / Tenopir 2000, S. 243). Das Verhältnis von individuellen und institutionellen Lizenzen hat sich gravierend verschoben: von insgesamt 6.100 Lizenzen waren 19753.700 individuelle Lizenzen; 1995 war die Zahl der durchschnittlichen Lizenzen pro Journal auf etwa 5.000 gesunken, davon waren nur noch 2.300 individuelle Lizenzen. Der einzelne Wissenschaftler - so die Interpretation der Ökonomen - reagiert preissensitiv auf Preissteigerungen. Allerdings nicht, weil er die Journale nicht benötigt, sondern weil der Bedarf zunehmend durch die Inanspruchnahme von Bibliotheken gedeckt wird. Immer weniger Wissenschaftler sind bereit für den Zugang zu Journalen steigende Preise zu zahlen, sondern sie nutzen stattdessen den kostengünstigen, alternativen Zugang über Bibliotheken. Dass die Auflagen vieler wissenschaftlicher Journale in den letzten beiden Jahrzehnten so stark zurückgegangen ist, hängt also mit Veränderungen in der Struktur der Nachfrage zusammen, die sich

148 Während in anderen Bereichen der Medienbranche die veränderte Kostenstruktur durch neue Geschäftsmodelle und Nachfragesteigerungen aufgefangen wurde, haben die neuen technischen Möglichkeiten im wissenschaftlichen Publikationswesen nicht zur Ausweitung der Nachfrage geführt. 
zunehmend auf die Bibliotheken verschoben hat. Je stärker die Preise stiegen, umso wichtiger wurden die Bibliotheken als institutionelle Nachfrager.

Tenopir / King erklären die exorbitanten Preissteigerungen seit Mitte der 90er Jahre damit, dass die (durchschnittliche) Auflagenhöhe der meisten Journale unter einen Schwellenwert gesunken ist, bei dem die gestiegenen Fixkosten nicht mehr durch - aus Nachfragesicht - vertretbare Preise refinanziert werden können. Sie belegen anhand einer Kostenrechnung mit realistischen (d. h. durchschnittlichen) Kostenparametern, dass eine 500er Auflage Kosten von 775 \$ pro Lizenz verursacht, eine 1.000er Auflage 404 \$; erst bei einer Auflage von 2.500er sinkt der Preis auf 181 \$, bei 5.000 verkauften Exemplaren kostet die Einzellizenz noch 107 \$. Demgegenüber ist die durchschnittliche Auflage der US-Journale von 2.900 im Jahr 1975 auf 1.900 in 1995 gesunken ${ }^{149}$. Dieser Effekt trifft nicht alle Journale/ Verlage gleichermaßen, sondern die Kluft zwischen den wenigen großen Journalen und einer wachsenden Zahl immer kleinerer Journale wird immer größer. Die kleinen Journale werden immer kleiner, die wenigen großen hingegen können ihre Auflagen halten ${ }^{150}$.

Die hohen Preissteigerungen der vergangenen Jahre sind auf grundlegende Veränderungen der Produktions- und Distributionsformen durch IuKTechnologien zurückzuführen. Die Verlage haben offenbar - so könnte man die skizzierte Entwicklung zugespitzt interpretieren - die ungleiche Marktposition der Bibliotheken ausgenutzt, um Geschäftsmodelle aufrecht zu erhalten, die nicht mehr zu den veränderten Produktions- und Distributionsformen passen. Mittlerweile hat die Spirale aus Preissteigerungen und sinkender Nachfrage für viele Bibliotheken ein Niveau erreicht, dass ihre Finanzierungsmöglichkeiten und die Finanzierungsbereitschaft der sie tragenden Forschungseinrichtungen zu sprengen droht. Die bisher bestehende komplementäre Interdependenz zwischen Wissenschaft und Verlagen bricht von der Nachfrageseite her auf. Zwar bedienen die Verlage weiterhin die Funktionen des Journals in Bezug auf Qualitätssicherung, Selektion, Bündelung und formalisierte Generierung von Anerkennung. Aber die wissenschaftlichen Nachfrager scheinen immer weniger bereit, den dafür von den Verlagen verlangten Preis zu zahlen - zumal dies in einer Situation geschieht, in der durch das Internet die Kosten für die Distribution gegen Null gehen, und daher von den Nachfragern, bzw. von der Wissenschaft möglicherweise auch in Eigenregie übernommen werden könnten.

\footnotetext{
149 Siehe dazu auch Meier (2002), der sich ebenfalls auf US-Untersuchungen stützt.

150 Dieser Trend erinnert verblüffend an den vertrauten Matthäus Effekt, der sich somit auch in der Auflagenentwicklung der Journale widerspiegelt: die Großen mit hoher Reputation werden immer größer und die Kleinen mit geringere Reputation hingegen immer kleiner.
} 


\section{Enabling Technology: neue Rezeptionsformen der Leser}

Ein Wissenschaftler kann nur einen begrenzten Teil seiner Arbeitszeit auf die Rezeption von Publikationen verwenden, wenn er als Autor nicht unproduktiv werden will. Obwohl das produzierte Wissen ständig ansteigt, bleibt der Zeitaufwand des Wissenschaftlers für die Rezeption nahezu konstant (siehe Abbildung 46). Allerdings kann der Wissenschaftler in dieser Zeit mehr oder weniger Artikel lesen. Dies hängt einerseits vom Umfang der Publikationen ab, je kürzer ein Aufsatz, um weniger Zeit benötigt man für das Lesen. Andererseits aber auch vom Aufwand des Wissenschaftlers für den Zugang zum Text (Suche, Bestellung, Beschaffung). Für Wissenschaftler als Leser bedeutete die Nutzung der Bibliotheken zunächst einmal einen Mehraufwand für die Beschaffung. Bibliotheken haben IuK-Technologien eingesetzt, um die Wissenschaftler bei der Beschaffung zu unterstützen. Die Untersuchungen von King / Tenopir haben ergeben, dass seit Mitte der 90er Jahre die Zahl der gelesenen Veröffentlichungen steigt (siehe Abbildung 46).

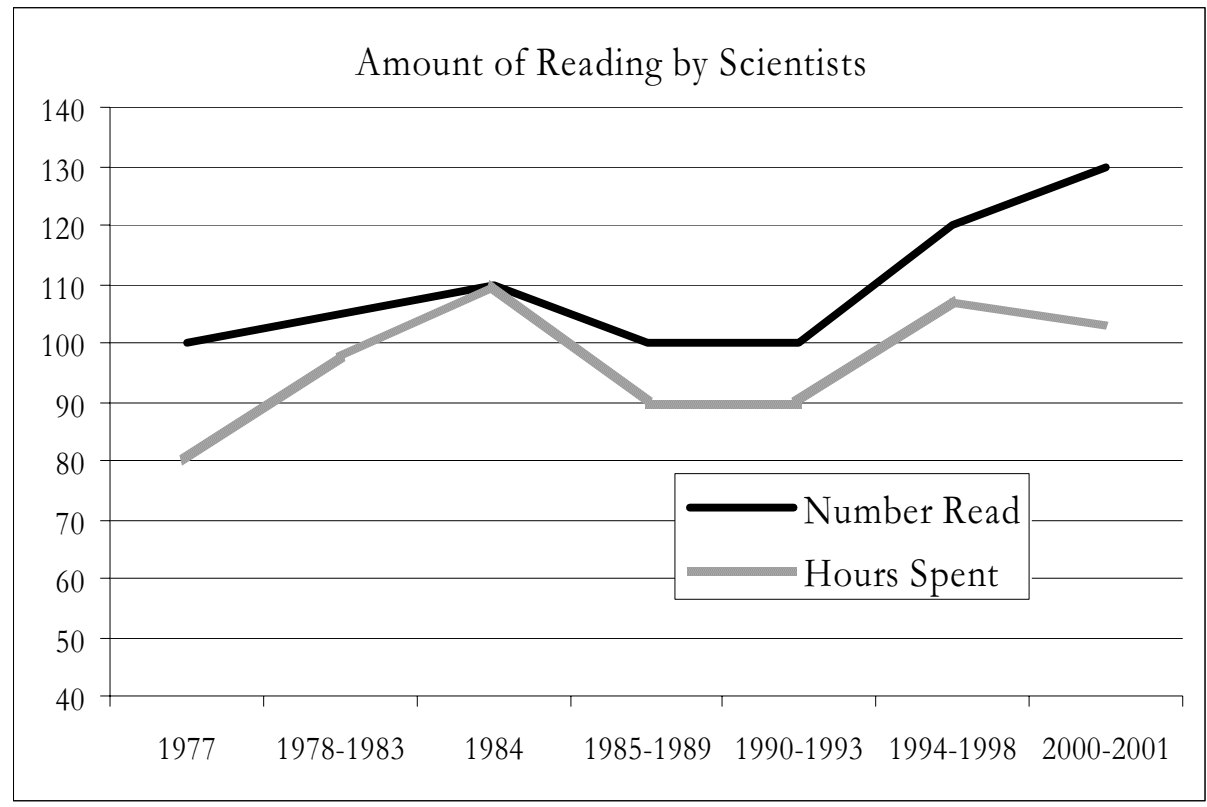

Abbildung 46: Average Time Spent and Number of Articles Read Per Year Per Scientist (Tenopir/King 2004: 23)

Wissenschaftler haben daher ein Interesse an der Verbesserung des Zugangs zu wissenschaftlichen Journalen. Sie haben die neuen Technologien, insbesondere das Internet für diesen Zweck vergleichsweise rasch und intensiv genutzt. Dabei 
verändern sich die Rezeptionsformen. Digitale Kataloge und elektronische Journale erleichtern dem Wissenschaftler die Suche und die Auswahl der zu lesenden Aufsätze. Der Aufwand für die Selektion durch den Leser sinkt, er kann die Selektionskriterien besser selbst steuern. Möglicherweise verliert die Selektionsleistung der Verlage in der bisherigen Form für den Leser an Bedeutung.

Gleichzeitig wird die Bündelungsfunktion der Journale zumindest teilweise obsolet. Ein Journal, das der Wissenschaftler selbst abboniert hat, blättert er vermutlich durch, bevor er es ins Regal stellt. Wenn er etwas zum Thema sucht, wird er zuerst auf das vorhandene Journal zurückgreifen. Die Bündelung der Aufsätze im Journal hat hier eine unmittelbare Wirkung auf die Rezeption. Diese Bündelungswirkung der Journale verliert hingegen an Bedeutung wenn die Aufsätze im Internet nur noch einen Mausklick weit auseinander liegen und womöglich mittels Suchabfrage in einer Datenbank gleichzeitig Aufsätze aus unterschiedlichen Journalen zugänglich sind. Für die Recherche ist es möglicherweise nicht mehr so wichtig in welchem Journal sie erschienen sind. Ob diese These zutrifft ist nicht wirklich erwiesen. Unbestritten ist jedoch, dass die neuen Technologien die Rezeptionsgewohnheiten verändert haben. Das früher vorherrschende Blättern in Journalen und Katalogen (Browsing: 58\%) wird nur noch von 21\% der Wissenschaftler verwendet, fast 40\% nutzen 2001/2003 Online-Suchmöglichkeiten.

\begin{tabular}{|l|l|l|l|}
\hline & $1990-1993$ & $2000-2002$ & $2001-2002$ \\
\hline Browsing & $58 \%$ & $46 \%$ & $21 \%$ \\
\hline Online Search & $9 \%$ & $14 \%$ & $39 \%$ \\
\hline Colleagues & $16 \%$ & $22 \%$ & $21 \%$ \\
\hline Citations & $6 \%$ & $13 \%$ & $16 \%$ \\
\hline
\end{tabular}

Abbildung 47: Veränderung von Such- und Rezeptionsformen von Wissenschaftlern (How Scientists Learned About Articles, Tenopir/King 2003)

Die veränderten Kommunikations- und Rezeptionsformen von Wissenschaftlern bringen neue Ansprüche und Anforderungen an das Publikationssystem hervor, die auf verbesserte Such- und Rezeptionsmöglichkeiten am eigenen Arbeitsplatz, sowie auf uneingeschränkten und weltweiten Zugang zielen. Diese Ansprüche werden durch die intensive Nutzung des Internets durch Wissenschaftler angetrieben. Bibliotheken und Forschungseinrichtungen unterstützen die neuen Rezeptionsformen, unter anderem auch durch den Aufbau umfangreicher Publikationsverzeichnisse auf Homepages. Auch die großen Verlage reagieren auf die veränderten Rezeptionsformen und verwenden ihrerseits Anstrengungen darauf umfassende Datenbanken aufzubauen, die dem Leser komfortable Möglichkeiten zur Recherche bis hin zum Volltextzugriff bieten. Zugriff auf diese Datenbanken haben jedoch i. d. R. nur diejenigen, die das jeweilige Journal abboniert haben oder Wissenschaftler einer Forschungseinrichtung mit institutioneller Lizenz. Die Daten- 
banken der Verlage sind dann besonders attraktiv, wenn eine Forschungseinrichtung alle Journale des Verlages lizenziert hat, denn deren Angehörige können über alle Journale hinweg suchen und dabei ggf. auch Querverweise zwischen den Aufsätzen verfolgen. Bezeichnend für die gegenwärtige Entwicklung scheint zu sein, dass Verlage sich nur begrenzt an den weltweiten Anstrengungen zum Aufbau offener, standardisierter Datenbanken beteiligen, sondern stattdessen proprietäre Systeme aufbauen, deren Attraktivität sie durch Zusatzservices (wie redaktionelle Querverweise) für den Leser steigern. Dafür verlangen sie allerdings bisher hohe Lizenzpreise, die für viele Einrichtungen nicht finanzierbar sind. Auch von Seiten der Leser wissenschaftlicher Publikationen wachsen daher die Widersprüche zu den Verlagen. Wissenschaftler als Leser haben vielfach Sympathien für die Ideen der Open Access Bewegung.

\section{Alternative Entwicklungspfade der Wissenschafts- kommunikation: Open Access Publikationsplattformen}

Open Access (OA) Publikationsplattformen können als eine Reaktion innerhalb der Wissenschaft verstanden werden, mit der Teile derselben auf die - aus der Sicht vieler OA-Vertreter - überschießende Wertschöpfung kommerzieller Verlagen einerseits und die neuen technischen Möglichkeiten andererseits reagieren. Die programmatische Forderung nach Open Access - also nach freiem, kostenlosem Zugang zu wissenschaftlichen Veröffentlichungen - ist aus der Perspektive des Wissenschaftssystems allerdings weder neu noch besonders radikal, sondern entspricht - so haben wir oben argumentiert - den normativen Grundprinzipien der Wissensproduktion im Wissenschaftssystem, wonach Wissen ein öffentliches Gut zu sein hat. Weitreichende Veränderungen impliziert die Forderung nach kostenlosem Zugang jedoch in Bezug auf die Geschäftsmodelle der Verlage, deren Erlösmodell auf dem Verkauf des Journals an die meist wissenschaftlichen Leser und die Bibliotheken als institutionelle Nachfrager basiert ${ }^{151}$.

Ein denkbarer Weg zur Verwirklichung von Open Access wäre, wenn man die Verwandlung des neuen Wissens in eine Ware gänzlich umgehen könnte. Wenn z. B. die „Wissenschaft“ den Publikationsprozess weitgehend selbst organisieren würde (ohne Verlage bzw. an den Verlagen vorbei). Zweifellos ist dies durch Digitalisierung und Internet entscheidend einfacher und kostengünstiger geworden.

151 Für die Diskussion alternativer Geschäftsmodelle ist hierbei wichtig, dass die Leistungen der Verlage bei der Publikation - also Verbreitung, Qualitätssicherung. Selektion und die Mechanismen zur Generierung formalisierter Anerkennung - nicht unterschiedliche Erlösquellen konstituieren, sondern sich als vermischtes Leistungsbündel im Preis des Journals niederschlagen. Der Leser als Nachfrager bezahlt bisher alle Leistungen, also auch die Leistungen, die für den Wissenschaftler als Autor von größerer Bedeutung sind, wie z. B. die Mechanismen zur Generierung formalisierter Anerkennung. 
Die infrastrukturellen Voraussetzungen für eine fast kostenneutrale Verbreitung über das Internet sind in der Wissenschaft vorhanden und werden bereits intensiv für die Kommunikation und den Austausch unter den Wissenschaftlern genutzt. Bereits heute wird das Internet für die Verbreitung wissenschaftlicher Beiträge und Publikationen genutzt, z. B. durch Homepages der Autoren und/oder der Forschungseinrichtungen, durch Online-Archive von Bibliotheken, Webseiten von Fachgesellschaften, Tagungen und Workshops. In einigen Disziplinen haben (traditionell) Preprints eine große Bedeutung; hier sind bedeutende PreprintServer entstanden. Forschungsinstitutionen haben auf ihren Homepages elektronische Archive aufgebaut, deren Inhalt neben den Aufsätzen eigener Wissenschaftler häufig auch Texte von kooperierenden Wissenschaftlern umfasst (z. B. Cern, MIT u.a.). Von besonderer Bedeutung in der aktuellen Diskussion ist das Konzept vernetzter institutioneller Repositories, die von Forschungsinstitutionen betrieben werden und in die qua Organisationszugehörigkeit alle Texte der angehörigen Wissenschaftler aufgenommen werden sollen. Neben grauer Literatur zählen dazu auch Working Papers, Unterrichtsmaterial, Vorträge, aber auch Aufsätze in wissenschaftlichen Journalen (z. B. MPG, einen aktuellen Überblick findet man auf den Seiten der DINI-Initiative ${ }^{152}$ ). Open Access-Publikationsplattformen finden bei den Wissenschaftsorganisationen wachsende Unterstützung (siehe dazu die Berlin Deklaration $\left.{ }^{153}\right)$.

Diese Entwicklung wird in der Open Access-Diskussion als Self Archiving oder die ,green road“ bezeichnet (vgl. Budapest Open Access Initiative ${ }^{154}$ ). Beim Self Achiving stellen Autoren selbst ihre Aufsätze im Internet zur Verfügung. Im Unterschied zur informellen Kommunikation hat jeder Wissenschaftler weltweit Zugang zu den Aufsätzen und Papers ${ }^{155}$. Die oben beschriebenen Archive und Repositories stellen die technische und organisatorische Infrastruktur für das Self Archiving bereit. Sie bieten nicht nur den offenen, weltweiten Zugang, sondern auch eine einheitliche Datenstruktur, sowie weltweite, standardisierte Suchmöglichkeiten. Zunehmend erlauben spezielle Suchmaschinen das Auffinden der Aufsätze in weltweit verteilten Archiven.

Dabei sind allerdings noch einige Probleme zu überwinden. Erstens ist die nachträgliche Veröffentlichung von Aufsätzen, die bereits in etablierten Journalen von Verlagen veröffentlicht wurden, nach Ansicht vieler Verlage nicht durch die rechtlichen Beziehungen zwischen Autor und Verlag gedeckt. Einige große Verlage (z. B. Elsevier) haben auf den Druck der Open Access Bewegung reagiert und den Autoren das Recht zugestanden ihre Aufsätze auf der eigenen Homepage frei zugänglich zu machen. Dieser Konflikt ist nicht entschieden (siehe Dorschel in

\footnotetext{
$152 \mathrm{http}: / /$ www.dini.de.

153 http://www.zim.mpg.de/openaccess-berlin/berlindeclaration.html.

154 Siehe http://www.soros.org/openaccess/read.shtml,, Download am 15.03.05.

155 Man könnte auch sagen ,invisible colleges“ (Crane 1972) werden „visible“.
} 
diesem Band). Für Autoren ist die Beseitigung dieser Unsicherheiten allerdings wie wir unten belegen werden - von zentraler Bedeutung.

Auch die Such- und Rezeptionsmöglichkeiten sind je nach Plattform unterschiedlich benutzerfreundlich. Für Insider des speziellen Themengebietes mögen simple Suchprozeduren nach Autor und Schlagwort hinreichend sein. Wissenschaftler, die mit dem spezifischen Thema weniger vertraut sind, könnten allerdings von manchem Pre- oder Postprint-Server enttäuscht sein, wenn sie wie bei klassischen Journalen eine Vorselektion und thematische Bündelung erwarten. Aus Sicht der Leser wären verbesserte Recherchetools und einheitliche Standards $\mathrm{zu}$ wünschen. Es ist zu erwarten, dass die Entwicklung von wissenschaftsbezogenen Suchmaschinen ${ }^{156}$ z. B. wesentliche Verbesserungen bringt. Zum einem weil sie plattformübergreifend genutzt werden, zum andern weil sie Volltextsuche ermöglichen und damit etwas mehr Unabhängigkeit von komplexen bibliografischen Metadaten verschaffen. Die thematische Bündelung von Beiträgen, die bei Journalen üblicherweise von den Editoren mit der inhaltlichen Gestaltung des Journals geleistet wird, kann auf OA-Publikationsplattformen durch Diskussionsforen oder Mailinglisten übernommen werden. Dem Leser wird hier neben dem Text gleichzeitig auch die darauf bezogene fachinterne Diskussion präsentiert (z. B. clio-online ${ }^{157}$ ). Eine solche enge Verkopplung von Publikationsplattformen mit den Diskussionsprozessen innerhalb der Scientific Community könnte die Attraktivität der Plattformen für die Leser erhöhen.

Die bisherigen Erfahrungen mit Self Archiving Repositories weisen auf ein zentrales Problem dieser Open Access Plattformen hin: Autoren beteiligen sich bisher nur sehr begrenzt am Self Archiving. Da dieses Konzept so stark auf die Eigeninitiative der Wissenschaftler setzt, bleiben viele Archive und Repositories bisher vergleichsweise leer und damit für Leser uninteressant. Zweifellos belegen die erfolgreichen Beispiele von Pre- und Postprint Servern (siehe Bargheer in diesem Band), dass dieses Konzept funktionieren kann. Doch viele andere Beispiele zeigen, dass es unter anderen Bedingungen bzw. in anderen Fachdiziplinen nicht einfach ist, Autoren für das Self Archiving zu gewinnen (vgl. Foster / Gibbbons 2005, Vorträge auf der DINI-Tagung $\left.{ }^{158}\right)$. Eine repräsentative Umfrage der DFG unter DFG-geförderten Wissenschaftlern ergab, dass im Herbst 2004 erst etwa $15 \%$ der befragten Wissenschaftler ihre publizierten Aufsätze zusätzlich im Inter-

156 Z. B. Google Scholar und andere, siehe auch IUK-Tagung 2005, Session 6, http://www.iukinitiative.de; sowie http://www.ForschungsPortal.Net.

157 http://www.clio-online.de/

158 Klaus Franken: Gute Ideen und Technik reichen nicht aus - das Einwerben von Beiträgen für ein Institutional Repository am Beispiel der Universität Konstanz; Joanne Yeomans: How to fill an institutional repository - winning scientists over - the example from CERN; Vorträge auf dem DINI-Symposium "Wissenschaftliches Publizieren der Zukunft - Open Access Vom 23. 24.05.2005 in Göttingen http://www.dini.de/veranstaltung/workshop/goettingen_2005-05-23/, zuletzt abgerufen am 10.06.05. 
net veröffentlicht haben (Postprints). Naturwissenschaftler liegen mit 20\% über dem Durchschnitt, Geistes- und Sozialwissenschaftler mit 6\% deutlich darunter (Mugabushaka 2005).

An den Untersuchungsbefunden ist besonders interessant, dass das geringe Engagement der Wissenschaftler als Autoren in deutlichem Kontrast zur allgemeinen Befürwortung von OA-Plattformen aus der Perspektive der Wissenschaftler steht (Mugabushaka 2005, Fournier 2005). Für den Wissenschaftler als Autor steht beim Publizieren der Ertrag der Veröffentlichung gemessen in Aufmerksamkeit, Anerkennung und Reputation im Vordergrund. Open Access-Archive bzw. Repositories haben in der Regel nicht den Anspruch diese Funktion klassischer Journale zu übernehmen. Abbildung 48 veranschaulicht, dass beim Self Archiving die für Autoren zentralen Instrumente zur Generierung formalisierter Anerkennung nicht bedient werden. Im Unterschied zu Journalen wird von den Betreibern eines Archivs keine Qualitätskontrolle durchgeführt, sondern die Aufsätze werden von Autoren eigenverantwortlich im Archiv abgelegt. Aus der Perspektive der Open Access Forderung mag es ausreichen die Aufsätze auch aus etablierten Journalen (Postprints) frei zugänglich zu machen. Aus der Perspektive der Autoren reicht das allein nicht aus. Ihn interessiert auf welche Weise er/sie formelle Anerkennung und Reputation erhalten kann. Die Aufmerksamkeit, die den in einem Archiv abgelegten Aufsätzen zuteil kommt, wird weder bei der Berechnung von Zitationsraten noch bei Impact Faktoren berücksichtigt. Die formalisierte Anerkennung ,klebt“ weiterhin am klassischen Journal. Gezählt wird nur der Aufsatz bzw. das Zitat, das in einem anerkannten Journal erscheint. Self Archiving könnte man daher als einen parallelen Verbreitungsweg beschreiben, der neben den etablierten Journalen eröffnet wird und ausschließlich der Verbreitung dient.

In der oben bereits zitierten DFG-Umfrage meinen über zwei Drittel der Wissenschaftler (nach Fachgebiet schwankend), dass OA-Publikationen seltener zitiert werden, seltener bibliografisch nachgewiesen werden und auch bei Anträgen auf Fördermittel nicht hinreichend berücksichtigt werden. Dies zeigt den Widerspruch, in dem sich Wissenschaftler befinden. Solange das wissenschaftsinterne System der Verteilung von Anerkennung und Reputation auf die formalisierte Generierung von Anerkennung durch etablierte Journale ausgerichtet ist, ist es schwierig, Autoren für alternative Wege der Verbreitung zu gewinnen. Veröffentlichungen außerhalb der Mechanismen über die Anerkennung generiert werden, sind gerade angesichts der immer stärker leistungsorientierten Ressourcenverteilung im (deutschen) Wissenschaftssystem ein Luxus, den sich vornehmlich diejenigen leisten können, die bereits über Reputation verfügen. Zumindest solange, wie die schiere Anzahl der Veröffentlichungen in High Impact Journalen in vielen Disziplinen als ausschlaggebender Leistungsindikator benutzt wird. 


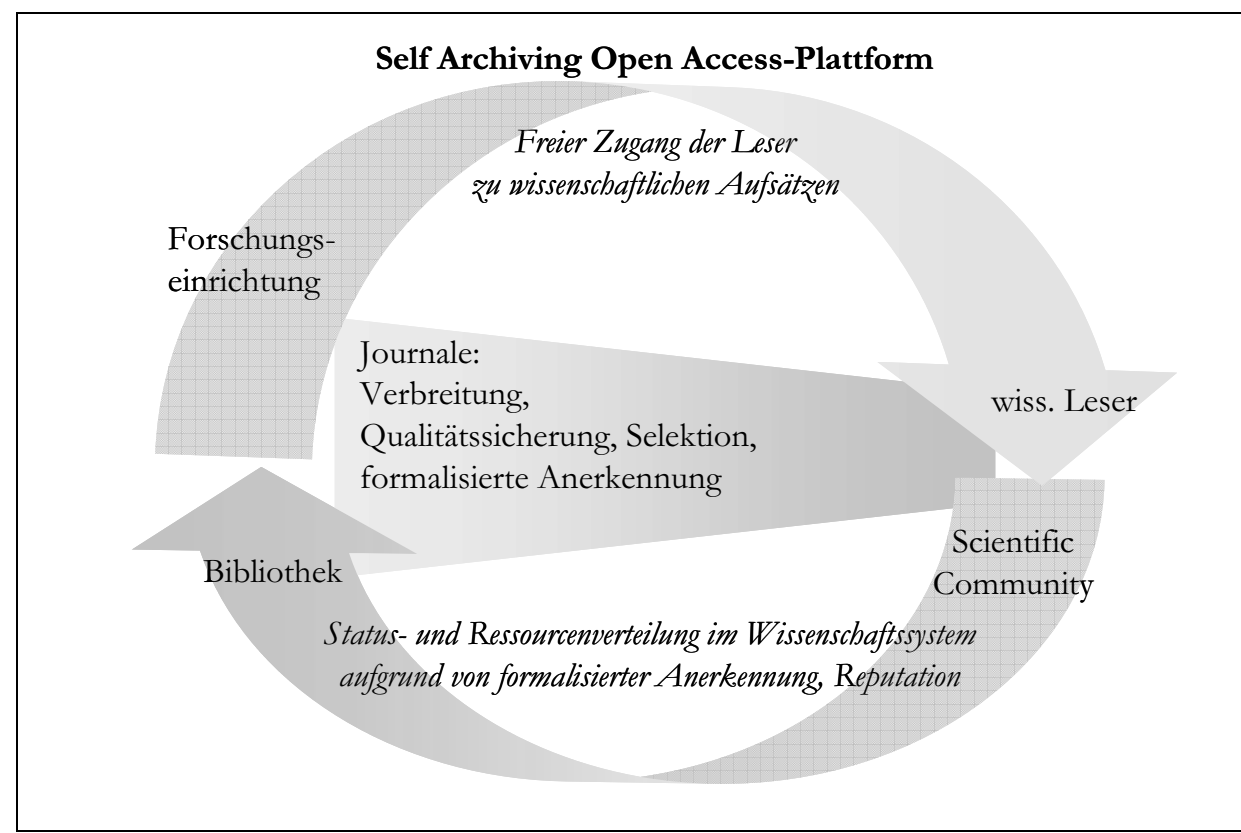

Abbildung 48: Self Archiving Open Access-Plattformen als paralleler Verbreitungsweg für wissenschaftliche Publikationen

Empirische Untersuchungen (Lawrence 2001, Harnad 2004, Brody / Harnad 2004) belegen, dass die ergänzende Verbreitung von Aufsätzen über OA-Archive durchaus geeignet sein kann, die Zitationshäufigkeit in klassischen Journalen zu erhöhen, weil diese Aufsätze von mehr Wissenschaftlern gelesen werden. Auf diese Weise werde - zwar indirekt, aber durchaus wirkungsvoll - auch über Open Access Plattformen formalisierte Anerkennung generiert. Allerdings ist dieser $\mathrm{Zu}$ sammenhang noch vielfach unbekannt und möglicherweise ist die Wirkung auch nicht stark genug, um beim Autor ggf. vorhandene Zweifel über die Risiken in Bezug auf zukünftige Publikationschancen auszuräumen. Mindestens ebenso wichtig für die Akzeptanz von Autoren wäre aus unserer Sicht die Anpassung der wissenschaftsinternen Mechanismen und Indikatoren zur Messung von wissenschaftlicher Qualität und Anerkennung. Würden OA-Publikationen bei der Verteilung von Status und Ressourcen im Wissenschaftssystem stärker berücksichtigt, wäre das Engagement von Autoren vermutlich wesentlich größer.

Wenn man Self Archiving als ergänzenden Verbreitungsweg betrachtet und nicht als Substitution von Journalen müsste es zu einem neuen institutionellen Arrangement - einer neuen Form der Arbeitsteilung in Bezug auf die Funktionen der Publikation zwischen Wissenschaft und Verlagen kommen. Roosendaals Vorschlag einer Trennung von Zugang (availability) und Selektionsfunktion (selection) 
(siehe Roosendaal in diesem Band) weist in diese Richtung. Der neuralgische Punkt dieser Modelle ist aus unserer Sicht, ob und wie eine neue institutionelle Verschränkung zwischen Wissenschaft und Verlagen erreicht werden kann. Verlage scheinen derzeit vor allem darum bemüht zu sein eine solche Trennung von $\mathrm{Zu}-$ gang und Selektion zu verhindern. Für diesen Eindruck spricht aus unserer Sicht, dass insbesondere die großen Verlage große Anstrengungen darauf verwenden, umfassende und serviceorientierte Online-Datenbanken aus ihren Journalen aufzubauen. Diese sind als proprietäre Systeme angelegt und stellen daher eine neue (internetadäquate) Form der Bündelung von Zugang und Selektion dar. Offen ist daher, wie das Konzept institutioneller Repositories mit den Strategien der großen Verlage in Einklang zu bringen ist, wie ein solches Arrangement konkret aussehen könnte. Andererseits hat gerade das Modell institutioneller Repositories den besonderen Charme, dass bisher weitergehende Interessen von Forschungseinrichtungen und Forschungsgemeinschaften in Bezug auf ihre Außendarstellung und -wirkung erfüllt werden könnten (Leistungsnachweis, Reputation, Konkurrenz).

Die Alternative zum Self Archiving sind elektronische Open Access Journale im Internet, die den Lesern freien, kostenlosen Zugang gewähren - die so genannte „gold road“ (Budapest Open Access Initiative ${ }^{159}$ ). Open Access Journale sind im Funktionsumfang etablierten Journalen vergleichbar. Im Unterschied zum Self Archiving geht der Veröffentlichung der Aufsätze hier ein Peer Review Verfahren voraus. OA-Journale leisten neben der Verbreitung im Internet ebenfalls Qualitätskontrolle, Selektion und i. d. R. auch die Messung von Zitations- und Impact Indices. Sie bedienen sich renommierter Editoren und Gutachter und vermitteln ebenso wie klassische Journale Anerkennung und Reputation in der Scientific Community. Insofern stehen sie (potentiell) in Konkurrenz zu klassischen Journalen von Verlagen. Neben dem freien Online-Zugang für Leser nutzen sie die Vorteile des Internets bei der Distribution (verkürzte Reproduktions- und Distributionsstufen) und neuartige internetbasierte und offene Möglichkeiten des Peer Review. Dies soll sich nicht zuletzt positiv auf die Kostenstruktur der OA-Journale auswirken und somit neue Erlösmodelle erleichtern.

Die schematische Darstellung (Abbildung 49) zeigt, dass das Modell in Bezug auf die Funktion der Publikation ähnlich aussieht wie das Schema eines klassischen Journals - mit dem entscheidenden Unterschied, dass der Leser keinen finanziellen Beitrag leisten muss.

159 Siehe http://www.soros.org/openaccess/read.shtml, Download am 15.03.05. 
Finanzierung der Dienstleistung durch

\section{Open Access Journale}

Autoren oder

Forschungseinrichtungen

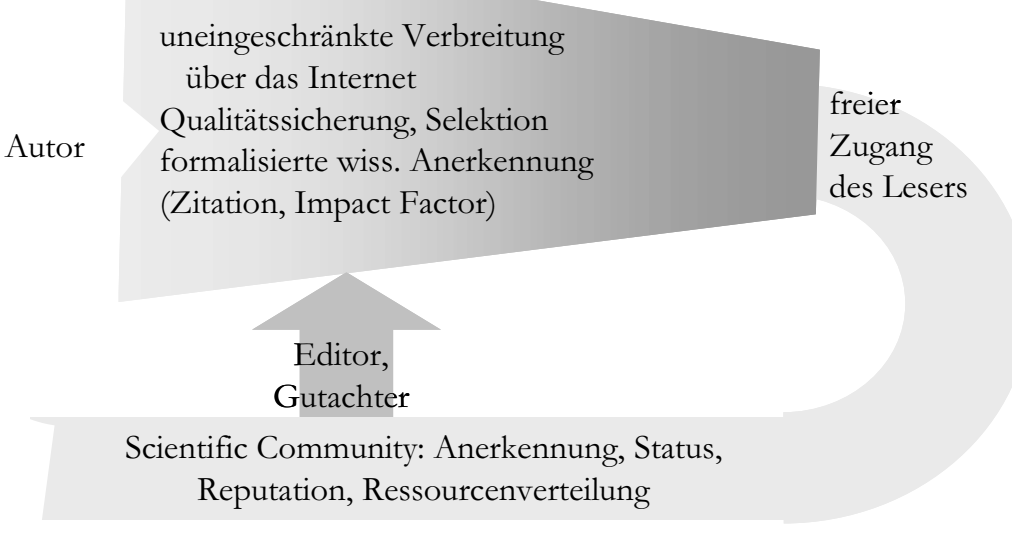

Abbildung 49: Open Access Journale

Um den kostenfreien Zugang des Lesers gewähren zu können, bedarf es neuer institutioneller und vor allem auch ökonomischer Modelle. Die Frage, wer diese Leistungen erbringt und wie sie finanziert werden, wird in den verschiedenen Modellen unterschiedlich gelöst. Einige OA-Journale werden von Fachgesellschaften oder Forschungsorganisationen getragen oder finanziert. Die OA-Journale werden in diesem Fall von „der Wissenschaft“ übernommen (z.B. einige Journale der European Geosciences Union EGU). Auch dieses Modell ist im Prinzip nicht neu, sondern knüpft an die Erfahrungen von Fachgesellschaften, z.B. in den USA an, die ihre Journale in eigenen Verlagen publizieren. Natürlich stellt sich auch im wissenschaftsbasierten Modell das Problem der Finanzierung - denn auch Leistungen, die von Einrichtungen des Wissenschaftssystems erbracht werden, erfordern technische und vor allem personelle Ressourcen. Diese müssten nicht nur kurzfristig über den Weg der Projektfinanzierung, sondern auch mittel- und langfristig von den entsprechenden Wissenschaftseinrichtungen aufgebracht werden.

Andere OA-Journale verfolgen ein Finanzierungsmodell, das die bisherige Wertschöpfungskette auf den Kopf stellt: Die Autoren statt der Leser sollen zumindest die Kosten für Qualitätssicherung und Selektion tragen (,author pays“). Die Begründung ist nicht unplausibel, denn der Autor würde auf diese Weise die Dienstleistungen des Journal-Betreibers (bzw. des Verlages) zahlen: das Qualitätszertifikat für seinen Aufsatz und die Generierung von formalisierten Anerkennung. Gegenüber dem traditionellen (Subskriptions-)Geschäftsmodell der Verlage 
hat das ,author pays“-Modell für Autoren den Nachteil, dass sie neben der Arbeit an dem Aufsatz auch noch zusätzlich zur Kasse gebeten werden.

Die bisherigen Erfahrungen mit OA-Journalen zeigen, dass es auch für Open Access Journale schwierig ist, Autoren zu gewinnen. In der o.g. DFG-Umfrage im Herbst 2004 geben über 80\% der Autoren an, noch nie in einem Open Access Journal publiziert zu haben (Fournier 2005). Über zwei Drittel wären auch nicht bereit, eine Autorengebühr zu zahlen (Ausnahme sind die Lebenswissenschaften, in denen Autoren auch bei konventionellen Journalen Gebühren zahlen). Daneben gibt es einige erfolgreiche Open Access Journale, die in ihrer Fachdisziplin auch in der Hierarchie der High Impact Journale in der obersten Liga mitspielen (z. B. einige Journale der Public Library of Science ${ }^{160}$, z. B. plos biology; Atmospheric Chemistry and Physics ${ }^{161}$, einige Journale von Biomed Central ${ }^{162}$ ). Diese Erfolgsfälle machen zweierlei deutlich: Erstens dass Autoren dann gewonnen werden können, wenn das OA-Journal bei der Verteilung von Anerkennung und Reputation eine Rolle spielt. Denn anders als OA-Archive und Repositories können sich OA Journale an den etablierten Mechanismen zur Generierung von formalisierter Anerkennung (Citation Index, Impact Factor) beteiligen. Ihr Problem ist, dass sie normalerweise als Journal Newcomer sind und als solche (wie konventionelle Newcomer auch) darum kämpfen müssen, sich in der Hierarchie der Journale einen Platz zu erobern. OA-Journale haben wie andere neue Journale zunächst keinen hohen Impact Faktor aufzuweisen. Unter rationalen Abwägungsgründen würden viele Autoren schon aus dieser Überlegung heraus die Veröffentlichung in einem der etablierten Journale vorziehen. Das für Autoren weniger attraktive Geschäftsmodell könnte verschärfend wirken. Der negative Matthäus-Effekt träfe die OA-Journale in besonderer Weise: weil sie wenig Reputation haben, wäre es schwer Reputation zu erlangen. In neuen Themenfeldern hingegen, die nicht bereits von etablierten Journalen besetzt sind, könnten OAJournale ein interessantes Modell sein. Bleibt es allerdings dabei, dass die interessantesten Aufsätze in etablierten Journalen erscheinen, könnten OA-Journale (bis auf weiteres) ein Modell für neue oder „kleine“ Journale bleiben.

Protagonisten von OA-Journalen argumentieren demgegenüber, dass Open Access Journale für Autoren (zumindest auf mittlere Sicht) attraktiver sind, weil sie häufiger gelesen werden, mehr Beachtung finden und somit auch mehr Anerkennung und Reputation versprechen (Harnad 2005). Sie verweisen auf die Vorteile für Leser und Rezipienten und auf den größeren Kreis von Wissenschaftlern, denen sie zugänglich sind - eben nicht nur einem ausgewählten Kreis von Wissenschaftlern an finanziell gut ausgestatteten Forschungseinrichtungen. Man könnte

\footnotetext{
160 http://www.plos.org/.

$161 \mathrm{http}: / /$ www.copernicus.org/EGU/acp/.

162 http://www.biomedcentral.com/.
} 
auch sagen, dass Open Access der wissenschaftlichen Arbeit der Autoren den größtmöglichen Verbreitungsgrad verspricht und daher im ureigenen Interesse der Autoren liegen könnte. Diesem Argument kann man entgegenhalten, dass die Verteilung von Reputationschancen ein stark institutionalisierter Prozess ist, in dem die großen Verlage mit ihren etablierten Journalen eine Schlüsselposition einnehmen. Für die Generierung von Reputation reicht die bloße Verbreitung des Aufsatzes nicht aus, wichtig ist vielmehr die formalisierte Messung von Anerkennung durch die klassischen Instrumente, mittels derer Anerkennung (z. B. Citation Index) bewertet wird. Allerdings spricht einiges dafür, dass Open Access Journale mit innovativen Verfahren (z. B. transparentem Peer Review, siehe Pöschel (2004 und 2005), renommierten Wissenschaftlern als Editoren und enger Anbindung an die jeweilige Scientific Community durchaus in der Lage sein können, die Position der etablierten Journale ins Wanken zu bringen.

\section{Das wissenschaftliche Publikationssystem im Umbruch}

Ausgangspunkt unserer Argumentation war die These, dass die Veränderung des wissenschaftlichen Publizierens weder allein aus der Perspektive des Wissenschaftssystems noch allein aus der Logik der Wertschöpfung heraus erklärt und beurteilt werden könne. Im ersten Teil des Textes haben wir ein Modell des bisherigen Publikationssystems skizziert, in dem scheinbar konträre Organisations- und Steuerungsprinzipien von Wissenschaft einerseits und kommerziellen Verlagen andererseits (kollektive uneigennützige Wissensproduktion vs. ökonomische Verwertungslogik) zu einer komplementären Interdependenz miteinander verwoben sind. Den Verlagen ist es gelungen, die Funktionen wissenschaftlicher Publikationen und wissenschaftlicher Journale für das Wissenschaftssystem zu erfüllen, indem sie diese Funktionen zu ökonomischen Verwertungsinteressen umformulieren. Der Widerspruch zwischen kollektiver Wissensproduktion und ökonomischer Verwertung wurde solange überbrückt, wie beide (Wissenschaft und Verlage) in dieser Interdependenz eine Win-Win-Situation sahen. Dies scheint sich seit einigen Jahren zu ändern.

Teile der Wissenschaft, sowohl Wissenschaftler als auch Forschungseinrichtungen und Wissenschaftsorganisationen, sehen die bisherige Form der Arbeitsteilung mit den Verlagen zunehmend als Verlustgeschäft für die Wissenschaft. Der Widerspruch ist an der Stelle aufgebrochen, an der die Wissenschaft selbst ihn bisher durch die Bibliotheken überbrückt hat. Denn die Bibliotheken garantieren im bisherigen Modell den für die Wissensproduktion unabdingbaren freien Zugang der Wissenschaftler zu den Publikationen ihrer Fachkollegen. Viele Bibliotheken sind nicht mehr bereit und/oder in der Lage die steigenden Preise insbesondere für STM-Journale aufzubringen. Damit wird die Rückverwandlung des von den Verlagen in eine Ware verwandelten Wissens in ein öffentliches Gut (Dekommodifizierung) in Frage gestellt. Der uneingeschränkte Zugang der Wissen- 
schaftler zu den Ergebnissen der Fachkollegen ist nicht mehr gewährleistet. Wissenschaftler fordern demgegenüber einen, den neuen Möglichkeiten des Internet entsprechenden, verbesserten Zugang zu wissenschaftlichen Journalen. Das „alte“ Modell der Interdependenz droht auseinander zu brechen.

Die Trendwende wurde durch die technische Entwicklung, insbesondere die Digitalisierung des Produktionsprozesses und die Distribution über das Internet, angestoßen und vorangetrieben. Auch die so genannte Zeitschriftenkrise hängt ursächlich mit den neuen Produktions- und Distributionsverfahren von wissenschaftlichen Publikationen und den daraus resultierenden Veränderungen von Kostenstruktur und Geschäftsmodellen zusammen. Wissenschaftliche Publikationen werden bereits heute weitgehend als digitale Produkte erstellt und vielfach auch über das Internet distribuiert (entweder kostenpflichtig durch Verlage oder auch als Open Access). Ob die Publikationen bzw. Journale ausschließlich als elektronische Version oder auch als Druckausgabe produziert und vermarktet werden, hängt vornehmlich von den beteiligten Akteuren ab, insbesondere von den Geschäftsmodellen der Verlage bzw. Herausgeber. Auch die Suche und Rezeption der Leser erfolgt weitgehend über internetbasierte Kataloge und Datenbanken.

Das wissenschaftliche Journal hat sich grundlegend verändert - damit scheint auch die Aufrechterhaltung des bisherigen Publikationsmodells immer weniger sinnvoll. Man kann die gegenwärtige Situation daher als Umbruch betrachten - als eine Übergangsphase, in der sich neue Publikationsmodelle und Strukturen herausbilden und die Akteure sich neu positionieren. Die großen kommerziellen Verlage tun dies u. a. durch den Aufbau von umfassenden und für Leser komfortablen Online-Datenbanken und Publikationssystemen (z. B. SCOPUS 163 von Elsevier). Die Protagonisten neuer Publikationsmodelle in der Wissenschaft sammeln sich gegenwärtig hinter der Forderung nach Open Access. Unverkennbar handelt es sich bei den Open Access Publikationsplattformen (mindestens) um zwei unterschiedliche Entwicklungsmodelle. Das eine Modell ist Self Archiving in OnlineArchiven bzw. Repositories, deren Funktion sich vor allem auf die freie, internetbasierte Verbreitung wissenschaftlicher Veröffentlichungen konzentriert und die übrigen Funktionen wissenschaftlicher Publikationen (Peer Review, Selektion und Generierung von Reputation) den (klassischen oder neuen) Journalen überlässt. Self Archiving ist ein paralleler Verbreitungskanal, der voraussetzt, dass die übrigen Funktionen wissenschaftlicher Publikationen auf andere Weise gewährleistet werden. Es impliziert daher eine neue Form der Interdependenz bzw. Arbeitsteilung zwischen dem Wissenschaftssystem - das diesen Verbreitungskanal organisiert - und Verlagen, die die übrigen Funktionen erfüllen. Wie eine neue Form der Interdependenz aussehen könnte, ist gegenwärtig noch umstritten.

163 http://www.scopus.com/. 
Open Access Journale hingegen umfassen alle Funktionen einer wissenschaftlichen Publikation, insofern sind sie nicht komplementär zu den klassischen Journalen, sondern substitutiv. Dies impliziert jedoch, dass sie sich gegenüber denn etablierten Journalen der Verlage durchsetzten müssen - sie sind Newcomer. Die Konkurrenz wird über die Gewinnung von Autoren ausgetragen, deren Interessen auf die Generierung formalisierter Anerkennung ausgerichtet sind. Die großen Verlage haben hier bisher eine Schlüsselstellung inne. Für die weitere Entwicklung wird es wichtig sein, ob es den Open Access Journale gelingt sich zu etablieren und damit ,ihren“ Autoren auch bei der Generierung formalisierte Anerkennung (über Citationindex und Impact Faktoren) nützlich zu sein. Möglicherweise können sie sich dabei neue Formen des Publikationsprozesses (Vorabveröffentlichung von Preprints bzw. Discussionpapers, neue Formen des Peer Review) und eine enge Einbindung in den wissenschaftsinternen Kommunikationsprozess zunutze machen.

Die bisherige Schlüsselstellung der etablierten Journale bei der Generierung von Anerkennung würde möglicherweise auch durch die Überprüfung und Erweiterung der wissenschaftsinternen Mechanismen zur Messung von Anerkennung und zur Verteilung von Ressourcen aufgebrochen werden. Das bisher relativ hohe Gewicht von Citationindex und Impactfaktoren in wissenschaftsinternen Evaluationsverfahren wäre möglicherweise durch andere Verfahren der Messung von Qualität und Anerkennung zu ergänzen. Insofern ist die Diskussion über neue Publikationsmodelle nicht unabhängig von der Diskussion über das wissenschaftsinterne System der Evaluation und Ressourcenverteilung. In diesem Sinne hat der Wandel des Publikationssystems möglicherweise gerade erst begonnen.

\section{Literatur}

Bergstrom (2004): Bergstrom, T. C.: The Costs and Benefits of Library Site Licenses to Academic Journals. In: PNAS (Proceedings of the National Academy of Sciences of the United States of America), Vol. 101, No. 3 (January 20, 2004), S. 897-902. http://www.pnas.org/cgi/doi/10.1073/pnas.0305628101 (Download vom 28.02.05).

Brody / Harnad (2004): Brody, T., Harnad, S.: Using Web Statistics as a Predictor of Citation Impact http://www.ecs.soton.ac.uk/ harnad/Temp/timcorr.doc (Download vom 28.02.05).

Buss / Wittke (2001): Buss, K.-P., Wittke, V.: Veränderungen im Verhältnis von Wissenschaft und Gesellschaft. In: Bender, Gerd (Hrsg.): Neue Formen der Wissenserzeugung. Frankfurt a. M. / New York, Campus Verlag, S.123-146.

Crane (1972): Crane, D.: Invisible Colleges. Diffusion of Knowledge in Scientific Communities. Chicago \& London, The University of Chicago Press. 
Foster / Gibbons (2005): Foster, N., Gibbons, S.: Understanding Faculty to Improve Content Recruitment for Institutional Repositories. D-Lib Magazine January 2005, Volume 11 Number 1.

Fournier (2005): Fournier, J.: The DFG Study of Author Experience of Open Access. http://www.eprints.org/berlin3/ppts/05-JohannesFournier.ppt.

Gibbons / Limoges / Nowotny / Schwartzman / Scott / Trow (1994): Gibbons, M., Limoges, C. Nowotny, H. Schwartzman, S., Scott, P., Trow, M.: The New Production of Knowledge: The Dynamics of Science and Research in Contemporary Societies. London, Sage.

Harnad (2005): Harnad, S.: Fast-Forward on the Green Road to Open Access: The Case Against Mixing Up Green and Gold. Ariadne 42. http://www.ariadne.ac.uk/issue42/harnad/ (Download vom 15.03.05).

Harnad / Brody / Vallieres / Carr / Hitchcock / Gingras / Oppenheim / Stamerjohanns / Hilf (2004): Harnad, S., Brody, T., Vallieres, F., Carr, L., Hitchcock, S., Gingras, Y, Oppenheim, C., Stamerjohanns, H., Hilf, E.: The Access/Impact Problem and the Green and Gold Roads to Open Access. Serials Review 30. http://www.ecs.soton.ac.uk/ harnad/Temp/impact.html (Download vom 28.02.05).

Harnad (2000): Harnad, S.: The Invisible Hand of Peer Review. http://exploitlib.org/issue5/peer-review/ (Download vom 28.02.05).

Lawrence (2001): Lawrence, S.: Online or Invisible? http://www.neci.nec.com/ lawrence/ papers/online-nature01 (Download 29.04.03).

Luhmann (1992): Luhmann, N.: Die Wissenschaft der Gesellschaft. Frankfurt a. M., Suhrkamp.

McCabe (2002): McCabe, M. J.: A Portfolio Approach to Print Legal Serials Pricing. http://www.prism.gatech.edu/ mm284/LegalPub.pdf (Download vom 28.02.05).

McCabe / Snyder (2004): McCabe, M. J., Snyder Ch. M.: A Model of Academic Journal Quality with Applications to Open-Access Journals. http://www.prism.gatech.edu/ mm284/OA2.pdf (Download vom 28.02.05).

Meier (2002): Meier, M.: Returning Science to the Scientists. Der Umbruch im STMZeitschriftenmarkt unter Einfluß des Electronic Publishing. In: Jäger, Georg (Hrsg.): Buchhandel der Zukunft. Aus der Wissenschaft für die Praxis. Münchner Buchwissenschaft an der Ludwig-Maximilians-Universität, Band 2. Peniope, München.

Merton (1985): Merton, R. K.: Entwicklung und Wandel von Forschungsinteressen. Aufsätze zur Wissenschaftssoziologie. Frankfurt a. M., Suhrkamp.

Mugabushaka (2005): Mugabushaka, A-M.: Open Access: Die strategische Kluft? Eine empirische Untersuchung von Determinanten des Publikationsverhaltens von Wissenschaftlern. Vortrag auf der IUK-Tagung 2005 am 09.05.2005 in Bonn; http://www.iuk-initiative.de. 
Nowotny / Scott / Gibbons (2004): Nowotny, H., Scott, P., Gibbons, M.: Wissenschaft neu denken. Wissen und Öffentlichkeit in einem Zeitalter der Ungewissheit. Weilerswist, Velbrück Wissenschaft.

Odlyzko (2000): Odlyzko, A.: The Future of Scientific Communication. http://www.dtc.umn.edu/ odlyzko/doc/future.scientific.comm.pdf (Download vom 28.02.05).

Pöschl (2004): Pöschl, U.: Interactive journal concept for improved scientific publishing and quality assurance. In: Learned Publishing (2004)17, S. 105-113. http://www.isn-oldenburg.de/ hilf/poeschl-publishing.pdf zuletzt abgerufen am 10.06.05.

Pöschl (2005): Pöschl, U.: Verbesserung wissenschaftlicher Kommunikation und Qualitätssicherung durch Open Access Publishing. Vortrag auf DINISymposium „Wissenschaftliches Publizieren der Zukunft - Open Access“, 23. 24.05.2005 in Göttingen. http://www.dini.de/veranstaltung /workshop/ goettingen_2005-05-23/programm.php zuletzt abgerufen am 10.06.05.

Roosendaal / Geurts / van der Vet (2002): Roosendaal, H. E., Geurts, P., Vet, P. E. van der: Integration of Information for Research and Education: Changes in the Value Chain? In: Serials Vol. 15, No. 1 (March 2002), S. 51-56.

Roosendaal (2005): Roosendaal, H. E.: Scientific Information: The Split between Availability and Selection; in diesem Band.

Solla Price (1974): Solla Price, D. J. de: Little Science, Big Science. Von der Studierstube zur Großforschung. Frankfurt a. M., Suhrkamp.

Tenopir / King (2000): Tenopir, C., King, D.W.: Towards Electronic Journals. Realities for Scientists, Librariens, and Publishers. Washington DC, SLA Publishing.

Tenopir / King / Boyce / Grayson / Zhang / Ebuen (2003): Tenopir, C., King, D. W., Boyce, P., Grayson, M., Zhang, Y., Ebuen, M.: Patterns of Journal Use by Scientists through Three Evolutionary Phases. http://www.dlib.org/dlib/ may03/king/05king.html (Download vom 28.02.05).

Tenopir / King / Boyce / Grayson / Paulson (2004): Tenopir, C., King, D. W.; Boyce, P., Grayson, M.; Paulson, K.-L.: Relying on Electronic Journals: Reading Patterns of Astronomers.

http://web.utk.edu/ tenopir/eprints/tenopir_jasist_article_042503_preprint.pdf

(Download vom 28.02.05).

Weingart (2003): Weingart, P.: Wissenschaftssoziologie. Bielefeld, Transcript Verlag. 



\title{
Open Access und Urheberrecht: Open Source in neuem Gewand?
}

\author{
Joachim Dorschel
}

\section{Vorbemerkung}

Das Prinzip des freien Zugangs zu wissenschaftlichen Informationen folgt, neben anderen, dem Gedanken, dass die Kommunikation wissenschaftlicher Erkenntnis nicht durch ein Mauerwerk starrer Zuweisungen ausschließlicher Eigentumsrechte an geistigen Leistungen blockiert werden darf, welche deren Rezipienten in der Nutzung und Auswertung beschränken und mit haftungsrechtlichen Unwägbarkeiten überziehen. ${ }^{164}$ Die technischen, wissenschaftstheoretischen, ökonomischen,

164 So heißt es etwa in der „Göttinger Erklärung für Bildung und Wissenschaft“ vom 5.7.2004: „In einer digitalisierten und vernetzten Informationsgesellschaft muss der Zugang zur weltweiten Information für Jedermann zu jeder Zeit von jedem Ort für Zwecke der Bildung und Wissenschaft sichergestellt werden! [...] Es gilt, im Urheberrecht faire und ausgewogene Bedingungen gesetzlich so zu regeln, dass die Nutzung von geschützten Werken angemessen vergütet, aber gleichzeitig deren Zugänglichkeit für Zwecke der Bildung und Wissenschaft nicht behindert wird.", Erklärung abrufbar unter http://www.urheberrechtsbuendnis.de/index.html; In die gleiche Richtung geht der Ansatz der „Science Commons“ - Initiative der Creative Commons- Bewegung, siehe http://science.creativecommons.org/, hier heißt es u.a.: "Progress in modern technology combined with a legal system designed for a different technology-based environment is now leading to 
sozial- und bibliothekswissenschaftliche Fragen, die sich aus dem Zusammenspiel neuer innovativer Publikationstechniken, traditioneller Wertschöpfungsketten und der Ressourcenknappheit der öffentlichen Hand im wissenschaftlichen Umfeld ergeben und die sich nur zum Teil unter dem viel bemühten Begriff der „Publication Crisis" rubrizieren lassen (zum Begriff der Publication Crisis vgl. Bargheer in diesem Band), sind zentraler Gegenstand des Mediaconomy- Projekts und wurden in den vorhergehenden Beiträgen eingehend behandelt. Die vorliegende Arbeit setzt sich daher mit den spezifisch juristischen Fragestellungen auseinander, die die Implementierung von Publikationsmodellen auf Basis von Open Access aufwerfen.

Bei der Suche nach juristischen Ansatzpunkten muss das Rad nicht neu erfunden werden. Der Umgang mit Open Content ist im Zuge der zunehmenden Etablierung von Open Source Software auch im gewerblichen und verwaltungswirtschaftlichen Bereich seit Jahren Gegenstand juristischer Analyse. Freilich sind die rechtlichen Probleme auch in diesem Bereich in keiner Weise konsolidiert (vgl. Jäger / Metzger 2005; Spindler 2004; Spindler in Büllesbach / Dreier 2004) ${ }^{165}$. Die Vielzahl der ungelösten Fragen konfrontiert damit die im Aufbau befindlichen praktischen Umsetzungen der Open Access-Bewegung mit ähnlichen Rechtsunsicherheiten wie auch die Lieferanten und Nutzer von Open Source Software. Gleichzeitig können aber die rechtlichen Lösungen, die die Rechtswissenschaft im Bereich der Open Source Software anbietet, nicht ohne weiteres auf Fragen der Wissenschaftskommunikation übertragen werden. Denn letztere ist in hohem Maße von den Freiheitsrechten des Art. 5 Abs. 3 GG geprägt, deren objektivrechtlicher Gehalt auch das Immaterialgüter- und Vertragsrecht durchsetzt (vgl. hierzu unten).

Der vorliegende Beitrag beruht auf ersten Überlegungen und Recherchen, die zu Beginn des Mediaconomy-Projektes durchgeführt wurden. Vornehmliches Ziel ist es daher (noch) nicht, konkrete Lösungen für die mannigfachen rechtlichen Detailprobleme anzubieten - dies soll im Rahmen eines umfassenden Leitfadens geschehen, dessen Veröffentlichung Anfang 2006 geplant ist. Vorliegend soll zunächst ein gewisser Überblick über die verschiedenen juristischen Probleme sowie der bisher entwickelten Lösungsansätze gegeben werden.

some unintended consequences. One of these is a kind of legal "friction" that hinders reuse of scientific discoveries and could lead to discouraging innovation."

165 Siehe nur Jäger / Metzger: Open Source Software: Rechtliche Rahmenbedingungen der freien Software, 2. Aufl. 2005; Spindler (Hg.), Rechtsfragen bei Open Source, 2004; Spindler in Büllesbach / Dreier, Wem gehört die Information im 21. Jahrhundert? Proprietäre versus nicht proprietäre Verwertung digitaler Inhalte, 2004; Spindler, K\&R 2004, 528. Spindler / Wiebe, CR 2003, 873 ff.; Spindler in v. Taeger / Wiebe, Festschrift für Wolfgang Kilian, S. 353 ff. 


\section{Einführung}

Die mannigfachen Gestaltungsmöglichkeiten von Open Access-Modellen (vgl. hierzu eingehend Bargheer in diesem Band) lassen sich für die juristische Bewertung in zwei Kategorien zusammenfassen. Die einfachste Form bilden jene Konstellationen, in denen der Verfasser eines Werkes dieses selbst im Wege des „Open Access-Archiving“, z. B. auf Pre- oder Postprintservern zum kostenlosen Abruf bereitstellt.

Unter der Prämisse, dass auch Open Access im Hinblick auf die Renommeestrukturen des etablierten Verlagswesens einer gewissen Institutionalisierung bedarf, gilt es aber vor allem jene Modelle zu untersuchen, in denen Open AccessInstitutionen, sei es der Anbieter eines speziellen Open Access-Medienproduktes oder auch die Universität des wissenschaftlichen Autors (z. B. in der Form von Institutional Repositories) das Werk für die Öffentlichkeit bereithält. Denn während der Verfasser im ersten Fall seine originären Rechte selbst ausübt, bedarf es im zweiten Fall schon auf Seiten des Anbieters eines Rechtsgeschäftes - was Fragen aufwirft, welche weniger aus dem Open Source Bereich als aus dem Bereich des klassischen Verlagsrechts bekannt sind.

Parallelen zur Open Source Software ergeben sich dagegen auf Nutzerseite: Denn hier wie dort gilt es, das Spannungsfeld zwischen dem Prinzip des „offenen Zugangs" auf der einen Seite und dem Wunsch mancher Urheber, die Nutzung seines Werkes - teils aus ideellen, teils aus ökonomischen Motiven - auf bestimmte „gewünschte“ Nutzungsformen, z. B. die nicht- kommerzielle Nutzung zu beschränken, durch lizenzvertragliche Gestaltung in Einklang zu bringen (vgl. Spindler 2003). ${ }^{166}$

\section{Rechtsbeziehungen auf Seiten der Verfasser und Anbieter des Werkes}

\subsection{Vertragsrechtliche Ausgestaltung}

Grundansatz der vertraglichen Beziehungen auf Anbieterseite ist es, die für die Realisierung der Publikation zuständigen Institutionen mit den hierfür erforderlichen Nutzungsrechten auszustatten, insbesondere zur Bereithaltung zum Abruf sowie zur Vervielfältigung und Verbreitung auf Anfrage (Print on Demand).

\footnotetext{
$166 \mathrm{Vgl}$. für den Bereich der Open Source Software: Spindler: Rechtsfragen bei Open Source, 2003, S. 39 ff mit umfangreichen Nachweisen sowie LG München, Urteil v. 19.5.2004 - 21 O 6123/04, CR 2004, 774 ff.
} 
Im Verhältnis von Urheber und Publikationsintermediär unterscheiden sich daher Open Access-Modelle zunächst nicht von klassischen Publikationsformen. Während allerdings im klassischen Verlagsvertrag die Pflicht des Verfassers zur Einräumung des ausschließlichen Vervielfältigungs- und Verbreitungsrechts (Verlagsrecht, vgl. \ 8 VerlG) und die Pflicht des Verlegers, das Werk zu veröffentlichen, den Kern eines synallagmatischen Vertragsverhältnisses bilden ${ }^{167}$, liegt der Schwerpunkt in den Leistungsbeziehungen bei Open Access-Publikationen auf den technischen und vertrieblichen Dienstleistungen des Anbieters wie Aufbereitung des Werkes, Bereitstellung auf leistungsfähigen Servern, Einstellen in geeignete Nachweiskataloge, welche durch den Verfasser entsprechend vergütet werden. Das Vertragsverhältnis zwischen Verfasser und Open Access-Anbieter gleicht daher eher dem eines Web-Design- und Web-Hosting-Vertrages, bei dem miet- und werkvertragliche Elemente im Vordergrund stehen ${ }^{168}$. Allerdings hat der Verfasser, in der Regel, anders als bei Web-Hosting-Verträgen, keine Möglichkeit, etwa per ftp- Verbindung auf dessen Systeme zuzugreifen. Die physische Kontrolle über das Werk übt hier allein der Anbieter aus. In diesem Fall fehlt es an der für eine Anwendbarkeit der mietrechtlichen Vorschriften erforderlichen Gebrauchsgewährung. ${ }^{169}$ Die Online-Bereitstellung des Werkes mag daher - je nach vertraglicher Ausgestaltung - als Werk- oder Dienstvertrag einzuordnen sein. Die Rechtseinräumung stellt regelmäßig keine Hauptpflicht dar sondern bildet als notwendige Mitwirkungshandlung eine vertragliche Nebenpflicht des Verfassers.

Der entscheidende Unterschied zu klassischen Verlagsverträgen besteht jedoch im Umfang der Rechtsübertragung. So sehen Verlagsverträge in aller Regel die Einräumung des ausschließlichen Rechtes zur Vervielfältigung und Verbreitung vor, in Ermangelung einer ausdrücklichen Regelung ergibt sich diese Verpflichtung aus $\int 8$ Abs. 1 VerlG. ${ }^{170}$ Verträge über Open Access-Publikationen fordern vom Verfasser meist nur die Einräumung einfacher Nutzungsrechte (vgl. die Beispiele bei Bargheer in diesem Band). Dies gilt auch, in Anwendung des Zweck-

167 Schack, Urheber- und Urhebervertragsrecht, 3. Aufl. 2005, Rz. 994; Schricker, Verlagsrecht, 3. Aufl. 2001, J 1 Rz. 11; Ulmer, Urheber- und Verlagsrecht, 3. Aufl. 1980, S. 435.

$168 \mathrm{Vgl}$. M. Schmidt in Spindler, Vertragsrecht der Internetprovider, 2. Aufl., 2004, Teil VIII Rz. 4 ff.; M. Schuppert in Spindler, Vertragsrecht der Internetprovider, 2. Aufl., 2004, Teil V Rz. 1 ff., beide m.w.N.

169 Die Gebrauchsgewährung liegt bei Web-Hosting-Verträgen gerade in der Zugangsgewährung zu den vertraglich geschuldeten Speicherressourcen, vgl. Schuppert in Spindler, Vertragsrecht der Internetprovider, 2. Aufl., 2004, Teil V Rz. 4; Cichon, Internet- Verträge, 2. Aufl. 2005, Rz. 186; Schuster / Müller in Schuster, Vertragshandbuch Telemedia, 2001, Teil 6 Rz. 15.

170 Freilich ist die Einräumung des subjektiven Verlagsrechts kein begriffliches Merkmal des Verlagsvertrages, was sich nicht zuletzt daraus ergibt, dass Verlagsverträge auch über gemeinfreie Werke geschlossen werden können (vgl. \ 39 VerlG) und auch die Verpflichtung aus \ 8 VerlG zur Bestellung des Verlagsrechts vertraglich abdingbar ist, Schricker, Verlagsrecht, 3. Aufl. 2001, ऽ 1 Rz. 11. 
übertragungsgrundsatzes, ${ }^{171}$ soweit keine ausdrückliche Bestimmung des Nutzungsrechtsumfangs erfolgt ist. Denn während im Rahmen klassischer Publikationsformen der Vertragszweck auf die Amortisation der Publikationskosten durch die exklusive Ausübung der Verwertungsrechte durch den Verleger gerichtet ist, bedarf der Open Access-Anbieter, dessen Finanzierungsmodell nicht auf Subskriptionsgebühren basiert (vgl. hierzu im einzelnen Bargheer in diesem Band), gerade keiner ausschließlichen, ein Verbietungsrecht gegenüber Dritten begründenden ${ }^{172}$ Rechte, an dem Werk.

\subsection{Kollision von Open Access und Verlagsverträgen}

Fälle, in denen Wissenschaftler ein Werk ausschließlich für den „elektronischen Markt" verfassen, sind nach wie vor in der Minderzahl. Veröffentlichungen auf Open Access Basis erfolgen, den Zwängen etablierter Verlagsstrukturen und Renommee-Erwartungen folgend, meist zusätzlich zu einer Publikation desselben Werkes auf traditionellem Weg. Der Konflikt zwischen dem Interesse des Verlegers an einer möglichst weitgehenden Monopolmacht über das verlegte Werk auf der einen und dem Interesse des Wissenschaftlers an einer Verfügbarmachung seiner Arbeit auf der anderen Seite liegt auf der Hand.

Tatsächlich kann in der Vertragspraxis hier jedoch ein gewisser Paradigmenwechsel verzeichnet werden:

Traditionell wird dem Autor neben der Einräumung der klassischen Hauptund Nebenrechte eine Übertragung auch der Online-/Offline-Rechte abverlangt, unabhängig davon, ob der Verlag diese Rechte tatsächlich zu nutzen gedenkt. Um der im Hinblick auf den Zweckübertragungsgrundsatz einschränkenden Auslegung von Generalklauseln zu entgehen, erfolgt dies meist über Buy-out-Verträge, in welchen enumerativ alle denkbaren Nutzungsrechte aufgelistet werden. Wenngleich es sich hierbei offensichtlich um eine bewusste Umgehung des $\int 31$ Abs. 5 S. 1 UrhG handelt, werden solche Verträge, soweit sie die Grenzen des \138 bzw. der $\iint 306$ ff. BGB nicht sprengen, von der h.M. wegen des Grundsatzes der Vertragsfreiheit als wirksam erachtet. ${ }^{173}$ Damit erwirbt der Verleger jedoch ein, die ergänzende Veröffentlichung auf Open-Access-Basis ausschließendes, Verbietungs-

171 Ist der Umfang der Rechtsübertragung im Vertrag nicht im Einzelnen bezeichnet, so bestimmt sich dieser gem. $\int 31$ Abs. 5 S. 1 UrhG nach dem zu Grunde liegenden Vertragszweck. Dies ist Ausdruck der grundsätzlichen Tendenz des Urheberrechts, soweit als möglich beim Urheber zurückzubleiben, vgl. Ulmer, Urheber- und Verlagsrecht, 3. Aufl. 1980, S. 365, so dass Unklarheiten bei Bestimmung der Reichweite der Rechtsübertragung zu Lasten des Lizenznehmers wirken.

172 BGH, Urt. v. 21.4.1953 - I ZR 110/52, BGHZ 9, 262 (264) - Lied der Wildbahn; BGH, Urt. v. 12.12.1991 - I ZR 165/89, GRUR 1992, 310 (311); Wandtke / Grunert in Wandtke / Bullinger, UrhG, 2002, \ 31 Rz. 29; Schulze in Dreier/Schulze, UrhG, 2004, \ 31 Rz. 56; Schricker in Schricker, UrhR, 2. Aufl. 1999, \$S 31/32 Rz. 5.

173 vgl. Wandtke / Grunert in Wandtke / Bullinger, UrhG, 2002, \31 Rz. 73. 
recht sowohl gegenüber dem Autor als auch gegenüber potenziellen Open AccessAnbietern.

Während diese Tradition von zahlreichen Verlegern mit einigem Nachdruck gepflegt wird, haben einige wissenschaftliche Verlage unter dem Rubrum des „grünen Wegs“ Ansätze entwickelt, vor- oder nachgelagerte Veröffentlichungen auf Pre- oder Postprintservern zu gestatten. ${ }^{174}$ Die vertragsrechtliche Ausgestaltung solcher Konzepte ist vielfältig und reicht von einer tatsächlichen Beschränkung der Rechtseinräumung auf das für den Vertragszweck erforderliche Maß bis zu einer „traditionellen“ vollständigen Übertragung, gepaart mit einer schuldrechtlichen Gestattung einzelner Nutzungshandlungen nach Ablauf einer gewissen Schutzfrist.

Aus Sicht des Open Access-Archiving ist vor allem letztgenannte Variante nachteilig. Denn die schuldrechtliche Gestattung wirkt im Gegensatz zu den (quasidinglichen) urheberrechtlichen Nutzungsrechten nur inter partes, ${ }^{175}$ wobei der wissenschaftliche Autor ohne Zustimmung des Verlages nicht berechtigt ist, Dritten, etwa seiner Universität, überhaupt Nutzungsrechte einzuräumen. Gleiches gilt, wenn dem Urheber lediglich ein einfaches Nutzungsrecht verbleibt. Auch dieses berechtigt seinen Inhaber nicht zu weiteren konstitutiven Verfügungen. ${ }^{176}$ So weit dem Verfasser bei der Gestaltung des Verlagsvertrages ein tatsächlicher Verhandlungsspielraum bleibt, sollte er darauf achten, die Rechtseinräumung so weit als möglich auf einfache Nutzungsrechte zu beschränken und die ausschließlichen Rechte bei sich zu behalten. Den berechtigten Amortisationsinteressen des Verlages kann durch geeignete schuldrechtlich vereinbarte Schutzfristen Rechnung getragen werden.

De lege ferenda wird bereits überlegt, diesen Weg urhebervertragsrechtlich indisponibel abzusichern.

\subsection{Sonderfall angestellte Wissenschaftler}

Eine andere Frage ist, inwieweit Dritten Partizipationsrechte an wissenschaftlichen Forschungsergebnissen zustehen. In Rede stehen hier insbesondere Universitäten und andere öffentliche Forschungseinrichtungen, welche durch den Einsatz ihrer Ressourcen, etwa des Arbeitsentgelts des Autors, wissenschaftlicher Hilfskräfte, der Infrastruktur der Universität usw. die Schaffung wissenschaftlicher Werke finanzieren. Dem Grunde nach geht es hier um die Partizipationsrechte der Allgemeinheit an öffentlich finanzierter Forschung.

\footnotetext{
${ }^{174}$ Einen Überblick über die Verlagspraxis im anglo-amerikanischen Raum gibt http://www.sherpa.ac.uk/romeo.php.

175 Schricker in Schricker, UrhG, 2. Aufl. 1999, Vor $\$ \int 28$ ff. Rz. 25 m.w.N.

176 Schricker a.a.O. $\int \$ 31 / 32$ Rz. 6 sowie Vor $\int S 28$ Rz. 23.
} 


\section{Arbeitsvertrag und Übertragung von Nutzungsrechten: Rechtslage de lege lata}

Nach \43 UrhG gilt für die Rechtsübertragung im Rahmen von Angestellten- und Dienstverhältnissen nichts anderes als für Rechtsübertragungen gegenüber Dritten: diese setzt eine ausdrückliche oder konkludente Zustimmung des Autors voraus, welche dieser erteilen oder auch verweigern kann. Zwar gilt im Grundsatz, dass der Urheber die Rechte an den in Ausübung seiner Arbeits- oder Dienstpflicht geschaffenen Werken auf seinen Arbeits- oder Dienstherrn zu übertragen hat ${ }^{177}$, wobei i.d.R. von einer stillschweigenden Rechtsübertragung bei Übergabe des Werkes ausgegangen wird. ${ }^{178}$ Bei wissenschaftlich tätigem Personal unterfällt diese Dienstpflicht jedoch dem verfassungsrechtlichen Grundsatz der freien Forschung und Lehre (Art. 5 Abs. 3 GG), was jenen die Freiheit gibt, die inhaltliche Ausgestaltung ihrer Lehr- und Forschungspflichten frei von Weisungen vorzunehmen. ${ }^{179}$

Eine Dienstpflicht von Hochschulprofessoren, ein bestimmtes urheberrechtlich geschütztes Werk herzustellen, besteht grundsätzlich nicht. ${ }^{180}$ Demzufolge ist ein Hochschullehrer auch nicht verpflichtet, der Universität die Nutzungsrechte an seinem Werk zu übertragen. ${ }^{181}$ Gleiches gilt für andere wissenschaftliche Angestellte, soweit diese selbstständig wissenschaftlich tätig sind. ${ }^{182}$

177 Dies ergibt i.d.R. schon der Zweck des Arbeitsvertrages, da der Arbeitgeber die Nutzungsrechte zur Verwertung des Werkes auf dem Markt benötigt, vgl. Spautz in Möhring / Nicolini, UrhG, 2. Aufl. 2000, \43 Rz. 7; Rojahn in Schricker, UrhG, 2. Aufl. 1999, \43 Rz. 38; Wandtke in Wandtke/Bullinger, UrhG, 2002, \43 Rz. 30; Haberstumpf, ZUM 2001, 819 (825).

178 RG, Urt. v. 8.4.1925 - I 251/24, RGZ 110, 393 (396) - Innenausstattung Riviera; BGH, Urt. v. 26.10.1951 - I ZR 93/51, GRUR 1952, 257 (258) - Krankenhauskartei; BGH; Urt. v. 12.4.1960 I ZR 173/58, GRUR 1960, 609 (612) - Wägen und Wagen; BGH, Urt. v. 22.2.1974 - I ZR 128/72, GRUR 1974, 480 (483) - Hummelrechte; BGH, Urt. v. 10.5.1984 - I ZR 85/82, GRUR 1985, 129 (130) - Elektrodenfabrik; BAG, Urt. v. 24.11.1960 - 5 AZR 261/60, GRUR 1961, 491 - Nahverkehrschronik; BAG, Urt. v. 13.9.1983 - 3 AZR 371/81, GRUR 1984, 429 (431) - Statikprogramme; BAG, Urt. v. 12.3.1997 - 5 AZR 669/95, DB 1997, 1571 f. - Schaufensterdekoration; KG, Urt. v. 29.11.1974 - 5 U 1736/74, GRUR 1976, 264 - gesicherte Spuren; Rojahn in Schricker, UrhG, 2. Aufl. 1999, \43 Rz. 40 f.; Vinck in Fromm / Nordemann, UrhG, 9. Aufl. 1998, \ 43 Rz. 3; Spautz in Möhring / Nicolini, UrhG, 2. Aufl. 2000, \43 Rz. 8; Wandtke in Wandtke / Bullinger, UrhG, 2002, \ 43 Rz. 50; Ulmer, Urheber- und Verlagsrecht, 3. Aufl. 1980, S. $402 \mathrm{f}$.

179 BVerfG, Beschl. v. 1.3.1978 - 1 BvR 333/75, 1 BvR 174/71, 1 BvR 178/71, 1 BvR 191/71, NJW 1978, 1621; Pernice in Dreier, GG, 2. Aufl. 2004, Art. 5 Abs. 3 Rz. 36 ff.; Haberstumpf, ZUM 2001, 819 (825); Dallinger, JZ 1971, 665 (667); Strauch JZ 1972, 43 ff.

180 BGH, Urteil v. 27.9.1990 - I ZR 244/88, NJW 1991, 1480 (1481) - Grabungsmaterialien; Moltke, Urheberrecht an den Werken der Wissenschaft, 1992, S. 226; Pflüger / Ertmann, ZUM 2004, 436 (439); Haberstumpf, ZUM 2001, S. 819 (825 f.).

181 BGH, Urt. v. 6.2.1985 - I ZR 179/82, GRUR 1985, 530 - Happening m. Anm. Jacobs; Haberstumpf, ZUM 2001, 819 (826); Wandtke in Wandtke / Bullinger, UrhG, 2002, \43 Rz. 43; etwas anderes mag etwa für Klausuren im universitären Prüfungsverfahren gelten, vgl. LG Köln, Urt. v. 1.9.1999 - 28 O 161/99, NJW-RR 2000, 1294 (1295), ebenso für Auftragsforschung, vgl. Rojahn in Schricker, UrhG, 2. Aufl. 1999, \43 Rz. 7 und langfristige Forschungsprojekte, vgl. BGH, Ur- 


\section{Zwangslizenzen, Urheberrechtsschranken und Vertragsrecht - Vorschläge de lege ferenda}

Während die Diskussion um die Partizipationsansprüche der Allgemeinheit an öffentlich finanzierten Werken auf politischer Ebene in vollem Gange ist, entwickelt die Rechtswissenschaft konkrete Vorschläge nur sehr zögerlich. Im Wesentlichen werden in der Literatur drei verschiedene Ansätze diskutiert, deren gemeinsames Ziel es ist, den Autor eines öffentlich finanzierten wissenschaftlichen Werkes zu zwingen, die Allgemeinheit in gewissem Umfang an diesem partizipieren zu lassen und den Verlagen die Möglichkeit zu nehmen, durch einseitige Vertragsgestaltungen eine solche (kostenlose) Partizipation zu verhindern. So wird zum einen vorgeschlagen, in Anlehnung an die Grabungsmaterialien-Entscheidung des BGH ${ }^{183}$, dem angestellten wissenschaftlichen Autor eine zeitlich befristete Anbietungspflicht zu Gunsten der Hochschule oder Forschungseinrichtung aufzuerlegen. ${ }^{184}$ Innerhalb einer gesetzlich bestimmten Frist habe der das Werk finanzierende Arbeitgeber die Möglichkeit (nicht die Pflicht), ein (wohl einfaches) Nutzungsrecht an dem Werk zu verarbeiten und dieses, etwa im hauseigenen Verlag oder in entsprechenden Repositorien, zu veröffentlichen. Der Sache nach handelt es sich hierbei letztlich um eine Zwangslizenz, wie sie das Urheberrecht bislang nur zur Herstellung von Tonträgern kennt (vgl. \42a UrhG). ${ }^{185}$

Ein weiterer Ansatzpunkt wäre die Implementierung einer neuen urheberrechtlichen Schranke zu Gunsten von Wissenschaft und Forschung, welche, nach Ablauf einer - je nach Wissenschaftsgebiet individuell zu bestimmenden - Sperrfrist, die öffentliche Zugänglichmachung eines überwiegend aus öffentlichen Mitteln finanzierten Werkes für wissenschaftliche Zwecke erlaubt. ${ }^{186}$

Ein dritter Vorschlag sieht eine Verzahnung öffentlich-rechtlicher und urhebervertragsrechtlicher Regelungen vor. ${ }^{187}$ So könnte per zwingender gesetzlicher Anordnung dem Urheber auch im Falle einer ausschließlichen Übertragung der betreffenden Rechte das einfache Recht zur öffentlichen Zugänglichmachung, etwa auf entsprechenden Repositorien des Instituts, ggf. nach Ablauf einer gewissen Sperrfrist, verbleiben. Die Pflicht zu einer solchen öffentlichen Zugänglichmachung könnte sich dann aus entsprechenden dienst- oder hochschulrechtlichen Bestimmungen ergeben.

teil v. 27.9.1990 - I ZR 244/88, NJW 1981, 1480 (1482) - Grabungsmaterialien; dagegen Haberstumpf, ZUM 2001, 819 (827).

182 Wandtke in Wandtke / Bullinger, UrhG, 2002, \43 Rz. 42; Dreier in Dreier / Schulze, UrhG, 2004, \43 Rz. 12; Rojahn in Schricker, UrhG, 2. Aufl. 1999, \43 Rz. 132; Haberstumpf, ZUM 2001, 819 (827).

183 So o. Fn. 180.

184 So Pflüger / Ertmann, ZUM 2004, 436 (441).

185 Schulze in Dreier / Schulze, UrhG, 2004, Vor $\$ 31$ Rz. 3; in Dreier / Schulze, UrhG, 2004, Vor IS 44a ff. Rz. 12.

186 Vgl. hierzu Hansen, GRUR Int. 2005, 378 (383 ff.).

187 So Hansen a.a.O. 386 f. 


\section{Verfassungs-, europa- und völkerrechtliche Grenzen der urheber- rechtlichen Gestaltungsmöglichkeiten.}

Freilich muss bei Bewertung der denkbaren gesetzgeberischen Handlungsalternativen berücksichtigt werden, dass das Urheberrecht, wie auch das Recht der Wissenschaft und Forschung, einer Vielzahl vor- oder gleichrangiger Rechtsbestimmungen unterliegt, welche den Handlungsspielraum des Gesetzgebers beschränken.

Aus verfassungsrechtlicher Sicht setzt vor allem Art. 5 Abs. 3 GG dem Gesetzgeber enge Grenzen. Denn die Freiheit von Wissenschaft und Forschung ist als vorbehaltloses Grundrecht durch einfachgesetzliche Regelungen nicht, auch nicht zu Gunsten des Allgemeinwohls, beschränkbar. ${ }^{188}$ Einschränkungen ergeben sich lediglich aus der Verfassung selbst, so etwa dem Schutz anderer Rechtsgüter von Verfassungsrang. ${ }^{189}$

Das Bedürfnis nach kostenloser Verfügbarkeit urheberrechtlich geschützter wissenschaftlicher Werke genießt aber per se einen solchen Verfassungsrang nicht. Denn weder ergibt sich ein unmittelbarer Anspruch auf nicht öffentlich zugängliche Informationen, auf die sonst kein Rechtsanspruch besteht, zu Gunsten der Wissenschaft aus Art. 5 Abs. 3, ${ }^{190}$ noch lässt sich ein solcher aus Art. 5 Abs. 1 S. 1 GG herleiten, da insofern das Urheberrecht als einfachgesetzliche Schranke wirkt. ${ }^{191}$

Auch aus dem objektiv-rechtlichen Gehalt des Art. 5 Abs. 3 GG wird man einen solchen Verfassungsrang nicht herleiten können. Zwar mag man die Verfügbarkeit wissenschaftlicher Informationen durchaus unter die aus Art. 5 Abs. 3 abgeleitete Staatsaufgabe der „Schaffung und Beachtung von Rahmenbedingungen der Kreativität“ 192 subsumieren, gleichwohl wird man hieraus Konsequenzen allenfalls auf der leistungsstaatlichen Ebene ziehen können. ${ }^{193}$

Damit reduzieren sich die Handlungsoptionen des Gesetzgebers auf Maßnahmen jenseits des Schutzbereichs der Wissenschaftsfreiheit. ${ }^{194}$ Dies verbietet jede gesetzliche Regelung, gleich ob urheber- oder dienstrechtlicher Natur, die dem Autor die Entscheidung für oder gegen eine Veröffentlichung seines Werkes als

\footnotetext{
188 Statt aller Pernice in Dreier, GG, 2. Aufl. 2004, Art. 5 Abs. 3 Rz. 39.

189 Starck in v. Mangoldt / Klein / Starck, GG, 4. Aufl. 1999, Art. 5 Abs. 3 Rz. 375; Pernice in Dreier, GG, 2. Aufl. 2004, Art. 5 Abs. 3 Rz. 39 f.; BVerfG, 1 Beschl. v. 1.3.1978 - 1 BvR 333/75, BVerfGE, 47, 327 (369); s. auch Pflüger / Ertmann, ZUM 2004, 436 (440).

190 Vgl. für Akteneinsichtsansprüche gegenüber Behörden BVerwG, Beschl. v. $9.10 .1985-7$ B 188/85, NJW 1986, 1277 (1278); Wendt in von Münch / Kunig, GG, 5. Aufl. 2000, Art. 5 Rz. 105; Starck in v. Mangoldt / Klein / Starck, GG, 3. Aufl. 1999, Art. 5 Abs. 3 Rz. 332.

191 Schulze-Fielitz in Dreier, GG, 2. Aufl. 2004, Art. 5 I, II Rz. 85; Hoffmann-Riem in AK-GG, 2001, Art. 5 I, II Rz. 116; OLG Köln, Urt. v. 1.3.1978 - 2 U 133/77, DVBl. 1979, 523 (524).

192 Blankenagel, AöR 105 (1980), 35 (65 ff., 70); Pernice in Dreier, GG, 2. Aufl. 2004, Art. 5 III Rz. 55.

193 S. hierzu Pernice in Dreier, GG, 2. Aufl. 2004, Art. 5 Abs. 3 Rz. 55.

194 Ebenso Pflüger / Ertmann, ZUM 2004, 436 (440 f.).
} 
solche nimmt. Denn aufgrund seiner negativen Publikationsfreiheit ist ein Wissenschaftler grundsätzlich berechtigt, seine Arbeit, auch dauerhaft, geheim zu halten, sei sie nun öffentlich finanziert oder nicht. ${ }^{195}$ Ebenso unzulässig wäre selbstverständlich eine, auch befristete, Regelung, welche dem Autor verbietet, sein Werk zu veröffentlichen (positive Publikationsfreiheit). ${ }^{196}$

Unklar ist, inwieweit der Gesetzgeber berechtigt ist, auf die Art und Weise der Publikation Einfluss zu nehmen, insbesondere dann, wenn es um die Publikation in einem kommerziellen Verlag geht. Denn ob die kommerzielle Verwertung von Forschungsergebnissen von Art. 5 Abs. 3 GG umfasst ist, ist in der Literatur streitig. ${ }^{197}$

Verneint man dies, so stellt sich die Frage, ob die Veröffentlichung von „dienstlich“ gewonnenen Forschungsergebnissen bei einem kommerziellen Verlag auch dieser „dienstlichen“ Forschung oder aber einer, außerhalb der Freiheit von Wissenschaft und Forschung liegenden, rein kommerziellen Nebentätigkeit zuzuordnen ist. ${ }^{198}$ Dagegen spricht jedoch, dass die Wahl der Publikationsform, etwa des Publikationsmediums und des Publikationsorgans, Teil des Prozesses der Verbreitung und Bewertung der gewonnenen wissenschaftlichen Erkenntnisse ist - und damit integraler Bestandteil der wissenschaftlichen Forschung selbst. ${ }^{199}$ Auch der „kommerzielle“ Charakter der Veröffentlichung muss, zumindest für das Urheberrecht ${ }^{200}$, bezweifelt werden. Die Veröffentlichung dient im wissenschaftlichen Bereich gerade nicht der Gewinnerzielung dies zeigen Finanzierungsmodelle auf der Basis von Autorengebühren und Druckkostenzuschüssen überdeutlich (vgl. Bargheer in diesem Band) - sondern der Verbreitung von Forschungsergebnissen und damit letztlich dem wissenschaftlichen Renommee des Autors. Daher wird man der (zweifelsfrei von der hauptamtlichen Tätigkeit des angestellten Wissenschaftlers und der Frei-

195 S. auch Pflüger / Ertmann a.a.O.; Brehm / Zimmerling, WissR 2001329 (347); Thieme, Deutsches Hochschulrecht, 3. Aufl. 2004 Rz. 127; vgl. für die gleiche Frage bei $\int 42$ ArbEG Beyerlein, NZA 2002, 1020; Körting / Kummer, RdA 2003, 279 (284); Leuze, GRUR 2005, 27 (28); ebenso die amtl. Begr. zum ArbEG, BT-Drs. 14/5975 S. 5 und 7; a.A. Pernice in Dreier, GG, 2. Aufl. 2004, Art. 5 III Rz. 28, der die negative Publikationsfreiheit dem Art. 12 Abs. 1 zuordnen will.

196 Beyerlein, NZA 2002, 1020 (1022); Körting / Kummer, RdA 2003, 279 (282); Thieme, Deutsches Hochschulrecht, 3. Aufl. 2004. Rz. 776; unklar Pflüger / Ertmann, ZUM 2004, 436 (441).

197 Dagegen etwa Kraßer, in: Hartmer / Detmer, Hochschulrecht, 2004, S. 463 Fußn. 33; auf den objektiv-rechtlichen Gehalt des Art. 5 Abs. 3 abstellend und damit für eine Einbeziehung der kommerziellen Verwertung streitend Pernice in Dreier, GG, 2. Aufl. 2004, Art. 5 Abs. 3, Rz. 62; s. auch Beier/Straus, Der Schutz wissenschaftlicher Forschungsergebnisse, 1982, S. 7 ff. 85 f.

198 Freilich kann ein Hochschullehrer auch bei Ausübung einer Nebentätigkeit den Schutz des Art. 5 Abs. 3 GG in Anspruch nehmen vgl. Thieme, Deutsches Hochschulrecht, 3. Aufl. 2004, Rz. 770.

199 Tettinger / Lux-Wesener, in: Hartmer / Detmer, Hochschulrecht, 2004, S. 226 Rdnr. 65 sprechen von einem ,konstitutiven Element wissenschaftlicher und damit durch Art. 5 III GG geschützter Tätigkeit“; s. hierzu auch Leuze, GRUR 2005, 27 (28).

200 Etwas anderes mag etwa im Patent- und Gebrauchsmusterrecht gelten. 
heit von Forschung und Lehre erfassten) Freiheit der Veröffentlichung ${ }^{201}$ auch die Wahl einer kommerziellen Publikationsform zuordnen müssen.

Dies zu Grunde gelegt sind die Möglichkeiten, den freien Zugang zu wissenschaftlichen Werken zwangsweise durchzusetzen, außerordentlich begrenzt. Denn letztlich wird jede Regelung, die den Autor in irgendeiner Weise dazu zwingt, sein Werk bei einem bestimmten Publikationsorgan, z. B. einem Universitätsrepositorium, zu veröffentlichen, mit der Freiheit von Wissenschaft und Forschung kollidieren.

Verfassungsrechtlich weniger bedenklich wäre dagegen die Implementierung einer entsprechenden Urheberrechtsschranke. Zwar würde auch eine solche de facto dazu führen, dass der wissenschaftliche Autor die Weiterverbreitung eines einmal veröffentlichten Werkes über ihm möglicherweise nicht genehme Kanäle zu dulden hätte, eine solche faktisch-mittelbare Beeinträchtigung der Publikationsfreiheit bliebe jedoch schon von ihrer Intensität her unter der grundrechtlich relevanten Eingriffsschwelle. ${ }^{202}$ Der freilich nicht unerhebliche Eingriff in die Eigentumsgarantie des Art. 14 GG wäre, zumindest in Grenzen, von der Sozialbindung des Eigentums gedeckt. ${ }^{203}$

Probleme bestehen hier allerdings auf europa- und völkerrechtlicher Ebene: So enthält die InfoSoc-Richtlinie ${ }^{204}$ in Art. 5 einen erschöpfenden ${ }^{205}$ Katalog gemeinschaftsrechtlich zulässiger Urheberrechtsschranken. Die hier diskutierte Beschränkung der Ausschließlichkeitsrechte des Urhebers / Rechtsinhabers könnte auf Art. 5 Abs. 3 Lit. InfoSoc-RL gestützt werden, was freilich bedeutete, den Tatbestand an die Nutzung zu Gunsten von Wissenschaft und Forschung koppeln zu müssen. ${ }^{206}$

Daneben gilt es, die Anforderungen des Dreistufentests zu berücksichtigen. Nach dieser, mittlerweile allen Vorschriften des internationalen Urheberrechts

201 So die ganz h.M. vgl. BVerfG, Beschluss v. 1.3.1978 - 1 BvR 333/75 und 174, 178, 191/71, BVerfGE 47, 327 (375 f.); VG Berlin, Urt. v. 31.3.1977 - VII A 96/76, DÖV 1977, 643; Jarrass in Jarass / Pieroth, GG, 7. Aufl. 2004, Art. 5 Rz. 122; Blümel / Scheven, Nebentätigkeitsrecht des wissenschaftlichen und künstlerischen Personals in Fälming, Handbuch des Wissenschaftsrechts, 2. Aufl. 1996, S. 459; Kehler in Denninger, HRG, 1984, \& 43 Rz. 34, S 52 Rz. 11, Hufen, MittHV, 1985, 288 (292); Rohrmann, Die Abgrenzung von Hauptamt und Nebentätigkeit, 1988, S. 89 f., 91 f.; Müller in Hailbronner / Geis, HRG, 2003, \52 Rz. 10; Haberstumpf, ZUM 2001, 819 (826).

$202 \mathrm{Zu}$ den Voraussetzungen faktisch-mittelbarer Eingriffe vgl. BVerfG, Beschl. v. 26.6.2002 - 1 BvR 670/91, BVerfGE 105, 279 (301).

203 Voraussetzung einer „verfassungsgemäßen Urheberrechtsschranke“ ist, dass diese „die grundsätzliche Zuordnung des vermögenswerten Ergebnisses der schöpferischen Leistung an den Urheber" sowie die „die im Interesse des Gemeinwohls erforderlichen Grenzen“" in Ausgleich bringt, vgl. BVerfGE 31, 229 (241).

204 Richtlinie 2001/29/EG des Europäischen Parlaments und des Rates vom 22. Mai 2001 zur Harmonisierung bestimmter Aspekte des Urheberrechts und der verwandten Schutzrechte in der Informationsgesellschaft, ABl. EG L 167 vom 22.6.2001, S. 10.

$205 \mathrm{Vgl}$. Erwägungsgrund 32.

206 So zutreffend Hansen, GRUR Int. 2005, 378 (384 f.). 
immanenten ${ }^{207}$ urheberrechtlichen „Schranken-Schranke“ müssen Urheberrechtschranken (1.) an einen bestimmten Sonderfall anknüpfen, dürfen (2.) die normale Auswertung des Werkes nicht beeinträchtigen und (3.) die berechtigten Interessen des Urhebers nicht ungebührlich verletzen. ${ }^{208}$ Welche Handlungsalternativen diese Bestimmungen dem Gesetzgeber letztlich noch belassen, kann an dieser Stelle nicht abschließend geklärt werden. ${ }^{209}$ Sie reichen jedoch, dies mag an dieser Stelle bereits konstatiert werden, nicht annähernd so weit wie die Forderung mancher Open Content-Lobbyisten nach freiem Zugang zu jeder Art von Information.

\subsection{Universitätsrepositorien, Universitätsverlage und angemessene Vergütung}

Bislang völlig ungeklärte Fragen ergeben sich aus dem Zusammenspiel von Open Access-Publikationsmodellen und dem Anspruch auf angemessene Vergütung nach $\iint 32,32 \mathrm{a}$ UrhG. ${ }^{210}$ Denn während das geltende Urhebervertragsrecht in diesen Normen eine grundsätzliche Vergütungspflicht jeder Nutzung eines Werkes statuiert, 211 sehen die Finanzierungsmodelle von Open Access-Publikationen ein Autorenhonorar gerade nicht vor. \ 32 UrhG gibt dem Urheber für jede Einräumung eines Nutzungsrechtes einen Anspruch auf eine angemessene Vergütung. Ist eine Vergütung vertraglich vereinbart, diese aber nicht angemessen, so besteht ein Anspruch auf Vertragsanpassung (§ 32 Abs. 1 S. 3 UrhG).

\section{Angemessene Vergütung angestellter Autoren?}

Für die Vergütung der Nutzungsrechtseinräumung von angestellten wissenschaftlichen Autoren stellen sich dabei zunächst dieselben Fragen wie für die Nutzungsrechtseinräumung selbst. Nach der herrschenden sog. „Abgeltungslehre“ werden

207 Vgl. Art. 9 Abs. 2 RBÜ, Art. 13 TRIPS, Art. 10 Abs. 1 u. 2 WCT, Art. 16 Abs. 2 WPPT, Art. 5 Abs. 5 Richtlinie 2002/29/EG.

208 Vgl. hierzu eingehend Senftleben in Hilty / Peukert, Interessenausgleich im Urheberrecht, 2004, 159 (173 ff.); Spindler, GRUR 2002, 105 (111).

209 Eine eingehende Analyse einschließlich eines konkreten Gesetzesvorschlags findet sich bei Hansen, GRUR Int. 2005, 378 (383 ff.).

210 Grundsätzlich zu den Neuerungen der Urhebervertragsrechtsnovelle Hertin, MMR 2003, 16 ff.; Berger, ZUM 2003, 521 ff.; Schmidt, ZUM 2002, 781 ff.; zur verfassungsrechtlichen Bewertung der Neuregelungen Grzeszick, AfP 2002, 383 ff.

211 Vgl. Wandtke / Grunert und Wandtke / Bullinger, UrhG, 2002, \ 32 Rz. 1; diesem bestreben folgte die Rechtsrechung schon vor der Novellierung des Urhebervertragsrechts, vgl. etwa RG, Urt. v. 12.5.1926 - I 287/25, RGZ 113, 413 - Der Thor und der Tod; RG, Urt. v. 5.4.1933 - I 175/32, RGZ 140, 231 - Tonfilm; RG, Urt. v. 14.11.1936 - I 124/36, RGZ 153, 1 - Schallplattensendung; BGH, Urt. v. 6.11.1953 - I ZR 97/52, BGHZ 11, 135 (143) - Lautsprecherübertragung; BGH, Urt. v. 18.5.1955 - I ZR 8/54, BGHZ 17, 266 (267) - Grundig-Reporter; BGH, Urt. v. 12.12.1991 - I ZR 210/89, BGHZ 116, 305 (308) - Altenwohnheim II; BGH, Urt. v. 8.7.1993 - I ZR 124/91, BGHZ 123, 149 (155) - Verteileranlagen. 
durch das Arbeitsentgelt eines Arbeitnehmer-Urhebers nicht nur dessen arbeitsvertragliche Leistungen abgegolten, sondern auch die dabei entstandenen urheberrechtlich geschützten Arbeitsergebnisse, auch wenn sie auf seine vertraglich geschuldete Arbeitsleistung zurückgehen. ${ }^{212}$ Eine gesonderte Vergütung erhält der Arbeitnehmer-Urheber nur bei tätigkeitsbezogenen quantitativen und qualitativen Mehrleistungen, die über seine arbeitsvertraglichen Pflichten hinausgehen (sog. tätigkeitsbezogene Sonderleistungstheorie ${ }^{213}$ ). Die Nutzung eines im Zuge der arbeitsvertraglichen Pflichten geschaffenen Werkes im Rahmen des Betriebszwecks wird auch nach dem neuen Urhebervertragsrecht als durch den Lohn abgegolten angesehen, der damit zugleich als angemessene Vergütung i.S.d. \32 Abs. 1 S. $3 \mathrm{UrhG}^{214}$ angesehen werden muss ${ }^{215}$. Soweit die Vergütung tarifvertraglich geregelt ist, ist ein Vertragsanpassungsanspruch nach $\$ 32$ Abs. 4 UrhG ohnehin ausgeschlossen.

Allerdings besteht bei Autoren, die im Rahmen ihrer Wissenschafts- und Forschungsfreiheit tätig sind, keine dienstvertragliche Pflicht zur Schaffung bestimmter Werke. Die Nutzungsrechtsübertragung beruht hier auf einer freien Entscheidung des Verfassers (vgl. o. 0). Damit ist diese Nutzungsrechtsübertragung jedoch grundsätzlich auch angemessen zu vergüten, ${ }^{216}$ auf eine Vereinbarung, dass die Nutzungsrechtseinräumung kostenlos erfolgt sei, kann sich der Open AccessAnbieter grundsätzlich nicht berufen. ${ }^{217}$ Ein gewisser Abschlag kann sich allenfalls bei Bestimmung der Vergütungshöhe ergeben, so etwa aus dem beamtenrechtlichen Treueverhältnis verbeamteter Hochschullehrer (Art. 33 GG) oder aus der Nutzung von Universitätsressourcen, einschließlich der (von der Universität oder Forschungseinrichtung vergüteten) Arbeitszeit des Verfassers. ${ }^{218}$

\section{Angemessene Vergütung und Open Access}

Damit wären die (zwingenden) Vorschriften des Urhebervertragsrechts jedoch ohne weiteres geeignet, das Modell „Open Access“ zu torpedieren. Denn dieses fußt ja gerade darauf, dass der Autor sein Werk dem Open Access-Anbieter

212 OLG Hamburg, Urt. v. 22.1.1976 - 3 U 77/75, GRUR 1977, 556, 558 - Zwischen Marx und Rothschild; BAG, Urt. v. 12.3.1997 - 5 AZR 669/95, NZA 1997, 765 (766) - Lieferantenwettbewerb; Zirkel, ZUM 2004, 626, 630; Balle, NZA 1997, 868 (871); Berger, ZUM 2003, 173 (174); Bayreuther, GRUR 2003, 570 (572).

213 Zirkel, ZUM 2004, 626 (630).

214 Dieser ist nach mittlerweile herrschender Meinung, ebenso wie $\int 32$ a UrhG, auch auf Arbeitnehmer- Urheber anwendbar, vgl. Dreier / Schulze, \43 UrhG Rz. 30; Zentek / Meinke, Urheberrechtsreform 2002, 97; Bayreuther, GRUR 2003, 570; Haas, Urhebervertragsrecht, 110 ff. a.A. Ory, AfP 2002, 93 (95); differenzierend Berger, ZUM 2003, 173 (177 ff.).

215 Dreyer / Kotthoff / Meckel, \ 43 Rn. 23; Berger, ZUM 2003, 173 (176); Wimmers / Rode, CR 2003, 399 (402 f.); Zirkel, WRP 2003, 59 (63).

216 Vgl. Veddern, Multimediarecht für die Hochschulpraxis, 2. Aufl. 2004, S. 149.

217 Wandtke / Grunert und Wandtke/Bullinger, UrhG, 2002, § 32 Rz. 11.

${ }^{218} \mathrm{Vgl}$. Veddern a.a.O. 
kostenlos zur Verfügung stellt und für dessen Serviceleistungen selbst bezahlt. Die Regelung des $₫ 32$ Abs. 3 S. 3 UrhG, wonach der Urheber ein einfaches Urheberrecht zu Gunsten jedermann einräumen kann („Open-Source-Klausel“), verspricht hier nur in solchen Konstellationen Rettung, in denen der Nutzer, wie im Regelfall der Open-Source-Distribution, sein Nutzungsrecht direkt vom Verfasser erwirbt. ${ }^{219}$ Überträgt der Verfasser die entsprechenden Rechte zunächst an dritte Lizenzgeber, z. B. seinen „hauseigenen“ Universitätsverlag, welcher dann selbst das Recht „,ür jedermann" vergibt, so bleibt der Anspruch auf angemessene Vergütung im Verhältnis zu Open-Access-Anbieter bestehen.220 Unklar ist, inwieweit sich dieses Problem bei Bestimmung der Vergütungshöhe entschärfen lässt. Diese richtet sich, soweit keine gemeinsamen Vergütungsregeln (\$ 32 Abs. 2 S. 1 UrhG) bzw. eine einschlägige Branchenübung 221 vorhanden sind, nach den Umständen des Einzelfalles. Dabei sind alle relevanten Umstände zu berücksichtigen, etwa Art und Umfang der Nutzung, Marktverhältnisse, Investitionen, Risikotragung, Kosten, Zahl der hergestellten Werkstücke oder öffentlichen Wiedergaben und zu erzielende Einnahmen.222 Selbst dann, wenn die Nutzung des Werkes keinen unmittelbaren Ertrag abwirft, kann nach der Rechtsprechung des BGH aus dem Beteiligungsgrundsatz ein urheberrechtlicher Vergütungsanspruch folgen. ${ }^{223}$

Allerdings ist der Open Access-Vertrag, anders als der klassische Verlagsvertrag, nicht auf die wirtschaftliche Verwertung von Nutzungsrechten ausgelegt (vgl. o 3.1 sowie Bargheer in diesem Band). Vielmehr geht es dem Verfasser hier um eine möglichst weite Verbreitung seiner wissenschaftlichen Arbeitsergebnisse. Dies zu Grunde gelegt scheint es angemessen, den, dem Grunde nach bestehenden, Vergütungsanspruch für Nutzungsrechtseinräumungen zu Gunsten von Open Access-Veröffentlichungen, ähnlich etwa wie bei druckkostenzuschussfinanzierten Dissertationsveröffentlichungen ${ }^{224}$ auf Null schrumpfen zu lassen. Hierfür spricht auch, dass Open Access-Verträge, anders als klassische Verlagsverträge, eine Nutzungsrechtseinräumung nur in dem Maße vorsehen, als die für die Veröffentlichung des Werkes auch tatsächlich erforderlich ist und dem Verfasser damit das Recht belassen, sein Werk auch anderweitig zu verwerten. ${ }^{225}$

\footnotetext{
219 Also der „klassischen“ Form des Open Content

220 Schulze in Dreier / Schulze, UrhG, 2004, \32 Rz. 81.

221 S. hierzu Wandtke / Grunert und Wandtke/Bullinger, UrhG, 2002, \ 32 Rz. 27 ff.

222 So die amtl. Begr. BT-Drs. 14/6433, 44.

223 BGH, Urt. v. 18.5.1955 - I ZR 8/54, BGHZ 17, 266 (267) - Grundig-Reporter.

224 S. hierzu Wandtke / Grunert und Wandtke / Bullinger, UrhG, 2002, \ 32 Rz. 34; sowie die amtl. Begr. BT-Drs. 14/6433, 45.

${ }^{225}$ Vgl. o.13.1; zum Zusammenhang zwischen Umfang der Nutzungsrechtsübertragung und angemessener Vergütung vgl. Berger, ZUM 2003, 525.
} 


\section{Rechtsbeziehungen auf Seiten der Nutzer des Werkes}

Die bisherigen Betrachtungen bezogen sich auf die Bereitstellung des Werkes auf Basis von Open Access-Publikationsmodellen. Hiervon $\mathrm{zu}$ trennen sind jene Rechtsbeziehungen zwischen dem Verfasser und/oder dem Open AccessAnbieter und dem Endnutzer des Werkes, welche sich auf die Nutzung des Werkes beziehen bzw. diese ermöglichen.

\subsection{Urheberrechtsschutz und Urheberrechtsschranken}

Die Prämisse des folgenden Abschnitts ist, dass auch an Open Access publizierten Werken ein urheberrechtlicher Schutz besteht, welcher den Verfasser bzw. Rechtsinhaber mit entsprechenden Verbietungsrechten ausstattet.

Dies mag zunächst widersprüchlich erscheinen, kommt es doch Verfassern, Anbietern und Nutzern gerade darauf an, das Werk ungehindert von solchen Verbietungsrechten nutzbar zu machen. Dennoch ist die vollständige Aufgabe des Urheberrechts dem deutschen UrhG nicht immanent ${ }^{226}$, auch dann nicht, wenn es sich um ein ausländisches Werk handelt und das Urheberrecht unter der ausländischen Rechtsordnung wirksam aufgegeben wurde. ${ }^{227}$ Zwar ist ein Verzicht auf die (materiellen) Verwertungsrechte der $\iint 15 \mathrm{ff}$. UrhG grundsätzlich möglich ${ }^{228}$, jedoch wird man in aller Regel davon ausgehen können, dass auch der Verfasser eines auf Open Content-Basis veröffentlichten Beitrags eine Mindest-Kontrolle über sein Werk behalten möchte, etwa um dessen kommerzielle Ausbeutung oder eine Werkmonopolisierung durch Dritte zu verhindern, so dass von einem konkludenten Verzicht auf die urheberrechtlichen Verwertungs- (und damit Verbietungs-)rechte nicht ausgegangen werden kann. ${ }^{229}$

Wie auch bei der kommerziellen Werkverwertung finden die Einflussmöglichkeiten des Urhebers ihre Grenzen in den einschlägigen Urheberrechtsschranken

${ }^{226}$ BGH, Urt. v. 23.2.1995 - I ZR 68/93, GRUR 1995, 673 (675) - Mauer-Bilder; Spindler in Spindler, Rechtsfragen bei Open Source, 2004, C Rz. 6 ff. Ahlberg in Möhring / Nicolini, UrhG, 2 Aufl. 2000, J 7 Rz. 5; Spautz in Möhring / Nicolini, UrhG, 2 Aufl. 2000, § 29 Rz. 7; Schricker in Schricker, UrhG, 2. Aufl. 1999, \29 Rz. 15; Plaß, GRUR 2002, 670.

227 Dies folgt aus dem Schutzlandprinzip des internationalen Immaterialgüterrechts, vgl. BVerfG, Beschl. v. 23. 1. 1990 - 1 BvR 306/86, BVerfGE 81, 208 (222) - Bob Dylan; BGH NJW 1981, 1906 (1907) - Schallplattenimport; Katzenberger in Schricker, UrhG, 2. Aufl. 1999, Vorb. JS 120 ff. Rz. 120 ff. m.w.N.; Plaß GRUR 2002, 670 (671).

228 Str. vgl. BGH, Urt. v. 23.2.1995 - I ZR 68/93, GRUR 1995, 673 (675) - Mauer-Bilder; Ahlberg in Möhring / Nicolini, UrhG, 2 Aufl. 2000, \ 7 Rz. 5; a.A. Marly, Softwareüberlassungsverträge, 3. Aufl. 2000, Rz. 289.

229 Spindler in Spindler, Rechtsfragen bei Open Source, 2004, C Rz. 6; Plaß, GRUR 2002, 670 (673); Spautz in Möhring/Nicolini, UrhG, 2 Aufl. 2000, \ 29 Rz. 7; Veddern, Multimediarecht für die Hochschulpraxis, 2. Aufl. 2004, S. 69; s. auch zur Auslegung der GPL LG München I, Urt. v. 19.5.2004 - 21 O 6123/04, MMR 2004, 693 (694). 
der $\iint 44 a$ ff. UrhG, Art. 5 Richtlinie 2002/29/EG. Insbesondere die $\iint 44 a, 53$ UrhG enthalten weit reichende Beschränkungen des urheberrechtlichen Ausschließlichkeitsrechts, welche die reine Werknutzung grundsätzlich abdecken was auf der Gegenseite dazu führt, dass der Nutzer für die betreffenden Handlungen an die Bestimmungen einer Lizenz nicht mehr gebunden ist.

Jeder Nutzung wird zunächst eine Vervielfältigungshandlung (\$16 UrhG) vorausgehen, denn um das Werk auf dem Endgerät sichtbar zu machen, muss es vom Server herunter geladen und zumindest temporär in einem Speicher des Endgerätes hinterlegt werden. ${ }^{230}$ Dieser Vorgang wird grundsätzlich als Browsing von der Schranke des $\ 44$ a Ziff. 2 UrhG erfasst. ${ }^{231}$ Allerdings setzt dies eine „rechtmäßige“" Nutzung und damit eine Zustimmung des Rechtsinhabers voraus. ${ }^{232}$ Zwar wird man bei „Open Access“ publizierten Werken, ähnlich wie bei frei abrufbaren Webseiten, von einem konkludenten Einverständnis in den Abruf des Werkes ausgehen können ${ }^{233}$, so dass eine i.S.d Vorschrift rechtmäßige Nutzung i.d.R. vorliegt. Allerdings kann die der Zustimmung zu Grunde liegende Willenserklärung an Bedingungen geknüpft sein, insbesondere an die Beachtung bestimmter Open Access-Lizenzbestimmungen. ${ }^{234}$ Dies führt jedoch letztlich dazu, dass die aus $\int 44 a$ Ziff. 2 UrhG legitimierten Nutzungsrechte nicht weiter reichen als jene, die auch aufgrund der lizenzierten Nutzungsrechte zulässig sind.

Ist mit der Nutzung eine dauerhafte Perpetuierung des Werkes auf einem nicht nur flüchtigen Speichermedium verbunden, etwa eine Speicherung auf magnetischen oder optischen Datenträgern oder ein Ausdruck auf Papier oder Mikrofiche, so liegt hierin eine weitere Vervielfältigung, welche nicht mehr unter den Begriff des Browsing gefasst werden kann.

Von besonderer Bedeutung ist in diesem Zusammenhang das Recht auf Privatkopie ( 53 UrhG). So sind auch weitere Vervielfältigungshandlungen ohne Zu-

230 H.M. vgl. Loewenheim in Schricker, UrhG, 2. Aufl. 1999, S 16 Rz. 9; Nordemann in Fromm / Nordemann, UrhG, 9. Aufl. 1998, \16 Rz. 2; Schack, Urheber- und Urhebervertragsrecht, 3. Aufl. 2005, Rz. 380; Kreutzer, GRUR 2001, 193 (197); Sosnitza CR 2001, 693 (698); Schack, JZ 1998, 753 (755); Becker, ZUM 1995, 231 (243); Völker in Ensthaler/Bosch/Völker, Handbuch Urheberrecht und Internet, 2002, S. 187; Dreier in: Schricker, Urheberrecht auf dem weg in die Informationsgesellschaft, 1991, S. 112; Klett, Urheberrecht im Internet aus deutscher und amerikanischer Sicht, 1998, S. 123; Burmeister, Urheberrechtsschutz gegen Framing im Internet, 2000, 104 ff.

231 v. Welser in Wandtke / Bullinger, UrhG Ergänzungsband, 2003, \44a Rz. 3; vgl. auch Erwägungsgrund 33 der Richtlinie 2001/29/EG.

232 v. Welser in Wandtke / Bullinger, UrhG Ergänzungsband, 2003, \44a Rz. 17.

233 LG München I, Urt. v. 1.3.2002 - 21 O 9997/01, K\&R 2002, 258 (259); Hart EIPR 2002, 58 (59); Sosnitza CR 2001, 693 (699); Leupold CR 1998, 234 (239); Ernst BB 1997, 1057 (1059); a.A. Intveen, Internationales Urheberrecht und Internet, 1999, S. 41 f.

234 Von einer solchen bedingten Zustimmung wird man insbesondere dann ausgehen müssen, wenn der Verfasser das Werk im Internet mit einer entsprechenden Lizenz versieht. 
stimmung des Rechtsinhabers zulässig, wenn diese zum eigenen wissenschaftlichen Gebrauch erfolgen ( $\int 53$ Abs. 2 S. 1 Nr. 1 UrhG). ${ }^{235}$

Voraussetzung ist jedoch, dass die Vervielfältigung zu privatem Gebrauch geboten ist, was regelmäßig dann nicht der Fall ist, wenn das betreffende Werk ohne erheblichen Aufwand beschafft werden kann. ${ }^{236}$ Dies wird man bei Werken, die auf Open Access-Basis veröffentlicht sind, so dass eine rechtmäßige Vervielfältigung lediglich die Beachtung der entsprechenden Lizenzbestimmungen voraussetzt, in der Regel kaum annehmen können. \53 UrhG ist daher ebenfalls nicht geeignet, den Nutzer von den Bestimmungen der Lizenz zu entbinden.

Damit ist der „verwertungsrechtsfreie“ Raum bei Open Access-Publikationen zur wissenschaftlichen Nutzung wesentlich enger als bei anderen Werkformen. Mit gewisser Pauschalisierung mag konstatiert werden, dass jede Nutzung eines solchen Werkes von der Open Access-Lizenz des Rechtsinhabers abhängt.

Grundsätzlich an die Lizenz gebunden sind selbstverständlich auch solche Nutzungshandlungen, die über diese ,alltäglichen“ Formen der Nutzung hinausgehen - etwa Vervielfältigungen in größerem Umfang, die Weiterverbreitung an die Öffentlichkeit oder die eigene öffentliche Zugänglichmachung des Werkes, z. B. durch dessen Einbindung in den eigenen Internet-Auftritt.

\subsection{Lizenzvertragsrechtliche Ausgestaltungen}

\section{Vertragsrechtliche Grundlagen}

Grundlage der Nutzung eines Werkes ist in aller Regel ein Lizenzvertrag, welcher neben der eigentlichen Rechtseinräumung die Art und Weise der zulässigen Werknutzung regelt. Im Falle der Selbstpublikation kommt dieser Vertrag zwischen Verfasser und Nutzer zu Stande. Tritt ein Open Access-Anbieter als Distributor $^{237}$ zwischen Verfasser und Nutzer, so stehen zwei unterschiedliche Wege der Lizenzierung zur Verfügung: Denkbar ist zum einen, dass der Verfasser jedem Endnutzer die zur Werknutzung erforderlichen Rechte selbst einräumt, und sich

235 Dies schließt die Weitergabe des Werkes an Dritte mit ein, wenn diese innerhalb einer geschlossenen Einheit, z. B. einer Wissenschaftseinrichtung erfolgt, vgl Dreier in Dreier / Schulze, UrhG, 2004, \53 Rz. 23; Decker in Möhring / Nicolini, UrhG, 2. Aufl. 2000, \53 Rz. 18; Veddern, Multimediarecht für die Hochschulpraxis, 2. Aufl. 2004, S. 69.

236 v. Weser in Wandtke / Bullinger, UrhG Ergänzungsband, 2003, \53 Rz. 23; Dreier in Dreier / Schulze, UrhG, 2004, \53 Rz. 23; Loewenheim in Schricker, UrhG, 1999, \53 Rz. 23.

${ }^{237}$ Der Begriff stammt aus dem Bereich der Open Source Software und bezeichnet Personen, die Programme gegen Erstattung des Materialwertes auf geeigneten Datenträgern zur Verfügung stellen. 
ggf. des Open Access-Anbieters als Boten bedient. ${ }^{238}$ Dieser Weg, der in der Praxis der Open Content-Distribution im Internet, welche sich weniger über zentrale Distributoren als über peer-to-peer-Strukturen organisiert, weithin üblich ist, ${ }^{239}$ hat den Vorteil dass jeder Nutzer ,sein“ Nutzungsrecht direkt vom Verfasser ableitet, so dass dessen Wirksamkeit nicht von der Wirksamkeit der Vorlizenz abhängt und auch unzulässige Änderungen der Nutzungsbedingungen in der Lizenzkette ausgeschlossen werden können. ${ }^{240}$.

Liegt dagegen die technische Bereitstellung des Werkes, einschließlich der Wahl des Formats und der Implementierung von Funktionsbeschränkungen in der Hand eines zentralen Distributors (z. B. eines Universitätsverlages oder eines zentralen Repositoriums), so zeigt dieses System einige entscheidende Schwächen: denn hier übt, anders als bei den im Bereich der Open Source-Software üblichen Strukturen, der Distributor die entscheidende Kontrolle über die Verbreitung des Werkes aus, so dass er, schon im Hinblick auf die Gefahren einer Mitstörerverantwortlichkeit, darauf angewiesen ist, dass nicht nur er selbst, sondern auch die Nutzer, die auf seine Ressourcen zugreifen, über die erforderlichen Nutzungsrechte verfügen. Hier mag es sich als nachteilig erweisen, wenn der Anbieter sich auf eine wirksame Lizenzierung zwischen Verfasser und Nutzer verlassen muss, auf die er unmittelbar keinen Einfluss hat.

Nahe liegender scheint es daher hier, dem Anbieter ein Recht zur Unterlizenzierung einzuräumen. Der Nutzer erwirbt dann ein abgeleitetes Nutzungsrecht vom Anbieter in dem vom Verfasser vorgesehenen Umfang. Die technische und die rechtliche Ausgestaltung der Nutzung liegen damit, wie beim klassischen Verlagsvertrag, in der Hand des Open Access-Anbieters.

\section{Reichweite des Nutzungsrechtes bei fehlender ausdrücklicher Lizenzierung}

Nimmt der Autor oder die wissenschaftliche Einrichtung die Bereitstellung des Werkes selbst vor, so reduziert sich die Publikation häufig auf die tatsächliche technische Freischaltung des Werkes - allenfalls noch verbunden mit dem Hin-

\footnotetext{
238 S. hierzu Spindler in Spindler, Rechtsfragen bei Open Source, 2004, C Rz. 41; von diesem Weg gehen auch Jaeger / Metzger, MMR 2003, 431 (434) aus, die weiterhin annehmen, dass der Nutzer den Lizenzvertrag u.U. erst nach Erwerb des Werkexemplares abschließt, etwa dann wenn er von der Lizenz Kenntnis erlangt bzw. deren Rechte in Anspruch nimmt; vgl zu dieser Form der Lizenzierung auch Plaß, GRUR 2002, 670 (676 f.). Zwar mag ein solche Form der Kontrahierung unter bestimmten Voraussetzungen möglich sein (vgl. \151 BGB), allerdings bleibt unklar, wie denn der Nutzer hier konkret von der Lizenz Kenntnis erlangen und sich damit vom Umfang seiner (noch zu erwerbenden) Nutzungsrechte Gewissheit verschaffen soll, vgl. zur Kritik, insbesondere auch aus AGB-rechtlicher Sicht auch Strobel, MMR 2003, 778 (779 f.); zum Erfordernis der Kenntnis von den konkreten Vertragsbestimmungen vgl. Spindler a.a.O. Rz. 43.

239 Vgl. nur das Modell der Craetive Commons, http:/ / creativecommons.org/.

240 Plaß, GRUR 2002, 670 (676 f.).
} 
weis, das Werk sei „frei nutzbar“ o. ä., so dass der Umfang der konkludent eingeräumten Rechte im konkreten Fall durch Auslegung ermittelt werden muss. Selbstverständlich ist dabei zunächst, dass der Rechtsinhaber und/oder Verfasser nur einfache Nutzungsrechte einräumen will - ist es doch gerade Sinn des Open Access-/Open-Content - Modells, das Werk nicht zu monopolisieren.

Weiterhin gilt es zu berücksichtigen, dass bei wissenschaftlichen Sprachwerken, anders als etwa bei Open Source-Software, die persönlichkeitsrechtlichen Elemente stark im Vordergrund stehen. ${ }^{241}$ Dies ist vor allem beim Recht zur Bearbeitung von Bedeutung. Denn während bei Software das Bearbeitungsrecht konstitutiver Bestandteil Open Source-Modells ist, ${ }^{242}$ mag ein solches den Interessen eines wissenschaftlichen Autors gerade zuwiderlaufen. Ob und unter welchen Voraussetzungen von einer konkludenten Einräumung des Bearbeitungsrechts ausgegangen werden kann, wird man letztlich von der Art des Werkes und der konkreten Publikationsform abhängig machen müssen. Entscheidend ist dabei, inwieweit das Werk einer Weiterentwicklung durch Dritte zugänglich ist ${ }^{243}$ - was bei wissenschaftlichen Werken eher die Ausnahme als die Regel sein wird. So weit man hier tatsächlich in besonders gelagerten Fällen ${ }^{244}$ von einem Bearbeitungsrecht ausgehen will, so wird dieses jedenfalls an die Pflicht gebunden sein, Änderungen in geeigneter Weise kenntlich zu machen und den ursprünglichen Verfasser mit diesen nicht in Verbindung zu bringen. ${ }^{245}$

Gleiches gilt bei Anwendung des Zweckübertragungsgrundsatzes. Denn keineswegs geht es dem Verfasser, der sein Werk Open Access veröffentlicht, immer und vornehmlich um dessen weitere Verwertung durch die wissenschaftliche Community. ${ }^{246}$ Zweck der öffentlichen Zugänglichmachung wird für ihn i.d.R. vielmehr der erhöhte Bekanntheitsgrad und der hierdurch bedingte Renommeegewinn einer vergütungsfreien Publikation sein - ein Zweck, der eine extensive Einräumung von Nutzungsrechten in keiner Weise bedingt.

241 Vgl. Jaeger / Metzger, MMR 2003, 431.

242 Vgl. Jaeger / Metzger, MMR 2003, 431 (433); für die GPL vgl. Spindler in Spindler, Rechtsfragen bei Open Source, 2004, C Rz. 74.

243 Jaeger / Metzger, MMR 2003, 431 (433).

244 Insbesondere im populärwissenschaftlichen Bereich, vgl. etwa die Online- Enzyklopädie Wikipedia, http://de.wikipedia.org/wiki/Hauptseite.

245 Vgl. Jaeger / Metzger a.a.O.; dies folgt letztlich auch aus dem Recht des Urhebers auf Anerkennung der Urheberschaft, vgl. Schulze in Dreier/Schulze, UrhG, 2004, § 13 Rz. 32.

246 So aber Plaß, GRUR 2002, 670 (673). 


\section{Ausdrückliche Rechtsübertragungen durch Standard-Lizenz- Verträge}

Ein anderer Weg der Spezifikation der Nutzungsrechte ist die Verwendung eines Standard-Lizenzvertrages. Nach gängiger Praxis im E-Commerce kann einer solcher über einen Hyperlink wirksam in den Nutzungsvertrag eines Repositoriums oder einer Instituts-Homepage eingebunden werden. ${ }^{247}$ Entsprechende Lizenztexte sind mittlerweile in großer Zahl im Internet verfügbar. ${ }^{248}$

Ein Lizenzvertrag soll nach den Bestimmungen der meisten Lizenztexte dadurch zu Stande kommen, dass der Nutzer das Werk in einer urheberrechtlich relevanten Art und Weise nutzt. ${ }^{249}$ Im Allgemeinen enthalten diese Lizenzen eine Generalklausel, in welcher dem Nutzer die wichtigsten Nutzungsrechte, Vervielfältigung, Verbreitung, öffentliche Zugänglichmachung etc. eingeräumt werden. 250 Die Rechtseinräumungsklauseln unterscheiden sich vor allem darin, ob ein Bearbeitungsrecht sowie ein Recht zur Vergabe von Unterlizenzen eingeräumt wird oder nicht. Z.T. ist die Rechtseinräumung mit der Bedingung versehen, dem Werk den Lizenztext beizufügen. 251 Soweit eine Lizenzierung des Bearbeitungsrechtes vorgesehen ist, so steht dieses i.d.R. unter dem Vorbehalt, die Änderungen gegenüber dem Ursprungswerk kenntlich zu machen ${ }^{252}$ sowie, ganz nach dem Vorbild

247 Vgl. Köhler / Arndt, Recht des Internet, 4. Aufl. 2003, S. 85; Holzbach / Süßenberger in Moritz / Dreier, Rechtshandbuch zum E-Commerce, 2002, C Rz. 280; Stempfle in Bräutigam / Leupold, Online-Handel, 2003, B III Rz. 310; Koehler, MMR 1998, 289; Ernst CoR, 1997, 165.

248 Einen umfassenden Überblick, sowohl für den Bereich Open Source als auch Open Content allgemein, gibt http://www.ifross.de/.

${ }^{249}$ Vgl. z. B. Ziff. 1 der GNU Free Documentation License, V1.2, abrufbar unter http://www.fsf.org/licensing/licenses/fdl.html ; Ziff. 2 der OpenOffice Public Documentation License, abrufbar unter http://www.openoffice.org/licenses/PDL.html; ebenso der Creative Commons Legal Code, der auch Publikationen der Public Library of Science zu Grunde liegt, abrufbar unter http://creativecommons.org; Ziff. 1 der Lizenz für freie Inhalte des Kompetenznetzwerks Universitätsverbund Multimedia NRW, abrufbar unter http://www.uvm.nrw.de/kunden/uvm/; freilich setzt dies voraus, dass der Nutzer vorher von der Lizenz Kenntnis genommen hat.

250 Ziff. 2. der Apple Common Documentation License, (http://www.opensource.apple.com/cdl/) Ziff. 2 der GNU Free Documentation License, V1.2 (Fn. 249); \$ 1 der ifrOSS' Freie Lizenz für Texte und Textdatenbanken, abrufbar unter http://www.ifross.de/ifross_html/ifl.html; Ziff. 2 der OpenOffice Public Documentation License (Fn. 249); Ziff. 3 der National Imports Design Science License, abrufbar unter http://www.rare-earth-magnets.com/magnet_university/ design_science_license.htm; Ziff. I der OPENCONTENT Open Publication License, abrufbar unter http://opencontent.org/opl.shtml; Ziff. 3 des Creative Commons Legal Code (Fn. 249); Ziff. 2 der Lizenz für freie Inhalte (Fn. 249).

251 Ziff. 2. der Apple Common Documentation License (Fn. 250); Ziff. 2 u. 3 der GNU Free Documentation License, V1.2 (Fn. 249); Ziff. 3.1 der OpenOffice Public Documentation License (Fn. 249); Ziff I der OPENCONTENT Open Publication License (Fn. 250); Ziff. 4a des Creative Commons Legal Code (Fn. 249).

252 Vgl. z. B. Ziff. 4 der GNU Free Documentation License, V1.2 (Fn. 249); \ 1 der ifrOSS’ Freie Lizenz für Texte und Textdatenbanken (Fn. 250); Ziff. 3.3 der OpenOffice Public Documentation License (Fn. 249); Ziff. 4b der National Imports Design Science License (Fn. 250); Ziff III der OPENCONTENT Open Publication License (Fn. 250); Ziff. 4b des Creative Commons Legal Code (Fn. 249); Ziff. 3c der Lizenz für freie Inhalte (Fn. 249). 
der GPL, abhängig geschaffene Werke wieder unter dieser Lizenz zu veröffentlichen (sog. Copyleft- Klausel). ${ }^{253}$

Weiterhin sind Änderungen des Lizenztextes selbst in aller Regel ausgeschlossen. ${ }^{254}$ Zum Teil sehen die Standard- Lizenztexte einen Haftungsausschluss des Verfassers für Schäden vor, welche dem Nutzer aus der Nutzung des Werkes entstehen könnten. ${ }^{255}$ Zuwiderhandlungen gegen einzelne Bestimmungen des Lizenzvertrages werden durch eine automatische Vertragsbeendigung und einen Rückfall der Rechte an den Lizenzgeber sanktioniert. ${ }^{256}$

Zwei Lizenzmodelle haben für die Open Access-Bewegung in Deutschland besondere Bedeutung erlangt:

Die Musterlizenzen der Creative Commons-Initiative ${ }^{257}$ können den Wünschen des Lizenzgebers angepasst werden. So kann der Verfasser wählen, ob eine kommerzielle Nutzung seines Werkes sowie eine unfreie Bearbeitung gestattet sein und diese unter der Bedingung einer Copyleft-Klausel stehen soll. Weiterhin kann der Verfasser zwischen verschiedenen Werkarten, z. B. Musik, Text, Software usw. wählen. Verfügbar ist auch eine Lizenzfassung auf der Grundlage deutschen Urheberrechts. Diese lässt die Befugnisse des Nutzers, die sich aus den Urheberrechtsschranken sowie dem Erschöpfungsgrundsatz ergeben, ausdrücklich unberührt. Eine speziell für wissenschaftliche Inhalte konzipierte Lizenz wird zurzeit von der Creative Commons Initiative entwickelt. ${ }^{258}$

Auch die Digital Peer Publishing Lizenz der Open Access-Initiative Digital Peer Publishing Nordrhein-Westfahlen ${ }^{259}$, die gemeinsam mit dem Institut für

${ }^{253}$ So Ziff. 3 der Apple Common Documentation License (Fn. 250); Ziff. 4 GNU Free Documentation License, V1.2 (Fn. 249); \ 3 der ifrOSS' Freie Lizenz für Texte und Textdatenbanken (Fn. 250); Ziff. 3.1 der OpenOffice Public Documentation License (Fn. 249); Ziff. 4 der National Imports Design Science License (Fn. 250); Ziff. 4 des Creative Commons Legal Code (Fn. 249); Ziff. 4 der Lizenz für freie Inhalte (Fn. 249).

254 Vgl. etwa die Apple Common Documentation License (Fn. 250); Ziff. 4a des Creative Commons Legal Code (Fn. 249).

255 Ziff. 5 u. 6 . der Apple Common Documentation License (Fn. 250); Ziff. 6 u. 8 der OpenOffice Public Documentation License (Fn. 249); Ziff. 7 der OpenOffice Public Documentation License (Fn. 249); Ziff. 7 u. 8 der National Imports Design Science License (Fn. 250); Ziff III der OPENCONTENT Open Publication License (Fn. 250); Ziff. 5 u. 6 des Creative Commons Legal Code (Fn. 249).

256 Ziff. 9. der Apple Common Documentation License (Fn. 250); \5 der ifrOSS' Freie Lizenz für Texte und Textdatenbanken (Fn. 250); Ziff. 4 der National Imports Design Science License (Fn. 250); Ziff. 7 a des Creative Commons Legal Code (Fn. 249); Ziff. 9 der Lizenz für freie Inhalte (Fn. 249); eine solche Regelung stellt keine unangemessene Benachteiligung des Vertragspartners des Verwenders dar, vgl. LG München I, Urt. v. 19.5.2004 - 21 O 6123/04, MMR 2004, 693 (695).

257 http://creativecommons.org/.

${ }^{258} \mathrm{Vgl}$. http://science.creativecommons.org/; vorgesehen ist auch eine an das deutsche Recht angepasste Fassung, s. hierzu: http://www.heise.de/newsticker/meldung/54824.

259 http://www.dipp.nrw.de/. 
Rechtsfragen der freien und Open Source-Software entwickelt wurde, sieht verschiedene Stufen des Umfangs der Rechtsübertragung vor. So kann die Rechtseinräumung auf die Vergabe elektronischer Rechte beschränkt werden. Dieses Modell ist insbesondere für eine Preprint- oder Postprint-Veröffentlichung neben einem klassischen Verlagsvertrag gedacht. In zwei „Ausbaustufen“ sieht das Lizenzmodell daneben auch eine Einräumung des Bearbeitungsrechts vor, wobei unterschieden werden kann, ob das gesamte Dokument oder nur bestimmte, vom Verfasser freigegebene Teile veröffentlicht werden dürfen.

\section{Funktionsbeschränkter freier Zugang?}

Wie bereits angedeutet, bedeutet Open Access nicht zwangsläufig, dass der Verfasser mit jedweder Nutzung seines Werkes einverstanden ist - vielmehr mag gerade die kostenlose Zurverfügungstellung seiner Arbeit dazu führen, dass eine Nutzung nur zu bestimmten, von ihm gebilligten Zwecken, etwa nichtkommerzieller oder wissenschaftlicher Art, erfolgen soll. Während der Ausschluss einzelner Verwertungsrechte, etwa des Bearbeitungsrechtes, lizenzvertraglich ohne weiteres möglich ist, ist eine auf bestimmte Nutzungszwecke beschränkte Lizenzierung einzelner Nutzungshandlungen urheberrechtlich nur in Grenzen zulässig. Zwar kann ein Nutzungsrecht im Grundsatz örtlich, zeitlich und inhaltlich beschränkt werden, ${ }^{260}$ eine dingliche Wirkung entfaltet eine solche Beschränkung jedoch nur dann, wenn sie sich auf eine wirtschaftlich-technisch selbständige Nutzungsart bezieht. ${ }^{261}$ Ohne weiteres möglich ist freilich eine Vereinbarung schuldrechtlicher Art, das Werk nur in der vom Verfasser vorgegebenen Art und Weise zu nutzen. ${ }^{262}$ Eine urheberrechtliche Wirkung kann mittelbar dadurch erzielt werden, dass eine Verletzung dieses „Nutzungsvertrages“ zu einem Rückfall sämtlicher Rechte an den Lizenzgeber führt. ${ }^{263}$

Der Möglichkeit einer ausdrücklichen oder konkludenten schuldrechtlichen Beschränkung der Werknutzung steht auch nicht das Prinzip der „offenen Nutzung“ von Open Access-Publikationen entgegen. Dies gilt auch dann, wenn der Anbieter / Verfasser keine Vorsorgemaßnahmen zur Rechtsdurchsetzung wie etwa die namentliche Registrierung der Nutzer vorsieht. ${ }^{264}$ Denn auch wenn der

\footnotetext{
260 Vgl. \ 31 Abs. 1 S. 2 UrhG.

261 BGH, Urt. v. 12.12.1991 - I ZR 165/89, GRUR 1992, 310 (311) - Taschenbuch- Lizenz; BGH, Urt. v. 6.7.2000 - I ZR. 244/97, OEM-Version; BGH, Urt. v. 24.10.2002 - I ZR 3/00 GRUR 2003416 (418) - CPU-Klausel, Schulze in Dreier/Schuze, UrhG, 2004, § 31 Rz. 28.

262 BGH, Urt. v. 24.10.2002 - I ZR 3/00 GRUR 2003416 (418) - CPU-Klausel; OLG Düsseldorf, Urt. v. 3.3.1998 - 20 U 76/97, CR 1999, 150; Schulze in Dreier / Schulze, UrhG 2004, \& 31 Rz. 29; Plaß, GRUR 2002, 670 (675).

263 Vgl. auch für die GPL LG München I, Urt. v. 19.5.2004 - 12 O 6123/04, MMR 2004, 693 (695), welches von einer aufschiebend bedingten dinglichen Einigung ausgeht.

264 A.A. wohl Plaß, GRUR 2002, 670 (675).
} 
Open Access-Anbieter seine Nutzer nicht wie etwa ein Anbieter kostenpflichtiger Online-Publikationen persönlich registriert, so mag die schuldrechtliche wie die dingliche Einigung bestimmender Erklärungswille dennoch darauf gerichtet sein, einem Dritten den kommerziellen Vertrieb seines Werkes oder dessen Einbindung in einen unerwünschten Kontext zu verbieten.

\section{Ausdrückliche Lizenzierung und AGB-Kontrolle}

$\mathrm{Zu}$ berücksichtigen ist, dass eine formularmäßige Ausgestaltung der Nutzungsbedingungen durch den Open Access-Anbieter als allgemeine Geschäftsbedingung den Anforderungen der $\iint 305$ ff BGB zu genügen hat, ${ }^{265}$ auch dann, wenn es sich beim Anbieter um eine Non-Profit-Organisation oder einen Universitätsverlag bzw. ein Hochschulrepositorium handelt, soweit nur die Nutzung privatrechtlich ausgestaltet ist. ${ }^{266}$ So wäre eine Bestimmung, die eine Restriktion der Nutzung des Werkes (bewusst oder unbewusst) in den erlaubnisfreien Bereich der $\int S 44 \mathrm{a}$ ff UrhG ausdehnt, im Hinblick auf \ 307 Abs. 2 Nr. 1 UrhG in aller Regel unwirksam. ${ }^{267}$ Umstritten ist auch die Wirksamkeit formularmäßiger Nutzungsbeschränkungen bez. Werkexemplaren, an denen sich das Urheberrecht nach \17 Abs. 2 UrhG erschöpft hat. (z. B. eine pauschale Beschränkung des Verbreitungsrechts auf unentgeltliche Verbreitungshandlungen). ${ }^{268}$

Unklar ist auch, inwieweit sich eine AGB-rechtlich unwirksame Beschränkung einer Lizenz auf die Einräumung des Nutzungsrechtes selbst auswirkt. So könnte das Verbot der geltungserhaltenden Reduktion ${ }^{269}$ hier dazu führen, dass unzulässig beschränkte Rechtseinräumungs- Klauseln unwirksam werden, mit dem gleichwohl paradoxen Ergebnis, dass die gesamte Rechtsübertragung für die jeweilige Nutzungsart hinfällig wäre. So weit die Beschränkung nur schuldrechtliche Wirkung entfaltet, ist deren Unwirksamkeit für das Bestehen des Nutzungsrechtes jedoch unschädlich. Denn eine bloße Unwirksamkeit schuldrechtlicher Vereinba-

265 Plaß, GRUR 2002, 670 (678); für die entsprechenden Open-Source-Lizenzen vgl. Spindler in Spindler, Rechtsfragen bei Open Source, 2004, C Rz. 45; F.A.Koch, CR 2000, 333 (339); Omsels in FS Hertin, 2000, 141 (147); Jaeger / Metzger, Open Source Software, 2002, S. 147; Jaeger/Metzger, GRUR Int. 1999, 839 (846); Deike, CR 2003, 9 (13).

266 BGH, Urt. v. 7.3.1989 - KZR 15/87, NJW 1989, 3010; BGH, Urt. v. 30.5.1990 - IV ZR 266/89, NJW 1990, 2686; OLG Düsseldorf, Urt. v. 14.11.1991 - 6 U 91/91, WM 1992, 1895, S. Roloff in Erman, BGB, 11. Aufl. 2004, \305 Rz. 8.

267 Plaß, GRUR 2002, 670 (679); Dreier in Dreier/Schulze, UrhG 2004, Vor \$S 44a ff. Rz. 9;

${ }^{268}$ Für eine Wirksamkeit Spindler in Spindler, Rechtsfragen bei Open Source, C Rz. 98, dagegen $\mathrm{Plaß}$, a.a.O.

${ }^{269}$ S. hierzu BGH, Urteil vom 17.5.1982 - VII ZR 316/81, BGHZ 84, 109, 114f.; BGH, Urteil vom 20.1.1983 - VII ZR 105/81, BGHZ 86, 284, 297; BGH, Urteil vom 1.2.1984 - VIII ZR 54/83, BGHZ 90, 69, 73; BGH, Urteil vom 20.6.1984 - VIII ZR 337/82, BGHZ 91, 375, 384; Urteil vom 16.10.1984 - X ZR 97/83, NJW 1985, 319; BGH, Urteil vom 24.9.1985 - VI ZR 4/84, NJW 1986, 1610, 1611; Schmidt in Ulmer / Brandner / Hensen, AGBG, 9. Aufl. 2001, \6 Rn. 14ff.; Lindacher in Wolf / Horn / Lindacher, AGBG, 4. Aufl. 1999, \ 6 Rn. 31. 
rungen kann die dingliche Rechtslage, d.h. den Bestand eines wirksam eingeräumten Nutzungsrechts, nicht beeinflussen, so dass dieses letztlich ohne die Beschränkung fortbesteht. ${ }^{270}$ Aber auch die unangemessene Benachteiligung durch eine dinglich wirkende Beschränkung wird das Nutzungsrecht in aller Regel nicht als ganzes entfallen lassen. Denn nach der Rechtsprechung ist die Teilung einer Klausel in einen wirksamen und einen unwirksamen Teil dann zulässig, wenn diese ,inhaltlich voneinander trennbare, einzeln aus sich heraus verständliche Regelungen enthält"،, d.h. wenn der unwirksame Teil der Klausel gestrichen werden kann, ohne dass der Sinn des anderen Teils darunter leidet. ${ }^{271}$ Dies wird in den allermeisten vorliegend interessierenden Fällen jedoch anzunehmen sein, zumal die wirtschaftlich-technische Selbstständigkeit ja gerade Voraussetzung einer dinglichen Wirkung der Beschränkung ist.

Unwirksam sind auch Klauseln, welche eine Haftung des Verfassers, soweit diese überhaupt auf dispositivem Recht beruht, generell ausschließen (vgl. \309 Ziff. 7 BGB).

\section{Grenzen der Lizenzierbarkeit}

Nicht zuletzt gilt es zu beachten, dass das deutsche Recht einen indisponiblen Kern des Urheberrechts kennt, der dem Verfasser auch durch eine Open AccessLizenz nicht genommen werden kann. ${ }^{272}$ Hierzu zählen insbesondere das Recht auf Anerkennung der Urheberschaft ( $\int 13 \mathrm{UrhG}$ ) sowie das Entstellungsverbot $\left(\int 14\right.$ UrhG). Dies mag insbesondere im Verhältnis von Verfasser und Anbieter relevant sein: Soweit sich der Anbieter formularmäßig ein Bearbeitungsrecht einräumen lässt, empfiehlt es sich, das Entstellungsverbot ausdrücklich unberührt zu lassen. ${ }^{273}$

\section{Zusammenfassung}

Bei aller Schwierigkeit, aus dem Überblick über die inhomogenen juristischen Problembereiche im Zusammenhang mit Open Access eine einheitliche Schlussfolgerung zu ziehen, mag doch konstatiert werden, dass die eingangs angedeuteten Feststellungen zum Verhältnis von Open Source und Open Access sich auch bei näherer Betrachtung bestätigen: Während auf Seite der Nutzung Open Access pub-

\footnotetext{
270 OLG Nürnberg, Urt. v. 20.6.1989 - 3 U 1342/88, NJW 1989, 2634 (2635); Plaß, GRUR 2002, 670 (682); dies folgt letztlich aus dem Abstraktionsprinzip, welches auch im Urheberrecht gilt, vgl. Schricker in Schricker, UrhG, 2. Aufl. 1999, Vor. $\int \$ 28$ ff. Rz. 58 ff.

271 St. Rspr. vgl. nur BGH, Urt. v. 18.4.1989 - X ZR 31/88, NJW 1989, 3215 (3216) m.w.N.; Basedow in MünchKommBGB, 4. Aufl. 2003, \ 306 Rz. 18.

272 Vgl. hierzu Schulze in Dreier/Schulze, UrhG, 2004 Vor $\$ 12$ Rz. 10; Dietz in Schricker, UrhG, 2. Aufl. 1999, Vor $\$ \$ 12$ ff. Rz. 26 f.

273 S. hierzu Jaeger / Metzger, MMR 2003, 431 (435).
} 
lizierter Werke in viele Bereichen die Erkenntnisse aus dem Bereich der Open Source-Software fruchtbar gemacht werden können, unterscheiden sich auf Anbieterseite die rechtlichen Fragestellungen und Lösungsansätze doch erheblich. Dies resultiert zum einen aus der unterschiedlichen Interessenlage, die bei Open Source-Software in hohem Maße von einem Community-Gedanken und letztlich altruistischen Motiven geprägt ist, während bei Open Access die Ressourcenknappheit der öffentlichen Hand sowie die individuellen Renommeeinteressen des Wissenschaftlers im Vordergrund stehen. Zum andern weist wissenschaftliche Publikationstätigkeit anders als die IT-Anwendungsentwicklung eine Durchsetzung mit grundrechtlichen Freiheitsbefugnissen auf. Ob und inwieweit sich die unterschiedlichen Rechtsfragen von Open Source und Open Access letztlich auf einer Meta-Ebene des Open Content zusammenführen lassen, wird die Diskussion der Zukunft zeigen.

\section{Literatur}

Balle (1997): Balle: Der urheberrechtliche Schutz von Arbeitsergebnissen, Neue Zeitschrift für Arbeitsrecht 1997, S. 868 ff.

Bayreuther (2003): Bayreuther: Zum Verhältnis zwischen Arbeits-, Urheber- und Arbeitnehmererfindungsrecht, Gewerblicher Rechtsschutz und Urheberrecht 2003, S. $570 \mathrm{ff}$.

Becker (1995): Becker: Neue Übertragungstechniken und Urheberrechtschutz, Zeitschrift für Urheber- und Medienrecht 1995, S. 231 ff.

Beier / Straus (1982): Beier, Straus: Der Schutz wissenschaftlicher Forschungsergebnisse, Verl. Chemie, Weinheim 1982.

Berger (2003): Berger: Der Anspruch auf angemessene Vergütung gemäß \ 32 UrhG - Konsequenzen für die Vertragsgestaltung, Zeitschrift für Urheberund Medienrecht 2003, S. 521 ff.

Berger (2003a): Berger: Zum Anspruch auf angemessene Vergütung ( $\$ 32$ UrhG) und weitere Beteiligung $(\$ 32 \mathrm{a} U \mathrm{rhG})$ bei Arbeitnehmer-Urhebern, Zeitschrift für Urheber- und Medienrecht 2003, S. 173 ff.

Beyerlein (2002): Beyerlein: Wegfall des "Hochschullehrerprivilegs" (\$ 42 ArbEG) - eine Erleichterung für die Forschung an deutschen Hochschulen? Neue Zeitschrift für Arbeitsrecht 2002, S. 1020 ff.

Blankenagel (1980): Blankenagel: Wissenschaftsfreiheit aus der Sicht der Wissenschaftssoziologie, Archiv des öffentlichen Rechts 105 (1980), S. 35 ff.

Blümel / Scheven (1996): Blümel, Scheven: Nebentätigkeitsrecht des wissenschaftlichen und künstlerischen Personals in Fläming (Hrsg.), Handbuch des Wissenschaftsrechts, 2. Aufl., Springer, Berlin, New York 1996.

Bräutigam / Leupold (2003): Bräutigam, Leupold (Hrsg.): Online-Handel, Beck, München 2003. 
Burmeister (2000): Burmeister: Urheberrechtsschutz gegen Framing im Internet, Eul 2000.

Cichon (2005): Cichon: Internet- Verträge, 2. Aufl., Schmidt, Köln 2005.

Dallinger (1971): Dallinger: Wissenschaftsfreiheit und Mitbestimmung, Juristenzeitung 1971, S. 665ff.

Deike (2003): Deike: Open Source Software - IPR-Fragen und Einordnung ins deutsche Rechtssystem, Computer und Recht 2003, S. 9 ff.

Denninger (1984): Denninger (Hrsg.): Hochschulrahmengesetz: Kommentar, Beck, München, 1984.

Dreier (2004): Dreier (Hrsg.): Grundgesetz: Kommentar, 2. Aufl., Mohr Siebeck, Tübingen 2004.

Dreier / Schulze (2004): Dreier, Schulze: Urheberrechtsgesetz: Urheberrechtswahrnehmungsgesetz, Kunsturhebergesetz; Kommentar, Beck, München 2004.

Ensthaler / Bosch / Völker (2002): Ensthaler, Bosch, Völker (Hrsg.): Handbuch Urheberrecht und Internet, Verl. Recht und Wirtschaft, Heidelberg 2002.

Erman / Westermann (2004): Erman (Begr.), Westermann (Hrsg.): Bürgerliches Gesetzbuch, 11. Aufl., Aschendorff u.a., Münster 2004.

Ernst (1997): Ernst: Wirtschaftsrecht im Internet, Betriebs-Berater 1997, S. 1057 ff.

Fromm / Nordemann (1998): Fromm, Nordemann: Kommentar zum Urheberrechtsgesetz und zum Urheberrechtswahrnehmungsgesetz, 9. Aufl., Kohlhammer, Stuttgart 1998.

Grzeszick (2002): Grzeszick: Der Anspruch des Urhebers auf angemessene Vergütung - Zulässiger Schutz jenseits der Schutzpflicht, Archiv für Presserecht 2002, S. 383 ff.

Haas (2002): Haas: Das neue Urhebervertragsrecht, Beck, München 2002.

Haberstumpf (2001): Haberstumpf: Wem gehören Forschungsergebnisse? Zeitschrift für Urheber- und Medienrecht 2001, $819 \mathrm{ff}$.

Hansen (2005): Hansen: Zugang zu wissenschaftlichen Informationen - alternative urheberrechtliche Ansätze, Gewerblicher Rechtsschutz und Urheberrecht International 2005, S. 378 ff.

Hartmer / Detmer (2004): Hartmer, Detmer (Hrsg.): Hochschulrecht, Müller, Heidelberg 2004.

Hertin (2003): Hertin: Urhebervertragsnovelle 2002: Up-Date von Urheberrechtsverträgen, MultiMedia und Recht 2003, S. 16 ff.

Intveen (1999): Intveen: Internationales Urheberrecht und Internet, Nomos, Baden-Baden 1999. 
Jaeger / Metzger (2003): Jaeger, Metzger: Open Content-Lizenzen nach deutschem Recht, MultiMedia und Recht 2003, S. 431 ff.

Jäger / Metzger (2005): Jaeger, Metzger: Open Source Software: Rechtliche Rahmenbedingungen der freien Software, 2. Aufl., Beck, München 2005.

Klett (1998): Klett: Urheberrecht im Internet aus deutscher und amerikanischer Sicht, Nomos, Baden-Baden 1998.

Koch (2000): Koch, F.A.: Urheber- und kartellrechtliche Aspekte der Nutzung von Open-Source-Softwarem Computer und Recht 2000, 333 ff.

Köhler / Arndt (2003): Köhler, Arndt: Recht des Internet, 4. Aufl., Müller, Heidelberg 2003.

Körting / Kummer (2003): Körting, Kummer: Von der Hochschullehrererfindung zur Hochschulerfindung, Recht der Arbeit 2003, 279 ff.

Kreutzer (2001): Kreutzer: Napster, Gnutella \& Co.: Rechtsfragen zu FilesharingNetzen aus der Sicht des deutschen Urheberrechts de lege lata und de lege ferenda - Teil 1, Gewerblicher Rechtsschutz und Urheberrecht 2001, S. 193 ff.

Leupold (1998): Leupold: Auswirkungen der Multimedia-Gesetzgebung auf das Urheberrecht, Computer und Recht 1998, 234 ff.

Leuze (2005): Leuze: Kritische Anmerkungen zu \ 42 ArbEG, Gewerblicher Rechtsschutz und Urheberrecht 2005, 27 ff.

Marly (2000): Marly: Softwareüberlassungsverträge, 3. Aufl., Beck, München 2000.

Möhring / Nicolini (2000): Möhring, Nicolini (Hrsg.): Urheberrechtsgesetz, 2. Aufl., Vahlen, München 2000.

Moltke (1992): Moltke: Urheberrecht an den Werken der Wissenschaft, Nomos, Baden-Baden 1992.

Münch, von / Kunig (2001): von Münch, Kunig (Hrsg.): Grundgesetz, 5. Aufl., Beck, München 2001.

Ory (2002): Ory: Das neue Urhebervertragsrecht, Archiv für Presserecht 2002, S. $93 \mathrm{ff}$.

Pflüger / Ertmann (2004): Pflüger, Ertmann: E-Publishing und Open Access Konsequenzen für das Urheberrecht im Hochschulbereich, Zeitschrift für Urheber- und Medienrecht 2004, S. 436 ff.

Plaß (2002): Plaß: Open Contents im deutschen Urheberrecht, Gewerblicher Rechtsschutz und Urheberrecht 2002, S. 670 ff.

Rohrmann (1988): Rohrmann: Die Abgrenzung von Hauptamt und Nebentätigkeit, Erlangen-Nürnberg, Univ., Diss., 1988.

Schack (1998): Schack: Neue Techniken und Geistiges Eigentum, Juristenzeitung 1998, S. $753 \mathrm{ff}$.

Schack (2005): Schack: Urheber- und Urhebervertragsrecht, 3. Aufl., Mohr Siebeck, Tübingen 2005. 
Schmidt (2002): Schmidt: Der Vergütungsanspruch des Urhebers nach der Reform des Urhebervertragsrechts, Zeitschrift für Urheber- und Medienrecht 2002, S. $781 \mathrm{ff}$.

Schricker (1999): Schricker (Hrsg.): Urheberrecht: Kommentar, 2. Aufl., Beck, München 1999.

Schricker (2001): Schricker: Verlagsrecht, 3. Aufl., Beck, München 2001.

Schuster (2001): Schuster (Hrsg.): Vertragshandbuch Telemedia, Beck, München 2001.

Senftleben (2004): Senftleben: Die Bedeutung der Schranken des Urheberrechts in der Informationsgesellschaft und ihre Begrenzung durch den Dreistufentest, in Hilty/Peukert (Hrsg.), Interessenausgleich im Urheberrecht, Nomos, Baden-Baden 2004, $159 \mathrm{ff}$.

Sosnitza (2001): Sosnitza: Das Internet im Gravitationsfeld des Rechts - Zur rechtlichen Beurteilung so genannter Deep Links, Computer und Recht 2001, S. $693 \mathrm{ff}$.

Spindler (2004): Spindler (Hrsg.): Rechtsfragen bei Open Source, Schmidt, Köln 2004.

Spindler (2004a) Spindler (Hrsg.): Vertragsrecht der Internetprovider, 2. Aufl., Schmidt, Köln 2004.

Spindler (2004b): Spindler: Ausgewählte urheberrechtliche Probleme von Open Source Software unter der GPL, in Büllesbach/Dreier (Hrsg.), Wem gehört die Information im 21. Jahrhundert? Proprietäre versus nicht proprietäre Verwertung digitaler Inhalte, O. Schmidt, Köln 2004.

Spindler (2004c): Spindler in v. Taeger / Wiebe (Hrsg.): Informatik - Wirtschaft Recht: Regulierung in der Wissensgesellschaft; Festschrift für Wolfgang Kilian zum 65. Geburtstag, Nomos, Baden-Baden 2004, S. 353 ff.

Spindler (2004d): Spindler: Europäisches Urheberrecht in der Informationsgesellschaft, Gewerblicher Rechtsschutz und Urheberrecht 2002, S. 105 ff.

Spindler (2004e): Spindler: Open Source Software auf dem gerichtlichen Prüfstand - Dingliche Qualifikation und Inhaltskontrolle, Kommunikation und Recht 2004, S. 528 ff.

Spindler / Wiebe (2003): Spindler, Wiebe: Open Source-Vertrieb, Computer und Recht 2003, $873 \mathrm{ff}$.

Strobel (2003): Strobel: So content with Open Content - Zufriedenheit dank Open-Content-Lizenz? MultiMedia und Recht 2003, S. 778 ff.

Thieme (2004): Thieme: Deutsches Hochschulrecht, 3. Aufl., Heymann, Köln 2004.

Ulmer (1980): Ulmer: Urheber- und Verlagsrecht, 3. Aufl., Springer, Berlin, Heidelberg New York, 1980. 
Ulmer / Brandner / Hensen (2001): Ulmer, Brandner, Hensen: AGB-Gesetz: Kommentar zum Gesetz zur Regelung des Rechts der Allgemeinen Geschäftsbedingungen, 9. Aufl., Schmidt, Köln 2001.

Veddern (2004): Veddern: Multimediarecht für die Hochschulpraxis, 2. Aufl. 2004 abrufbar unter http://www.cec.nrw.de/kunden/uvm/www.nsf/ 0/FA103BA5756C8483C1256E240055C4CF/\%24file/ratgeber.pdf.

Wandtke / Bullinger (2002): Wandtke, Bullinger (Hrsg.): Praxiskommentar zum Urheberrecht, Beck, München 2002.

Wimmers / Rode (2003): Wimmers, Rode: Der angestellte Softwareprogrammierer und die neuen urheberrechtlichen Vergütungsansprüche, Computer und Recht 2003, S. 399 ff.

Wolf / Horn / Lindacher (1994): Wolf, Horn, Lindacher (Hrsg.): AGB-Gesetz: Gesetz zur Regelung des Rechts der allgemeinen Geschäftsbedingungen; Kommentar, 3. Aufl., Beck, München 1994.

Zentek / Meinke (2002): Zentek, Meinke: Urheberrechtsreform, Haufe, Freiburg 2002.

Zirkel (2004): Zirkel: Der angestellte Urheber und \$ 31 Abs.4 UrhG, Zeitschrift für Urheber- und Medienrecht 2004, S. 626 ff. 



\section{Vierter Teil: \\ Empirische Befunde}





\title{
Internet und Beschäftigung: Quantitative Effekte in der Medienbranche
}

\author{
Carmen Lanfer / Kai Marquardsen
}

\section{Einleitung}

Den gesellschaftlichen und wirtschaftlichen Auswirkungen der Informations- und Kommunikationstechnologien - insbesondere des Internets - wird in der Öffentlichkeit nach wie vor ein hoher Stellenwert beigemessen, schließlich sind sie allgegenwärtig: Der Handel über das Internet dehnt sich aus; kein Produkt, das sich hier nicht schnell und einfach per Mausklick bestellen ließe. Unternehmen agieren über weltweit vernetzte Systeme rund um die Uhr; sie flexibilisieren und intensivieren Transaktionen und Kontakte. Mehr als 50\% der Deutschen setzen das Internet für berufliche und private Zwecke ein; sie beschaffen sich Informationen und nutzen Kommunikationsmöglichkeiten via E-Mail, Chat etc..

Verschiedene Konzepte gehen als Resultat der zunehmenden Nutzung und Verbreitung von Informations- und Kommunikationstechnologien von einem tiefgreifenden Strukturwandel in nahezu allen gesellschaftlichen Bereichen aus. In den Überlegungen zur „Wissensgesellschaft“ (vgl. Stehr 1994, vgl. Willke 1998) oder „Informationsgesellschaft“ (vgl. EU-Kommission 2003) wird in diesem Zu- 
sammenhang betont, dass die Verarbeitung und Distribution von Information und Wissen zu zentralen gesellschaftlichen Bestimmungsfaktoren geworden seien - wesentlich forciert durch den Einsatz von Informationstechnologien und ihre Vernetzung.

Neben der Wirtschaft gilt die Arbeitswelt im Kontext solcher Zeitdiagnosen als zentraler gesellschaftlicher Bereich, für den Informations- und Kommunikationstechnologien als wichtige Impulsgeber wirksam werden: Mit der Zunahme von „Informationsarbeit“ sei ein Wandel der Arbeitskräftestruktur verbunden, denn wachsende Beschäftigtengruppen seien mit dem Sammeln, Aufbereiten und Verarbeiten von Informationen beschäftigt (vgl. Dostal 1995) und gleichzeitig gehe der Anteil fertigungsbezogener Tätigkeiten zurück. Neben solchen quantitativen Effekten wird im Zuge der zunehmenden „Informationsarbeit“ von neuen bzw. veränderten Arbeitsanforderungen ausgegangen, die aus dem Umgang mit den neuen Technologien resultierten (vgl. Baukrowitz et al. 2001; vgl. Kleemann et al. 2003).

Von diesen technologischen Wandlungsprozessen und Weiterentwicklungen zweifellos besonders tangiert wurde und wird die Medienbranche: Die verbesserte Informations- und Kommunikationstechnik beeinflusst sowohl Organisation und Management von Medienunternehmen als auch die Produktion, Redaktion, Distribution und Konsumption von Medienprodukten. Was bisher über spezifische, physische Trägermedien vertrieben wurde, lässt sich nun durch das universelle Medium Internet digital übertragen (vgl. Hass 2004, S. 33 f.). Dadurch verändern sich die klassischen Wertschöpfungsprozesse, denn Mediendistribution und -konsum werden vereinfacht und unter Umständen auch verbilligt. Maßgeblich vorangetrieben wird diese Entwicklung von den sich in ihren Kapazitäten scheinbar ins Grenzenlose entwickelnden Aufnahme-, Speicher- und Distributionstechniken, auf die Medienprodukte aufsetzen.

Ziel dieses Beitrags ist es, ausgehend von einer sekundäranalytischen Betrachtung von Beschäftigtendaten der Medienbranche zu Aussagen über die Relevanz des Internets für die Beschäftigungsentwicklungen der vergangenen Jabre zu gelangen. Da die Medienbranche als ausnehmend prädestiniert für Veränderungen durch internetbasierte Technologien gilt, werden Beschäftigungswirkungen zwar kaum in Zweifel gezogen, doch über Richtung und Umfang der gegenwärtigen wie zukünftigen Entwicklung im Mediensektor herrscht Uneinigkeit:

Auf der einen Seite wird von negativen Beschäftigungswirkungen ausgegangen. Die Charakteristika der Branche und ihrer Produkte erlauben es, dass sich das Internet als zusätzlicher bzw. möglicherweise auch alternativer Distributionsweg für Medienprodukte etabliert. Beispielsweise entfallen bei der Direktvermarktung von Medienprodukten über das Internet bestimmte Zwischenhandels- und Produktionsstufen („Disintermediation“; vgl. Hass 2004), wenn sich der Kunde eine CD im Internet selbst zusammenstellt und brennt. Gleiches gilt für Peer-to-Peer- 
Netzwerke, bei denen verteilte Rechnerressourcen direkt gemeinschaftlich genutzt werden können (z. B. bei den Musiktauschbörsen Kazaa oder Gnutella). ${ }^{274}$ Aus der Verkürzung von Wertschöpfungsketten in der Medienproduktion und -distribution werden Beschäftigungsverluste für eben diese Bereiche abgleitet.

Auf der anderen Seite werden positive Auswirkungen auf die Beschäftigungsentwicklung der Branche im Zusammenhang mit erweiterten Distributionsmöglichkeiten über das Internet konstatiert: Der zusätzliche Vertriebskanal Internet bewirke eine gesteigerte Nachfrage nach Medienprodukten. Darüber hinaus entstünden neue Aufgabenfelder und Funktionsbereiche (bzw. die Erweiterung bestehender Felder) und damit zusätzliche Arbeitsplätze, die sich aus der Notwendigkeit redaktioneller Anpassungen an das Internet ergäben. Ob sich die o.g. möglichen negativen Beschäftigungseffekte durch neu geschaffene Tätigkeitsfelder kompensieren lassen oder es sogar zu einem Beschäftigungsplus kommt, ist fraglich.

Als Ausgangspunkt der Analysen konstatieren wir folglich eine Zweischneidigkeit zwischen womöglich negativen Beschäftigungswirkungen aufgrund verkürzter Wertschöpfungsprozesse und potentiell positiven aufgrund von Kompensationseffekten durch die Schaffung neuer Tätigkeitsfelder. Diese Wechselwirkungen verdeutlichen bereits, dass die Bestimmung der Relevanz des Internets für die Beschäftigungsentwicklung in der Medienbranche kein triviales Problem darstellt. Überdies wirkt eine Vielzahl weiterer Triebkräfte wie bspw. die allgemeine Wettbewerbssituation der Firmen und Unternehmen, die betrieblichen Organisationsformen von Arbeit und Produktion, Konzentrationsprozesse in den Printmedien oder auch die Krise am Werbemarkt.

Vor dem Hintergrund der skizzierten Unsicherheiten in Bezug auf die Beschäftigtenentwicklung erscheint es uns sinnvoll, bei der Sekundäranalyse zuvorderst allgemeine Trends zur Beschäftigung der Gesamtbranche festzustellen. Darauf folgend wird die Entwicklung in den einzelnen Teilsektoren (vgl. die Ausführungen zur Branchenabgrenzung in Abschnitt 2) betrachtet werden, um evtl. Unterschiede bezüglich ihrer Veränderungsdynamiken aufzudecken. Der Differenzierung in Teilsektoren liegt die These zu Grunde, dass das Internet einen je unterschiedlichen Wirkungsgrad entfaltet: In diesem Zusammenhang wird davon ausgegangen, dass sich durch das Internet der Entscheidungsspielraum bezüglich der Festlegung von Erlösformen, die für die klassischen Medienangebote vorgegeben waren, verändert, weil sowohl eine neue Verwertungsstufe der Inhalte als auch neue Erlösmodelle möglich werden (vgl. Zerdick et al. 1999, S. 24). Für den Printsektor bspw. eröffnen sich durch das Internet Zusatzangebote wie der Neugewinn von Abonnenten von E-paper-Ausgaben, insbesondere zur Erschließung

${ }^{274}$ Ein Peer-to-Peer-Netzwerk ist ein Kommunikationsnetzwerk zwischen Rechnern, in dem jeder Teilnehmer sowohl Client- als auch Server-Aufgaben durchführen kann. Es dient einem gleichberechtigten und dezentralen Austausch von Daten (File-Sharing-System); vgl. dazu auch den Beitrag Seidenfaden in diesem Band. 
regionaler Teilmärkte. Auch Optionen wie Archiv- und Datenbankzugänge sind bereits verbreitet. Den neuen Verwertungskonzepten steht die mögliche Konkurrenz durch den sich ausdehnenden Anzeigenmarkt im Internet gegenüber, weil Zielgruppensicherheit und Gestaltungsmöglichkeiten im Internet größer sind als in den klassischen Printmedien (vgl. Sennewald 1998, S. 128). Auch für den Musikmarkt ergibt sich durch das Internet eine gewisse Ambivalenz in Bezug auf die Verwertungsstrategie: Zum einen sind verschiedene Internetmusikportale mit Downloadmöglichkeiten für Kaufmusik auf den Markt gekommen. ${ }^{275}$ Zum anderen wird mit Klageandrohungen und neuen Sicherheitstechnologien Druck auf die Konsumenten ausgeübt, die über Peer-to-Peer-Netzwerke Musikstücke austauschen. ${ }^{276}$ Die Beispiele deuten darauf hin, dass der Einbezug des Internets in die Wertschöpfung und die sich daraus ergebenden Konsequenzen für die Beschäftigtenentwicklung in den verschiedenen Medienteilsektoren nicht gleichläufig sind. Weil wir davon ausgehen, dass eine ausschließlich aggregierte Betrachtung über evtl. Unterschiede hinwegtäuschen würde, werden wir die Beschäftigtenentwicklung der Medienbranche sektorenspezifisch analysieren.

Die Betrachtung der Beschäftigungsdynamiken wird zudem von der Frage geleitet, welche weiteren Faktoren für die jeweiligen Entwicklungen verantwortlich sein könnten, um zu einer Einschätzung darüber zu gelangen, inwiefern das Internet tatsächlich zu Dynamiken in der Beschäftigungsentwicklung der Medienbranche beiträgt bzw. in welchem Verhältnis es zu anderen Ursachen steht.

Zwar existiert eine Vielzahl an Untersuchungen zu möglichen Veränderungen, die das Internet auch in der Arbeitswelt bereits bewirkt hat bzw. noch bewirken wird, diese fokussieren allerdings überwiegend die qualitativen Veränderungen von Arbeit (vgl. z. B. Baukrowitz et al. 2001).277 Es lassen sich kaum greifbare Bezüge zur Relevanz des Internets für die Beschäftigungsentwicklung auftun. Prognosen aus Mitte/Ende der 1990er Jahre278 nehmen zwar die erwarteten Beschäftigungswirkungen der IuK-Technologien (bzw. die TIME-Branche ${ }^{279}$ ) in den Blick, aber empirische Untersuchungen zur Medienbranche finden sich nicht. Insofern können wir für unsere Untersuchungen auf keine konkreten Vergleichsdaten oder -untersuchungen zurückgreifen.

\footnotetext{
275 Vgl. http://www.musicload.de; http://www.apple.com/de/itunes; http://www.medionmusic.com.

276 Vgl. die aktuelle Berichterstattung bei www.heise.de.

277 Qualitative Veränderungen von Arbeit schließen Veränderungen hinsichtlich der Aufgabenzuschnitte, Arbeitsverhältnisse, Tätigkeitsprofile, Qualifikationsanforderungen etc. ein; sie beziehen sich folglich auf die Ebene der konkreten Tätigkeitsausführung und die dafür erforderlichen Qualifikationen.

$278 \mathrm{Zu}$ den vier großen Studien in diesem Bereich zählen die Metier-Studie von 1995, die DIW/Prognos-Studie von 1996 (vgl. Schrape et al. 1996, vgl. DIW 1996), die Studie von Arthur D. Little von 1996 (vgl. Little 1996) und die BIPE Counseil-Studie von 1997 (vgl. EUKommission 1997); für einen Vergleich der Studien: vgl. Bosch (1998).

279 TIME: Telekommunikation, Informationstechnologie, Medien und Elektronik.
} 


\section{Zur Eingrenzung des Untersuchungsfeldes}

\subsection{Abgrenzung der Medienbranche}

Ein zunächst notwendiger Schritt beinhaltet die Abgrenzung der Medienbranche; dieser Schritt ist insbesondere in diesem Fall unentbehrlich, denn es existiert keine allgemein gültige oder verbindliche Definition der Branche. In der Literatur finden sich zahlreiche Abgrenzungen mit je unterschiedlichen Schwerpunkten. Den Ausgangspunkt bildet vielfach der Bereich der „traditionellen“ Medien, zu denen Hörfunk, Fernsehen, Zeitungen, Zeitschriften und Nachrichtenagenturen gezählt werden (vgl. Altmeppen 1999). Zerdick et al. (1999) erweitern diese Abgrenzung, indem sie der Medienbranche sowohl die klassischen Printmedien (Zeitungen, Zeitschriften und Buch) und den Rundfunkbereich (Hörfunk und Fernsehen) als auch den Bereich Kino- und Videofilme und die Tonträgerbranche zurechnen. Kennzeichnend für alle Bereiche ist die Produktion und Verbreitung von Inhalten (vgl. Zerdick et al. 1999, S. 36 f.).

In einer Reihe von Publikationen wird der Fokus weniger auf die Medienbranche gelegt, sondern die Informations- und Medienbranche als Querschnittsbranche betrachtet (vgl. NIW 2002, vgl. RWI 2000). Dies hat zur Folge, dass neben den Inhalteproduzenten (Content Industries: Verlagsgewerbe, Werbewirtschaft, traditionelle AV-Produzenten [Rundfunk- und Filmwirtschaft], Musikproduktion) auch die Kernbereiche des IuK-Sektors in die Abgrenzung aufgenommen werden, d. h. neben den Informations- und Kommunikationsdienstleistungen werden auch die Informations- und Kommunikationstechnik und somit die Endgerätehersteller einbezogen (vgl. NIW 2002, S. 11 f). Wir interessieren uns dagegen für die Auswirkungen des Internet auf die Bereiche der ContentProduktion und -Distribution und deren evtl. Beschäftigungseffekte.

Unsere Eingrenzung der Medienbranche greift verschiedene Elemente der oben genannten Abgrenzungen auf, indem sie die klassischen Inhalteproduzenten zum Ausgangspunkt nimmt. Hierzu zählen wir die Printmedien, den Rundfunkbereich, die Filmbranche und die Musikbranche. Hinzu kommt die Werbung als Produzent und Finanzier einer spezifischen Form des Mediencontent, denn Werbung beeinflusst die Gestaltung des Mediengesamtprodukts entscheidend mit. Eine besonders enge Verknüpfung besteht zu den Printmedien und zum Bereich Rundfunk; die beiden Segmente vereinen den überwiegenden Teil der Werbeinvestitionen auf sich und sind folglich in erheblichem Maße auf Werbung als Einnahmequelle angewiesen. ${ }^{280}$ Neben der Werbung sind als weiterer (teil-)sektorübergreifender Bereich die Korrespondenz- und Nachrichtenbüros und selbständigen Journalisten einzubeziehen.

\footnotetext{
${ }^{280}$ Für das Jahr 2003 machen die Ausgaben für Werbung in den Segmenten Zeitschriften und Zeitung zusammen 58,4\% der Werbeausgaben aus, im Segment Rundfunk (Hörfunk und Fernsehen) sind es 34,2\%; Quelle: vgl. PricewaterhouseCoopers 2004, S. 7; eigene Berechnungen.
} 
Der Bereich, der sich mit der Produktion und Verbreitung von Inhalten beschäftigt wird in unserer Abgrenzung ergänzt um die Branchen, die die nötige Infrastruktur entwickeln und bereitstellen: Hierzu gehören die Fernmeldedienste, der Bereich Softwareberatung und -entwicklung sowie die Datenverarbeitungsdienste. Ihnen ist gemein, dass sie ihre Produkte und Dienstleistungen nicht exklusiv für den Bereich Medien erstellen, sondern ebenso Leistungen für andere Branchen bereitstellen. Daraus ergibt sich folgende Schwierigkeit: Die Anteile derjenigen, die in der bzw. für die Medienbranche tätig sind, lassen sich nicht zweifelsfrei ermitteln (SAP-Lösungen werden z. B. für verschiedenste Branchen entwickelt).

Als Resultat dieser Abgrenzungsproblematik operieren wir im Folgenden mit zwei Definitionen der Medienbranche: Die erste orientiert sich am engen Branchenbegriff; dieser ist insofern als eng zu betrachten als er diejenigen Sektoren einschließt, die den in der Medienbranche wirksamen Dynamiken zweifelsfrei ausgesetzt sind. Die zweite Definition des erweiterten Branchenbegriffs schließt zusätzlich weitere relevante Bereiche ein, bei denen aber nicht eindeutig klar ist, inwiefern ihre spezifischen Entwicklungsdynamiken mit denen der Medienbranche in Zusammenhang stehen. Deshalb steht bei unseren Analysen vornehmlich die Beschäftigungsentwicklung des engen Branchenbegriffs im Mittelpunkt.

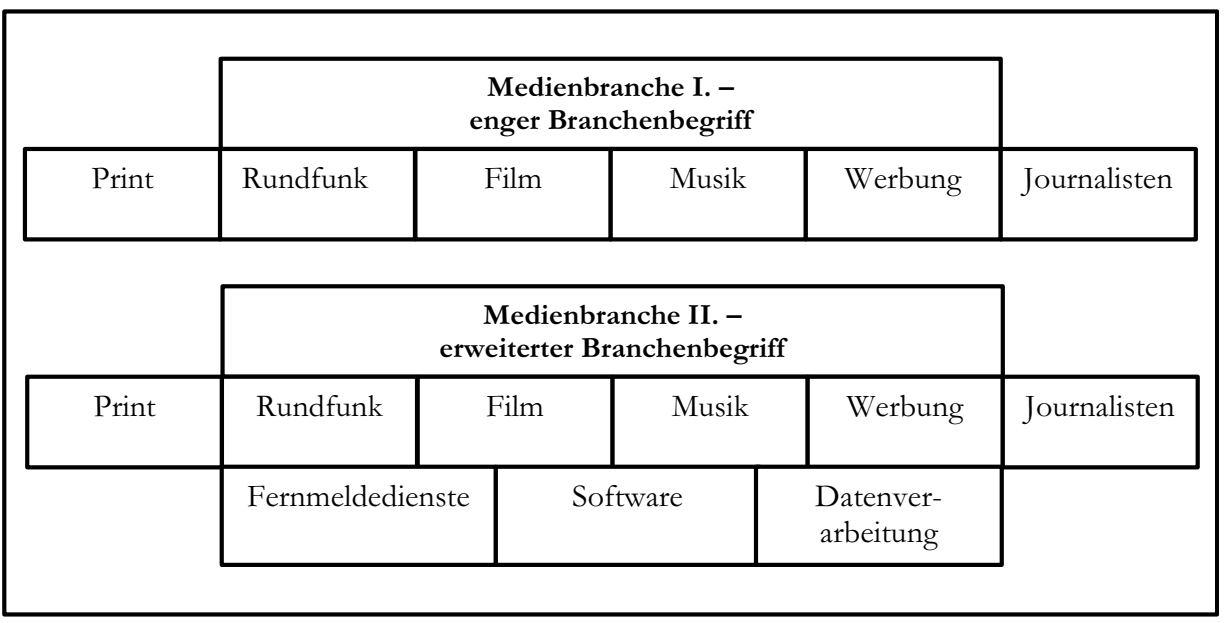

Abbildung 50: Abgrenzung der Medienbranche - enger und erweiterter Branchenbegriff 


\subsection{Datenbasis und Untersuchungszeitraum}

\section{Datenbasis}

Die Sekundäranalyse von Beschäftigtendaten zur Medienbranche bezieht sich auf die Beschäftigungsstatistik der Bundesagentur für Arbeit (BA). Als Datengrundlage wurden die Beschäftigtendaten nach Wirtschaftszweigen (WZ) herangezogen, um unsere Abgrenzungen der Medienbranche möglichst detailliert abbilden zu können. Bei der Klassifikation der Wirtschaftszweige handelt es sich um ein System, über das die wirtschaftliche Schwerpunkttätigkeit von Unternehmen, Betrieben und anderen statistischen Einheiten erfasst wird. ${ }^{281}$ Um unsere Branchendefinitionen mit ihren Teilsektoren vollständig über dieses Klassifikationssystem abbilden zu können, benötigen wir stark differenzierte Datenbestände. ${ }^{282}$ Die amtlich veröffentlichten Statistiken weisen diese Differenzierungen jedoch in der Regel nicht aus. Als Folge stellte die Bundesagentur für Arbeit im Rahmen eines Datenauftrags eigens die von uns benötigten Datenbestände zusammen, so dass wir über Beschäftigtendaten für die Jahre 1999-2003 mit je zwei Messpunkten (30.06. und 31.12.) verfügen, deren Differenzierung unsere Branchenabgrenzungen abbilden können. ${ }^{283}$

Die BA-Daten beziehen sich auf sozialversicherungspflichtig Beschäftigte. Die Medienbranche ist allerdings von jeher durch Beschäftigungsverhältnisse wie freie Mitarbeitertätigkeit, „Feste Freie“, auf Produktions-/Projektdauer Beschäftigte oder auch Selbständige mit möglicherweise mehreren Auftraggebern (z. B. in den Bereichen audio-visuelle Medien, Werbung, Journalisten in den Printmedien etc.;

281 Mit dieser Vorgehensweise knüpfen wir an bestehende Definitionen und Abgrenzungen zur Informations- und Medienbranche an (vgl. OECD 1998, vgl. DIW 1999, vgl. DIW 2002). Das WZKlassifikationssystem ist hierarchisch gegliedert und ermöglicht eine weitreichende Differenzierung bis auf die fünfte Gliederungsebene. Die hierarchische Gliederung der Wirtschaftsklassifikation umfasst 17 Abschnitte, 31 Unterabschnitte, 60 Abteilungen, 222 Gruppen, 513 Klassen und 1041 Unterklassen; vgl. http://www.destatis.de/allg/d/klassif/wz2003.htm. Ende 2002 hat es eine Umstellung des Wirtschaftszweigklassifikationssystems gegeben, die bislang gültige Version WZ 93 wurde durch die neue Klassifikation der Wirtschaftszweige WZ 2003 ersetzt. Eine solche Umstellung erfüllt den notwendigen und wichtigen Zweck, bereits bestehende Klassifikationen anzupassen und neue Wirtschaftsbereiche in die Klassifikation aufzunehmen.

282 Die allgemein und öffentlich zugänglichen Datenbestände weisen in der Regel lediglich Differenzierungen bis auf die dritte bis höchstens vierte Gliederungsebene aus; für unsere Definition und Abgrenzung der Medienbranche ist es wichtig, Daten bis zur kleinsten Unterklasse (5-Steller) auswerten zu können. Ein Beispiel mag diese Problematik illustrieren: Als zwei zentrale Medienteilsektoren unterscheiden wir den Print- und den Musikbereich, zu beiden Teilbranchen werden zwar Daten für das entsprechende Verlagswesen ausgewiesen (Musikverlage, Zeitungs- und Zeitschriftenverlage), beide Gruppen werden in der allgemein verfügbaren Beschäftigtenstatistik aber der Oberkategorie „Verlagsgewerbe“ (differenziert bis zum 4-Steller) zugeordnet, so dass eine Differenzierung zwischen Musik- und Printbereich nicht vorgenommen werden kann. Diese wird aber über die Ebene des 5-Stellers wieder möglich.

283 Frühere Daten sind momentan nicht verfügbar. Die Daten für das Jahr 2004 stehen ab Herbst 2005 zur Verfügung. 
vgl. Marrs/Boes 2003) gekennzeichnet. Solche Beschäftigungsverhältnisse werden nicht über die BA erfasst, d. h. für die quantitativen Analysen der Beschäftigungsentwicklung ergibt sich die Schwierigkeit, dass die BA-Daten die Beschäftigtenentwicklung der Medienbranche nicht vollständig abbilden können. Diese Lücke füllt zu erheblichen Teilen die Versichertenbestandsentwicklung der Künstlersozialkasse (KSK), die Selbständigen und Freien in den Tätigkeitsbereichen „Wort“, „Musik“, „bildende Kunst“ und „darstellende Kunst“ Zugangsmöglichkeiten zur Renten-, Kranken- und Pflegeversicherung eröffnet. ${ }^{284}$ Für die Analyse der Beschäftigtenentwicklung der Medienbranche sind vor allem die Angaben der KSK zu den Bereichen Wort und Musik aufschlussreich. ${ }^{285}$ Die Versichertenbestandsentwicklung für diese Bereiche liegt uns ebenfalls für den Zeitraum 1999-2003 vor (mit einem Messpunkt pro Jahr).

Das Referat „Freie und Selbstständige“ von Ver.di nimmt an, dass nur ca. 20\% der Freischaffenden nicht über die KSK versichert sind; d. h. addiert man zu den Angaben der KSK 20\% hinzu, erhält man näherungsweise die Anzahl der Freiberufler. Auch wenn die Versichertenbestandsentwicklung der KSK nicht der Beschäftigtenentwicklung der Freien und Selbständigen entspricht, lässt sie doch Rückschlüsse in Bezug auf generelle Entwicklungstrends in einzelnen Berufsfeldern zu und liefert notwendige Ergänzungen zu den Beschäftigtenzahlen: Es ist zu vermuten, dass in einigen Fällen, wie z. B. bei der Gruppe der Journalisten, die Daten der Beschäftigtenstatistik nicht annähernd die tatsächliche Zahl der Beschäftigten wiedergeben und die KSK-Angaben hier sinnvolle und notwendige Ergänzungen liefern (vgl. unten).

\section{Untersuchungszeitraum}

Bei der Auswahl des Untersuchungszeitraums haben wir uns am zentralen Zeitraum der Internetentwicklung orientiert: Wir gehen davon aus, dass für die Frage nach der Relevanz des Internets für die Beschäftigtenentwicklung in der Me-

${ }^{284}$ Die Künstlersozialkasse (KSK) ordnet die Freien und Selbständigen der vier Bereiche nach Berufsgruppen. Sie hat zwei zentrale Aufgabenbereiche: Sie prüft die Zugehörigkeit zum versicherungspflichtigen Personenkreis und zieht den Beitragsanteil der Versicherten, die Künstlersozialabgabe der abgabepflichtigen Unternehmen sowie den Bundeszuschuss ein. Das am 01.01.1983 in Kraft getretene Künstlersozialversicherungsgesetz (KSVG) bietet selbständigen Künstlern und Publizisten sozialen Schutz in der Renten-, Kranken- und Pflegeversicherung. Wie Arbeitnehmer zahlen sie nur eine Hälfte der Versicherungsbeiträge; die andere Beitragshälfte trägt die Künstlersozialkasse. Die hierfür erforderlichen Mittel werden aus einem Zuschuss des Bundes und aus einer Abgabe der Unternehmen finanziert, die künstlerische und publizistische Leistungen verwerten (Künstlersozialabgabe); vgl. www.kuenstlersozialkasse.de.

285 Bereich „Wort“: Schriftsteller, Autor, Lektor, Journalist, Bildjournalist, wissenschaftlicher Autor, PR-Fachmann, Übersetzer, sonstige Tätigkeit, Vertragstätigkeit; Bereich „Musik“: Komponist, Texter, Musikbearbeiter, Dirigent, Chorleiter, Instrumentalsolist, Orchestermusiker, Sänger (unterschiedliche Musikrichtungen), sonstige künstlerisch-technischer Mitarbeiter, Pädagoge, Ausbilder im Bereich Musik, Diskjockey, sonstige Tätigkeit im Bereich Musik. 
dienbranche die Entwicklung der Internetnutzung in Deutschland als Indikator herangezogen werden kann.

Die Daten zur Internetnutzung zeigen erwartungs- wie erfahrungsgemäß, dass die Onlinenutzung seit Ende der 1990er Jahre stark angestiegen ist und insbesondere für den genannten Zeitraum 1999-2003 wesentlich zugenommen hat (vgl. Abb. 51). Das Datenmaterial bezieht sich demnach auf den Hauptausbreitungszeitraum des Internets und bescheinigt dem Zeitraum der aktuellen Datenverfügbarkeit damit eine sinnvolle zeitliche Dimensionierung.

\begin{tabular}{|l|c|c|c|c|c|c|c|c|c|c|}
\hline & $\mathbf{1 9 9 7}^{1}$ & $\mathbf{1 9 9 8}^{1}$ & $\mathbf{1 9 9 9}^{1}$ & $\mathbf{2 0 0 0}^{1}$ & $\mathbf{2 0 0 1}^{1}$ & $\mathbf{2 0 0 2}^{1}$ & $\mathbf{2 0 0 3}^{1}$ & $\mathbf{2 0 0 3}^{2}$ & $\mathbf{2 0 0 4}^{1}$ & $\mathbf{2 0 0 4}^{2}$ \\
\hline in \% & 6,5 & 10,4 & 17,7 & 28,6 & 38,8 & 44,1 & 53,1 & 51,5 & 55,5 & 52,6 \\
\hline $\begin{array}{l}\text { Zuwachs } \\
\text { in \% }\end{array}$ & - & +61 & +68 & +64 & +36 & +14 & +22 & +17 & +4 & +2 \\
\hline
\end{tabular}

$1=$ gelegentliche Online-Nutzung $\quad 2=$ Onlinenutzung innerhalb der letzten 4 Wochen

Abbildung 51: Entwicklung der Online-Nutzung in Deutschland 1997 bis $2004^{286}$

\section{Befunde zur Beschäftigtenentwicklung in der Medienbranche}

\subsection{Die Entwicklung der Gesamtbranche}

Um allgemeine Trends zur Beschäftigung der Gesamtbranche feststellen zu können, haben wir die Daten der entsprechenden Wirtschaftszweige aggregiert. Die Summe der Beschäftigten der Medienteilsektoren Printmedien, Musik, Film, Rundfunk, Werbung und der Korrespondenz- und Nachrichtenbüros/selbständige Journalisten bildet die Beschäftigtenentwicklung für die Medienbranche im Sinne der engen Branchendefinition ab (vgl. Abb. 52).

286 Quelle: vgl. ARD/ZDF-Online-Studie 2004. 


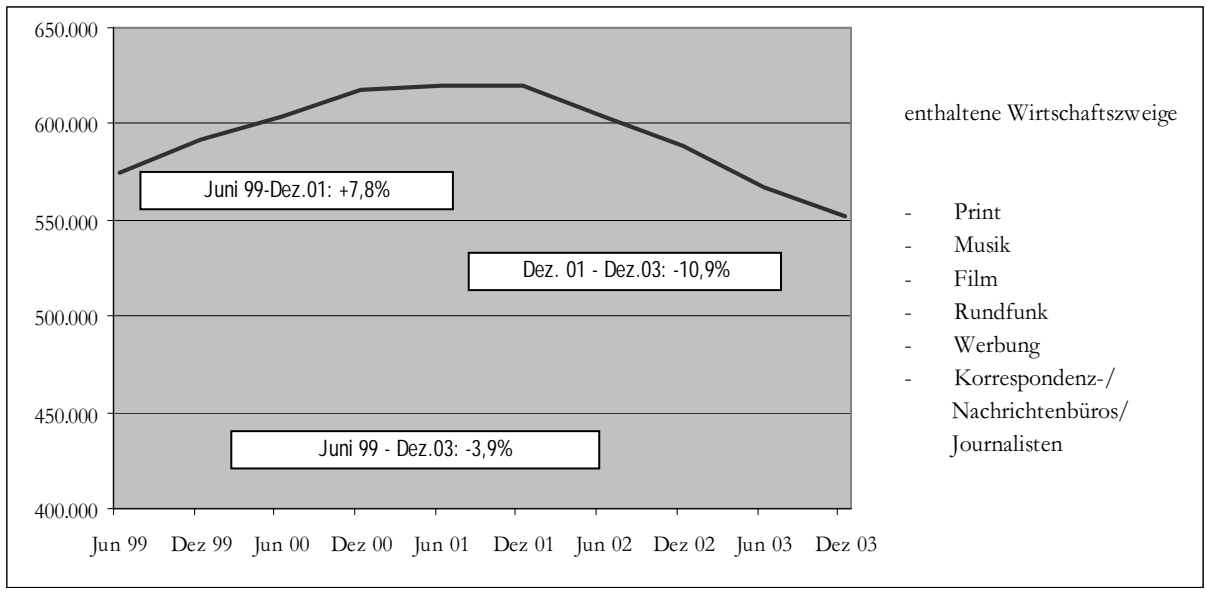

Abbildung 52: Sozialversicherungspflichtig Beschäftigte in der Medienbranche 1999-2003 (enge Branchendefinition)

Die Beschäftigtenentwicklung verläuft zunächst ansteigend, danach stagniert sie für ein Jahr auf hohem Niveau und fällt schließlich stark ab. Die Beschäftigtenzahlen sinken dabei zwischen Ende 2001 und Ende 2003 um fast 11\%. Für den gesamten Zeitraum ergeben sich Verluste von fast 4\%. Zum Vergleich: Im gleichen Zeitraum verlief die Entwicklung der sozialversicherungspflichtig Beschäftigten über alle Wirtschaftszweige in Deutschland ganz ähnlich (ohne Abbildung). Nach Anstieg der Beschäftigtenzahlen bis ins Jahr 2000/2001 kam es zu überdurchschnittlichen Einbrüchen bis 2003 (-3\% zwischen 2001 und 2003). Für den Betrachtungszeitraum 1999-2003 ergeben sich für die Gesamtentwicklung der Beschäftigten in Deutschland trotz des ähnlichen Verlaufs der Entwicklung Verluste von nur -1,9\% (vgl. http://www.destatis.de/basis/d/erwerb/erwerbtab5.php; eigene Berechnungen). Der Vergleich verweist also darauf, dass sich die Beschäftigungsentwicklung der Medienbranche bezüglich ihres grundsätzlichen Verlaufs an der Gesamtentwicklung ausrichtet, die Effekte in der Medienbranche jedoch insgesamt stärker ausfallen.

Betrachtet man dagegen die Entwicklung für den erweiterten Branchenbegriff (d. h. zu dem oben angegebenen kommen die Wirtschaftszweige Software, Datenverarbeitung und Fernmeldedienste hinzu), fällt auf, dass die Beschäftigtenentwicklung bei weitem steiler ansteigt (vgl. Abb. 53): Das Beschäftigtenplus beträgt zwischen 1999 und Ende 2001 fast 20\%. Seitdem brechen die Beschäftigtenzahlen ähnlich stark ein wie die der engen Branchendefinition (hier: -9,7\%). Durch das starke Plus zu Beginn des Betrachtungszeitraums fällt die Bilanz aber mit $+8,2 \%$ insgesamt positiv aus. 
Unabhängig von der jeweiligen Grenzziehung der Medienbranche lässt sich festhalten: Seit 2001 gehen die Beschäftigtenzahlen stark zurück, während sie bis dahin angestiegen waren, zum Teil sogar erheblich. Für den Betrachtungszeitraum markiert das Jahr 2001 demnach gleichzeitig den Höhe- wie auch den Wendepunkt der Beschäftigtenentwicklung der Branche.

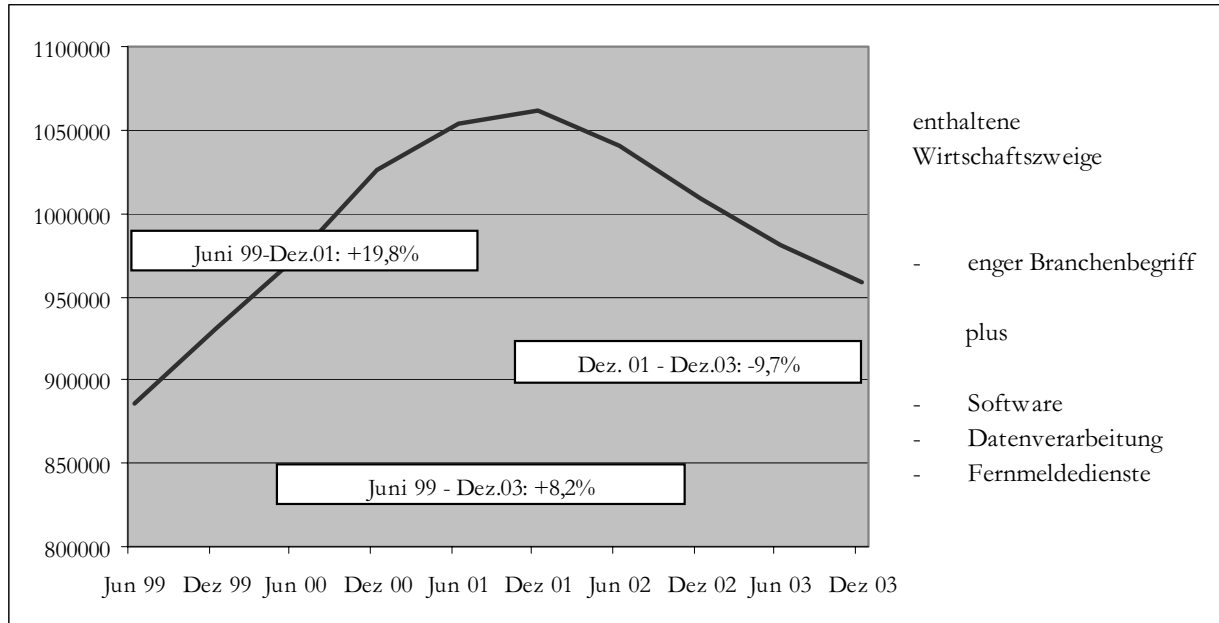

Abbildung 53: Sozialversicherungspflichtig Beschäftigte in der Medienbranche 1999-2003 (erweiterte Branchendefinition)

Unsere Hypothese ist, dass die Frage danach, wie das Internet - im Unterschied $\mathrm{zu}$ anderen möglichen Einflussfaktoren - auf die Beschäftigungsentwicklung in der Medienbranche wirkt, für die verschiedenen Medienteilsektoren jeweils gesondert zu stellen ist. Die dargestellte unterschiedliche Entwicklung der beiden Branchenabgrenzungen liefert bereits Hinweise für sektorenbedingte Beschäftigungsentwicklungen. Die Disaggregation der Branchenentwicklungen kann Aufschluss darüber geben, welche der inkludierten Teilsektoren die jeweiligen Verläufe in besonderer Weise dominieren: Welches quantitative Gewicht bringen die Sektoren jeweils mit? Welche der Mediensektoren weisen eine besonders hohe Beschäftigtendynamik auf, welche eine eher geringe? Welche weiteren Unterschiede wie auch Parallelen zwischen den Teilsektoren sind erkennbar? Eine solche differenzierte Darstellung der Beschäftigungsverläufe stellt damit die Basis für eine vergleichende Analyse der einzelnen Sektoren dar. Da sich die Gründe für die jeweiligen Beschäftigungsentwicklungen jedoch nicht aus den bloßen Verläufen erschließen lassen, gilt es, auf der Grundlage der jeweils einschlägigen, aktuellen Literatur nach möglichen Ursachen der jeweiligen Entwicklungen zu fragen und auf den Einfluss und die Relevanz internetbasierter Technologien für die verschiedenen Teilsektoren einzugehen. 


\subsection{Entwicklungstrends der Teilbranchen - ein uneinheitliches Bild}

Die Überblicksanalyse der Beschäftigtenentwicklung in den Bereichen, die zum engen Branchenbegriff zusammengefasst wurden, (vgl. Abb. 54/55) verdeutlicht, dass die Gesamtentwicklung wesentlich vom Bereich Printmedien bestimmt wird; dieser repräsentiert den beschäftigungsstärksten Bereich der Medienbranche ${ }^{287}$ und nimmt mit $-11 \%$ über den gesamten Betrachtungszeitraum eine im Vergleich zur Gesamtentwicklung stark negative Entwicklung. Der Bereich Werbung wirkt sich durch sein quantitatives Gewicht ebenfalls wesentlich auf die Gesamtentwicklung der Branche aus, zeigt aber eine insgesamt positive Entwicklung (+11,6\%).

Ebenfalls positiv entwickeln sich die Bereiche Rundfunk (+6,0\%) und Film $(+9,2 \%)$. Besonders stark stiegen die Beschäftigtenzahlen für den Bereich Korrespondenz- und Nachrichtenbüros/selbständige Journalisten an $(+26,8 \%)$, der aber quantitativ kaum ins Gewicht fällt. Eine ähnlich geringe Beschäftigtenzahl weist der Bereich Musik auf. Hier sind allerdings Beschäftigungsrückgänge um mehr als $7 \%$ zu verzeichnen.

Insgesamt fallen die Beschäftigungsentwicklungen durchaus unterschiedlich aus: Während der dominante Sektor Print erhebliche Beschäftigungsverluste zu verzeichnen hat und auch der Bereich Musik sich insgesamt negativ entwickelt, ziehen die übrigen Sektoren über den Gesamtbetrachtungszeitraum hinweg, wenn es auch mehrheitlich seit 2001 zu Beschäftigungseinbußen gekommen ist, eine positive Bilanz. Um Gemeinsamkeiten und Unterschiede genauer analysieren zu können, betrachten wir die Teilsektoren im Folgenden jeweils einzeln.

${ }^{287}$ In Bezug auf die enge Branchendefinition macht der Bereich Printmedien einen Anteil von ca. $62 \%$ (je nach zu Grunde liegendendem Berechungsjahr) am Gesamtverlauf aus. 

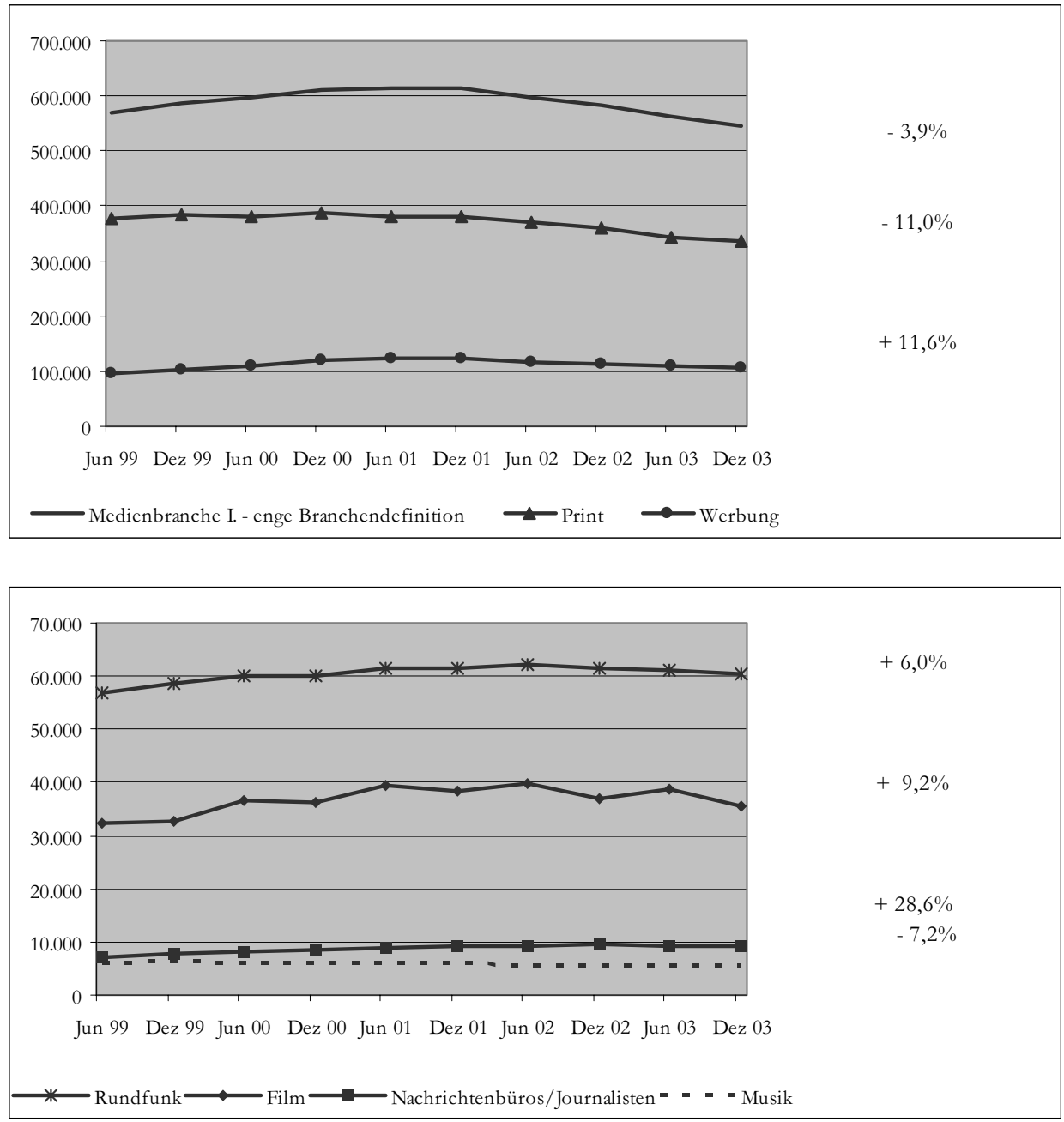

Abbildung 54 / Abbildung 55: Sozialversicherungspflichtig Beschäftigte in der Medienbranche 1999-2003 (enger Branchenbegriff) nach Teilsektoren

\subsection{Printmedien und Rundfunk: negative gegen positive Beschäftigungsentwicklung}

Dem für die gesamte Branchenentwicklung prägendsten Wirtschaftszweig Printmedien werden das Verlagsgewerbe (Buch-, Zeitungs-, Zeitschriften- und Adressbuchverlage, sonstiges Verlagsgewerbe; nicht Musikverlage), das Druckgewerbe sowie die Druck- und Medienvorstufe (Satz, Reproduktion etc.) zugeordnet. Vor dem Hintergrund der Annahme zunehmender Informatisierung von Arbeit und 
Produktion sowie der verstärkten Verbreitung internetbasierter Technologien stellt sich die Frage, in welchen Bereichen des Printsektors technologische Neuerungen, wie das Internet, ihre Wirkungskraft besonders entfalten könnten. Der Bereich der Distribution - d. h. der Druckbereich und seine vorbereitenden Stufen - wird bereits seit Jahrzehnten stark automatisiert betrieben. Computerbasierte Workflowsysteme ermöglichen es hier, Arbeitsabläufe zu vereinfachen und zu standardisieren, aber auch bestimmte Tätigkeitsfelder gänzlich aufzulösen. ${ }^{288} \mathrm{Im}$ Gegensatz dazu lässt sich der Bereich der Produktion von Content nur bedingt automatisieren, weil z. B. die Erstellung eines aktuellen Zeitungsartikels komplexe Recherche-, Selektions- und sonstige kreative Bearbeitungsaufgaben beinhaltet, die sich einer vollständigen Technisierung entziehen. Zwar ist davon auszugehen, dass das Internet die Contentproduktion verändert, indem es z. B. erweiterte Rechercheformen oder eine bessere Vernetzung der verschiedenen Verlagseinheiten (Redaktionen, Vertrieb, Marketing, freie Journalisten etc.) ermöglicht. Die tatsächliche Produktion des Content kann jedoch nicht von technologischer Seite erfolgen und insofern auch nicht substituiert werden. Möglicherweise eröffnet der Bereich der Content-Gestaltung für Online-Medien sogar neue Tätigkeitsfelder, wie z. B. die Arbeit in Online-Redaktionen. Diese würden zunehmen, wenn eine Online-Redaktion originären Content produziert, wenn also z. B. die Online-Ausgabe einer Tageszeitung echte Zusatzangebote zur gedruckten Ausgabe liefert, die eigener journalistischer Recherchen und Bearbeitung bedürften. Zunächst liegt aufgrund dieser Überlegungen jedoch die Vermutung nahe, dass sich die negativen Beschäftigungsentwicklungen des Printsektors eher im Bereich der Distribution wiederfinden.

Die Annahme, dass die Beschäftigungsentwicklung im Bereich Distribution sich im Vergleich zur Content-Produktion negativer entwickeln würde, wird durch die Daten bestätigt (vgl. Abb. 56). Allerdings fallen die Unterschiede zwischen den Bereichen schwächer aus als erwartet. Der Bereich der Distribution büßt über den gesamten Betrachtungszeitraum mehr als 13\% der Beschäftigten ein. Aber auch im Bereich der Content-Produktion ist es zu Beschäftigtenverlusten gekommen. Waren bis Ende 2001 noch leichte Anstiege der Beschäftigtenzahlen zu verzeichnen, fallen sie seitdem ebenso stark ab wie im Bereich Distribution (insgesamt um $-7,0 \%)$. Der Content-Bereich ist also stärker von Beschäftigungsverlusten betroffen als vermutet. Ob diese dem zunehmenden Einsatz von Netzwerktechnologien

${ }^{288}$ Ein aktuelles Beispiel ist das sog. JDF (Job Definition Format), eine Art elektronische „Auftragstasche“, die den gesamten Produktionsumfang umfasst und außerdem einen Standard zur Beschreibung von Meldungen und ein Austauschprotokoll beinhaltet. Dass solche technischen Entwicklungen zu Beschäftigungseinsparungen führen können, ist offensichtlich: JDF ermöglicht es z. B., dass Kunden einen Druck über ein JDF-fähiges Formular im Internet in Auftrag geben, das dem Drucker dann als elektronische Auftragsvorlage dient und direkt in seinen Arbeitsprozess einbezogen werden kann. Auch wenn sich aktuell noch nicht feststellen lässt, inwiefern sich solche Systeme flächendeckend durchsetzen werden, ebnen sie möglicherweise den Weg in Richtung einer „Ein-Personen-Druckerei“, d. h. hin zu Möglichkeiten, den Druckprozess möglichst personalextensiv zu betreiben. 
und Internet zuzuschreiben sind, ist schwer feststellbar. Allerdings wird z. B. darauf hingewiesen, dass durch die Online-Angebote von Tageszeitungen kaum zusätzliche Arbeitsplätze entstünden. Online-Redaktionen beschäftigten in der Regel nur sehr wenige Mitarbeiter (vgl. Neuberger 2002a, S. 129). Ergebnisse einer Berufsfeldstudie zählten im Frühjahr 2000 lediglich knapp 2000 Online-Journalisten (vgl. Neuberger 2002b, S. 102). Häufig arbeiten die Mitarbeiter nicht exklusiv für den Online-Bereich, sondern sowohl für die Print- als auch für die Online-Ausgabe einer Zeitung. Insofern wirkt das Internet trotz der neuer Tätigkeitsfelder nur begrenzt beschäftigungsfördernd; seine Einflussnahme bezieht sich demnach vornehmlich auf die Veränderung von Tätigkeitsprofilen und Aufgabenzuschnitten.

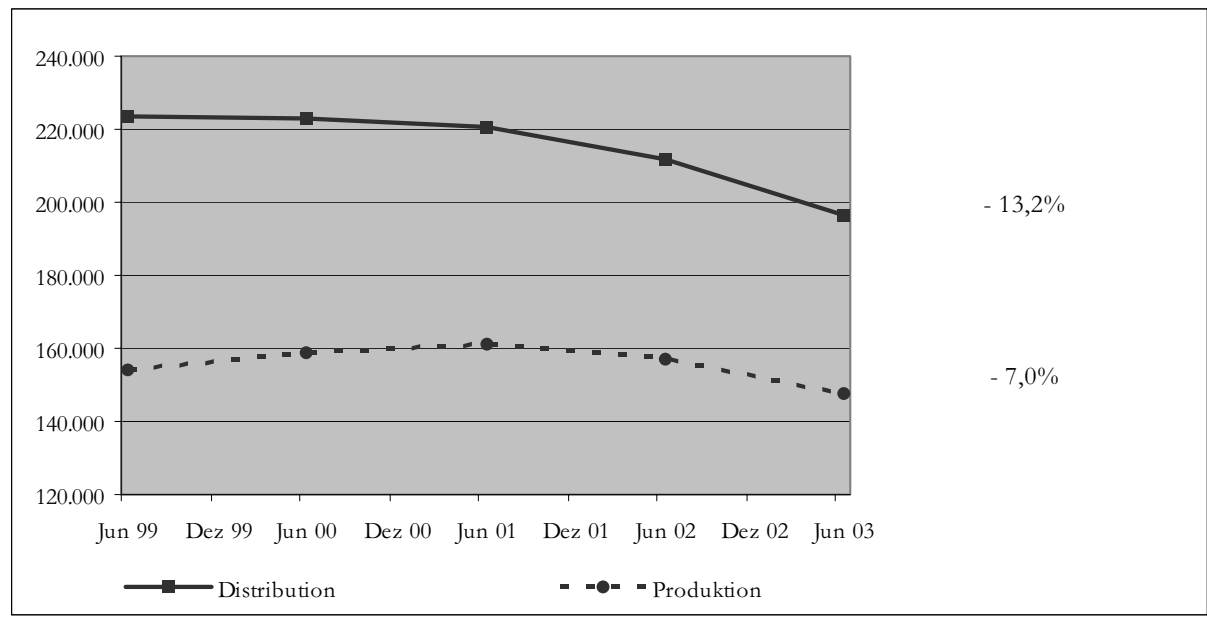

Abbildung 56: Sozialversicherungspflichtig Beschäftigte in den Printmedien Teilbereiche Distribution und Produktion (1999-2003)

Neben den Bereichen Content-Produktion und Distribution gilt es zusätzlich den Bereich der Buchverlage und des Buchdrucks als Teil des Printsektors zu berücksichtigen, weil hier ebenfalls Beschäftigungseffekte durch das Internet zu erwarten sind. In diesem Bereich stellt sich die Situation etwas anders dar als im Zeitungsund Zeitschriftengewerbe; unter dem Einfluss internetbasierter Technologien entstehen z. B. neue Geräte und Formate elektronischer Bücher (vgl. Riehm et al. 2001, S. 111 ff.). Ob diese Entwicklung zu einer Substitution gedruckter Werke und damit zu einer Reduktion von Beschäftigung beim Buchdruck führen könnte, ist eine offene Frage, da sich der tatsächliche wirtschaftliche Erfolg dieser Angebote erst noch erweisen muss. Darüber hinaus besteht ein wichtiger Einfluss des Internets in diesem Bereich in der zunehmenden Bedeutung des Online-Buchhandels. Die Umsätze im klassischen Sortimentsbuchhandel sind zwischen 2002 und 2003 um 2,5\% auf 5,13 Mrd. $€$ zurückgegangen, während sie beim Online-Buchhandel im 
gleichen Zeitraum um 10\% auf ca. 400 Mio. $€$ gestiegen sind (vgl. http://www.boersenverein.de/de/64584). Mit dieser Entwicklung entstehen neue Formen der Direktvermarktung von Büchern, die eine Disintermediation - also eine Ausschaltung von Zwischenhandelsstufen zwischen Verlag und Kunden bewirken (vgl. Riehm et al. 2001, S. 154). ${ }^{289}$

Im Teilbereich der Buchverlage zeigen die Beschäftigtenzahlen allerdings einen deutlichen Negativtrend (ohne Abbildung): Nach einem Anstieg der Beschäftigung auf über 49.000 Mitarbeiter Ende 1999 und einer Phase relativer Stagnation bis Ende 2001 ist es zu einem starken Einbruch der Beschäftigtenzahlen auf 43.480 Mitarbeiter gekommen. Dies entspricht über den gesamten Betrachtungszeitraum einem Beschäftigungsrückgang von 11\%. Hier wäre noch genauer zu prüfen, inwieweit es sich dabei tatsächlich um eine Wirkung des Internets handelt. In diesem Zusammenhang könnte z. B. die Tatsache von Bedeutung sein, dass das Lesen von Büchern als Freizeitaktivität zunehmend durch die Nutzung anderer Medien zurückgedrängt wird bzw. die deutliche Zunahme der täglichen Nutzungsdauer von Medien nur in sehr geringem Umfang dem Medium Buch zu gute kommt. ${ }^{290}$

Obwohl sich unseren Analysen zu Folge im gesamten Printsektor eine z. T. deutliche Abnahme von Beschäftigung verzeichnen lässt, bleibt es schwierig, diese Veränderungen auf die Auswirkungen des Internets zurückzuführen. Als weitere Ursache für den Beschäftigungsrückgang lässt sich die Krise auf dem Werbemarkt identifizieren, die für die Zeitungs- und Zeitschriftenverlage eine z.T. existentielle Bedeutung hat. Das Anzeigengeschäft bei den Tageszeitungen ist zwischen 2000 und 2002 um fast ein Viertel (-1,6 Mrd. €) eingebrochen. Die Werbeeinnahmen sind 2002 auf das Niveau von 1993 gefallen. Als Gründe dafür werden vor allem konjunkturelle Probleme angeführt, die bei der werbetreibenden Wirtschaft zu massiven Kürzungen des Werbebudgets geführt haben. Andererseits wird als mögliche Ursache für den Einbruch des Anzeigengeschäfts auch die Abwanderung von Werbekunden ins Internet diskutiert (vgl. Keller 2003, S. 20 f.). Das Internet ist aufgrund steigender Nutzerzahlen und zielgruppensicherer Angebote für die werbetreibende Wirtschaft längst zu einer ernsthaften Alternative geworden:

289 Hier ist allerdings zu beachten, dass der klassische Buchhandel in den folgenden Beschäftigtenzahlen nicht berücksichtigt ist. Unsere Abgrenzung der Medienbranche schließt den Handel mit Medienprodukten nicht mit ein, weil dieser in unserem Verständnis den Bereichen der Produktion und Distribution nachgelagert ist und auch bezüglich des Einflusses durch das Internet anderen Entwicklungsdynamiken unterliegen dürfte. Insofern sollen die oben genannten Zahlen weniger auf einen Verlust von Beschäftigung im Buchhandel hinweisen, sondern vielmehr auf mögliche Potentiale wachsender Beschäftigung bei den Verlagen durch neue Formen der Direktvermarktung.

290 Zwischen 1990 und 2000 hat die durchschnittliche Mediennutzungsdauer kontinuierlich von 380 auf 502 Minuten pro Tag zugenommen. Dieser Zuwachs bezieht sich vor allem auf das Fernsehen (von 135 auf 185 Minuten pro Tag), den Hörfunk (von 170 auf 206 Minuten pro Tag) und die Tonträgermedien (von 14 auf 36 Minuten pro Tag). Die Nutzungsdauer bei Büchern ist dagegen zwischen 1990 und 2000 mit 18 Minuten pro Tag konstant geblieben (vgl. Media Perspektiven Basisdaten 2004, S. 64). 
Während in den verschiedenen Mediensegmenten (Fernsehen, Rundfunk, Zeitschriften und Zeitungen) die Ausgaben für Werbung in den vergangenen Jahren deutlich zurückgingen, stiegen die Ausgaben für Werbung im Segment Internet auch während der Werbekrise weiter an. ${ }^{291}$ Für den Bereich der Printmedien ist denkbar, dass das Internet auch deshalb zu negativer Beschäftigungswirkung beiträgt, weil die verminderten Werbeeinnahmen zu Einsparungen im redaktionellen Bereich führen und sich folglich in Beschäftigungsrückgängen niederschlagen können, während sich die verbleibenden Beschäftigten in den Redaktionen zugleich veränderten Arbeitsanforderungen gegenüber gestellt sehen.

Schließlich lassen sich als mögliche Ursachen für den Beschäftigungsrückgang im Bereich der Printmedien aber auch Auswirkungen von Konzentrationsprozessen im Verlagsgewerbe und Internationalisierungstendenzen benennen. Die weltweit stark ausgeprägte Konzentration des Medienmarkts lässt sich auf GröBenvorteile (Economies of Scale) und Verbundvorteile (Economies of Scope) bei der Produktion redaktioneller Inhalte, der Werbung und der Distribution zurückführen. In beiden Fällen besteht das Ziel in der Realisierung von Kostenvorteilen, wobei sich Größenvorteile bei der Produktion eines Gutes und Verbundvorteile bei der Produktion mehrerer Güter und den daraus resultierenden Synergieeffekten erzielen lassen (vgl. Sennewald 1998, S. 51 f.). In diesem Sinne verweist Röper (2004) darauf, dass sich im deutschen Zeitungsmarkt gegenwärtig eine beschleunigte Konzentration vollziehe, an der vor allem die Großverlage teilhaben. Solche Konzentrationsprozesse können zu Entlassungen in den betreffenden Unternehmen führen, weil sie in der Regel mit grundlegenden Umstrukturierungsmaßnahmen in Verwaltung und Produktion verbunden sind.

Im Zusammenhang mit den zunehmenden Tendenzen der Internationalisierung in der Medienbranche sind es ebenfalls die Großverlage, die verstärkt auf internationalen Märkten agieren. So lag bei den Großverlagen Gruner + Jahr (Bertelsmann), Springer, Bauer und Burda der Anteil des Auslandsumsatz im Jahr 2002 zusammen bei 37,2\%, was gegenüber dem Jahr 1996 einer Zunahme von fast 10\% entspricht (vgl. Röper 2004, S. 54). Allein der Verlag Gruner + Jahr erwirtschaftete 2003 nach eigenen Angaben 63\% des Gesamtumsatzes im Ausland (vgl. ebd. S. 61). Ein Zusammenhang mit der Beschäftigungsentwicklung ergibt sich dann, wenn international tätige Unternehmen Beschäftigung im Inland durch Beschäftigung im Ausland substituieren bzw. neue Unternehmenseinheiten von vornherein im Ausland aufbauen. Dabei ist allerdings zu beachten, dass diese Möglichkeit für den Bereich der Contentproduktion nicht gleichermaßen gegeben ist wie im Druckbereich. Insofern ist im Falle der Printverlage - im Unterschied z. B. zu den Musikverlagen - nur eine begrenzte Internationalisierung der Produktionsstrukturen zu erwarten, weil es sich hier weitaus stärker um regional- bzw.

291 Ausgabenzuwächse für Werbung im Segment Internet: 1999: $+450 \%$; 2000: $+98,7 \%$; 2001: $+20,9 \%$; 2002: $+22,7 \%$; 2003: $+8,4 \%$; vgl. PricewaterhouseCoopers 2004, S. 7. 
nationalspezifische Inhalte handelt. Prozesse der Konzentration und Internationalisierung werden durch das Internet unterstützt, indem es z. B. die Voraussetzung für eine verbesserte Vernetzung zwischen den räumlich verstreuten Unternehmenseinheiten und Kooperationspartnern schafft.

Die Korresponden₹- und Nacbricbtenbüros und selbständigen Journalisten gehören auch zum Bereich der Content-Produktion, da sie aber Inhalte für unterschiedliche Medien produzieren (sowohl für die Printmedien als auch für den Rundfunk) und sich insofern keinem der „traditionellen“ Mediensektoren exklusiv zuordnen lassen, werden sie hier getrennt aufgeführt.292 Der Entwicklungsverlauf verdeutlicht, dass es zwischen 1999 und 2003 zu dem beträchtlichen Beschäftigungszuwachs von $27 \%$ gekommen ist (vgl. Abb. 57). Den Löwenanteil hieran halten die Korrespondenz- und Nachrichtenbüros, die mehr als $80 \%$ der Beschäftigten dieses Wirtschaftszweigs ausmachen $(+27,6 \%)$, aber auch bei der kleineren Gruppe der selbständigen Journalisten ist es zu einem Beschäftigtenplus von $22 \%$ gekommen.

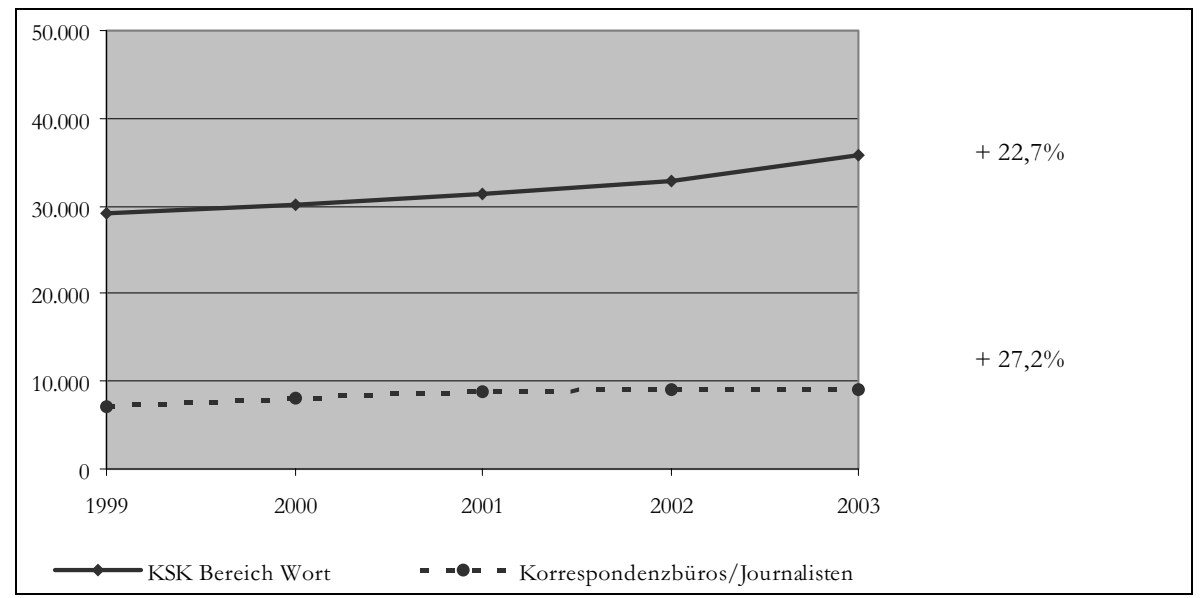

Abbildung 57: Versichertenbestandsentwicklung der KSK zum Bereich Wort und Beschäftigte im Bereich Korrespondenz- und Nachrichtenbüros/selbständige Journalisten (1999-2003)

Es ist offensichtlich, dass die Zahl der Journalisten in Deutschland deutlich höher liegen dürfte als die Daten der Beschäftigtenstatistik angeben, denn insbesondere in diesem Tätigkeitsbereich ist freie Tätigkeit stark verbreitet. Dies belegt die Versichertenbestandsentwicklung der KSK für den Bereich „Wort" ${ }^{\star 293}$ (vgl. Abb. 57);

${ }^{292}$ Auch in der WZ-Klassifikation stellen sie einen eigenen Zweig.

293 Die Berufsgruppen, die die KSK dem Bereich „Wort“ zuordnet, überschreiten zwar den Tätigkeitsbereich Journalismus, gehören aber zur Medienbranche und dienen in diesem Fall weniger als direkte Vergleichszahlen, sondern vielmehr um den Trend zu freier Tätigkeit für die Medien- 
auch wenn die Angaben der KSK nicht der tatsächlichen Beschäftigtenentwicklung der Freien und Selbständigen entsprechen, lassen sie doch Rückschlüsse in Bezug auf generelle Entwicklungstrends dieses Beschäftigungsbereichs zu. Im Bereich „Wort“ ist es zwischen 1999 und 2003 zu Zuwächsen von fast $23 \%$ gekommen. Die Versichertenbestandsentwicklung der KSK belegt damit, dass die Angaben der Beschäftigtenstatistik - insbesondere für bestimmte Tätigkeitsbereiche der Medienbranche wie dem Journalismus - die Zahl der hier Tätigen nicht annähernd erfassen. Die Vernachlässigung freier Journalisten und Redakteure würde demnach zu groben Fehleinschätzungen der Beschäftigtenentwicklung führen. Darüber hinaus - und für unseren Untersuchungskontext zentraler - verdeutlichen diese Daten den sich über die vergangenen Jahre weiter verstärkenden Trend hin zu freier Tätigkeit (in den Mediensektoren Print und Rundfunk, denn hier lässt sich der Bereich „Wort“ überwiegend ansiedeln).

Die Relevanz des Internets für die Entwicklung bei den Beschäftigten der Korrespondenz- und Nachrichtenbüros und selbstständigen Journalisten isoliert zu betrachten, fällt schwer, weil der Bereich stark von der Entwicklung in den Bereichen Print und Rundfunk abhängig ist, denn beide Bereiche sind „Zulieferer" des Content dieser Sektoren. Grundsätzlich kann man davon ausgehen, dass sich durch den zunehmenden Einsatz von Internet und Computertechnologie v. a. die journalistischen Tätigkeitsbereiche, d. h. die Art und Weise wie Inhalte aufgearbeitet werden, verändern. Dies beträfe demnach eher Veränderungen der Arbeit, deren Einfluss auf die Beschäftigtenzahlen schwer feststellbar ist. Das Potential des Internets ein neues und zusätzliches Arbeitsfeld für Journalisten ist zumindest umstritten (s.o., vgl. Neuberger 2002a).

Auf die Beschäftigungsentwicklung im Bereich der Printmedien wirkt neben dem Internet offenbar eine Reihe weiterer Faktoren (Werbekrise, Konzentrationsprozesse, Internationalisierung, Umstrukturierung von Beschäftigungsverhältnissen) ein. Um den Stellenwert des Interneteinflusses genauer gewichten zu können, ziehen wir zum Vergleich den Rundfunksektor heran. Auch in diesem Bereich eröffnen sich evtl. neue Möglichkeiten der Content-Produktion und Distribution; auch hier dürfte die Krise am Werbemarkt relevant sein. Der Bereich Rundfunk beinhaltet die Wirtschaftszweige Rundfunk- und Fernsehanstalten sowie die Herstellung von Rundfunk- und Fernsehprogrammen (vgl. Abb. 58). ${ }^{294}$ Im Unterschied zum Printsektor haben wir es in diesem Bereich mit einem relativ stabilen Verlauf der Beschäftigtenzahlen zu tun. Bis Mitte 2002 nahmen die Beschäftigtenzahlen zu. Seitdem sind sie rückläufig und befanden sich Ende 2003 auf dem Niveau des Jahres 2000.

teilsektoren Print und Rundfunk zu verdeutlichen. Da die Versichertenbestandsentwicklung der KSK einen Messpunkt pro Jahr ausweist, haben wir die Daten der Beschäftigtenstatistik angeglichen und ebenfalls einen Messpunkt ausgewählt (jeweils 30.06.).

${ }^{294}$ Die Systematik der WZ unterscheidet nicht zwischen Hörfunk- und Fernsehveranstaltern. 
Allerdings sind Beschäftigungsverhältnisse wie freie Mitarbeiterschaft beim Rundfunk besonders stark verbreitet und müssen entsprechend bei der Frage nach der Beschäftigungsentwicklung berücksichtigt werden. ${ }^{295}$ Abbildung 58 zeigt die Verteilung der verschiedenen Beschäftigtengruppen beim privaten und öffentlichrechtlichen Hörfunk und Fernsehen für das Jahr 2002.

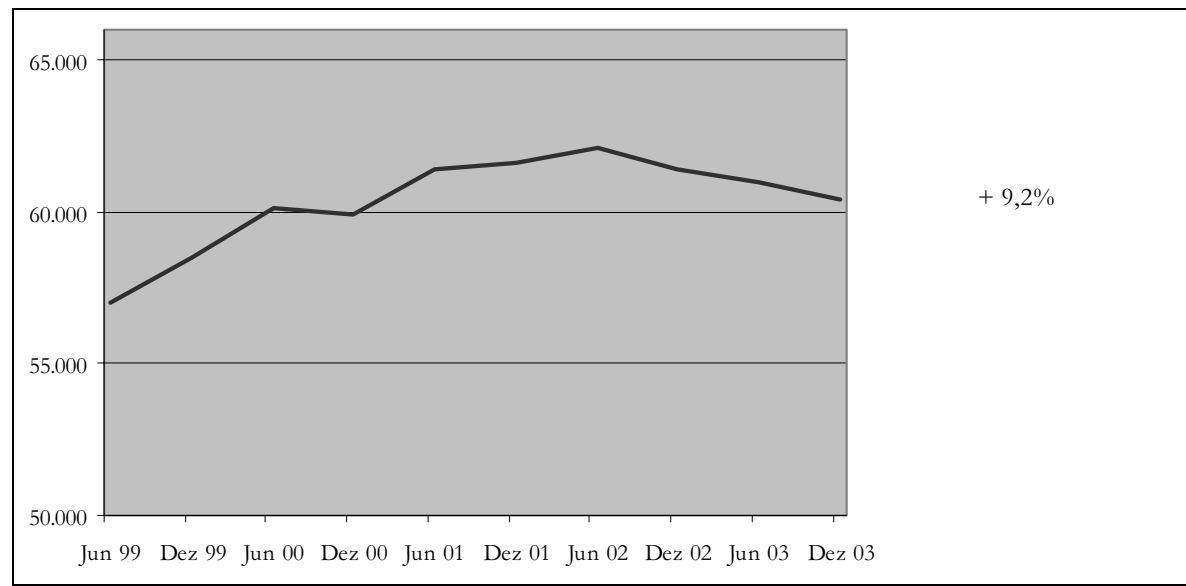

Abbildung 58: Sozialversicherungspflichtig Beschäftigte im Bereich Rundfunk (1999-2003)

\begin{tabular}{|l|l|l|l|l|l||l|}
\hline \multicolumn{2}{|c|}{} & $\begin{array}{l}\text { Feste Mitar- } \\
\text { beiter }\end{array}$ & Feste Freie & $\begin{array}{l}\text { Sonst. } \\
\text { Freie }\end{array}$ & Praktikanten & Summe \\
\hline \multirow{3}{*}{ Privat } & Hörfunk & $4.459(9,7 \%)$ & $\begin{array}{l}1.199 \\
(7,5 \%)\end{array}$ & $\begin{array}{l}607 \\
(8 \%)\end{array}$ & $\begin{array}{l}518 \\
(10,2 \%)\end{array}$ & $\begin{array}{l}6.783 \\
(9,1 \%)\end{array}$ \\
\cline { 2 - 7 } & Fernsehen & $11.982(26 \%)$ & $\begin{array}{l}3.614 \\
(22,7 \%)\end{array}$ & $\begin{array}{l}389 \\
(5,1 \%)\end{array}$ & $\begin{array}{l}539 \\
(10.6 \%)\end{array}$ & $16.524(22,1 \%)$ \\
\hline $\begin{array}{l}\text { Öffentlich- } \\
\text { rechtlich }\end{array}$ & Hörfunk & 12.993 & $\begin{array}{l}3.449 \\
(21,6 \%)\end{array}$ & $\begin{array}{l}2.107 \\
(27,8 \%)\end{array}$ & $\begin{array}{l}1.247 \\
(24,6 \%)\end{array}$ & $19.796(26,5 \%)$ \\
\cline { 2 - 7 } & Fernsehen & $\begin{array}{l}(28,2 \%) \\
(36,65 \%)\end{array}$ & $\begin{array}{l}7.676 \\
(48,2 \%)\end{array}$ & $\begin{array}{l}4.477 \\
(59 \%)\end{array}$ & $\begin{array}{l}2.774 \\
(54,6 \%)\end{array}$ & $31.582(42,3 \%)$ \\
\hline \hline Summe & & $46.089(100 \%)$ & $\begin{array}{l}15.938 \\
(100 \%)\end{array}$ & $\begin{array}{l}7.580 \\
(100 \%)\end{array}$ & $\begin{array}{l}5.078 \\
(100 \%)\end{array}$ & $74.685(100 \%)$ \\
\hline
\end{tabular}

Abbildung 59: Beschäftigte im privaten und öffentlich-rechtlichen Rundfunk nach Statusgruppen für das Jahr $2002^{296}$

2002 waren 34\% der Beschäftigten beim privaten Hörfunk ohne festes Arbeitsverhältnis (d. h. „Feste Freie“, „,sonstige Freie“ oder „Praktikanten“), bei den privaten TV-Anbietern waren es $28 \%$. Damit ergibt sich für den privaten Rundfunk

\footnotetext{
295 Sonstige Vergleichszahlen aus der KSK-Versichertenbestandsentwicklung liegen uns für den Bereich Rundfunk nicht vor.

296 Quelle: vgl. Landesmedienanstalten 2004, S. 62/91; eigene Berechnungen.
} 
insgesamt ein Anteil von 29\% ohne festes Arbeitsverhältnis. Die absolute Zahl dieser Beschäftigtengruppe ist zwar seit 1998 kontinuierlich gesunken, während die Zahl der regulär Erwerbstätigen bis Ende 2001 kontinuierlich zugenommen hat. Seit 2001 ist aber auch bei den festen Mitarbeitern ein Rückgang um etwa 5\% zu verzeichnen, wodurch sich eine prozentuale Verschiebung zugunsten der verschiedenen Formen freier Beschäftigung ergibt (vgl. Landesmedienanstalten 2004, S. 61). Noch deutlicher stellt sich das Verhältnis beim öffentlich-rechtlichen Rundfunk dar. Im Bereich des Hörfunks waren 34\% ohne festes Arbeitsverhältnis, beim Fernsehen waren es sogar 47\%. Im Vergleich zu 2001 hat der Anteil der Beschäftigten ohne festes Arbeitsverhältnis um etwa 4\% zugenommen (vgl. Landesmedienanstalten 2004, S. 91).297 Auf den gesamten Rundfunksektor bezogen ergibt sich somit ein Anteil von 38\% der Beschäftigten, die nicht sozialversicherungspflichtig beschäftigt sind. Gegenwärtig wird eine weitere Zunahme von „freien Freien“ in der Rundfunkproduktion prognostiziert. Diese Gruppe umfasst jene Beschäftigte, die lediglich ein Honorar erhalten, nicht aber auf bestimmte Arbeitszeitregelungen, ein Mindesteinkommen sowie tarifvertragliche Ansprüche zurückgreifen können und sich folglich in einem besonders prekären Beschäftigungsstatus befinden (vgl. Marrs / Boes 2003, S. 194 f.).

Im Vergleich zum Printbereich schlägt sich die Werbekrise hier weniger stark nieder: Während insbesondere die Zeitungen (wegen ihrer Abhängigkeit vom Anzeigenmarkt) und Zeitschriften am schwersten von der Krise betroffen sind, haben Fernsehen und Radio zwar auch Rückgänge der Werbeausgaben hinzunehmen, jedoch in geringerem Ausmaß. ${ }^{298} \mathrm{Im}$ Bereich des Rundfunks ist insbesondere der privat-rechtliche Sektor erheblich werbefinanziert, bei den öffentlichrechtlichen Anstalten sind dagegen die Werbeanteile stark limitiert und werden von Seiten des Rundfunkrats kontrolliert. Die Werbekrise hat hier - im Unterschied zur Entwicklung bei zahlreichen großen, überregionalen Tageszeitungen nicht zu der Tendenz geführt, den Content zu reduzieren. Stattdessen sind neue Inhalteformate (z. B. Teleshopping) und Strategien der Mehrfachverwertung entstanden, bei denen zunehmend auch das Internet als ergänzendes Distributionsmedium einbezogen wird (vgl. Niggemeier 2001, vgl. Langer 2001).

Neue Chancen für Beschäftigung könnten in der Digitalisierung des Fernsehens liegen. Bis 2010 soll die analoge TV-Übertragung bundesweit vollständig durch die digitale Übertragung abgelöst werden. Beschäftigungspotentiale sind im Zusammenhang der Beseitigung von Kapazitätsengpässen und der daraus re-

297 Entsprechend machen die Personalkosten und Aufwendungen für Honorare für freie Mitarbeiter nur 13\% aller Aufwendungen im privaten Rundfunk aus, aber 38\% aller Aufwendungen im öffentlich-rechtlichen Rundfunk (vgl. Landesmedienanstalten 2004, S. 30 f.).

298 Ausgaben für Werbung 1999-2003 (Veränderung in \%): Fernsehen: -4,4\%; Hörfunk: -2,1\%; Zeitschriften: $-8,4 \%$; Zeitungen: $-23,9 \%$; vgl. PricewaterhouseCoopers 2004, S. 7 (eigene Berechnungen). 
sultierenden Ausweitung des Programmangebots denkbar sowie durch neue Möglichkeiten der Mehrfachverwertung auf Basis der Verknüpfung mit anderen digitalen Medien (vgl. ALM 2003, S. 275 f.). Allerdings verläuft die Verbreitung der verschiedenen Formen des digitalen Fernsehens bislang nur zögerlich und nicht flächendeckend. Seit dem Verkauf der Kabelnetze durch die Deutsche Telekom AG im Jahr 2000 hat es kaum Investitionen in die Erweiterung der Bandbreite und Rückkanalfähigkeit der Netze gegeben (vgl. ebd. S. 250 f.); zudem verfügten im Jahr 2003 nur 8,8\% der deutschen Haushalte über digitale Satellitenreceiver, was im europäischen Vergleich unterdurchschnittlich ist (vgl. ebd. S. 256). Beim interaktiven digitalen Fernsehen (iDTV) stellten bislang die uneinheitlichen DecoderStandards und hohen Preise für digitale Empfangsgeräte eine wichtige Ursache für die Zurückhaltung der Kunden dar (vgl. ALM 2003, S. 277 f.). Andererseits zeichnen sich bei der digitalen terrestrischen Übertragung (DVB-T) Erfolge ab - so z. B. in Berlin, wo bereits im August 2003 die analoge terrestrische Verbreitung komplett abgeschaltet wurde, was den Verkauf von Empfangsgeräten enorm ansteigen ließ und damit ein Sinken der Gerätepreise zur Folge hatte (vgl. ebd. S. 255 f.). Zudem wird dem Fernsehempfang via Internet durch die rasche Ausbreitung schneller Internetverbindungen ein hohes Entwicklungspotential zugesprochen (vgl. ebd. S. 258). Bei all diesen Entwicklungen sollte jedoch berücksichtigt werden, dass durch die Deregulierung des Fernsehmarktes eine enorme Konkurrenz um Zuschauermarktanteile und Werbeeinnahmen ausgelöst worden ist, die zu einem Verdrängungswettbewerb auf dem TV-Markt führt (vgl. Marrs / Boes 2003, S. 191).

\subsection{Die Werbebranche: Wichtiger Content-Produzent und -Finanzier}

Eine wechselseitige Abhängigkeit besteht - wie bereits angedeutet - zwischen der Entwicklung der Teilsektoren Rundfunk und Print auf der einen und der Entwicklung im Bereich der Werbung auf der anderen Seite. Die Werbebranche beinhaltet die Wirtschaftszweige Werbegestaltung, Werbemittelverbreitung sowie Werbevermittlung und ist neben dem Printsektor der zweite große Bestimmungsfaktor für die Beschäftigung in der Medienbranche. Zwischen ihr und den oben genannten Teilsektoren besteht insofern ein enger Zusammenhang, als die rückläufigen Ausgaben der werbetreibenden Wirtschaft zu einem rückläufigen Anzeigenaufkommen (bzw. einer Verbilligung der Preise für Werbung) und infolgedessen auch zu rückläufigen Umsätzen in der Werbebranche führen. Dies schlägt sich in der Beschäftigtenentwicklung der Werbebranche nieder (vgl. Abb. 60). Zwischen 1999 und 2001 haben die Beschäftigtenzahlen in der Werbebranche zunächst stark zugenommen (um 30\%) und erreichten Ende 2001 ihren Höhepunkt. Seitdem ist die Beschäftigtenentwicklung rückläufig - allein innerhalb der letzten zwei Jahre um mehr als -14\%. Dabei bestehen klare Parallelen 
zwischen der Entwicklung der Beschäftigung und der Entwicklung der Ausgaben für Werbung: So profitierte die Branche für die Jahre 1999 (+6,2\%) und 2000 $(+9,1 \%)$ zwar von satten Steigerungen der Werbeausgaben, seit 2001 ist es jedoch zu einem Abwärtstrend gekommen, der sich über die Jahre 2002 (-10,4\%) und $2003(-6,1 \%)$ fortsetzte. ${ }^{299}$ Im Jahre 2004 (+0,6\%) befand sich die Branche wieder in leichtem Aufwind (vgl. PricewaterhouseCoopers 2004, S. 7). Den Befunden der Deutschen Public Relations Gesellschaft zufolge erwartet die Werbebranche eine 20\%ige Auftragssteigerung für das Jahr 2005. Zwar sollen damit auch steigende Beschäftigtenzahlen einhergehen; allerdings bezieht sich die Aufstockung der Personalbestände vorwiegend auf freie Mitarbeiter, Volontäre und Praktikanten. Einstellungen fester Mitarbeiter sollen nur vereinzelt erfolgen (vgl. Pressemeldung http://www.mediafon.net/aktuelles.php3\#41eba55fcc3af).

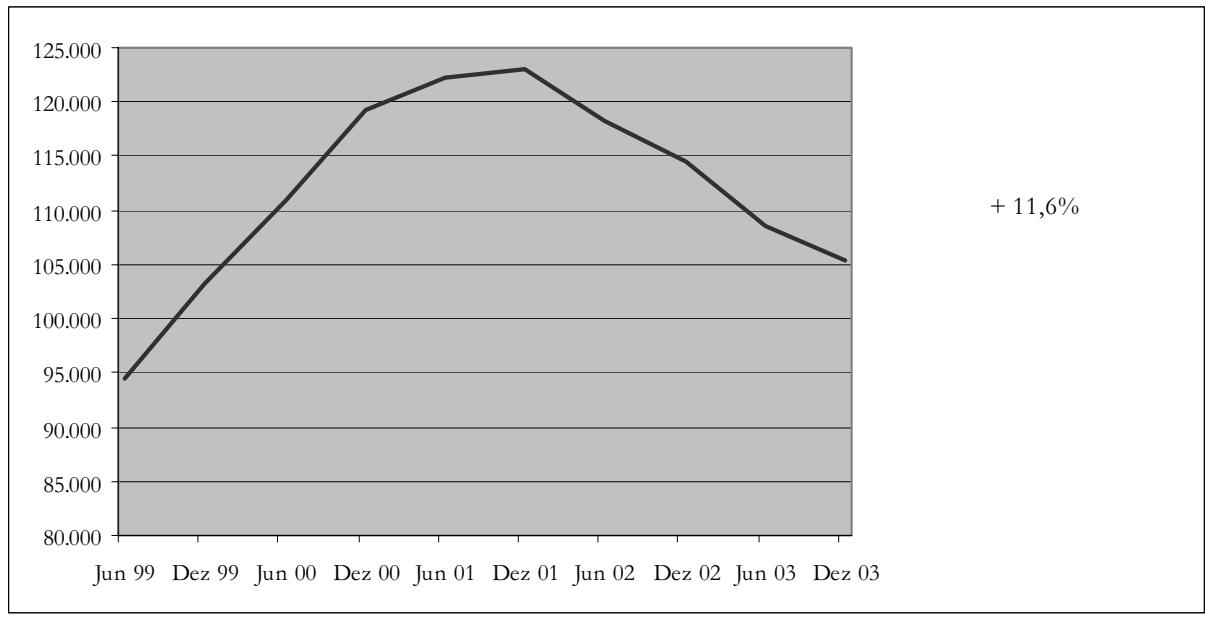

\section{Abbildung 60: Sozialversicherungspflichtig Beschäftigte im Bereich Werbung (1999-2003)}

Fragt man nach der Rolle des Internets für die Beschäftigtenentwicklung, sind folgende Faktoren für die Werbebranche relevant: Zwar ist die Werbebranche unmittelbar von den reduzierten Ausgaben der werbetreibenden Wirtschaft betroffen, zudem ist davon auszugehen, dass internetbasierte Technologien - ähnlich wie in anderen Bereichen der Medienbranche - zu Rationalisierungsprozessen und neuen Arbeitsanforderungen im Bereich der Contentproduktion führen. Die Abwanderung von Werbekunden ins Internet dürfte für die Beschäftigungsentwicklung der

${ }^{299}$ Dies dürfte auch auf die Krise der New Economy zurückzuführen sein. Viele der betroffenen Unternehmen haben ihre Werbeausgaben im Zeichen der Krise drastisch reduziert oder sogar vollständig zurückgefahren (vgl. DIE WELT vom 08.02.2001: „Krise der New Economy kostet Werbefirmen die Existenz"). 
Werbebranche gleichwohl kaum ins Gewicht fallen, weil der Bedarf an Werbevermittlung und -gestaltung unabhängig vom jeweiligen Distributionsmedium weiterhin gegeben bleibt. Insofern dürfte die Wirkung des Internets auf die Beschäftigung im Bereich der Werbung vor allem vermittelt über dessen Wirkung in den anderen Teilsektoren der Medienbranche verlaufen.

\subsection{Film- und Musikbranche: Beschäftigungsrückgänge in Folge illegaler Downloads?}

Relevant für die Entwicklung der Werbebranche ist auch der Filmsektor, zu dem sowohl die Wirtschaftszweige Vervielfältigung von bespielten Bildträgern, Filmund Videofilmherstellung und der Verleih von Filmen als auch die Kinos gehören. Wie auch in der TV-Produktion sind Beschäftigungsverhältnisse auf Produktionsdauer für den Bereich der Filmproduktion charakteristisch. Oftmals treffen sich innerhalb der zeitlich befristeten Projektteams jeweils dieselben Mitarbeiter wieder (vgl. Sydow/Windeler 1999). Die wellenförmige Beschäftigungsentwicklung des Filmsektors (vgl. Abb. 61) lässt sich vermutlich darauf zurückführen, dass es sich bei Filmproduktionen oftmals um „Saisonarbeiten“ handelt, am Ende einer Produktion werden die Beschäftigten dann wieder entlassen. Insgesamt zeigt sich, dass es bis ins Jahr 2002 Beschäftigungsanstiege in der Filmbranche gegeben hat, seitdem ist die Entwicklung rückläufig. Über den gesamten Betrachtungszeitraum verzeichnet die Filmbranche aber einen Beschäftigungszuwachs von mehr als $9 \%$.

Ob die jüngsten Beschäftigungsverluste mit der illegalen Verbreitung von Filmen über das Internet in Zusammenhang stehen, lässt sich nicht eindeutig klären. Zwar klagt die Filmindustrie über starke Gewinneinbußen aufgrund illegaler Raubkopien, jüngste Untersuchungen verweisen allerdings darauf, dass die Möglichkeit illegaler Downloads nur bedingt zu einem veränderten Verhalten der Konsumenten führt. Im Rahmen einer Befragung gaben 46,6\% an, dass die Möglichkeit des Brennens und Downloadens von Filmen keinen Einfluss darauf ausübe, auch weiterhin ins Kino zu gehen, 7,1\% gingen sogar häufiger ins Kino (vgl. GfK 2004). Dennoch lässt sich ein Rückgang der Zuschauerzahlen bei den Kinos beobachten, der aber auch auf das Fehlen erfolgreicher deutscher Filmproduktionen und die allgemein schlechte konjunkturelle Lage zurückgeführt wird (vgl. PricewaterhouseCoopers 2004, S. 11 f.). 


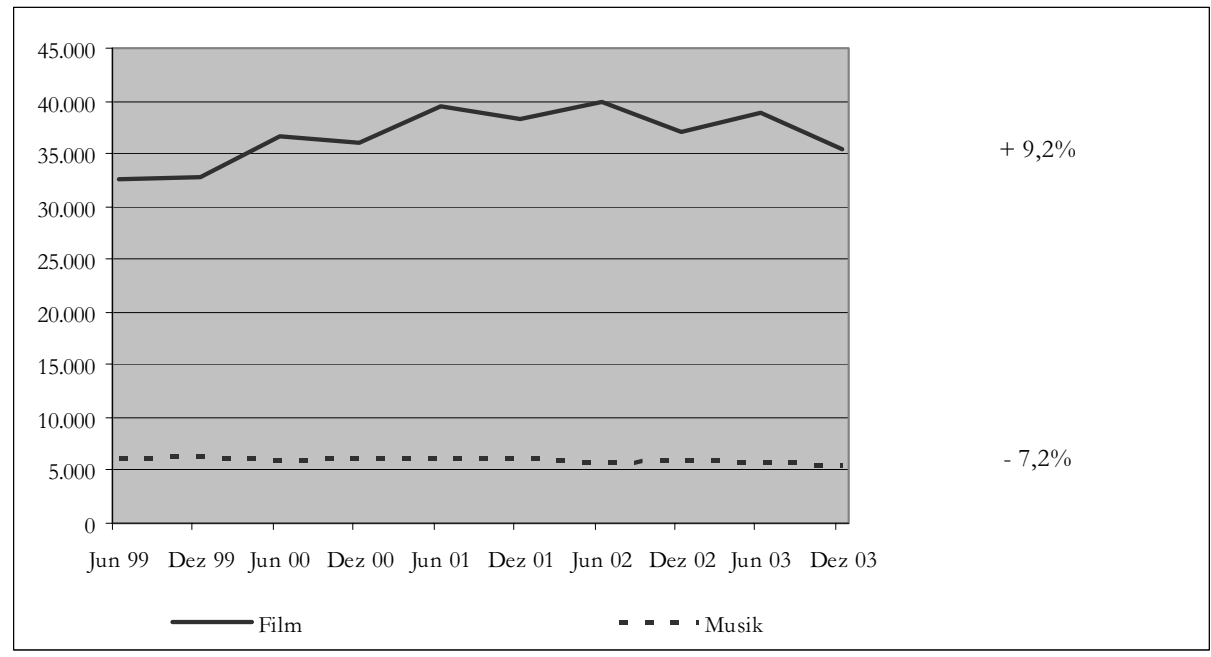

Abbildung 61: Sozialversicherungspflichtig Beschäftigte in den Bereichen Film und Musik (1999-2003)

Darüber hinaus können im Filmsektor Beschäftigungswirkungen aufgrund des Übergangs zum digitalen Kino erwartet werden: Bestimmte Tätigkeitsbereiche entfallen gänzlich, während daneben neue entstehen. Z. B. dürfte die Arbeit des klassischen Filmvorführers mit dem digitalen Kino obsolet werden, während zugleich neue Intermediäre entstehen, die eine verlustfreie Übertragung der Bildund Tondateien zwischen den einzelnen Stufen der Filmproduktion sowie zwischen dem Play-Out-Service-Center und dem Kinobetrieb gewährleisten (vgl. von Staden / Hundsdörfer 2003, S. 15f.).

Zum Bereich Musik wurden die Wirtschaftszweige Musikverlage, Musikalienverlage (Noten, Bühnenstücke u.ä.) sowie die Vervielfältigung bespielter Tonträger zusammengezogen. Hierbei handelt es sich - nach unserer Abgrenzung - um den Bereich der Medienbranche mit dem quantitativ betrachtet geringsten Beschäftigungsanteil (vgl. ebenfalls Abb. 61). ${ }^{300}$ Die deutsche Musikwirtschaft erwirtschaftet erhebliche Anteile im Ausland. Die Bertelsmann Music Group (BMG), ein bedeutender Player in der Musikbranche, erzielte z. B. im Jahre 2002 2.462 Mio. $€$ seines Umsatzes auf dem internationalen und nur 252 Mio. $€$ auf dem deutschen Markt (vgl. Bertelsmann Geschäftsbericht 2002). ${ }^{301}$ Entsprechend dieser internati-

300 Dieser Bereich entspricht nicht der Musikwirtschaft, zu der z. B. auch der gesamte Einzelhandel mit Tonträgern und einschlägiger Unterhaltungselektronik gehört. Im Sinne der oben dargestellten Branchenabgrenzung beschränken wir uns vor allem auf die Contentproduktion und distribution.

301 Im Jahre 2004 ist die Bertelsmann Music Group mit SONY Music fusioniert, wodurch nach dem Branchenführer UNIVERSAL der zweitgrößte Musikkonzern der Welt entstanden ist. 
onalen Ausrichtung der Musikbranche wird auch ein großer Teil der Mitarbeiter im Ausland beschäftigt.

Die Bilanzierung von $-7,2 \%$ über den Betrachtungszeitraum hinweg fällt mit Blick auf die Wehklagen der Musikindustrie noch moderat aus: Diese beklagt ähnlich wie die Filmindustrie - Umsatzeinbußen aufgrund von Raubkopien, illegalen Musikdownloads und Tauschbörsen im Internet. Nach Angaben des Deutschen Phonoverbands sank der Umsatz der deutschen Phonowirtschaft im Jahr 2003 aus diesem Grund um 20\% (vgl. http://www.idw-online.de/pages/de/ news96537). Allerdings schlägt sich dieser Umstand zumindest bis Ende 2003 in beschränktem Maße auf die Beschäftigungsentwicklung in der deutschen Musikbranche nieder. Mittlerweile haben die Unternehmen der Musikindustrie zahlreiche legale und kostenpflichtige Downloadmöglichkeiten im Internet geschaffen, die sich offenbar einer immer stärkeren Akzeptanz von Seiten der Konsumenten erfreuen. ${ }^{302}$ In diesem Sinne scheint sich der zunächst zögerliche Versuch der Branche, das Internet strategisch in die Distribution ihrer Produkte einzubeziehen, inzwischen in Richtung ökonomisch tragfähiger Modelle zu entwickeln. Wie sich dies auf die Entwicklung der Beschäftigung in der Musikbranche in Deutschland auswirken wird, lässt sich zu diesem Zeitpunkt nicht abschließend klären und bedarf genauerer Untersuchungen.

\subsection{Zwischenfazit}

Die Medienteilsektoren des engen Branchenbegriffs verzeichnen zum Teil erhebliche Effekte in der Beschäftigungsentwicklung. Insbesondere der Bereich der Printmedien weist vergleichsweise starke negative Beschäftigungseffekte auf. In anderen Bereichen ist die Beschäftigungsdynamik weniger stark ausgeprägt: Beim Film und im Rundfunk-Bereich stellten wir insgesamt betrachtet positive Entwicklungen bzw. geringe Entwicklungsdynamiken fest. Zur Werbung fällt die Gesamtbilanz der Beschäftigtenentwicklung über den Betrachtungszeitraum hinweg deutlich positiv aus, auch wenn seit 2001 Rückgänge zu verzeichnen sind. Der Bereich Musik verzeichnet eine negative Entwicklung. Insofern hat die Disaggregation der Medienteilsektoren gezeigt, dass wir es tatsächlich mit teilweise sehr unterschiedlichen Entwicklungsdynamiken zu tun haben. Auch über das quantitative Gewicht der einzelnen Sektoren haben wir Aufschluss erhalten. Hier hat sich u. a. gezeigt, dass der Bereich der Printmedien den Gesamtkurvenverlauf wesentlich dominiert.

Die Gründe für die Entwicklung der vergangenen Jahre lassen sich nicht ohne weiteres an den Beschäftigungsdaten ablesen und auch die diesbezügliche Relevanz des Internet konnte nicht eindeutig benannt werden. Allerdings legen unsere Analysen nahe, dass der Einfluss des Internet auf die Beschäftigungsentwicklung

302 Vgl. dazu die aktuelle Berichterstattung bei www.heise.de. 
überwiegend indirekt erfolgt, d. h. vermittelt über Prozesse wie die Internationalisierung von Märkten, Konzentrationsprozesse oder die Werbekrise.

\subsection{Beschäftigungsentwicklung in den Bereichen des erweiterten Branchenbegriffs}

Dass sich mit dem Internet klassische Distributionswege gewandelt haben bzw. an Bedeutung einbüßen, haben die Ausführungen zu den Bereichen Print und Musik verdeutlicht. Diejenigen Wirtschaftszweige, die die technische Infrastruktur bereitstellen, haben wir ebenfalls, wenn auch mit den genannten Problemen, der Medienbranche zugeordnet. Sie bilden neben den „traditionellen“ Mediensektoren den erweiterten Branchenbegriff: Softwareberatung und -entwicklung, Datenverarbeitung und Fernmeldedienste. Hier existiert die Schwierigkeit, dass die drei genannten Bereiche auch für andere Branchen aktiv sind, weshalb unklar ist, welche Beschäftigtenanteile exklusiv der Medienbranche zugerechnet werden können (vgl. vorne). Für diesen ersten, grundsätzlichen Zugriff betrachten wir alle Beschäftigten dieser Wirtschaftszweige, ohne eine Gewichtung hinsichtlich des tatsächlichen Beschäftigtenanteils vorzunehmen (vgl. Abb. 62).

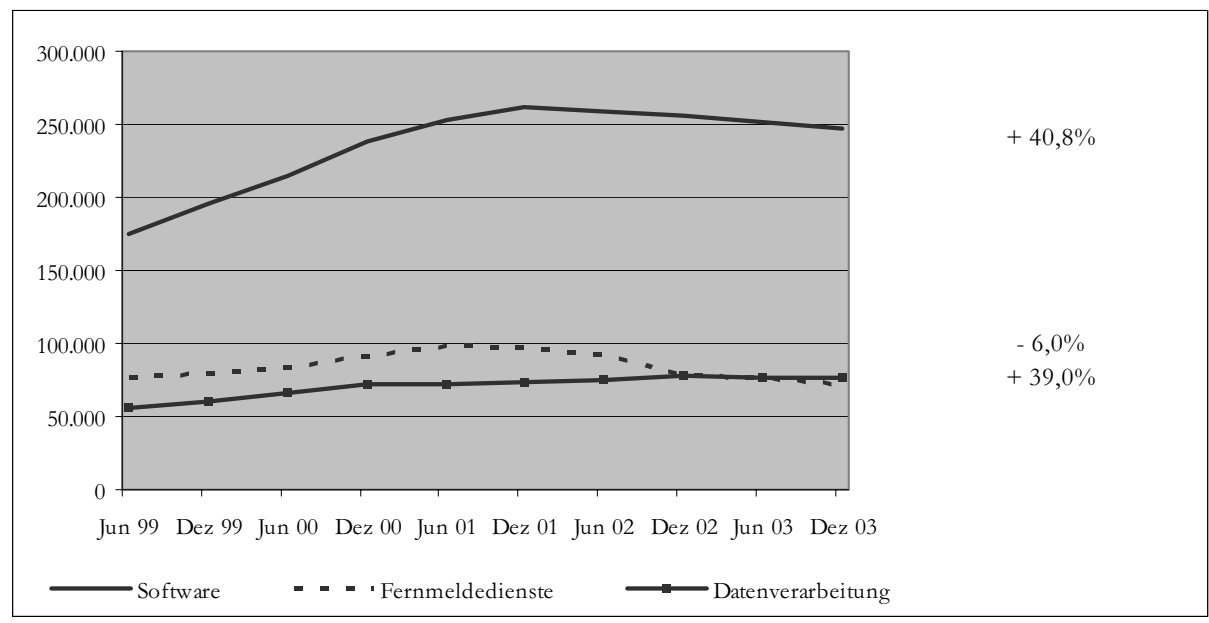

Abbildung 62: Sozialversicherungspflichtig Beschäftigte in den Bereichen Software, Fernmeldedienste und Datenverarbeitung (1999-2003) (erweiterte Branchendefinition)

Der Bereich Software gehörte zu Zeiten des New Economy-Booms zu den Wachstumsmotoren der Branche schlechthin. Dies zeigt der enorme Anstieg der Beschäftigtenzahlen zwischen 1999 und 2001 um 49,3\%. Das beispiellose Wachstum dürfte v. a. mit der Entstehung zahlreicher „Start-Ups“, Neugründungen junger Unternehmen im Softwarebereich, und dem Erfolg deutscher Software (z. B. füh- 
rend: SAP) in engem Zusammenhang stehen. Dass die Zahl der Beschäftigten in diesem Bereich seit Ende 2001 langsam sinkt, dürfte auch auf das Ende des Booms zurückzuführen sein, der bei einer Vielzahl der Unternehmen mit massiven Kürzungen im personellen Bereich bzw. ganzen Unternehmensschließungen einherging. Da jedoch der Rückgang an Beschäftigung seit Ende 2001 relativ moderat ausfällt, scheint in Bezug auf die Softwarebranche in den letzten Jahren eher eine Konsolidierung als ein dramatischer Einbruch stattgefunden zu haben.

Bei den Fernmeldediensten hat es seit Ende 2001 Einschnitte in der Beschäftigtenentwicklung gegeben, und der Rückgang hält seitdem an. Eine differenzierte Betrachtung der Entwicklung macht dabei deutlich, dass für diese Einschnitte insbesondere die Entwicklung der Beschäftigten beim Betrieb von leitungsgebundenen Fernmeldenetzen verantwortlich ist, was nicht zuletzt auf eine fortschreitende Substitution von Festnetz- durch Mobilkommunikation zurückzuführen sein dürfte. Offensichtlich hat dies jedoch nicht zu einem einfachen Transfer von Beschäftigung von einem Segment in das andere geführt, weil es auf den gesamten Bereich bezogen zu Rückgängen gekommen ist. Als weitere Ursache für die Beschäftigungsentwicklung in diesem Bereich kommt die allgemeine Tendenz zum Outsourcing bestimmter Tätigkeitsbereiche in Frage; nach der Auslagerung geringqualifizierter Arbeitsplätze, trifft es mittlerweile selbst die Kernkompetenzen und hochqualifizierte Dienstleistungsarbeiten (vgl. Boes 2004). Zu den umfangreichen Restrukturierungsmaßnahmen der Branche zählt auch die Privatisierung der Telekom vor 10 Jahren (am 01.01.1995). Seit der Umwandlung des Postbetriebs in eine Aktiengesellschaft wurde jeder zweite Mitarbeiter entlassen - bislang waren es etwa 112.000 (Die Zeit, 03.03.2005).

Im Bereich Datenverarbeitung ist es in den vergangenen Jahren zu beträchtlichen Beschäftigungszuwächsen gekommen ( $+39 \%)$, die interessanterweise weiter anhalten bzw. auf hohem Niveau verharren. Beide Sektoren - sowohl der Softwarebereich als auch die Datenverarbeitung - haben seit Beginn der Entwicklung der Informations- und Kommunikationstechnologien eine zentrale Rolle gespielt. Der deutlich kleinere Bereich der Datenverarbeitung scheint jedoch eher die Position des stillen Nutznießers an der zunehmenden Verbreitung digitaler Technologien auszufüllen: Zwar hat er in der Euphorie des New Economy-Booms nur wenig Aufmerksamkeit gefunden. Tatsächlich ist aber davon auszugehen, dass der Bedarf nach Datenverarbeitung mit einer erhöhten Verbreitung und Komplexität der Informationssysteme auch zukünftig weiter zunehmen wird.

\section{Zusammenfassung und Ausblick}

Der Blick auf die Beschäftigtenentwicklung in der Medienbranche ermöglichte es, in einem ersten Schritt sowohl allgemeine Entwicklungstrends der Branche und 
ihrer verschiedenen Teilsektoren aufzuzeigen als auch jene Sektoren zu identifizieren, in denen besonders hohe Veränderungsdynamiken zu verzeichnen sind. Durch dieses Vorgehen konnte verdeutlicht werden, dass für die gesamte Branche ein dachförmiger Verlauf in der Beschäftigtenentwicklung charakteristisch ist: Nach Anstiegen in den Jahren 1999/2000 erreichen die Beschäftigtenzahlen im Jahr 2001 ihren Höhe- und gleichzeitigen Wendepunkt, mit Ende des Jahres sinken die Beschäftigtenzahlen.

In den Einzelsektoren sind ähnliche Entwicklungsverläufe feststellbar, die Beschäftigtenzahlen gehen seit 2001 (mehr oder weniger) stark zurück. Diese Entwicklung bezieht sich jedoch lediglich auf die Abnahme sozialversicherungspflichtiger Beschäftigung. Die Versichertenbestandsentwicklung der KSK hingegen verweist auf die in den vergangenen Jahren stark zunehmende Zahl von Versicherten in freien Berufen, vor allem in den Bereichen „Wort“ und „Musik“, was als Indiz dafür gewertet werden kann, dass in bestimmten Bereichen der Medienbranche sozialversicherungspflichtige Tätigkeit durch freie Erwerbstätigkeit ersetzt wurde und wird.

Verschiedene Erscheinungsformen „atypischer“ Beschäftigung (freie Mitarbeit, Projektarbeit, [Schein]Selbständigkeit) sind für die Medienbranche - im Vergleich zu anderen Branchen (z. B. dem produzierenden Gewerbe) - schon seit längerem geradezu konstitutiv. Bereits für das Jahr 1998 kommt z. B. eine repräsentative Befragung in Nordrhein-Westfalen zu dem Ergebnis, dass zwei Drittel der Positionen in den audiovisuellen Medien ,unständig“ sind (vgl. Michel 1998, S. 32/43). Den vorliegenden, aktuellen Befunden nach dürfte sich dieser Anteil mittlerweile weiter erhöht haben. Neben den quantitativen Auswirkungen (der Ausweitung freier Tätigkeit in der Medienbranche sowie einer rückläufigen Beschäftigungsentwicklung) ist von qualitativen Effekten auf die Arbeitsbedingungen, Tätigkeitsprofile und Qualifikationsanforderungen auszugehen, die jedoch hinter den Daten verborgen bleiben.

Die Gründe für die Beschäftigungsentwicklung der Medienbranche lassen sich nicht eindeutig bestimmen, denn es ist davon auszugehen, dass neben der technologischen Entwicklung weitere Faktoren auf die Beschäftigung einwirken. Mit dem Ende der Boomjahre der New Economy (1999/2000) verschwanden zahlreiche Unternehmen - auch aus der Medienbranche - gänzlich vom Markt, und es kam zu einer Welle von Entlassungen. Andere Unternehmen kürzten ihre Werbeetats drastisch - mit weit reichenden Folgen für die Medienbranche, die in starker Abhängigkeit zur werbetreibenden Wirtschaft steht: Sie ist auf die Einnahmen durch Werbung in hohem Maße angewiesen, da sie sich überwiegend nicht allein durch den Verkauf ihrer Produkte finanzieren kann. Personalabbau, Gehaltskürzungen oder das Einstellen bestimmter Inhalteformate gehören zu den Konsequenzen der problematischen Lage. 
Als schwierig erweist es sich, eindeutige Aussagen zum Zusammenhang von Internet und Beschäftigungsentwicklung in der Medienbranche zu machen. Die Medienbranche hebt sich aufgrund ihres hohen Durchdringungsgrads mit digitalen Technologien von anderen Branchen ab. Die Effekte solcher Technologien sind bei der Erstellung des Contents (z. B. in Form erweiterter Medienangebote) deutlich spürbar, und auch für die Distribution haben sie unterstützende Wirkungen (z. B. durch die Entstehung neuer Vertriebswege). Dass informatisierte Wertschöpfungsprozesse Auswirkungen auf Arbeit und Beschäftigung implizieren, ist unbestritten; gleichwohl bleibt die Schwierigkeit, festzustellen, welche besondere Rolle das Internet in Bezug auf die Veränderungsdynamiken der Branche einnimmt. Das Problem des ursächlichen Zusammenhangs lässt sich aber auf Basis einer sekundäranalytischen Datenaufbereitung nicht hinlänglich angehen, weil die Gründe für den sich abzeichnenden negativen Trend in der Beschäftigtenentwicklung nicht ersichtlich werden.

Ergänzend ist es deshalb in einem weiteren Forschungsschritt erforderlich, auf der Basis betrieblicher Fallanalysen in ausgewählten Unternehmen Expertengespräche mit Unternehmensvertretern wie mit Beschäftigten zu führen. Eine wichtige Frage könnte in diesem Zusammenhang sein, ob sich der für manche Teilsektoren andeutende Trend zu freier Tätigkeit tatsächlich wieder findet. Zudem gilt es, die Relevanz des Internets für die Beschäftigtenentwicklung genauer zu erfassen. Hinter welchen Effekten tritt sie evtl. zurück? In welchen Wertschöpfungsbereichen zeigt sie sich deutlicher als in anderen? Wie lässt sie sich in den Rahmen betrieblicher Gestaltungsprozesse einordnen?

Wegen seiner hohen quantitativen Bedeutung und der starken Veränderungsdynamik liegt es nahe, zunächst den Bereich Printmedien als Feld für weitere Untersuchungen heranzuziehen. Die Automatisierungs- und Rationalisierungsprozesse v. a. im Druckbereich (z. B. in der Druckvorbereitung und Reproduktion) haben bereits zu Beschäftigungsrückgängen geführt. Gerade die Differenzierungen in den Bereichen Distribution und Content-Produktion, die der Sekundäranalyse zu Folge weniger gravierend sind als zuvor angenommen, können über den Zugriff auf der Basis von Einzelfallstudien genauer untersucht werden. Ausgehend von Analysen zu den gegenwärtigen Formen des Interneteinsatzes in Unternehmen des Printsektors gilt es Strategien zu benennen, die der Einbeziehung des Internet in die Wertschöpfungsstruktur zu Grunde liegen. Auf diese Weise kann es am Beispiel des Printsektors möglich werden, den Zusammenhang zwischen Unternehmensstrategien und Beschäftigungsentwicklung exemplarisch näher zu bestimmen und die Frage nach dem qualitativen $W$ andel der Arbeitsanforderungen im Zeichen eines forcierten Interneteinsatzes in der Medienbranche zu stellen. 


\section{Literatur}

Altmeppen (1999): Altmeppen, K.-D.: Arbeitsmarktentwicklung in Kommunikationsberufen. Zur Klassifikation journalistischer und journalismusnaher Arbeitsmärkte. In: Knoche, Manfred/Siegert, Gabriele (Hrsg.): Strukturwandel der Medienwirtschaft im Zeitalter digitaler Kommunikation, München, Reinhard Fischer, S. 69-87.

Arbeitsgemeinschaft der Landesmedienanstalten (ALM) (2003): ALM (Hrsg.): Privater Rundfunk in Deutschland 2003. Jahrbuch der Landesmedienanstalten, Berlin.

Baukrowitz / Boes / Schmiede (2001): Baukrowitz, A., Boes, A., Schmiede, R.: Die Entwicklung der Arbeit aus der Perspektive ihrer Informatisierung. In: Matuschek, Ingo/Henninger, Annette/Kleemann, Frank (Hrsg.): Neue Medien im Arbeitsalltag, Wiesbaden, VS Verlag für Sozialwissenschaften, S. 217-235.

Bertelsmann Gruppe (BMG) (2002): Bertelsmann Geschäftsbericht 2002, Gütersloh.

Boes (2004): Boes, A.: Industrialisierung und Internationalisierung von IT-Dienstleistungen. Voraussetzungen und Konsequenzen von Offshoring. Beitrag zur ITFachtagung des ver.di-Landesfachbereichs TK/IT/ DV in Baden-Wüttermberg, http://www.arb-it2.de/docs/Boes_Folien_040312_Offshoring.pdf Abruf am 22.03.2005.

Bosch (1998): Bosch, G.: 'Jobless Growth'? Die Auswirkungen der neuen Informationstechnologie auf die Beschäftigung. In: Arbeit, Heft 4, Jg. 7, S. 299-315.

Deutsches Institut für Wirtschaftsforschung (DIW) (1996): Multimedia: Beschäftigungszuwachs im Medien- und Kommunikationsbereich vielfach überschätzt. In: DIW-Wochenbericht 19/1996, http://www.diw.de/deutsch/produkte/publikationen/wochenberichte/docs/96-10-1.html, Abruf am 22.03.2005.

Deutsches Institut für Wirtschaftsforschung (DIW) (2002): Beschäftigte und wirtschaftliche Lage des Rundfunks in Deutschland 1999/2000, Berlin, http://www.diw.de/deutsch/produkte/publikationen/gutachten/jahrgang2002 /index.jsp, Abruf am 22.03.2005.

Deutsches Institut für Wirtschaftsforschung (DIW) (1999): Perspektiven der Medienwirtschaft in Niedersachsen, Berlin, http://www.diw.de/deutsch/produkte/ publikationen/gutachten/docs/ndspdf.pdf, Abruf am 22.03.2005.

Die WELT (2001): Krise der New Economy kostet Werbefirmen die Existenz, 08.02.2001.

Die ZEIT (2005): Aufbruch oder Abbruch? Seit der Privatisierung hat die Telekom mehr als 100.000 Stellen gestrichen. Aggressiver Wettbewerb und neue Technik werden weitere Jobs kosten. Ideereich steuert der Personalchef den Umbau, 03.03.2005, 10/2005.

Dostal (1995): Dostal, W.: Die Informatisierung der Arbeitswelt - Multimedia, offene Arbeitsformen und Telearbeit. In: Mitteilungen aus der Arbeitsmarkt- und Berufsforschung, 28. Jg., Heft 4, S. 527-543. 
EU-Kommission (2003): Empfehlung für eine Empfehlung des Rates zur Durchführung der Beschäftigungspolitik der Mitgliedsstaaten (KOM XXXX), Brüssel.

EU-Kommission (1997): Effects on employment of liberalization of the telecommunications sector, Luxemburg.

Gesellschaft für Konsumforschung (GfK) (2004): Brennerstudie 2004, http://www.ifpi.de/news/379/brennerstudie2004.pdf, Abruf am 22.03.2005.

Hass (2004): Hass, B. H.: Desintegration und Reintegration im Mediensektor: Wie sich Geschäftsmodelle durch Digitalisierung verändern. In: Zerdick, Axel/Picot, Arnold/Schrape, Klaus/Burgelmann, Jean-Claude/Silverstone, Roder/Feldmann, Valerie/Heger, Dominik K./Wolff, Carolin (Hrsg.) (2004): EMerging Media. Kommunikation und Medienwirtschaft der Zukunft, Berlin, Springer, S. 5-29.

Keller (2003): Keller, D.: Zur wirtschaftlichen Lage der deutschen Zeitungen. In: Bundesverband Deutscher Zeitungsverleger (BDZV): Zeitungen 2003, Berlin, ZV-Zeitungs-Verlag Service GmbH, S. 19-95.

Kleemann / Matuschek / Voß (2003): Kleemann, F., Matuschek, I., Voß, G. G.: Subjektivierung von Arbeit. Ein Überblick zum Stand der Diskussion. In: Moldaschl, Manfred/Voß, Günter G. (Hrsg.): Subjektivierung von Arbeit, München/Mering, Hampp, S. 53-111, 2. überarbeitete Auflage.

Kleemann (2000): Kleemann, F.: Informatisierung der Arbeit. Folgen für Arbeitsverhältnisse und subjektive Leistungen der Arbeitnehmenden. In: kommunikation@gesellschaft, Jg. 1, Beitrag 3.

Krömmelbein (2001): Krömmelbein, S.: Internet und Wissensgesellschaft - Visionen und Widersprüche eines „epochalen“ Wandels, Frankfurt a.M., http://www.soz.uni-frankfurt.de/arbeitslehre/WSI54Heft4.html, Abruf am 22.03.2005.

Landesmedienanstalten (Hrsg.) (2004): Beschäftigte und wirtschaftliche Lage des Rundfunks in Deutschland 2001/2002. Schriftenreihe der Landesmedienanstalten, Berlin, Vistas Verlag GmbH.

Langer (2001): Langer, U.: „Bitte rufen Sie jetzt an!“ Von der Glotze zur Geldverdienmaschine: Die Sender entdecken, dass man nicht nur mit Programmen Gewinn macht. In: ALM (Hrsg.): Programmbericht zur Lage und Entwicklung des Fernsehens in Deutschland 2000/2001, Konstanz, UVK Verlagsgesellschaft, S. 80-89.

Little (1996): Little, A D.: Innovation und Arbeit für das Informationszeitalter. Zusammenfassung der Ergebnisse, BMBF, Bonn.

Marrs / Boes (2003): Marrs, K., Boes, A.: Alles Spaß und Hollywood? Arbeits- und Leistungsbedingungen bei Film und Fernsehen. In: Pohlmann, Markus/Sauer, Dieter/Trautwein-Kalms, Gudrun/Wagner, Alexandra (Hrsg.) (2003): Dienstleistungsarbeit: Auf dem Boden der Tatsachen. Befunde aus Handel, Industrie, Medien und IT-Branche, Berlin, edition sigma, S. 187-242. 
Media Perspektiven Basisdaten (2004): Daten zur Mediensituation in Deutschland 2004, Frankfurt a.M., herausgegeben im Auftrag der Arbeitsgemeinschaft der ARD-Werbegesellschaften.

Metier Consortium (1995): The impact of advanced communication in European growth and trade, Studie im Auftrag der EU-Kommission.

Michel (1998): Michel, L. P.: AV-Medien in NRW. Arbeitsmarkt und Qualifizierung, Verv. Ms., Düsseldorf.

Neuberger (2002a): Neuberger, C.: Zurückhaltung im Netz. Das Engagement der Tageszeitungen im Internet. In: Theis-Berglmair, Aanna M. (Hrsg.) (2002): Internet und die Zukunft der Printmedien. Kommunikationswissenschaftliche und medienökonomische Aspekte, Münster, LIT, S. 117-133.

Neuberger (2002b): Neuberger, C.: Online-Journalismus: Akteure, redaktionelle Strukturen und Berufskontext. Ergebnisse einer Berufsfeldstudie. In: M\&K Medien und Kommunikationswissenschaft, Heft 1, S. 102-114.

Niedersächsisches Institut für Wirtschaftsforschung (NIW) (2002): Informationsund Medienwirtschaft in Niedersachsen 2002, Hannover, http://www.niw.de, Abruf am 22.03.2005.

Niggemeier (2001): Niggemeier, S.: Alles geht, wenig läuft. Vom Bild zum Text: Angebote der Fernsehsender im Internet.. In: ALM (Hrsg.): Programmbericht zur Lage und Entwicklung des Fernsehens in Deutschland 2000/2001, Konstanz, UVK Verlagsgesellschaft GmbH, S. 90-97.

OECD (1998): ICCP Statistical Panel. Definition for the Information and Communication Technology Sector, Paris.

PricewaterhouseCoopers (2004): German Media and Entertainment Outlook: 2003-2007, http://www.pwc.com/de/ger/ins-sol/publ/EuM_Outlook_5.pdf, Abruf am 22.03.2005.

Rheinisch-Westfälisches Institut für Wirtschaftsforschung (RWI) (2000): Wachstums- und Beschäftigungspotentiale der Informationsgesellschaft bis zum Jahre 2010, Essen, http://www.rwi-essen.de/pls/portal30/ docs/FOLDER/PUBLIKATIONEN/GUTACHTEN/INFOGESELLSCHAFT.PDF, Abruf am 22.03.2005.

Riehm / Orwat / Wingert (2001): Riehm, U., Orwat, C., Wingert, B.: OnlineBuchhandel in Deutschland. Die Buchhandelsbranche vor der Herausforderung des Internet, http://elib.uni-stuttgart.de/opus/volltexte/2004/1879/ pdf/AB192.pdf, Abruf am 22.03.2005.

Röper (2004): Röper, H.: Formationen deutscher Medienmultis 2003. In: Media Perspektiven 2/2004, S. 54-80.

Schrape / Seufert / Haas / Hürst / Gafke (1996): Schrape, K., Seufert, W., Haas, H., Hürst, D., Gafke, S.: Künftige Entwicklung des Medien- und Kommunikationssektors in Deutschland. In: Deutsches Institut für Wirtschaftsforschung (Hrsg.): Beiträge zur Strukturforschung, Berlin, Duncker \& Humblot. 
Sennewald (1998): Sennewald, N.: Massenmedien und Internet: zur Marktentwicklung in der Pressebranche, Wiesbaden, Gabler.

Stehr (1994): Stehr, N.: Arbeit, Eigentum und Wissen. Zur Theorie von Wissensgesellschaften, Frankfurt a.M., Suhrkamp.

Sydow / Windeler (1999): Sydow, J., Windeler, A.: Projektnetzwerke: Management von (mehr als) temporären Systemen. In: Engelhard, Johann/Sinz, Elmar J. (Hrsg.): Kooperation im Wettbewerb - Neue Formen und Gestaltungskonzepte im Zeichen von Globalisierung und Informationstechnologie, Wiesbaden, Gabler, S. 211-235.

van Eimeren / Gerhard / Frees (2004): van Eimeren, B., Gerhard, H., Frees, B.: Internetverbreitung in Deutschland: Potenzial vorerst ausgeschöpft? ARD/ZDFOnline-Studie 2004. In: Media Perspektiven 8/2004, S. 350-370.

von Staden / Hundsdörfer (2003): von Staden, I., Hundsdörfer, B.: „Majors planen digital roll-out...". Auswirkungen der digitalen Zukunft auf die Kinobranche, Berlin, http://www.filmfoerderungsanstalt.de/downloads/studie_digitales_kino _0309.pdf, Abruf am 22.03.2005.

Willke (1998): Willke, H.: Organisierte Wissensarbeit. In: Zeitschrift für Soziologie, Jg. 27, Heft 4, S. 161-177.

Zerdick et al. (1999): Zerdick, A., Picot, A., Schrape, K., Artopé, A., Goldhammer, K., Heger, D., Lange, U. T., Vierkant, E., Lopez-Escobar, E., Silverstone, R.: Die Internet-Ökonomie. Strategien für die digitale Wirtschaft, Berlin, Springer.

Zerdick et al. (2004): Zerdick, A., Picot, A., Schrape, K., Burgelmann, J.-C., Silverstone, R., Feldmann, V., Heger, D.K., Wolff, C.: E-Merging Media. Kommunikation und Medienwirtschaft der Zukunft, Berlin, Springer.

\section{Weitere Internetquellen (ohne Autor):}

http://www.apple.com/de/itunes, Abruf am 22.03.2005.

http://www.boersenverein.de/de/64584, Abruf am 22.03.2005.

http://www.destatis.de/allg/d/klassif/wz2003.htm, Abruf am 22.03.2005.

http://www.destatis.de/basis/d/erwerb/erwerbtab5.php, Abruf am 22.03.2005.

http://www.heise.de, Abruf am 22.03.2005.

http://idw-online.de/pages/de/news96537, Abruf am 22.03.2005.

http://www.kuenstlersozialkasse.de, Abruf am 22.03.2005.

http://www.mediafon.net/aktuelles.php3\#41eba55fcc3af, Abruf am 22.03.2005.

http://www.medionmusic.com, Abruf am 22.03.2005.

http://www.musicload.de, Abruf am 22.03.2005. 


\section{Controlling in Start-Up-Unternehmen: Empirische Untersuchungen zum Verhältnis von Planung und Unternehmenserfolg}

Björn Ortelbach

\section{Einleitung}

Als Antwort auf den zunehmenden Einzug der IuK-Technologie in die Medienbranche ist eine Vielzahl neuer Geschäftsmodelle entstanden, die von einem ergänzenden Inhalteangebot für klassische Print- und Rundfunkprodukte über Anbieter mit originär für das Internet erstellten Inhalten bis zu neuen Online-Intermediären reichen. Außerdem finden sich zahlreiche neue Geschäftsmodelle, die unterstützende Dienste für die zuvor genannte Gruppe von Unternehmen anbieten. Zu denken ist hier beispielsweise an Anbieter von Micropayment-Verfahren.

Die mit der Entwicklung des Online-Medien-Marktes verbundenen wirtschaftlichen Herausforderungen - aber auch deren Chancen - sind enorm. Die Branche ist gekennzeichnet durch eine dynamische Entwicklung der zugrunde liegenden IuK-Technologien, schnelle Marktein- und -austritte, Unternehmensübernahmen und -zusammenschlüsse sowie eine sich ständig verändernde Wettbewerberland- 
schaft. Den neuen Erlösformen stehen die für Medienunternehmen charakteristischen hohen Fixkosten bei oftmals noch nicht erreichter Break-Even-Schwelle gegenüber. Daraus erwächst insgesamt die Notwendigkeit einer streng am wirtschaftlichen Ergebnis orientierten Steuerung, die hilft, diesen Herausforderungen aktiv zu begegnen, anstatt sich von ihnen treiben zu lassen.

Bezogen auf die Praxis wird insbesondere am Beispiel der „geplatzten Internetblase" deutlich, dass betriebswirtschaftliche Instrumentarien notwendig sind, um Unternehmen mit innovativen Geschäftsmodellen zielgerichtet steuern zu können. In der Rückschau wird deutlich, dass viele Geschäftsideen in der Internetwelt unter anderem auch aufgrund mangelnder Steuerung in Form von Controlling und Kostenrechnung erfolglos geblieben bzw. die betroffenen Unternehmen mittelfristig nicht richtig ausgerichtet worden sind.

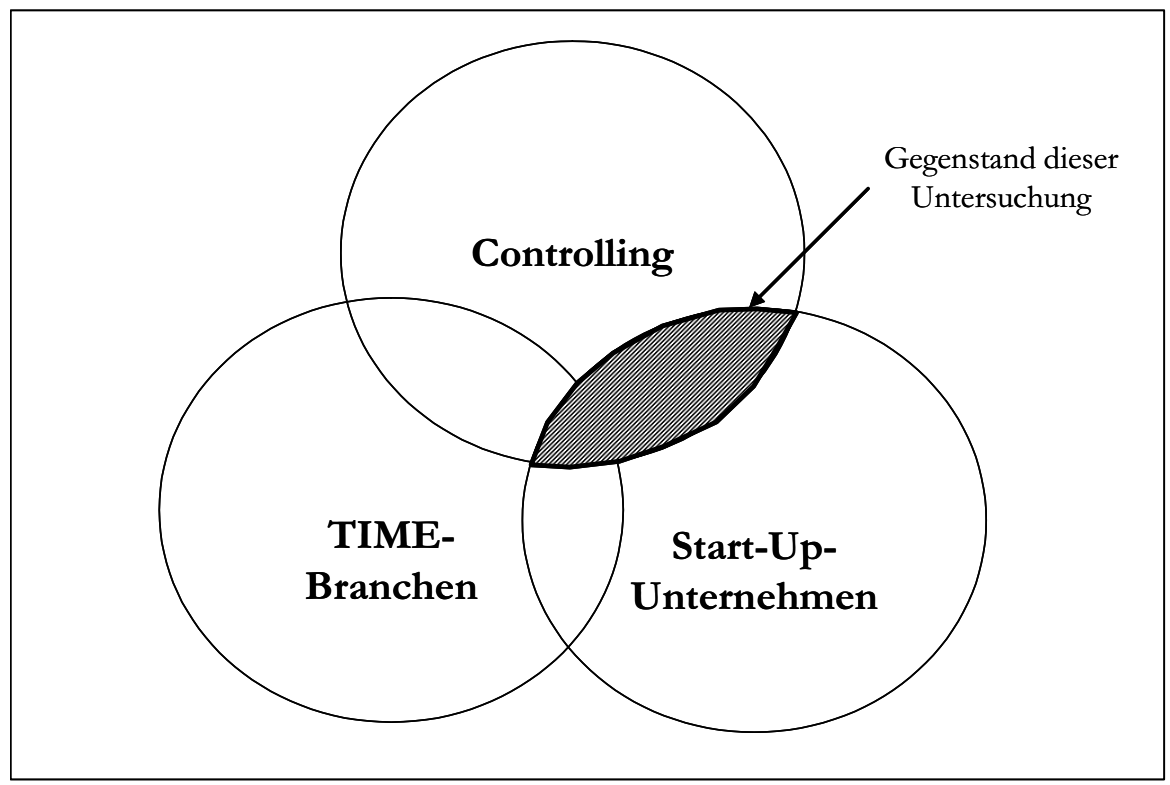

Abbildung 63: Bezugspunkt und Fokussierung der vorliegenden Untersuchung

Für die Forschung in diesem Bereich ergeben sich drei Bezugspunkte: das Controlling als funktionale Sicht, die TIME-Branchen als branchenbezogene Sicht sowie Start-Up-Unternehmen als organisationsbezogene Sicht (vgl. Abb. 63). Will man sich dem skizzierten Problem - das als Schnittmenge der genannten Bezugspunkt anzusehen ist - nähern, ist es notwendig, sich zunächst nicht speziell auf die TIME-Branche zu beschränken, sondern zunächst im Allgemeinen die Problemstellung des Controlling in Start-Up-Unternehmen zu untersuchen, weil selbst dieser weiter gefassten Fragestellung in der Forschung erst seit kurzer Zeit explizit Aufmerksamkeit gewidmet wird. 
Achtleitner und Bassen (2002) machen bereits im Titel ihres Beitrags „Controllingsysteme in jungen Wachstumsunternehmen - terra incognita“ auf eine entsprechende Forschungslücke aufmerksam. Gleichermaßen leisten sie selbst in der Folge mit dem Herausgeberband „Controlling in jungen Unternehmen“ (vgl. Achtleitner / Bassen 2003) einen wichtigen Beitrag, diese Lücke zu verkleinern. Es ist jedoch festzustellen, dass dieses Werk nahezu keine empirischen Ergebnisse zur untersuchten Problemstellung vorstellt. Vor dem Hintergrund der vielfach vorgebrachten Forderung nach einer stärkeren empirischen Ausrichtung des Controlling (vgl. z. B. Küpper / Weber / Zünd 1990, Schäffer / Weber 2003, Weber / Kunz 2003, Brockhoff 2003) scheint diese Perspektive jedoch besonders wichtig zu sein.

Die vorliegende Untersuchung befasst sich mit einer grundlegenden Fragestellung des Themengebiets „Controlling in Start-Up-Unternehmen“. Im Folgenden soll der Frage nachgegangen werden, ob bzw. in welcher Form und unter welchen Bedingungen Planung positiv auf den Erfolg Start-Up-Unternehmen wirkt. Das Ziel dieser Untersuchung ist die Aufarbeitung von Studien, die einen empirisch fundierten Beitrag zur Beantwortung dieser Frage leisten. Hierbei liegt der Schwerpunkt darauf, insbesondere Studien aus dem nicht-deutschsprachigen Raum zu berücksichtigen, um diese in die Diskussion der deutschsprachigen Forschung einzubringen.

Das zweite Kapitel behandelt den Begriff des Controlling. Die aktuelle wissenschaftliche Diskussion über die konzeptionelle Ausrichtung dieses Fachgebiets wird nachgezeichnet und die Argumentation in den speziellen Kontext der Untersuchung eingeordnet. Das dritte Kapitel der Untersuchung befasst sich mit der Abgrenzung des Untersuchungsobjekts. Zu diesem Zweck sollen die Charakteristika junger Unternehmen herausgearbeitet werden. Im vierten Kapitel werden die zur genannten Fragestellung vorliegenden empirischen Untersuchungen zusammenfassend dargestellt und ihre Methodiken und Ergebnisse diskutiert. Das fünfte Kapitel fasst die Ergebnisse der Untersuchung zusammen und identifiziert mögliche zukünftige Forschungsfragen.

\section{Der Begriff des Controlling}

\subsection{Einführung}

Der Begriff des Controlling ist trotz seiner weiten Verbreitung in der Literatur nicht einheitlich definiert. Er wird vielfach auch als „schillernd“ (z. B. Küpper/Weber/Zünd 1990, S. 282) charakterisiert. Abgeleitet vom englischen Verb „to control“ versteht man darunter im Allgemeinen die Steuerung eines Unternehmens. In seinem angloamerikanischen Ursprung wird controlling in funktionaler Sicht als ein Element des Managementprozesses neben coordination, organizing oder di- 
recting gesehen (vgl. Weber 2002, S. 1). In institutioneller Sicht (im englischsprachigen Raum als „Controllership“ bezeichnet) bezieht sich das Controlling auf das Aufgabenspektrum von Stelleninhabern, die diese Bezeichnung führen.

Seit den 1970er Jahren versucht die fachspezifische deutschsprachige Literatur, den „Kern des Controlling“ bzw. seine „theoretische Fundierung“ herauszuarbeiten. In diesem Zusammenhang entwickelten sich verschiedene konkurrierende Konzeptionen des Controlling. Eine solche Controlling-Konzeption ,ist die grundlegende Auffassung über Controlling. Sie ist vergleichbar mit der Verfassung eines Staates und umfasst alle Grundgedanken über Zweck, Funktionsweise und Zusammenwirken mit anderen Systemen“ (Eschenbach / Niedermayr 1996, S. 65). Es handelt sich um ein Aussagensystem, das eine Zweck-Mittel-Relation zwischen dem Zweck eines funktional verstandenen Controlling und den zu diesem Zweck eingesetzten Mittel herstellen. Sie sind damit keine Beschreibung der Realität, sondern ein Denkmodell (vgl. Harbert 1982, S. 140).

\subsection{Konzeptionen}

Unter der Vielzahl der Konzeptionen lassen sich traditionell folgende Grundtypen unterscheiden:

- informationsorientierte Konzeptionen

- planungs- und kontrollorientierte Konzeptionen

- führungsgesamtsystemorientierte Konzeptionen

Bei informationsorientierten Controlling-Konzeptionen steht die Funktion im Vordergrund, die Unternehmensführung mit entscheidungsrelevanten Informationen zur versorgen. Das Controlling wird hierbei weitgehend mit der betrieblichen Informationswirtschaft gleichgesetzt, wobei der Fokus des Controlling in der Abstimmung bzw. Koordination von Informationsbedarf und Informationsbereitstellung liegt. Begründet wird dies mit der in der Praxis häufig festzustellenden Unbrauchbarkeit und mangelnden Entscheidungsrelevanz der traditionell zur Verfügung stehenden Daten. Als Begründer bzw. wesentliche Vertreter dieses Ansatzes sind Müller (1974) und Reichmann (1985) zu nennen.

Die planungs- und kontrollorientierten Konzeptionen, die häufig auch als eingeschränkt koordinationsorientierte Konzeption bezeichnet werden, differenziert das System Unternehmung in ein Führungs- und ein Ausführungssystem. Die Aufgabe der Führung liegt in der Koordination des Ausführungssystems. Das Führungssystem gliedert sich in Führungsteilsysteme. Diese Arbeitsteilung der Führung macht auch hier eine Koordination erforderlich. Dem Controlling als eines der Führungsteilsysteme kommt nach dieser Auffassung die Funktion der Koordination der Führungsteilsysteme Planung, Kontrolle und Information zu. 
Dabei ist Controlling primär formalzielorientiert; Sachziele werden jedoch mittelbar über ihren Bezug zu den Ergebniszielen berücksichtigt. Begründer und wesentlicher Vertreter dieses Ansatzes ist Horváth (1979).

Der führungsgesamtsystemorientierte Ansatz ist der vorhergehenden Konzeption sehr ähnlich. Auch hier wird der Begriff der Koordination als zentrales Charakteristikum des Controlling herausgestellt. In dieser Sichtweise wird Controlling jedoch nicht auf Planung, Kontrolle und Information beschränkt, sondern bezieht sich auf alle Führungsteilsysteme, insbesondere auch auf das Personalführungs- und das Organisationssystem. Begründet wird dieses mit der hohen Interdependenz zwischen den Führungsteilsystemen. In dieser Sichtweise wird der Koordinationsbegriff enger gefasst: Er bezieht sich lediglich auf die Interdependenzen zwischen den Teilsystemen, nicht jedoch auf den Entwurf der Systeme (vgl. Zenz 1999, S. 31). Eine weitere Annahme dieser Konzeption besteht darin, dass Koordination primär durch Pläne erfolgt (vgl. Eschenbach/Niedermayr 1996b, S. 61).303 Wesentlicher Vertreter dieser Sichtweise ist Küpper (1995).

Durch zahlreiche neue Anregungen zur Konzeption des Controlling entstand während der letzten Jahre eine erneute Diskussion über den Untersuchungsgegenstand dieser Disziplin. An erster Stelle ist dabei das von Weber und Schäffer vertretene rationalitätsorientierte Verständnis des Controlling zu nennen (vgl. Weber / Schäffer / Langenbach 1999). Zu berücksichtigen ist weiterhin die reflexionsorientierte Konzeption von Pietsch und Scherm (2000).

Die rationalitätsorientierte Konzeption wurde Ende der 1990er Jahre von Weber und Schäffer als Modell zur Integration der traditionellen Ansätze vorgeschlagen, da diese nach Meinung dieser Wissenschaftler verschiedene Schwächen aufweisen. Sie postulieren eine neue Sichtweise, in der sie die „Sicherstellung der Rationalität der Führung“ als gemeinsamen Nenner aller Controlling-Konzeptionen herausstellen (vgl. Weber / Schäffer / Langenbach 1999; Weber 2000; Weber / Schäffer 2001; Schäffer / Weber 2003). Rationalität wird dabei als Zweckrationalität verstanden, die sich an einer effizienten Mittelverwendung bei gegebenem Ziel bemisst. Dabei ist der Zweck i. d. R. selbst ein Mittel zum Erreichen eines übergeordneten Ziels. Der Ausgangspunkt ist die Feststellung, dass durch Wollens- bzw. Könnensdefizite von Führungsverantwortlichen Rationalitätsdefizite entstehen können. Als Rationalitätssicherung der Führung sind Handlungen zu verstehen, die die Wahrscheinlichkeit erhöhen, dass die Führungshandlungen den MittelZweck-Beziehungen trotz dieser Defizite entsprechen. Der Sicherstellungsfunktion liegt das Subsidiaritätsprinzip zugrunde: sein Umfang und seine Ausprägung leiten sich aus den Rationalitätsdefiziten der Führung ab (vgl. Weber 2002b, S. 65). Die

303 Es wird unterschieden zwischen Koordination durch Programme, Koordination durch Pläne, Koordination durch Selbstabstimmung sowie Koordination durch persönliche Weisungen. Dabei ist kein Koordinationsmechanismus grundsätzlich den anderen überlegen; die Vorteilhaftigkeit ist vielmehr kontextabhängig (vgl. Kieser/Kubicek 1992). 
Rationalitätssicherung ist ein Zusammenspiel von Intuition und Reflexion in einem (idealtypischen) Führungsprozess, der sich aus den Elementen Willensbildung, Willensdurchsetzung und Kontrolle zusammensetzt. Dabei wird der Willensbildungsprozess bei ausreichendem explizitem Wissen und hohem Bewusstseinsgrad als Reflexion, bei begrenztem impliziten Wissen und niedrigem Bewusstseinsgrad als Intuition bezeichnet (vgl. Weber / Schäffer 1999).

Im Gegensatz zu Webers Versuch, einen integrativen Ansatz für das Controlling zu entwickeln, beabsichtigen Pietsch und Scherm mit ihrer reflexionsorientierten Konzeption die Abgrenzung des Controlling als Führungs- und Führungsunterstützungsfunktion zu präzisieren und damit zu einer enger eingegrenzten Sichtweise zu gelangen (vgl. Pietsch / Scherm 2000; Pietsch / Scherm 2001a; Pietsch / Scherm 2001b). Eine wesentliche Annahme des Modells ist, dass das Handlungsfeld „Unternehmung“ funktional nicht nur in Führung und Ausführung, sondern in Führung, Führungsunterstützung und Ausführung gegliedert wird. Die Aufgabe der Ebene der Führungsunterstützung ist die Beschaffung und Aufbereitung von führungsrelevanten Informationen. Nach Pietsch und Scherm beinhaltet Controlling sowohl Führungs- als auch Führungsunterstützungsfunktionen. In ihrer Sicht ist das Controlling als Führungsfunktion durch eine Reflexionsaufgabe gekennzeichnet. Im Gegensatz zur Selektion, die sowohl bewusste Überlegungen als auch intuitive Elemente enthält, ,stellt die Reflexion immer eine distanzierend-kritische und somit spezielle Gedankenarbeit dar" (Pietsch / Scherm 2000). Sie argumentieren, dass zum Erfüllen der Reflexionsaufgabe als Führungsfunktion des Controlling eine „funktionsübergreifende, informatorische Gesamtsicht“ (Pietsch / Scherm 2000, S. 407) benötigt wird. Die Bereitstellung und Aktualisierung dieser Informationsbasis ist die Führungsunterstützungsfunktion des Controlling.

\subsection{Zum Controllingverständnis im Kontext dieser Untersuchung}

In der Mehrzahl der Veröffentlichungen zum Controlling in Start-Up-Unternehmen wird die rationalitätsorientierte Konzeption als für diesen Kontext am besten geeignet angesehen (vgl. z. B. Achtleitner / Bassen 2002). Der wesentliche Grund, diese Konzeption zu befürworten, ist, dass dieser Ansatz die Kontextabhängigkeit des Controlling explizit betont (vgl. Weber 2003) und damit bereits auf konzeptioneller Ebene darauf hindeutet, „dass eine besondere Ausgestaltung des Controlling in jungen Wachstumsunternehmen notwendig sein könnte“ (Achtleitner / Bassen 2002, S. 1192).

Bereits die Definition des Begriffs der Controlling-Konzeption zeigt aber auch, dass es sich hierbei um ein Konstrukt auf hohem Abstraktionsniveau handelt. Trotz aller Differenzen im inhaltlichen und begrifflichen Detail darf nicht überse- 
hen werden, dass die dargestellten Konzeptionen auch große Schnittmengen aufweisen. Dies gilt insbesondere, wenn sich die Betrachtungsebene vom Abstrakten dem Konkreten nähert. Eine solche Konkretisierung der theoretischen Ebene dient nicht nur dazu, dem Anwendungsbezug der Forschung Rechnung zu tragen, sondern ist auch originär für die Forschung notwendig. Dies ist der Fall, wenn die Controllingforschung der häufig gestellten Forderung einer stärkeren empirischen Ausrichtung nachkommen will, da hierfür der Betrachtungsgegenstand „Controlling“ operationalisierbar gemacht und die Begrifflichkeit an die Praxis angenähert werden muss.

Allen Konzeptionen ist gemeinsam, dass sie Controlling als ein Mittel zur Unterstützung der Unternehmensführung sehen. Die Kernbestandteile des Controlling sind folgende Elemente:

- die Informationsversorgung (Beschaffung und Aufbereitung führungsrelevanter Informationen),

- die Planung (Vorbereitung und Durchführung der Planung, insbesondere auch deren methodische Unterstützung),

- die Kontrolle (kontinuierliche Prüfung der Planungsprämissen, Vergleichen der Plan- mit der Ist-Situation).

Im Folgenden werden unter Controlling alle Aktivitäten verstanden, die der Informationsversorgung, der Planung und der Kontrolle in Start-Up-Unternehmen dienen. Diese bewusst weite Begriffsauffassung trägt dem geringen Erkenntnisstand in diesem Forschungsfeld insoweit Rechnung, dass sie eine breite Annäherung an die Problemstellung ermöglicht.

\section{Start-Up-Unternehmen als Untersuchungsobjekt der Controllingforschung}

Bei „Start-Ups“ handelt sich um Unternehmen, die sich in der Phase der Gründung befinden bzw. deren Gründungszeitpunkt erst eine bestimmte Zeit zurückliegt. Prägend für alle betrieblichen Funktionen - und damit auch für das Controlling - einer solch jungen Organisation ist es, dass interne Prozesse und die Stellung des Unternehmens am Markt kaum etabliert sind.

Zur Beschreibung des Entwicklungsverlaufs von Start-Up-Unternehmen werden in der Literatur häufig Phasenschemata verwendet. Bassen und Gröne (2003) unterscheiden beispielsweise zwischen der Early Stage und der Expansion-Stage, wobei Start-Up-Unternehmen im engeren Sinn nur der erst genannten Phase zuzurechnen sind. Die Early Stage wird weiter differenziert in die Unterphasen Seed Stage (Entwicklung und Erprobung des Geschäftsmodells), Start Up Stage 
(Unternehmensgründung und Schaffung organisatorischer Grundlagen für unternehmerisches Handeln) sowie First Stage (erstmalige marktliche Nutzung der Geschäftsidee und Entwicklung der Kundenbeziehungen).

Problematisch an Phasenkonzepten wie dem zuvor vorgestellten ist, dass es sich um deduktive Modelle handelt. Der wesentliche Kritikpunkt an diesen Schemata ist ihre Willkür in der Abgrenzung der einzelnen Phasen. Den Gegensatz hierzu bilden empirisch-induktiv entwickelte Modelle, wie z. B. das Konzept von Kazanzjian (1988). Auf Basis einer Fallstudienuntersuchung US-amerikanischer Hightech-Wachstumsunternehmen werden die dominierenden Führungsprobleme als zentrales Kriterium zur Abgrenzung der einzelnen Phasen identifiziert. Hieraus ergeben sich folgende vier Phasen und jeweils zugehörige dominierende Führungsprobleme: Konzeption und Entwicklung (Ressourcenbeschaffung und Technologieentwicklung), Kommerzialisierung (Probleme der Produktionsanlagen und des Produktionsanlaufs), Wachstum (Probleme des Absatz- und Umsatzwachstums) sowie Stabilität (Probleme der Profitabilität, der internen Steuerung sowie zukünftiger Wachstumsressourcen). Insbesondere die theoretische Rolle der phasenspezifischen Dominanz bestimmter Probleme wird betont - nicht nur zur Abgrenzung der Phasen, sondern auch zum Verständnis der Transformation der Organisationen von einer Phase zur anderen. Bei einer Prüfung des Modells in einer weiteren Studie (vgl. ebenso Kazanzjian 1988) zeigte sich, dass bestimme Probleme generell (also phasenunabhängig) wichtiger sind als andere. Als wichtiges Ergebnis dieser Folgestudie soll für die vorliegende Untersuchung herausgestellt werden, dass Controlling-spezifischen Problemen insbesondere in der Wachstumsphase eines jungen Unternehmens verstärkte Aufmerksam zu widmen ist.

Die neueste Zusammenstellung der Forschungsergebnisse zum Controlling in jungen Wachstumsunternehmen stammt von Achleitner und Bassen (2002). Auf Basis einer Literaturanalyse identifizieren sie typische Charakteristika dieser Unternehmen und leiten hieraus einen Katalog mit Anforderungen an das Controlling ab. Ein zentrales Charakteristikum von Start-Up-Unternehmen wird hierbei jedoch nicht berücksichtigt: diese Unternehmen sind - auch wenn sie schnell wachsen - Kleinunternehmen. Geht man von der Feststellung aus, dass die betriebswirtschaftliche Forschung zum Controlling in Start-Up-Unternehmen noch wenig entwickelt ist, erscheint ein Blick in eng verwandte betriebswirtschaftliche Themengebiete nahe liegend. Hierbei ist insbesondere an Untersuchungen über das Controlling in kleinen und mittelständischen Unternehmen zu denken. Diese weisen aufgrund ihrer Größe (als ein wesentliches organisatorisches Strukturmerkmal) große Gemeinsamkeiten mit Start-Up-Unternehmen auf.

Zu beachten ist hierbei allerdings, dass zwischen Kleinunternehmen und StartUp-Unternehmen auch strukturelle Unterschiede bestehen, so dass sich durch einen unkritischen Austausch von Erkenntnissen zwischen diesen Teilgebieten falsche Schlussfolgerunge ergeben können. Dass solche Differenzen bestehen und 
auch in der Wissenschaft wahrgenommen werden, zeigt die Aussage von Robinson und Pearce (1984, S. 135):

"Is the application of planning the main ingredient that separates the growing (entrepreneurial) business from the small, static business? Researchers as well as policymakers for some time have sought to distinguish between two 'types' of small businesses. Increasingly, government programs and policies at federal and state level are seeking to target growing, entrepreneurial businesses rather than small businesses."

Unterscheiden kann man somit Kleinunternehmen, die ein kontinuierliches Wachstum anstreben und typischerweise als Start-Up-Unternehmen bezeichnet werden, und solche, die dauerhaft in weitgehend gleich bleibender Größe operieren. Trotz möglicher Schwierigkeiten, die bei der Übertragung von Erkenntnissen bezüglich Kleinunternehmen auf das Gebiet der Start-Up-Unternehmen bestehen, ist eine Isolierung beider Bereiche nicht zu rechtfertigen. Dieses wird besonders deutlich, wenn die Charakteristika und die daraus abgeleiteten Anforderung von Achtleitner / Bassen (2002) betrachtet werden. Insbesondere die Forderung nach einem „einfachen System“ zur Steuerung, die in der genannten Untersuchung einzig aus dem geringen Alter des Unternehmens abgeleitet wird, trifft ohne Einschränkung auch auf Kleinunternehmen zu, denn auch für Start-Up-Unternehmen gilt: „A small business is not a little big business“ (Welsh / White 1981, S. 18). Es ist somit nicht das junge Alter, sondern die geringe Größe der wichtigere Grund für die Forderung nach einem einfachen Controllingsystem.

Die folgende synoptische Betrachtung beider Bereiche ist damit in der Tradition des von Wortman (1983, S. 273) postulierten ,unified framework for the interface between entrepreneurship and small business" zu sehen. Entsprechend betrachtet die folgende Untersuchung das Verhältnis von Planung und Erfolg sowohl für Klein- als auch Start-Up-Unternehmen.

\section{Empirische Ergebnisse zum Verhältnis von Planung und Erfolg in Start-Up-Unternehmen}

\subsection{Methodik}

Der geringe Erkenntnisstand über das Controlling in Start-Up-Unternehmen und insbesondere die mangelnde empirische Fundierung vieler Aussagen sind die primäre Motivation der vorliegenden Untersuchung. Stellt man die Argumentation der deutschsprachigen Literatur in den Kontext der internationalen Forschung, ist festzustellen, dass die zuvor genannte Kritik keinesfalls neu ist. 
"The literature on planning in small business has grown considerably over the past decade. Yet, as a number of writers ... have found, it is not easy to bring together coherently either in theory or practice. There are a number of major reasons for this. In the first place much literature is normative without being extensively grounded; at the very least, planning is assumed to be desirable and associated with success. Secondly, comparative analysis is difficult because of looseness of definitions - of the terms associated with planning as well as what constitutes a 'small business." (Gibb/Scott 1985, S. 597f.)

Die Aufarbeitung empirischer Ergebnisse, die die dargestellte Problemstellung betreffen, ist aus zwei Gründen sinnvoll. Zum einen kann sie einen Beitrag zur stärkeren empirischen Fundierung des Controlling leisten. Dabei wird insbesondere das Ziel verfolgt, Erkenntnisse der internationalen Forschung in die Diskussion der deutschen Betriebswirtschaftslehre einzubringen. Zum anderen ermöglichen der Vergleich und die Zusammenfassung vorliegender Ergebnisse die Identifikation zukünftiger Forschungsfragen.

Im Folgenden soll der Frage nachgegangen werden, ob bzw. in welcher Form und unter welchen Bedingungen Planung einen Beitrag zum Unternehmenserfolg in Start-Up-Unternehmen leistet. Hierbei handelt es sich um eine fundamentale Frage für das Controlling in diesen Unternehmen, da sie die grundsätzliche Sinnhaftigkeit dieser betrieblichen Funktion thematisiert. In die nachfolgende Aufarbeitung werden entsprechend Untersuchungen einbezogen, die einen empirischen Beitrag zur Beantwortung dieser Frage leisten. Berücksichtigt werden dabei alle Studien, die im Zeitraum von 1980-2004 in den relevanten internationalen Fachzeitschriften im Rahmen entsprechender wissenschaftlicher Konferenzen veröffentlicht wurden. Hierbei handelt es sich um folgende Zeitschriften:

- Academy of Management Journal

- American Journal of Small Business

- British Journal of Management

- Entrepreneuship Theory and Practice

- Journal of Business and Economic Studies

- Journal of Business Venturing

- Journal of Small Business Management

- Long Range Planning

- Strategic Management Journal

- Small Enterprise Research

Bezüglich der Konferenzen werden die Annual National Conference der United States Association for Small Business and Entrepreneurship (USASBE) sowie die 
Babson Entrepreneurship Research Conference einbezogen. Diese Selektion orientiert sich an früheren Metaanalysen in diesen Forschungsgebiet (vgl. Schwenk / Shrader 1993).

Die Studien werden zunächst zusammenfassend dargestellt. Es werden insbesondere die folgenden Aspekte der Untersuchungen wiedergegeben: das exakte Untersuchungsziel, die untersuchte Branche, das Land, in dem die Untersuchung durchgeführt wurde, die Merkmale der verwendeten Stichprobe, die Untersuchungsmethodik sowie die wesentlichen Ergebnisse. Diese qualitative Aufarbeitung ist notwendig, da eine aggregierte Auswertung der Ergebnisse nur möglich ist, wenn die jeweilige Ausrichtung der Studie, ihr Kontext sowie die Operationalisierung der Untersuchungskonstrukte berücksichtigt werden.

\subsection{Aufarbeitung über vorliegende empirische Studien}

Entsprechend des zuvor genannten Selektionskriteriums wurden folgende Studien identifiziert und in die Untersuchung einbezogen:

- Robinson (1982)

- Robinson / Pearce (1983)

- Ackelsberg / Arlow (1985)

- Orpen (1985)

- Robinsion / Logan / Salem (1986)

- Shuman / Seeger (1986) und Shuman / Shaw / Sussmann (1985)

- Boag (1987)

- Smith et al. (1988)

- Bracker / Keats / Pearson (1988)

- Cragg / King (1988)

- Shrader / Mulford / Blackburn (1989)

- Duchesneau / Gartner (1990)

- Lyles et al. (1993)

- McKiernan / Morris (1994)

- Olson / Bokor (1995)

- Berman / Gordon / Sussman (1997)

- Rue / Ibrahim (1998) 
- Lumpkin / Shrader / Hills (1998)

- Rauch / Frese (1998)

- Mazzarol (2001)

- Perry (2001)

- Gibson/ Cassar / Wingham (2001)

- Wijewardena et al. (2004)

\section{Robinson (1982)}

Robinson geht der Frage nach, inwieweit der Erfolg von Kleinunternehmen verbessert werden kann, wenn der strategische Planungsprozess durch externe Berater unterstützt wird. Hierzu vergleicht er in einem Experiment die Profitabilität von 101 US-amerikanische Kleinunternehmen verschiedener Branchen, denen eine externe Beratung zur Verfügung gestellt wird, mit zwei Kontrollgruppen, die Unternehmen mit ähnlichen Größen- und Erfolgsmerkmalen enthalten, denen jedoch keine externe Beratung zur Verfügung gestellt wird. Alle untersuchten Unternehmen sind nicht Teil eines Konzerns, haben weniger als 50 Beschäftigte und einen Jahresumsatz von weniger als 3 Mio. US\$. Die Beratungsleistung umfasst eine Erörterung der allgemeinen Unternehmensstrategie, Analysen und Entscheidungsunterstützungen in mindestens zwei Funktionsbereichen sowie mindestens zehn Beratungskontakte während des Versuchszeitraums. Als Erfolgsindikatoren dienen das relative Umsatzwachstum, die Umsatzrentabilität, der Umsatz pro Mitarbeiter sowie die relative Veränderung der Anzahl der Beschäftigten. Diese Erfolgsgrößen werden zwischen der Beratungsgruppe und den beiden Testgruppen sowohl vor als auch nach Durchführung des Experiments verglichen. Während sich vor dem Experiment keine signifikanten Unterschiede ergaben, zeigten sich nach der Durchführung deutliche Abweichungen. Bei Vergleich der Veränderungen zwischen prä- und postexperimentellem Zeitpunkt zeigen sich signifikanten Unterschiede zur Kontrollgruppe bezüglich Umsatzwachstum, Umsatzrentabilität und Mitarbeiterzahl. Lediglich beim Umsatz pro Mitarbeiter kann kein signifikanter Unterschied ermittelt werden.

Robinson folgert aus den Ergebnissen seiner Untersuchung, dass strategische Planung auch für Kleinunternehmen bedeutsam ist und dass Geschäftsführer bzw. Eigentümer, die solche Aktivitäten aus Zeitmangel auslassen, Umsatz und Ertragskraft ihres Unternehmens schwächen. Da die Untersuchung zeigt, dass externe Berater die strategische Planung erfolgreich unterstützen können, wird ein solches Vorgehen für Kleinunternehmen empfohlen. Robinson schränkt allerdings ein, dass eine Reihe von Fragen in diesem Zusammenhang unbeantwortet bleiben - beispielsweise die optimale Intensität und Zeitdauer der Beratungsleistung. 


\section{Robinson und Pearce (1983)}

Robinson und Pearce untersuchen den Einfluss des Formalisierungsgrads der strategischen Planung auf die Ertragslage kleiner Banken. Ihre Stichprobe besteht aus 85 US-amerikanischen Geschäftsbanken, deren Einlagen kleiner als 25 Mio. US\$ sind und somit in dieser Branche als kleine Unternehmen angesehen werden können. Die Untersuchung wird in Form einer zweistufigen schriftlichen Befragung sowie eines nachfolgenden Telefoninterviews durchgeführt. Der Formalisierungsgrad der Planung wird durch einen Fragebogen gemessen, der bereits für eine Reihe früherer Studien verwendet wurde. Dieser fokussiert insbesondere auf den Umfang der Verschriftlichung der Planung sowie die darin enthaltenen Elemente. Auf Basis dieser Daten wird die Stichprobe in zwei Klassen eingeteilt: die „formal planners“ und die „non-formal planners”. Der Erfolg wird anhand der Umsatzrentabilität, der Gesamt- und Eigenkapitalrentabilität sowie des Wachstums des Kreditvolumens gemessen. Als Hypothese wird formuliert, dass die stärker formal planenden Unternehmen bei Betrachtung drei aufeinander folgender Jahre signifikant erfolgreicher sind als solche, die weniger formal planen. Diese Hypothese muss jedoch verworfen werden, da ein signifikanter Zusammenhang zwischen dem Formalisierungsgrad der strategischen Planung und dem monetären Erfolg eines Unternehmens nicht nachgewiesen werden kann. In der weiteren Untersuchung wird der Frage nachgegangen, wie dieses Ergebnis zu erklären ist. Eine Erklärung wird in der Charakteristik des strategischen Entscheidungsprozesses kleiner Unternehmen gesehen. Es wird angenommen, dass Planung in kleinen Unternehmen auch ohne Formalisierung durchführbar ist und durchgeführt wird. Um dies zu überprüfen, wird die Geschäftsführung der Unternehmen gebeten, die Wichtigkeit von sechs Elementen des strategischen Managements für den Entscheidungsprozess in ihrem Unternehmen zu beurteilen. Hierbei handelt es sich im Einzelnen um Umweltbeobachtungen, Zielformulierungen, das Erlangen besonderer Unternehmenskompetenzen, Ausrichten organisatorischer Strukturen, Ressourcenallokation sowie Kontrolle. Die Hypothese hierzu lautet, dass die Bedeutung der einzelnen Elemente von formal planenden Unternehmen nicht anders eingeschätzt wird als von nicht-formal planenden Unternehmen. Mit Ausnahme der Bedeutung der Zielformulierung zeigen sich keine signifikanten Abweichungen zwischen den beiden Gruppen, d. h. den Elementen des strategischen Managements wird in den beiden Klassen eine gleich große Bedeutung zugemessen. Dieses Ergebnis wird als Bestätigung dafür gesehen, dass eine Formalisierung der Planung und Zielvereinbarungen für kleine Unternehmen von nachrangiger Bedeutung sind. Abschließend betonen Robinson und Pearce jedoch, dass der Erfolg informeller Planung nicht so interpretiert werden sollte, dass weniger Planung notwendig ist, sondern in dem Sinne, dass ein geringerer Grad der Formalisierung ausreichend ist. 


\section{Ackelsberg und Arlow (1985)}

Ackelsberg und Arlow untersuchen den Zusammenhang zwischen Planung und Unternehmenserfolg. Ihre Studie basiert auf einer Stichprobe von 135 USamerikanischen Kleinunternehmen ${ }^{304}$ verschiedener Branchen, die schriftlich befragt wurden. Der Fragebogen dient zur Messung zum einen der analytischen und zum anderen der formalen Elemente der Planung. ${ }^{305}$ Als Erfolgsindikatoren dienen das Umsatz- und Gewinnwachstum der vergangenen drei Jahre. Als Hypothese wird zum einen formuliert, dass Planung und Unternehmenserfolg einen signifikant positiven Zusammenhang aufweisen und zum anderen dass dieser Zusammenhang für verschiedene Branchen signifikant unterschiedlich ist.

Die erste Hypothese kann grundsätzlich für beide Erfolgsgrößen bestätigt werden, wobei sich für das Umsatzwachstum eine höhere Signifikanz ergibt als für das Gewinnwachstum. Weiterhin wird eine Korrelationsanalyse zwischen dem Unternehmenserfolg und den beiden Skalen der Planung (analytische bzw. formale Planungsdimension) durchgeführt. Bezüglich des Zusammenhangs zwischen der analytischen Planungsdimension und dem Umsatzwachstum kann eine positive Korrelation festgestellt werden. Gleiches gilt für den Zusammenhang mit dem Gewinnwachstum. Für die Korrelation zwischen der formalen Planungsdimension und den beiden Erfolgsgrößen ergibt sich kein signifikanter Zusammenhang. Die erste Hypothese kann somit als grundsätzlich bestätigt angesehen werden. Für die zweite Hypothese kann lediglich eine partielle Unterstützung gefunden werden. Letzteres Ergebnis wird primär auf die geringe Stichprobengröße zurückgeführt.

Abschließend weisen Ackelsberg und Arlow auf den explorativen Charakter ihrer Untersuchung hin. Sie betonen die hohe Komplexität des Zusammenhangs von Planung und Unternehmenserfolg. Als weitgehend gesichert sehen sie die Erkenntnis an, dass der Formalisierungsgrad kein kritischer Einflussfaktor für den Erfolg von Kleinunternehmen ist.

\section{Orpen (1985)}

Orpen untersucht die Auswirkungen langfristiger Planung auf den finanziellen Erfolg kleiner Unternehmen ${ }^{306}$ unterschiedlicher Branchen. Die Stichprobe besteht

\footnotetext{
304 Als Abgrenzungskriterium wird die Definition der US-amerikanischen Small Business Administration (SBA) verwendet. Dieser liegt eine branchenspezifische Abgrenzung kleiner Unternehmen zugrunde. Die Obergrenzen liegen zwischen 100 und 1000 Beschäftigten bzw. zwischen 6 und 30 Mio US\$ Jahresumsatz (vgl. United States Small Business Adminstration 2004).

305 Unter der formalen Dimension der Planung wird die Schriftlichkeit und Vorhandensein von Budgets verstanden, unter der analytischen Dimension die Berücksichtigung eigener Stärken und Schwächen für die Planung, die Ausarbeitung mehrerer Handlungsalternativen sowie die regelmäßige Überarbeitung der Pläne.

306 Eine exakte Definition für Kleinunternehmen wird nicht angegeben.
} 
aus 58 Geschäftsführern australischer Kleinunternehmen verschiedener Branchen. Die Untersuchung erfolgt zweistufig. Zum einen müssen die teilnehmenden Geschäftsführer über einen Zeitraum von sechs Wochen ihre tägliche Arbeit protokollieren. Diese Tagebücher werden im Anschluss von unabhängigen Gutachtern mit dem Ziel ausgewertet, den Zeiteinsatz für langfristige Planung zu bestimmen. Zum anderen werden die Geschäftsführer direkt schriftlich befragt. Hierbei werden sie einerseits gebeten, zu schätzen, welchen Zeitanteil ihrer Arbeitszeit sie für langfristige Planung verwenden, andererseits, Angaben zur Organisation der Planungsfunktion, zu ihrer Einstellung zur Planung sowie zu den Inhalten und Problemen zu machen. Als Erfolgsindikatoren werden das Umsatzwachstum und die Gesamtkapitalrentabilität verwendet. Auf Basis dieser Angaben werden die Firmen in erfolgreiche (Erfolgsindikatoren oberhalb des Median) und weniger erfolgreiche (Erfolgsindikatoren unterhalb des Median) gruppiert.

Das erste zentrale Ergebnis der Analyse ist, dass keine signifikanten Unterschiede zwischen den beiden Gruppen bezüglich des Zeitaufwands für langfristige Planung festzustellen sind. Abweichungen bestehen jedoch bei der Mehrzahl der darüber hinaus erhobenen Planungsdimensionen. Es zeigt sich, dass erfolgreiche Unternehmen einen höheren Formalitätsgrad in ihrer langfristigen Planung und eine positivere Einstellung hierzu aufweisen. Auch führen sie häufiger Prämissenkontrollen und Wettbewerberanalysen durch. Schließlich ist auch ihr Planungshorizont länger und ihr Planungsinhalt breiter. Orpen folgert hieraus, dass es weniger auf den zeitlichen Aufwand für langfristiger Planung ankommt als vielmehr auf ihre Qualität.

\section{Robinsion, Logan und Salem (1986)}

Die Studie von Robinsion, Logan und Salem untersucht die Auswirkungen operativer im Vergleich zu strategischer Planung auf den Unternehmenserfolg kleiner Einzelhändler. Hierzu befragen sie schriftlich 81 unabhängige, US-amerikanische Einzelhandelsunternehmen. Die Unternehmen der Stichprobe weisen durchschnittlich einen Umsatz von 1,97 Mio. US\$ auf und beschäftigen im Mittel 12 Vollzeit- und 9 Teilzeitkräfte. Die Intensität der operativen Planung wird in die Funktionsbereiche Finanzen, Marketing, Personal und Lagerhaltung differenziert. Als Indikatoren für den Unternehmenserfolg werden das Umsatzwachstum, die relative Veränderung der Umsatzrentabilität sowie der Umsatz pro Mitarbeiter der Jahre 1981 und 1983 verwendet. Darüber hinaus werden die Geschäftsführer nach ihrer persönlichen (subjektiven) Wahrnehmung des Unternehmesserfolgs gefragt.

Generell zeigen die Daten, dass operative Planung von den Geschäftsführern der entsprechenden Unternehmen als wichtiger eingeschätzt wird als die strategische Planung. 63\% der Befragten halten operative Planung für „wichtig“ bzw. „sehr wichtig“, während der strategischen Planung nur 28\% einen solchen Bedeu- 
tungsgrad zumessen. Bei $65 \%$ der Unternehmen wird eine hohe operative Planungsaktivität im Bereich des Marketings festgestellt. Für den Bereich Lagerhaltung ergibt sich eine Quote von 54,8\%, für den Bereich Finanzen eine Quote von 49,4\% und für den Personalbereich eine Quote von 37\%. Lediglich 15,6\% führen Planungen auf strategischer Ebene durch.

Beim Vergleich zwischen Unternehmen, die in allen vier Funktionsbereichen eine detaillierte Planung durchführen, und den Unternehmen, die dies nicht tun, zeigen sich für die erste Gruppe bei drei der vier Erfolgsindikatoren (Veränderung der Umsatzrentabilität, Umsatz pro Mitarbeiter und subjektive Erfolgseinschätzung durch den Geschäftsführer) signifikant bessere Werte. Bezüglich des Umsatzwachstums konnten keine signifikanten Unterschiede festgestellt werden. Beim Vergleich zwischen Unternehmen mit hoch entwickelter operativer Planung in allen Funktionsbereichen, die darüber hinaus über eine strategische Planung verfügen, mit Unternehmen auf die dies nicht zutrifft, zeigen sich ähnliche Ergebnisse wie bei vorhergehenden Tests. Um die Wirkung der strategischen Planung zu isolieren, wird außerdem ein Vergleich zwischen Unternehmen mit und ohne strategische Planung durchgeführt. Bei diesem Test zeigt sich keiner der objektiven Erfolgsindikatoren signifikant abweichend. Lediglich die subjektive Einschätzung der Geschäftsführer ist bei strategisch planenden Unternehmen signifikant höher. Eine mögliche Erklärung in der Abweichung zwischen objektiven und subjektiven Erfolgsgrößen vermuten Robinsion, Logan und Salem darin, dass die subjektive Einschätzung in höherem $\mathrm{Maß}$ eine langfristige Sichtweise widerspiegelt und so der strategischen Planung besser entspricht.

\section{Shuman und Seeger (1986)}

Shuman und Seeger untersuchen die Unterschiede zwischen normativen Modellen der strategischen Planung und deren praktische Umsetzung in kleinen, schnell wachsenden Unternehmen. Ihre Analyse basiert auf einer Befragung von 220 der 500 am schnellsten wachsenden US-amerikanischen Unternehmen (bezogen auf den Zeitraum von 1978 bis 1982), deren Umsatz 1978 zwischen 100.000 und 25 Mio. US\$ lag (vgl. zur Erhebung auch Shuman/Shaw/Sussman 1985). Die Untersuchung kommt zu folgenden Ergebnissen:

- Während nur etwa die Hälfte der Unternehmen zum Zeitpunkt ihrer Gründung über eine formale Planung verfügte, wurde diese mehrheitlich im laufenden Geschäftsbetrieb eingeführt.

- Mit wachsendem Umsatz steigt der Formalisierungsgrad der Planung. Dennoch sind die Planungssysteme der Praxis i. d. R. weit weniger hoch entwickelt als dies in der Fachliteratur empfohlen wird. 
- Ca. 66\% der befragten Unternehmen fokussieren ihre strategische Planung auf den Absatzbereich, ca. 20\% auf betriebliche Abläufe, lediglich 4\% auf den Finanzbereich.

- Die Mehrheit der Geschäftsführer bevorzugt selbst eine aktive Rolle im Planungsprozess, statt diese Verantwortlichkeit zu delegieren.

- Ca. 15\% der Unternehmen verfügen über eine separate Planungsabteilung, die typischerweise über 2-4 Mitarbeiter verfügt.

- Die in der Literatur genannten Elemente der strategischen Planung 307 finden sich auch in kleinen Unternehmen wieder.

Es stellt sich die zentrale Frage, ob die höhere Formalisierung und Intensität der Planung durch die steigenden Anforderungen aufgrund des Unternehmenswachstums verursacht werden oder ob das Wachstum freie Ressourcen generiert, die eine verbesserte Planung ermöglichen. Aus der statistischen Analyse der Daten lassen sich hierüber keine Aussagen machen. Shuman und Seeger argumentieren, dass Interviews, die sie ergänzend zur Studie durchführten, eher die zweite Alternative unterstützen. Ein signifikanter Zusammenhang zwischen dem Planungsprozess und dem Wachstum des Umsatzes bzw. des Umsatzes pro Mitarbeiter konnte nicht gefunden werden. Während Studien typischerweise den direkten Zusammenhang zwischen Planung und Unternehmenserfolg untersuchen, schlagen sie vor, freie Ressourcen als intervenierende Variable zu berücksichtigen. Hieraus leiten sie die Handlungsempfehlung ab, dass es notwendig ist, ein Teil der durch Wachstum frei werdenden Ressourcen für Planungsanstrengungen zur Verfügung zu stellen.

\section{Boag (1987)}

Die Untersuchung von Boag befasst sich mit dem Einfluss des MarketingControlling auf den Markterfolg junger Wachstumsunternehmen aus dem HighTech-Sektor. Auf Basis einer konzeptionellen Untersuchung schlägt er ein vierstufiges Modell zur Charakterisierung des Entwicklungstands des MarketingControlling vor. Mit diesem Modell gruppiert er eine Stichprobe von 20 kanadischen Unternehmen der genannten Branche, die zwischen 15 und 150 Beschäftigte haben. Es zeigen sich signifikante Unterschiede zwischen den vier Modellkategorien bezüglich der absoluten Werte für Umsatz und Umsatzwachstum sowie für Firmenalter und Beschäftigtenzahl. Boag betont, dass es seine Untersuchung primär explorativ und deskriptiv ist. Dennoch sieht er in seinen Ergebnissen ein In-

\footnotetext{
307 Hierbei handelt es sich um folgende Elemente: Grundsatzentscheidung zur Planung, Situationsanalyse, persönliche und unternehmerische Ziele, Problemdefinition, Entwicklung von Handlungsalternativen, Bewertung und Auswahl der Alternativen, Implementation, Kontrolle und Rückkopplung.
} 
diz, dass bestimmte Elemente eines formalen Steuerungssystems das Wachstum in Kleinunternehmen begünstigen könnten.

\section{Smith et al. (1988)}

In der Untersuchung von Smith et al. werden Unterschiede im Entscheidungsverhalten von Unternehmern in kleineren Firmen und professionellen Managern in größeren Firmen betrachtet. Die Basis der Untersuchung ist das Konzept der analytischen Entscheidung („comprehensive decision making ${ }^{\circ}$ ). Hierunter wird ein intensiv analysierender, rationaler Prozess verstanden, bei dem systematisch die Problemursache bestimmt und Handlungsalternativen entwickelt und bewertet werden. Im Allgemeinen wird angenommen, dass ein solches Verhalten eher bei professionellen Managern als bei Unternehmern zu finden ist. Die Persönlichkeit des Unternehmers wird dagegen typischerweise als entscheidungsfreudig und individualistisch beschrieben. Es stellt sich die Frage, wie diese Charakteristika auf das Entscheidungsverhalten wirken. Obwohl im Allgemeinen die Vorteile der analytischen Entscheidung betont werden, könnte es auch Situationen geben, in denen sie kontraproduktiv ist. Fredrickson und Mitchell (1984) legen dar, dass analytische Entscheidungsprozesse bei unstabilen Umweltbedingungen dysfunktional sein können, da sie zu zeit- und kostenintensiv sind.

Um diesen Zusammenhang tiefer zu ergründen, befragen Smith et al. mündlich 28 Unternehmer bzw. Manager der Elektronikindustrie. Auf Basis der Unternehmensgröße und der persönlichen Auffassung der entsprechenden Personen werden diese in kleine, unternehmergeführte und große, professionell geführte Unternehmen eingeteilt. Die Akteure werden bezüglich ihres Entscheidungsverhaltens befragt und auf dieser Basis in die Kategorien ,stark analytische Entscheider“" und „schwach analytische Entscheider“ eingeteilt. Mit diesen beiden Dimensionen wird eine 2x2-Matrix gebildet und eine zweidimensionale Varianzanalyse durchgeführt. Untersuchungsgegenstand sind hierbei das Umsatzwachstum, die Umsatzrentabilität, die Gesamtkapitalrentabilität sowie ein aggregierter Gesamtwert für die drei Erfolgsgrößen.

Es zeigt sich, dass analytische Entscheider den nicht-analytischen Entscheidern bezüglich der Umsatzrentabilität, der Kapitalrentabilität sowie des Gesamterfolgs signifikant überlegen sind. Zwischen kleineren und größeren Unternehmen konnte kein signifikanter Unterschied festgestellt werden. Auch zeigten sich statistisch keine Wechselwirkungen zwischen den beiden Dimensionen. Dies bedeutet, dass analytische Entscheidungen unabhängig von der Unternehmensgröße zu bevorzugen sind und somit auch in Inhabergeführten Unternehmen stärker zum Einsatz kommen sollten. 


\section{Bracker, Keats und Pearson (1988)}

Bracker, Keats und Pearson untersuchen den Zusammenhang zwischen dem Entwicklungsgrad des Planungssystems und dem finanziellen Erfolg von kleinen Unternehmen in Wachstumsbranchen, die mindestens fünf Jahre am Markt bestehen konnten. Die Studie basiert auf einer Stichprobe von 97 US-amerikanischen Unternehmen der Elektroindustrie. Diese bestehen im Mittel seit 9,2 Jahren, weisen einen durchschnittlich Umsatz von 4,25 Mio. US\$ auf und beschäftigen durchschnittlich 71 Mitarbeiter. Als Indikatoren für den Unternehmenserfolg werden das Umsatzwachstum, das Gewinnwachstum, das Wachstum des Unternehmenswerts sowie der Gehaltszuwachs des Geschäftsführers über einen Zeitraum von fünf Jahren verwendet. Der Entwicklungsgrad des Planungssystems wird durch eine Klassifikation in drei Kategorien von Planern ${ }^{308}$ gemessen: strukturiert strategisch planende Unternehmen, strukturiert operativ planende Unternehmen sowie unstrukturiert planende Unternehmen. Als Hypothese wird erstens formuliert, dass sich ein hoher Entwicklungsgrad des Planungssystems positiv auf den Unternehmenserfolg auswirkt, und zweitens, dass Erfolgsunterschiede zwischen Unternehmen mit länger Planungstradition und solchen mit kürzerer Planungstradition bestehen. Eine Varianzanalyse kann die erste Hypothese für drei der vier Erfolgsindikatoren bestätigen: strukturiert strategisch planende Unternehmen sind signifikant erfolgreicher als die Unternehmen der anderen beiden Kategorien. Die zweite Hypothese musste im Rahmen der Varianzanalyse dagegen verworfen werden. Betrachtet man jedoch ausschließlich Unternehmen mit langer Planungstradition, wird der Unterschied zwischen strukturiert strategisch planenden Unternehmen und den anderen Kategorien noch deutlicher. Hieraus wird gefolgert, dass eine längere Planungstradition nur dann positiv wirkt, wenn ein hoch entwickeltes Planungssystem vorliegt. Bracker, Keats und Pearson (1988, S. 601) interpretieren ihre Ergebnisse zusammenfassend wie folgt: "It appears from the study that planning for the sake of planning does not lead to increased financial performance. What does seem important is the type of planning process employed, and how long the firm has been planning."

\section{Cragg und King (1988)}

Cragg und King untersuchen den Zusammenhang zwischen organisatorischen Charakteristika und finanziellem Erfolg in Kleinunternehmen unter Berücksichtigung intermediärer Faktoren. Sie stellen fest, dass die in früheren Studien gefundenen Zusammenhänge zwischen einzelnen organisatorischen Charakteristika und dem Unternehmenserfolg den Einfluss korrelierender Variablen vernachlässigen. Als Beispiel führen sie das Alter des Unternehmers ein: Hat eine Studie einen po-

308 Zur Operationalisierung dieser Kategorien vgl. Bracker/Pearson 1986. 
sitiven Zusammenhang zwischen dem Einsatzgrad von Planungsinstrumenten und dem monetären Erfolg identifiziert, könnte hierbei vernachlässigt worden sein, dass insbesondere jüngere Unternehmer moderne Planungsverfahren einsetzen. Es ist daher im Einzelnen zu untersuchen, wie die Interdependenzen zwischen den Einflussfaktoren sind.

Für ihre eigene Untersuchung verwenden Cragg und King eine Stichprobe mit 157 britischen Unternehmen der metallverarbeitenden Industrie, die weniger als 50 Mitarbeiter beschäftigen. Diese werden mit Hilfe eines schriftlichen Fragebogens untersucht. Zur Operationalisierung der zu messenden Konstrukte werden Fragen verwendet, die bereits in früheren Studien eingesetzt wurden, um eine Vergleichbarkeit zu gewährleisten. Als Indikatoren für den Unternehmenserfolg werden die Umsatzrentabilität, die quantitative Veränderung der des Umsatzes auf Jahres- und Fünfjahresbasis sowie die Tendenz der Veränderung des Gewinns auf Jahresbasis verwendet.

Eine Analyse der Korrelationen zwischen den gemessenen unabhängigen Variablen und dem Unternehmenserfolg zeigten nur vereinzelt signifikante Ergebnisse. Bezogen auf die Planungsaktivitäten wurden keinerlei positive Korrelationen festgestellt; in zwei Fällen zeigten sich sogar negative Korrelationen. Auch bei den anderen vermuteten Einflussfaktoren (u. a. Alter des Unternehmers) zeigten sich widersprüchliche Ergebnisse. Bestätigt sehen sich Cragg und King in ihrer Forderung, in zukünftigen Studien bestimmte Kontrollvariablen zu berücksichtigen. In ihrem Fazit betonen sie, dass es außerdem notwendig ist, die Richtung des Zusammenhangs zweier Größen zu untersuchen: „For example, does the use of formal planning methods cause good performance, or does good performance enable or encourage a firm to adopt formal methods?"“ (Cragg/King 1988, S. 60). Sie führen aus, dass der Aufbau eines Kausalmodells nur durch Langzeitstudien realisierbar sei.

\section{Shrader, Mulford und Blackburn (1989)}

Die Studie von Shrader, Mulford und Blackburn analysiert die Zusammenhänge zwischen operativer und strategischer Planung und dem Unternehmenserfolg. Hierzu wird eine geschichtete Stichprobe mit 97 US-amerikanischen Unternehmen verwendet, deren Beschäftigtenzahl zwischen 10 und 100 liegt, von denen 31 im produzierenden Gewerbe, 35 im Einzelhandel und 31 in anderen Dienstleistungsbranchen tätig sind. Adressaten der schriftlichen Befragung sind die Geschäftsführer der Unternehmen. Der Grad der Planung auf strategischer Ebene wurde mit Hilfe einer von Wood und Laforge (1981) vorgeschlagenen ordinalen Skala gemessen und die Unternehmen entsprechend in drei Klassen kategorisiert. Der Detaillierungsgrad der operativen Planung wird separat für folgende Bereiche gemessen: Absatz, Budget, Lagerbestand, Personal und Marketing. Als Erfolgsmaß werden Umsatz, Mitarbeiteranzahl und Nachsteuergewinn für drei aufeinan- 
der folgende Jahre verwendet. Die deskriptive Analyse der Daten zeigt, dass die Mehrheit der Unternehmen auf eine formale strategische Planung verzichtet. Die wichtigsten Gründe hierfür sind Zeitmangel, mangelnde Qualifikation sowie zu hohe Kosten. Auf Basis der zum Untersuchungszeitpunkt vorliegenden Literatur werden $u$. a. folgende Hypothesen formuliert:

- Hypothese 1: Operative Planung wird in kleinen Unternehmen häufiger verwendet als strategische Planung.

- Hypothese 2: Der Formalisierungsgrad der strategischen Planung hat keinen Einfluss auf den Erfolg kleiner Unternehmen.

- Hypothese 3: Die operative Planung hat einen positiven Einfluss auf den Erfolg eines Kleinunternehmens.

Die Signifikanz wird auf Basis einer Korrelationsanalyse überprüft. Eindeutig bestätigt werden kann nur die erste Hypothese. Bei den anderen beiden Zusammenhängen zeigt sich ein wechselhaftes Bild: Die Korrelationen bzw. ihre Signifikanz ist unterschiedlich in Abhängigkeit von der Branche, dem Bereich der operativen Planung, der verwendeten Erfolgsgröße sowie dem Betrachtungzeitpunkt. Shrader, Mulford und Blackburn folgern, dass der Zusammenhang zwischen Planung und Erfolg für kleine Firmen sehr komplex ist. Obwohl ihre Ergebnisse die Eignung operativer Planung für Kleinunternehmen teilweise bestätigen konnten, gestehen sie ein, dass strategische Planung unter bestimmten Bedingungen wertvoll für diese Unternehmen sein kann. Weiterhin stellen sie fest, dass strategische Planung in nahezu allen Unternehmen allein durch den Geschäftsführer durchgeführt wird, wodurch diese - aufgrund der Zeitintensität - zu einer „alles oder nichts“ Angelegenheit wird. Für die operative Planung identifizieren sie die Absatzplanung als wichtigstes Element.

\section{Duchesneau und Gartner (1990)}

Die Studie von Duchesneau und Gartner verfolgt das Ziel, Faktoren für den Erfolg junger Unternehmen zu identifizieren. Als Hauptfaktoren werden auf Basis einer Auswertung früherer Untersuchungen die Eigenschaften des Unternehmers, das Gründungsverhalten sowie die Unternehmensstrategie angenommen. Diese Hauptfaktoren werden durch jeweils mehrere Einzelfaktoren repräsentiert. Als Einzelfaktoren des Gründungsverhaltens werden unter anderem die Nutzung umfassender Planungsprozesse, der zeitliche Aufwand der Planung sowie die Planungsbreite (gemessen an der Anzahl einbezogener Funktionsbereiche) verwendet.

Grundlage der Studie ist eine Stichprobe von 26 jungen, US-amerikanischen Unternehmen der Frischsaftdistributionsbranche, von denen 13 erfolgreich operieren und 13 mit ihrer unternehmerischen Tätigkeit gescheitert sind. Die betrachteten 
Unternehmen weisen Umsätze zwischen 200.000 und 6.000.000 US\$ auf. Die Befragung erfolgt sowohl schriftlich als auch mündlich; es werden sowohl quantitative als auch qualitative Daten erhoben.

Alle drei zuvor genannten Einzelfaktoren zeigen signifikante Unterschiede zwischen den beiden Kategorien. Dies bedeutet, dass sowohl die Strukturiertheit der Planung, der Zeitaufwand hierfür als auch die Breite der Planung positiv auf den Erfolg eines jungen Unternehmens wirken. Die drei Größen weisen untereinander eine deutliche Korrelation auf. Betrachtet man die Korrelationen dieser mit den anderen in der Studie erhobenen Faktoren, zeigt sich, dass die planungsbezogenen Faktoren außerdem deutliche Korrelationen mit der Neigung der Unternehmers zur Risikovermeidung und der Klarheit der Geschäftsidee zu verzeichnen sind. Eine mögliche Schlussfolgerung hieraus ist, dass die unternehmerischen Planungstätigkeit wesentlich durch die persönlichen Eigenschaften des Unternehmers determiniert werden.

\section{Lyles et al. (1993)}

Lyles et al. untersuchen den Zusammenhang zwischen Formalisierungsgrad, Inhalten und Entscheidungsprozessen in der strategischen Planung in Kleinunternehmen. Die Studie basiert auf strukturierten Interviews mit 188 US-amerikanischen Kleinunternehmen verschiedener Branchen, die weniger als 500 Beschäftigte haben, jedoch einen Jahresumsatz von mindestens 1 Mio. US\$.

Unter strategischen Entscheidungsprozessen wird in dieser Untersuchung das Durchlaufen bestimmter idealtypischer Teilschritte in der Entscheidungssituation verstanden (vgl. hierzu auch Robinson/Pearce 1983). Hierbei handelt es sich im Einzelnen um folgende Elemente: Umweltbeobachtungen, Zielformulierungen, das Erlangen besonderer Unternehmenskompetenzen, Ausrichten organisatorischer Strukturen, Ressourcenallokation sowie Kontrolle. Es wird argumentiert, dass eine formalisierte Planung (gemessen durch z. B. die Schriftlichkeit der Planung) nicht notwendigerweise zu einem umfassenderen Entscheidungsprozesses führt, weshalb diese beiden Konstrukte separat betrachtet werden. Dennoch wird ein positiver Zusammenhang zwischen beiden Konstrukten vermutet (Hypothese 1). Bezüglich der Relation von Strategieinhalt und Formalisierungsgrad wird angenommen, dass formale Planung zu einer Berücksichtigung eines breiteren Spektrums strategischer Optionen führt (Hypothese 2). Schließlich wird angenommen, dass Formalisierung zu einer höheren Effektivität führt, d. h. formale Planung mit einer höheren Eigen- und Gesamtkapitalrentabilität und einem größeren Umsatzwachstum assoziiert ist (Hypothese 3).

Eine erste Auswertung der Daten zeigt, dass sich Unterschiede zwischen formal und nicht-formal planenden Unternehmen ergeben. Während erstere durch- 
schnittlich 101 Beschäftigte und einen Umsatz von 9 Mio. US\$ aufweisen, beschäftigen letztere im Mittel lediglich 47 Mitarbeiter und generieren einen Umsatz von 6 Mio. US\$. Somit zeigt auch diese Untersuchung, dass der Formalisierungsgrad mit wachsender Größe des Unternehmens zunimmt.

Bezüglich der ersten Hypothese kann für fünf der sechs Teilschritte des strategischen Planungsprozesses (Ausnahme ist die Umweltbeobachtung) bei formal planenden Unternehmen eine signifikante Betonung dieser Elemente festgestellt werden. Auch wenn die erste Hypothese damit grundsätzlich bestätigt wird, müssen einschränkend die zuvor genannten Größenunterschiede zwischen den beiden Gruppen beachtet werden.

Die Analyse der Unterschiede, die die beiden Gruppen einzelnen Strategien beimessen, zeigt, dass die formal planenden Unternehmen bei nahezu allen Strategieoptionen höhere Werte aufweisen. Dieses wird dahingehend interpretiert, dass formal planende Unternehmen eine größere Breite von Strategiealternativen in ihre Planung einbeziehen.

Bezüglich der Gesamt- und Eigenkapitalrentabilität zeigen sich keine signifikanten Unterschiede zwischen den beiden Gruppen. Jedoch ist das durchschnittliche Umsatzwachstum bei formal planenden Unternehmen signifikant höher als bei der anderen Gruppe. Zu berücksichtigen ist hierbei, dass sich dieses Ergebnis nur auf die 67 Unternehmen bezieht, die in der Befragung bereit waren, entsprechende Informationen zur Verfügung zu stellen.

\section{McKiernan und Morris (1994)}

McKiernan und Morris untersuchen den Zusammenhang zwischen dem Formalisierungsgrad der strategischen Planung und dem langfristigen finanziellen Erfolg von Kleinunternehmen. Ihre Studie basiert auf einer schriftlichen Befragung im Jahr 1980 und einer Sekundäranalyse der Finanzdaten mit Hilfe einer externen Datenbank für den Zeitraum von 1983-1987 von 100 zufällig ausgewählten Kleinunternehmen verschiedener Branchen mit weniger als 500 Beschäftigten. Die Unternehmen wurden auf Basis der Selbsteinschätzung des Geschäftsführers in formal und nicht-formal planende Unternehmen differenziert. Zur Unterstützung der Selbsteinschätzung wurden den Befragten Beschreibungen der beiden Planungstypen und Fragen, die die Elemente der beiden Kategorien betonten, vorgelegt. Als Messgröße für den Erfolg dienen das Umsatzwachstum, die Umsatzrentabilität, die Gesamt- und Eigenkapitalrentabilität sowie der Umsatz pro Mitarbeiter. Für alle Größen wird der Durchschnitt für den Zeitraum von 1983 bis 1987 verwendet.

Zur Auswertung werden die Unternehmen auf der Basis des Mittelwerts aller Unternehmen in über- und unterdurchschnittlich erfolgreiche gruppiert. Sowohl für die gesamte Stichprobe als auch für die nach Branchen gegliederten Teilstich- 
proben werden für jede Erfolgsgröße Chi-Quadrat-Tests durchgeführt. Von diesen insgesamt zwanzig Tests, die den Zusammenhang zwischen Planung und Erfolg prüfen, ist nur einer signifikant. Somit findet sich in der analysierten Stichprobe kein Zusammenhang zwischen dem Formalisierungsgrad der strategischen Planung und dem finanziellen Erfolg der Unternehmen. Um ihr Ergebnis, das von der Mehrzahl früherer Befunde abweicht, zu erklären, vermuten sie, das die Erfolgswirkung formaler Planungssystem nur bei sehr dynamischen Umweltbedingungen feststellbar ist.

\section{Olson und Bokor (1995)}

Die Studie von Olson und Bokor untersucht die Wechselbeziehung zwischen dem Prozess und dem Inhalt strategischer Planung und dessen Wirkung auf das Umsatzwachstum in Start-Up-Unternehmen. Der Ausgangspunkt ihrer Untersuchung ist die Feststellung, dass frühere Untersuchungen sich entweder mit dem Prozess oder mit dem Inhalt strategischer Planung auseinandergesetzt haben, dass die Wechselwirkungen zwischen beiden Dimensionen jedoch vernachlässigt wurden. Genau einen solchen Zusammenhang vermuten Olson und Bokor jedoch. Zu Untersuchung dieser Hypothese werden die 500 am schnellsten wachsenden USamerikanischen Kleinunternehmen (bezogen auf den Zeitraum von 1982-1986) angeschrieben. Nachdem von den antwortenden Unternehmen diejenigen ausgeschlossen wurden, die älter als acht Jahre sind, ergibt sich eine Stichprobe von 89 Unternehmen verschiedener Branchen.

Die Prozessdimension wird durch Fragen zum Formalisierungsgrad der Planung gemessen. Für die Auswertung der Daten werden die Stufen „keine formale Planung" und „formale Planung" unterschieden. Bezüglich der Inhaltsdimension werden die Geschäftsführer nach der grundlegenden Wettbewerbsstrategie zum Zeitpunkt der Unternehmensgründung befragt. In dieser Dimension wird zwischen einer Innovationsstrategie (Angebot eines Produkts, das dem Kunden in mehrfacher Hinsicht einen deutlichen Mehrwert bietet) und einer Imitationsstrategie (Nachahmung eines bestehenden Produkts) unterschieden.

Zur Auswertung wird ein lineares Regressionsmodell mit (Dummy-)Variablen für die Prozessdimension, für die Inhaltsdimension sowie für das Kreuzprodukt beider Dimensionen verwendet. Von diesen zeigt sich nur der letzte Parameter signifikant. Auch liegt das Bestimmtheitsmaß des Modells lediglich bei 0.089. Dennoch sehen Olson und Bokor ihre Hypothese in der Tendenz bestätigt und fordern daher, dass in zukünftigen Untersuchungen stets beide Dimensionen gemeinsam untersucht werden sollten. Zur weiteren Analyse stellen sie das Umsatzwachstum in Abhängigkeit von den beiden Dimensionen in einer 2x2-Matrix dar. Für Unternehmen mit einer Innovationsstrategie führt formale Planung zu deut- 
lich ${ }^{309}$ höheren Umsatzzuwächsen. In Unternehmen mit Imitationsstrategie scheint die Wirkung dagegen genau anders herum: Imitatoren ohne formale Planung weisen im Mittel einen höheren Umsatzzuwachs auf als Imitatoren mit formaler Planung. Da dieses Ergebnis im Widerspruch zur Erwartung bzw. Literaturmeinung steht, wird versucht das Ergebnis durch das Vorhandensein anderer Kontextfaktoren zu erklären. Eine folgende Analyse des Einflusses von Erfahrung und Motivation der Geschäftsführer kann jedoch nicht zur Aufklärung des Widerspruchs beitragen.

\section{Berman, Gordon und Sussman (1997)}

Berman, Gordon und Sussman untersuchen den Nutzen höher entwickelter Planungssysteme für das Umsatzwachstum in Kleinunternehmen. Die Basis der Untersuchung ist eine schriftliche Befragung von 161 US-amerikanischen Kleinunternehmen $^{310}$ verschiedener Branchen. In der Befragung werden vier Entwicklungsstufen von Planungssystemen („Krisenmanagement“, „Planung durch Budgets“, „langfristige Planung“, „strategische Planung“) verbal beschrieben und die Befragten gebeten, die Beschreibung zu identifizieren, die auf ihr Unternehmen am besten zutrifft. Zur Auswertung werden die Unternehmen lediglich in den aggregierten „Unternehmen mit schwach entwickelten Planungsprozessen“ und „Unternehmen mit hoch entwickelten Planungsprozessen" differenziert. Die Auswertung zeigt eine signifikante Korrelation zwischen der Entwicklungsstufe des Planungssystems und dem Umsatzwachstum.

\section{Rue und Ibrahim (1998)}

Rue und Ibrahim untersuchen den Ausbaugrad des Planungssystems und dessen Auswirkungen auf den Erfolg kleiner Unternehmen. Die Untersuchung basiert auf einer schriftlichen Befragung von 253 US-amerikanischer Unternehmen verschiedener Branchen mit einer Beschäftigtenzahl zwischen 15 und 500. Befragt werden die Unternehmen danach, ob sie eine schriftliche strategische Planung durchführen und falls dies zutrifft, welche Größen darin quantitativ geplant sind und welche externen Faktoren beobachtet werden. Erhoben wird weiterhin, ob die Planung Maßnahmen zum Erkennen und Behandeln von Planabweichungen enthält. Als Maßgröße für den Unternehmenserfolg dienen die Tendenz der Umsatzentwicklung der zurückliegenden drei Jahre, das quantifizierte Umsatzwachstum des vorhergehenden Jahres sowie der Return on Investment (ROI) des vorhergehenden Jahres.

Zur Auswertung werden die Unternehmen entsprechend ihrer Planungsaktivitäten in drei Gruppen kategorisiert. Unternehmen der ersten Kategorie (39,9\% der

\footnotetext{
${ }^{309}$ Ein Signifikanztest der Abweichungen wird - unverständlicherweise - nicht durchgeführt.

310 Eine exakte Definition für Kleinunternehmen wird nicht angegeben.
} 
Unternehmen) verfügen über keinerlei schriftliche strategische Planung; Unternehmen der zweiten Kategorie (35,2\%) verfügen über ein Planungssystem mittleren Ausbaugrades, d. h. die führen eine schriftliche strategische Planung durch, die mindestens einige quantitative Element enthält; Unternehmen der dritten Kategorie $(24,9 \%)$ verfügen über ein hoch entwickeltes Planungssystem, das über quantifizierte Zielgrößen, Budgets, Umweltbeobachtungen und Kontrollmechanismen umfasst.

Die Untersuchung des Zusammenhangs zwischen der Tendenz der Umsatzentwicklung im Branchenvergleich und dem Ausbaugrad des Planungssystems zeigt ein schwach signifikantes Ergebnis. Eine Anwendung der gleichen Methodik, in der das Umsatzwachstum des vergangen Jahres als Erfolgsindikator verwendet wird, zeigt ebenfalls signifikante Unterschiede. Bezüglich des Zusammenhangs zwischen Ausbaugrad des Planungssystems und Return on Investment kann dagegen kein signifikanter Zusammenhang identifiziert werden. Eine positive Wirkung des Ausbaugrades des Planungssystems konnte somit für das Umsatzwachstum nachgewiesen werden, nicht jedoch für die Kapitalrentabilität. Rue und Ibrahim sehen in diesem Ergebnis zwei Interpretationsmöglichkeiten. Eine mögliche Erklärung liegt in einer potenziellen zeitlichen Differenz zwischen Umsatzsteigerung und Rentabilitätswirkung. Möglich ist es jedoch auch, dass Unternehmen mit besserem Planungssystem zwar höhere Umsätze erzielen, diese jedoch aufgrund unternehmensinterner Ineffizienzen nicht in höheren Gewinn umsetzen können. Abschließend wird eingeschränkt, dass die Studie zwar Zusammenhänge nachweisen konnte, hieraus jedoch nicht auf Kausalitäten geschlossen werden kann: "The question is: did a sophisticated planning process lead to higher sales? Or, did higher sales encourage the top executives to engage in a more sophisticated planning process?” (Rue/Ibrahim 1998, S. 30).

\section{Lumpkin, Shrader und Hills (1998)}

Lumpkin, Shrader und Hills untersuchen den Zusammenhang zwischen formaler Planung in verschiedenen Funktionsbereichen und verschiedenen Erfolgsdimensionen in Kleinunternehmen. Ihre Studie basiert auf einer schriftlichen Befragung von 94 amerikanischen Kleinunternehmen ${ }^{311}$. Zur Analyse werden diese in neue ${ }^{312}$ und etablierte Unternehmen gruppiert. Zur Messung der Planungsaktivitäten werden die Befragten gebeten, die Wichtigkeit von Marktanalysen, Wettbewerberanalysen, Absatzplanung, Organisationsplanung und Finanzplanung auf einer Sieben-Punkt-Skala zu bewerten. Als Indikatoren für den Unternehmenserfolg dienen das Umsatzwachstum, die Umsatzrentabilität, der Rohertrag sowie die Finanzkraft (Liquidität und Fähigkeit zur Kapitalbeschaffung). Die Befragten werden gebeten, die Entwicklung dieser drei Größen relativ zur Entwicklung ihrer Wettbewerber zu beurteilen.

${ }^{311}$ Eine exakte Definition für Kleinunternehmen wird nicht angegeben.

312 Hierunter werden Unternehmen verstanden, die weniger als sieben Jahre Geschäftstätigkeit aufweisen. 
Eine Korrelationsanalyse zwischen den Planungsaktivitäten und den Erfolgsgrößen zeigt kaum signifikante Ergebnisse. Lediglich zwischen der Finanzplanung und der Finanzkraft kann eine schwach signifikante Korrelation festgestellt werden. Im Weiteren werden für die beiden Gruppen (neue und etablierte Unternehmen) schrittweise multiple Regressionsanalysen durchgeführt. Für junge Unternehmen zeigen sich schwach signifikante Zusammenhänge zwischen Finanzplanung einerseits sowie Finanzkraft und Rohertrag andererseits. Für Wettbewerberanalysen ergibt sich erstaunlicherweise sogar eine negative Wirkung auf die Finanzkraft. In etablierten Unternehmen sind Wettbewerberanalysen dagegen das einzige Planungsgebiet, das positiv mit einer Erfolgsdimension (Finanzkraft) assoziiert ist.

\section{Rauch und Frese (1998)}

Rauch und Frese untersuchen den moderierenden Effekt eines feindlichen und unsicheren Wettbewerbsumfelds auf das Verhältnis von Planung und Unternehmenserfolg. Für die erste Stufe der Untersuchung ( $\mathrm{t}_{1}$ : Oktober 1993 bis Februar 1995) werden deutsche 201 Unternehmen mit weniger als fünfzig Beschäftigten befragt, die zwischen 1990 und 1992 gegründet wurden. Dieselben Unternehmen werden in der zweiten Stufe ( $\mathrm{t}_{2}: 1997$ bis 1998) erneut befragt. An der zweiten Erhebung beteiligen sich 107 Unternehmen. Die Befragung erfolgt in beiden Zeitpunkten in kombinierter mündlicher und schriftlicher Form.

Die Planungsaktivitäten der Unternehmen werden auf Basis offener mündlicher Interviews ermittelt, die von zwei unabhängigen Gutachtern ausgewertet werden. In der ersten Untersuchungsstufe wird zunächst geprüft, ob eine Planung zum Zeitpunkt der Unternehmensgründung vorhanden ist. Für die Unternehmen, für die dies zutrifft, wird darüber hinaus ein Punktwert für die Qualität und den Detaillierungsgrad der Planung bestimmt. In der zweiten Untersuchungsstufe werden das Vorhandensein und die Qualität der aktuellen Planung auf einer dreistufigen Skala gemessen. Die Umweltbedingungen (Feindlichkeit und Unsicherheit des Wettbewerbsumfelds) werden mit einem selbst entwickelten Konstrukt gemessen. Zur Prüfung des Messinstruments werden in der zweiten Stufe der Untersuchung zusätzlich die Messkonstrukte von Miller und Droege (1986) und Khandwalla (1976) verwendet. Zur Messung des Erfolgs wird ein multiples Messkonstrukt verwendet, das Größe und Wachstum von Umsatz und Beschäftigtenzahl, das Einkommen des Unternehmers, seine Zufriedenheit und seine Selbsteinschätzung des Erfolgs beinhaltet. Als Kontrollvariablen werden außerdem die Höhe des Gründungskapitals, das Alter des Unternehmens sowie die Branche erhoben.

Als zentrale Hypothese der Untersuchung wird angenommen, dass in einem feindlichen Wettbewerbsumfeld eine positive Relation zwischen Planung und Erfolg besteht, während in einem nicht-feindlichen Wettbewerbsumfeld keine solche Beziehung bzw. sogar eine negative Beziehung besteht. 
Im ersten Schritt werden die Korrelationen zwischen den Variablen ermittelt. Hierbei zeigt sich, dass eine hohe Korrelation zwischen dem Erfolg in $t_{1}$ und Erfolg in $t_{2}$ bestehen. Weiterhin zeigt sich für $t_{1}$ ein direkter positiver Zusammenhang zwischen Planungsaktivitäten und Erfolg. Dieses Ergebnis kann für $\mathrm{t}_{2}$ jedoch nicht repliziert werden. Im Weiteren wird die formulierte Hypothese mit Hilfe einer multiplen Regressionsanalyse geprüft, bei der schrittweise zunächst die Kontrollvariablen, dann die Umweltbedingungen, folgend das Planungsverhalten und schließlich das Interaktionsverhalten von Umweltbedingungen und Planungsverhalten einbezogen wurden. Diese bestätigt die Hypothese der moderierenden Wirkung der Umweltbedingungen auf das Verhältnis von Planung und Erfolg weitgehend. Während der Zusammenhang bei feindlichen bzw. unsicheren Umweltbedingungen positiv ist, ist er in nicht-feindlichen bzw. sicheren Umweltbedingungen deutlich geringer bzw. sogar negativ. Weitergehend wird geprüft, ob Planung bei gegebenem Wettbewerbsumfeld eine positive Erfolgsentwicklung bewirkt. Zu diesem Zweck wird ein weiteres hierarchisches Regressionsmodell mit dem Erfolg in $t_{2}$ als abhängige Variable verwendet, das als unabhängige Variable den Erfolg in $t_{1}$ einbezieht. In zwei von sechs Fällen zeigt sich eine positive Wirkung der Planung bei feindlichen bzw. unsicheren Umweltbedingungen. Das Einbeziehen dieser Variable in das Modell kann die Erklärbarkeit der Varianz um 58\% erhöhen. In diesem Ergebnis sehen Rauch und Frese einen Hinweis auf die Kausalität im Verhältnis von Planung und Erfolg, da die Wirkung des früheren Erfolgs eliminiert wurde. Dies bedeutet, dass der Unternehmenserfolg in einem feindlichen bzw. unsicheren Wettbewerbsumfeld durch Planung verbessert werden kann.

\section{Mazzarol (2001)}

Die Untersuchung von Mazzarol (2001) befasst sich mit dem Zusammenhang zwischen formalen Plänen und Unternehmenserfolg kleiner Unternehmen. Eine Stichprobe von 55 australischen Kleinunternehmen verschiedener Branchen mit weniger als 100 Mitarbeitern wurde hierzu schriftlich befragt. Die Unternehmen werden danach gruppiert, ob sie über einen formalen Plan verfügen (51\% der Unternehmen) oder nicht (49\%). Die erste Gruppe der Unternehmen weist in absoluten Werten im Mittel höhere Umsätze, Umsatzzuwächse und Gewinne auf. Hieraus wird gefolgert, dass der Formalisierungsgrad der Planung mit zunehmender Größe des Unternehmens zunimmt. ${ }^{312}$

\footnotetext{
312 Unverständlich ist, warum die Untersuchung nur absolute und keine relativen Erfolgsindikatoren verwendet. Erstaunlicherweise zeigt sich nämlich, dass die (auf Basis der veröffentlichten Daten näherungsweise) ermittelte Umsatzrentabilität und das relative Umsatzwachstum bei Unternehmen ohne formale Planung deutlich besser sind.
} 


\section{Perry (2001)}

Perry untersucht den Zusammenhang zwischen schriftlicher Planung und dem Scheitern von Kleinunternehmen. Grundlage der Studie ist eine gepaarte Stichprobe US-amerikanischer Kleinunternehmen verschiedener Branchen mit weniger als 500 Mitarbeitern. Als ,gescheiterte“ Unternehmen werden solche bezeichnet, die Insolvenz angemeldet und einen Kreditausfall verursacht haben. Mit einer Stichprobe zufällig ausgewählter Unternehmen wird telefonisch Kontakt aufgenommen und um Teilnahme an der Untersuchung gebeten. Aus diesem Prozess ergibt sich eine Stichprobe mit 152 Unternehmen. Zu diesen gescheiterten Unternehmen wird nun mit Hilfe Kreditrating-Datenbank jeweils ein Unternehmen gesucht, das bezüglich Alter, Branche, Umsatz, Mitarbeiterzahl und Standort möglichst ähnlich ist, so dass sich eine gepaarte Stichprobe mit 152 Elementen ergibt. Der Median der Mitarbeiterzahl liegt in der Stichprobe bei 5 für die gescheiterten Unternehmen bzw. bei 4 für die nicht-gescheiterten Unternehmen.

Zur Messung der Planungsaktivitäten wird ein Fragebogen mit lediglich fünf Fragen verwendet. Zur Auswertung wird jede Ja-Antwort mit einem Punkt bewertet, so dass sich eine Skala von 0 bis 5 ergibt. Als erstes Ergebnis wird festgestellt, dass die Planungsaktivität in Kleinunternehmen in beiden Gruppen sehr gering ist. Die durchschnittliche Planungsaktivität in gescheiterten Unternehmen ist schwach signifikant höher als bei nicht-gescheiterten Unternehmen Das Planungsverhalten erklärt ca. 2-3\% der Varianz zwischen den beiden Gruppen. Interessante Ergebnisse ergeben sich jedoch bei weitergehenden Analysen. In beiden Gruppen zeigen sich Häufungen am unteren und oberen Ende der Skala. Dieses kann so interpretiert werden, dass man bei Geschäftsführern von Kleinunternehmen charakterisierend „Planer“ und „Nicht-Planer“ unterscheiden kann. Die Studie zeigt weiterhin, dass die Planungsaktivität mit steigender Mitarbeiterzahl zunimmt. Perry folgert, dass schriftliche Planungen unterhalb einer bestimmten Unternehmensgröße (ca. 5-15 Mitarbeiter) nicht durchgeführt werden und vermutlich auch kaum notwendig bzw. hilfreich sind. Er schlägt daher vor, zukünftige Studien zum Planungsverhalten auf Unternehmen mit mindestens 15 Mitarbeitern zu beschränken, da unterhalb dieser Grenze keine sinnvollen Ergebnisse zu erwarten sind.

\section{Gibson, Cassar und Wingham (2001)}

Gibson, Cassar und Wingham untersuchen in einer Langzeitstudie die Interdependenzen zwischen Planung und Erfolg in Kleinunternehmen. Diese basiert auf einer vom Australian Bureau of Statistics (ABS) durchgeführten Befragung von 2.956 australischen Unternehmen, die zu Beginn der Untersuchung weniger als 200 Mitarbeiter beschäftigten. Die Befragung wurde von 1995-1998 jährlich wiederholt. Es handelt sich somit um eine Studie, die sowohl aufgrund ihrer großen Stichprobe als auch aufgrund ihres Langzeitcharakters eine hohe Validität auf- 
weist. Als Erfolgsindikatoren dienen das Wachstum der Beschäftigtenzahl und des Umsatzes. Aufgrund ihrer Angaben zum Planungsverhalten wurden die Unternehmen in regelmäßig planende und regelmäßig nicht-planende Unternehmen differenziert. Zu ersteren werden diejenigen Unternehmen gezählt, die in allen Betrachtungsjahren angaben, eine formale Planung durchzuführen. Zu letzteren zählen diejenigen, die in keinem Jahr formal planten. Als "planning introducers“ werden diejenigen Unternehmen bezeichnet, die im Jahr $\mathrm{t}$ angaben, eine formale Planung durchzuführen, während sie dies im vorhergehenden Jahr t-1 nicht getan hatten. Alle Hypothesen werden mit vier unterschiedlichen statistischen Verfahren (Chi-Quadrat-Test, T-Test, Mann-Whitney-Test sowie multiple Regressionsanalyse) getestet, um eine Verzerrung aufgrund des statistischen Verfahrens auszuschließen.

Zunächst wird die grundlegende Hypothese überprüft, die annimmt, dass regelmäßig planende Unternehmen erfolgreicher sind als regelmäßig nicht-planende Unternehmen. Zur Überprüfung der Hypothese werden die vier genannten Verfahren für beide Erfolgsindikatoren für alle Zeitpunkte bzw. Zeiträume angewendet. Bei nahezu allen Test zeigen sich signifikante Unterschiede zwischen den beiden Gruppen, so dass die Hypothese als bestätigt angesehen wird.

Aufgrund der Bestätigung ersten Hypothese kann somit von einem positiven Zusammenhang zwischen Planung und Erfolg ausgegangen werden. In der weiteren Untersuchung wird der Frage nach der Kausalität der Beziehung nachgegangen, d. h. es wird untersucht, ob die Planung erfolgsfördernd wirkt oder ob Erfolg eine Ausdehnung der Planungstätigkeiten ermöglicht. Hierzu wird der Erfolg der Unternehmen, die formale Planung eingeführt haben, mit dem Erfolg derjenigen, die zum gleichen Zeitpunkt keine Planung eingeführt haben, verglichen. Dem traditionellen Verständnis folgend, dass Planung erfolgsfördernd ist, wird als Hypothese formuliert, dass sich der Erfolg in Unternehmen, die eine formalen Planung einführen, in den Folgeperioden besser entwickelt als in Unternehmen, die dies nicht tun. Auch diese Hypothese wird mit den vier genannten Tests für beide Erfolgsgrößen und alle Zeitpunkte überprüft. Jedoch ergibt sich nur bei einem der insgesamt zweiunddreißig Tests ein leicht signifikanter Zusammenhang, so dass die Hypothese verworfen wird. Es wird nun geprüft, ob für die umgekehrte Wirkungsrichtung ein Nachweis gefunden werden kann. Die Hypothese hierzu lautet, dass Unternehmen, die eine formale Planung eingeführt haben, zuvor bessere Erfolgswerte aufweisen konnten als Unternehmen, die dies nicht getan haben. Es wird wiederum das gleiche Testverfahren verwendet. Es ergeben sich deutliche Hinweise, dass der Einführung formaler Planungen ein Wachstum in der Beschäftigtenzahl vorausgeht. Die Ergebnisse bezüglich des Umsatzwachstums sind nicht eindeutig, tendieren jedoch in die gleiche Richtung.

Die Studie unterstützt die bereits mehrfach nachgewiesene positive Beziehung zwischen Planung und Unternehmenserfolg. Jedoch finden sich deutliche Widersprüche zur traditionellen Annahme, dass der unternehmerische Erfolg durch 
formale Planung unterstützt wird. Vielmehr finden sich klare Hinweise, dass der entgegengesetzte Wirkungszusammenhang vorliegt. Somit könne die generelle Forderung von wissenschaftlicher Seite, dass Kleinunternehmen formale Planung einsetzen sollten, nicht mehr aufrechterhalten werden.

\section{Wijewardena et al. (2004)}

Wijewardena et al. untersuchen die Auswirkungen des Detaillierungsgrads von Budgetplanung und -kontrolle auf die Umsatzentwicklung in kleinen- und mittelständischen Unternehmen. Die Studie basiert auf einer Stichprobe von 168 Unternehmen aus Sri Lanka, die in der verarbeitenden Industrie tätig sind und weniger als 300 Beschäftigte haben. Auf Basis der Befragung werden die Unternehmen zunächst in drei Kategorien entsprechend des Detaillierungsgrads ihrer Budgetplanung (keine schriftlich fixierte Budgets, einfache schriftlich fixierte Budgets, detaillierte schriftliche Budgets) eingeteilt. Die Unternehmen der zweiten und dritten Kategorie werden entsprechend ihrer Budgetkontrolle in drei weitere Klassen eingeteilt (keine Abweichungsanalysen, gelegentliche Nutzung von Abweichungsanalysen, regelmäßige Nutzung von Abweichungsanalysen). Als Erfolgsindikator dient die Umsatzentwicklung des letzten Jahres, die ebenfalls von der Geschäftsführung erhoben wird. Diese wird zum einen quantitativ (relative Veränderung des Umsatzes) zum anderen - bei den Unternehmen, die zu einer Quantifizierung nicht bereit sind in ihrer Tendenz gemessen. Zur Auswertung der Daten werden Chi-QuadratTests sowie Varianzanalysen eingesetzt. Die Studie kann sowohl für den Detaillierungsgrad der Budgetplanung als auch für den Detaillierungsgrad der Budgetkontrolle eine positive Erfolgswirkung nachweisen.

\subsection{Zusammenfassung der Ergebnisse}

In Abbildung 64 werden die Darstellungen des vorhergehenden Abschnitts noch einmal tabellarisch zusammengefasst. Die in der letzten Spalte der Tabelle angegebene Tendenz bezieht sich auf das grundsätzliche Ergebnis der Untersuchung. Alle Studien, in denen keinerlei Zusammenhang zwischen Planung und Unternehmenserfolg gefunden werden konnte, werden mit „,-“ gekennzeichnet. Diejenigen Studien, in denen mindestens für bestimmte Planungselemente oder für eine Erfolgsdimension ein Zusammenhang festgestellt wurde, werden mit „,"“ gekennzeichnet. 


\begin{tabular}{|c|c|c|c|c|c|c|c|}
\hline Studie & $\begin{array}{l}\text { Stichproben- } \\
\text { größe }\end{array}$ & $\begin{array}{l}\text { Branche } \\
\text { (Land) }\end{array}$ & $\begin{array}{l}\text { Verwendente } \\
\text { Abgrenzung }\end{array}$ & Methodik & $\begin{array}{l}\text { Untersuchungs- } \\
\text { schwerpunkt }\end{array}$ & Wichtigste Erkenntnisse & $\begin{array}{l}\text { Ten- } \\
\text { denz }\end{array}$ \\
\hline 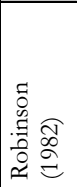 & $\begin{array}{l}101 \text { (sowie } \\
\text { zwei ähnlich } \\
\text { große Kon- } \\
\text { trollgruppen) }\end{array}$ & $\begin{array}{l}\text { Verschie- } \\
\text { dene Bran- } \\
\text { chen (USA) }\end{array}$ & $\begin{array}{l}\text { Weniger als } 50 \\
\text { Beschäftigte und } \\
\text { weniger als } 3 \\
\text { Mio. US\$ Um- } \\
\text { satz }\end{array}$ & $\begin{array}{l}\text { Experi- } \\
\text { ment }\end{array}$ & $\begin{array}{l}\text { Einfluss und Erfolgs- } \\
\text { wirkung externer Bera- } \\
\text { ter für strategische } \\
\text { Planung in Kleinunter- } \\
\text { nehmen }\end{array}$ & $\begin{array}{l}\text { Durch externe Beratung bei } \\
\text { Planungsaktivitäten können } \\
\text { Umsatz und Ertragskraft in } \\
\text { höherem Maß gesteigert } \\
\text { werden als ohne diese Un- } \\
\text { terstützung möglich ist. }\end{array}$ & + \\
\hline 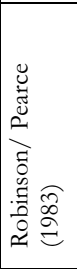 & 85 & $\begin{array}{l}\text { Banken } \\
\text { (USA) }\end{array}$ & $\begin{array}{l}\text { Einlagen kleiner } \\
\text { als } 25 \text { Mio. US\$ }\end{array}$ & $\begin{array}{l}\text { Schriftli- } \\
\text { che und } \\
\text { mündli- } \\
\text { che Be- } \\
\text { fragung }\end{array}$ & $\begin{array}{l}\text { Einfluss des Formali- } \\
\text { sierungsgrads der stra- } \\
\text { tegischen Planung auf } \\
\text { die Ertragslage kleiner } \\
\text { Banken }\end{array}$ & $\begin{array}{l}\text { Eine positive Wirkung eines } \\
\text { hohen Formalisierungsgrad } \\
\text { der strategischen Planung } \\
\text { auf den Erfolg kann nicht } \\
\text { nachgewiesen werden. } \\
\text { Dennoch ist Planung (in } \\
\text { nicht-formaler Form) auch } \\
\text { für kleine Organisationen } \\
\text { von Bedeutung. }\end{array}$ & - \\
\hline 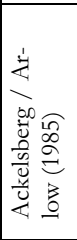 & 135 & $\begin{array}{l}\text { Verschie- } \\
\text { dene Bran- } \\
\text { chen (USA) }\end{array}$ & $\begin{array}{l}\text { Entsprechend } \\
\text { der Definition } \\
\text { der U. S. Small } \\
\text { Business Ad- } \\
\text { minstration }\end{array}$ & $\begin{array}{l}\text { Schriftli- } \\
\text { che Be- } \\
\text { fragung }\end{array}$ & $\begin{array}{l}\text { Zusammenhang zwi- } \\
\text { schen Planung und } \\
\text { Unternehmenserfolg in } \\
\text { Kleinunternehmen }\end{array}$ & $\begin{array}{l}\text { Der Zusammenhang zwi- } \\
\text { schen Planung und Unter- } \\
\text { nehmenserfolg ist sehr kom- } \\
\text { plex. } \\
\text { Die analytische Planungsdi- } \\
\text { mension ist von größerer } \\
\text { Bedeutung als die formale } \\
\text { Planungsdimension. }\end{array}$ & + \\
\hline 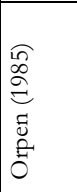 & 58 & \begin{tabular}{|l|} 
Verschie- \\
dene Bran- \\
chen (Aust- \\
ralien)
\end{tabular} & keine Angabe & $\begin{array}{l}\text { Schriftli- } \\
\text { che Be- } \\
\text { fragung }\end{array}$ & $\begin{array}{l}\text { Auswirkungen langfris- } \\
\text { tiger Planung auf fi- } \\
\text { nanziellen Erfolg klei- } \\
\text { ner Unternehmen }\end{array}$ & $\begin{array}{l}\text { Für den Erfolg ist weniger } \\
\text { der zeitliche Aufwand für } \\
\text { langfristige Planung als viel- } \\
\text { mehr ihre Qualität relevant. }\end{array}$ & + \\
\hline 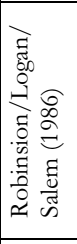 & 81 & $\begin{array}{l}\text { Einzelhan- } \\
\text { del (USA) }\end{array}$ & $\begin{array}{l}\text { Entsprechend } \\
\text { der Definition } \\
\text { der U. S. Small } \\
\text { Business Ad- } \\
\text { minstration }\end{array}$ & $\begin{array}{l}\text { Schriftli- } \\
\text { che Be- } \\
\text { fragung }\end{array}$ & $\begin{array}{l}\text { Zusammenhang zwi- } \\
\text { schen operativer Pla- } \\
\text { nung und Unterneh- } \\
\text { menserfolg kleiner } \\
\text { Einzelhandelsunter- } \\
\text { nehmen }\end{array}$ & $\begin{array}{l}\text { Unternehmen mit breiter } \\
\text { operativer Planung sind } \\
\text { erfolgreicher als solche mit } \\
\text { geringeren operativen Pla- } \\
\text { nungsaktivitäten. } \\
\text { Strategische Planung allein } \\
\text { zeigt keinen Einfluss auf den } \\
\text { Unternehmenserfolg. } \\
\end{array}$ & + \\
\hline 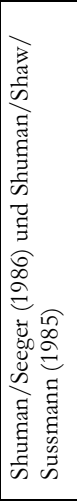 & 220 & $\begin{array}{l}\text { Verschie- } \\
\text { dene Bran- } \\
\text { chen (USA) }\end{array}$ & $\begin{array}{l}\text { Umsatz zwi- } \\
\text { schen } 100.000 \\
\text { und } 25 \text { Mio. } \\
\text { US\$ }\end{array}$ & $\begin{array}{l}\text { Schriftli- } \\
\text { che Be- } \\
\text { fragung }\end{array}$ & $\begin{array}{l}\text { Unterschiede zwischen } \\
\text { normativen Modellen } \\
\text { der strategischen Pla- } \\
\text { nung und deren prakti- } \\
\text { sche Umsetzung in } \\
\text { kleinen, schnell wach- } \\
\text { senden Unternehmen }\end{array}$ & $\begin{array}{l}\text { Die in der Literatur genann- } \\
\text { ten Elemente der strategi- } \\
\text { schen Planung finden sich } \\
\text { auch in kleinen Unterneh- } \\
\text { men wieder. } \\
\text { Ein signifikanter Zusam- } \\
\text { menhang zwischen dem } \\
\text { Planungsprozess und dem } \\
\text { Wachstum des Umsatzes } \\
\text { bzw. des Umsatzes pro Mit- } \\
\text { arbeiter kann nicht gefunden } \\
\text { werden. } \\
\text { Freie Ressourcen sind eine } \\
\text { intervenierende Variable des } \\
\text { Verhältnisses zwischen Pla- } \\
\text { nung und Unternehmenser- } \\
\text { folg. }\end{array}$ & |- \\
\hline
\end{tabular}




\begin{tabular}{|c|c|c|c|c|c|c|c|}
\hline Studie & $\begin{array}{l}\text { Stichproben- } \\
\text { größe }\end{array}$ & $\begin{array}{l}\text { Branche } \\
\text { (Land) }\end{array}$ & \begin{tabular}{|l|} 
Verwendente \\
Abgrenzung \\
\end{tabular} & Methodik & \begin{tabular}{|l|}
$\begin{array}{l}\text { Untersuchungs- } \\
\text { schwerpunkt }\end{array}$ \\
\end{tabular} & Wichtigste Erkenntnisse & $\begin{array}{l}\text { Ten- } \\
\text { denz }\end{array}$ \\
\hline 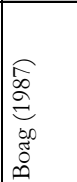 & 20 & $\begin{array}{l}\text { High Tech } \\
\text { (Kanada) }\end{array}$ & $\begin{array}{l}\text { Mehr als } 15 \text { und } \\
\text { weniger als } 150 \\
\text { Beschäftigte }\end{array}$ & $\begin{array}{l}\text { Mündli- } \\
\text { che Be- } \\
\text { fragung }\end{array}$ & $\begin{array}{l}\text { Einfluss des Marketing- } \\
\text { Controlling auf den } \\
\text { Markterfolg junger } \\
\text { Wachstumsunterneh- } \\
\text { men }\end{array}$ & $\begin{array}{l}\text { Bestimmte Elemente eines } \\
\text { formalen Steuerungssystems } \\
\text { könnten das Wachstum in } \\
\text { Kleinunternehmen begünsti- } \\
\text { gen. }\end{array}$ & + \\
\hline 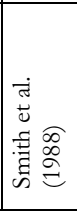 & 20 & $\begin{array}{l}\text { Elektroin- } \\
\text { dustrie } \\
\text { (USA) }\end{array}$ & $\begin{array}{l}\text { Kleine Unter- } \\
\text { nehmen: } 2-50 \\
\text { Beschäftigte, } \\
\text { große Unter- } \\
\text { nehmen: } 100- \\
2350 \text { Beschäftig- } \\
\text { te }\end{array}$ & $\begin{array}{l}\text { Mündli- } \\
\text { che Be- } \\
\text { fragung }\end{array}$ & $\begin{array}{l}\text { Unterschiede im Ent- } \\
\text { scheidungsverhalten } \\
\text { von Unternehmern in } \\
\text { kleineren Firmen und } \\
\text { professionellen Mana- } \\
\text { gern in größeren Fir- } \\
\text { men }\end{array}$ & $\begin{array}{l}\text { Unabhängig von der Unter- } \\
\text { nehmensgröße sind analyti- } \\
\text { sche Entscheidungen nicht- } \\
\text { analytischen überlegen. }\end{array}$ & + \\
\hline 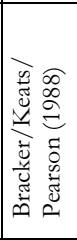 & 97 & $\begin{array}{l}\text { Elektroin- } \\
\text { dustrie } \\
\text { (USA) }\end{array}$ & $\begin{array}{l}\text { Weniger als } 100 \\
\text { Beschäftigte }\end{array}$ & $\begin{array}{l}\text { Schriftli- } \\
\text { che Be- } \\
\text { fragung }\end{array}$ & $\begin{array}{l}\text { Zusammenhang zwi- } \\
\text { schen dem Entwick- } \\
\text { lungsgrad des Pla- } \\
\text { nungssystems und dem } \\
\text { finanziellen Erfolg von } \\
\text { kleinen Unternehmen } \\
\text { in Wachstumsbranchen }\end{array}$ & $\begin{array}{l}\text { Strukturiert strategisch pla- } \\
\text { nende Unternehmen sind } \\
\text { erfolgreicher als Unterneh- } \\
\text { men mit weniger entwickel- } \\
\text { ten Planungssystemen. } \\
\text { Bei Unternehmen mit langer } \\
\text { Planungstradition verstärkt } \\
\text { sich der vorgenannte Effekt. }\end{array}$ & + \\
\hline 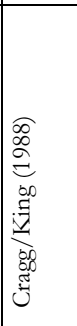 & 157 & $\begin{array}{l}\text { Metall- } \\
\text { verarbei- } \\
\text { tende In- } \\
\text { dustrie } \\
\text { (UK) }\end{array}$ & $\begin{array}{l}\text { Weniger als } 50 \\
\text { Beschäftigte }\end{array}$ & $\begin{array}{l}\text { Schriftli- } \\
\text { che Be- } \\
\text { fragung }\end{array}$ & $\begin{array}{l}\text { Zusammenhang zwi- } \\
\text { schen organisatori- } \\
\text { schen Charakteristika } \\
\text { und finanziellem Erfolg } \\
\text { in Kleinunternehmen } \\
\text { unter Berücksichtigung } \\
\text { intermediärer Faktoren }\end{array}$ & $\begin{array}{l}\text { Ein positiver Zusammen- } \\
\text { hang zwischen Planung und } \\
\text { Unternehmenserfolg kann } \\
\text { nicht nachgewiesen werden. } \\
\text { Zukünftige Studien müssen } \\
\text { zum einen Korrelationen } \\
\text { zwischen Einflussfaktoren, } \\
\text { zum anderen die Wirkungs- } \\
\text { richtungen der Zusammen- } \\
\text { hänge stärker berücksichti- } \\
\text { gen. }\end{array}$ & - \\
\hline 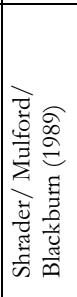 & 115 & $\begin{array}{l}\text { Verschie- } \\
\text { dene Bran- } \\
\text { chen (USA) }\end{array}$ & $\begin{array}{l}\text { Mehr als } 10 \text { und } \\
\text { weniger als } 100 \\
\text { Beschäftigte }\end{array}$ & $\begin{array}{l}\text { Schriftli- } \\
\text { che Be- } \\
\text { fragung }\end{array}$ & $\begin{array}{l}\text { Zusammenhang zwi- } \\
\text { schen operativer bzw. } \\
\text { strategischer Planung } \\
\text { und Unternehmenser- } \\
\text { folg }\end{array}$ & $\begin{array}{l}\text { Operative Planung wird } \\
\text { häufiger verwendet als stra- } \\
\text { tegische. } \\
\text { Eine eindeutig positive Wir- } \\
\text { kung von Planung auf Un- } \\
\text { ternehmenserfolg kann nicht } \\
\text { nachgewiesen werden. } \\
\text { Auf operativer Ebene ist die } \\
\text { Absatzplanung am wichtigs- } \\
\text { ten. }\end{array}$ & + \\
\hline 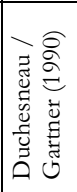 & 26 & $\begin{array}{l}\text { Frischsaft- } \\
\text { distributi- } \\
\text { onsbranche } \\
\text { (USA) }\end{array}$ & $\begin{array}{l}\text { Umsatz zwi- } \\
\text { schen } 200.000 \\
\text { und } 6 \text { Mio. US\$ }\end{array}$ & $\begin{array}{l}\text { Mündli- } \\
\text { che und } \\
\text { schriftli- } \\
\text { che Be- } \\
\text { fragung }\end{array}$ & $\begin{array}{l}\text { Identifikation von Fak- } \\
\text { toren für den Erfolg } \\
\text { junger Unternehmen }\end{array}$ & $\begin{array}{l}\text { Strukturiertheit der Planung, } \\
\text { hoher zeitlicher Aufwand für } \\
\text { Planung und die Breite der } \\
\text { Planung wirken positiv auf } \\
\text { den Erfolg junger Unter- } \\
\text { nehmen. }\end{array}$ & + \\
\hline
\end{tabular}




\begin{tabular}{|c|c|c|c|c|c|c|c|}
\hline Studie & $\begin{array}{l}\text { Stichproben- } \\
\text { größe }\end{array}$ & \begin{tabular}{|l}
$\begin{array}{l}\text { Branche } \\
\text { (Land) }\end{array}$ \\
\end{tabular} & \begin{tabular}{|l} 
Verwendente \\
Abgrenzung \\
\end{tabular} & Methodik & $\begin{array}{l}\text { Untersuchungs- } \\
\text { schwerpunkt }\end{array}$ & Wichtigste Erkenntnisse & $\begin{array}{l}\text { Ten- } \\
\text { denz }\end{array}$ \\
\hline 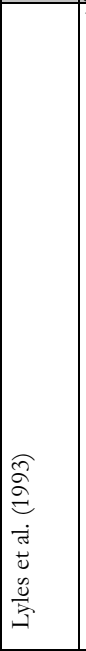 & 188 & $\begin{array}{l}\text { Verschie- } \\
\text { dene Bran- } \\
\text { chen (USA) }\end{array}$ & $\begin{array}{l}\text { Weniger als } 500 \\
\text { Beschäftigte, } \\
\text { mindestens } \\
1 \text { Mio. US\$ Um- } \\
\text { satz }\end{array}$ & $\begin{array}{l}\text { Mündli- } \\
\text { che Be- } \\
\text { fragung }\end{array}$ & $\begin{array}{l}\text { Zusammenhang zwi- } \\
\text { schen Formalisierungs- } \\
\text { grad, Inhalten und } \\
\text { Entscheidungsprozes- } \\
\text { sen in der strategischen } \\
\text { Planung kleiner Unter- } \\
\text { nehmen }\end{array}$ & $\begin{array}{l}\text { Der Grad der Betonung } \\
\text { einzelner Element des stra- } \\
\text { tegischen Planungsprozesses } \\
\text { ist in formal planenden } \\
\text { Kleinunternehmen höher als } \\
\text { in nicht-formal planenden. } \\
\text { Formal planende Kleinun- } \\
\text { ternehmen berücksichtigen } \\
\text { ein breiteres Spektrum an } \\
\text { Strategiealternativen als } \\
\text { nicht-formal planende. } \\
\text { Bezüglich Eigen- und Ge- } \\
\text { samtkapitalrentabilität zeigen } \\
\text { sich keine Unterschiede } \\
\text { zwischen formal und nicht- } \\
\text { formal planenden Kleinun- } \\
\text { ternehmen. } \\
\text { Formal planende Kleinun- } \\
\text { ternehmen weisen ein höhe- } \\
\text { res Umsatzwachstum als } \\
\text { nicht-formal planende Un- } \\
\text { ternehmen auf. }\end{array}$ & + \\
\hline 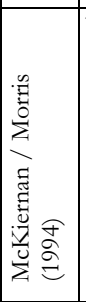 & 100 & $\begin{array}{l}\text { Verschie- } \\
\text { dene Bran- } \\
\text { chen (UK) }\end{array}$ & $\begin{array}{l}\text { Weniger als } 500 \\
\text { Beschäftigte }\end{array}$ & $\begin{array}{l}\text { Schriftli- } \\
\text { che Be- } \\
\text { fragung } \\
\text { und Se- } \\
\text { kundära- } \\
\text { nalyse } \\
\text { von Un- } \\
\text { terneh- } \\
\text { mens- } \\
\text { daten }\end{array}$ & $\begin{array}{l}\text { Zusammenhang zwi- } \\
\text { schen Formalisierungs- } \\
\text { grad der strategischen } \\
\text { Planung und dem lang- } \\
\text { fristigen finanziellen } \\
\text { Erfolg von Kleinunter- } \\
\text { nehmen }\end{array}$ & $\begin{array}{l}\text { Ein Zusammenhang zwi- } \\
\text { schen dem Formalisierungs- } \\
\text { grad der strategischen Pla- } \\
\text { nung und dem finanziellen } \\
\text { Erfolg der Unternehmen ist } \\
\text { nicht feststellbar. }\end{array}$ & - \\
\hline 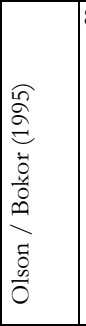 & 89 & $\begin{array}{l}\text { Verschie- } \\
\text { dene Bran- } \\
\text { chen (USA) }\end{array}$ & $\begin{array}{l}\text { Entsprechend } \\
\text { der Definition } \\
\text { der U. S. Small } \\
\text { Business Ad- } \\
\text { minstration }\end{array}$ & $\begin{array}{l}\text { Schriftli- } \\
\text { che Be- } \\
\text { fragung }\end{array}$ & $\begin{array}{l}\text { Wechselbeziehung } \\
\text { zwischen Prozess und } \\
\text { Inhalt strategischer } \\
\text { Planung und dessen } \\
\text { Wirkung auf das Um- } \\
\text { satzwachstum in Start- } \\
\text { Up-Unternehmen }\end{array}$ & $\begin{array}{l}\text { Wechselbeziehung zwischen } \\
\text { Prozess und Inhalt strategi- } \\
\text { scher Planung haben Ein- } \\
\text { fluss auf das Umsatzwachs- } \\
\text { tum in Start-Up- } \\
\text { Unternehmen. } \\
\text { Es wird vermutet, dass ande- } \\
\text { re Kontextfaktoren Einfluss } \\
\text { auf die Erfolgswirksamkeit } \\
\text { der Wechselbeziehung aus- } \\
\text { üben. }\end{array}$ & + \\
\hline 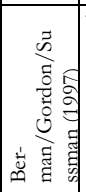 & 161 & \begin{tabular}{|l|} 
Verschie- \\
dene Bran- \\
chen (USA)
\end{tabular} & keine Angabe & $\begin{array}{l}\text { Schriftli- } \\
\text { che Be- } \\
\text { fragung }\end{array}$ & $\begin{array}{l}\text { Nutzen höher entwi- } \\
\text { ckelter Planungssyste- } \\
\text { me für das Umsatz- } \\
\text { wachstum in Kleinun- } \\
\text { ternehmen }\end{array}$ & $\begin{array}{l}\text { Es existiert eine signifikante } \\
\text { Korrelation zwischen der } \\
\text { Entwicklungsstufe des Pla- } \\
\text { nungssystems und dem Um- } \\
\text { satzwachstum. }\end{array}$ & + \\
\hline 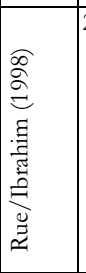 & 253 & $\begin{array}{l}\text { keine An- } \\
\text { gaben (U- } \\
\text { SA) }\end{array}$ & $\begin{array}{l}\text { Mehr als } 15 \text { und } \\
\text { weniger als } 500 \\
\text { Beschäftigte }\end{array}$ & $\begin{array}{l}\text { Schriftli- } \\
\text { che Be- } \\
\text { fragung }\end{array}$ & $\begin{array}{l}\text { Auswirkungen des } \\
\text { Ausbaugrads des Pla- } \\
\text { nungssystems auf den } \\
\text { Erfolg kleiner Unter- } \\
\text { nehmen }\end{array}$ & $\begin{array}{l}\text { Es existiert ein Zusammen- } \\
\text { hang zwischen Ausbaugrad } \\
\text { des Planungssystems und } \\
\text { Umsatzwachstum. } \\
\text { Ein Zusammenhang zwi- } \\
\text { schen Ausbaugrad des Pla- } \\
\text { nungssystems und Return on } \\
\text { Investment wird dagegen } \\
\text { nicht nachgewiesen. }\end{array}$ & + \\
\hline
\end{tabular}




\begin{tabular}{|c|c|c|c|c|c|c|c|}
\hline \begin{tabular}{|l|} 
Studie \\
\end{tabular} & $\begin{array}{l}\text { Stichproben- } \\
\text { größe }\end{array}$ & $\begin{array}{l}\text { Branche } \\
\text { (Land) }\end{array}$ & $\begin{array}{l}\text { Verwendente } \\
\text { Abgrenzung }\end{array}$ & \begin{tabular}{|l|} 
Methodik \\
\end{tabular} & $\begin{array}{l}\text { Untersuchungs- } \\
\text { schwerpunkt }\end{array}$ & Wichtigste Erkenntnisse & $\begin{array}{l}\text { Ten- } \\
\text { denz }\end{array}$ \\
\hline 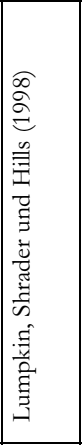 & 94 & $\begin{array}{l}\text { Verschie- } \\
\text { dene Bran- } \\
\text { chen (USA) }\end{array}$ & keine Angabe & $\begin{array}{l}\text { Schriftli- } \\
\text { che Be- } \\
\text { fragung }\end{array}$ & $\begin{array}{l}\text { Zusammenhang zwi- } \\
\text { schen formaler Planung } \\
\text { in verschiedenen Funk- } \\
\text { tionsbereichen und } \\
\text { verschiedenen Erfolgs- } \\
\text { dimensionen in Klein- } \\
\text { unternehmen }\end{array}$ & $\begin{array}{l}\text { Junge Unternehmen: } \\
\text { schwach signifikanter positi- } \\
\text { ver Zusammenhang zwi- } \\
\text { schen Finanzplanung einer- } \\
\text { seits und Finanzkraft und } \\
\text { Rohertrag andererseits, für } \\
\text { Wettbewerberanalysen dage- } \\
\text { gen negativ signifikanter } \\
\text { Zusammenhang. } \\
\text { Etablierte Unternehmen: } \\
\text { Wettbewerberanalysen sind } \\
\text { das einzige Planungsgebiet, } \\
\text { das positiv mit einer Er- } \\
\text { folgsdimension (Finanzkraft) } \\
\text { assoziiert ist. }\end{array}$ & + \\
\hline 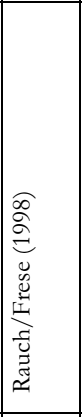 & 107 & $\begin{array}{l}\text { Verschie- } \\
\text { dene Bran- } \\
\text { chen } \\
\text { (Deutsch- } \\
\text { land) }\end{array}$ & $\begin{array}{l}\text { Weniger als } 50 \\
\text { Beschäftigte }\end{array}$ & \begin{tabular}{|l|} 
Mündli- \\
che und \\
schriftli- \\
che Be- \\
fragung, \\
Erhe- \\
bung zu \\
zwei \\
Zeit- \\
punkten \\
im Ab- \\
stand von \\
vier Jah- \\
ren \\
\end{tabular} & $\begin{array}{l}\text { Moderierende Wirkung } \\
\text { des Wettbewerbsum- } \\
\text { felds auf das Verhältnis } \\
\text { von Planung und Un- } \\
\text { ternehmenserfolg }\end{array}$ & $\begin{array}{l}\text { In einem feindlichen Wett- } \\
\text { bewerbsumfeld besteht eine } \\
\text { positive Relation zwischen } \\
\text { Planung und Erfolg, wäh- } \\
\text { rend in einem nicht- } \\
\text { feindlichem Wettbewerbs- } \\
\text { umfeld keine solche Bezie- } \\
\text { hung bzw. sogar eine negati- } \\
\text { ve Beziehung besteht. } \\
\text { Eine kausale Wirkung von } \\
\text { Planung auf den Erfolg kann } \\
\text { ebenso bestätigt werden. }\end{array}$ & + \\
\hline 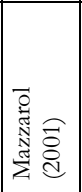 & 55 & $\begin{array}{l}\text { Verschie- } \\
\text { dene Bran- } \\
\text { chen (Aust- } \\
\text { ralien) }\end{array}$ & $\begin{array}{l}\text { Weniger als } 100 \\
\text { Beschäftigte }\end{array}$ & \begin{tabular}{l|} 
Schriftli- \\
che Be- \\
fragung
\end{tabular} & $\begin{array}{l}\text { Zusammenhang zwi- } \\
\text { schen formalen Busi- } \\
\text { ness Plänen und Un- } \\
\text { ternehmenserfolg klei- } \\
\text { ner Unternehmen }\end{array}$ & $\begin{array}{l}\text { Der Formalisierungsgrad der } \\
\text { Planung steigt mit zuneh- } \\
\text { mender Unternehmensgröße }\end{array}$ & + \\
\hline 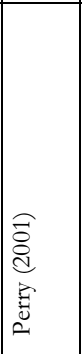 & $2 \times 152$ & $\begin{array}{l}\text { Verschie- } \\
\text { dene Bran- } \\
\text { chen (USA) }\end{array}$ & $\begin{array}{l}\text { Weniger als } 500 \\
\text { Beschäftigte }\end{array}$ & \begin{tabular}{|l|} 
Telefoni- \\
sche \\
Befra- \\
gung
\end{tabular} & $\begin{array}{l}\text { Zusammenhang zwi- } \\
\text { schen schriftlicher Pla- } \\
\text { nung und Scheitern } \\
\text { von Kleinunternehmen }\end{array}$ & $\begin{array}{l}\text { Die Planungsaktivtät ist in } \\
\text { Kleinunternehmen generell } \\
\text { gering. } \\
\text { Die Planungsaktivtät ist in } \\
\text { gescheiterten niedriger als in } \\
\text { nicht-gescheiterten Unter- } \\
\text { nehmen. } \\
\text { Schriftliche Planung sind in } \\
\text { Kleinunternehmen erst ab } \\
\text { einer Größe von ca. 5-15 } \\
\text { Mitarbeiter notwendig bzw. } \\
\text { nützlich. }\end{array}$ & + \\
\hline
\end{tabular}




\begin{tabular}{|c|c|c|c|c|c|c|c|}
\hline Studie & $\begin{array}{l}\begin{array}{l}\text { Stichproben- } \\
\text { größe }\end{array} \\
\end{array}$ & \begin{tabular}{|l}
$\begin{array}{l}\text { Branche } \\
\text { (Land) }\end{array}$ \\
\end{tabular} & \begin{tabular}{|l} 
Verwendente \\
Abgrenzung \\
\end{tabular} & Methodik & $\begin{array}{l}\text { Untersuchungs- } \\
\text { schwerpunkt }\end{array}$ & Wichtigste Erkenntnisse & \begin{tabular}{|l} 
Ten- \\
denz
\end{tabular} \\
\hline 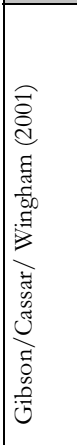 & 2.956 & $\begin{array}{l}\text { Verschie- } \\
\text { dene Bran- } \\
\text { chen (Aust- } \\
\text { ralien) }\end{array}$ & $\begin{array}{l}\text { Weniger als } 200 \\
\text { Beschäftigte }\end{array}$ & $\begin{array}{l}\text { Schriftli- } \\
\text { che Be- } \\
\text { fragung }\end{array}$ & $\begin{array}{l}\text { Interdependenzen zwi- } \\
\text { schen Planung und } \\
\text { Erfolg in Kleinunter- } \\
\text { nehmen }\end{array}$ & $\begin{array}{l}\text { Formale Planung und } \\
\text { Wachstum des Umsatzes } \\
\text { und der Beschäftigtenanzahl } \\
\text { weisen einen positiven Zu- } \\
\text { sammenhang auf. } \\
\text { Die vermutete Wirkungs- } \\
\text { richtung, dass formale Pla- } \\
\text { nung erfolgsfördernd wirkt, } \\
\text { kann nicht bestätigt werden. } \\
\text { Die entgegengesetzte Wir- } \\
\text { kungsrichtung (mit zuneh- } \\
\text { mendem Wachstum nimmt } \\
\text { der Umfang formaler Pla- } \\
\text { nung zu) wird dagegen in der } \\
\text { Tendenz bestätigt. }\end{array}$ & + \\
\hline 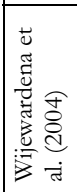 & 168 & $\begin{array}{l}\text { Verarbei- } \\
\text { tende In- } \\
\text { dustrie (Sri } \\
\text { Lanka) }\end{array}$ & $\begin{array}{l}\text { Weniger als } 300 \\
\text { Beschäftigte }\end{array}$ & $\begin{array}{l}\text { Schriftli- } \\
\text { che Be- } \\
\text { fragung }\end{array}$ & \begin{tabular}{|l|} 
Auswirkungen des \\
Detaillierungsgrads von \\
Budgetplanung und \\
-kontrolle auf die Um- \\
satzentwicklung
\end{tabular} & $\begin{array}{l}\text { Sowohl bei Planung als auch } \\
\text { bei Kontrolle zeigen sich } \\
\text { positive Wirkungen auf den } \\
\text { Umsatz. }\end{array}$ & + \\
\hline
\end{tabular}

Abbildung 64: Übersicht über empirische Untersuchungen zum Verhältnis von Planung und Erfolg in Kleinunternehmen

\subsection{Analyse und Diskussion der Ergebnisse der Studien}

Die Darstellung der Studien, die sich mit dem Verhältnis von Planung und Unternehmenserfolg in Kleinunternehmen befassen, illustriert die Komplexität dieses Forschungsgebiets in mehrfacher Hinsicht. Im Folgenden werden gemeinsame Tendenzen der Untersuchungen herausgestellt sowie die in den Studien am häufigsten berücksichtigten Einflussfaktoren auf das Verhältnis von Planung und Erfolg analysiert. Hierbei handelt es sich um die Unternehmensgröße, die Branche, das Wettbewerbsumfeld sowie die Charakteristika des Unternehmers.

Grundsätzlich lässt sich festhalten, dass die Mehrheit der Studien einen positiven Zusammenhang zwischen Planung und Erfolg konstatiert. Die in der normativen Literatur i. d. R. pauschal unterstellte positive Erfolgswirkung der Planung und die daraus resultierende uneingeschränkte Empfehlung eines möglichst intensiven Einsatzes von Planungsinstrumenten muss jedoch relativiert werden. Es ist anzuerkennen, dass Planung in diesem Umfeld nicht unbedingt in formaler Form durchgeführt werden muss. Dies gilt insbesondere in Kleinstunternehmen mit weniger als 15 Mitarbeitern. Dieses Ergebnis steht in Einklang mit der theoretischen Literatur zur Planung, die das Entstehen von formalisieren Planungssystemen auf die wachsende Komplexität des Unternehmens als Organisationssystem zurückführt. Dort wo die Koordination durch persönliche Weisung an ihre Grenzen stößt, wird sie durch Pläne als Koordinationsmechanismus substituiert. 
Allgemein zeigen die empirischen Untersuchungen, dass das tatsächliche Verhältnis durch eine Reihe von Faktoren beeinflusst wird. Generell festgestellt werden muss außerdem, dass die Kausalität in Relation von Planung und Unternehmenserfolg nicht eindeutig ist. Als weitgehend gesicherte Erkenntnis kann angenommen werden, dass der Umfang und der Formalisierungsgrad mit steigender Größe eines Unternehmens zunehmen. Dies lässt vermuten, dass sich Planung und Unternehmenserfolg gegenseitig bedingen, d. h. Kausalitäten in beiden Richtungen vorliegen. Während der Unternehmenserfolg von einer Vielzahl von Einflussfaktoren abhängig und somit nicht direkt beeinflussbar ist, ist die Ausgestaltung der Planung durch die Handelnden im Unternehmen direkt steuerbar. Daher muss das Interesse von Forschung und Praxis primär in der folgenden Wirkungsrichtungen liegen: Es interessiert die Frage, wie bzw. unter welchen Bedingungen Planung positiv auf den Erfolg eines Kleinunternehmens wirkt. Im Folgenden wird diese Frage auf Basis der Ergebnisse der dargestellten Studien analysiert.

Die 23 dargestellten empirischen Untersuchungen kommen zu unterschiedlichen Ergebnissen. Während in fünf Untersuchungen kein Zusammenhang zwischen Planung und Erfolg beobachtet werden kann, liefern 18 eine zumindest partielle Unterstützung der Hypothese. Erstere werden im Nachfolgenden als Untersuchungen mit „,negativem Ergebnis“ bezeichnet, letztere als Untersuchungen mit „positivem Ergebnis“. Es ist zu vermuten, dass die Abweichungen in den Ergebnissen auf bestimmte inhaltliche oder formale Unterschiede in den Untersuchungen zurückzuführen sind. Dieses wird im Folgenden geprüft.

Eine Grundannahme der hier vorliegenden Ausarbeitung ist es, dass die Bedeutung der Planung von der Unternehmensgröße abhängig ist. Damit ist auch anzunehmen, dass auch innerhalb der Gruppe der Kleinunternehmen größenabhängig differenziert werden muss. Die Studien verwenden unterschiedliche Kriterien zur Abgrenzung des Begriffs Kleinunternehmen, wodurch die Unternehmen in den analysierten Stichproben deutliche Größenunterschiede aufweisen. Während die Unternehmen in der Untersuchung von Duchesneau und Gartner (1990) beispielsweise im Mittel 6,5 Mitarbeiter aufweisen, beschäftigen die von Smith et al. (1988) untersuchten Unternehmen durchschnittlich 342 Mitarbeiter.

Es ist zu vermuten, dass für Untersuchungen, bei denen Unternehmen mit weniger Beschäftigten analysiert werden, die Wahrscheinlichkeit geringer ist, einen Zusammenhang zwischen Planung und Erfolg festzustellen. Um dies zu prüfen, bietet es sich an, die Mittelwerte der Beschäftigtenzahl der Stichprobenunternehmen in Studien mit positivem mit denen mit negativem Ergebnis zu vergleichen. Da ein großer Teil der Untersuchungen keine detaillierten Angaben zu den Unternehmen der Stichprobe macht, ist ein solcher Vergleich nicht möglich. Von den Untersuchungen mit negativem Ergebnis geben lediglich Shuman und Seeger (1986) die durchschnittliche Mitarbeiterzahl der Unternehmen in der Stichprobe an. Sie beträgt in diesem Fall 22. Von den 18 Untersuchungen mit positivem Er- 
gebnis geben elf die durchschnittlichen Mitarbeiter der Unternehmen in der Stichprobe an. Der Mittelwert beträgt für diese Gruppe 62,5. Eine begründete Schlussfolgerung ist somit aufgrund der Datenlage auf Metaebene nicht möglich.

Eine Reihe von Studien kann jedoch bestätigen, dass der Formalisierungsgrad der Planung mit steigender Unternehmensgröße zunimmt (vgl. z. B. Boag 1987, Mazzarol 2001). In diesem Zusammenhang ist insbesondere noch einmal auf ein Ergebnis von Perry (2001) zu verweisen. Er folgert, dass schriftliche Planungen unterhalb einer Unternehmensgröße von 5-15 Mitarbeitern nicht durchgeführt werden und vermutlich auch kaum notwendig bzw. hilfreich sind. Er schlägt daher vor, Studien zum Planungsverhalten auf Unternehmen mit mindestens 15 Mitarbeitern zu beschränken, da unterhalb dieser Grenze keine sinnvollen Ergebnisse zu erwarten sind. Relativiert werden muss diese Aussage jedoch vor dem Hintergrund des Ergebnisses von Smith et al. (1988), die feststellen, dass analytische Entscheidungen intuitiven Entscheidungen unabhängig von der Unternehmensgröße überlegen sind.

Häufig berücksichtigt wird außerdem der Einfluss der Branche. Sowohl bei der Betrachtung einzelner Untersuchungen, in denen Branchenvergleiche durchgeführt wurden (vgl. Ackelsberg / Arlow 1985, Shrader / Mulford / Blackburn 1989, McKiernan / Morris 1994, Berman / Gordon / Sussman 1997), als auch beim Vergleich zwischen Untersuchungen, die auf eine einzelne Branche begrenzt sind (vgl. Robinson / Pearce 1983, Robinsion / Logan / Salem 1986, Boag 1987, Smith et al. 1988, Bracker / Keats / Pearson 1988, Cragg / King 1988, Duchesneau / Gartner 1990, Wijewardena et al. 2004), zeigt sich jedoch kein Hinweis auf einen Einfluss der Branche auf das Verhältnis von Planung und Erfolg. Positiv formuliert bedeutet dies, dass aus den vorliegenden Studien geschlossen werden muss, dass der Einfluss der Planung auf den Erfolg in allen Branchen tendenziell gleich hoch ist.

Ein weiterer potenzieller Einflussfaktor auf das Verhältnis von Planung und Erfolg ist das Wettbewerbsumfeld des Kleinunternehmens. Während Shrader, Mulford und Blackburn (1989) feststellen, dass Unternehmen bei zunehmender Umweltunsicherheit ihre operative und strategische Planungsintensität erhöhen, gelingt es Rauch und Frese (1998) den moderierenden Effekt des Wettbewerbsumfelds auf Verhältnis von Planung und Erfolg explizit nachzuweisen. Die Erfolgswirkung der Planung wirkt umso stärker, je größer die Unsicherheit der Unternehmensumwelt.

Häufig wird außerdem ein moderierender Einfluss des Unternehmertyps vermutet. Dieser wird untersucht von Smith et al. (1988), Bracker, Keats und Pearson (1988) sowie von Cragg und King (1988). Ein signifikanter Einfluss wird jedoch in keiner der Studien festgestellt. 


\section{Fazit}

Die Gründung eines Unternehmens ist immer mit einem besonderen Risiko verbunden. Bezogen auf die Praxis führt insbesondere das Beispiel der ,geplatzten Internetblase“ zur Annahme, dass Controllingsysteme notwendig sind, um Unternehmen mit innovativen Geschäftsmodellen zielgerichtet steuern zu können. Für die Forschung in diesem Bereich ergeben sich drei Bezugspunkte: das Controlling als funktionale Sicht, die TIME-Branchen als branchenbezogene Sicht sowie StartUp-Unternehmen als organisationsbezogene Sicht. Es zeigt sich, dass es notwendig ist, sich zunächst nicht speziell auf die TIME-Branche zu beschränken, sondern zunächst im Allgemeinen die Problemstellung des Controlling in Start-UpUnternehmen zu untersuchen, weil selbst dieser weiter gefassten Fragestellung in der Forschung erst seit kurzer Zeit explizit Aufmerksamkeit gewidmet wird. Somit befasst sich die vorliegende Untersuchung mit einer grundlegenden Fragestellung des Themengebiets „Controlling in Start-Up-Unternehmen“. Es wird der Frage nachgegangen, ob bzw. in welcher Form und unter welchen Bedingungen Planung einen Beitrag zum Unternehmenserfolg in Start-Up-Unternehmen leistet. Das Ziel dieses Beitrags ist die Aufarbeitung von Untersuchungen, die einen empirisch fundierten Beitrag zur Beantwortung dieser Frage leisten.

Zur Begründung der Notwendigkeit, Controlling in Start-Up-Unternehmen zu untersuchen, wird insbesondere auf die Arbeiten von Achleitner und Bassen (2002) verwiesen. Diese entwickeln aus den charakteristischen Merkmalen junger Wachstumsunternehmen einen Anforderungskatalog für das Controlling in diesem Unternehmen. In diesem Katalog wird ein wesentliches Strukturmerkmal jedoch nicht explizit berücksichtigt: Start-Up-Unternehmen sind - auch wenn sie schnell wachsen - Kleinunternehmen. Geht man von der Feststellung aus, dass die betriebswirtschaftliche Forschung zum Controlling in Start-Up-Unternehmen noch wenig entwickelt ist, erscheint jedoch ein Blick in eng verwandte betriebswirtschaftliche Themengebiete nahe liegend. Auch wenn Unterschiede zwischen Kleinunternehmen und Start-Up-Unternehmen zu berücksichtigen sind, ist eine Isolierung beider Bereiche nicht zu rechtfertigen.

Im Hauptteil des Beitrags werden 23 empirische Untersuchungen dargestellt, die sich mit dem Zusammenhang von Planung und Erfolg in Kleinunternehmen befassen. Die hohe Komplexität und Vielschichtigkeit der Fragestellung zeigt sich u. a. in der Vielfalt der Untersuchungsansätze. Da sowohl die Planung als auch der Erfolg mehrdimensionale Konstrukte sind, ist auch ihr Zusammenhang sehr vielschichtig. Entsprechend sind auch die Ergebnisse der untersuchten Studien sehr heterogen. Trotz partieller Widersprüchlichkeiten lassen sich bestimmte empirisch gestützte Erkenntnisse festhalten: 
- Planung findet grundsätzlich auch in Kleinunternehmen statt. Sie weist jedoch i. d. R. einen wesentlich geringeren Formalisierungsgrad auf als in der Literatur häufig gefordert wird.

- Die geringere Formalisierung ist jedoch nicht unbedingt auf fehlendes Know-How oder mangelnde Ressourcen zurückzuführen, sondern scheint dem Kontext angepasst. Die Untersuchungen zeigen, dass ein geringerer Formalisierungsgrad von Planung kein Erfolgshemmnis für Kleinunternehmen ist.

- Weiterhin zeigt sich, dass Planung besonders bei hoher Umweltunsicherheit erfolgskritisch ist.

- Besonders wichtig scheint dagegen die analytische Dimension der Planung, die insbesondere dazu dient, Handlungsoptionen des Unternehmens zu identifizieren und zu bewerten.

Diese Ergebnisse lassen sich grundsätzlich auch auf den speziellen Untersuchungsbereich - das Controlling innovativer Geschäftsmodelle in den TIME-Branchen - übertragen. Insbesondere die letzten beiden Erkenntnisse sind hierbei von zentraler Bedeutung. Geht man für diese konvergierenden Branchen von einer hohen Unsicherheit der Umwelt aus, unterstreicht das Ergebnis dieser Untersuchung die vielfach erhobene Forderung nach einer Intensivierung des Controlling in diesem Umfeld. Das letzt genannte Ergebnis - die Bedeutung der analytischen Dimension der Planung - kann als Leitgedanke weiterer konzeptioneller Forschung im betrachteten Gebiet dienen. Als konkretes Forschungsziel ergibt sich die Aufgabe, Methoden zu konzipieren, mit denen Handlungsoptionen für Unternehmen der TIME-Branchen systematisch identifiziert und bewertet werden können.

\section{Literatur}

Achleitner / Bassen (2002): Achleitner, A., Bassen, A.: Controllingsysteme in jungen Wachstumsuntemehmen - terra incognita. In: Betriebs-Berater, 57, S. 1192-1197.

Achleitner / Bassen (2003): Achleitner, A., Bassen, A. (Hrsg.): Controlling von jungen Unternehmen, Stuttgart.

Ackelsberg / Arlow (1985): Ackelsberg, R., Arlow, P.: Small Businesses do plan and it pays off. In: Long Range Planning, 18, S. 61-67.

Berman / Gordon / Sussman (1997): Berman, J. A., Gordon, D. D., Sussman, G.: A study to determine the benefits small business firms derive from sophistication planning versus less sophisticated types of planning. in: Journal of Business and Economic Studies, 3, S. 1-11. 
Bassen / Gröne (2003): Bassen, A., Gröne, F.: Controlling von Start-up-Unternehmen aus der Perspektive von Vernture-Capital-Gesellschaften. In: Controlling 15, S. 293-299.

Boag (1987): Boag, D. A.: Marketing control and performance in early growth companies. In: Journal of Business Venturing, 3, S. 365-379.

Bracker / Keats / Pearson (1988): Bracker, J. S., Keats, B. W., Pearson, J. N.: Planning and financial performance among small firms in a growth industry. In: Strategic Management Journal 9, S. 591-603.

Cragg / King (1988): Cragg, P. B., King, M.: Organizational Characteristics and small firms' performance revisited. In: Entrepreneuship Theory and Practice, 13, S. 4964.

Duchesneau / Gartner (1990): Duchesneau, D. A., Gartner, W. B.: A profile of new venture success and failure in an emerging industry. In: Journal of Business Venturing, 5, S. 297-312.

Eschenbach / Niedermayr (1996): Eschenbach, R., Niedermayr, R.: Die Konzeption des Controlling. In: Eschenbach, R. (Hrsg.): Controlling, 2. Aufl., Stuttgart 1996, S. 65-93.

Fredrickson / Mitchell (1984): Fredrickson, J. W., Mitchell, T. R.: Strategic decision process: comprehensiveness and performance in a unstable industry. In: Academy of Management Journal, 27, S. 399-423.

Filley / Aldag (1978): Filley, A. C., Aldag, R. J.: Characteristics and measurement of an organization typology. in: Academy of Management Journal, 31, S. 578-591.

Gibson / Cassar / Wingham (2001): Gibson, B., Cassar, G., Wingham, D.: Longitudinal analysis of relationship between planning and performance in small Australian firms. Paper presented at the 2001 USASBE/SBIDA Annual National Conference, Orlando.

Harbert (1982): Harbert, L.: Controlling-Begriffe und Controlling-Konzeptionen: eine kritische Betrachtung des Entwicklungsstandes des Controlling und Möglichkeiten seiner Fortentwicklung, Bochum.

Horváth (1979): Horváth, P.: Controlling, München.

Kazanjian (1988): Kazanjian, R.: Relation of dominant problems to stages of growth in technology-based new ventures. In: Academy of Management Journal, 31, S. 257-279.

Khandwalla (1976): Khandwalla, P. N.: Some top management styles, their context and performance. In: Organization and Administrative Science, 7, S. 21-51.

Kieser / Kubicek (1992): Kieser, A., Kubicek, H.: Organisation, 3. Aufl., Berlin u. a.

Küpper (1995): Küpper, H.: Controlling: Konzeption, Aufgaben und Instrumente, Stuttgart. 
Küpper / Weber / Zünd (1990): Küpper, H., Weber, J., Zünd, A.: Zum Verständnis und Selbstverständnis des Controlling: Thesen zur Konsensbildung. In: ZfB, 74, S. 281-293.

Lumpkin / Shrader / Hills (1998): Lumpkin, G. T., Shrader, R. C., Hills, G. E.: Does formal business planning enhance the performance of new ventures? Paper presented at the 1998 Babson Entrepreneurship Research Conference, Gent.

Lyles / Baird / Orris / Kuratko (1993): Lyles, M. A., Baird, I. S., Orris, J. B., Kuratko, D. F.: Formalized planning in small business: increasing strategic choices. In: Journal of Small Business Management, 31, S. 38-50.

Mazzarol (2001): Mazzarol, T.: Do formal business plans really matter? A survey of small business owners in Australia. In: Small Enterprise Research, 9, S. 32-45.

McKiernan / Morris (1994): McKiernan, P./Morris, C.: Strategic planning and financial performance in UK SMEs: Does formality matter? In: British Journal of Management, 5 (Special Issue), S. S31-S41.

Miller / Droege (1986): Miller, D./Droege, C.: Psychological and traditional determinants of structure. In: Administrative Science Quarterly 31, S. 539-560.

Müller (1974): Müller, W.: Die Koordination von Informationsbedarf und Informationsbeschaffung als Zentrale Aufgabe des Controlling. In: ZfbF, 27, S. 683-693.

Olson / Bokor (1995): Olson, P. D./Bokor, D. W.: Strategy process-content interaction: effects on growth performance in small, start-up firms. In: Journal of Small Business Management, 33, S. 34-43.

Orpen (1985): Orpen, C.: The effects of long-range planning on small business performance: a further examination. In: Journal of Small Business Management, 23, S. $16-23$.

Pietsch / Scherm (2000): Pietsch, G., Scherm, E.: Die Präzisierung des Controlling als Führungs- und Führungsunterstützungsfunktion. In: Die Unternehmung, 54, S. 395-411.

Pietsch / Scherm (2000a): Pietsch, G., Scherm, E.: Controlling - Rationalitätssicherung versus Führungs- und Führungsunterstützungsfunktion. In: Die Unternehmung, 55, S. 81-84.

Pietsch / Scherm (2001): Pietsch, G., Scherm, E.: Die Reflexionsaufgabe im Zentrum des Controlling. In: krp (2001), S. 307-313.

Rauch / Frese (1998): Rauch, A., Frese, M.: A contingency approach to small scale business success: a longitudinal study on the effects of environmental hostility an uncertainty on the relationship of planning and success, Paper presented at the 1998 Babson Entrepreneurship Research Conference, Gent.

Reichmann (1985): Reichmann, T.: Controlling mit Kennzahlen: Grundlagen einer systemgestützten Controlling-Konzeption, München.

Robinson (1982): Robinson, R. B.: The importance of "outsiders" in small firm strategic planning. In: Academy of Management Journal, 25, S. 80-93. 
Robinson / Logan / Salem (1986): Robinson, B. R., Logan, J. E., Salem, M. Y.: Strategic versus operational planning in small retail firms. In: American Journal of Small Business, 11, S. 7-16.

Robinson / Pearce (1983): Robinson, R. B./Pearce, J. A.: The impact of formalized planning on financial performance in small organization. In: Strategic Management Journal 4 (3), S. 197-207.

Rue / Ibrahim (1998): Rue, L. W., Ibrahim, N. A.: The relationship between planning sophistication and performance in small businesses. In: Journal of Small Business Management, 36, S. 24-32.

Schäffer / Weber (2003): Schäffer, U., Weber, J.: Thesen zum Controlling (II), CCMForschungspapier Nr. 8, Vallendar.

Schwenk / Shrader (1993): Schwenk, C. R., Shrader, C. B.: Effects of Formal Strategic Planning on Financial Performance in Small Firms: A Meta-Analysis. in: Entrepreneuship Theory and Practice 17, S. 53-64.

Shrader / Mulford / Blackburn (1989): Shrader, C. B., Mulford, C. L., Blackburn, V. L.: Strategic and operational planning, uncertainty, and performance in small firms. In: Journal of Small Business Management, 27, S. 46-60.

Shuman / Seeger (1986): Shuman, J. C., Seeger, J. A.: The theory and practice of strategic management in smaller rapid growth firms. In: American Journal of Small Business, 11 (Summer), S. 7-18.

Shuman / Shaw / Sussman (1985): Shuman, J. C., Shaw, J. J., Sussman, G.: Strategic planning in smaller rapid growth companies. In: Long Range Planning, 18, S. 4853.

Smith / Gannon / Grimm / Mitchell (1988): Smith, K. G., Gannon, M. T., Grimm, C., Mitchell, T. R.: Decision making behavior in smaller entrepreneurial and larger professionally managed firms. In: Journal of Business Venturing, 3, S. 223 232.

United States Small Business Adminstration (USSBA) (2004): Small Business Size Standards matched to North American Industry Classification System, http://www.sba.gov/size/sizetable2002.html, Abruf: 2005-01-10.

Weber (2000): Weber, J.: Neue Perspektiven des Controlling, WHU-Forschungspapier Nr. 77, Vallendar.

Weber (2003): Weber, J.: Controlling in unterschiedlichen Führungskontexten - ein Überblick. in: Controlling \& Management, 47, S. 183-192.

Weber / Kunz (2003): Weber, J., Kunz, J.: Empirische Controllingforschung: Begründung - Beispiele - Ergebnisse, Wiesbaden.

Weber / Schäffer (1999): Weber, J., Schäffer, U.: Rationalitätssicherung in der Willensbildung durch die Nutzung des fruchtbaren Spannungsverhältnisses von Reflexion und Intuition. In: Zeitschrift für Planung, 10, S. 205-244. 
Weber / Schäffer (2001): Weber, J./Schäffer, U.: Controlling als Rationalitätssicherung der Führung. In: Die Unternehmung, 55, S. 75-79.

Weber / Schäffer / Langenbach (1999): Weber, J., Schäffer, U., Langenbach, W.: Gedanken zur Rationalitätskonzeption des Controlling, WHUForschungspapiere Nr. 70, Vallendar.

Welsh / White (1981): Welsh, J. A., White, J. F.: A small business is not a little big business. In: Harvard Business Review, 59, July/August, S. 18-32.

Wijewardena / de Zoysa / Fonseka / Perera (2004): Wijewardena, H., de Zoysa, A., Fonseka, T., Perera, B.: The impact of planning and control sophistication on performance of small and medium-sized enterprises: evidence from Sri Lanka. In: Journal of Small Business Management, 42, S. 209-217.

Wood / LaForge (1981): Wood, D. R., LaForge, R. L.: Toward the planning scale: an example from the banking industry. In: Strategic Management Journal, 2, S. 209219.

Zenz (1999): Zenz, A.: Strategisches Qualitätscontrolling: Konzeption als Metaführungsfunktion, Wiesbaden. 


\section{Autoren}

\section{Bargheer, Margo, M.A.}

Jahrgang 1963, Magister der Sozial- und Medienwissenschaften mit den Schwerpunkten „Qualitative Sozialforschung“ und „Angewandte Medienforschung“. Seit 2002 an der Niedersächsischen Staats- und Universitätsbibliothek Göttingen für den Universitätsverlag und im Bereich des elektronischen Publizierens zuständig. Lehrtätigkeit zu Dokumentenservern, Informations- und Medienkompetenz sowie zum elektronischen Publizieren an der FH Köln, der Bibliotheksschule München sowie der Georg-August-Universität.

bargheer@mail.sub.uni-goettingen.de • http://www.univerlag.uni-goettingen.de

\section{Dorschel, Joachim, Dipl.-Jur.}

Jahrgang 1978, Studium der Rechtswissenschaft, von 1999 - 2003 in Freiburg und Tübingen; Wissenschaftlicher Mitarbeiter am Lehrstuhl für Bürgerliches Recht, Handels- und Wirtschaftsrecht, Multimedia- und Telekommunikationsrecht, Rechtsvergleichung der Universität Göttingen, seit 2004 Doktorand bei Prof. Dr. Gerald Spindler, Thema der Dissertation „Auskunftsansprüche gegen InternetService-Provider".

http://lehrstuhl.jura.uni-goettingen.de/gspindler/ 


\section{Hanekop, Heidemarie, Dipl.-Sozialwirtin}

Jahrgang 1953, Studium der Sozialwissenschaften, von 1983-85 wissenschaftliche Mitarbeiterin am Institut für Publizistik und Kommunikationswissenschaften an der Universität Göttingen; seit 1986 wissenschaftliche Mitarbeiterin am Soziologischen Forschungsinstitut, Forschungsprojekte im Themenfeld IT, neue Medien, Internet, Innovationsforschung.

hhaneko@sofi.uni-goettingen.de• http://www.sofi-goettingen.de

\section{Hess, Thomas, Prof. Dr.}

Jahrgang 1967, Studium der Wirtschaftsinformatik an der TU Darmstadt, Promotion zum Dr. oec. an der Universität St. Gallen/Schweiz in 1995 inkl. Forschungsaufenthalt an der Nanyang University of Singapore, Vorstandsassistent bei Bertelsmann in 1996 und 1997, Arbeitsgruppenleiter am Institut für Wirtschaftsinformatik der Universität Göttingen von 1998-2001, im Sommersemester 2001 Lehrstuhlvertretung an der Universität Augsburg, Habilitation an der Universität Göttingen in 2001, Berufung zum C4-Professor und Direktor des Instituts für Wirtschaftsinformatik und Neue Medien der Ludwig-Maximilians-Universität München in 2001, Mitherausgeber der Zeitschrift für Controlling und Management, der MedienWirtschaft und des International Journal on Media Management, Koordinator des Münchner Forschungsschwerpunkts intermedia, des ZIM und Mitglied im CDTM.

thess@bwl.uni-muenchen.de • http://www.wim.bwl.uni-muenchen.de

\section{Kaspar, Christian, Dipl.-Kfm.}

Jahrgang 1976, Studium der Betriebswirtschaftslehre, seit 2002 wissenschaftlicher Mitarbeiter und Doktorand am Institut für Wirtschaftsinformatik an der Universität Göttingen und in der Forschungsgruppe Konvergente Märkte der Internetökonomie. Titel der Doktorarbeit: Individualisierung und mobile Dienste in der Medienbranche.

ckaspar@uni-goettingen.de • http://wi2.wiso.uni-goettingen.de

\section{Lanfer, Carmen, M.A.}

Jahrgang 1975, Studium der Soziologie und Deutschen Philologie an den Universitäten Göttingen und Bristol/GB, Abschluss im Jahr 2002; seitdem wissenschaftliche Mitarbeiterin am Soziologischen Forschungsinstitut (SOFI) an der Georg- 
August-Universität Göttingen; Forschungsschwerpunkte: Entwicklung der Informationsgesellschaft, Weiterbildungsforschung; Promotionsprojekt am SOFI zu den Auswirkungen der Internetökonomie auf Arbeit und Beschäftigung in der Medienbranche.

clanfer@gwdg.de • http://www.sofi.uni-goettingen.de

\section{Marquardsen Kai M.A.}

Jahrgang 1974, Studium der Soziologie, Wirtschafts- und Sozialpsychologie und Medien- und Kommunikationswissenschaften, 2003-2005 studentische Hilfskraft und wissenschaftlicher Mitarbeiter am Soziologischen Forschungsinstitut (SOFI) an der Georg-August-Universität Göttingen; Forschungsschwerpunkte: Auswirkung des Internet auf Arbeit und Beschäftigung in der Medienbranche, Entgrenzung von Arbeit; Promotionsprojekt zum Wandel von Arbeit und Beschäftigung im Zeichen der aktivierenden Arbeitsmarktpolitik.

ka.mar@stud.uni-goettingen.de • http:// www.sofi.uni-goettingen.de

\section{Mönch, Matthias, Dipl.-Jur.}

Jahrgang 1978, Studium der Rechtswissenschaft, von 1999 - 2004 in Göttingen; Wissenschaftlicher Mitarbeiter am Lehrstuhl für Bürgerliches Recht, Handels- und Wirtschaftsrecht, Multimedia- und Telekommunikationsrecht, Rechtsvergleichung der Universität Göttingen, seit Oktober 2004 Doktorand bei Prof. Dr. Gerald Spindler, Thema der Dissertation „Rechtliche Aspekte von Dialern und Mehrwertdiensten".

Matthias.Moench@jur.uni-goettingen.de •

http://lehrstuhl.jura.uni-goettingen.de/gspindler/

\section{Ortelbach, Björn, Dipl.-Wirtsch.-Inf.}

Jahrgang 1978, Studium der Wirtschaftsinformatik an der Universität Göttingen sowie der National University of Ireland, Galway, seit April 2004 wissenschaftlicher Mitarbeiter und Doktorand am Institut für Wirtschaftsinformatik an der Universität Göttingen in der Forschungsgruppe Konvergente Märkte der Internetökonomie. Arbeitstitel der Doktorarbeit: Controlling in der Fachverlagsbranche.

bortelb2@uni-goettingen.de • http://wi2.wiso.uni-goettingen.de 


\section{Rauscher, Barbara, Dipl.-Kffr.}

Jahrgang 1978, Studium der Betriebswirtschaftslehre an der Ludwig-MaximiliansUniversität München und der Universidad de Castilla - La Mancha, Toledo, Spanien, seit 2004 wissenschaftliche Mitarbeiterin am Institut für Wirtschaftsinformatik und Neue Medien an der Ludwig-Maximilians-Universität München.

brauscher@bwl.uni-muenchen.de • http://www.wim.bwl.uni-muenchen.de

\section{Riedel, André, Dipl.-Math.}

Jahrgang 1976, Studium der Mathematik und Informatik an der Westfälischen Wilhelms-Universität Münster, seit 2002 wissenschaftlicher Mitarbeiter am Institut für Informatik an der Universität Göttingen.

riedel@informatik.uni-goettingen.de • http://www.tmg.informatik.uni-goettingen.de

\section{Roosendaal, Hans E., Prof. Dr.}

Jahrgang 1947, Studium der Physik, Promotion 1974, von 1974-1983 wissenschaftlicher Mitarbeiter an der Universität Bielefeld,. Managementpositionen bei Elsevier 1983-1998, Professor of Scientific Information at the University of Twente in the Netherlands.

h.e.roosendaal@utwente.nl • http: www.scineo.de

\section{Seidenfaden, Lutz, Dipl.-Wirtsch.-Inf.}

Jahrgang 1978, Studium der Wirtschaftsinformatik, seit 2004 wissenschaftlicher Mitarbeiter am Institut für Wirtschaftsinformatik an der Universität Göttingen. Tätig in der Forschungsgruppe Konvergente Märkte der Internetökonomie.

lseiden@uni-goettingen.de • http://wi2.wiso.uni-goettingen.de

\section{Zibull, Marco, Dipl.-Inf.}

Jahrgang 1976, Studium der Informatik an der Technischen Universität Clausthal, seit 2002 wissenschaftlicher Mitarbeiter am Institut für Informatik an der Universität Göttingen.

zibull@informatik.uni-goettingen.de• http://www.tmg.informatik.uni-goettingen.de. 


\section{Buch- und Reihenherausgeber}

\section{Hagenhoff, Svenja, Dr.}

Jahrgang 1971, Studium der Betriebswirtschaftslehre, von 1997-2002 wissenschaftliche Mitarbeiterin am Institut für Wirtschaftsinformatik an der Universität Göttingen, 2001 Promotion zum Dr. rer. pol., seit September 2002 wissenschaftliche Assistentin am Institut für Wirtschaftsinformatik an der Universität Göttingen, Leiterin der Forschungsgruppen Konvergente Märkte der Internetökonomie und Wissens- und Bildungsmanagement, seit Wintersemester 2004 / 2005 Lehrstuhlvertretung an der Universität Hildesheim.

shagenh@uni-goettingen.de • http://www.wi2.wiso.uni-goettingen.de

\section{Hogrefe, Dieter, Prof. Dr.}

Jahrgang 1958, Studium der Informatik und Mathematik an der Universität Hannover, 1986 Promotion zum Dr. rer. nat., Wissenschaftlicher Assistent im Bereich Software Engineering und verteilte Systeme an der Universität Hamburg, 1989 Habilitation in Informatik, 1989-1995 Professur für angewandte Computerwissenschaften an der Universität Bern, 1995-2002 Direktor des Instituts für Telematik der Universität Lübeck, seit 2002 Lehrstuhlinhaber (C4) am Institut für Informatik und Leiter des Zentrums für Informatik an der Universität Göttingen.

hogrefe@informatik.uni-goettingen.de • http://www.tmg.uni-goettingen.de 


\section{Mittler, Elmar, Prof. Dr.}

Jahrgang 1940, Studium der Germanistik und Geschichte, Professur für Buchwissenschaften an der Universität Mainz, außerdem am Seminar für Mittlere und Neue Geschichte der Georg-August-Universität Göttingen tätig. 1971-74 Stellvertretender Leiter der Universitätsbibliothek Freiburg i.Br., 1974-79 Direktor der Badischen Landesbibliothek, 1979-1990 Direktor der Universitätsbibliothek Heidelberg, seit 1990 Direktor der Niedersächsischen Staats- und Universitätsbibliothek Göttingen. Elmar Mittler ist zudem federführend in nationalen und internationalen Gremien und Initiativen tätig, die sich um den Fortschritt der wissenschaftlichen Informationsversorgung, der digitalen Bibliothek und des elektronischen Publizierens bemühen.

mittler@mail.sub.uni-goettingen.de • http:// www.sub.uni-goettingen.de

\section{Schumann, Matthias, Prof. Dr.}

Jahrgang 1959, Studium der Betriebswirtschaftslehre an der Universität Göttingen. Wissenschaftlicher Mitarbeiter am Lehrstuhl für Betriebswirtschaftslehre, insbesondere Wirtschaftsinformatik an der Universität Erlangen-Nürnberg. Postdoctoral Fellow am IBM Scientific Center Los Angeles, Akademischer Rat an der Wirtschafts- und Sozialwissenschaftlichen Fakultät an der Universität ErlangenNürnberg. Professor für Wirtschaftsinformatik, Leiter der Abteilung Wirtschaftsinformatik II, Geschäftsführender Leiter des Instituts für Wirtschaftsinformatik und Leiter des Rechenzentrums der Wirtschaftswissenschaftlichen und Sozialwissenschaftlichen Fakultäten. Von April 2001 bis März 2005 Vizepräsident der Universität Göttingen.

mschuma1@uni-goettingen.de• http://www.wi2.wiso.uni-goettingen.de

\section{Spindler, Gerald, Prof. Dr.}

Jahrgang 1960, Dipl.-Ökonom, hat Rechtswissenschaften und Wirtschaftswissenschaften in Frankfurt aM, Hagen, Genf und Lausanne studiert und beide Staatsexamina absolviert. Nach einer Assistentenzeit bei Prof. Dr. Mertens, Frankfurt, sowie am Institut für Internationales und Ausländisches Wirtschaftsrecht, Frankfurt, promovierte er 1993 mit einer rechtsvergleichenden Arbeit über das Thema "Recht und Konzern". Die Habilitation erfolgte 1996 mit einer Arbeit über Unternehmens-Organisationspflichten, wobei ihm die Lehrbefugnis für Bürgerliches Recht, Handels- und Wirtschaftsrecht, Internationales Privatrecht, Rechtsvergleichung und Arbeitsrecht verliehen wurde. Herr Spindler ist seit 1997 nach Ablehnung von Rufen an die Universitäten zu Köln und Bielefeld Ordinarius für Bür- 
gerliches Recht, Handels- und Wirtschaftsrecht, Rechtsvergleichung, Multimediaund Telekommunikationsrecht an der Universität Göttingen.

lehrstuhl.spindler@jura.uni-goettingen.de

http://www.lehrstuhl-spindler.uni-goettingen.de

\section{Wittke, Volker, Prof. Dr.}

Jahrgang 1957, Studium der Sozialwissenschaften, von 1985-1986 wissenschaftlicher Mitarbeiter am Soziologischen Seminar der Universität Göttingen, 1986-1996 wissenschaftlicher Mitarbeiter am Soziologischen Forschungsinstitut, Promotion zum disc. pol., seit 1996 Direktor des Soziologischen Forschungsinstitutes, Habilitation 2004, seit 2005 Professor am Institut für Soziologie der Universität Göttingen.

vwittke@sofi-goettingen.de • http://www.sofi-goettingen.de 